\title{
A base modulated synthesis of indoles and quinolines, an expedient synthesis of salviadione, and chemoselective couplings en route to indoles and pyrroloindoles
}

Matthew Marshall Cummings

West Virginia University

Follow this and additional works at: https://researchrepository.wvu.edu/etd

\section{Recommended Citation}

Cummings, Matthew Marshall, "A base modulated synthesis of indoles and quinolines, an expedient synthesis of salviadione, and chemoselective couplings en route to indoles and pyrroloindoles" (2013). Graduate Theses, Dissertations, and Problem Reports. 388.

https://researchrepository.wvu.edu/etd/388

This Dissertation is protected by copyright and/or related rights. It has been brought to you by the The Research Repository @ WVU with permission from the rights-holder(s). You are free to use this Dissertation in any way that is permitted by the copyright and related rights legislation that applies to your use. For other uses you must obtain permission from the rights-holder(s) directly, unless additional rights are indicated by a Creative Commons license in the record and/ or on the work itself. This Dissertation has been accepted for inclusion in WVU Graduate Theses, Dissertations, and Problem Reports collection by an authorized administrator of The Research Repository @ WVU.

For more information, please contact researchrepository@mail.wvu.edu. 


\title{
A Base Modulated Synthesis of Indoles and Quinolines, An Expedient Synthesis of Salviadione, and Chemoselective Couplings en route to Indoles and Pyrroloindoles
}

\author{
Matthew Marshall Cummings \\ Dissertation submitted to the Eberly College of Arts and Sciences at West Virginia \\ University in partial fulfillment of the requirements for the degree of \\ Doctor of Philosophy \\ in \\ Chemistry
}

\author{
Björn C. G. Söderberg, Ph.D., Chair \\ Robert K. Griffith, Ph.D. \\ John H. Penn, Ph.D. \\ Jeffrey L. Petersen, Ph.D. \\ Kung K. Wang, Ph.D. \\ C. Eugene Bennett Department of Chemistry \\ Morgantown, West Virginia \\ 2013
}

Keywords: indole, quinoline, Salviadione, Stille coupling, palladium-catalyzed, pyrroloindole

Copyright 2013 Matthew Marshall Cummings 


\begin{abstract}
A Base Modulated Synthesis of Indoles and Quinolines, An Expedient Synthesis of Salviadione, and Chemoselective Couplings en route to Indoles and Pyrroloindoles

\section{Matthew Marshall Cummings}

Palladium-catalyzed reductive $N$-heteroannulation of ortho-nitrostyrenes has become a synthetically useful method for the construction of indoles and indole-based heterocycles. Söderberg's elaboration of this methodology has been utilized in the synthesis of indoles and quinolines from a common precursor. The same protocol has also been employed in the synthesis of the indole alkaloid Salviadione. A systematic investigation of chemoselectivity in Kosugi-Migita-Stille coupling reactions provided the basis for the synthesis of isomeric pyrroloindoles through palladium-catalyzed reductive double $N$ heteroannulation of dinitro-dialkenyl benzenes
\end{abstract}




\section{Acknowledgements}

This work is dedicated to the memory of my best friend Connor O'Halloran, who passed away during my first year of graduate school. You've always been there for me and I wish you were here to witness my accomplishments.

I would like to thank my advisor, Dr. Bjorn Söderberg, for his support, guidance, patience, and friendship during my time in his research group. I would also like to thank Dr. Phillip Perischini III, my undergraduate advisor who sparked my interest in Organic Chemistry. In addition, I would like to thank the faculty of the C. Eugene Bennett Department of Chemistry, especially Dr. John Penn, Dr. Jeffrey Petersen, and Dr. Kung Wang for serving on my doctoral defense committee. I would also like to thank Dr. Robert Griffith for serving on my committee as well. In addition, I owe many thanks to Albert Taylor, Jr., Barbara Foster, and Rebecca Secrist for their constant help over the past few years.

Much appreciation is extended to Dr. Novruz Akhmedov, whose NMR expertise was vital at numerous points of my graduate work.

Most importantly, I would like to thank my family for all of their love and support over the past five years. To my parents, Debbie and Gary, and my sister, Allison, I cannot thank you enough for everything that you've done for me. Your constant support and encouragement helped me more than you'll ever know and I cannot thank you enough. A huge thanks is extended to my friends, who were always there for me. I would also like to thank my girlfriend, Blair, for her love and support over the last two years. Lastly, I would like to thank my fellow research group members, both past and present, for their advice and companionship. In particular, much appreciation is extended to Dr. Christopher Dacko for his friendship and mentorship. 


\section{Table of Contents}

Title Page

Abstract $\quad$ ii

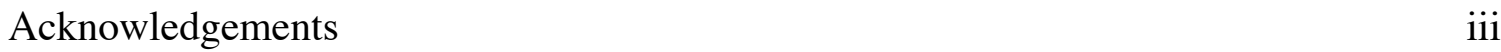

Table of Contents $\quad$ iv

List of Figures viii

List of Schemes $\quad$ xvii

List of Tables $\quad$ xxiv

\section{Chapter 1}

Introduction to Indole, History of Indole, and Historical Routes to the

\section{Indole Core}

1.1 Indole Background and Notable Indoles 2

1.2 Classical Routes to the Indole Core 6

1.3 Transition Metal Catalyzed Indole Synthesis $\quad 8$

1.4 Indole Synthesis via Reductive Cyclization 10

1.5 Indole Preparation via Palladium-Catalyzed Reductive Heterocyclization 12

Chapter 2

A Base Modulated Synthesis of Indoles and Quinolines

2.1 Introduction

2.2 Results and Discussion

2.3 Conclusions 


\section{Chapter 3}

\section{An Expedient Synthesis of Salviadione}

3.1 Salviadione Background 35

3.2 Expedient Synthesis of Salviadione $\quad 35$

3.2.1 Retrosynthetic Analysis 36

$\begin{array}{ll}\text { 3.2.2 Results and Discussion } & 37\end{array}$

$\begin{array}{ll}3.3 \text { Conclusions } & 43\end{array}$

\section{Chapter 4}

\section{Investigation of Chemoselectivity in Kosugi-Migita-Stille Coupling}

\section{Reactions}

4.1 Kosugi-Migita-Stille Reaction Background 46

4.2 Chemoselective Stille Coupling Reactions 46

4.3 Investigation of Nitro Effect in Stille Coupling Reactions 48

4.3.1 Preparation of Isomeric Substrates 49

4.3.2 Results and Discussion $\quad 53$

$\begin{array}{ll}4.3 \text { Conclusions } & 64\end{array}$

\section{Chapter 5}

Preparation of Pyrroloindoles via Palladium-Catalyzed Reductive

\section{Double $N$-Heterocyclization}

5.1 Pyrroloindole Introduction and Background

5.2 Historical Routes to Pyrroloindoles of Type I 69

5.3 Pyrroloindole Preparation using Reductive Double $N$-Heterocyclization 73 
5.3.1 Retrosynthetic Analysis

5.3.2 Banini Route to Pyrroloindoles

5.3.3 Synthesis of Symmetrical Pyrroloindoles

5.4 Synthesis of Non-Symmetrical Pyrroloindoles

5.4.1 Preparation of Cyclization Precursors

5.4.1.1 2,4-Dinitro-1,5-Dialkenyl Benzene Isomer

5.4.1.2 2,5-Dinitro-1,4-Dialkenyl Benzene Isomer

5.4.1.3 2,3-Dinitro-1,4-Dialkenyl Benzene Isomer

5.4.1.4 2,4-Dinitro-1,3-Dialkenyl Benzene Isomer

100

5.4.1.5 1,4-Dinitro-2,3-Dialkenyl Benzene Isomer

106

5.4.2 Pyrroloindole Synthesis via Reductive Double $N$-Heterocyclization

110

5.5 Conclusions

115

\section{Chapter 6}

\section{Synthesis of Functionalized Aromatics}

6.1 Brominations of 2-Nitrobenzaldehyde

6.1.1 Introduction

6.1.2 Results and Discussion

6.1.3 Conclusions

6.2 Synthesis of Functionalized Indoles

126

6.2.1 Synthesis of 6-Methyl-Nitroindoles

6.2.2 Synthesis of 5-Methoxy-Nitroindoles 


\section{Chapter 7}

\section{Supporting Information: Experimental Procedures}

7.1 Supporting Information for Chapter 2: Indole/Quinoline

7.2 Supporting Information for Chapter 3: Salviadione

7.3 Supporting Information for Chapter 4: Chemoselective Coupling

7.4 Supporting Information for Chapter 5: Pyrroloindoles

7.5 Supporting Information for Chapter 6.1: 2-Nitrobenzaldehyde Brominations

References and Notes

\section{Appendix}

\section{${ }^{1} \mathrm{H}$ and ${ }^{13} \mathrm{C}$ NMR Spectra}

- $\quad{ }^{1} \mathrm{H}$ and ${ }^{13} \mathrm{C}$ NMR for Chapter 2: Indole/Quinoline

- $\quad{ }^{1} \mathrm{H}$ and ${ }^{13} \mathrm{C}$ NMR for Chapter 3: Salviadione

- $\quad{ }^{1} \mathrm{H}$ and ${ }^{13} \mathrm{C}$ NMR for Chapter 4: Chemoselective Coupling

- $\quad{ }^{1} \mathrm{H}$ and ${ }^{13} \mathrm{C}$ NMR for Chapter 5: Pyrroloindoles

- $\quad{ }^{1} \mathrm{H}$ and ${ }^{13} \mathrm{C}$ NMR for Chapter 6.1: Bromination

- $\quad{ }^{1} \mathrm{H}$ and ${ }^{13} \mathrm{C}$ NMR for Chapter 6.2: Functionalized Indoles 


\section{List of Figures}

Figure 1: Structure and Numbering of Indole $\quad 2$

Figure 2: Structures of L-Tryptophan, Serotonin, and Melatonin 4

Figure 3: Structures of Dragmacidin, Eudistomin K, and Flustramine D 4

Figure 4: Structures of Sumtriptan, Tadalafil, Rizatriptan, and Fluvastatin 5

Figure 5: Structure and Atom Numbering of Salviadione 35

$\begin{array}{ll}\text { Figure 6: Types of Isomeric Pyrroloindoles } & 67\end{array}$

$\begin{array}{ll}\text { Figure 7: Physostigmine and Phenserine } & 67\end{array}$

Figure 8: CC-1065, Duocarmycin, and Yatakemycin 68

Figure 9: Isomeric Pyrroloindoles of Type I $\quad 69$

$\begin{array}{ll}\text { Figure 10: Pyrroloindole Numbering } & 69\end{array}$

Figure 11: 2,4-Dinitro-1,5-Dialkenyl Benzene $\quad 79$

Figure 12: Modified Work-up Procedure for Coupling of Phenol 84

Figure 13: 2,5-Dinitro-1,4-Dialkenyl Benzene $\quad 88$

Figure 14: 2,3-Dinitro-1,4-Dialkenyl Benzene 93

Figure 15: 2,4-Dinitro-1,3-Dialkenyl Benzene 100

Figure 16: 1,4-Dinitro-2,3-Dialkenyl Benzene 106

Figure 17: ${ }^{1} \mathrm{H}$ NMR of 3-Cyclohexyl-2-iodo-2-propenenitrile (110) 209

Figure 18: ${ }^{13} \mathrm{C}$ NMR of 3-Cyclohexyl-2-iodo-2-propenenitrile (110) 210

Figure 19: ${ }^{1} \mathrm{H}$ NMR of 3-Cyclohexyl-2-(2-nitrophenyl)-2-propenenitrile (111) 211

Figure 20: ${ }^{13} \mathrm{C}$ NMR of 3-Cyclohexyl-2-(2-nitrophenyl)-2-propenenitrile (111) 212

Figure 21: ${ }^{1} \mathrm{H}$ NMR of 3-Cyano-2-cyclohexylindole (112) 213

Figure 22: ${ }^{13} \mathrm{C}$ NMR of 3-Cyano-2-cyclohexylindole (112) 214 
Figure 23: ${ }^{1} \mathrm{H}$ NMR of 3-cyano-2-(1-hydroxycyclohexyl)indole (113)

Figure 24: ${ }^{13} \mathrm{C}$ NMR of 3-cyano-2-(1-hydroxycyclohexyl)indole (113)

Figure 25: ${ }^{1} \mathrm{H}$ NMR of 5,5-Dimethyl-6-(4-methoxyphenylethyl)-3-(1-methylethoxy)cyclohex-2-enone (127)

Figure 26: ${ }^{13} \mathrm{C}$ NMR of 5,5-Dimethyl-6-(4-methoxyphenylethyl)-3-(1-methylethoxy)cyclohex-2-enone (127)

Figure 27: ${ }^{1} \mathrm{H}$ NMR of 10,10a-Dihydro-1,1-dimethyl-6-methoxy-7-(1-methylethyl)-5nitrophenanthren-3(1H,2H,9H)-one (132)

Figure 28: ${ }^{13} \mathrm{C}$ NMR of 10,10a-Dihydro-1,1-dimethyl-6-methoxy-7-(1-methylethyl)-5- 220 nitrophenanthren-3(1H,2H,9H)-one (132)

Figure 29: ${ }^{1} \mathrm{H}$ NMR of 10,10a-Dihydro-1,1-dimethyl-6-hydroxy-7-(1-methylethyl)-5nitrophenanthren-3(1H,2H,9H)-one (125)

Figure 30: ${ }^{1} \mathrm{H}$ NMR of $8 a, 9,10,10 a$-Tetrahydro-1,1-dimethyl-8a-hydroxy-7-(1methylethyl)-5-nitrophenanthrene-3,6(1H,2H)-dione (133)

Figure $31:{ }^{13} \mathrm{C}$ NMR of $8 a, 9,10,10 a$-Tetrahydro-1,1-dimethyl- $8 a$-hydroxy-7-(1methylethyl)-5-nitrophenanthrene-3,6(1H,2H)-dione (133)

Figure 32: ${ }^{1} \mathrm{H}$ NMR of 1,8,9,9a-Dihydro-1,1-dimethyl-5-methoxy-6-(1-methylethyl)4H-benzo[def]carbazol-3(2H)-one (134)

Figure 33: ${ }^{13} \mathrm{C}$ NMR of 1,8,9,9a-Dihydro-1,1-dimethyl-5-methoxy-6-(1-methylethyl)225 4H-benzo[def]carbazol-3(2H)-one (134)

Figure 34: ${ }^{1} \mathrm{H}$ NMR of 1,8,9,9a-Tetrahydro-1,1-dimethyl-5-methoxy-6-(1226 methylethyl)-8-hydroxy-4H-benzo[def]carbazol-3(2H)-one (136) 
Figure 35: ${ }^{13} \mathrm{C}$ NMR of 1,8,9,9a-Tetrahydro-1,1-dimethyl-5-methoxy-6-(1-

methylethyl)-8-hydroxy-4H-benzo[def]carbazol-3(2H)-one (136)

Figure 36: ${ }^{1} \mathrm{H}$ NMR of 1,8,9,9a-Tetrahydro-1,1-dimethyl-5-hydroxy-6-(1-methylethyl)- 228 4H-benzo[def]carbazol-3(2H)-one (124)

Figure 37: ${ }^{13} \mathrm{C}$ NMR of 1,8,9,9a-Tetrahydro-1,1-dimethyl-5-hydroxy-6-(1methylethyl)-4H-benzo[def]carbazol-3(2H)-one (124)

Figure 38: ${ }^{1} \mathrm{H}$ NMR of Salviadione (123) 230

Figure 39: ${ }^{13} \mathrm{C}$ NMR of Salviadione (123) 231

Figure 40: ${ }^{1} \mathrm{H}$ NMR of 2-Bromo-5-trifluoromethanesulfonyloxy-nitrobenzene (163) 232

Figure 41: ${ }^{13} \mathrm{C}$ NMR of 2-Bromo-5-trifluoromethanesulfonyloxy-nitrobenzene (163) 233

Figure 42: ${ }^{1} \mathrm{H}$ NMR of 2-Bromo-6-trifluoromethanesulfonyloxy-nitrobenzene (166) 234

Figure 43: ${ }^{13} \mathrm{C}$ NMR of 2-Bromo-6-trifluoromethanesulfonyloxy-nitrobenzene (166) 235

Figure 44: ${ }^{1} \mathrm{H}$ NMR of 3-Bromo-2-trifluoromethanesulfonyloxy-nitrobenzene (151) 236

Figure 45: ${ }^{13} \mathrm{C}$ NMR of 3-bromo-2-trifluoromethanesulfonyloxy-nitrobenzene (151) 237

Figure 46: ${ }^{1} \mathrm{H}$ NMR of 3-Bromo-5-trifluoromethanesulfonyloxy-nitrobenzene (157) 238

Figure 47: ${ }^{13} \mathrm{C}$ NMR of 3-Bromo-5-trifluoromethanesulfonyloxy-nitrobenzene (157) 239

Figure 48: ${ }^{1} \mathrm{H}$ NMR of 4-Bromo-3-trifluoromethanesulfonyloxy-nitrobenzene (159) 240

Figure 49: ${ }^{13} \mathrm{C}$ NMR of 4-Bromo-3-trifluoromethanesulfonyloxy-nitrobenzene (159) 241

Figure 50: ${ }^{1} \mathrm{H}$ NMR of 2-Bromo-3-ethenyl-nitrobenzene (170) 242

Figure 51: ${ }^{13} \mathrm{C}$ NMR of 2-Bromo-3-ethenyl-nitrobenzene (170) 243

Figure 52: ${ }^{1} \mathrm{H}$ NMR of 2-Ethenyl-4-trifluoromethanesulfonyloxy-nitrobenzene (171) 244

Figure 53: ${ }^{13} \mathrm{C}$ NMR of 2-Ethenyl-4-trifluoromethanesulfonyloxy-nitrobenzene (171) 245

Figure 54: ${ }^{1} \mathrm{H}$ NMR of 2-Bromo-4-ethenyl-nitrobenzene (172) 246 
Figure 55: ${ }^{13} \mathrm{C}$ NMR of 2-Bromo-4-ethenyl-nitrobenzene (172)

Figure 56: ${ }^{1} \mathrm{H}$ NMR of 2-Ethenyl-5-trifluoromethanesulfonyloxy-nitrobenzene (173) 248

Figure 57: ${ }^{13} \mathrm{C}$ NMR of 2-Ethenyl-5-trifluoromethanesulfonyloxy-nitrobenzene (173) 249

Figure 58: ${ }^{1} \mathrm{H}$ NMR of 2-Ethenyl-6-trifluoromethanesulfonyloxy-nitrobenzene (175) 250

Figure 59: ${ }^{13} \mathrm{C}$ NMR of 2-Ethenyl-6-trifluoromethanesulfonyloxy-nitrobenzene (175) 251

Figure 60: ${ }^{1} \mathrm{H}$ NMR of 2-Bromo-6-ethenyl-nitrobenzene (176) 252

Figure 61: ${ }^{13} \mathrm{C}$ NMR of 2-Bromo-6-ethenyl-nitrobenzene (176) 253

Figure 62: ${ }^{1} \mathrm{H}$ NMR of 3-Ethenyl-2-trifluoromethanesulfonyloxy-nitrobenzene (177) 254

Figure 63: ${ }^{13} \mathrm{C}$ NMR of 3-Ethenyl-2-trifluoromethanesulfonyloxy-nitrobenzene (177) 255

Figure 64: ${ }^{1} \mathrm{H}$ NMR of 3-Ethenyl-4-trifluoromethanesulfonyloxy-nitrobenzene (178) 256

Figure 65: ${ }^{13} \mathrm{C}$ NMR of 3-Ethenyl-4-trifluoromethanesulfonyloxy-nitrobenzene (178) 257

Figure 66: ${ }^{1} \mathrm{H}$ NMR of 3-Bromo-4-ethenyl-nitrobenzene (179) 258

Figure 67: ${ }^{13} \mathrm{C}$ NMR of 3-Bromo-4-ethenyl-nitrobenzene (179) 259

Figure 68: ${ }^{1} \mathrm{H}$ NMR of 3-Ethenyl-5-trifluoromethanesulfonyloxy-nitrobenzene (180) 260

Figure 69: ${ }^{13} \mathrm{C}$ NMR of 3-Ethenyl-5-trifluoromethanesulfonyloxy-nitrobenzene (180) 261

Figure 70: ${ }^{1} \mathrm{H}$ NMR of 3-Bromo-5-ethenyl-nitrobenzene (181) 262

Figure 71: ${ }^{13} \mathrm{C}$ NMR of 3-Bromo-5-ethenyl-nitrobenzene (181) 263

Figure 72: ${ }^{1} \mathrm{H}$ NMR of 3-Ethenyl-6-trifluoromethanesulfonyloxy-nitrobenzene (182) 264

Figure 73: ${ }^{13} \mathrm{C}$ NMR of 3-Ethenyl-6-trifluoromethanesulfonyloxy-nitrobenzene (182) 265

Figure 74: ${ }^{1} \mathrm{H}$ NMR of 5-Bromo-2-ethenyl-nitrobenzene (183) 266

Figure 75: ${ }^{13} \mathrm{C}$ NMR of 5-Bromo-2-ethenyl-nitrobenzene (183) 267

Figure 76: ${ }^{1} \mathrm{H}$ NMR of 4-Ethenyl-2-trifluoromethanesulfonyloxy-nitrobenzene (184) 268

Figure 77: ${ }^{13} \mathrm{C}$ NMR of 4-Ethenyl-2-trifluoromethanesulfonyloxy-nitrobenzene (184) 269 
Figure 78: ${ }^{1} \mathrm{H}$ NMR of 4-Ethenyl-3-trifluoromethanesulfonyloxy-nitrobenzene (186) 270

Figure 79: ${ }^{13} \mathrm{C}$ NMR of 4-Ethenyl-3-trifluoromethanesulfonyloxy-nitrobenzene (186) 271

Figure 80: ${ }^{1} \mathrm{H}$ NMR of 4-Bromo-3-ethenyl-nitrobenzene (187) 272

Figure 81: ${ }^{13} \mathrm{C}$ NMR of 4-Bromo-3-ethenyl-nitrobenzene (187) 273

Figure 82: ${ }^{1} \mathrm{H}$ NMR of 3-Iodo-6-ethenyl-nitrobenzene (198) 274

Figure 83: ${ }^{13} \mathrm{C}$ NMR of 3-Iodo-6-ethenyl-nitrobenzene (198) 275

Figure 84: ${ }^{1} \mathrm{H}$ NMR of 3-Bromo-6-trifluoromethanesulfonyloxy acetophenone (201) 276

Figure 85: ${ }^{13} \mathrm{C}$ NMR of 3-Bromo-6-trifluoromethanesulfonyloxy acetophenone (201) 277

Figure 86: ${ }^{1} \mathrm{H}$ NMR of 3-Ethenyl-6-trifluoromethanesulfonyloxy acetophenone (202) 278

Figure 87: ${ }^{13} \mathrm{C}$ NMR of 3-Ethenyl-6-trifluoromethanesulfonyloxy acetophenone (202) 279

Figure 88: ${ }^{1} \mathrm{H}$ NMR of 6-Ethenyl-3-bromoacetophenone (203) 280

Figure 89: ${ }^{13} \mathrm{C}$ NMR of 6-Ethenyl-3-bromoacetophenone (203) 281

Figure 90: ${ }^{1} \mathrm{H}$ NMR of 3-Ethenyl-4-trifluoromethanesulfonyloxy anisole (209) 282

Figure 91: ${ }^{13} \mathrm{C}$ NMR of 3-Ethenyl-4-trifluoromethanesulfonyloxy anisole (209) 283

Figure 92: ${ }^{1} \mathrm{H}$ NMR of 2,3-dinitro-1,4-ethenylbenzene (241) 284

Figure 93: ${ }^{13} \mathrm{C}$ NMR of 2,3-dinitro-1,4-ethenylbenzene (241) 285

Figure 94: ${ }^{1} \mathrm{H}$ NMR of 2,3-dinitro-1,4-di(prop-1-en-2-yl)benzene (257) 286

Figure 95: ${ }^{13} \mathrm{C}$ NMR of 2,3-dinitro-1,4-di(prop-1-en-2-yl)benzene (257) 287

Figure 96: ${ }^{1} \mathrm{H}$ NMR of 5-iodo-2,4-dinitrophenol (263) 288

Figure 97: ${ }^{13} \mathrm{C}$ NMR of 5-iodo-2,4-dinitrophenol (263) 289

Figure 98: ${ }^{1} \mathrm{H}$ NMR of 2,4-dinitro-5-(prop-1-en-2-yl)phenol (271) 290

Figure 99: ${ }^{13} \mathrm{C}$ NMR of 2,4-dinitro-5-(prop-1-en-2-yl)phenol (271) 291 
Figure 100: ${ }^{1} \mathrm{H}$ NMR of 2,4-dinitro-5-(prop-1-en-2-yl)phenyl

trifluoromethanesulfonate (261)

Figure 101: ${ }^{13} \mathrm{C}$ NMR of 2,4-dinitro-5-(prop-1-en-2-yl)phenyl

trifluoromethanesulfonate (261)

Figure 102: ${ }^{1} \mathrm{H}$ NMR of 1-chloro-2,4-dinitro-5-(prop-1-en-2-yl)benzene (273)

Figure 103: ${ }^{13} \mathrm{C}$ NMR of 1-chloro-2,4-dinitro-5-(prop-1-en-2-yl)benzene (273)

Figure 104: ${ }^{1} \mathrm{H}$ NMR of 1,5-dinitro-2,4-di(prop-1-en-2-yl)benzene (260)

Figure 105: ${ }^{13} \mathrm{C}$ NMR of 1,5-dinitro-2,4-di(prop-1-en-2-yl)benzene (260)

Figure 106: ${ }^{1} \mathrm{H}$ NMR of 1,5-dinitro-2-(prop-1-en-2-yl)-4-(prop-1-enyl)benzene (274) 298

Figure 107: ${ }^{13} \mathrm{C}$ NMR of 1,5-dinitro-2-(prop-1-en-2-yl)-4-(prop-1-enyl)benzene (274)

Figure 108: ${ }^{1} \mathrm{H}$ NMR of (E)-1,5-dinitro-2-(1-phenylprop-1-enyl)-4-(prop-1-en-2-

300

yl)benzene (276)

Figure 109: ${ }^{13} \mathrm{C}$ NMR of (E)-1,5-dinitro-2-(1-phenylprop-1-enyl)-4-(prop-1-en-2-

yl)benzene (276)

Figure 110: ${ }^{1} \mathrm{H}$ NMR of 2,5-dinitro-4-ethenylphenol (278)

Figure 111: ${ }^{13} \mathrm{C}$ NMR of 2,5-dinitro-4-ethenylphenol (278)

303

Figure 112: ${ }^{1} \mathrm{H}$ NMR of 2,5-dinitro-4-ethenylphenyl trifluoromethanesulfonate (277)

Figure 113: ${ }^{13} \mathrm{C}$ NMR of 2,5-dinitro-4-ethenylphenyl trifluoromethanesulfonate (277)

305

Figure 114: ${ }^{1} \mathrm{H}$ NMR of 4-bromo-2,3-dinitrophenol (292)

Figure 115: ${ }^{13} \mathrm{C}$ NMR of 4-bromo-2,3-dinitrophenol (292)

Figure 116: ${ }^{1} \mathrm{H}$ NMR of (Z)-methyl 2-(4-bromo-2,3-dinitrophenyl)but-2-enoate (301) 308

Figure 117: ${ }^{13} \mathrm{C}$ NMR of (Z)-methyl 2-(4-bromo-2,3-dinitrophenyl)but-2-enoate (301) 309 
Figure 118: ${ }^{1} \mathrm{H}$ NMR of (Z)-methyl 2-(2,3-dinitro-4-(prop-1-en-2-yl)phenyl)but-2enoate (302)

Figure 119: ${ }^{13} \mathrm{C}$ NMR of $(Z)$-methyl 2-(2,3-dinitro-4-(prop-1-en-2-yl)phenyl)but-2enoate (302)

Figure 120: ${ }^{1} \mathrm{H}$ NMR of 1-iodo-3-methyl-2,4-dinitrobenzene (308)

Figure 121: ${ }^{13} \mathrm{C}$ NMR of 1-iodo-3-methyl-2,4-dinitrobenzene (308)

Figure 122: ${ }^{1} \mathrm{H}$ NMR of 2-(3-iodo-2,6-dinitrophenyl)ethanol (312)

Figure 123: ${ }^{13} \mathrm{C}$ NMR of 2-(3-iodo-2,6-dinitrophenyl)ethanol (312)

Figure 124: ${ }^{1} \mathrm{H}$ NMR of 1-iodo-2,4-dinitro-3-ethenylbenzene (313)

Figure 125: ${ }^{13} \mathrm{C}$ NMR of 1-iodo-2,4-dinitro-3-ethenylbenzene (313)

Figure 126: ${ }^{1} \mathrm{H}$ NMR of 1,3-dinitro-4-(prop-1-en-2-yl)-2-ethenylbenzene (314)

Figure 127: ${ }^{13} \mathrm{C}$ NMR of 1,3-dinitro-4-(prop-1-en-2-yl)-2-ethenylbenzene (314)

Figure 128: ${ }^{1} \mathrm{H}$ NMR of 3,5-dimethyl-1,7-dihydropyrrolo[3,2-f]indole (326)

Figure 129: ${ }^{13} \mathrm{C}$ NMR of 3,5-dimethyl-1,7-dihydropyrrolo[3,2-f]indole (326)

Figure 130: ${ }^{1} \mathrm{H}$ NMR of 3-methyl-2,6-dihydropyrrolo[2,3-g]indole (330)

Figure 131: ${ }^{13} \mathrm{C}$ NMR of 3-methyl-2,6-dihydropyrrolo[2,3-g]indole (330)

Figure 132: ${ }^{1} \mathrm{H}$ NMR of Methyl 2,6-dimethyl-1,8-dihydropyrrolo[3,2-g]indole-3carboxylate (331):

Figure 133: ${ }^{13} \mathrm{C}$ NMR of Methyl 2,6-dimethyl-1,8-dihydropyrrolo[3,2-g]indole-3carboxylate (331):

Figure 134: ${ }^{1} \mathrm{H}$ NMR of 2,5-dimethyl-1,7-dihydropyrrolo[3,2-f]indole (332)

Figure $135:{ }^{13} \mathrm{C}$ NMR of 2,5-dimethyl-1,7-dihydropyrrolo[3,2-f]indole (332)

Figure 136: ${ }^{1} \mathrm{H}$ NMR of 2,5-dimethyl-3-phenyl-1,7-dihydropyrrolo[3,2-f]indole (333) 
Figure 137: ${ }^{13} \mathrm{C}$ NMR of 2,5-dimethyl-3-phenyl-1,7-dihydropyrrolo[3,2-f]indole (333) 329

Figure 138: ${ }^{1} \mathrm{H}$ NMR of 3,4-dibromo-2-nitrobenzaldehyde (343) 330

Figure 139: ${ }^{13} \mathrm{C}$ NMR of 3,4-dibromo-2-nitrobenzaldehyde (343) 331

Figure 140: ${ }^{1} \mathrm{H}$ NMR of 4,5-dibromo-2-nitrobenzaldehyde (344) 332

Figure 141: ${ }^{13} \mathrm{C}$ NMR of 4,5-dibromo-2-nitrobenzaldehyde (344) 333

Figure 142: ${ }^{1} \mathrm{H}$ NMR of 3,4-dibromo-2-nitrobenzylalcohol (349) 334

Figure 143: ${ }^{13} \mathrm{C}$ NMR of 3,4-dibromo-2-nitrobenzylalcohol (349) 335

Figure 144: ${ }^{1} \mathrm{H}$ NMR of 4,5-dibromo-2-nitrobenzylalcohol (350) 336

Figure 145: ${ }^{13} \mathrm{C}$ NMR of 4,5-dibromo-2-nitrobenzylalcohol (350) 337

Figure 146: ${ }^{1} \mathrm{H}$ NMR of 3,6-dibromo-2-nitrobenzylalcohol (351) 338

Figure 147: ${ }^{13} \mathrm{C}$ NMR of 3,6-dibromo-2-nitrobenzylalcohol (351) 339

Figure 148: ${ }^{1} \mathrm{H}$ NMR of 2,3-dinitro-4-ethenyltoluene (355) 340

Figure 149: ${ }^{13} \mathrm{C}$ NMR of 2,3-dinitro-4-ethenyltoluene (355) 341

Figure 150: ${ }^{1} \mathrm{H}$ NMR of 6-methyl-7-nitroindole (356) 342

Figure 151: ${ }^{1} \mathrm{H}$ NMR of 3,5-dinitro-4-ethenyltoluene (355) 343

Figure 152: ${ }^{13} \mathrm{C}$ NMR of 3,5-dinitro-4-ethenyltoluene (355) 344

Figure 153: ${ }^{1} \mathrm{H}$ NMR of 6-methyl-4-nitroindole (356) 345

Figure 154: ${ }^{13} \mathrm{C}$ NMR of 6-methyl-4-nitroindole (356) 346

Figure 155: ${ }^{1} \mathrm{H}$ NMR of 5-iodo-2,4-dinitroanisole (363) 347

Figure 156: ${ }^{13} \mathrm{C}$ NMR of 5-iodo-2,4-dinitroanisole (363) 348

Figure 157: ${ }^{1} \mathrm{H}$ NMR of 5-ethenyl-2,4-dinitroanisole (365) 349

Figure 158: ${ }^{13} \mathrm{C}$ NMR of 5-ethenyl-2,4-dinitroanisole (365) 350

Figure 159: ${ }^{1} \mathrm{H}$ NMR of 2,4-dinitro-5-(prop-1-en-2-yl)anisole (369) 351 
Figure 160: ${ }^{13} \mathrm{C}$ NMR of 2,4-dinitro-5-(prop-1-en-2-yl)anisole (369)

Figure 161: ${ }^{1} \mathrm{H}$ NMR of 3-methyl-5-methoxy-6-nitroindole (370)

Figure 162: ${ }^{1} \mathrm{H}$ NMR of 3-iodo-2,4-dinitroanisole (364)

Figure 163: ${ }^{13} \mathrm{C}$ NMR of 3-iodo-2,4-dinitroanisole (364)

Figure 164: ${ }^{1} \mathrm{H}$ NMR of 3-ethenyl-2,4-dinitroanisole (367)

Figure 165: ${ }^{13} \mathrm{C}$ NMR of 3-ethenyl-2,4-dinitroanisole (367)

Figure 166: ${ }^{1} \mathrm{H}$ NMR of 3-(prop-1-en-2-yl)-2,4-dinitroanisole (371)

Figure 167: ${ }^{13} \mathrm{C}$ NMR of 3-(prop-1-en-2-yl)-2,4-dinitroanisole (371)

Figure 168: ${ }^{1} \mathrm{H}$ NMR of 3-methyl-5-methoxy-4-nitroindole (372)

Figure 169: ${ }^{13} \mathrm{C}$ NMR of 3-methyl-5-methoxy-4-nitroindole (372) 


\section{List of Schemes}

Scheme 1: Initial Preparation of Indole from Indigo 3

$\begin{array}{lr}\text { Scheme 2: Fischer Indole Synthesis } & 6\end{array}$

Scheme 3: Fischer Cyclization en route to Haplophytine $\quad 6$

$\begin{array}{ll}\text { Scheme 4: Madelung Indole Synthesis } & 7\end{array}$

$\begin{array}{ll}\text { Scheme 5: Houlihan Modification to Madelung Indole Synthesis } & 7\end{array}$

Scheme 6: Madelung Methodology in the Synthesis of Penitrem D 8

Scheme 7: Hegedus Palladium-catalyzed Cyclization of $o$-Allylanilines 8

Scheme 8: Larock Heteroannulation of Internal Alkyne with $o$-Iodoaniline 9

Scheme 9: Baran's Synthesis of Psychotrimine via Larock Cyclization 9

Scheme 10: Buchwald Indole Synthesis via Intramolecular Amination 10

Scheme 11: Reissert Method for Indole Preparation $\quad 10$

Scheme 12: Leimgruber-Batcho Indole Synthesis 11

Scheme 13: Cadogan and Sundberg's Reductive Heterocyclization 11

Scheme 14: Mechanism of Cadogan-Sundberg Indole Synthesis 12

Scheme 15: Alternative Mechanism of Sundberg-Cadogan Cyclization 12

Scheme 16: Watanabe Palladium(II)-Catalyzed Reductive Heterocyclization 13

Scheme 17: Davies Reductive N-Heterocyclization of Isomeric Nitrostilbenes 14

Scheme 18: Söderberg Reductive Heterocyclization 14

Scheme 19: Proposed Mechanism(s) of Reductive $N$-Heteroannulation 16

Scheme 20: Potential Palladium-Olefin Coordination-Mediated Reduction 17

Scheme 21: ${ }^{18} \mathrm{O}$ Labeling Study of Cadogan-Sundberg Reductive Cyclization 18

Scheme 22: Deuterium-Labeling Study of [1,5]-Sigmatropic Rearrangement 19 
Scheme 23: Initial Cyclization of Cyanoalkene

Scheme 24: Akazome's Synthesis of Indoles and Quinolines

Scheme 25: Investigation of Cyclization Conditions

Scheme 26: Preparation of 2-Nitrostyrenes via Stille Coupling

Scheme 27: Preparation and Cyclization of Cyanoalkene 105

Scheme 28: Preparation and Cyclization of Cyanoalkene 111

Scheme 29: Attempt to Trap Reactive Intermediates

Scheme 30: Potential Mechanism(s) for Cyclization in Absence of Base

Scheme 31: Proposed Mechanism for Quinoline Formation

Scheme 32: Mechanistic Analysis of Quinoline Formation

Scheme 33: Proposed Mechanism Accounting for Fission, Oxidation, Elimination

Scheme 34: Proposed Retrosynthetic Outline for Salviadione

Scheme 35: Alkylation of Dimedone

Scheme 36: Preparation of Alkyliodide

Scheme 37: Preparation of Cyclization Precursor

Scheme 38: Acid-Induced Intramolecular Cyclization-Friedel-Crafts Alkylation

Scheme 39: Nitration of Tricyclic Diterpinoid Core

Scheme 40: Oxidation of Products to Quinole 133

Scheme 41: Palladium-Catalyzed Reductive $N$-Heteroannulation

Scheme 42: Attempted Cyclization of Quinole

Scheme 43: Benzylic Oxidation of Anisole Using DDQ

Scheme 44: DDQ Oxidation to Afford Salviadione

Scheme 45: Echavarren's Chemoselective Stille Coupling Conditions 
Scheme 46: Krolski Selective Coupling of 2-Bromo-3-Nitrophenyl Triflate

Scheme 47: Preparation of 2,4-Dinitro-5-Bromophenyl Triflate

Scheme 48: Attempted Selective Coupling of Dinitro-Bromophenyl Triflate

Scheme 49: Nitration of 2-Bromophenol

Scheme 50: Preparation of Isomeric Nitrophenyl Triflates

Scheme 51: Preparation of 2-Nitro-4-Bromophenyl Triflate

Scheme 52: Preparation of 3-Nitro-5-Bromophenyl Triflate

Scheme 53: Preparation of 2-Bromo-5-Nitrophenyl Triflate

Scheme 54: Preparation of 4-Bromo-3-Nitrophenyl Triflate

Scheme 55: Preparation of 3-Bromo-2-Nitrophenyl Triflate

Scheme 56: Preparation of $\mathbf{1 6 7}$ and $\mathbf{1 6 8}$

Scheme 57: Preparation of 2-Bromo-3-Nitrophenyl Triflate

Scheme 58: Preparation of Bromophenyl Triflates

Scheme 59: Echavarren's Coupling of $p$-Iodophenyl Triflate

Scheme 60: Preparation of 4-Iodo-2-Nitrophenyl Triflate

Scheme 61: Coupling of 4-Iodo-2-Nitrophenyl Triflate

Scheme 62: Preparation of 5-Bromo-2-Trifloxy Acetophenone

Scheme 63: Selectivity Under Conditions A and B

Scheme 64: Bromine-Selective Coupling Under Conditions B

Scheme 65: Preparation of 3-Bromo-4-Trifloxy Anisole

Scheme 66: Selectivity Under Conditions A and B

Scheme 67: Berlin's Approach to Pyrroloindoles

Scheme 68: Montmorillonite K-10 Catalyzed Synthesis of Pyrroloindoles 
Scheme 69: Samsoniya Route to Isomeric Pyrroloindoles

Scheme 70: Pyrroloindole Preparation from Aminoindoline

Scheme 71: Oxidative Bicyclization of Diimines

Scheme 72: Intramolecular Hydroamination of Di-yne

Scheme 73: Retrosynthetic Analysis of Pyrroloindoles

Scheme 74: Banini Route to Pyrroloindoles of Class B

Scheme 75: Banini Route to Pyrroloindoles of Class C

Scheme 76: Failed Preparation of Benzyl Bromide

Scheme 77: Attempted Mono-Coupling of Dibromide

Scheme 78: Preparation of Dialkenyl-Dinitrobenzene

Scheme 79: Proposed Retrosynthetic Outline to Cyclization Precursor

Scheme 80: Nitration of 3-Iodophenol

Scheme 81: Nitration of 5-Iodo-2-Nitrophenol

Scheme 82: Nitration of 3-Iodo-4-Nitrophenol

Scheme 83: Preparation of Aryl Triflate

Scheme 84: Attempted Kosugi-Migita-Stille Coupling

Scheme 85: Kosugi-Migita-Stille Coupling of Iodophenol

Scheme 86: Conversion of Phenol to Triflate

Scheme 87: Attempted Kosugi-Migita-Stille Coupling of Aryl Triflate

Scheme 88: Conversion of Aryl Triflate to Aryl Chloride

Scheme 89: Kosugi-Migita-Stille Coupling of Aryl Chloride

Scheme 90: Kosugi-Migita-Stille Coupling of Aryl Triflate

Scheme 91: Preparation of Cyclization Precursor 
Scheme 92: Proposed Retrosynthetic Outline to Cyclization Precursor

Scheme 93: Nitration of 4-Iodophenol

Scheme 94: Nitration of 3-Nitrophenol

Scheme 95: Preparation of Benzyltriphenylphosphonium Peroxymonosulfate

Scheme 96: Oxidative Iodination of 2,5-Dinitrophenol

Scheme 97: Iodination of Deactivated Arene

Scheme 98: Electrophilic Iodination of 2,5-Dinitrophenol

Scheme 99: Kosugi-Migita-Stille Coupling of 4-Iodo-2,5-Dinitrophenol

Scheme 100: Preparation of Aryl Triflate

Scheme 101: Kosugi-Migita-Stille Coupling of Aryl Triflate

Scheme 102: Proposed Retrosynthetic Outline to Cyclization Precursor

Scheme 103: Attempted Oxidative Iodination of 2,3-Dinitrophenol

Scheme 104: Attempted Electrophilic Iodination of 2,3-Dinitrophenol

Scheme 105: Preparation of 4-Bromo-2,3-Dinitrophenol

Scheme 106: Kosugi-Migita-Stille Coupling of Bromophenol

Scheme 107: Nitration of 1,4-Diiodobenzene

Scheme 108: Attempted Kosugi-Migita-Stille Coupling of Aryl Bromide

Scheme 109: Selective Mono-Coupling of Dibromide by Banini

Scheme 110: Mono-Coupling Using $\alpha$-Stannyl Ester

Scheme 111: Preparation of Non-Symmetrical Substrate

Scheme 112: Proposed Retrosynthetic Outline to Cyclization Precursor

Scheme 113: Preparation of Dinitrophenol Isomers 
Scheme 115: Attempted Coupling of Iodophenol

Scheme 116: Kawakami Methylation of 2,4-Dinitroaniline

Scheme 117: Preparation of Aryl Iodide

Scheme 118: Mundla's Preparation of 2,6-Dinitrostyrene

Scheme 119: Synthesis of Styrene

Scheme 120: Kosugi-Migita-Stille Coupling of Aryl Iodide

Scheme 121: Attempted Condensation with Hexanal

Scheme 122: Proposed Retrosynthetic Outline to Cyclization Precursor

Scheme 123: Attempted Nitration of 2-Iodo-3-Nitrophenol

Scheme 124: Proposed ortho-Functionalization of Phenol

Scheme 125: Mechanism of ortho-Selective Nitration of Phenol

Scheme 126: Attempted ortho-Selective Nitration of Phenol

Scheme 127: Gaude's ortho-Iodination of Phenol

Scheme 128: Attempted ortho-Iodination of Dinitrophenol

Scheme 129: Double Cyclization Using 1,10-phenanthroline/dppp

Scheme 130: Formation of Mixed Indole Dimer

Scheme 131: Intermolecular Dimerization of Pyrroloindole

Scheme 132: Proposed Substrate Preparation From Halobenzaldehyde

Scheme 133: Regioselective Bromination of 2-Nitrobenzaldehyde

Scheme 134: Bromination of 2-Nitrobenzaldehyde

Scheme 135: Sodium Borohydride Reduction of $\mathbf{3 4 0}$

Scheme 136: Reduction of Inseparable Mixture 
Scheme 138: Reduction of Aldehyde 344

Scheme 139: Reduction of Aldehyde 345

Scheme 140: Nitration of $p$-Bromotoluene

Scheme 141: Kosugi-Migita-Stille Coupling of Aryl Bromide

Scheme 142: Reductive $N$-Heterocyclization of Nitrostyrene 355

Scheme 143: Synthesis of 6-Methyl-4-Nitroindole

Scheme 144: Dinitration of 3-Haloanisole

Scheme 145: Preparation of 5-Methoxy-Nitroindoles

Scheme 146: Preparation of 3-Methyl-5-Methoxy-Nitroindoles 


\section{List of Tables}

Table 1: Knoevenagel Condensation of 2-Arylacetonitrile 23

Table 2: Preparation of Ester-Substituted Alkenes $\quad 24$

Table 3. Cyclizations to Afford Substituted Indoles and/or Quinolines 26

Table 4: ${ }^{1}$ HNMR Shift Comparison Between Synthetic and Natural Salviadione 42

Table 5: ${ }^{13} \mathrm{CNMR}$ Shift Comparison Between Synthetic and Natural Salviadione 43

Table 6: Isolated Yields From Reaction Under Conditions A and B 54

Table 7: Selective Coupling of Bromophenyl Triflates 58

Table 8: Reductive Double $N$-Heteroannulation $\quad 78$

Table 9: Reductive Double $N$-Heteroannulation Under Banini's Conditions 110

Table 10: Palladium-Catalyzed Reductive Double $N$-Heterocyclizations 112

Table 11: Molar Ratios of Brominated Products 120

Table 12: Isolated Yields of Brominated Products 121 


\section{Chapter 1}

\section{Introduction to Indole, History of Indole, and Historical Routes to the}

\section{Indole Core}

1.1 Indole Background and Notable Indoles

1.2 Classical Routes to the Indole Core $\quad 6$

1.3 Transition Metal Catalyzed Indole Synthesis $\quad 8$

1.4 Indole Synthesis via Reductive Cyclization $\quad 10$

1.5 Indole Preparation via Palladium-Catalyzed Reductive Heterocyclization 12 


\subsection{Indole Background and Notable Indoles}

Indole (1) is the common name for the molecule featuring a pyrrole ring fused to a benzene ring along the 2- and 3-positions of the pyrrole. ${ }^{1}$ The name indole itself is in relation to indigo, which is the parent compound from which indole was originally isolated through treatment with oleum.

The atoms of indole are numbered according to the following scheme ${ }^{2}$ : the nitrogen atom is designated atom 1 and proceeds around the pyrrole portion of the molecule in a counter-clockwise fashion (Figure 1). The carbons where the pyrrole is fused to the benzene ring are often times denoted " $3 \mathrm{a}$ " and " $7 \mathrm{a}$ ".

\section{Figure 1: Structure and Numbering of Indole}

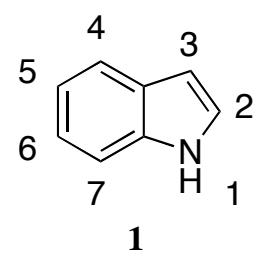

Indole is a planar heteroaromatic molecule composed of a ten-electron pi-system made up of two electrons from the nitrogen atom and eight electrons from the remaining eight carbon atoms. ${ }^{3}$ Due to it having more pi-electrons than atoms, indole is often referred to as a "pi-excessive" aromatic compound. ${ }^{4}$ Typically, the 3 -position of indole is highly reactive towards electrophilic species on the basis of pi-electron density, localization energy, and molecular orbital calculations. ${ }^{3}$ As expected, the amino-group of indoles is fairly acidic with a pKa of 17 , possessing the ability to form an anion in the presence of a strong base. ${ }^{4}$ While the resulting electron pair of the anion does not lie within the pi-system, it still serves as a basis for the increased reactivity of the 3-position toward electrophiles. ${ }^{5}$ 
The preparation of indoles and indole-based derivatives has been a topic of interest for more than a century, beginning with the initial development of indole triggered by the dye industry. Oxidation of indigo (2) to isatin (3) was achieved by Baeyer and Knopp ${ }^{6}$, who then reduced isatin (3) to oxindole (4) (Scheme 1). Pyrolysis of 4 in the presence of zinc dust afforded indole (1). Baeyer ${ }^{7}$ first reported the formula of indole, which is still accepted today, in 1869.

\section{Scheme 1: Initial Preparation of Indole from Indigo}

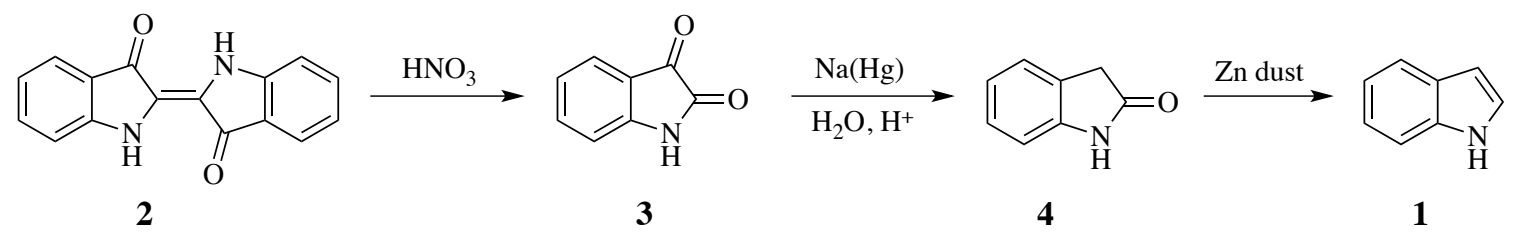

The main use of indoles through the end of the $19^{\text {th }}$ century was within the dyestuff industry. While the dye applications of indoles declined in the early $20^{\text {th }}$ century, organic dyes still account for approximately $\$ 14.4$ billion in global production. ${ }^{8}$

Interest in indole-based molecules was re-ignited in the 1930's as interest in alkaloid chemistry grew. ${ }^{9}$ The diverse structures of indole alkaloids leads to their wide range of biological activities and in turn triggered an increase in research toward the preparative methods of indoles. ${ }^{3}$

A number of indole-based molecules are involved in processes within the human body. The amino acid $L$-tryptophan $(\mathbf{5})^{10}$ is essential to the human diet while also functioning as a precursor for the neurotransmitter serotonin $(\mathbf{6})^{11}$, which can be further converted to the neurohormone melatonin (7) ${ }^{12}$ (Figure 2). 
Figure 2: Structures of L-tryptophan, Serotonin, and Melatonin<smiles>N[C@@H](Cc1c[nH]c2ccccc12)C(=O)O</smiles>

5<smiles>NCCc1c[nH]c2ccc(O)cc12</smiles>

6<smiles>COc1ccc2[nH]cc(CCNC(C)=O)c2c1</smiles>

7

Indole alkaloids make up a quarter of the marine alkaloids and are considered to be the most structurally complex molecules of this class. ${ }^{13}$ Indole alkaloids have been isolated from a number of marine species, including sponges, tunicates, red algae, acorn worms, and symbiotic bacteria. ${ }^{13}$ In addition, marine alkaloids often possess structural features uncommon to terrestrial species, such as halides (Figure 3). Dragmacidin $(\mathbf{8})^{14}$, isolated from the deep water sponge Dragmacidin sp., has exhibited in vitro cytotoxic activity against a number of cancer cell lines including lung, colon, and mammary. ${ }^{11}$ The novel oxathiazepine containing alkaloid Eudistomin $\mathrm{K}(\mathbf{9})^{15}$, isolated from the Caribbean tunicate E. olivaceum, inhibits herpes simplex virus-1 growth. ${ }^{13}$ Flustramine D $(\mathbf{1 0})^{16}$, isolated from the marine bryozoan $F$. foliacea, exhibits inhibition of a wide spectrum of bacterial including E. coli, S. typhimurium, S. aureus, and S.epidermidis. ${ }^{13}$

Figure 3: Structures of Dragmacidin, Eudistomin K, and Flustramine D<smiles>CC1C[C@H](c2c[nH]c3c(Br)c(Br)cc(O)c23)CN1</smiles>

8

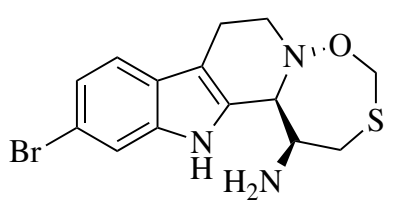

9<smiles>C=CC(C)(C)C12CCN(C)C1Nc1cc(Br)c(CC=C(C)C)cc12</smiles>

10

Synthetic indoles have also found applications in the medicinal field. Sumatriptan $(\mathbf{1 1})^{17}$, tadalafil $(\mathbf{1 2})^{18}$, rizatriptan $(\mathbf{1 3})^{19}$, and fluvastatin $(\mathbf{1 4})^{20}$, all containing an indole motif, accounted for over $\$ 3.2$ billion in sales in 2010 (Figure 4). ${ }^{21}$ Sumatriptan and rizatriptan have found use as anti-migrane $\operatorname{drugs}^{21}$, tadalafil (commonly known as Cialis) 
is used to treat erectile dysfunction ${ }^{22}$, and fluvastatin is used to prevent cardiovascular disease ${ }^{23}$.

Figure 4: Structures of Sumtriptan, Tadalafil, Rizatriptan, and Fluvastatin

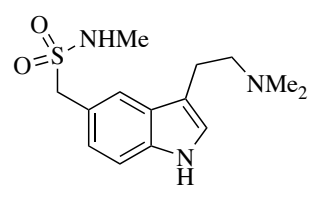

11

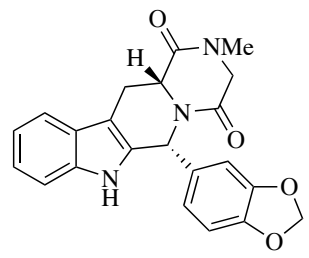

12

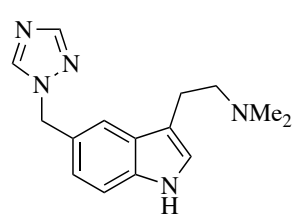

13

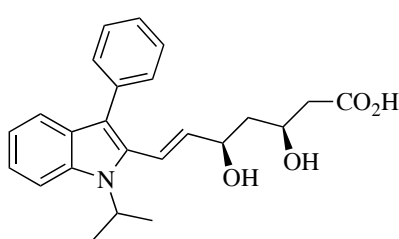

14

Indoles have found applications in a number of areas. Their initial use as dyestuffs gradually progressed to exploration of their uses as biological compounds. With advances in isolation and characterization techniques, numerous novel indole alkaloids are reported annually. This has in turn triggered a heightened interest in preparative methods of indoles, which will be discussed in the following section. 


\subsection{Classical Routes to the Indole Core}

One of the most widely applied methods for the preparation of indole intermediates en route to biologically active compounds ${ }^{25}$ is the Fischer indole synthesis. ${ }^{26,27}$ Developed in 1883 , it allowed for the relatively simple conversion of enolizable $N$-arylhydrazones to indoles. In a simplistic example, condensation of aryl hydrazine 15 with 2-butanone afforded intermediate hydrazone 16 which then underwent cyclization to afford indole $\mathbf{1 7}$ (Scheme 2). Addition of a protic or Lewis acid (typically $\mathrm{ZnCl}_{2}$ ) served two purposes: to assist in the tautomerization in order to form the enehydrazine as well as to facilitate cleavage of the N-N bond. ${ }^{4,25}$

\section{Scheme 2: Fischer Indole Synthesis}

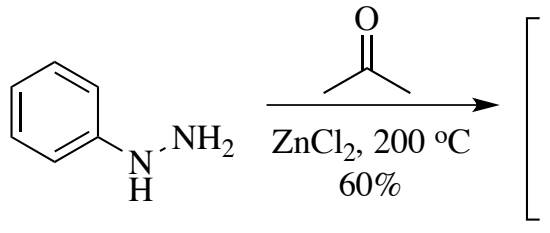

15

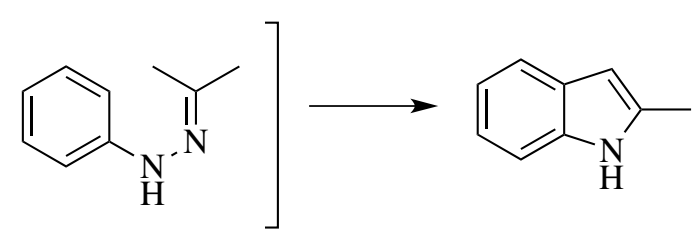

16
17

A Fischer cyclization was employed as a key step in Fukuyama's synthesis of haplophytine. ${ }^{28}$ Condensation of tricyclic ketone 18 with aryl hydrazine 19 followed by heating in the presence of $p$-toluenesulfonic acid afforded indoline $\mathbf{2 0}$ which was then converted to haplophytine in six additional steps (Scheme 3). The structural complexity of both substrates highlights the broad scope and functional group tolerance of the Fischer method.

Scheme 3: Fischer Cyclization en route to Haplophytine

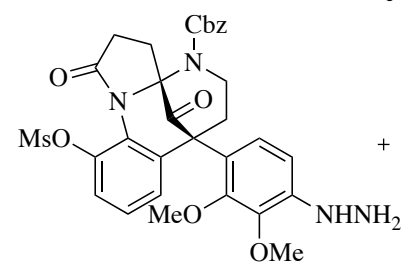

18

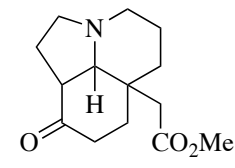

19

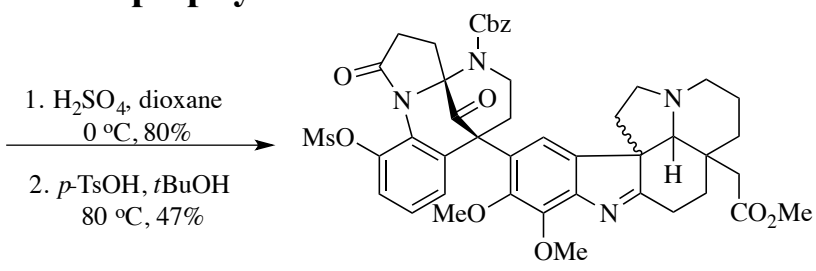

20 
The Madelung indole synthesis used high temperatures to convert $N$ phenylamides to indoles through intramolecular cyclization in the presence of a strong base. ${ }^{29}$ The first reported example prepared 2-methylindole (18) from $o$-methylacetanilide (21) through treatment with sodium amide and heating at $250^{\circ} \mathrm{C}$ (Scheme 4$){ }^{30}$

Scheme 4: Madelung Indole Synthesis

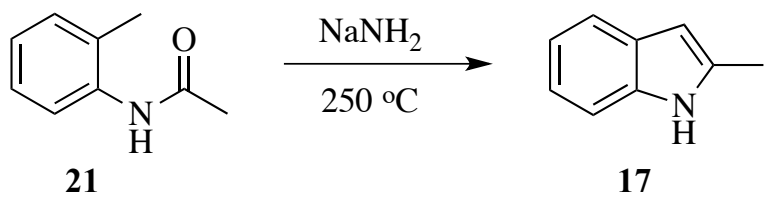

One downfall of these conditions was that they were extremely harsh and in turn limited the scope of functionality. ${ }^{4}$ However, a number of modifications which utilized much milder conditions were also been reported. The Houlihan modification ${ }^{31}$ employed the use of either $n$-butyllithium ( $n$-BuLi) or lithium $N, N$-diisopropylamide (LDA) as the base and required much lower reaction temperatures. Using these conditions, $N$-(4chloro-2-methylphenyl)benzamide (22) was converted to 5-chloro-2-phenylindole (23) through treatment with two equivalents of $n-\mathrm{BuLi}$ in tetrahydrofuran at $-20^{\circ} \mathrm{C}$ (Scheme $5){ }^{32}$

\section{Scheme 5: Houlihan Modification of Madelung Indole Synthesis}

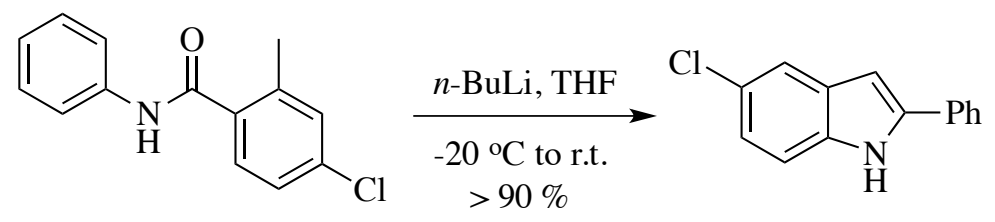

22

23

An elegant application of the Madelung route was presented in the synthesis of penitrem $\mathrm{D} .{ }^{33}$ This approach was daring in that it was applied at a late stage in the synthetic route. Condensation of aniline $\mathbf{2 4}$ with cyclic ester $\mathbf{2 5}$ was afforded heptacyclic indole 26 in excellent yield (Scheme 6). 


\section{Scheme 6: Madelung Methodology in the Synthesis of Penitrem D}

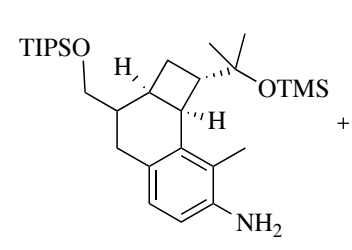

24

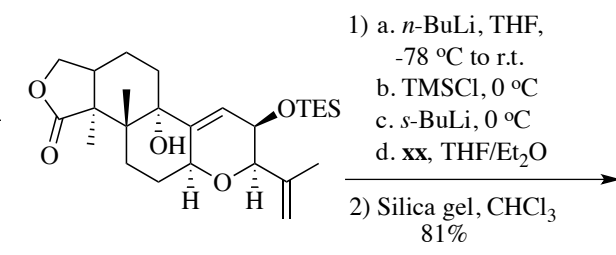

25

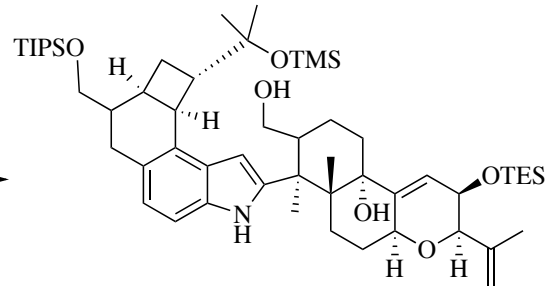

26

\subsection{Transition Metal Catalyzed Indole Synthesis}

An early example of palladium-catalyzed indole preparation was reported by Hegedus et al. in $1976 .^{34}$ This intramolecular elaboration of their earlier finding of palladium-assisted amination of olefins ${ }^{35}$ involved cyclization of an $o$-allylaniline 27 in the presence of palladium (II) catalysts to afford 2-substituted indole $\mathbf{2 8}$ (Scheme 7). The relatively mild reaction conditions accommodated a number of functional groups and was later applied in the preparation of ergot alkaloids. ${ }^{36}$

\section{Scheme 7: Hegedus Palladium-catalyzed Cyclization of o-Allylanilines}

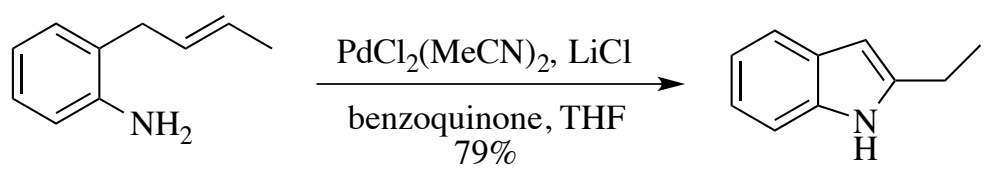

27

Larock et al. ${ }^{37}$ described a convenient preparation of 2,3-disubstituted indoles 31 via palladium-catalyzed heteroannulation between $o$-iodoaniline (30) and internal alkynes (Scheme 8). This methodology allowed for the preparation of highly functionalized indoles with regioselective control based on the identities of the substituents on the alkyne. The reaction's versatility has led to its application in the synthesis of a number of pharmaceutical compounds including Maxalt ${ }^{19}$ and Avitriptan. ${ }^{38}$ However, the Larock cyclization has been limited by the requirement of iodoaniline substrates, which are often 
more expensive or not commercially-available in comparison to the corresponding bromo- or chloroanilines. ${ }^{39}$

Scheme 8: Larock Heteroannulation of Internal Alkyne with o-Iodoaniline<smiles>CCCc1[nH]c2ccccc2c1C(C)C</smiles>

30

31

A practical application of Larock methodology was in the synthesis of psychotrimine by Baran et al. ${ }^{40}$ Attempts to use pre-formed indoles as nucleophiles to prepare intermediate 33 were unsuccessful, however, a Larock cyclization between 2iodoaniline derivative $\mathbf{3 2}$ and the properly substituted alkyne afforded bis-indole $\mathbf{3 3}$ in gram amounts (Scheme 9).

\section{Scheme 9: Baran's Synthesis of Psychotrimine via Larock Cyclization}<smiles>CC(=O)N1CC[C@]2(Nc3ccccc3I)c3cccc(Br)c3N[C@H]12</smiles>

32

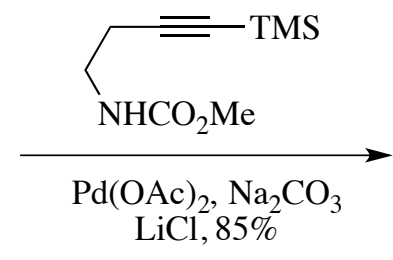

$\mathrm{LiCl}, 85 \%$<smiles>CC(=O)NCCc1cn([C@]2(CCN(C(C)=O)C(C)=O)CCNC2)c2ccccc12</smiles>

33

Buchwald's aryl amination methodology ${ }^{41}$ has also been applied toward the synthesis of indoles and indole derivatives. Palladium-catalyzed intermolecular amination of aryl bromide with inexpensive benzophenone hydrazone $\mathbf{3 4}$ afforded hydrazone 35 , which was converted to indole 36 upon heating under acidic conditions (Scheme 10). ${ }^{42}$ This method broadens the scope of the Fischer cyclization by providing an alterative method to prepare the requisite $N$-arylhydrazone precursors. 


\section{Scheme 10: Buchwald Indole Synthesis via Intramolecular Amination}

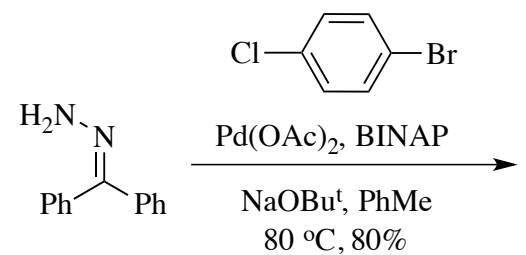

34

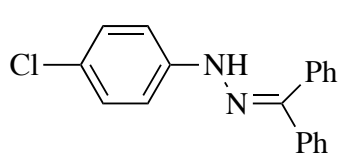

35

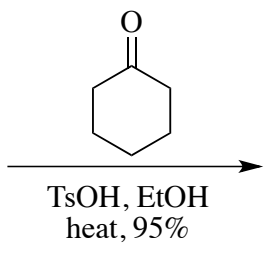<smiles>Clc1ccc2[nH]c3c(c2c1)CCCC3</smiles>

36

\subsection{Indole Synthesis via Reductive Cyclization}

An early example of indole synthesis via reductive heterocyclization was the Reissert method. ${ }^{43}$ In this route, an $o$-nitrobenzylcarbonyl compound 38, prepared through the condensation of an $o$-nitrotoluene (37) and an oxalic ester, underwent reductive cyclization the presence of zinc to afford indole-2-carboxylic acid derivatives, which then decarboxylated to give indole (1) (Scheme 11).

\section{Scheme 11: Reissert Method for Indole Preparation}

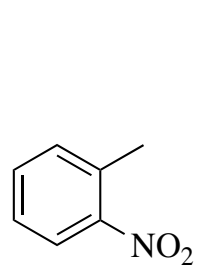

37

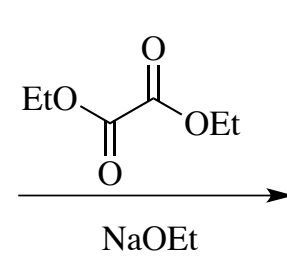<smiles>CCOC(=O)C(=O)Cc1ccccc1[N+](=O)[O-]</smiles>

38<smiles>CC(C)(C)O</smiles>

1

Another commonly used method in indole synthesis was the Leimgruber-Batcho route. ${ }^{44}$ This method, similar to the Reissert route, involved the condensation of $o$ nitrotoluenes 37 with dimethylformamide dimethyl acetal (DMFDMA), affording an intermediate $B$-(dimethylamino)-2-nitrostyrene 38 which then underwent reductive heterocyclization to afford indole (1) (Scheme 12). The success of the initial condensation was attributed to the increased acidity of the benzylic protons on account of the ortho nitro substituent. ${ }^{44}$ The addition of pyrrolidine resulted in the generation of a 
more reactive aminomethylenating reagent and was found to significantly shorten reaction times. $^{44}$

\section{Scheme 12: Leimgruber-Batcho Indole Synthesis}<smiles>Cc1ccccc1[N+](=O)[O-]</smiles>
37

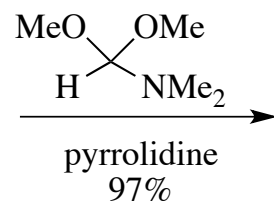

$97 \%$<smiles>CN(C)/C=C/c1ccccc1[N+](=O)[O-]</smiles>

38

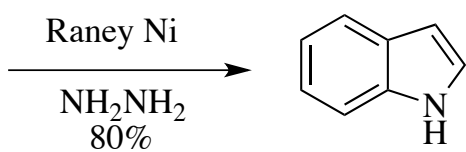

1

Another method for indole synthesis that has received considerable attention involves reductive cyclization of $o$-nitrostyrenes. This method was pioneered by Cadogan $^{45}$, who used trivalent phosphorous compounds to form indoles through deoxygenation of nitroaromatics. An early example involved heating $o$-nitrostyrene 39 in triethylphosphite, affording 2-phenylindole (40) in good yield (Scheme 13). ${ }^{45}$

\section{Scheme 13: Cadogan and Sundberg's Reductive Heterocyclization}

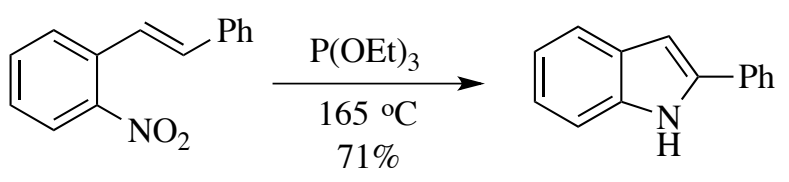

39

40

While this method is broadly applicable, it has been limited to small-scale applications due to its requirement of high temperatures $\left(>150{ }^{\circ} \mathrm{C}\right)$ as well as the generation of phosphorous waste. These conditions were also successful in preparing indoles from 2-nitrosobiphenyl compounds ${ }^{46}$, lending mechanistic evidence to the cyclization of analogous nitroaromatics proceeding through a nitroso intermediate. It was presumed that this cyclization proceeded through a nitrene intermediate generated by the sequential deoxygenation of the nitro group ${ }^{45}$ (Scheme 14). Addition of triethyl phosphite to nitroaromatic 39 followed by elimination of triethyl phosphite provides nitrosoarene 41, which was reduced to nitrene 42 through repetition of the addition- 
elimination sequence. Nitrene $\mathbf{4 2}$ then rapidly attacked the alkene to form heterocycle 43. Aromaticity was restored through $[1,5]$-sigmatropic rearrangement, producing indole 40.

\section{Scheme 14: Mechanism of Cadogan-Sundberg Indole Synthesis}<smiles>O=[N+]([O-])c1ccccc1/C=C/Pc1ccccc1</smiles>

39<smiles>O=Nc1ccccc1/C=C/P</smiles>

41
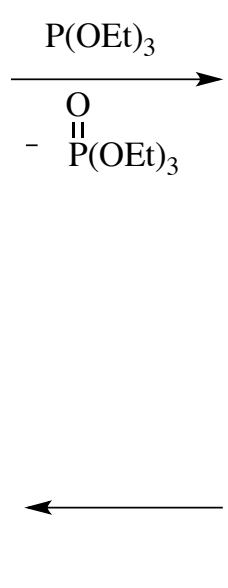

40<smiles>O=C1CCC(c2ccccc2)=CCc2ccccc2N1</smiles>

42<smiles>[Te][Te]</smiles><smiles>C1=c2ccccc2=NC1c1ccccc1</smiles>

43

An alternative mechanism has been proposed based on the isolation of hydroxyand ethoxy-indole by-products (Scheme 15 ). ${ }^{46}$ While the initial deoxygenation to afford nitroso compound $\mathbf{4 1}$ is widely accepted, the observation of oxy-indole side products could arise from direct cyclization of nitrosoaromatic $\mathbf{4 1}$ to afford hydroxyindole $\mathbf{4 4}$. It is then possible that hydroxyindole $\mathbf{4 4}$ could be further reduced to indole $\mathbf{4 0}$ by triethylphosphite.

Scheme 15: Alternative Mechanism of Sundberg-Cadogan Cyclization<smiles>O=Nc1ccccc1/C=C/c1ccccc1</smiles>

41<smiles>On1c(-c2ccccc2)cc2ccccc21</smiles>

44

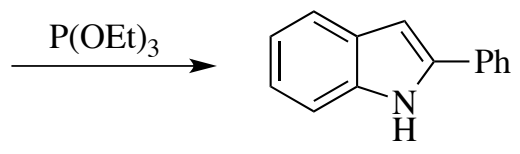

40

\subsection{Indole Preparation via Palladium-Catalyzed Reductive Heterocyclization}

Within the past few decades, interest in methods for indole synthesis has led to the discovery of numerous transition-metal catalysts capable of inducing the reductive 
heterocyclization of $o$-nitrostyrenes. ${ }^{47}$ While numerous metals have been found useful in such transformations, palladium-catalyzed routes employing carbon monoxide as a reducing agent have become the most popular. It is believed that these cyclizations proceed analogously to those reported by Cadogan and Sundberg, meaning that a nitrenoid intermediate likely forms via reduction of the nitro group by palladium/carbon monoxide. This transition-metal nitrene then attacks the ortho alkene to form the heterocycle, which undergoes [1,5]-sigmatropic rearrangement to give the indole. ${ }^{48}$ This mechanistic rationale is based on the proposed similarities between such reductive cyclizations and analogous cyclizations of azido aromatics. ${ }^{49}$

An early palladium-catalyzed reductive $N$-heterocyclization route to indoles and indazoles was reported by Watanabe et al. ${ }^{50}$ This method improved on the previous routes by providing milder reaction conditions involving palladium(II) and tin(II) chloride catalysts along with pressurized carbon monoxide. Under these conditions, $o$ nitrostyrene (45) was converted to indole (1) in moderate yield (Scheme 16). A selection of substituted indoles were prepared using this method, although yields were moderate at best even under fairly high carbon monoxide pressures.

\section{Scheme 16: Watanabe Palladium(II)-Catalyzed Reductive Heterocyclization}

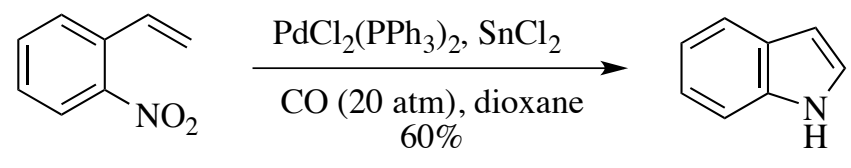

45

1

Davies $^{51}$ reported an improved route for similar reductive heterocyclizations at significantly lower carbon monoxide pressures. Such reactions were achieved using $0.1 \%$ catalyst loadings under $1 \mathrm{~atm}$ carbon monoxide (as opposed to $20 \mathrm{~atm}$ used by Watanabe ${ }^{50}$ ). It was also observed that the stereochemistry of the olefin did not have an 
effect on the reaction. Reaction of a 1:1 mixture of both $E$ - and $Z$-nitrostilbene isomers (39) afforded indole $\mathbf{4 0}$ in excellent yield (Scheme 17).

\section{Scheme 17: Davies Reductive $N$-Heterocyclization of Isomeric Nitrostilbenes}

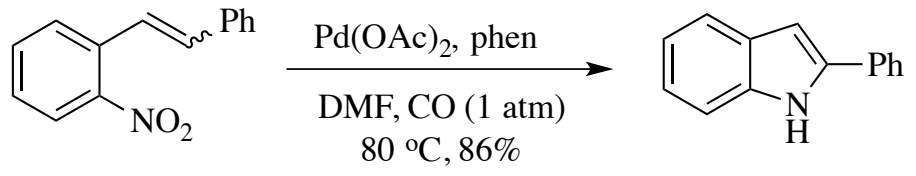

39

40

A similar transformation was reported by Söderberg in $1997 .{ }^{52}$ In an attempted methoxycarbonylation of styrene 46 , indole 47 was isolated as the sole product (Scheme 18). While this result was unexpected, it was also not unprecedented based on the previously mentioned examples.

\section{Scheme 18: Söderberg Reductive Heterocyclization}<smiles>C=Cc1c(Br)cccc1[N+](=O)[O-]</smiles>

46

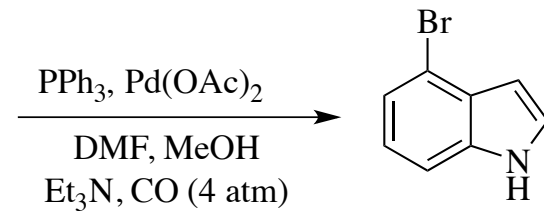

47

Through a detailed optimization study of the reaction conditions, it was found that methanol and triethylamine were not required to induce the cyclization. ${ }^{52}$ It was also found that a number of solvents, including DMF, acetonitrile, and methanol, all resulted in formation of the desired indole product. Numerous palladium sources were also found successful, including $\mathrm{Pd}(\mathrm{OAc})_{2}, \mathrm{PdCl}_{2}(\mathrm{MeCN})_{2}, \mathrm{Pd}(\mathrm{dba})_{2}$, and $10 \% \mathrm{Pd} / \mathrm{C}$. The array of plausible conditions makes this method highly attractive and broadly applicable.

The Söderberg method provides a number of significant improvements over previously reported conditions. In comparison to Watanabe's system ${ }^{50}$, the Söderberg conditions require significantly lower temperatures while also eliminating the need for a Lewis acid additive. It is also an improvement over alternative routes involving aniline 
derivatives ${ }^{34}$ in that (a) anilines are typically prepared through the reduction of the corresponding nitroarene, introducing an additional step to the route. In addition, the use of palladium (0) catalysts eliminates the need for an oxidant required when using palladium (II). From an environmental standpoint, the main by-product of the reaction is carbon dioxide, which is a significant improvement over previous routes using $\operatorname{tin}^{50}$ or phosphorous ${ }^{45}$ additives. This also leads to simplified workup procedures.

The plausible mechanisms for the reductive heterocyclization of $o$-nitrostyrenes are presented in Scheme $19 .^{53}$ The difficulty of isolating intermediates has prevented researchers from fully understanding the exact mechanism, so multiple plausible routes have been proposed based on numerous observations.

The mechanism initially proceeds through the palladium-catalyzed reduction of nitrostyrene 45 by carbon monoxide to give intermediate 48 . Carbon monoxide insertion into one of the palladium-oxygen bonds affords palladacycle 49 , which, upon reductive elimination of carbon dioxide, affords palladium-bound nitrosarene 50. From this point, the mechanism could proceed in three ways:

Path $\mathrm{A}^{51}$ involves liberation of palladium from nitrosarene $\mathbf{5 0}$ to afford nitrosostyrene 51, which can undergo 6-pi electrocyclization to afford nitronate $\mathbf{5 2}$. [1,5]-hydride shift of $\mathbf{5 3}$ followed by isomerization of intermediate $\mathbf{5 3}$ produces $\mathrm{N}$ hydroxyindole (54). Reduction of $\mathbf{5 4}$ by another equivalent of carbon monoxide then affords indole (1).

Path B begins with insertion of carbon monoxide to palladium-bound nitrosarene $\mathbf{5 0}$ to give palladacycle 55. Subsequent reductive elimination of carbon dioxide produces palladium-bound nitrene 56, which could undergo 6 pi-electrocyclization to afford six- 
membered palladacycle 57. Reductive elimination of palladium from $\mathbf{5 7}$ would furnish heterocycle 58, which could then isomerize to indole (1) through a [1,5]-hydrogen shift.

Path $\mathrm{C}$ is similar to Path $\mathrm{B}$, although initial reductive elimination of palladium from 56 affords free nitrene $\mathbf{5 9}$. Nitrene $\mathbf{5 9}$ could then undergo an electrocyclizationisomerization process (as described for Path B) to ultimately afford indole (1).

\section{Scheme 19: Proposed Mechanism(s) of Reductive $N$-Heteroannulation}

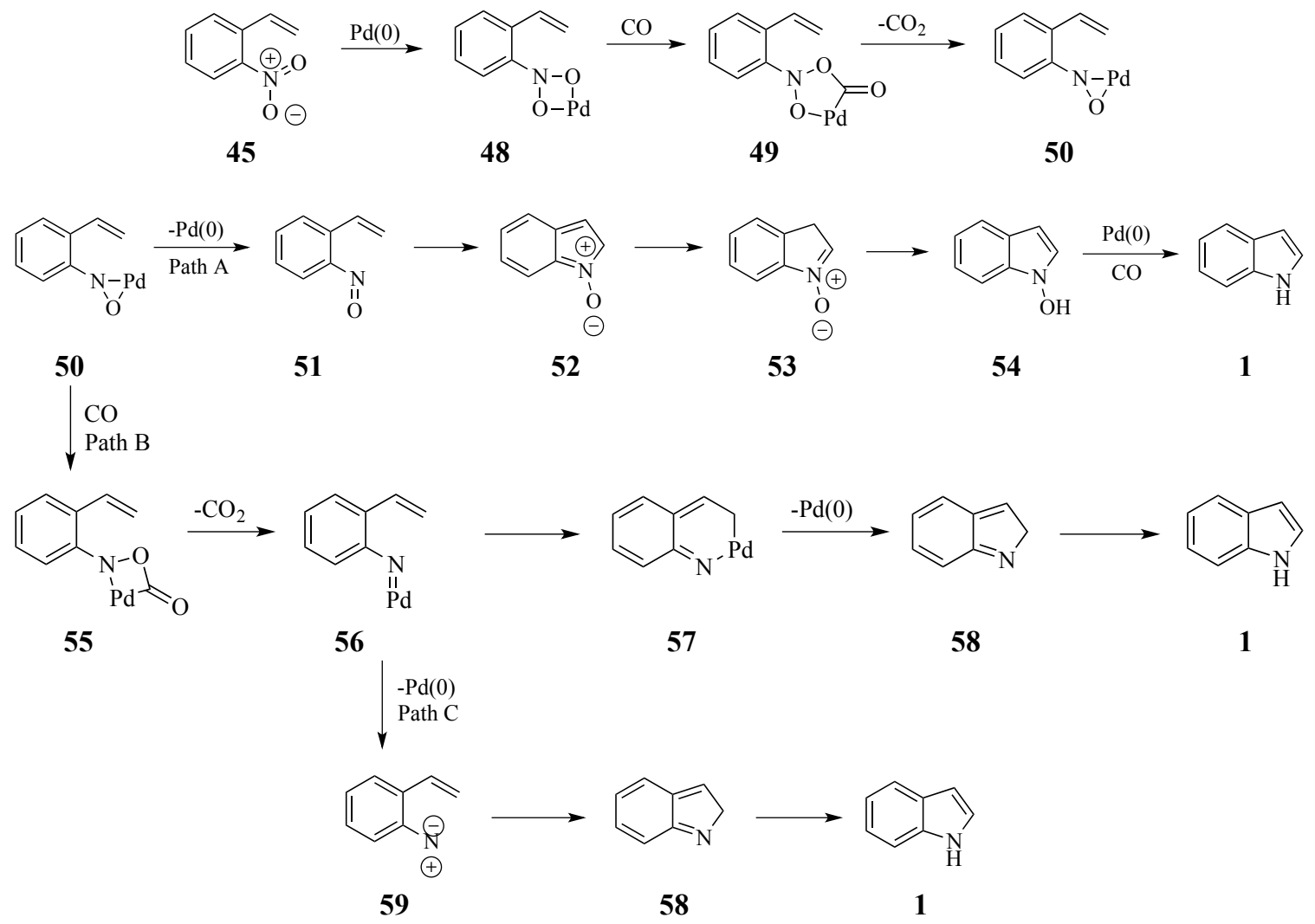

While the exact mechanism of this transformation is not fully understood, several observations lend some evidence to potential intermediates. It is believed that the proximity of the nitro group to the alkene is crucial in facilitating the reaction, as observed for two isomeric nitrostilbenes. ${ }^{52}$ While 2-nitrostilbene (39) reacts to give 2phenylindole (40) in quantitative yield, 4-nitrostilbene (60) is recovered unchanged, also 
in quantitative yield (Scheme 20). This result hints that initial coordination of the metal to the alkene is necessary for the initial reduction of the nitro group. ${ }^{52}$

\section{Scheme 20: Potential Palladium-Olefin Coordination-Mediated Reduction}<smiles>O=[N+]([O-])c1ccccc1/C=C/c1ccccc1</smiles>

39<smiles>O=[N+]([O-])c1ccc(/C=C/c2ccccc2)cc1</smiles>

60

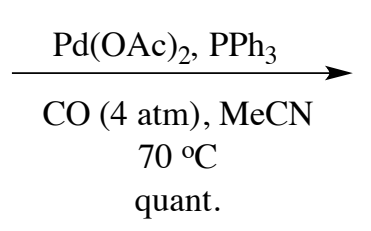

quant.

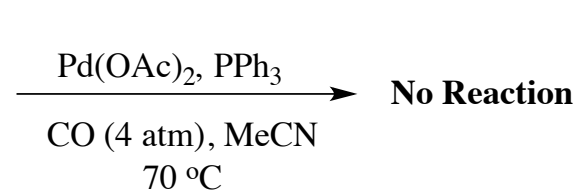<smiles>c1ccc(-c2cc3ccccc3[nH]2)cc1</smiles>

40

The prevalence of $\mathrm{N}$-ethoxyindoles as side products of the Cadogan-Sundberg indole synthesis provides justification for path $\mathrm{A} \cdot{ }^{54,55}$ While the origin of these products was not initially studied, recent work by Peet et $a l .{ }^{47}$ determined through ${ }^{18} \mathrm{O}$ labeling experiments that the oxygen found in the $N$-alkoxyindole originates from the nitro group, not from triethylphosphite (Scheme 21). This means that the labeled nitro group of $\mathbf{6 1}$ is only reduced to the corresponding nitroso compound $\mathbf{6 2}$ and not further reduced to the nitrene. Nitroso intermediate $\mathbf{6 2}$ then cyclizes to afford intermediate $\mathbf{6 3}$, which could lose a proton to give deprotonated $N$-hydroxyindole 64, which then attacks an ethyl group from the triethyl phosphate (produced in the initial deoxygenation of 61) to afford $N$ ethoxyindole $\mathbf{6 5}$. 


\section{Scheme 21: ${ }^{18}$ O Labeling Study of Cadogan-Sundberg Reductive Cyclization}

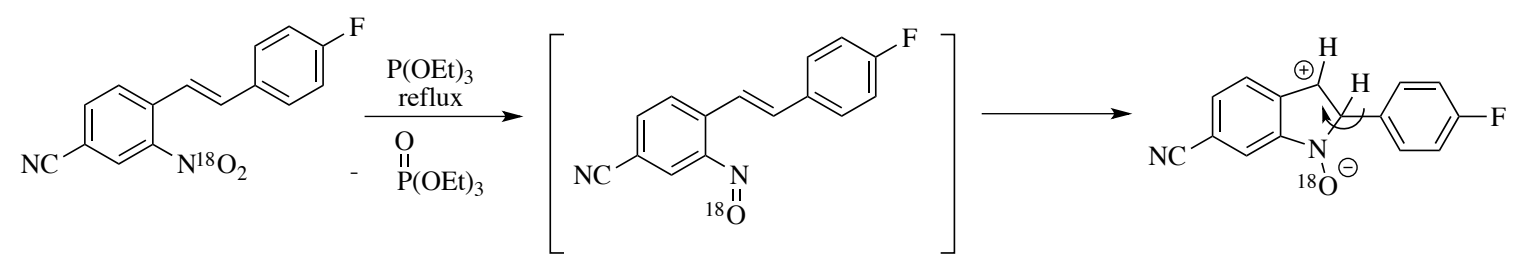

61

62

63

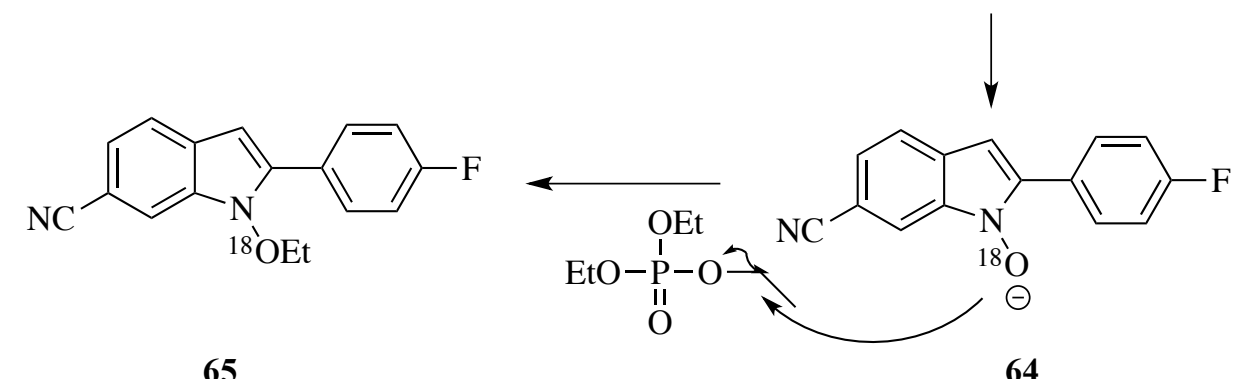

Watanabe $^{50}$ postulated that formation of the free nitrene (path C, Scheme 19) was

likely based on the evolution of carbon dioxide over the course of the reaction, although carbon dioxide is also generated in the initial reduction of the nitro group to the corresponding nitroso compound as well as in the reduction of nitrosarene $\mathbf{5 0}$ to palladium-bound nitrene 56 (path B, Scheme 19). This nitroso/nitrene species could then cyclize to form the five-membered ring, followed by a [1,5]-hydride shift to afford the indole product.

The aforementioned [1,5]-hydride shift was also studied by Watanabe ${ }^{50}$ (Scheme $^{-}$ 22). Through deuterium labeling of $o$-nitrostyrene $\mathbf{6 5}$, it was determined that the 3 methylindole product 67 was deuterated at both the 1- and 2-positions. This means that the nitrogen atom must abstract a deuterium from the $\beta$-carbon of the olefin during the cyclization process. This then became the basis for the proposed $[1,5]$-sigmatropic rearrangement of proposed intermediate $\mathbf{6 6}$ to afford indole $\mathbf{6 7 .}$ 


\section{Scheme 22: Deuterium-Labeling Study of [1,5]-Sigmatropic Rearrangement}

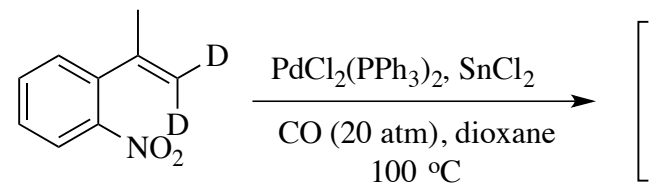

65

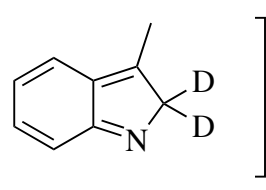

66

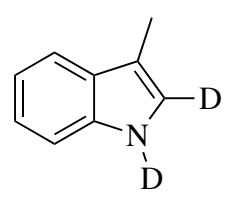

67

Söderberg's work over the past number of years has focused on applying this methodology toward the synthesis of a number of compounds including tryptophan derivatives $^{56}$, bicyclic heteroaromatics ${ }^{57}$, carbazole alkaloids $^{58}$, mushroom metabolites ${ }^{59}$, and various natural products ${ }^{60,61,62,63}$. The ensuing sections will discuss novel applications of palladium-catalyzed reductive $N$-heterocyclizations in the preparation of indoles and indole derivatives. 


\section{Chapter 2}

\section{A Base Modulated Synthesis of Indoles and Quinolines}

2.1 Introduction 


\subsection{Introduction}

One of the most challenging tasks in synthetic organic chemistry is finding selectivity in reactions. Often times, researchers struggle to target one specific functional group in the presence of other similar functionalities. The ideal reaction would only alter the desired functionality while leaving other reactive moieties untouched. In the same sense, the ability to prepare different products from a common starting material is also of great use.

Through previous work within Söderberg's group aimed at the preparation of 2,3substituted indoles, an interesting result was obtained. Upon cyclization of cyanosubstituted alkene 68 , the expected indole 69 was isolated in moderate yield along with a small amount of quinoline $\mathbf{7 0}$ (Scheme 23). ${ }^{64}$ This unexpected result was of interest for it presented the potential for preparation of two different yet useful products from a common starting material.

\section{Scheme 23: Initial Cyclization of Cyanoalkene}

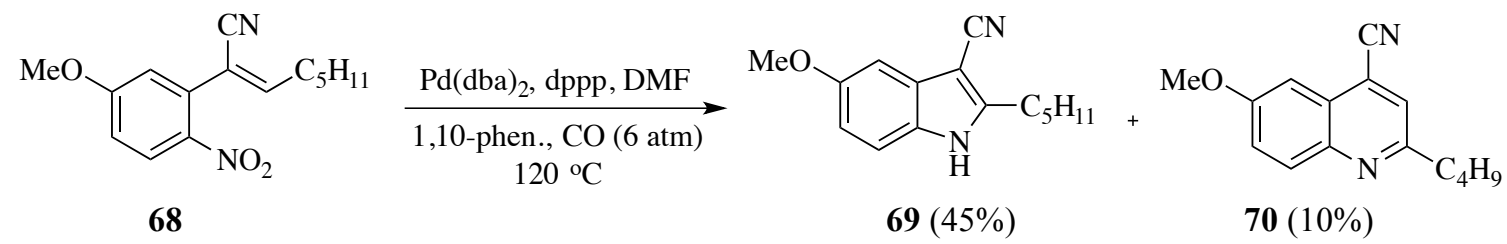

Upon examination of the literature, Akazome et al. ${ }^{50}$ reported a similar result. In the attempted cyclization of $o$-nitrocinnamaldehyde (71) using $\mathrm{PdCl}_{2}\left(\mathrm{PPh}_{3}\right)_{2}$ and $\mathrm{SnCl}_{2}$ under carbon monoxide, quinoline 72 was isolated albeit in low yield (Scheme 24). Similar reaction of $o$-nitrochalcone (73) afforded both indole 74 and quinoline 75. 


\section{Scheme 24: Akazome's Synthesis of Indoles and Quinolines}

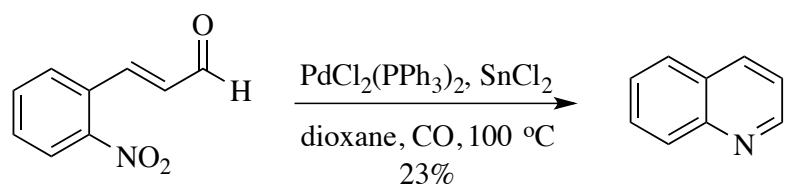

71

72

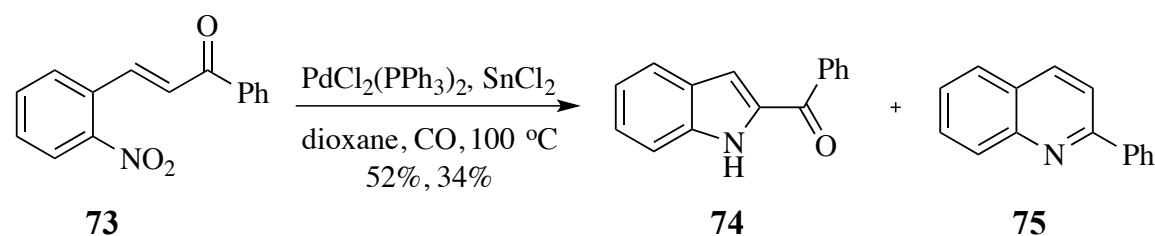

Although it can be envisioned that the aforementioned cyclizations may proceed through reduction of the nitro group to the corresponding aniline in a similar fashion to analogous indole syntheses reported by Hegedus, ${ }^{34}$ Akazome found that treatment of 2aminostilbene with the established conditions afforded neither indole $\mathbf{7 4}$ or quinoline 75. ${ }^{50}$ This clearly demonstrates a different mechanism, likely involving the reduction of the nitro group to a nitroso or nitrene (as discussed in Chapter 1).

\subsection{Results and Discussion}

An investigative study was launched based based on both Akazome's results ${ }^{50}$ as well as Söderberg's ${ }^{64}$. The formation of quinoline $\mathbf{7 0}$ from cyanoalkene $\mathbf{6 8}$ was unexpected, however, upon consideration of the reaction conditions, it was proposed that the presence of a base, specifically 1,10-phenanthroline, could have altered the predicted selectivity. It was decided to examine whether simply varying the additives used in the reaction could alter the selectivity. In screening various conditions for the cyclization of 76, Banini et al. found that the combination of $\mathrm{Pd}(\mathrm{OAc})_{2}$ and $\mathrm{PPh}_{3}$ (deemed "conditions A") produced exclusively indole 77, while the addition of DBU to the aforementioned conditions (deemed “conditions B”) afforded exclusively quinoline 78 (Scheme 25). This 
led to the proposition that the reaction selectivity and product observed could be modulated through addition or exclusion of base in the reaction mixture.

\section{Scheme 25: Investigation of Cyclization Conditions}

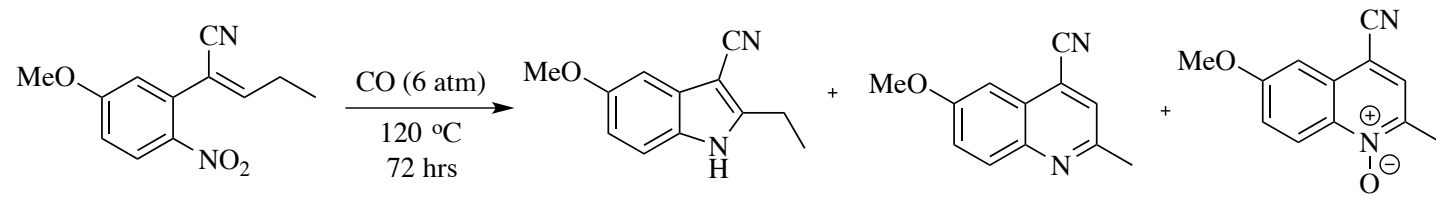

76

$1 \operatorname{Pd}(\mathrm{dba})_{2}-\mathrm{dppp}$

$2 \operatorname{Pd}(\mathrm{dba})_{2}-\mathrm{dppp}-\mathrm{NEt}_{3}$

$3 \operatorname{Pd}(\mathrm{dba})_{2}-\mathrm{dppp}$ - phen - DBU

$4 \mathrm{Pd}(\mathrm{OAc})_{2}-\mathrm{PPh}_{3}$

$5 \mathrm{Pd}(\mathrm{OAc})_{2}-\mathrm{PPh}_{3}-\mathrm{DBU}$
77

$6 \%$

$3 \%$

$---$

$79 \%$

$\mathrm{dppp}=1,3$-bis(diphenylphosphino)propane, $\mathrm{phen}=1,10$-phenanthroline, $\mathrm{DBU}=1,8$-diazabicyclo[5.4.0]undec-7-ene

To expand the scope of these findings, Banini et al. prepared a number of additional substrates to then subject to the two sets of cyclization conditions. Four unsaturated nitriles $(\mathbf{8 1}, \mathbf{6 8}, \mathbf{8 3}, \mathbf{8 5})$ were prepared through Knoevenagel condensation ${ }^{65}$ of the corresponding 2-arylacetonitrile with ethanal or hexanal (Table 1).

Table 1: Knoevenagel Condensation of 2-Arylacetonitrile ${ }^{\mathrm{a}}$

\section{Entry} Substrate

$\mathrm{MeO}$<smiles>N#CCc1ccccc1[N+](=O)[O-]</smiles>

1

80<smiles>COc1ccc([N+](=O)[O-])c(CC#N)c1</smiles>

2

80
Aldehyde

Product (yield)

$\mathrm{MeCHO}$<smiles>CC=C(C#N)c1cc(OC)ccc1[N+](=O)[O-]</smiles>

$81(41 \%)$

$\mathrm{C}_{5} \mathrm{H}_{11} \mathrm{CHO}$

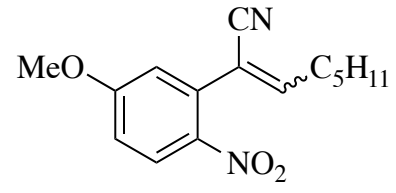

$68(89 \%)$ 
Table 1: Knoevenagel Condensation of 2-Arylacetonitrile ${ }^{\text {a }}$ (cont'd)

Entry Substrate<smiles>N#CCc1ccccc1[N+](=O)[O-]</smiles>

82<smiles>COc1ccc([N+](=O)[O-])c(CC#N)n1</smiles>

84

3

4
Aldehyde

$\mathrm{MeCHO}$

a) Conditions: $\mathrm{AcOH}$, piperidine, $\mathrm{PhH}$

In addition to the cyanoalkenes, two ester-functionalized alkenes $(\mathbf{8 7}, \mathbf{8 9})$ were
Product (yield)<smiles>CC=C(C#N)c1ccccc1[N+](=O)[O-]</smiles>

$83(21 \%)$

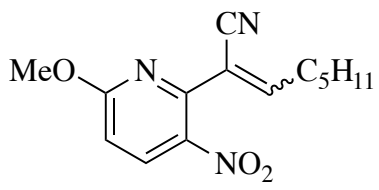

$85(62 \%)$ prepared using similar methodology ${ }^{65}$ (Table 2).

Table 2: Preparation of Ester-Substituted Alkenes ${ }^{a}$

Entry Substrate<smiles>CC(=O)Cc1ccccc1[N+](=O)[O-]</smiles>

86

2

88

1<smiles>COc1ccc([N+](=O)[O-])c(CC(=O)OC(C)(C)C)c1</smiles>
Aldehyde

$\mathrm{MeCHO}$

$\mathrm{MeCHO}$

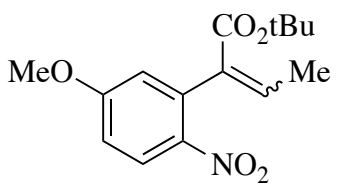

89 (73\%)

a) Conditions: $\mathrm{AcOH}$, piperidine, $\mathrm{PhH}$

Two additional substrates were prepared via Kosugi-Migita-Stille cross-coupling between 1-iodo-2-nitrobenzene (90) and either 1-propene-1-yltributyltin and 1-phenyl-1propen-1-yltributyltin (Scheme 26). 


\section{Scheme 26: Preparation of 2-Nitrostyrenes via Stille Coupling}

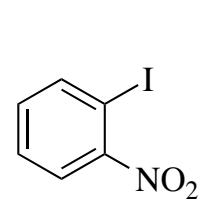

90

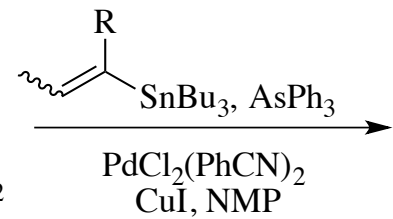

CuI, NMP

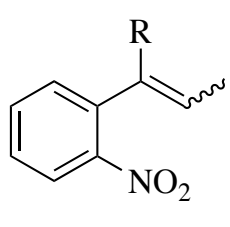

$91(\mathrm{R}=\mathrm{Ph}, 84 \%)$

$92(\mathrm{R}=\mathrm{H}, 73 \%)$

With a number of substrates prepared, Banini et al. then examined the basemodulated cyclization. Cyclization of each ortho-nitrostyrene derivative using conditions A afforded exclusively indoles $(69,77$, and 93-98, 17) in good isolated yields (Table 3 , entries 1-6 and 10,11,13). The corresponding quinolines were not observed in the crude spectra from these reactions. Reaction of the same substrates using Conditions B gave different results depending on the substituent on the alkene. As anticipated, the cyanosubstituted substrates furnished the corresponding quinolines 70, 78, 99-101 (entries 1-5). Disappointingly, ester $\mathbf{8 7}$ did not undergo cyclization to afford a quinoline under the basic conditions B. Three additional bases were examined for ester $\mathbf{8 7}$, however, indole 96 was formed in all cases (entries 6-9). This made it apparent that the formation of quinolines was limited to the cyano-substituted alkenes. In contrast, azaquinoline $\mathbf{1 0 3}$ was formed upon reaction of the pyridine derivative $\mathbf{8 9}$ (entry 10). In addition to $\mathbf{1 0 3}$, one additional product was isolated and identified as the azaindole 102. It is worth noting that $\mathbf{1 0 2}$ had lost a methyl group compared to azaindole $\mathbf{9 7}$ formed through reaction of $\mathbf{8 9}$ under conditions A. It was apparent that DBU was sufficiently basic to deprotonate all nitriles and esters examined as evidenced by the immediate change in color of the reaction mixtures upon addition of base, indicative of anion formation. In contrast, no color change was observed for the significantly less acidic substrates 91 and 92 upon addition of base. Reaction of 91 using DBU as the base furnished only indole 98 in $95 \%$ 
yield (entry 11). However, reaction of 91 under conditions $\mathrm{B}$ using $t$-BuOK as the base (as opposed to DBU) initially produced a deep blue color (indicative of anion formation) and furnished quinoline 104 (entry 12). The least acidic substrate studied (92) afforded exclusively indole 17 under both conditions A and conditions B, regardless of what base was used (entry 13), indicating that neither $t$-BuOK nor DBU were basic enough deprotonate the substrate.

Table 3. Cyclizations to Afford Substituted Indoles and/or Quinolines

\begin{tabular}{lll}
\hline Entry & Nitroalkene & Conditions A \\
\hline
\end{tabular}<smiles>COc1ccc([N+](=O)[O-])c(/C(C#N)=C/P)c1</smiles>

$76(\mathrm{R}=\mathrm{Et})$

$81(\mathrm{R}=\mathrm{Me})$ $68(\mathrm{R}=\mathrm{Pent})$<smiles>C/C=C(/C#N)c1ccccc1[N+](=O)[O-]</smiles>

4

5

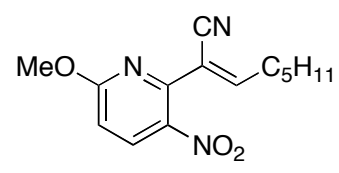

85<smiles>CC=C(C(C)=O)c1ccccc1[N+](=O)[O-]</smiles>

87

7

8

9

10<smiles></smiles>

89<smiles>[R]c1[nH]c2ccc(OC)cc2c1C#N</smiles>

$77(79 \%)$ $93(82 \%)$ $69(91 \%)$<smiles>Cc1[nH]c2ccccc2c1C#N</smiles>

$94(80 \%)$<smiles>CCCc1cc(C#N)c2cc(OC)ccc2n1</smiles>

$78\left(\mathrm{R}^{\prime}=\mathrm{Me}, 88 \%\right)$

$99\left(\mathrm{R}^{\prime}=\mathrm{H}, 88 \%\right)$

$70\left(\mathrm{R}^{\prime}=\mathrm{Bu}, 79 \%\right)$<smiles>N#Cc1ccnc2ccccc12</smiles>

$100(83 \%)$

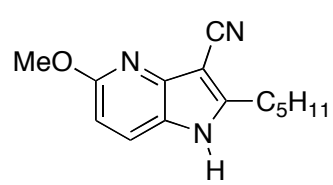

$95(74 \%)$<smiles>CC(=O)c1[nH]c2ccccc2c1C(C)=O</smiles>

$96(85 \%)$

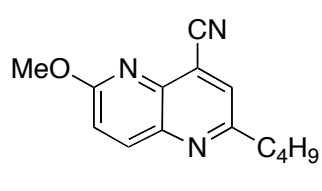

$101(69 \%)$<smiles>CC(=O)c1[nH]c2ccccc2c1C(C)=O</smiles>

96 (DBU, 87\%)

$96\left(\mathrm{NEt}_{3}, 78 \%\right)$

96 (t-BuOK, 35\%)

96 (NaHMDS, 84\%)<smiles>CCOC(=O)c1c(C)[nH]c2ccc(OC)nc12</smiles>

97 (89\%)<smiles>CCCCC(=O)c1ccnc2ccc(OC)nc12</smiles>

$102(24 \%)^{\mathrm{c}}$

$103(63 \%)^{\mathrm{c}}$ 
Table 3. Cyclizations to Afford Substituted Indoles and/or Quinolines (cont'd)

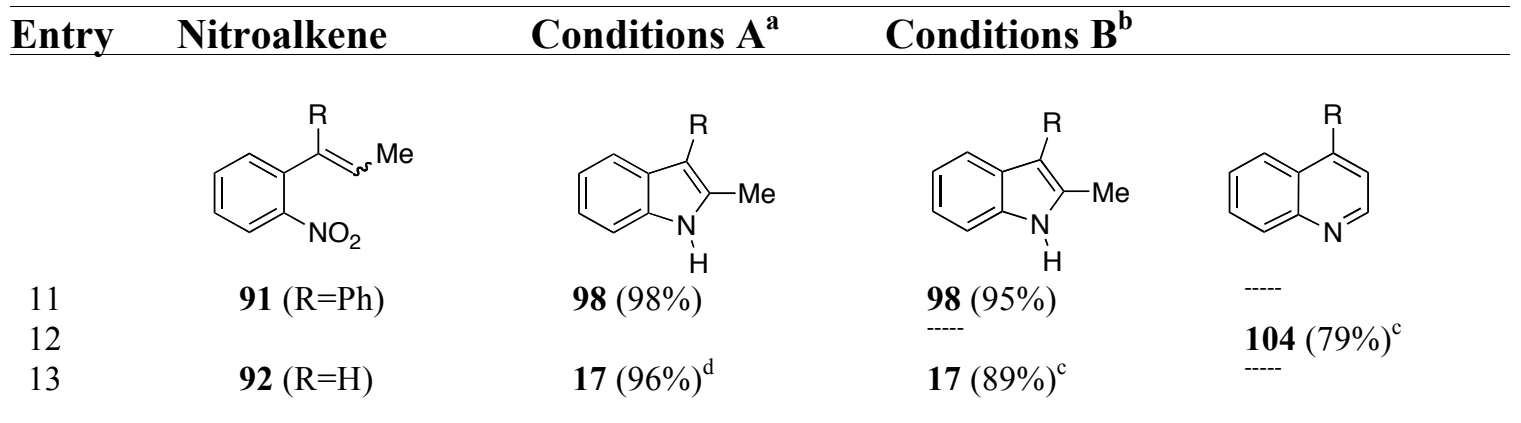

a) Conditions $\mathrm{A}: \mathrm{Pd}(\mathrm{OAc})_{2}, \mathrm{PPh}_{3}, \mathrm{DMF}, \mathrm{CO}(6 \mathrm{~atm}), 120^{\circ} \mathrm{C}, 72 \mathrm{~h}$.

b) Conditions B: $\mathrm{Pd}(\mathrm{OAc})_{2}, \mathrm{PPh}_{3}, \mathrm{DBU}, \mathrm{DMF}, \mathrm{CO}(6 \mathrm{~atm}), 120^{\circ} \mathrm{C}, 72 \mathrm{~h}$.

c) $\mathrm{t}-\mathrm{BuOK}$ in place of $\mathrm{DBU}$.

d) $\mathrm{Pd}(\mathrm{OAc})_{2}, \mathrm{PPh}_{3}, \mathrm{MeCN}, \mathrm{CO}(4 \mathrm{~atm}), 70{ }^{\circ} \mathrm{C}, 15 \mathrm{~h}$.

Two additional substrates $\mathbf{1 0 5}$ and $\mathbf{1 1 1}$, which cannot form fully aromatic quinolines without migration or loss of a carbon chain were also examined. Cyanoalkene 105 was prepared through condensation of 2-nitro-5-methoxy-1-cyanomethylbenzene (80) with 2-phenylpropanal (Scheme 27). Nitrile 105 was then subjected to reaction conditions A and B. Not surprisingly, under conditions A, the expected indole 106 was isolated in good yield (Scheme 27). In contrast, three different indoles, 107 and an inseparable mixture of $\mathbf{1 0 8}$ and $\mathbf{1 0 9}$ were obtained under the basic conditions B. The structures of 108 and 109 were elucidated using 2D NMR techniques including COSY, HMQC, HMBC, and NOESY. Interestingly, a significant part of the starting material was lost in the formation of indole $\mathbf{1 0 9}$. 


\section{Scheme 27: Preparation and Cyclization of Cyanoalkene 105}<smiles>COc1ccc([N+](=O)[O-])c(CC#N)c1</smiles>

80<smiles>COc1ccc([N+](=O)[O-])c(/C(C#N)=C/[C@H](C)c2ccccc2)c1</smiles>

105 (53\%)<smiles>COc1ccc2[nH]c(C(C)c3ccccc3)c(C#N)c2c1</smiles>

106 (76\%)

$\mathrm{Pd}(\mathrm{OAc})_{2} \mid \mathrm{DBU}$

$\mathrm{PPh}_{3}, \mathrm{CO}$<smiles>C=C(c1ccccc1)c1[nH]c2ccc(OC)cc2c1C#N</smiles>

107 (38\%)<smiles>COc1ccc2[nH]c(C(C)(O)c3ccccc3)c(C#N)c2c1</smiles>

$108(47 \%)$<smiles>COc1ccc2[nH]cc(C#N)c2c1</smiles>

$109(8 \%)$

Wittig reaction of cyclohexane carbaldehyde formed iodoalkene 110, which then underwent Kosugi-Migita-Stille cross-coupling with 2-nitrophenyl stannane to afford cyanoalkene 111 (Scheme 28). As anticipated, indole 112 was formed under conditions A, albeit in relatively low isolated yield. Under the basic conditions B, a low yield of indole 112 in addition to indole 113 featuring an oxidized cyclohexyl group were isolated. This outcome was interpreted as the result of a competing cyclization of $\mathbf{1 1 1}$ to $\mathbf{1 1 2}$ and cyclization of the anion formed through deprotonation of $\mathbf{1 1 1}$ to give $\mathbf{1 1 3 .}$

Scheme 28: Preparation and Cyclization of Cyanoalkene 111

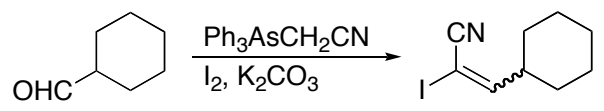

$110(17 \%)$

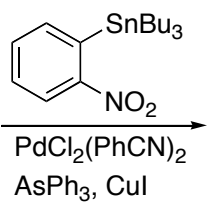

$$
\mathrm{AsPh}_{3}, \mathrm{Cul}
$$

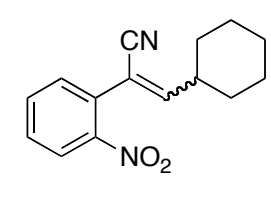

111 (48\%)

$\mathrm{Pd}(\mathrm{OAc})_{2} \mid \mathrm{DBU}$

$\mathrm{PPh}_{3}, \mathrm{CO}$

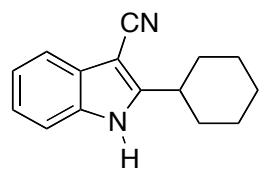

$112(7 \%)$

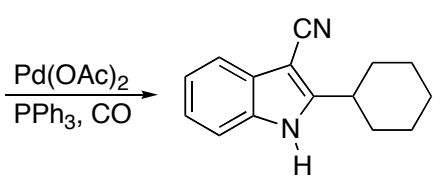

$112(48 \%)$

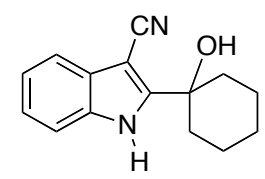

$113(31 \%)$ 
The difference in chemoselectivity between the nitriles and the esters was puzzling. It was unclear why either indoles or quinolines were obtained from the nitrilesubstituted substrates, while the esters afforded exclusively indoles under both sets of conditions. Presumably, the $\mathrm{pKa}$ for the substrates having either a nitrile or an ester moiety must be very similar in magnitude. For example, the pKa values for $\mathrm{CH}_{3} \mathrm{CN}$ and $\mathrm{CH}_{3} \mathrm{CO}_{2} \mathrm{Et}$ have been reported as 24.5 and 25.0, respectively. ${ }^{66}$ In addition, the color changes observed upon addition of base to both the ester and nitrile-substituted substrates likely indicated that the conjugate base was formed, thus raising questions regarding the resonance forms of each anion.

To probe the electronic distribution of anions formed, two similar substrates $\mathbf{8 1}$ and 114, differing only in the electron-withdrawing group, $\mathrm{CN}$ vs. $\mathrm{CO}_{2} \mathrm{Et}$, respectively, were compared. Each substrate was deprotonated with $t$ - $\mathrm{BuOK}$ in $t-\mathrm{BuOH}$, then methyl iodide was added to the mixture with the intent of trapping the intermediate to determine the identity of the anion formed through the initial deprotonation (Scheme 29). In doing such, two different products were obtained. After workup and purification, ester 114 gave exclusively $N$-methoxyindole 115, while on the other hand, nitrile $\mathbf{8 1}$ furnished quinoline- $N$-oxide 116. As was noticed for two previous indole products ( 25 and $\mathbf{3 2}$ ), part of the alkyl chain was lost during the formation of indole 115. The drastically different results obtained through these two experiments under identical reaction conditions confirmed that esters and the nitriles have different chemoselectivity. The reason for this difference is presently unknown. 


\section{Scheme 29: Attempt to Trap Reactive Intermediates}

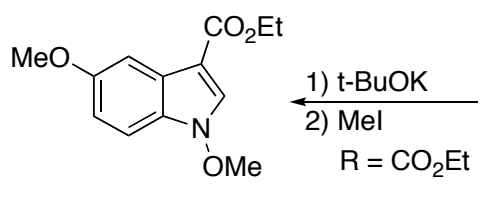

$--$

$115(83 \%)$

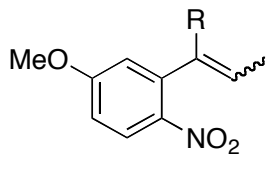

$81(\mathrm{R}=\mathrm{CN})$

$114\left(\mathrm{R}=\mathrm{CO}_{2} \mathrm{Et}\right)$

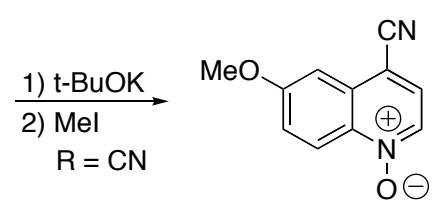

$116(53 \%)$

The mechanisms for the reactions leading to either indole or quinoline products and indoles wherein a carbon-carbon bond has been broken and/or a side-chain oxidized are not clear at this time. In the absence of a base, the reaction likely proceeds via a deoxygenation producing a nitroso compound followed by either a) a second deoxygenation to give a nitrenenoid intermediate and ultimately an indole or b) an electrocyclic ring closure to afford an $N$-hydroxy indole followed by a palladiumcatalyzed deoxygenation as discussed in Chapter 1 (Scheme 30). However, this mechanistic rationale accounts for the formation of indoles without carbon-carbon bond cleavage or side-chain oxidation, both of which were observed in a few of the previously mentioned examples.

\section{Scheme 30: Potential Mechanism(s) for Cyclization in Absence of Base}

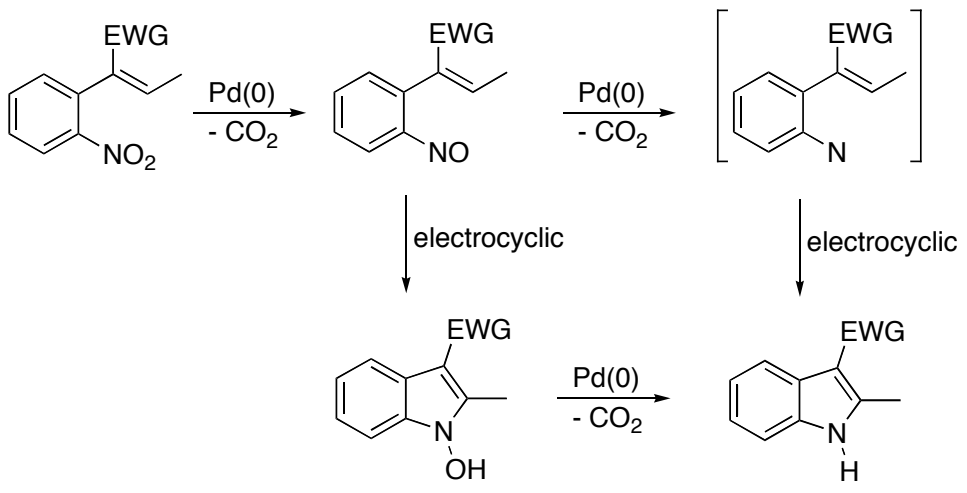

The mechanistic picture is slightly more complex in the presence of a base.

Addition of a sufficiently strong base to cyanoalkene $\mathbf{8 3}$ results in the formation of the conjugate base $\mathbf{1 1 7}$ as evidenced by the immediate formation of a deep blue solution 
(Scheme 31). The nitro group of $\mathbf{1 1 7}$ is likely deoxygenated by the catalyst system to form nitrosarene 118. Nucleophilic addition of the carbanion of $\mathbf{1 1 8}$ to the nitroso group followed by protonation-deprotonation and elimination of hydroxide (119 to 120) would furnish quinoline $\mathbf{1 0 0 .}$

\section{Scheme 31: Proposed Mechanism for Quinoline Formation}<smiles>C/C=C(\C#N)c1ccccc1[N+](=O)[O-]</smiles>

83<smiles>C=C[C-]C=C(C#N)c1ccccc1[N+](=O)[O-]</smiles>

117<smiles>N#CC(C=O)c1ccccc1[N+](=O)[O-]</smiles><smiles>N#C/C(=C/[O-])c1ccccc1[N+](=O)[O-]</smiles><smiles>N#C/C(=C/C=O)c1ccccc1N=O</smiles>

118<smiles>CC1(C)C=C(C#N)c2ccccc2N1O</smiles>

119

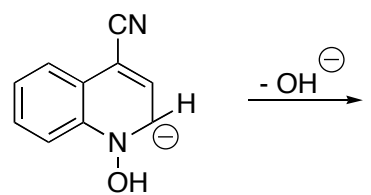

120<smiles>N#Cc1ccnc2ccccc12</smiles>

100

Makosza $e t a l^{67}$ have reported the formation of quinoline- $N$-oxides (such as 79,

Scheme 25) by treatment of compound 76, and related substrates, with triethylamine and trimethylsilyl chloride (Scheme 32). This result raised the question of whether the basemodulated cyclization forming quinolines (conditions B) was simply a palladiumcatalyzed reduction of the quinoline $N$-oxide $\mathbf{7 9}$ formed through nucleophilic attack of the initially formed carbanion to the nitro group of $\mathbf{7 6}$ without prior reduction of the nitro group to a nitrosarene. Submitting quinioline $N$-oxide 79 to conditions B did indeed furnish quinoline $\mathbf{7 6}$, however, the yield of quinoline $\mathbf{7 8}$ was only $24 \%$ after the same length of time required to produce quinoline $\mathbf{7 8}$ in $88 \%$ yield directly from $\mathbf{7 6}$ using our palladium-catalyzed methodology. 


\section{Scheme 32: Mechanistic Analysis of Quinoline Formation}

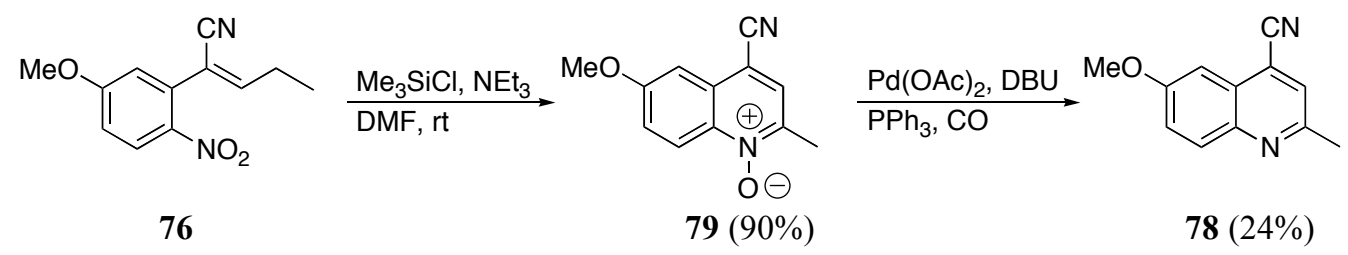

While this result is not conclusive, it indicates that the palladium-catalyzed reaction likely involves a different pathway than substrate (76) $\rightarrow$ quinoline- $N$-oxide (79) $\rightarrow$ quinoline (78). It is feasible that the palladium-catalyzed reduction of nitroaromatic 76, resulting in the formation of the nitroso-intermediate is faster in relation to nucleophilic addition to the nitro group in cases wherein a quinoline is formed. In the event, carbanion addition could then occur to the nitroso group (as depicted in Scheme 31) as opposed to the nitro group as reported by Makosza. ${ }^{67}$ Another explanation could be that nucleophilic addition to the nitro group may also be a reversible reaction under basic conditions while the reduction to a nitrosarene is not.

The final mechanistic question involved the formation of indoles with concurrent carbon-carbon bond fission $(\mathbf{1 0 2}, \mathbf{1 0 9})$, oxidation $(\mathbf{1 0 8}, \mathbf{1 1 3})$, or alkene formation $(\mathbf{1 0 7})$. The transformation may occur via addition of allylic carbanion 117 to one of the nitro group oxygens, affording the seven-membered intermediate 118 (Scheme 33). Ringopening of 118 would give nitrosarene 119, which following electrocyclic ring-closure would produce 120. Rearomatization of $\mathbf{1 2 0}$ either by loss of a proton would afford $N$ hydroxyindole 121 or through loss of acetophenone and subsequent protonation would provide $N$-hydroxyindole 122. Palladium-catalyzed deoxygenation of $\mathbf{1 2 1}$ would afford alcohol 107 after subsequent protonation. Elimination of water from 107 could then produce 108. Deoxygenation of $\mathbf{1 2 2}$ would result in the formation of indole $\mathbf{1 0 9}$. 


\section{Scheme 33: Proposed Mechanism Accounting for Fission, Oxidation, Elimination}<smiles>CC(C)(C)c1ccccc1/C(C#N)=C\C(C)(C)c1ccccc1[N+](=O)[O-]</smiles>

117

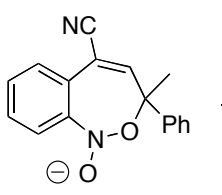

118

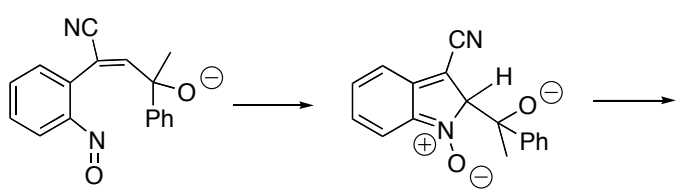

119
120

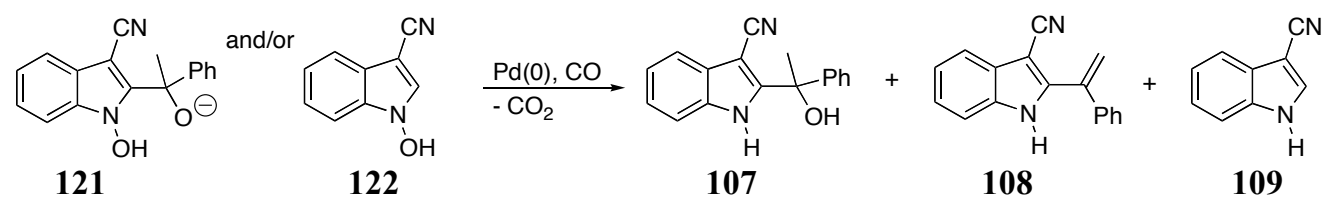

The addition of the carbanion to the nitro group of $\mathbf{1 1 7}$ proposed in Scheme 33 is supported by related observations reported in the literature. Nyerges et al ${ }^{68.69}$ reported novel 1,7 electrocyclizations of azomethine ylide to pendant nitro groups in their preparation of indazole- $N$-oxides. In addition, attack of carbanions to aromatic nitro groups has been proposed as one of the steps in the Bartoli indole synthesis using nitroarenes and excess alkenyl Grignard reagents. ${ }^{70}$

\subsection{Conclusions}

A synthetic methodology for the formation of various 4-cyanoquinolines and 3cyanoindoles from a common 1-cyano-1-(2-nitrophenyl)alkene precursor has been established. In the absence of a base, indoles are formed through palladium-catalyzed reductive $N$-heteroannulation. Quinolines are formed in the presence of a base, presumably through intramolecular nucleophilic addition of a carbanion to a nitrosarene. Analogous esters afford exclusively indoles regardless of the conditions used. Mechanistic rationales for the formation of both products and side products have been presented on the basis of literature precedence and experimental findings. 


\section{Chapter 3}

\section{An Expedient Synthesis of Salviadione}

3.1 Salviadione Background $\quad 35$

3.2 Expedient Synthesis of Salviadione $\quad 35$

3.2.1 Retrosynthetic Analysis 36

$\begin{array}{ll}\text { 3.2.2 Results and Discussion } & 37\end{array}$

$\begin{array}{ll}3.3 \text { Conclusions } & 43\end{array}$ 


\subsection{Introduction to Salviadione}

Traditional Chinese folk medicine has employed the use of the dried root of Salvia miltiorrhiza as treatment for a wide array of illnesses as Danshen or Tanshen. A number of biologically active compounds have been isolated from the dried root and can be classified as either water-soluble phenolic acids or lipophilic abietane-type diterpenes. These compounds have shown biological activity ranging from antioxidant, antiinflammatory, antifungal, anticoagulant, anti-HIV, antitumor, and antibacterial activity. ${ }^{71}$ Another structurally unique alkaloid, Salviadione, 1,1-dimethyl-6-(1-methylethyl)-1Hbenzo[def]carbazole-3,5-(2H,4H)-dione (123), was also isolated from these dried roots in 2005 (Figure 5). ${ }^{72}$ It is believed that this is the only naturally isolated benzo[def $]$ carbazole. ${ }^{73}$ and one of a small number prepared synthetically. ${ }^{74}$ The unique structure inspired us to commence a synthetic study.

Figure 5: Structure and Atom Numbering of Salviadione

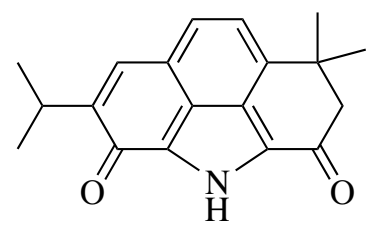

123

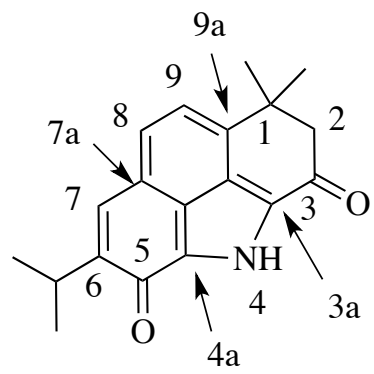

\subsection{Expedient Synthesis of Salviadione}

The unique structure of Salviadione made it an attractive candidate for a synthetic study. It was proposed that formation of the $N$-heterocyclic ring of the molecule could result from a Söderberg palladium-catalyzed reductive $N$-heterocyclization reaction. The goal was to highlight this methodology as a key step in the synthetic route. 


\subsubsection{Retrosynthetic Analysis}

The structure of Salviadione is deceptively complex, allowing us to propose a relatively concise synthetic route (Scheme 34). It was envisioned that Salviadione (123) could result from benzylic oxidation of phenol 124. The indole core of 124 could be prepared through reductive heterocyclization of nitroaromatic $\mathbf{1 2 5}$. Nitration of the tricyclic diterpenoid $\mathbf{1 2 6}$ would be expected to occur ortho to the methoxy group to give desired nitroaromatic $\mathbf{1 2 5}$. This tricyclic core could be prepared using the procedure previously outlined by Pan et al. ${ }^{75}$ Diterpenoid 126 would result from the acid-induced cyclization- Friedel-Craft's alkylation of para-substituted anisole 127, which could be prepared through reaction between iodide $\mathbf{1 2 9}$ and the carbanion derived from $\mathbf{1 2 8}$. Iodide 129 could be achieved through a simple iodination of commercially available alcohol 131, while isopropyl enol ether $\mathbf{1 2 8}$ could be prepared from $O$-alkylation of 5,5dimethyl-1,3-cyclohexanedione $\mathbf{1 3 0}$. 


\section{Scheme 34: Proposed Retrosynthetic Outline for Salviadione}<smiles>CC(C)C1=Cc2ccc3c4c([nH]c(c24)C1=O)C(C)(C)CC3=O</smiles>

Salviadione<smiles>C=C=CC(C)c1cc2c(c([N+](=O)[O-])c1O)C1=CC(=O)CC(C)(C)C1CC2</smiles>

124<smiles>COc1cc2c(cc1C(C)C)CCC1C2=CC(=O)CC1(C)C</smiles>

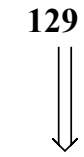

128<smiles>C=C=C</smiles>

127
126

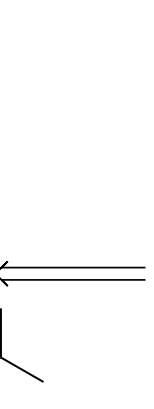<smiles>COc1ccc(CCO)cc1</smiles>

131<smiles>CC1(C)CC(=O)CC(=O)C1</smiles>

130

\subsubsection{Results and Discussion}

The synthetic route began with the alkylation of commercially available dimedone (5,5-dimethyl-1,3-cyclohexanedione) $\mathbf{1 3 0}$ to afford isopropyl enol ether $\mathbf{1 3 1}^{76}$ through reaction with isopropanol and a catalytic amount of $p$-toluenesulfonic acid (Scheme 35).

\section{Scheme 35: Alkylation of Dimedone}<smiles>CC1(C)CC(=O)CC(=O)C1</smiles>

130

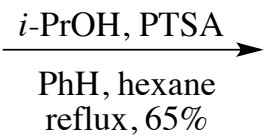<smiles>CC(C)OC1=CC(=O)CC(C)(C)C1</smiles>

128

Alcohol 131 was then converted to the corresponding iodide $129^{77}$ using typical Appel conditions (Scheme 36). 


\section{Scheme 36: Preparation of Alkyliodide}

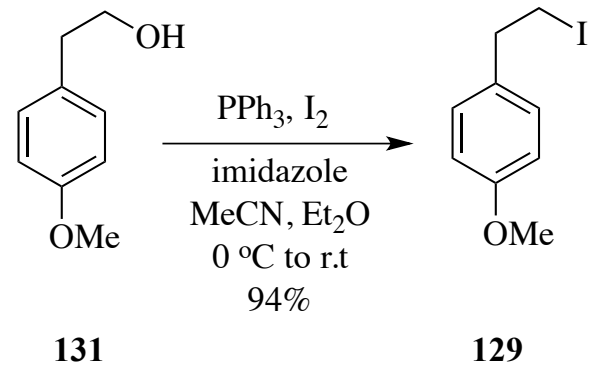

Iodide 129 then underwent alkylation with the carbanion formed through treatment of $\mathbf{1 2 8}$ with lithium $N, N$-diisopropylamine to give $\mathbf{1 2 7}$ (Scheme 37). ${ }^{78,79}$

\section{Scheme 37: Preparation of Cyclization Precursor}

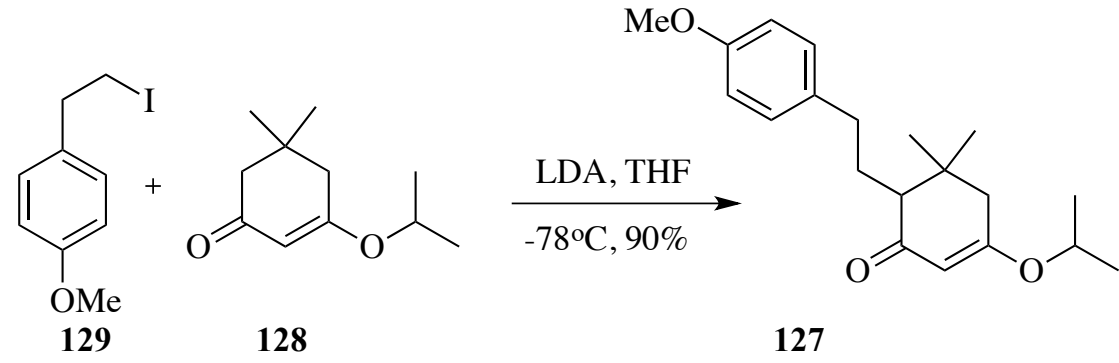

Intramolecular cyclization-Friedel-Crafts alkylation ${ }^{75}$ of $\mathbf{1 2 7}$ was achieved through heating in polyphosphoric acid, furnishing tricyclic terpenoid 126 (Scheme 38).

Scheme 38: Acid-Induced Intramolecular Cyclization-Friedel-Crafts Alkylation

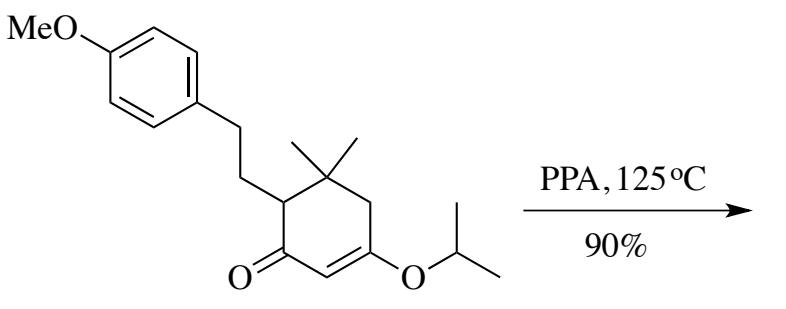

127<smiles>COc1cc2c(cc1C(C)C)CCC1C2=CC(=O)CC1(C)C</smiles>

126

The next major challenge was the nitration of tricyclic diterpenoid 126. The regioselectivity of the nitration was ortho to the methoxy-group as was expected, ${ }^{80}$ however, the observed product(s) were very sensitive to reaction conditions, work-up procedure, and speed of chromatographic purification. Given these variables, it was very hard to control the outcome of a given reaction even when using similar conditions. 
Nitration of $\mathbf{1 2 6}$ with a mixture of fuming nitric acid and sulfuric acid at $-78{ }^{\circ} \mathrm{C}$ provided nitro-phenol 125 in addition to desired anisole product 132 (Scheme 39). The absence of demethylation product $\mathbf{1 2 5}$ in reactions where the starting material was not fully consumed leads us to believe that the demethylation must occur after introduction of the nitro-group. Though unexpected, the demethylation turned out to be beneficial, as nitrophenol 125 provided a more direct route to salviadione (123).

\section{Scheme 39: Nitration of Tricyclic Diterpinoid Core}<smiles>COc1cc2c(cc1C(C)C)CCC1C2=CC(=O)CC1(C)C</smiles>

126

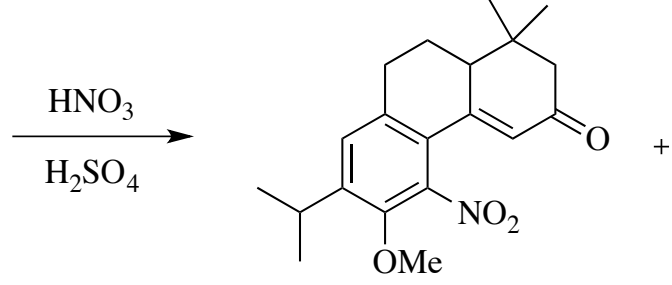

Temp $\left({ }^{0} \mathrm{C}\right)$

$-78^{\circ} \mathrm{C}$

$-20{ }^{\circ} \mathrm{C}$<smiles>CC(C)c1cc2c(c([N+](=O)[O-])c1O)C1=CC(=O)CC(C)(C)C1CC2</smiles>

125

$32 \%$

$77 \%$

When the nitration was performed at $-20{ }^{\circ} \mathrm{C}$, nitro-phenol 125 was the major product observed along with a trace amount of $\mathbf{1 3 2}$. Upon separation by column chromatography, both $\mathbf{1 2 6}$ and $\mathbf{1 3 2}$ were moderately stable, however, exposure to acidic conditions and open air or leaving the products absorbed onto silica gel for an extended amount of time resulted in oxidation to quinole $\mathbf{1 3 3}$ as a mixture of diastereomers (14:1 ratio). As an example, treatment of $\mathbf{1 3 2}$ with acetic acid while absorbed on silica gel afforded quinole $\mathbf{1 3 3}$ in $88 \%$ yield. This product was also observed when the nitration of 126 was performed at $0{ }^{\circ} \mathrm{C}$ and allowed to warm to room temperature, after which the crude product was absorbed onto silica gel and left open to the air (Scheme 40). 


\section{Scheme 40: Oxidation of Products to Quinole 133}

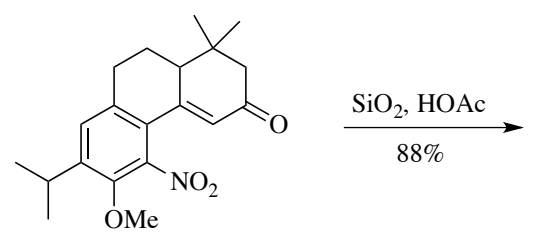

132

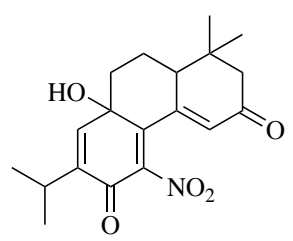

133

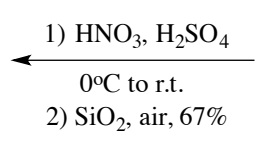

The relative stereochemistry of the major diastereomer of $\mathbf{1 3 3}$ was concluded

through NOE, COSY, and selective decoupling NMR experiments. The H9-axial proton and the hydroxy group displayed a four-bond spin-spin coupling $(J=1.5 \mathrm{~Hz})$, which is in accordance with values observed for similar compounds. ${ }^{81}$

Each of the three nitration products $(\mathbf{1 2 5}, \mathbf{1 3 2}, \mathbf{1 3 3})$ were subjected to the previously discussed palladium-catalyzed reductive $N$-heteroannulation conditions ${ }^{60}$ in hope of obtaining the expected benzo[def]carbazoles. Reaction of both anisole $\mathbf{1 3 2}$ and phenol 125 with carbon monoxide in the presence of a catalytic amount of palladium bis(dibenzylidenacetone) and 1,10-phenanthroline provided 134 and $\mathbf{1 2 4}$ in 87\% and 75\% yield, respectively (Scheme 41). Unlike their precursors, both cyclization products were stable towards oxidation when treated with acetic acid, air, and when adsorbed onto silica gel.

\section{Scheme 41: Palladium-Catalyzed Reductive $N$-Heteroannulation}<smiles>[R2]Oc1c(C(C)C)cc2c(c1[N+](=O)[O-])C1=CC(=O)CC(C)(C)C1CC2</smiles>

$132(\mathrm{R}=\mathrm{Me})$

$125(\mathrm{R}=\mathrm{H})$
$\mathrm{Pd}(\mathrm{dba})_{2}, \mathrm{CO}(6 \mathrm{~atm}), 120^{\circ} \mathrm{C}$

1,10-phenanthroline, DMF

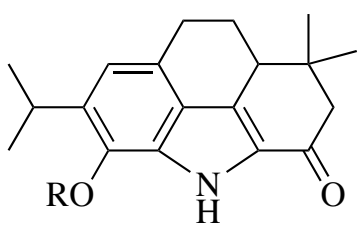

$134(\mathrm{R}=\mathrm{Me}, 87 \%)$

$124(\mathrm{R}=\mathrm{H}, 75 \%)$

Attempted palladium-catalyzed cyclization of quinole $\mathbf{1 3 3}$ was not as successful

as the two previous cases. Although a small amount (10\%) of salviadione (123) was isolated from a complex reaction mixture of which all of the starting material had been 
consumed, none of the expected cyclization product $\mathbf{1 3 5}$ was obtained (Scheme 42). Multiple attempts to improve the yield of this direct route to salviadione (123) were unsuccessful.

\section{Scheme 42: Attempted Cyclization of Quinole}

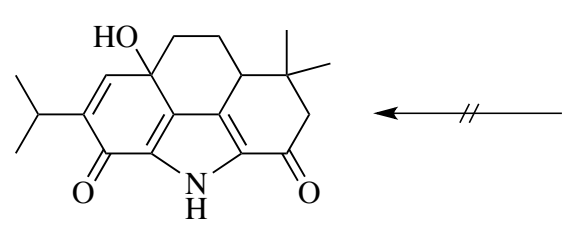

135

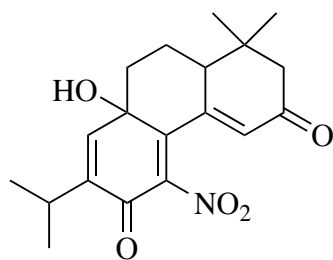

133

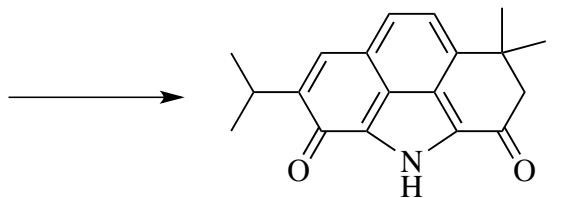

123

Conditions: 1,10-phenanthroline, $\mathrm{Pd}(\mathrm{dba})_{2}, \mathrm{CO}(6 \mathrm{~atm}), \mathrm{DMF}, 120{ }^{\circ} \mathrm{C}$

Methoxy-substituted compound $\mathbf{1 3 4}$ was also treated with 2,3-dichloro-5,6dicyanobenzoquinone (DDQ), resulting in benzylic oxidation to afford hydroxy substituted benzo[def]carbazole 136 (Scheme 43) ${ }^{82}$ Interestingly, 136 possesses the same relative stereochemistry between the hydroxy group and the $\mathrm{C} 9$-axial proton as reported for a number of similar naturally occurring compounds. ${ }^{83}$ In the case of $\mathbf{1 3 6}$, this relative stereochemistry was determined through NOE NMR experiments.

Unfortunately, conversion of this compound to salviadione (123) would require demethylation, elimination of water, and oxidation, so the route was not pursued.

\section{Scheme 43: Benzylic Oxidation of Anisole Using DDQ}

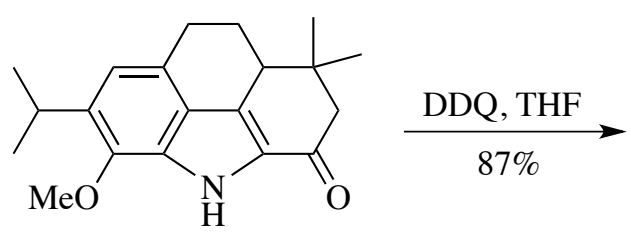

134

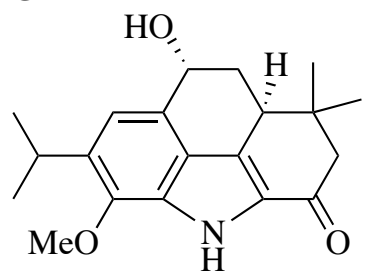

136

A more synthetically-useful route to salviadione (123) was achieved through treatment of hydroxy compound $\mathbf{1 2 4}$ with a four-fold excess of DDQ in tetrahydrofuran (THF), affording salviadione (123) in good yield (Scheme 44). 


\section{Scheme 44: DDQ Oxidation to Afford Salviadione}

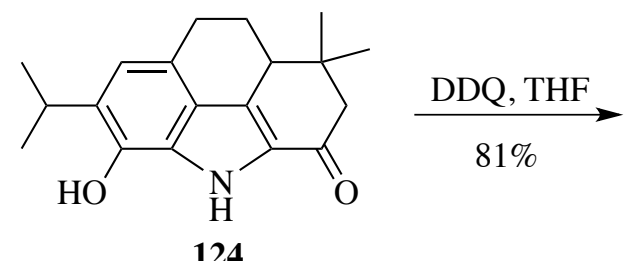

124

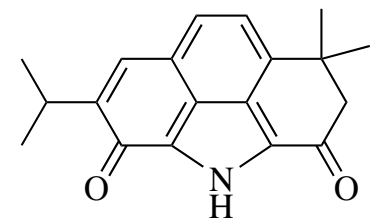

123

The structure of salviadione was confirmed through comparison of experimentally obtained melting point, IR, HRMS, and ${ }^{1} \mathrm{H}$ NMR data with that reported for the natural product. ${ }^{72}$ Table 4 provides a comparison of ${ }^{1} \mathrm{H}$ NMR data while ${ }^{13} \mathrm{C}$ NMR data is provided in Table 5. Every carbon resonance was found to be within $1 \mathrm{ppm}$ of the natural product aside from one (C3a), which was $1.2 \mathrm{ppm}$ off.

Table 4: ${ }^{1}$ HNMR Shift Comparison Between Synthetic and Natural Salviadione

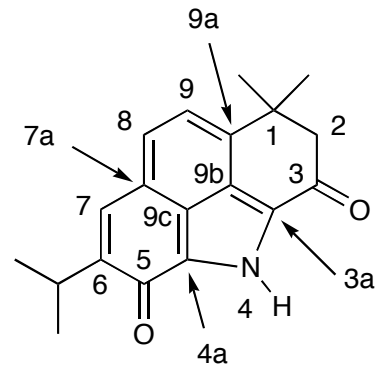

$\begin{array}{llll}\text { Proton } & \delta(\mathrm{ppm})^{\mathrm{a}, \mathrm{c}} & J(\mathrm{~Hz})^{\mathrm{a}, \mathrm{c}} & \Delta \delta(\mathrm{ppm}) \\ \mathrm{NH} & 13.24(14.23)^{\mathrm{b}} & & \\ \mathrm{H}-2 & 2.95(2.95) & & 0 \\ \mathrm{H}-6 & 7.55(7.56) & & 0.01 \\ \mathrm{H}-8 & 7.66(7.66) & 7.0(7.5) & 0 \\ \mathrm{H}-9 & 7.31(7.30) & 7.0(7.5) & -0.01 \\ \text { iPr-CH } & 3.49(3.54) & 6.9(7.0) & 0.05 \\ \text { iPr-Me } & 1.29(1.31) & 6.9(7.0) & 0.02 \\ \text { 1-Me } & 1.51(1.49) & & -0.02 \\ \text { a) In } \mathrm{CDCl}_{3} \text { b) In DMSO-d } & \text { c) Literature values in parenthesis. }\end{array}$


Table 5: ${ }^{13}$ CNMR Shift Comparison Between Synthetic and Natural Salviadione

\begin{tabular}{llc} 
Carbon & \multicolumn{1}{c}{$\delta(\mathrm{ppm})^{\mathrm{a}}$} & $\Delta \delta(\mathrm{ppm})$ \\
\hline 1 & $41.3(41.3)$ & 0 \\
2 & $57.3(57.4)$ & 0.1 \\
3 & $187.7(188.0)$ & 0.3 \\
3a & $127.0(128.2)$ & 1.2 \\
$4 \mathrm{a}$ & $126.7(126.9)$ & 0.2 \\
5 & $175.8(175.9)$ & 0.1 \\
6 & $151.3(151.4)$ & 0.1 \\
7 & $128.9(129.1)$ & 0.2 \\
$7 \mathrm{a}$ & $128.2(127.9)$ & -0.3 \\
8 & $129.5(129.7)$ & 0.2 \\
9 & $121.2(121.0)$ & -0.2 \\
$9 \mathrm{a}$ & $144.9(145.3)$ & 0.4 \\
$9 \mathrm{~b}$ & $124.0(123.9)$ & -0.1 \\
9c & $124.2(124.3)$ & 0.1 \\
iPr-Me & $22.7(22.8)$ & 0.1 \\
iPr-CH & $27.3(27.4)$ & 0.1 \\
C1-Me & $30.0(30.1)$ & -0.1 \\
\hline
\end{tabular}

a) $\mathrm{In} \mathrm{CDCl}_{3}$; Literature values in parenthesis.

To probe the potential biological uses of these compounds, salviadione (123), along with methoxy and hydroxy analogs 134 and 124, respectively, were tested for potential activity against liver cancer cell lines. However, initial results showed $\mathrm{LD}_{50}$ values similar to those typical for DMSO, which was used as the carrier solvent in the assays. Therefore, any cell death was attributed to the DMSO rather than the synthesized compounds. However, this preliminary examination was very narrow in focus and it is possible that compounds $\mathbf{1 2 3}, \mathbf{1 2 4}$, and 134 possess other biological uses.

\subsection{Conclusions}

A novel, concise synthetic route to Salviadione and two related derivatives has been developed utilizing a key palladium-catalyzed reductive $N$-heterocyclization to form the indole core. Through an unexpected yet advantageous demethylation encountered in 
the nitration of the tricyclic terpenoid intermediate, a more direct route than initially envisioned was established. This reaction sequence may allow facile preparation of additional structural analogues, which could prove useful in a wide array of biological applications. 


\section{Chapter 4}

\section{Investigation of Chemoselectivity in Kosugi-Migita-Stille Coupling}

\section{Reactions}

4.1 Kosugi-Migita-Stille Reaction Background

4.2 Chemoselective Stille Coupling Reactions 46

4.3 Investigation of Nitro Effect in Stille Coupling Reactions 48

4.3.1 Preparation of Isomeric Substrates $\quad 49$

4.3.2 Results and Discussion 53

$\begin{array}{ll}4.3 \text { Conclusions } & 64\end{array}$ 


\subsection{Kosugi-Migita-Stille Reaction Background}

Carbon-carbon bond forming reactions have been a key focus of many synthetic

organic chemists over the past number of decades. Kosugi, Shimizu, and Migita ${ }^{84,85,86}$ reported the first examples of palladium-catalyzed coupling between organotin compounds and carbon electrophiles in 1977. Stille's first study of similar reactions was published in $1978 .{ }^{87}$ While his work was preceded by that of Kosugi, Shimizu, and Migita, the coupling of organotin reagents with carbon electrophiles using palladium catalysts is often referred to as simply the "Stille Coupling" reaction as Stille's comprehensive mechanistic and synthetic investigations provided the basis for more recent studies. ${ }^{88}$

\subsection{Chemoselective Stille Coupling Reactions}

Selectivity is an ever-important yet challenging issue when employing a particular reaction. Chemoselectivity refers to the ability to react with one particular functional group in the presence of other potentially reactive groups ${ }^{89}$ A specific example of chemoselectivity was reported by Echavarren ${ }^{90}$, who studied Stille cross-coupling reactions between organostannanes and arenes featuring both a halide and a triflate. Through use of different palladium catalysts and additives, selective couplings were achieved through either the carbon-halide or carbon-triflate bond of bromophenyl triflate 137. These results are summarized in Scheme 45. The use of tetrakis(triphenylphosphine)palladium(0) as the catalyst in 1,4-dioxane favored reaction with the bromine, affording styrene 138, while using bis(triphenylphosphine)palladium(II) dichloride as the catalyst along with a three-fold excess of lithium chloride in $N, N$-dimethylformamide (DMF) afforded exclusively styrene 139 through reaction with the trifloxy group. 


\section{Scheme 45: Echavarren's Chemoselective Stille Coupling Conditions}

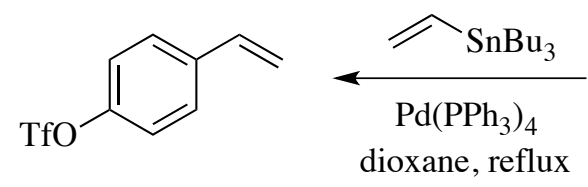

138<smiles>CCOc1ccc(Br)cc1</smiles>

137

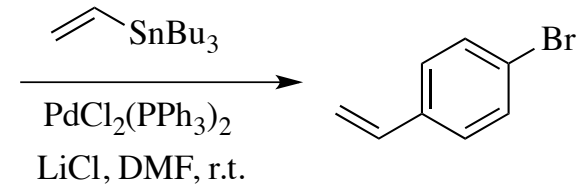

139

These initial studies suggest that using a palladium (II) catalyst as well as the addition of lithium chloride facilitates insertion of palladium into the carbon-oxygen bond of the triflate to afford bromostyrene 139. It was also proposed ${ }^{90}$ that coordination of the catalyst to the basic trifloxy group could direct the oxidative addition to occur into the triflate carbon-oxygen bond rather than to the carbon-bromine bond. The addition of lithium chloride facilitates ligand exchange of the trifluoromethanesulfonyloxy group for a chloride upon oxidative addition of palladium into the carbon-oxygen bond of the triflate, rendering the intermediate more reactive for subsequent transmetallation. ${ }^{91}$

Only one example of a chemoselective coupling reaction involving a nitrated bromophenyl triflate has been reported by Krolski. ${ }^{92}$ Using conditions similar to those previously discussed ${ }^{90}$ for the selective coupling of aryl bromides, chemoselective coupling through the carbon-bromine bond of 2-bromo-3-nitrophenyl triflate (140) accomplished, affording styrene 141 (Scheme 46).

\section{Scheme 46: Krolski Selective Coupling of 2-Bromo-3-Nitrophenyl Triflate}<smiles>CCOc1cccc([N+](=O)[O-])c1Br</smiles>

140

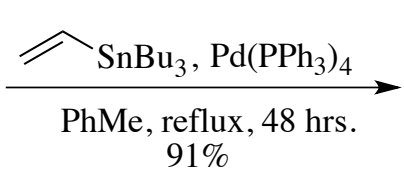
$91 \%$<smiles>C=Cc1c(OCC)cccc1[N+](=O)[O-]</smiles>

141

\subsection{Investigation of Nitro Effect in Stille Coupling Reactions}

Selective couplings were of interest for the potential use in the preparation of substrates en route to pyrroloindoles via reductive double $N$-heteroannulations. The first 
system examined was 2,4-dinitro-5-bromophenyl triflate (144), which was prepared in two steps from 3-bromophenol (142) (Scheme 47). Conversion of phenol 142 to the corresponding triflate $\mathbf{1 4 3}$ followed by nitration eliminated the formation of trinitrophenol as observed in the direct nitration of phenol 142.

\section{Scheme 47: Preparation of 2,4-Dinitro-5-Bromophenyl Triflate}<smiles>Oc1cccc(P)c1</smiles>

142<smiles>CCOC(=O)OCCO</smiles>
$88 \%$<smiles>CCOc1cccc(Br)c1</smiles>

143

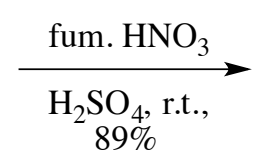

$89 \%$<smiles>CCOc1cc([N+](=O)[O-])c(Br)cc1[N+](=O)[O-]</smiles>

144

Application of Echavarren's conditions ${ }^{90}$ that should result in coupling through the carbon-bromine bond (Conditions A) using substrate $\mathbf{1 4 4}$ afforded a near 1:1 mixture of triflate-coupled product 145 along with bromine-coupled 146 (Scheme 48).

\section{Scheme 48: Attempted Selective Coupling of Dinitro-Bromophenyl Triflate}<smiles>C=Cc1cc(O)c([N+](=O)[O-])cc1[N+](=O)[O-]</smiles>

The lack of selectivity observed in the coupling of dinitroarene 144 was puzzling.

It was concluded that additional factors must have been influencing the erosion of chemoselectivity observed for the dinitro substrate 144. Considering that electronwithdrawing substituents often enhance the reactivity of $\mathrm{C}-\mathrm{X}$ bonds (with $\mathrm{X}$ referring to either a halide or pseudohalide) in coupling reactions,${ }^{90}$ it was proposed that the nitro groups were the root of the eroded selectivity. The effect was believed to be due to the nitro groups being in conjugation with the ortho and para X-groups, reducing the electron density at those positions. The more electrophilic X-groups would then be more 
prone to oxidative addition, potentially causing the observed loss of selectivity. Another proposal, based off Echavarren's explanation ${ }^{90}$ for the selectivity observed under triflateselective conditions $\mathrm{B}$, was that the nitro-group could facilitate the oxidative addition into each C-X bond through coordination with the catalyst. In the case of substrate 144 where each C-X bond is ortho to a nitro group, potential coordination between palladium and the nitro group could facilitate oxidative addition into either $\mathrm{C}-\mathrm{X}$ bond. To more explicitly examine the influence that placement of nitro groups had on coupling selectivity, it was deemed necessary to prepare each of the ten bromo-trifloxy nitrobenzene isomers in order to determine what effect the position of the nitro group relative to the triflate or bromine had on the selectivity of the coupling reaction. The plan was to subject each isomer to both the halide-selective conditions $\mathrm{A}\left(\mathrm{PPh}_{3}\right.$, $\operatorname{Pd}\left(\mathrm{PPh}_{3}\right)_{4}$, dioxane, reflux $)$ and triflate-selective conditions $\mathrm{B}\left(\mathrm{PdCl}_{2}\left(\mathrm{PPh}_{3}\right)_{2}, \mathrm{LiCl}, \mathrm{DMF}\right.$, room temperature) as established by Echavarren. ${ }^{90}$

\subsubsection{Preparation of Isomeric Substrates}

The first challenge was the preparation of the ten substitutional isomers using a variety of nitration and halogenation reactions.

2-bromo-4-nitrophenol 148 and 2-bromo-6-nitrophenol 149 were prepared through the nitration of 2-bromophenol $\mathbf{1 4 7}$ using sodium nitrite and oxalic acid along with wet silica gel (Scheme 49). ${ }^{93}$ The relatively mild reaction conditions provided solely mono-nitrated products with no evidence of di-nitration. 


\section{Scheme 49: Nitration of 2-Bromophenol}

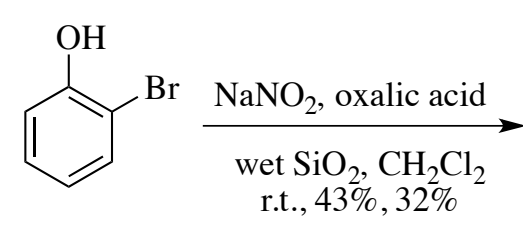

147<smiles>O=[N+]([O-])c1ccc(O)c(Br)c1</smiles>

148<smiles>O=[N+]([O-])c1cccc(Br)c1O</smiles>

149

Conversion to the corresponding triflates $\mathbf{1 5 0}$ and $\mathbf{1 5 1}$ was then accomplished through treatment with pyridine and triflic anhydride (Scheme 50). Filtration of the crude reaction mixture removed the pyridine salt byproduct, and evaporation of solvent provided nearly pure product in both cases.

Scheme 50: Preparation of Isomeric Nitrophenyl Triflates
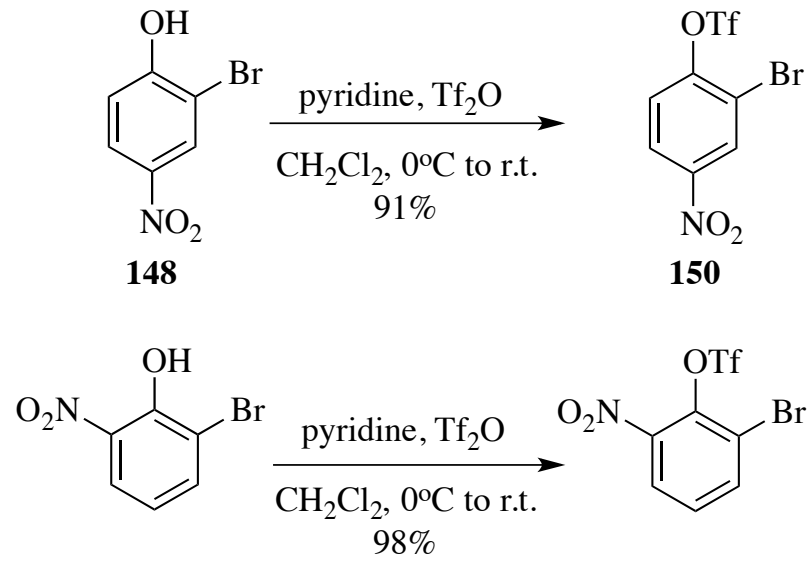

149

151

Nitration of 4-bromophenol (152) using the same conditions as above ${ }^{93}$ provided exclusively 2-nitro-4-bromophenol 153 (Scheme 51). Conversion to the corresponding triflate (as above) provided isomer 154 


\section{Scheme 51: Preparation of 2-Nitro-4-Bromophenyl Triflate}

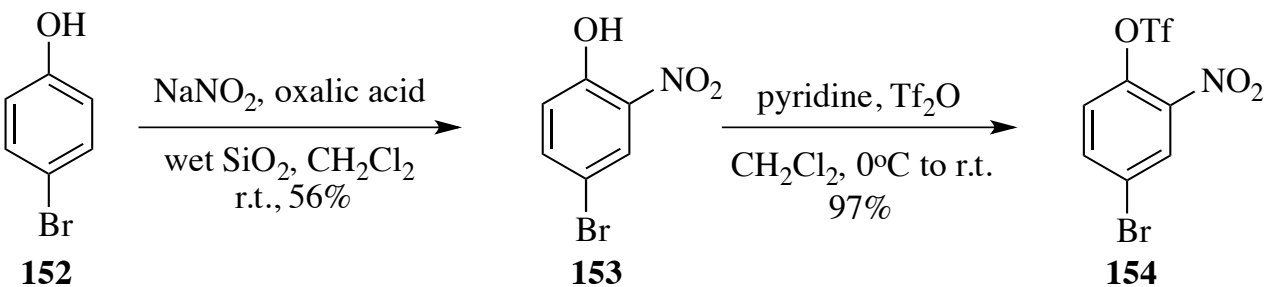

3-nitro-5-bromophenol 156 was prepared in a two-step process from 2-amino-5nitrophenol 155 (Scheme 52). ${ }^{94}$ Regioselective bromination using $N$-bromosuccinimide (NBS) followed by de-amination provided phenol 156, which was converted to triflate $\mathbf{1 5 7}$ through treatment with pyridine and triflic anhydride.

Scheme 52: Preparation of 3-Nitro-5-Bromophenyl Triflate

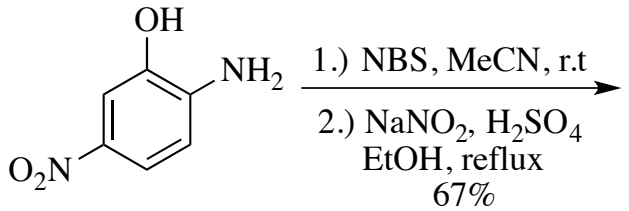

155<smiles>O=[N+]([O-])c1cc(O)cc(Br)c1</smiles>

156
157

2-bromo-5-nitrophenyl triflate 159 was also prepared from 2-amino-5-nitrophenol (155) (Scheme 53). Initial halogenation ${ }^{95}$ with copper (II) bromide provided aryl bromide 158, which was subsequently converted to triflate 159 using previously established conditions.

Scheme 53: Preparation of 2-Bromo-5-Nitrophenyl Triflate<smiles>CCOc1cc([N+](=O)[O-])ccc1Br</smiles>
155 158

159

3-nitro-4-bromophenyl triflate 163 was prepared in three steps from 3-nitro-4aminoanisole 160 (Scheme 54). Halogenation ${ }^{95}$ of aniline 160 using Sandmeyer-type 
conditions provided aryl bromide 161, which was then treated with boron tribromide to cleave the phenyl methyl ether, providing phenol $\mathbf{1 6 2}$. Treatment of 162 with base and triflic anhydride afforded triflate $\mathbf{1 6 3 .}$

\section{Scheme 54: Preparation of 4-Bromo-3-Nitrophenyl Triflate}

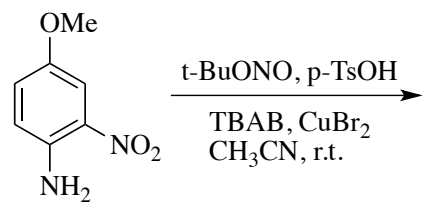

160

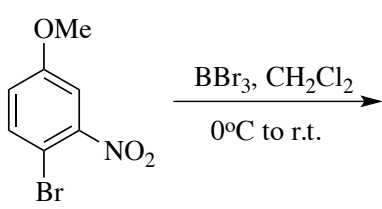

161

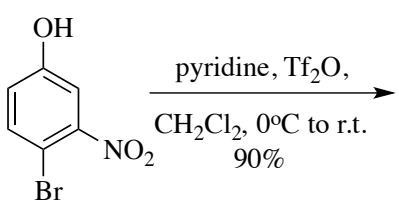

162<smiles>CCOc1ccc(Br)c([N+](=O)[O-])c1</smiles>

163

Boron tribromide demethylation ${ }^{95}$ of anisole 164 provided phenol $\mathbf{1 6 5}$, which was then converted to triflate $\mathbf{1 6 6}$ using previously discussed conditions (Scheme 55).

\section{Scheme 55: Preparation of 3-Bromo-2-Nitrophenyl Triflate}

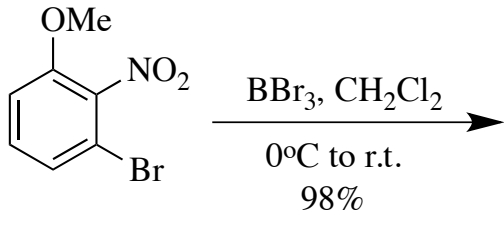

164

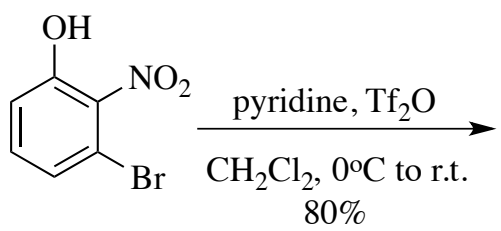

165<smiles>CCOc1cccc(Br)c1[N+](=O)[O-]</smiles>

166

Two additional isomers (167 and $\mathbf{1 6 8})$ were prepared through the mono-nitration of 3-bromophenyl triflate $\mathbf{1 4 3}$ (Scheme 56).

Scheme 56: Preparation of 167 and 168

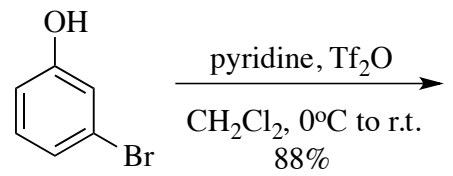

142

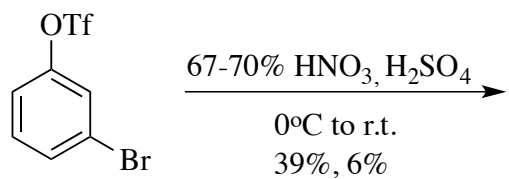

143<smiles>CCOc1ccc([N+](=O)[O-])c(Br)c1</smiles>

167<smiles>CCOc1cc(Br)ccc1[N+](=O)[O-]</smiles>

168

The final isomer 140 was prepared in near quantitative yield from phenol $\mathbf{1 6 9}$, which was on hand from previous work within the laboratory (Scheme 57). 


\section{Scheme 57: Preparation of 2-Bromo-3-Nitrophenyl Triflate}

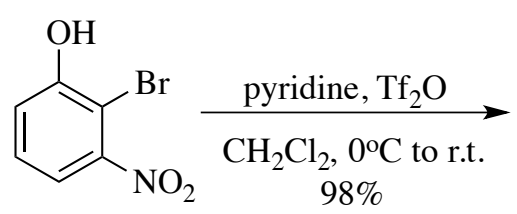

169<smiles>CCOc1cccc([N+](=O)[O-])c1Br</smiles>

140

\subsubsection{Results and Discussion}

With each of the possible structural isomers in hand, focus was turned to the coupling reaction, each substrate was treated with vinyl stannane in the presence of either bromine-selective conditions $\mathrm{A}\left(\mathrm{Pd}\left(\mathrm{PPh}_{3}\right)_{4}\right.$, dioxane, reflux $)$ and triflate-selective conditions $\mathrm{B}\left(\mathrm{PdCl}_{2}\left(\mathrm{PPh}_{3}\right)_{2}, \mathrm{LiCl}, \mathrm{DMF}, \mathrm{r.t}\right)$. The results of this systematic investigation are summarized in Table 6 . In some cases, unreacted starting material and/or coupled product was isolated as the corresponding phenol due to hydrolysis during column chromatography. In addition, minor amounts of di-coupled products were also detected in some cases. All reactions were run for 24 hours to allow direct comparison between substrates by limiting potential variables. In a slight variation from Echavarren's work ${ }^{90}$ which directly used $\mathrm{Pd}\left(\mathrm{PPh}_{3}\right)_{4}$, the same complex was generated in situ by mixing $\mathrm{Pd}(\mathrm{dba})_{2}$ with $\mathrm{PPh}_{3}$. For clarity, the substrates in Table 6 are arranged according to the position of the bromine relative to the nitro group. Entries 1-4 are isomers where the bromine is ortho to the nitro group, entries 5-8 possess a meta relationship between the bromine and nitro group, and entries 9-10 feature the bromine para to the nitro group. 
Table 6: Isolated Yields From Reaction Under Conditions A and B

\section{Entry Substrate Br-coupling TfO-coupling Hydrolysis}
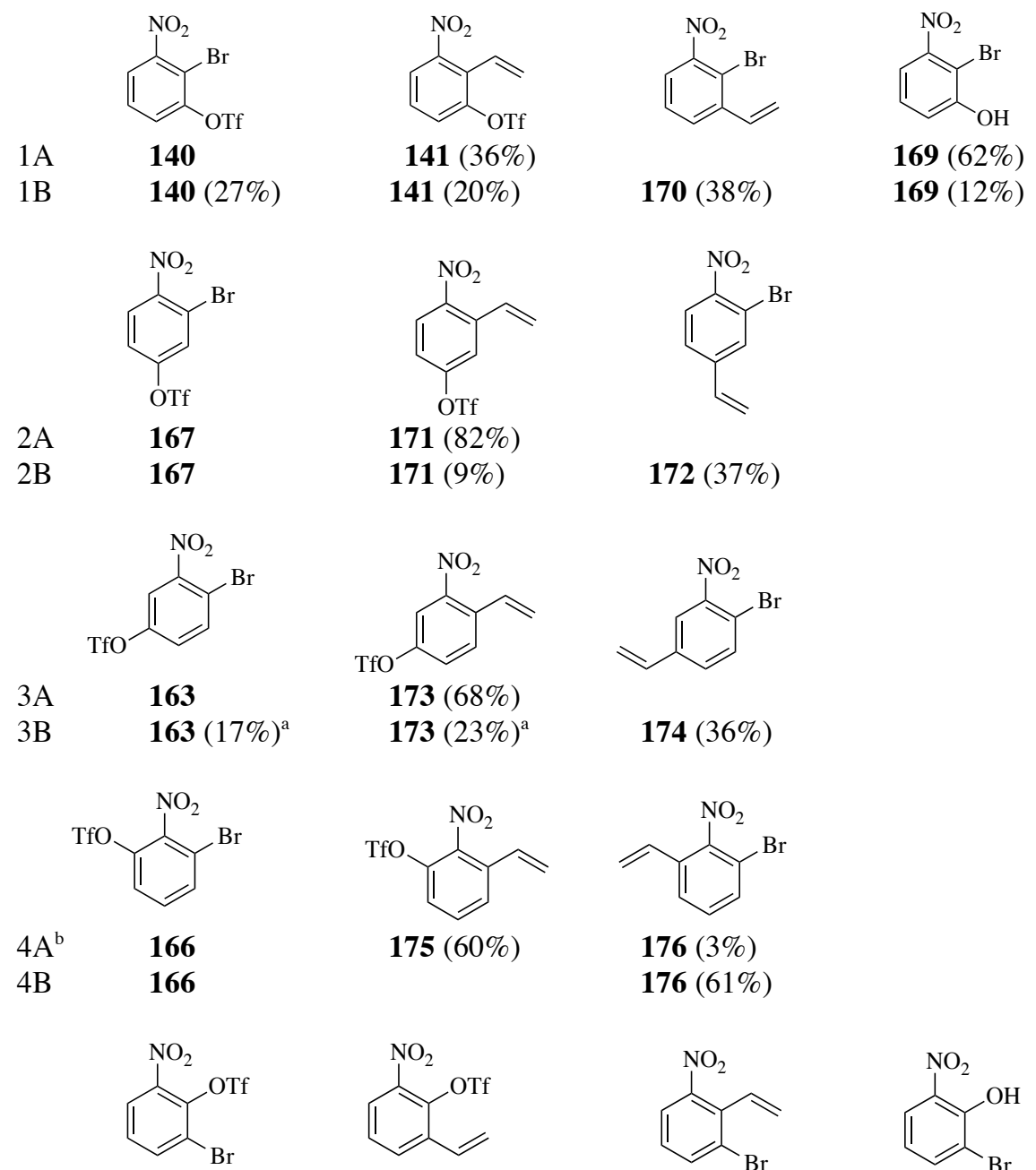
$5 \mathrm{~A}$
$5 \mathrm{~B}$

$$
151(9 \%)
$$$$
151(5 \%)
$$

$$
175(60 \%)
$$

$176(3 \%)$

$176(61 \%)$<smiles>CCOc1ccc([N+](=O)[O-])cc1Br</smiles><smiles>C=Cc1cccc([N+](=O)[O-])c1OCC</smiles>

177 (24\%)<smiles>C=Cc1cc([N+](=O)[O-])ccc1OCC</smiles>

$6 \mathrm{~A}$

$6 \mathrm{~B}$

$$
\begin{aligned}
& 150(7 \%) \\
& 150(8 \%)
\end{aligned}
$$

$178(22 \%)$<smiles>C=Cc1c(Br)cccc1[N+](=O)[O-]</smiles>

$$
\begin{aligned}
& 46(5 \%) \\
& 46(43 \%)
\end{aligned}
$$

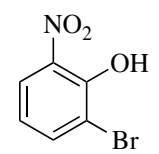

149 (18\%)

$149(12 \%)$
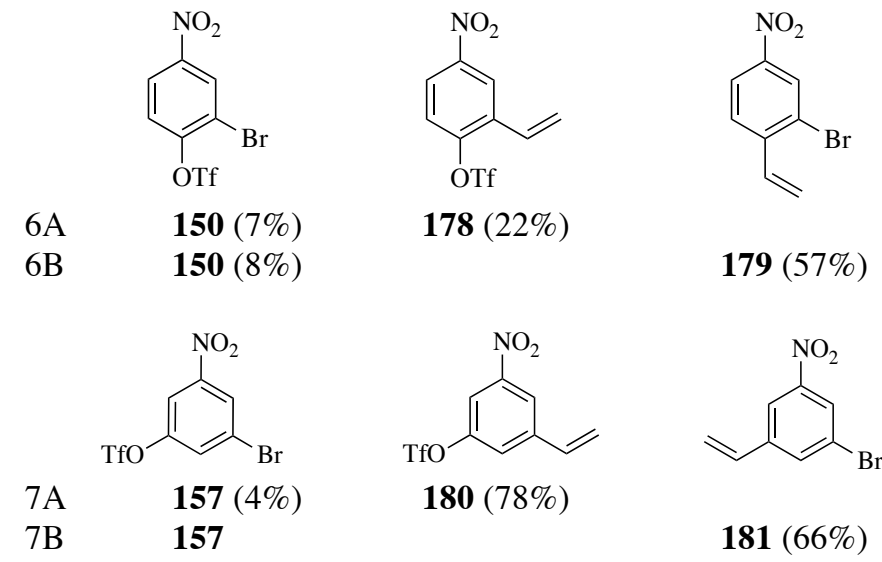

$179(57 \%)$
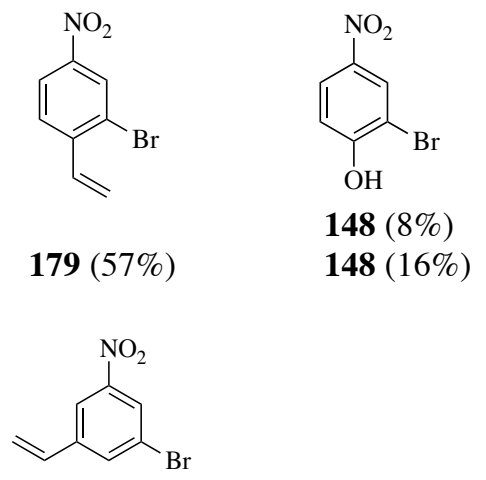

$181(66 \%)$ 
Table 6: Isolated Yields From Reaction Under Conditions A and B (cont'd)

\section{Entry Substrate Br-coupling TfO-coupling Hydrolysis}

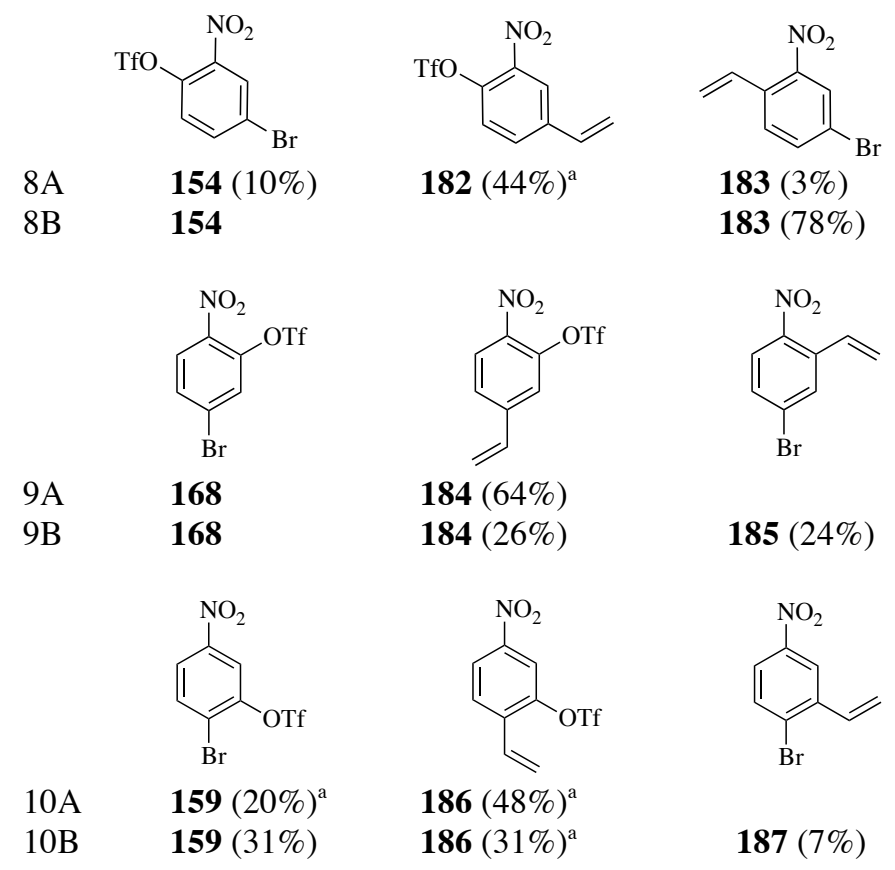

Cond. A: vinyl stannane, $\mathrm{PPh}_{3}, \mathrm{Pd}(\mathrm{dba})_{2}, 1,4$-dioxane, $105^{\circ} \mathrm{C}$

Cond. B: vinyl stannane, $\mathrm{PdCl}_{2}\left(\mathrm{PPh}_{3}\right)_{2}$, $\mathrm{LiCl}$, DMF, r.t.

a) Yield calculated based on integration of ${ }^{1} \mathrm{H}$ NMR spectrum; b) Also isolated was $31 \%$ di coupled product

Under conditions A, all but one substrate featuring a bromine ortho to the nitro group (table 6, entries 1-4) exhibited high selectivity, affording exclusively brominecoupled products $(\mathbf{1 4 1}, \mathbf{1 7 1}, \mathbf{1 7 3})$. The only isomer of this group that was not completely selective was that which had both a bromine and a trifloxy group ortho to the nitro group (entry 4). In this case, a small amount of trifloxy-coupled product $\mathbf{1 7 6}$ was isolated in addition to the expected bromine-coupled product 175. This result could be explained by the trifloxy group also being activated toward oxidative addition through conjugation with the ortho nitro substituent.

Interestingly, substrates with a bromine meta to the nitro group (entries 5-8) also exhibited high selectivity under bromine-selective conditions A. Only isomer $\mathbf{1 5 1}$ (entry 
5) was not completely selective for bromine coupling. It can be envisioned that the trifloxy group ortho to the nitro group would be activated through conjugation with the nitro group, while the bromine meta to the nitro group would not, leading to erosion of selectivity. However, substrates 150 and 154 (entries 6 and 8, respectively) in which the trifloxy group was also in conjugation with the nitro group do not exhibit the same erosion of selectivity. Also of interest is entry 7 where both the bromine and trifloxy group were meta to the nitro group. Surprisingly, the isolated yield of bromine-coupled 180 (78\%) was among the highest of any isomer examined. This result may be attributed to the overall electron-deficient nature of the nitro-substituted aromatic system even though the nitro group was not in conjugation with the bromine or trifloxy group.

The last two substrates, 168 and 159 (entries 9 and 10, respectively), in which the bromine was positioned para to the nitro group were completely selective for bromine coupling under bromine-selective conditions A. This was surprising for isomer 168 in which the trifloxy group was activated ortho to the nitro group, although the bromine was in the para position, which would also be activated through conjugation with the nitro group. In this case, the observed selectivity could be attributed to a faster transmetalation between palladium and tin upon oxidative addition of the catalyst to the carbon-bromine bond as opposed to the carbon-oxygen bond of the triflate. ${ }^{91}$

Under triflate-selective conditions B, three of the four isomers in which the trifloxy group was ortho to the nitro group (entries $4,5,8)$ exhibited absolute selectivity for triflate coupling. In contrast, isomer $\mathbf{1 6 8}$ (entry 9) was actually counter-selective, affording bromine-coupled 184 in higher yield than triflate-coupled $\mathbf{1 8 5}$. This lack of selectivity could be attributed to the bromine being activated through conjugation with 
the para nitro group, although the trifloxy group would also be activated ortho to the nitro group.

With the exception of entry 7 (where both the triflate and bromine were meta to the nitro group), isomers in which the trifloxy group was meta to the nitro group (entries $1,3,10)$ exhibited the lowest selectivities out of all of the substrates studied under conditions B. While this could be attributed to each substrate featuring an activated bromine ortho or para to the nitro group, it is also an indication that conditions B were not ideal for triflate-selective coupling. However, isomer 157 (entry 7) afforded exclusively triflate-coupled $\mathbf{1 8 1}$ on account of the bromine also being in an inactivated meta position relative to the nitro group.

The last two substrates were those where the trifloxy group was para to the nitro group (entries 2,6). While isomer 150 was completely selective for triflate-coupling, isomer 167 exhibited eroded selectivity. This contrast could be attributed to the orientation of the bromine relative to the nitro group in each substrate, with the bromine of isomer 150 being in an unactivated meta position while the bromine of isomer 167 was activated by the ortho nitro group.

From the numerous examples presented in Table 6, it was apparent that the nitro group was key in influencing the selectivity of the reaction. In general, substrates in which the nitro group was ortho or para to the triflate or bromine exhibited significantly higher conditions-dependent selectivity than those where the nitro-group was meta relative to the targeted coupling moiety. In cases where the targeted C-X bond was meta to the nitro group, the selectivity was reduced and, in some cases, reversed from what was expected. The reversal of predicted selectivity in these cases could then attributed to electronic activation of the other $\mathrm{C}-\mathrm{X}$ bond through conjugation with the nitro group. 
In addition to the ten mono-nitrated cases mentioned above, two additional

isomeric bromophenyl triflates 188 and 137 were prepared in near quantitative yield from the corresponding phenols (Scheme 58).

\section{Scheme 58: Preparation of Bromophenyl Triflates}

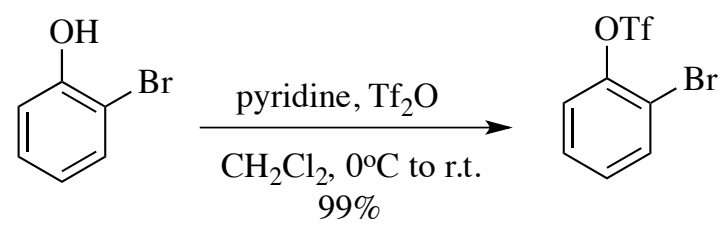

147<smiles>Oc1ccc(Br)cc1</smiles>

152
188<smiles>CCOc1ccc(Br)cc1</smiles>

137

Each of these substrates (along with 3-bromophenyl triflate 143 from above) were then subjected to coupling conditions A and B. The selectivity observed in each of these reactions is summarized in Table 7.

\section{Table 7: Selective Coupling of Bromophenyl Triflates}

Entry Substrate<smiles>CCOc1ccccc1Br</smiles>

1A

$1 \mathrm{~B}$

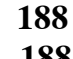<smiles>CCOc1cccc(Br)c1</smiles>

2A

2B Br-coupling<smiles>C=Cc1ccccc1O</smiles>

189 (1.0)

$189(0.15)$<smiles>C=Cc1cccc(O)c1</smiles>

191 (1.0)

$191(0.15)$

\section{TfO-coupling}<smiles>C=Cc1ccccc1Br</smiles>

$190(0.85)$

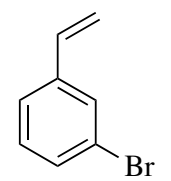

$192(0.85)$ 


\section{Table 7: Selective Coupling of Unsubstituted Bromophenyl Triflates (cont'd)}

\section{Entry Substrate $\quad$ Br-coupling $\quad$ TfO-coupling}

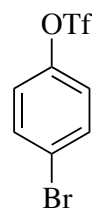

3A $\quad \mathbf{1 3 7}$

3B 137

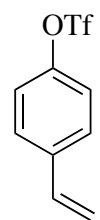

$138(0.85)$

$138(0.15)$

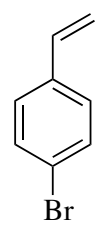

$139(0.15)$

$139(0.85)$

Cond. $\mathrm{A}: \mathrm{PPh}_{3}, \mathrm{Pd}(\mathrm{dba})_{2}$, 1,4-dioxane, $105^{\circ} \mathrm{C}$

Cond. B: $\mathrm{PdCl}_{2}\left(\mathrm{PPh}_{3}\right)_{2}$, LiCl, DMF, r.t.

Each of these substrates exhibited high selectivity under bromine-selective conditions A. However, conditions B were not as selective, with each substrate affording an approximately 6:1 ratio of the expected triflate-coupled product to the bromidecoupled product. This again demonstrated that conditions B were not ideal for selective coupling of triflates. Pure products were only isolated in some cases, however, due to the tendency of the styrene products to polymerize upon purification, particularly the bromostyrenes. In these cases, the coupled products were present in the NMR spectra obtained after work-up of the reaction mixture; however, no product was isolated upon purification using column chromatography regardless of whether silica gel, basic alumina, or neutral alumina were used as the stationary phase. For these substrates, the selectivities were inferred from integration of the ${ }^{1} \mathrm{H}$ NMR spectra.

Echavarren ${ }^{90}$ also examined the cross-coupling of iodophenyl triflate 193 (Scheme 59). In contrast to the results obtained for bromophenyl triflate $\mathbf{1 3 7}$, styrene $\mathbf{1 3 8}$ was the only product observed regardless of the catalyst conditions used. This was also in accordance with the established trend ${ }^{97}$ that iodides undergo oxidative addition faster than triflates. 


\section{Scheme 59: Echavarren's Coupling of $p$-Iodophenyl Triflate}

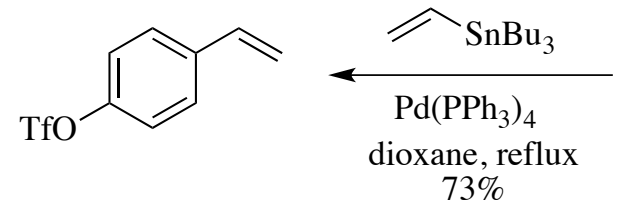

138<smiles>COc1ccc(I)cc1</smiles>

193

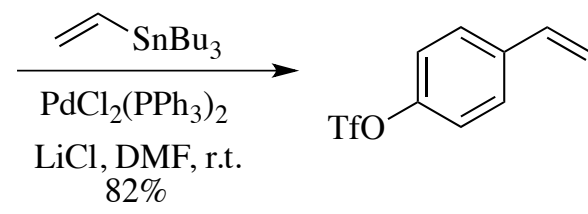

138

4-iodo-2-nitrophenyl triflate $\mathbf{1 9 6}$ was prepared as a model to examine whether an iodide would still be more reactive than a trifloxy group even if the iodide was in an unactivated position (meta to the nitro group) and the trifloxy group was in an activated position (ortho to the nitro group). Nitration ${ }^{93}$ of 4-iodophenol (194) afforded nitrophenol 195, which was then converted to triflate 196 using previously established conditions (Scheme 60).

\section{Scheme 60: Preparation of 4-Iodo-2-Nitrophenyl Triflate}<smiles>Oc1ccc(I)cc1</smiles>

194

$$
\underset{\underset{29 \%}{\mathrm{SiO}_{2}, \mathrm{NaNO}_{2}, \mathrm{Cl}_{2}, \mathrm{H}_{2} \mathrm{O}}}{\stackrel{\mathrm{CHalic}_{2}}{\longrightarrow}}
$$<smiles>CCOC(=O)C(C)CC</smiles>

195<smiles>CCOc1ccc(I)cc1[N+](=O)[O-]</smiles>

196

Under both conditions A and B, iodine-coupled 197 was the major product isolated (Scheme 61). Even though the triflate was in an activated position ortho to the nitro group, the iodine was still more reactive towards coupling, even under triflateselective conditions $\mathrm{B}$, which afforded only a trace amount of triflate-coupled product 198. This result was drastically different than that observed for the analogous bromide 154 (table 6, entry 8), which afforded exclusively triflate-coupled $\mathbf{1 8 3}$ in $78 \%$ yield under conditions B. In addition, iodide 196 afforded exclusively iodine-coupled 197 under conditions A, whereas the analogous bromide 154 (table 6, entry 8) exhibited erosion of 
selectivity under the same conditions. This direct comparison demonstrated the substantial difference in reactivity between aryl bromides and aryl iodides.

\section{Scheme 61: Coupling of 4-Iodo-2-Nitrophenyl Triflate}

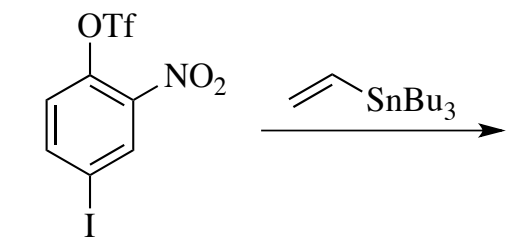

196

$\mathrm{Pd}(\mathrm{dba})_{2}, \mathrm{PPh}_{3}$, dioxane, reflux

$\mathrm{PdCl}_{2}\left(\mathrm{PPh}_{3}\right)_{2}, \mathrm{LiCl}, \mathrm{DMF}$, r.t.

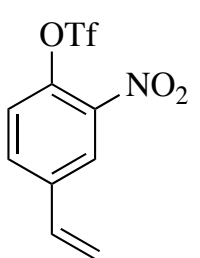

197

$40 \%$

$50 \%$<smiles>C=Cc1ccc(I)cc1[N+](=O)[O-]</smiles>

198

$4 \%$

To examine whether the generalized chemoselectivity could be extended to electron-withdrawing substituents aside from a nitro group, 5-bromo-2-trifloxy acetophenone (201) was prepared (Scheme 62). Bromination of phenol 199 afforded the desired bromide 200, which was then converted to triflate 201.

\section{Scheme 62: Preparation of 5-Bromo-2-Trifloxy Acetophenone}

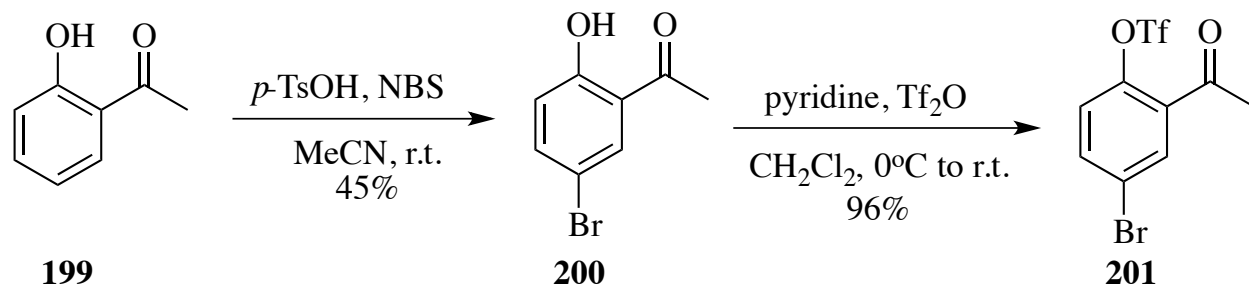

Based on the observations for nitro-substituted arenes, it was proposed that coupling of 201 under conditions A would exhibit erosion of selectivity due to the triflate being activated by the ortho electon withdrawing substituent. However, conditions A afforded only 202 (albeit in poor yield), and conditions B afforded only 203, as expected (Scheme 63). This suggests that weaker electron-withdrawing substituents have little influence on the overall selectivity. However, it is also important to note that for the analogous nitro substrate 154 (Table 6, entry 8), conditions A were not completely selective for bromine-coupling, while conditions B were completely selective for triflate- 
coupling. This result is in accordance with the weaker electron-withdrawing acetyl group present in $\mathbf{2 0 1}$ having a smaller activation effect relative to the nitro group in $\mathbf{1 5 4}$.

\section{Scheme 63: Selectivity Under Conditions A and B}<smiles>CCOc1ccc(Br)cc1C(=O)COc1ccccc1</smiles>

201

$\mathrm{Pd}(\mathrm{dba})_{2}, \mathrm{PPh}_{3}$, dioxane, reflux $\mathrm{PdCl}_{2}\left(\mathrm{PPh}_{3}\right)_{2}, \mathrm{LiCl}, \mathrm{DMF}$, r.t.<smiles>C=Cc1ccc(OC)c(C(C)=O)c1</smiles>

202

$14 \%$<smiles>C=Cc1ccc(Br)cc1C(C)=O</smiles>

203

$58 \%$

Previous work ${ }^{98}$ reported bromine-selective coupling under "Conditions B" for bromo-trifloxybenzene 204, affording 205 in moderate yield (Scheme 64). The observed selectivity was postulated to be due to the trifloxy group being deactivated by the ortho and para electron-donating methoxy and alkyl substituents, as well as the increased steric crowding around the triflate. The bromine positioned meta to the donating groups would be unaffected electronically, rendering it more reactive regardless of the catalyst conditions.

\section{Scheme 64: Bromine-Selective Coupling Under Conditions B}<smiles>CCOc1c(Br)cc(CO)cc1OC</smiles>

204

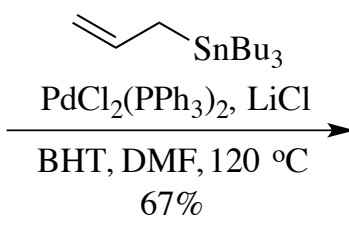

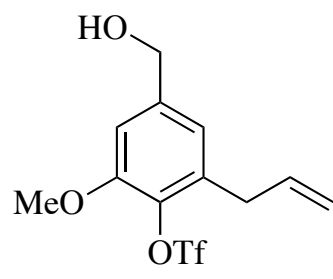

205

To study the effect of electron-donating substituents in a more simple, direct example, 3-bromo-4-trifloxy anisole (208) was prepared (Scheme 65). Bromination of phenol 206 exhibited high regioselectivity, affording bromide 207, which was then converted to triflate $\mathbf{2 0 8}$. 


\section{Scheme 65: Preparation of 3-Bromo-4-Trifloxy Anisole}

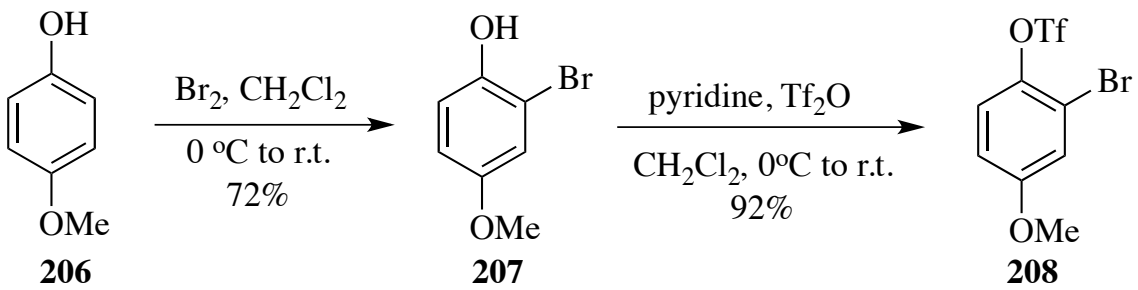

Based on previously reported results, ${ }^{98}$ it was proposed that the electron-donating methoxy group would deactivate the para-trifloxy substituent, while the meta-bromine would be unaffected since it was not in conjugation with the methoxy group. Indeed, bromine-coupled 209 was the only product observed under conditions A, while erosion of selectivity was observed under conditions B, resulting in a mixture of both $\mathbf{2 0 9}$ and $\mathbf{2 1 0}$ (Scheme 66). While the ratio of $\mathbf{2 1 0}: \mathbf{2 0 9}$ under conditions B was nearly 11:1, it was apparent that the para-methoxy group did indeed deactivate the triflate.

\section{Scheme 66: Selectivity Under Conditions A and B}

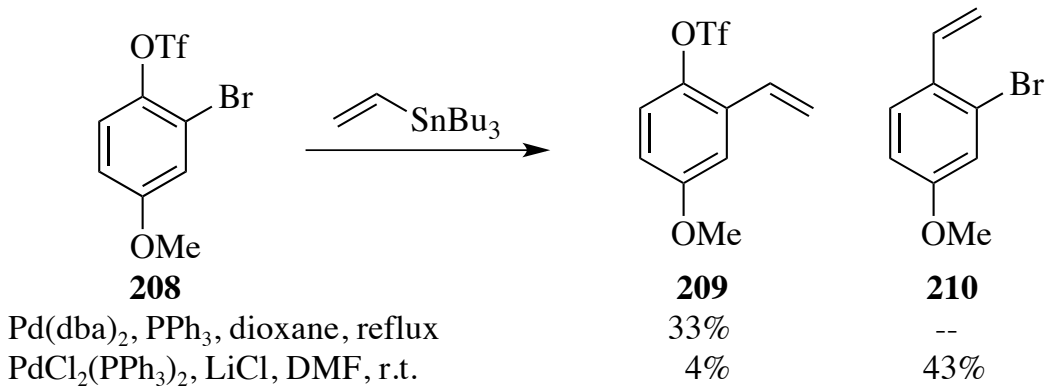

While this study of the selectivity in Stille coupling reactions allowed direct comparison between numerous substituted aromatics, there are still some issues that remain. For one, the yield in some cases was moderate at best. Although the goal was to study the selectivity only using the previously established conditions, ${ }^{90}$ it would be careless to make broad generalizations based on examples that were completely selective, yet the coupled product was isolated in poor yield. In addition, the role of the different reaction conditions is not fully explained. Though conditions B use a palladium (II) 
catalyst, this complex is presumably rapidly reduced to palladium (0) to afford a similar $\operatorname{Pd}\left(\mathrm{PPh}_{3}\right)_{\mathrm{x}}$ species to that formed under conditions A. ${ }^{99}$ Rather, the major difference between the two catalysts is the ratio of ligand : palladium in solution. ${ }^{100}$ The use of a more polar solvent (DMF vs. dioxane) under conditions B may also play a key role in stabilizing the intermediate formed through oxidative addition of palladium to the carbon-oxygen bond of the triflate. ${ }^{101}$ These numerous factors presumably had an effect on the observed selectivity.

\subsection{Conclusions}

Through this exhaustive study of selectivity in Stille coupling reactions, some important conclusions were drawn. It was clear that the presence of a nitro group as well as the positioning of the nitro group relative to the halide or trifloxy group had a direct effect on the chemoselectivity of the Stille coupling reaction. It was observed that reactivity was enhanced for groups positioned ortho and para to the nitro group, while groups meta to the nitro group were not affected. In the absence of a nitro group, selectivities were the direct result of the conditions themselves. In these cases, halideselective conditions A were highly selective, while triflate-selective conditions B exhibited slight erosion of selectivity, affording approximately 6:1 ratios of the expected triflate-coupled products relative to the bromine-coupled products. In accordance with the general reactivity series, iodides were more reactive than triflates regardless of the conditions used and independent the location of a nitro group in the molecule. In contrast to nitro groups, the weaker deactivating acetyl group did not exhibit a similar trend in activating ortho and para groups towards coupling reaction. On the contrary, an electron-donating group such as a methoxy group was found to deactivate the ortho and 
para positions through resonance. Based on these observations, the selectivity (or lack thereof) of a coupling reaction may be predicted. 


\section{Chapter 5}

\section{Preparation of Pyrroloindoles via Palladium-Catalyzed Reductive}

\section{Double $N$-Heterocyclization}

5.1 Pyrroloindole Introduction and Background

5.2 Historical Routes to Pyrroloindoles of Type I

5.3 Pyrroloindole Preparation using Reductive Double $N$-Heterocyclization

5.3.1 Retrosynthetic Analysis

5.3.2 Banini Route to Pyrroloindoles

5.3.3 Synthesis of Symmetrical Pyrroloindoles

5.4 Synthesis of Non-Symmetrical Pyrroloindoles

5.4.1 Preparation of Cyclization Precursors

5.4.1.1 2,4-Dinitro-1,5-Dialkenyl Benzene Isomer

5.4.1.2 2,5-Dinitro-1,4-Dialkenyl Benzene Isomer

5.4.1.3 2,3-Dinitro-1,4-Dialkenyl Benzene Isomer

5.4.1.4 2,4-Dinitro-1,3-Dialkenyl Benzene Isomer

100

5.4.1.5 1,4-Dinitro-2,3-Dialkenyl Benzene Isomer

106

5.4.2 Pyrroloindole Synthesis via Reductive Double $N$-Heterocyclization

110

5.5 Conclusions

115 


\subsection{Pyrroloindole Introduction and Background}

Pyrroloindoles have been attractive synthetic targets over the past few decades particularly due to their wide array of potential uses as biological agents as well as electroconductive materials ${ }^{102}$ and bidentate ligands. ${ }^{103}$ The name pyrroloindole refers to a molecule that features a pyrrole moiety fused onto an indole core. This fusion can come in one of three ways: Type I are those in which the pyrrole is attached to the benzene portion of the indole, Type II feature a pyrrole fused to the nitrogen-containing heterocyclic portion of the indole, and Type III which involve a common nitrogen atom (Figure 6).

Figure 6: Types of Isomeric Pyrroloindoles<smiles>c1cc2cc3cc[nH]c3cc2[nH]1</smiles>

Type I<smiles>c1ccc2c(c1)[nH]c1[nH]ccc12</smiles>

Type II<smiles>c1cc2ccn3ccc(c1)c23</smiles>

Type III

A number of pyrroloindoles have gained attention on account of their biological activities. Physostigmine (211), a natural product of Type II, is believed to possess therapeutic effects in the treatment of Alzheimer's disease (Figure 7). ${ }^{104}$ Phenserine (212), also a natural product of Type II, was another potential Alzheimer drug, although it failed in Phase III development (Figure 7).

\section{Figure 7: Physostigmine and Phenserine}<smiles>CNC(=O)Oc1ccc2c(c1)[C@]1(C)CCN(C)[C@H]1N2C</smiles>

211<smiles>CN1CC[C@]2(C)c3cc(OC(=O)Nc4ccccc4)ccc3N(C)[C@]12C</smiles>

212

One field in which pyrroloindole based compounds of Type I have found recent application is in the preparation of biological agents, specifically in anti-tumor 
treatments. Recent work focusing on the preparation of pyrroloindole based natural products CC-1065 (213), Duocarmycin (214) and Yatakemycin (215) has been reported by Boger et al (Figure 8). ${ }^{105}$ These compounds and their derivatives are members of a class of anti-tumor compounds whose mode of action centers around the sequenceselective alkylation of duplex DNA within the tumor through nucleophilic opening of the cyclopropane functionality of the anti-tumor agent.

\section{Figure 8: CC-1065, Duocarmycin, and Yatakemycin}<smiles></smiles>

213<smiles>COC(=O)[C@]1(C)NC(=O)C2=C1C(=O)C=C1[C@@H]2CCN1C(=O)c1cc2cc(OC)c(OC)c(OC)c2[nH]1</smiles>

214<smiles>C[C@]12CCN(C(=O)c3cc4cc(O)c(O)cc4[nH]3)C1=CC(=O)c1[nH]c(C(=O)N3CCc4c3c(O)c(O)c3[nH]c(C(=O)S)cc43)cc12</smiles>

215

Recent work in Soderberg's laboratory has focused on the preparation of pyrroloindoles of Type I in which the pyrrole is fused to the benzene portion of the indole. Within this class, there are five different isomers, which are shown in Figure 9. 
Figure 9: Isomeric Pyrroloindoles of Type I

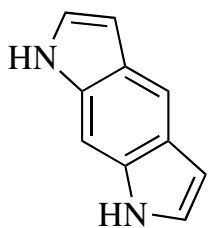

Class A

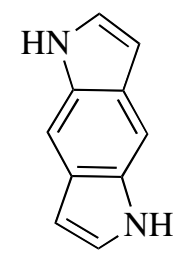

Class B<smiles>c1cc2ccc3cc[nH]c3c2[nH]1</smiles>

Class C

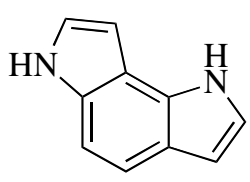

Class D<smiles>c1cc2c(ccc3[nH]ccc32)[nH]1</smiles>

Class E

Pyrroloindoles of type I are named based on the orientation of the pyrrole ring relative to the indole core (Figure 10). Similar to the numbering of the atoms of simple indole, the numbering ascends counter-clockwise around the heterocycle with the nitrogen being atom 1. In a similar fashion, each bond of the indole core is assigned a letter, with the bond between atoms 1 and 2 being bond $\mathrm{A}$. The manner in which the pyrrole unit is fused to the indole core is represented by $[\mathrm{x}, \mathrm{y}-z]$, where $\mathrm{x}$ and $\mathrm{y}$ are the atoms of the pyrrole and $\mathrm{z}$ is the bond of the indole core that atoms $\mathrm{x}$ and $\mathrm{y}$ are attached to. For example, pyrroloindoles of class $\mathrm{C}$ are named 1,8-dihydropyrrolo[3,2-g]indoles.

\section{Figure 10: Pyrroloindole Numbering}
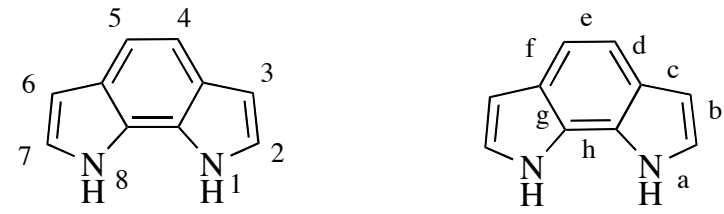

\subsection{Historical Routes to Pyrroloindoles of Type I}

One of the earliest preparations of symmetrical pyrroloindoles was reported by Berlin et al. in 1987 in the study of oxidative polymerization to afford electroconductive materials. ${ }^{106}$ This two-step route involved condensation of di-nitro xylene (216) with $N, N$ dimethylformamide diethyl acetal (DMF-DEA) to give the bis-enamine 217 which then 
underwent reductive cyclization catalyzed by palladium on carbon to afford dihydropyrroloindole 218 (Scheme 67). Although the route was concise, it was quite limited in scope in that only symmetrical, non-substituted pyrroloindoles were accessible.

\section{Scheme 67: Berlin's Approach to Pyrroloindoles}

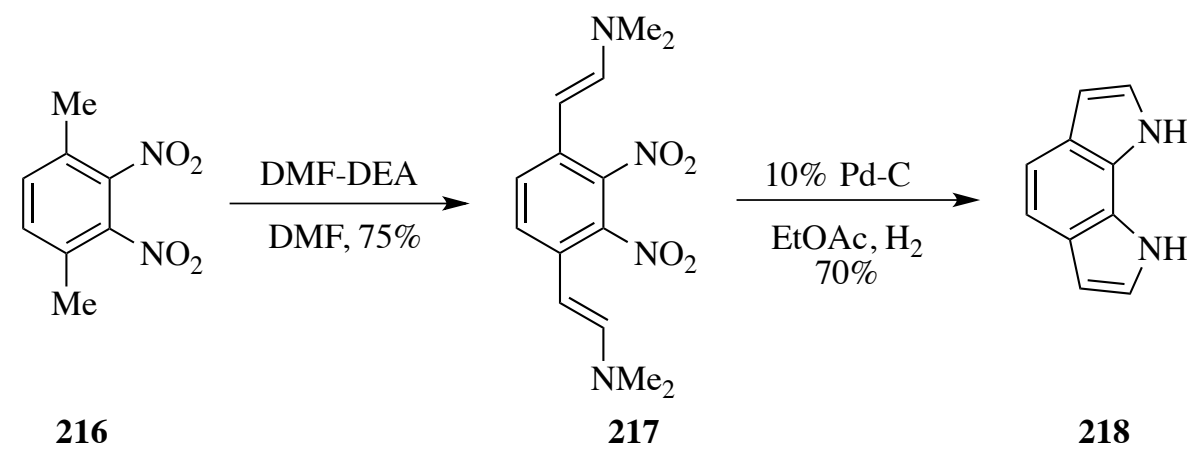

Shannon et al. prepared pyrrolo[3,2-f]- (220) and -[2,3-f]-indoles (221) through the Montmorillonite K-10 clay catalyzed Vilsmeier formylation of dipyrrole 219 (Scheme 68). ${ }^{107} \mathrm{~A}$ mixture of isomers was obtained due to free-rotation about the methylene bridge.

\section{Scheme 68: Montmorillonite K-10 Catalyzed Synthesis of Pyrroloindoles}

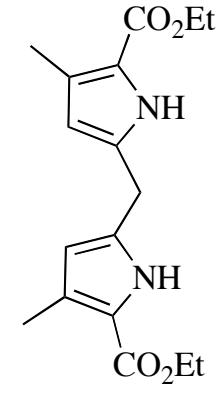

219

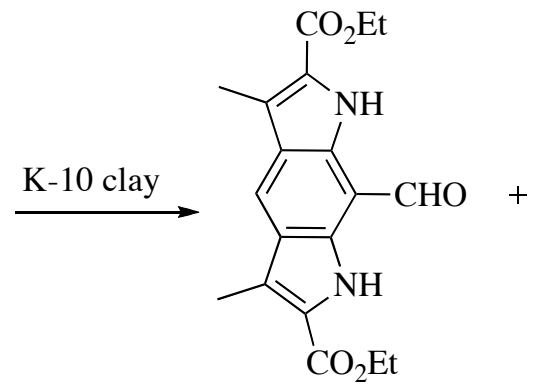

220<smiles>CCOC(=O)c1[nH]c2c(C=O)c3c(C)c(C(=O)OCC)[nH]c3cc2c1C</smiles>

221

Samsoniya et al. reported numerous methods to prepare and characterize isomeric pyrroloindoles ${ }^{108,109}$ The first method utilized a Fischer-type bicyclization of bishydrazone 222 to form the two pyrrole rings (Scheme 69). ${ }^{108}$ The authors noted that due to the harsh conditions required for this transformation, a considerable amount of tar formed over the course of the reaction, presumably from decomposition of the reactants 
and/or products. Another drawback was that due to the nature of the cyclization, one starting material 222 produced four isomeric pyrroloindole products $(\mathbf{2 2 3 - 2 2 6})$ with little control over selectivity.

\section{Scheme 69: Samsoniya Route to Isomeric Pyrroloindoles}

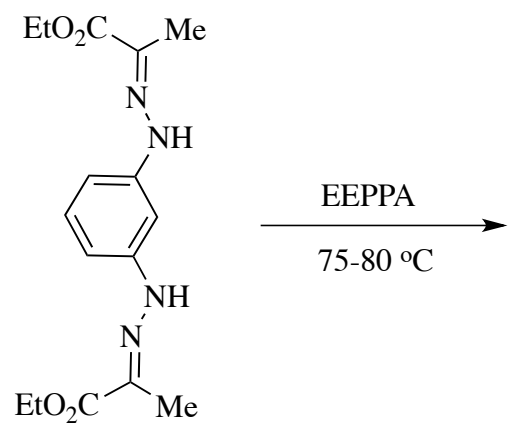

222<smiles>CCOC(=O)c1cc2cc3cc(C(=O)OCC)[nH]c3cc2[nH]1</smiles>

223<smiles>CCOC(=O)c1cc2cc3cc(C(=O)OCC)n(CC)c3cc2[nH]1</smiles>

224<smiles>CCOC(=O)c1cc2c(ccc3cc(C(=O)OCC)[nH]c32)[nH]1</smiles>

225<smiles>CCOC(=O)c1cc2c(ccc3cc(C(=O)OCC)n(CC)c32)[nH]1</smiles>

226

The second method reported by Samsoniya et al. circumvented the previously mentioned issue of isomer formation by using a pre-formed aminoindoline $\mathbf{2 2 7}$ (Scheme 70). ${ }^{109}$ Fischer-type cyclization of hydrazone $\mathbf{2 2 7}$ produced two isomeric pyrroloindolines $\mathbf{2 2 8}$ and $\mathbf{2 2 9}$ with a preference for linear isomer 228. Hydrolysis of the ester and acetyl groups followed by decarboxylation and dehydrogenation then afforded the non-substituted pyrroloindoles 230 and 231.

Scheme 70: Pyrroloindole Preparation from Aminoindoline

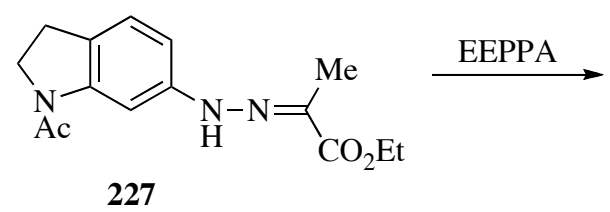

227
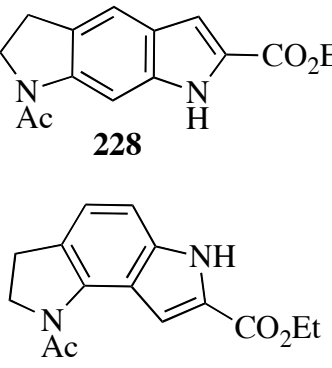

229<smiles>c1cc2cc3cc[nH]c3cc2[nH]1</smiles>

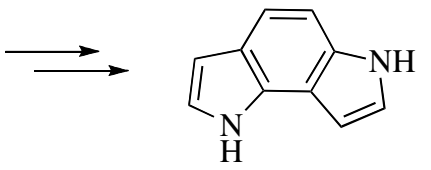

231

Yoshikai et al. have applied their palladium-catalyzed aerobic oxidative bicyclization method to the preparation of pyrroloindoles from $\mathrm{N}$-aryl imines. ${ }^{110}$ 
Palladium(II)-catalyzed dicyclization of diimines $\mathbf{2 3 2}$ and $\mathbf{2 3 4}$ afforded corresponding pyrroloindoles 233 and 235 (Scheme 71). While the authors did not attempt to prepare non-symmetrical compounds, the ease of preparation and wide range of aniline and ketone starting materials afford this method a very broad scope, although the yields of the pyrroloindole products were modest at best. In addition, the regioselectivities observed in the cyclizations were not explained

\section{Scheme 71: Oxidative Bicyclization of Diimines}

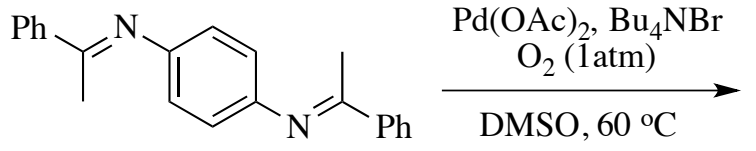

232<smiles>C/C(=N\c1cccc(/N=C(\C)c2ccccc2)c1)c1ccccc1</smiles>

234<smiles>c1ccc(-c2cc3cc4[nH]c(-c5ccccc5)cc4cc3[nH]2)cc1</smiles>

233<smiles>c1ccc(-c2cc3c(ccc4cc(-c5ccccc5)[nH]c43)[nH]2)cc1</smiles>

235

Fujii and Ohno reported the preparation of mesylated pyrroloindole 237 through copper-catalyzed bis-cyclization of di-alkynyl-dimesylamide 236 (Scheme 72). ${ }^{111}$ The authors state that the cyclization was highly dependent on the substituents on the nitrogen atoms, with mesylates giving the highest conversion. In addition, this approach was limited to terminal alkynes, affording only non-substituted pyrroloindoles.

\section{Scheme 72: Intramolecular Hydroamination of Di-yne}<smiles>C#Cc1cc(C#C)c(NN)cc1NC</smiles>

236

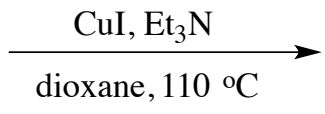

dioxane, $110{ }^{\circ} \mathrm{C}$<smiles></smiles>

237

One inherent limitation of many previous synthetic routes is that they only allow for preparation of symmetrical pyrroloindoles. This is in part due to limitations 
associated with the methods used to prepare the required starting materials, most of which also involve symmetrical compounds. Therefore, methods to prepare nonsymmetrical substrates that can be converted to non-symmetrical pyrroloindoles are highly desirable.

\subsection{Pyrroloindole Preparation via Reductive Double $N$-Heteroannulation}

As discussed in the preceding chapters, Soderberg's laboratory has studied the applications of palladium-catalyzed reductive $N$-heterocyclizations extensively. It was

envisioned that this methodology could be applied in the synthesis of pyrroloindoles from dinitro-dialkenyl benzenes. The generally mild conditions used in such reactions could allow for broad functional group compatibility and offer significant improvements over the previously discussed methods.

\subsubsection{Retrosynthetic Analysis}

Retrosynthetically, it was proposed that each of the five isomeric pyrroloindoles of type I could be prepared through the palladium-catalyzed reductive double heterocyclization of the corresponding dinitro-dialkenyl benzene (Scheme 73). 


\section{Scheme 73: Retrosynthetic Analysis of Pyrroloindoles}<smiles>c1cc2cc3cc[nH]c3cc2[nH]1</smiles>

230<smiles>C=CCc1cc(C=C)c([N+](=O)[O-])cc1C=C</smiles>

238<smiles>C=Cc1ccc(C=C)c([N+](=O)[O-])c1[N+](=O)[O-]</smiles>

241<smiles>c1cc2cc3[nH]ccc3cc2[nH]1</smiles>

239<smiles>C=CO[N+](=O)[O-]</smiles>

240<smiles>C=C</smiles><smiles>c1cc2c(ccc3[nH]ccc32)[nH]1</smiles>

243<smiles>C=COc1ccc([N+](=O)[O-])c(C=C)c1C=C</smiles>

231 242<smiles>C=Cc1c([N+](=O)[O-])ccc([N+](=O)[O-])c1C=C</smiles>

\subsubsection{Banini Route To Pyrroloindoles}

Banini et al. successfully prepared pyrroloindoles using Soderberg's palladiumcatalyzed reductive $N$-heteroannulation methodology. Pyrroloindoles of class B such as 239 were achieved in six steps from $p$-bromotoluene (245) (Scheme 74). Nitration ${ }^{112}$ of 245 afforded a four-product mixture from which isomer 246 was separated and subjected to radical benzylic bromination ${ }^{113}$ using $N$-bromosuccinimide and benzoyl peroxide. Benzyl bromide 247 was then converted to phosphine salt 248 before undergoing Wittig condensation to afford styrene 249. Kosugi-Migita-Stille coupling between bromide 249 and vinyl stannane then afforded dinitro-dialkenyl benzene 240. Reductive double $N$ heterocyclization was achieved through reaction with $\mathrm{PPh}_{3}$ and $\mathrm{Pd}(\mathrm{dba})_{2}$ in $\mathrm{DMF}$ at 120 ${ }^{\circ} \mathrm{C}$ in a sealed tube pressurized with carbon monoxide gas, affording pyrroloindole 239 in 
excellent yield. In addition to symmetrical pyrroloindole 239, non-symmetrical pyrroloindoles were also prepared using this route.

\section{Scheme 74: Banini Route to Pyrroloindoles of Class B}
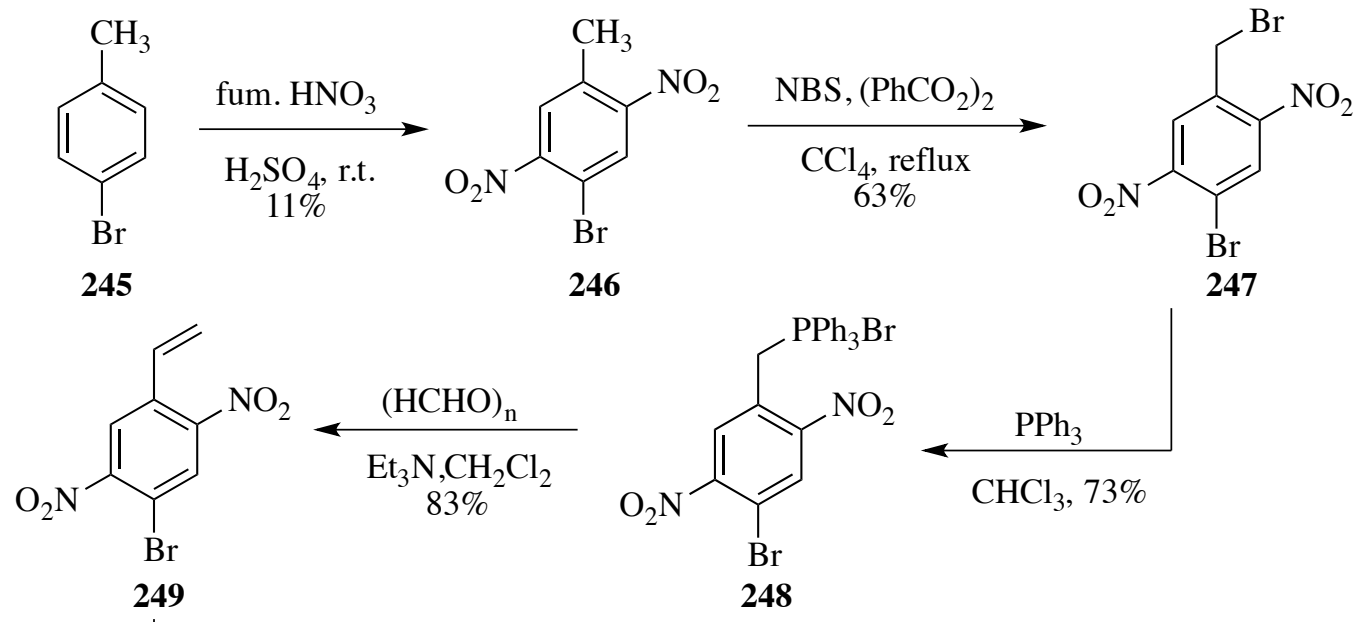

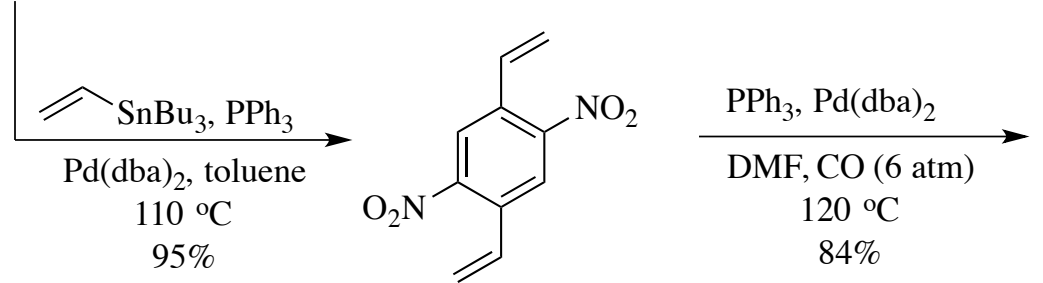

240<smiles>c1cc2cc3[nH]ccc3cc2[nH]1</smiles>

239

A slightly different approach was employed to prepare pyrroloindoles of class C

(Scheme 75). Nitration of 1,4-dibromobenzene (250) provided three dinitrobenzene isomers, including 2,3-dinitro-1,4-dibromobenzene (251). Kosugi-Migita-Stille crosscoupling between $\mathbf{2 5 1}$ and vinyl stannane afforded bromostyrene 252, which underwent a second Kosugi-Migita-Stille coupling with $\alpha$-stannyl ester to afford cyclization precursor 253. Exposure of $\mathbf{2 5 3}$ to altered Soderberg reductive heteroannulation conditions $\left(\mathrm{PPh}_{3}\right.$, $\left.\mathrm{Pd}(\mathrm{OAc})_{2}\right)$ afforded pyrroloindole 254 in excellent yield. 


\section{Scheme 75: Banini Route to Pyrroloindoles of Class C}<smiles>Brc1ccc(Br)cc1</smiles>

250
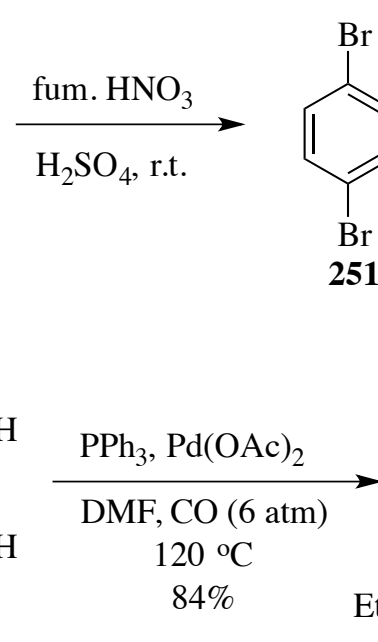

254<smiles>O=[N+]([O-])c1c(Br)ccc(Br)c1[N+](=O)[O-]</smiles>

251

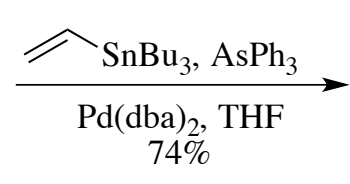<smiles>C=Cc1ccc(Br)c([N+](=O)[O-])c1[N+](=O)[O-]</smiles><smiles>CCOC(=O)c1c[nH]c2c1ccc1cc[nH]c12</smiles>

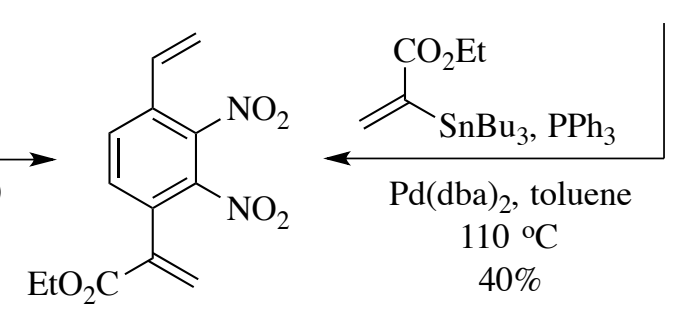

253

\subsubsection{Synthesis of Symmetrical Pyrroloindoles}

Initial attempts to elaborate on Banini's work encountered a number of issues. While Banini reported the benzylic bromination of dinitrotoluene 246 to afford benzyl bromide $\mathbf{2 4 7}$ in moderate yield, in our hands, the reaction afforded near quantitative recovery of starting material (Scheme 76).

\section{Scheme 76: Failed Preparation of Benzyl Bromide}<smiles>Cc1cc([N+](=O)[O-])c(Br)cc1[N+](=O)[O-]</smiles>

246<smiles>O=[N+]([O-])c1cc(CBr)c([N+](=O)[O-])cc1Br</smiles>

247

Replication of the reported mono-Kosugi-Migita-Stille cross-coupling of dinitrodibromobenzene 251 with vinyl stannane was also unsuccessful (Scheme 77). While Banini achieved styrene $\mathbf{2 5 2}$ in moderate yield, in our hands, a mixture of mono- and dicoupled products 252 and 241 was obtained. 


\section{Scheme 77: Attempted Mono-Coupling of Dibromide}

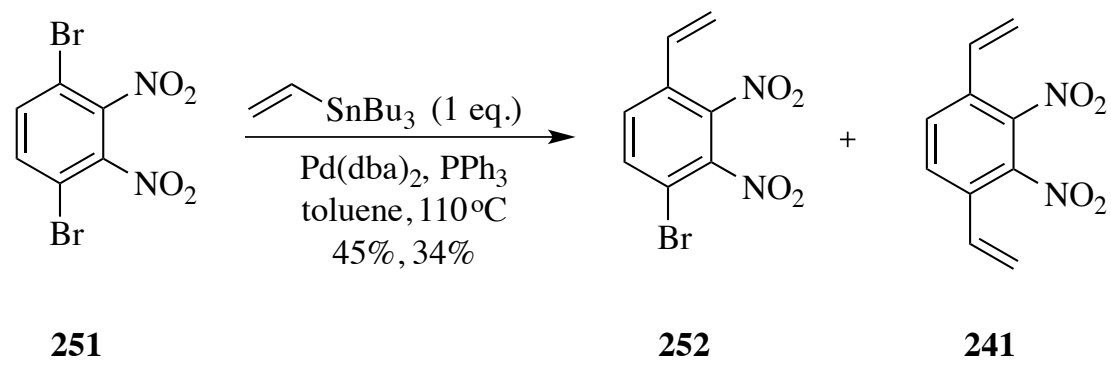

To circumvent this issue, the coupling reaction was attempted using a three-fold excess of stannane with the goal of preparing exclusively di-coupled arene 241 . In the event, di-vinyl compound $\mathbf{2 4 1}$ was prepared in moderate yield (Scheme 78).

\section{Scheme 78: Preparation of Dialkenyl-Dinitrobenzene}<smiles>O=[N+]([O-])c1c(Br)ccc(Br)c1[N+](=O)[O-]</smiles>

251

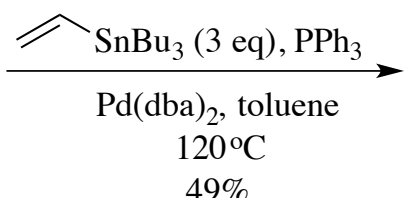

$49 \%$<smiles>C=Cc1ccc(C=C)c([N+](=O)[O-])c1[N+](=O)[O-]</smiles>

241

Reductive double $N$-heteroannulation of $\mathbf{2 4 1}$ was achieved using alternate conditions reported by Banini to afford pyrroloindole $\mathbf{2 1 8}$ in moderate yield (table 8 , entry 1). Two additional cyclization precursors 255 and 257 were also prepared through dicoupling of $\mathbf{2 4 1}$ with propenyl- and isopropenyl stannane, respectively. Palladiumcatalyzed cyclization of $\mathbf{2 5 5}$ afforded exclusively symmetrical pyrroloindole $\mathbf{2 5 6}$ in moderate yield (entry 2). Cyclization of $\mathbf{2 5 7}$ afforded solely indole $\mathbf{2 5 9}$ when the catalyst conditions were 1,10-phenanthroline and $\mathrm{Pd}(\mathrm{OAc})_{2}$, however, pyrroloindole 258 was achieved through use of $\mathrm{PPh}_{3}$ and $\mathrm{Pd}(\mathrm{dba})_{2}$, although indole 259 was the major product. 
<smiles>C=Cc1ccc(C=C)c([N+](=O)[O-])c1[N+](=O)[O-]</smiles>

$1^{\mathrm{b}}$

241

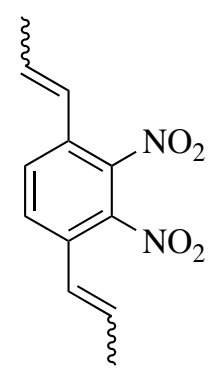

$2^{c}$

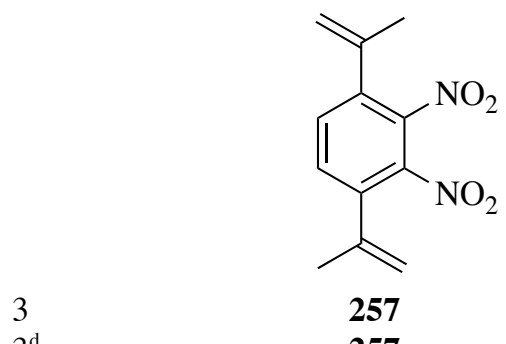<smiles></smiles>

218 (56\%)

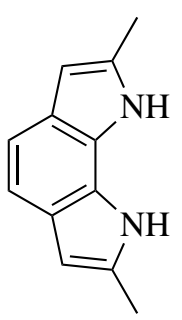

$256(43 \%)$

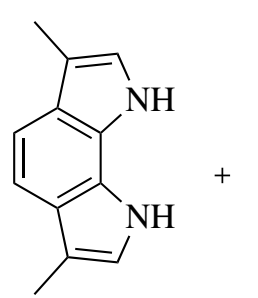

$-$<smiles>C=C(C)c1ccc2c(C)c[nH]c2c1[N+](=O)[O-]</smiles>

$259(12 \%)$ $259(54 \%)$

a) 1,10-phenanthroline, $\mathrm{Pd}(\mathrm{OAc})_{2}, \mathrm{CO}(6 \mathrm{~atm}), \mathrm{DMF}, 120^{\circ} \mathrm{C}$; b) Reaction run for 143 hours; c) Reaction run for 20 hours; d) $\mathrm{PPh}_{3}, \mathrm{Pd}(\mathrm{dba})_{2}, \mathrm{CO}(6 \mathrm{~atm}), \mathrm{DMF}, 120^{\circ} \mathrm{C}, 96$ hours

Dihydropyrroloindole 218 (entry 1) was isolated in moderate yield after 143 hours, although the cyclization was likely complete at an earlier time on account of the lack of unreacted starting material or mono-cyclized product. It was expected that the simple vinyl groups would present less steric repulsion toward the palladium in comparison to propenyl-substituted substrates 255 and 257 and would therefore react faster. Surprisingly, di-propenyl substrate 255 (entry 2) afforded pyrroloindole 256 in moderate yield after only 20 hours. On the contrary, 257 (entry 3) appeared to undergo mono-cyclization with ease, producing indole $\mathbf{2 5 9}$, however, the second cyclization to afford pyrroloindole $\mathbf{2 5 8}$ was much slower as evidenced by the significantly lower yield 
relative to indole 259. The decreased rate of the second cyclization could potentially be attributed to weaker coordination of the catalyst to the isopropenyl moiety of $\mathbf{2 5 9}$.

\subsubsection{Synthesis of Non-Symmetrical Pyrroloindoles}

While symmetrical pyrroloindoles were prepared using Soderberg's palladiumcatalyzed reductive double $N$-heteroannulation methodology, numerous limitations were apparent, including the inability to prepare non-symmetrical pyrroloindoles and the incomplete conversion to pyrroloindoles. In addition, only two of the five isomeric pyrroloindoles were achieved through the routes. Efforts to overcome the limitations in the preparation of non-symmetrical pyrroloindoles are presented in the sections hereafter.

\subsubsection{Preparation of Cyclization Precursors}

With the goal of preparing non-symmetrical pyrroloindoles using Soderberg's reductive annulation methodology, efforts were made to establish routes to prepare the requisite dinitro-dialkenyl benzenes. Specifically, efforts focused on synthesizing nonsymmetrical cyclization precursors in a controlled, sequential manner. For organizational clarity, each isomeric substrate is presented separately, although numerous commonalities were encountered in the preparation of each substrate.

\subsubsection{2,4-Dinitro-1,5-Dialkenyl Benzene Isomer}

The first and most extensively examined cyclization precursors were 2,4-dinitro1,5-dialkenyl benzenes, such as $\mathbf{2 3 8}$ (Figure 11).

Figure 11: 2,4-Dinitro-1,5-Dialkenyl Benzene

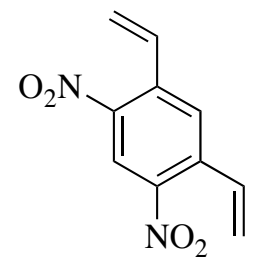


Retrosynthetically, it was envisioned that dinitro-dialkenyl benzene $\mathbf{2 6 0}$ could be prepared through Kosugi-Migita-Stille coupling reaction of triflate 261 (Scheme 79). The isopropenyl moiety of $\mathbf{2 6 1}$ could be introduced by Kosugi-Migita-Stille cross coupling between aryl iodide $\mathbf{2 6 2}$ and isopropenyl stannane. Dinitrophenyl triflate $\mathbf{2 6 2}$ could arise from dinitrophenol $\mathbf{2 6 3}$, which could be prepared through the nitration of 3iodophenol (264).

Scheme 79: Proposed Retrosynthetic Outline to Cyclization Precursor<smiles>C=C(C)c1cc(C(=C)C)c([N+](=O)[O-])cc1[N+](=O)[O-]</smiles>

260<smiles>C=COc1cc(C(=C)C)c([N+](=O)[O-])cc1OCC</smiles>

261<smiles>C=COc1cc(I)c([N+](=O)[O-])cc1OCC</smiles>

262<smiles>O=[N+]([O-])c1cc([N+](=O)[O-])c(I)cc1O</smiles>

Banwell et al..$^{114}$ have reported the nitration of 3-iodophenol (264) using sodium nitrate in a solution of aqueous methanol and sulfuric acid, providing both 3-iodo-4nitrophenol (265) and 5-iodo-2-nitrophenol (266) (Scheme 80). The relatively mild conditions employed prevent the formation of trinitrophenols as reported under harsher conditions. $^{115,116}$

\section{Scheme 80: Nitration of 3-Iodophenol}<smiles>Oc1cccc(I)c1</smiles><smiles>O=C(O[Na])[W](=O)O</smiles>
$22 \%, 34 \%$<smiles>O=[N+]([O-])c1ccc(O)cc1I</smiles>

265<smiles>O=[N+]([O-])c1ccc(I)cc1O</smiles>

266 
With isomers 265 and 266 in hand, a second nitration was examined. The methanol/water conditions used in the initial nitration (Scheme 80 ) proved to be too mild for a second nitration to occur, as evidenced by the absence of di-nitrated products. Therefore, a solution of sodium nitrate in sulfuric acid, believed to be slightly more acidic than the conditions used to introduce the first nitro group, yet milder than the fuming nitric acid/sulfuric acid system, was used. Gratifyingly, treatment of 5-iodo-2nitrophenol (266) with sodium nitrate in sulfuric acid provided di-nitrophenols 263 and 267 with no evidence of tri-nitration (Scheme 81). It should be noted that in some cases, solely isomer 263 was formed, while in other cases, a near 1:1 mixture of isomers 263 and 267 was obtained.

\section{Scheme 81: Nitration of 5-Iodo-2-Nitrophenol}

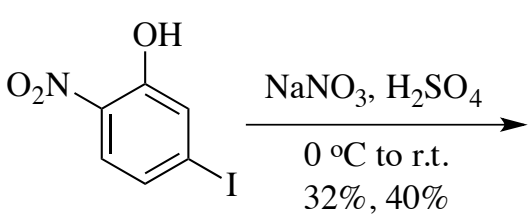

266<smiles>O=[N+]([O-])c1cc(I)c([N+](=O)[O-])cc1O</smiles>

263<smiles>O=[N+]([O-])c1ccc(I)c([N+](=O)[O-])c1O</smiles>

267

Nitration of 3-iodo-4-nitrophenol (265) using these same conditions afforded dinitrophenols 263 and 268 (Scheme 82). Once again, 5-iodo-2,4-dinitrophenol (263) was the major product observed in most reactions, although in some cases, isomer $\mathbf{2 6 8}$ was also formed. Unfortunately, dinitrophenols 263 and 268 were nearly inseparable using column chromatography.

\section{Scheme 82: Nitration of 3-Iodo-4-Nitrophenol}

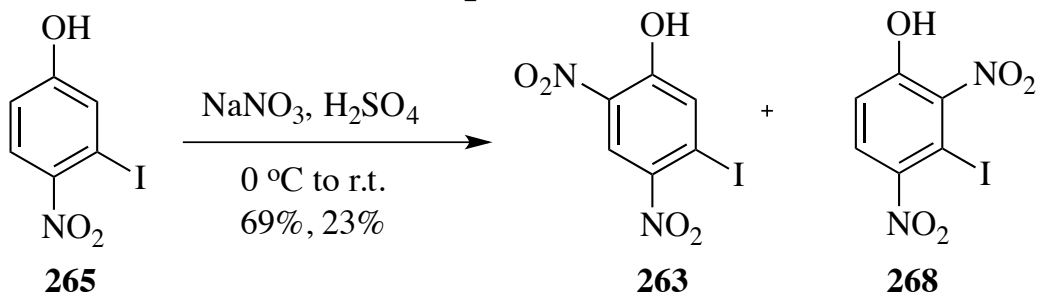


Subsequent treatment of dinitrophenol 263 with pyridine and triflic anhydride afforded aryl triflate $\mathbf{2 6 2}$ in near quantitative yield (Scheme 83).

\section{Scheme 83: Preparation of Aryl Triflate}<smiles>O=[N+]([O-])c1cc(I)c([N+](=O)[O-])cc1O</smiles>

263

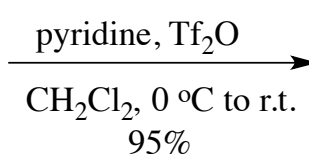
$95 \%$<smiles>CCOc1cc([N+](=O)[O-])c(I)cc1[N+](=O)[O-]</smiles>

262

With the desired functionalized starting material in hand, focus was turned to the coupling reactions to prepare the target non-symmetrical dialkenyl-dinitrobenzenes. Attempts to selectively couple the iodine of substrate $\mathbf{2 6 2}$ were successful in producing styrene 146, however, purification using column chromatography resulted in the hydrolysis of the trifloxy group of $\mathbf{1 4 6}$ and lead to the isolation of phenol 247 (Scheme 84). Similar hydrolysis of electron-deficient aryl triflates has been documented in the literature. $^{117}$

\section{Scheme 84: Attempted Kosugi-Migita-Stille Coupling}<smiles>CCOc1cc(I)c([N+](=O)[O-])cc1[N+](=O)[O-]</smiles>

262

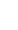

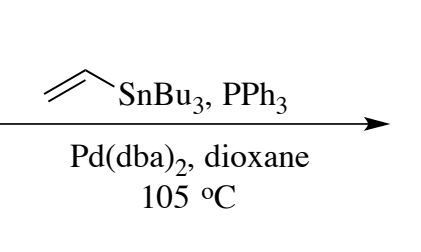<smiles>C=Cc1cc(OCC)c([N+](=O)[O-])cc1[N+](=O)[O-]</smiles>

146<smiles>C=Cc1cc(O)c([N+](=O)[O-])cc1[N+](=O)[O-]</smiles>

270

This counter-productive result forced reconsideration of the proposed synthetic

route. While phenols are typically poor substrates for Stille coupling reactions due to the electron-rich nature of the aromatic system, the two nitro-groups of phenol 263 would potentially provide enough activation to allow for coupling of the dinitro-iodophenol and circumvent the issue of hydrolysis. This hypothesis was tested using 4,6-dinitro-3iodophenol (263) (Scheme 85). Initially, attempted Kosugi-Migita-Stille coupling of 
iodophenol 263 under conditions $\mathrm{A}^{90}\left(\mathrm{PPh}_{3}, \mathrm{Pd}(\mathrm{dba})_{2}\right.$, dioxane, reflux $)$ afforded no product. Subsequently, copper (I) iodide was added and the solution was heated at reflux. Delightfully, complete conversion of the starting material to coupled phenol 271 was achieved in four hours. However, after workup with aqueous ammonia, no product was detected in the crude mixture via ${ }^{1} \mathrm{H}$ NMR. On account of the ortho and para nitro groups, the acidity phenolic hydrogen of $\mathbf{2 7 1}$ was substantially lower than that of phenol itself, rendering the phenol soluble in aqueous base. In the event, lowering the $\mathrm{pH}$ of the aqueous phase from $\sim 10$ to $\sim 4$ followed by extraction provided the desired coupled phenol 271. Optimization of conditions led to shortened reaction times (3 hours) while affording phenol 271 in good yield.

\section{Scheme 85: Kosugi-Migita-Stille Coupling of Iodophenol}<smiles>O=[N+]([O-])c1cc(I)c([N+](=O)[O-])cc1O</smiles>

263

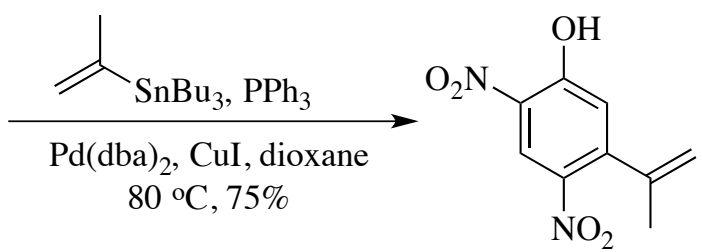

271

This result not only afforded the desired compound, but it also resulted in purification of the product using a simple extraction process, which is depicted in Figure 12. Initially, the phenol product was extracted using an aqueous base, "washing" away the unwanted organic components from the reaction including dba and tributyltin iodide. Acidification of the aqueous phase protonated the phenoxide, allowing for extraction of the nearly pure phenol product prior to chromatography. 
Figure 12: Modified Work-up Procedure for Coupling of Phenol

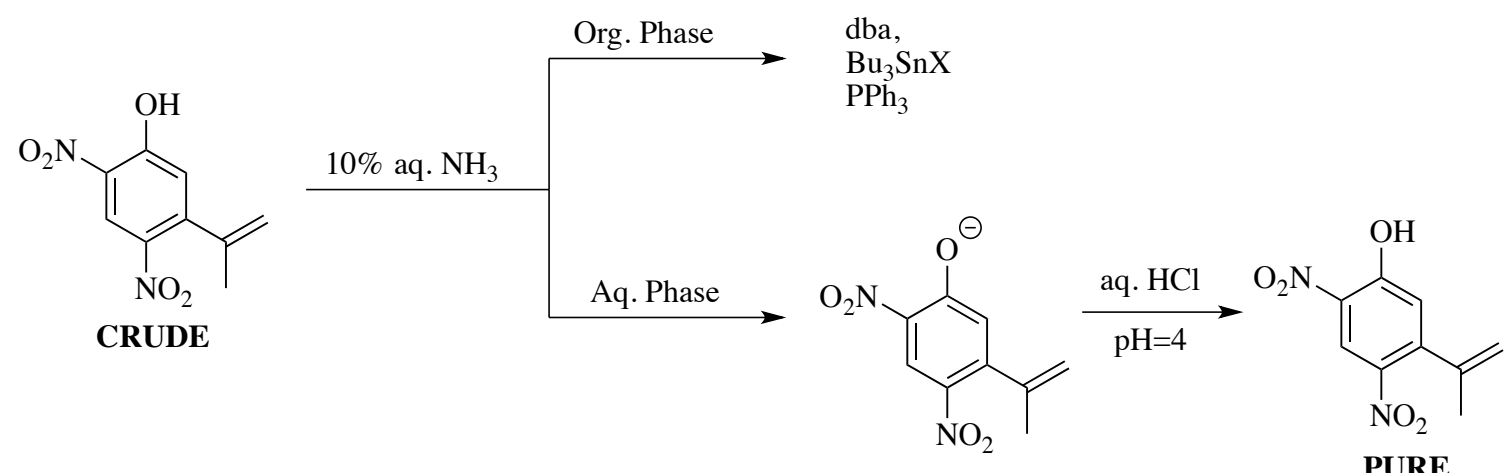

The success of the coupling reaction was attributed to the addition of copper iodide. The role of copper salts in Stille coupling reactions has been studied extensively. ${ }^{118,119,120}$ Liebeskind et al. reported significant rate enhancement when adding CuI to $\mathrm{Pd}(0) / \mathrm{PPh}_{3}$ catalyst systems, however, no rate enhancement was observed when using soft ligands such as $\mathrm{AsPh}_{3}{ }^{120}$ This has been attributed to $\mathrm{CuI}$ acting as a ligand scavenger, so the reported rate enhancement in the $\mathrm{Pd}(0) / \mathrm{PPh}_{3}$ system is presumably due to scavenging of excess $\mathrm{PPh}_{3}$, as $\mathrm{PPh}_{3}$ is known to inhibit the rate-limiting transmetalation step. Generation of organocopper species could also be generated through initial tin-copper transmetalation. ${ }^{118}$ The formed organocopper complex would then undergo tranmetalation to palladium faster in comparison to the initial stannane. Similar palladium-copper co-catalytic Stille conditions were employed by Hudgens et $a l .^{121}$ in the preparation of novel methylated tyrosine derivates.

Conversion of phenol 271 to triflate 261 was achieved using standard conditions (Scheme 86). Rapid purification using column chromatography afforded triflate 261 with no evidence of hydrolysis. 


\section{Scheme 86: Conversion of Phenol to Triflate}<smiles>C=C(C)c1cc(O)c([N+](=O)[O-])cc1[N+](=O)[O-]</smiles>

271

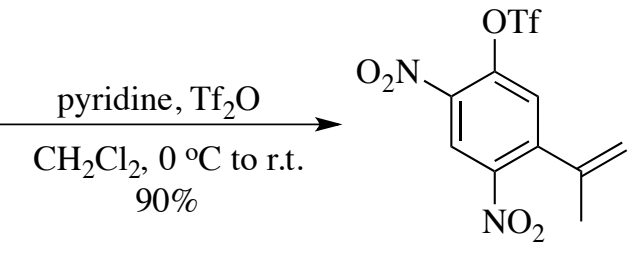

261

Initially, issues were encountered in applying conditions $\mathrm{B}^{90}\left(\mathrm{PdCl}_{2}\left(\mathrm{PPh}_{3}\right)_{2}, \mathrm{LiCl}\right.$, DMF, r.t.) to couple the trifloxy group of $\mathbf{2 6 1}$. The attempted coupling of triflate $\mathbf{2 6 1}$ with stannane $\mathbf{2 7 2}$ afforded exclusively aryl chloride 273 (Scheme 87).

\section{Scheme 87: Attempted Kosugi-Migita-Stille Coupling of Aryl Triflate}<smiles>C=C(C)c1cc(OCC)c([N+](=O)[O-])cc1[N+](=O)[O-]</smiles>

261

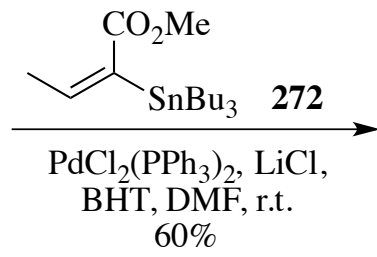

$60 \%$<smiles>C=C(C)c1cc(Cl)c([N+](=O)[O-])cc1[N+](=O)[O-]</smiles>

273

To probe the mechanism of this unexpected result, triflate $\mathbf{2 6 1}$ was treated with excess lithium chloride in DMF without the addition of palladium (Scheme 88). This would reveal whether the reaction involved a simple nucleophilic aromatic substitution mechanism or whether it was a palladium-catalyzed process. Interestingly, chloride $\mathbf{2 7 3}$ was achieved in moderate yield in the absence of a palladium catalyst, confirming the nucleophilic aromatic substitution mechanism. While unexpected, this result was reasonable considering highly activated nature of the trifloxy group on the basis of the ortho and para nitro substituents. Exploration of the literature also produced examples of similar observations. ${ }^{122}$ 


\section{Scheme 88: Conversion of Aryl Triflate to Aryl Chloride}

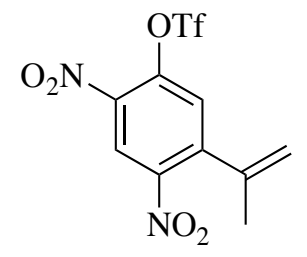

261

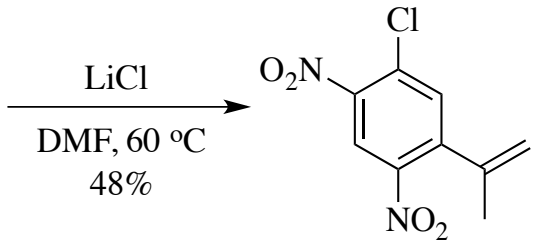

273

Aryl chloride $\mathbf{2 7 3}$ was then coupled with isopropenyl stannane under conditions $\mathrm{A}^{90}$ to afford the desired dinitro-dialkenyl substrate $\mathbf{2 6 0}$ (Scheme 89 ). This result is significant in that aryl chlorides are typically sluggish in Kosugi-Migita-Stille reactions and often require more tailored catalyst and ligand systems. The electron-deficient nature of the system on account of the two nitro groups likely augments the reactivity of the chloride towards oxidative addition of palladium.

\section{Scheme 89: Kosugi-Migita-Stille Coupling of Aryl Chloride}<smiles>C=C(C)c1cc(Cl)c([N+](=O)[O-])cc1[N+](=O)[O-]</smiles>

273

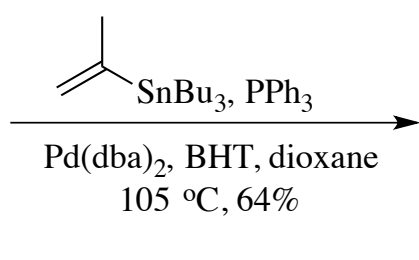

$05{ }^{\circ} \mathrm{C}, 64 \%$

While the successful coupling of aryl chloride 273 was encouraging, the overall process was not atomically efficient in going from phenol 271 to triflate $\mathbf{2 6 1}$ then to chloride 273. Rather, direct coupling of triflate 261 was desired. Gratifyingly, use of conditions $\mathrm{A}^{90}$ in DMF rather than dioxane afforded coupled product 274 in good yield (Scheme 90). While the role of changing solvent was not investigated, the exclusion of lithium chloride from the reaction mixture avoided potential triflate replacement. 


\section{Scheme 90: Kosugi-Migita-Stille Coupling of Aryl Triflate}

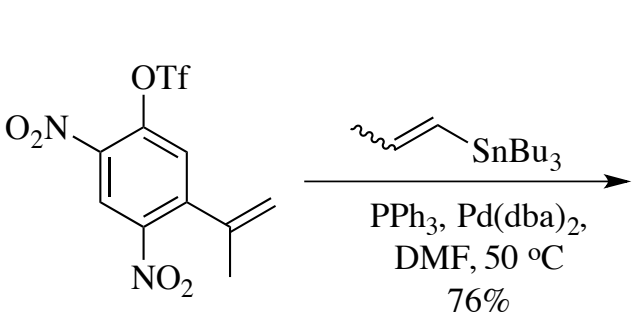

261

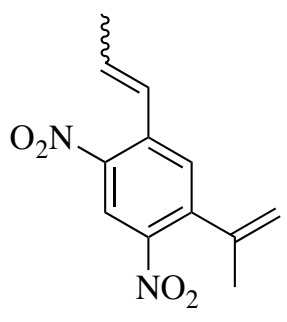

274

Similarly, cross-coupling between triflate 261 and $\alpha$-phenyl alkenylstannane 275 afforded cyclization precursor 276 (Scheme 91).

\section{Scheme 91: Preparation of Cyclization Precursor}<smiles>C=C(C)c1cc(OCC)c([N+](=O)[O-])cc1[N+](=O)[O-]</smiles>

261

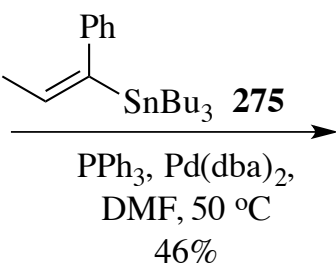

$46 \%$<smiles>C=C(C)c1cc(/C(=C/C)c2ccccc2)c([N+](=O)[O-])cc1[N+](=O)[O-]</smiles>

276

Some of the advantages of Stille coupling reactions include the vast array of stannanes, their ease of preparation either from Grignard reagents ${ }^{123}$ or hydrostannation of alkynes, ${ }^{124}$ and their stability ${ }^{125}$ relative to alternative reagents. Rather than delve too far into these different options, only the readily available stannanes described above were employed. However, variation of the alkenyl stannane used in either coupling step would allow for preparation of a number of structurally diverse pyrroloindoles.

A straightforward method to prepare non-symmetrical 2,4-dinitro-1,5-dialkenyl benzene derivatives has been established. This concise process involving sequential Kosugi-Migita-Stille cross-coupling reactions overcomes a number of obstacles previously encountered in the preparation of the described substrates. In addition, the 
generality of cross-coupling reaction allows for preparation of a diverse array of novel highly functionalized substrates.

\subsubsection{2,5-Dinitro-1,4-Dialkenyl Benzene Isomer}

The next isomeric cyclization precursor examined was 2,5-dinitro-1,4-dialkenyl benzene, such as divinylbenzene 240 (Figure 13). While Banini prepared compounds of this type (Section 3, this chapter), a more synthetically useful route was desired.

\section{Figure 13: 2,5-Dinitro-1,4-Dialkenyl Benzene}

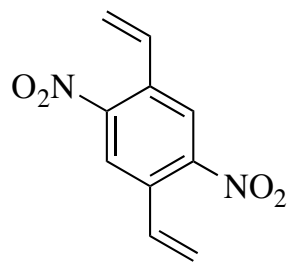

240

Retrosynthetically, it was envisioned that cyclization precursor $\mathbf{2 4 0}$ could be prepared through Kosugi-Migita-Stille coupling between triflate 277 and vinyl stannane (Scheme 92). Triflate 277 could be achieved from phenol 278. Styrene 278 would result from Kosugi-Migita-Stille cross-coupling between iodophenol 279 and vinyl stannane.

Dinitrophenol 279 could arise through the di-nitration of 4-iodophenol (194)

\section{Scheme 92: Proposed Retrosynthetic Outline to Cyclization Precursor}<smiles>C=Cc1cc([N+](=O)[O-])c(C=C)cc1[N+](=O)[O-]</smiles>

240<smiles>C=COc1cc([N+](=O)[O-])c(C=C)cc1[N+](=O)[O-]</smiles>

277<smiles>C=CC</smiles><smiles>C=C=C</smiles>

194<smiles>C=Cc1cc([N+](=O)[O-])c(O)cc1[N+](=O)[O-]</smiles>

278<smiles>C=CC=C</smiles><smiles>O=[N+]([O-])c1cc(I)c([N+](=O)[O-])cc1O</smiles>

279 
Preparation of isomer $\mathbf{2 4 0}$ was not as simple as initially proposed. It was envisioned that this isomer would be achieved through di-nitration of 4-iodophenol (194) (Scheme 93). However, after multiple attempts using a number of conditions, none of the desired di-nitrated product $\mathbf{2 7 9}$ was isolated. Rather, it appeared that the electrondonating hydroxy group para- to the iodine activated the iodine for ipso substitution, with 4-nitrophenol (280) being the isolated product. Under forcing conditions, 2,4dinitrophenol (281) was also isolated. Similar substitutions of aryl halides has also been reported by Hodgson ${ }^{126}$ in his exhaustive examination of nitration reactions.

\section{Scheme 93: Nitration of 4-Iodophenol}<smiles>O=[N+]([O-])c1ccc(O)cc1</smiles>

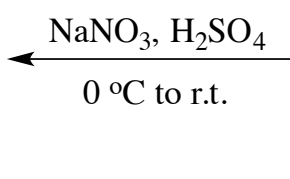

280<smiles>Oc1ccc(I)cc1</smiles>

194<smiles>CCCNOS(=O)(=O)c1cc(O)ccc1[N+](=O)[O-]</smiles>

280<smiles>O=[N+]([O-])c1ccc(O)c([N+](=O)[O-])c1</smiles>

281

Based on these results, a revised route was proposed that capitalized on the position para- to the alcohol being activated through conjugation with the electrondonating hydroxyl group. The proposed route involved preparation of 2,5-dinitrophenol (283), then introducing the iodine in order to overcome the issue of ipso substitution encountered in the case of 4-iodophenol (194). Nitration of 3-nitrophenol (282) using sodium nitrate in sulfuric acid afforded both the desired 2,5-dinitrophenol (283) along with 2,3-dinitrophenol (284) (Scheme 94). ${ }^{127}$ Fortunately, these isomers were separable using column chromatography, as it was envisioned that isomer 284 could be used in the preparation of another substrate (Section 5.4.3). 


\section{Scheme 94: Nitration of 3-Nitrophenol}

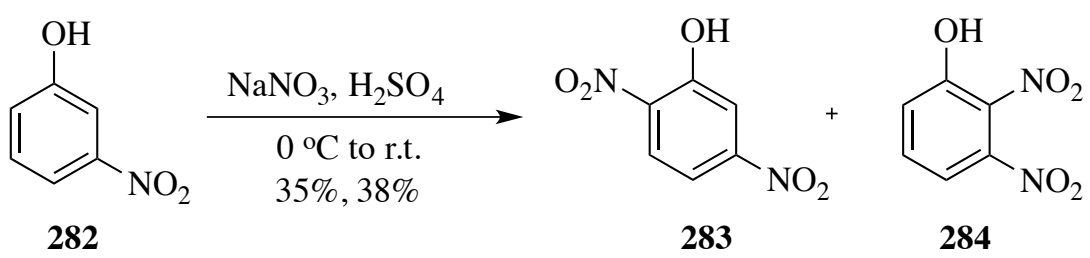

Oxidative iodination of phenols has been reported using a number of oxidants, with one of the most recent being benzyltriphenylphosphonium peroxymonosulfate (BTPPMS) (286), prepared through treatment of benzyltriphenylphosphonium chloride (285) with Oxone (Scheme 95). ${ }^{128}$

\section{Scheme 95: Preparation of Benzyltriphenylphosphonium Peroxymonosulfate}

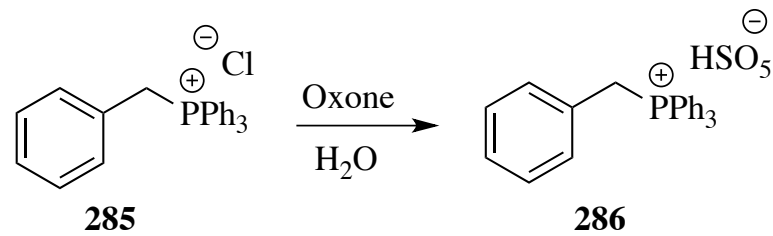

Hajipour ${ }^{129}$ has reported the para-selective iodination of a number of phenols using postassium iodide (KI) and BTPPMS (286). In fact, it was reported that 4-iodo2,5-dinitrophenol (279) was prepared in good yield from 2,5-dinitrophenol (283) using these conditions (Scheme 96).

\section{Scheme 96: Oxidative Iodination of 2,5-Dinitrophenol}<smiles>O=[N+]([O-])c1ccc([N+](=O)[O-])c(O)c1</smiles>

Attempts to replicate these results were unsuccessful in our hands. It was suspected that reagent $\mathbf{2 8 6}$ was highly impure and therefore not capable of oxidizing the substrate 283. The reported preparation of BTPPMS (286) (also reported by Hajipour ${ }^{128}$ ) 
provided no specific work-up or characterization data, leaving questions regarding this reagent.

Iodination of deactivated aromatics using $N$-iodosuccinimide (NIS) under acidic conditions has been reported by Olah. ${ }^{130}$ Treatment of nitrobenzene (287) with NIS and two equivalents of trifluoromethanesulfonic acid afforded 3-nitroiodobenzene (288) in excellent yield, confirming that the reaction proceeded through an electrophilic mechanism based on the regioselectivity observed in the product (Scheme 97). It was proposed that a super electrophilic iodine-trifluoromethanesulfonate, generated in situ, was the highly reactive iodinating species.

\section{Scheme 97: Iodination of Deactivated Arene}

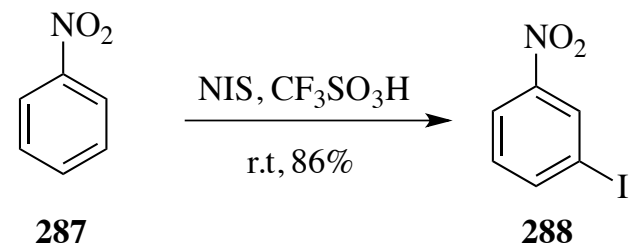

In a slight modification of Olah's protocol, ${ }^{130}$ treatment of 2,5-dinitrophenol (283) with NIS in sulfuric acid afforded the desired iodophenol 279 (Scheme 98). The ability to substitute expensive trifluoromethanesulfonic acid with sulfuric acid was an improvement to Olah's ${ }^{130}$ conditions.

\section{Scheme 98: Electrophilic Iodination of 2,5-Dinitrophenol}

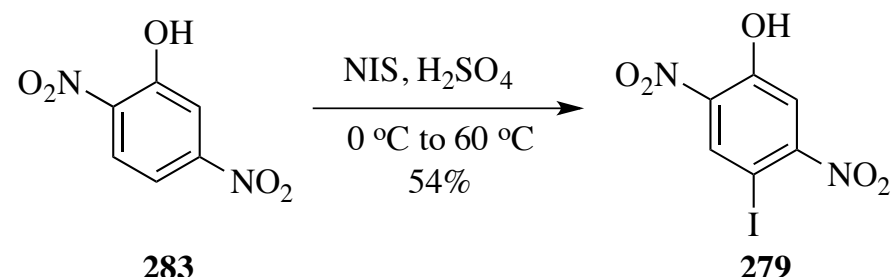

Coupling of iodophenol 279 was then attempted using the previously discussed modified Kosugi-Migita-Stille conditions (Scheme 85, Chapter 5). In the event, coupling 
between iodophenol $\mathbf{2 7 9}$ and vinyl stannane afforded styrene $\mathbf{2 7 8}$ in good yield (Scheme 99). Typical radical inhibitor 2,6-di-t-butyl-4-methylphenol (BHT) was added to the reaction in order to prevent polymerization of the styrene product.

\section{Scheme 99: Kosugi-Migita-Stille Coupling of 4-Iodo-2,5-Dinitrophenol}

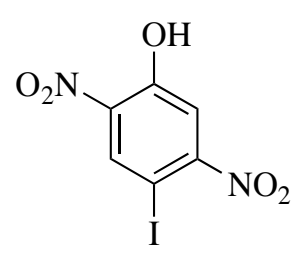

279

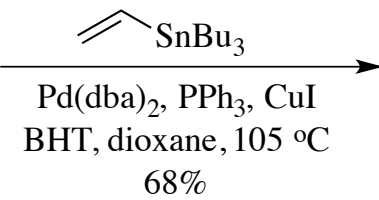

$68 \%$<smiles>C=Cc1cc([N+](=O)[O-])c(O)cc1[N+](=O)[O-]</smiles>

278

Phenol 278 was then converted to the corresponding triflate 277 using typical conditions (Scheme 100).

\section{Scheme 100: Preparation of Aryl Triflate}<smiles>C=Cc1cc([N+](=O)[O-])c(OCC)cc1C=CCCCCC(=O)OCC</smiles>

Kosugi-Migita-Stille cross-coupling of triflate $\mathbf{2 7 7}$ with vinyl stannane was achieved using typical conditions B. ${ }^{90}$ In contrast to the case of 261 (Scheme 87, Chapter 5), no chloride product was observed and the cross-coupled product $\mathbf{2 4 0}$ was achieved in moderate yield (Scheme 101).

\section{Scheme 101: Kosugi-Migita-Stille Coupling of Aryl Triflate}<smiles>C=Cc1cc([N+](=O)[O-])c(OCC)cc1[N+](=O)[O-]</smiles>

277

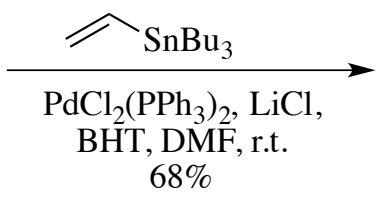
$68 \%$<smiles>C=Cc1cc([N+](=O)[O-])c(C=C)cc1[N+](=O)[O-]</smiles>

240 
The established method employing sequential cross-coupling reactions offers a number of improvements in comparison to Banini's method to prepare substrate $\mathbf{2 4 0}$ (Scheme 74, Chapter 5). Though both methods employed an initial nitration step, the nitration of 3-nitrophenol 282 (Scheme 94) afforded isomers 283 and 284 in moderate yield, whereas Banini's nitration step afforded a mixture of four isomers, each in low yield. Additionally, 2,3-dinitrophenol (284) was of interest for preparation of another isomer. This method also avoids the often unsuccessful benzyl bromination step employed by Banini to convert dinitrotoluene 246 to benzyl bromide 247 .

The desired 2,5-dinitro-1,4-diethenyl isomer 240 was achieved in five steps from 3-nitrophenol (282) using a series of electrophilic aromatic substitution and KosugiMigita-Stille coupling reactions. While only one symmetrical substrate was prepared, non-symmetrical cyclization precursors would be accessible by varying the stannane used in either coupling step.

\subsubsection{2,3-Dinitro-1,4-Dialkenyl Benzene Isomer}

Efforts were made to establish a synthetically useful route to prepare nonsymmetrical 2,3-dinitro-1,4-dialkenyl cyclization precursors, such as divinylbenzene $\mathbf{2 4 1}$ (Figure 14).

\section{Figure 14: 2,3-Dinitro-1,4-Dialkenyl Benzene}

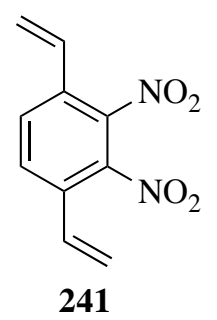

Initial attempts to prepare non-symmetrical substrates based on $\mathbf{2 4 1}$ through Kosugi-Migita-Stille coupling between 1,4-dibromo-2,3-dinitrobenzene (251) and vinyl 
stannane failed due to the inability to achieve selectivity for mono-coupling (as discussed in Section 5.3.3). Based on the successful coupling of halophenols in the preparation of other isomers in this study (Sections 5.4.1 and 5.4.2), a route involving similar ideology was sought.

Retrosynthetically, it was envisioned that cyclization precursor $\mathbf{2 4 1}$ could be prepared through Kosugi-Migita-Stille coupling between aryl triflate $\mathbf{2 8 9}$ and vinyl stannane (Scheme 102). Aryl triflate 289 could arise from dinitrophenol 290. Styrene 290 could result from the Kosugi-Migita-Stille coupling between iodophenol 291 and vinyl stannane. Dinitrophenol 291 was envisioned through di-nitration of 4-iodophenol (194).

\section{Scheme 102: Proposed Retrosynthetic Outline to Cyclization Precursor}<smiles>C=C=Cc1ccc(C=C)c([N+](=O)[O-])c1[N+](=O)[O-]</smiles>

241<smiles>C=Cc1ccc(OCC)c([N+](=O)[O-])c1[N+](=O)[O-]</smiles>

289<smiles>Oc1ccc(I)cc1</smiles>

194<smiles>C=Cc1ccc(O)c([N+](=O)[O-])c1[N+](=O)[O-]</smiles><smiles>C=C</smiles><smiles>O=[N+]([O-])c1c(O)ccc(I)c1[N+](=O)[O-]</smiles>

291

Unfortunately, attempts to prepare 2,3-dinitro-4-iodophenol (291) through nitration of $p$-iodophenol (194) were unsuccessful (as discussed in Section 5.4.2). It became clear that iodoarenes were highly prone to substitution, therefore, alternative routes to prepare iodophenol 291 were explored. One such route involved using Hajipour's ${ }^{129}$ methodology for the oxidative para iodination of phenols (described in 
greater detail in Section 5.4.2). 2,3-dinitrophenol (284) was prepared through the nitration ${ }^{127}$ of 3-nitrophenol (282) using sodium nitrate in sulfuric acid (as also described in Section 5.4.2). However, treatment of dinitrophenol 284 with BTPPMS and potassium iodide failed to produce the desired iodophenol 291 (Scheme 103).

\section{Scheme 103: Attempted Oxidative Iodination of 2,3-Dinitrophenol}<smiles>CN(C)CCSCC(=O)N(C)Br</smiles>

284<smiles>O=[N+]([O-])c1c(O)ccc(I)c1[N+](=O)[O-]</smiles>

291

Similar to the analogous case of 2,5-dinitrophenol (283) (Section 5.4.2), electrophilic iodination of $\mathbf{2 8 4}$ was also attempted using $N$-iodosuccinimide in triflic $\operatorname{acid}^{130}$ (Scheme 104). While the crude ${ }^{1} \mathrm{H}$ NMR spectrum appeared to show evidence of two iodinated products, neither was isolable through chromatographic purification. Trials using sulfuric acid in place of triflic acid were also unsuccessful.

\section{Scheme 104: Attempted Electrophilic Iodination of 2,3-Dinitrophenol}<smiles>O=[N+]([O-])c1cccc(O)c1[N+](=O)[O-]</smiles>

It was envisioned that the two nitro groups of $\mathbf{2 5 1}$ would render the bromines highly activated towards nucleophilic aromatic substitution $\left(\mathrm{S}_{\mathrm{N}} \mathrm{Ar}\right)$. Upon examination of the literature, examples of $S_{N} A r$ reactions using sodium hydroxide as a hydroxide source were discovered. ${ }^{116}$ Using this ideodology, treatment of di-bromide $\mathbf{2 5 1}$ with excess sodium hydroxide provided 4-bromo-2,3-dinitrophenol 292 in good yield (Scheme 105). 


\section{Scheme 105: Preparation of 4-Bromo-2,3-Dinitrophenol}

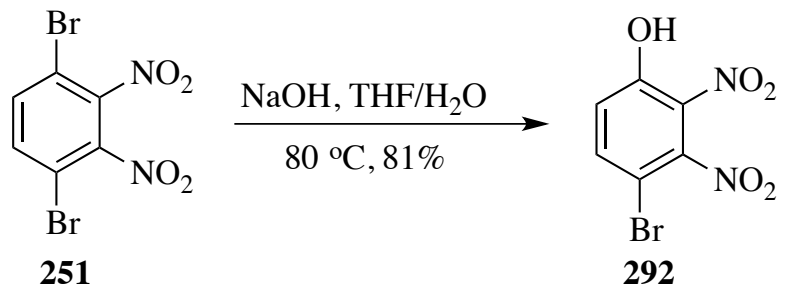

With phenol 292 in hand, Kosugi-Migita-Stile coupling was attempted using modified conditions $\mathrm{A}^{90}\left(\mathrm{PPh}_{3}, \mathrm{Pd}(\mathrm{dba})_{2}, \mathrm{CuI}\right.$, dioxane, reflux $)$, which were successful in coupling 2,4-dinitro-5-iodophenol 263 (Section 5.4.1) as well as 2,5-dinitro-4-iodophenol 279 (Section 5.4.2). Regrettably, the established conditions proved unsuccessful in the coupling of bromophenol 292, returning only unreacted starting material (Scheme 106). Interestingly, treatment of 292 with $\mathrm{PdCl}_{2}\left(\mathrm{PPh}_{3}\right)_{2}$ and $\mathrm{CuI}$ in toluene afforded isopropenyl-substituted phenol 293, albeit in poor yield.

\section{Scheme 106: Kosugi-Migita-Stille Coupling of Bromophenol}

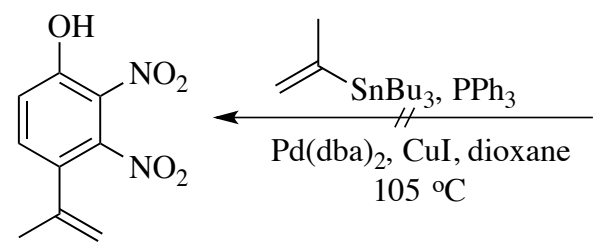

293

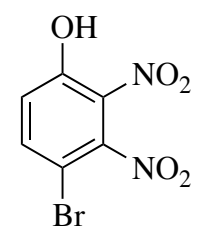

292

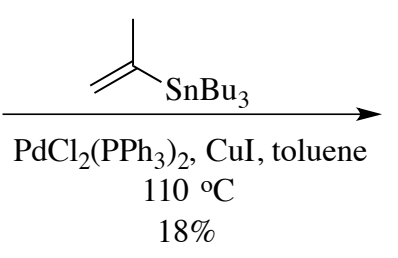

$18 \%$<smiles>C=C(C)c1ccc(O)c([N+](=O)[O-])c1[N+](=O)[O-]</smiles>

293

The drastic difference in reactivity observed for bromophenol 292 compared to coupling of iodophenol 279 (Section 5.4.2) was puzzling. Both 292 and 279 were expected to be similar in electronics on account of the bromine of 292 and the iodine of 279 being ortho and meta to nitro groups and para to a hydroxy group. However, iodophenol 279 underwent coupling while the coupling of bromophenol 292 was extremely sluggish. Therefore, it was postulated that the difference in reactivity must have been the result of using an aryl bromide rather than an aryl iodide. It is widely accepted that aryl iodides are more reactive than aryl bromides under a range of typical 
coupling conditions, ${ }^{97}$ so it was plausible that the combination of a less reactive aryl bromide that was also deactivated through conjugation with an electron-donating hydroxyl group caused the lack of reactivity observed for aryl bromide 292.

In an attempt to explore whether the analogous iodophenol would prove successful in the coupling step, a similar route to that previously presented was pursued using 1,4-diiodobenzene (294). Unfortunately, attempts to di-nitrate 294 affored 4nitroiodobenzene (295) as the lone product (Scheme 107).

\section{Scheme 107: Nitration of 1,4-Diiodobenzene}

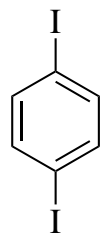

294

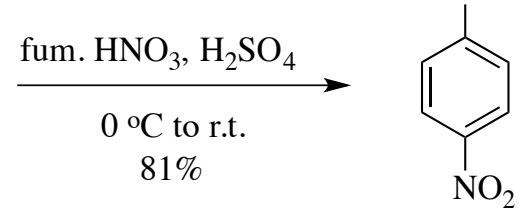

295

This result provided more insight into the highly reactive nature of aryl iodides relative to bromides. Comparison of the nitrations of 1,4-dibromobenzene 250 and 1,4diiodobenzene 294 provides a direct example. Di-nitration of dibromide $\mathbf{2 5 0}$ using mixed acid $90{ }^{\circ} \mathrm{C}$ afforded three di-nitrated isomers, whereas treatment of diiodide 294 with the same mixed acid conditions at room temperature resulted in ipso substitution of iodine by a nitro group. Considering these drastically different results, the differences in reactivity encountered in the aforementioned coupling reaction were not so surprising.

A revised route was sought to increase the reactivity of bromophenol 292 while limiting the number of additional steps required. It was envisioned that conversion of phenol 292 to tosylate 296 would decrease the electron-donor ablility of the phenolic oxygen and potentially render the bromine less deactivated. This could then facilitate the oxidative addition necessary for coupling while also adding only one additional step as 
tosylates are potentially suitable substrates for Kosugi-Migita-Stille coupling. ${ }^{132,133}$ Phenol 292 was converted to the corresponding tosylate 296 through treatment with $p$ toluenesulfonyl chloride in the presence of triethylamine ${ }^{134}$ (Scheme 108). Unfortunately, Kosugi-Migita-Stille coupling between aryl bromide 296 and isopropenyl stannane using conditions $\mathrm{A}^{90}$ did not afford isopropenylbenzene 297. However, a more extensive examination of conditions may provide conditions capable of facilitating coupling reaction of this substrate.

\section{Scheme 108: Attempted Kosugi-Migita-Stille Coupling of Aryl Bromide}<smiles>O=[N+]([O-])c1c(O)ccc(Br)c1[N+](=O)[O-]</smiles>

292

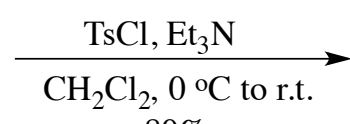
$80 \%$<smiles>COc1ccc(Br)c([N+](=O)[O-])c1[N+](=O)[O-]</smiles>

296

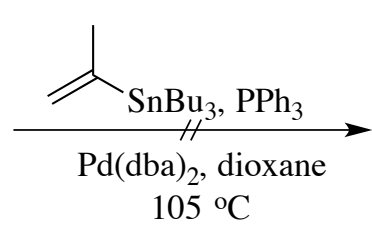<smiles>C=C(C)c1ccc(OC(F)(F)F)c([N+](=O)[O-])c1[N+](=O)[O-]</smiles>

297

Re-examination of previous work by Banini turned up an initially overlooked example. Kosugi-Migita-Stille coupling between dibromobenzene 251 and $\alpha$-stannyl ester 298 afforded exclusively mono-coupled product 299 (Scheme 109).

\section{Scheme 109: Selective Mono-Coupling of Dibromide by Banini}

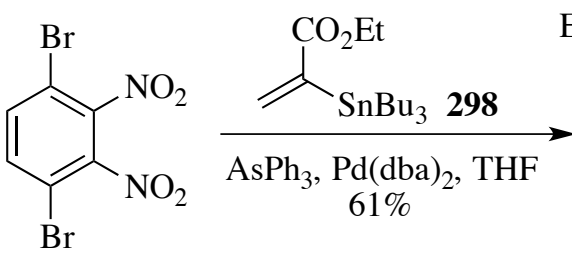

251<smiles>C=C(C(=O)OCC)c1ccc(Br)c([N+](=O)[O-])c1[N+](=O)[O-]</smiles>

299

It was envisioned that the presence of an electron-withdrawing substituent on the benzyl position of $\mathbf{2 9 9}$ rendered the mono-coupled product less reactive towards a second coupling reaction. This was in contrast to other examples employing simple alkenyl 
stannanes (as discussed in Section 5.3), in which the mono-coupled product appeared to be more reactive than the starting material (251) towards a second coupling reaction.

Gratifyingly, Kosugi-Migita-Stille coupling between 1,4-dibromo-2,3-

dinitrobenzene $\mathbf{2 5 1}$ and $\alpha$-stannyl ester $\mathbf{3 0 0}$ was achieved using Banini's conditions afforded mono-coupled arene $\mathbf{3 0 1}$ in moderate yield with no detected di-coupling (Scheme 110). Though not employed in Banini's example, it was also found that the addition of copper iodide aided the reaction, presumably through generation of a more reactive organocopper complex through tin-copper transmetalation. ${ }^{118}$

\section{Scheme 110: Mono-Coupling Using $\alpha$-Stannyl Ester}

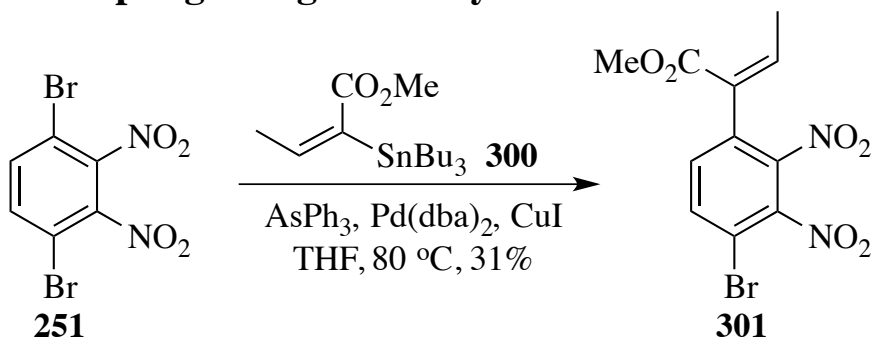

While the requirement of an electron-deficient stannane such as $\mathbf{3 0 0}$ was viewed as a limitation of this route, the broad range of functional groups that could still be employed provide a wide range of possible substrates that could be prepared using this method.

Cross-coupling between substrate $\mathbf{3 0 1}$ and isopropenyl stannane was achieved using previously described Kosugi-Migita-Stille conditions, ${ }^{90}$ affording the desired nonsymmetrical cyclization precursor $\mathbf{3 0 2}$ in moderate yield (Scheme 111).

\section{Scheme 111: Preparation of Non-Symmetrical Substrate}<smiles>C/C=C(\C(C)=O)c1ccc(Br)c([N+](=O)[O-])c1[N+](=O)[O-]</smiles>

301

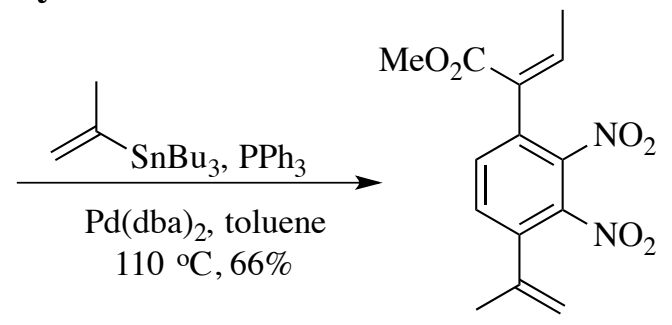

302 
Ultimately, non-symmetrical dinitro-dialkenyl benzene 302 was prepared in three steps from 1,4-dibromobenzene (250) through sequential Kosugi-Migita-Stille coupling reactions. While the first coupling step requires the use of an electron-withdrawing substituted stannane, a number of functional groups are still tolerated, providing a concise route to prepare otherwise inaccessible non-symmetrical substrates. The unusual reactivity of iodoarenes prevented the preparation of cyclization precursors such as $\mathbf{3 0 2}$ through coupling of 4-iodo-2,3-dinitrophenol (291).

\subsubsection{2,4-Dinitro-1,3-Dialkenyl Benzene Isomer}

The next target isomer was consisting of alternating nitro and alkenyl substituents, such as 242 (Figure 15). While this class could potentially lead to two different types of pyrroloindoles, they both faced the same obstacles in both the preparation of the substrate as well as the subsequent coupling steps.

\section{Figure 15: 2,4-Dinitro-1,3-Dialkenyl Benzene}

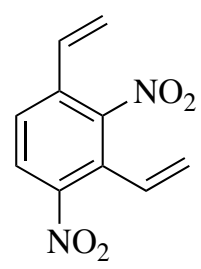

242

Retrosynthetically, it was initially envisioned that cyclization precursor $\mathbf{2 4 2}$ could be prepared through Kosugi-Migita-Stille coupling between aryl triflate $\mathbf{3 0 3}$ and vinyl stannane (Scheme 112). Aryl triflate 303 could be accessed through phenol 304. Styrene 304 would result from Kosugi-Migita-Stille coupling between iodophenol 268 and vinyl stannane. Dinitrophenol 268 was previously prepared through nitration of 3-iodophenol (264). 


\section{Scheme 112: Proposed Retrosynthetic Outline to Cyclization Precursor}

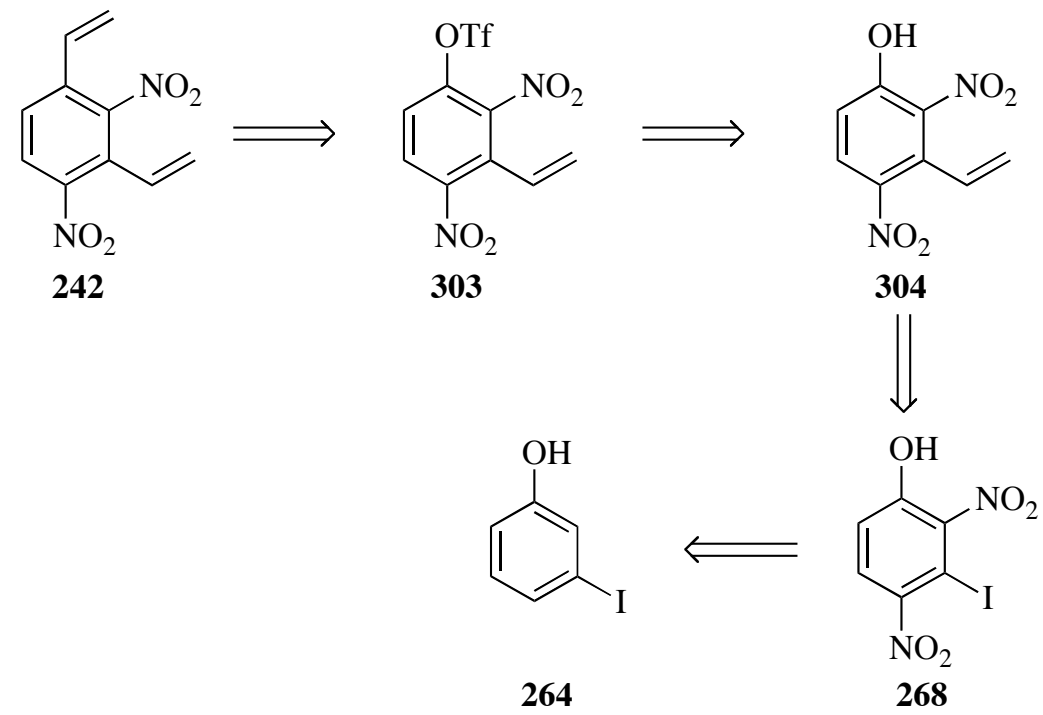

The sequential nitration ${ }^{114}$ of 3-iodophenol (264) (as discussed in Section 5.4.1). afforded a separable mixture of mono-nitrated isomers 265 and 266, which then underwent a second nitration using slightly stronger conditions (Scheme 113). Once again, 4,6-dinitro isomer $\mathbf{2 6 3}$ was the major product obtained, however, tailoring the conditions resulted in a slight increase in the amount of desired isomers 267 and $\mathbf{2 6 8}$ obtained (based on the starting material used). Unfortunately, the nitration of 3-iodo-4nitrophenol (265) afforded an inseparable mixture of isomers 263 and 268. Surprisingly, in the nitration of 2-nitro-5-iodophenol (266), dinitro isomer 267 in which the nitro group adds to the more hindered carbon was the major product and was also separable from 263.

Scheme 113: Preparation of Dinitrophenol Isomers<smiles>O=[N+]([O-])c1ccc(I)cc1ON(O)O[Na]</smiles> 


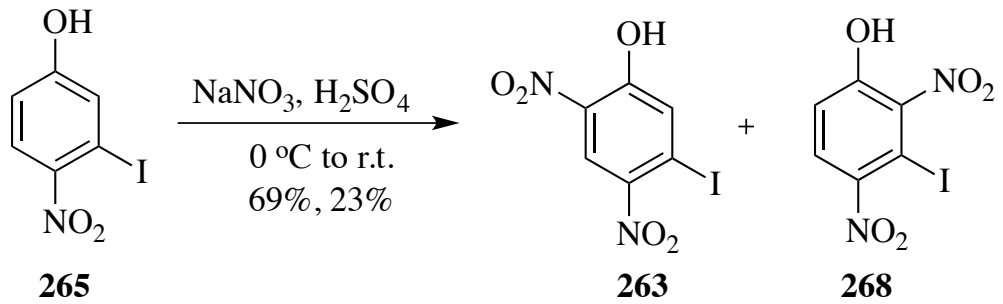

A mixture of phenols 263 and 268 were subjected to coupling conditions $\mathrm{A}^{90}$ with the addition of copper (I) iodide (as discussed in Chapter 5.4.1) (Scheme 114). While coupled products 271 and $\mathbf{3 0 5}$ were isolated, the yield of desired phenol $\mathbf{3 0 5}$ was very low $(8 \%)$. Attempts to improve yields were futile, partially because the starting material consisted of two isomers, which made it difficult to optimize conditions.

\section{Scheme 114: Attempted Coupling of Isomeric Mixture}<smiles>O=[N+]([O-])c1cc(I)c([N+](=O)[O-])cc1O</smiles>

263<smiles>O=[N+]([O-])c1ccc(I)c([N+](=O)[O-])c1O</smiles>

268

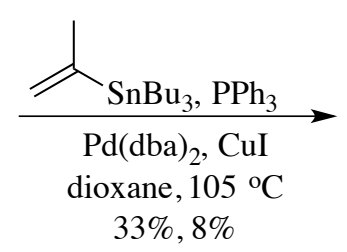
$33 \%, 8 \%$<smiles>C=C(C)c1cc(O)c([N+](=O)[O-])cc1[N+](=O)[O-]</smiles>

271<smiles>C=C(C)c1ccc([N+](=O)[O-])c(O)c1[N+](=O)[O-]</smiles>

305

Attempted Kosugi-Migita-Stille coupling between 3-iodo-2,4-dinitrophenol (268) and isopropenyl stannane using conditions $\mathrm{A}^{90}$ in addition to copper (I) iodide afforded none of the coupled product (Scheme 115). Rather, 2,4-dinitrophenol (281) was isolated.

\section{Scheme 115: Attempted Coupling of Iodophenol}<smiles>O=[N+]([O-])c1ccc(O)c([N+](=O)[O-])c1I</smiles>

268

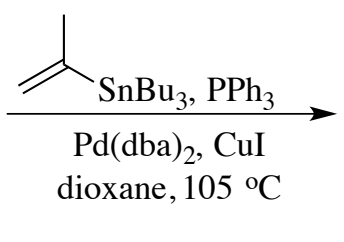

dioxane, $105{ }^{\circ} \mathrm{C}$<smiles>O=[N+]([O-])c1ccc(O)c([N+](=O)[O-])c1</smiles>

281

While it was suspected that the iodine of 3-iodo-2,4-dinitrophenol (268) would be highly activated towards oxidative addition on account the two ortho nitro substituents, it appeared that the neighboring nitro groups also provided steric hindrance, preventing the 
transmetalation of the isopropenyl group from tin to palladium. However, the formation of dinitrophenol $\mathbf{2 8 1}$ was evidence that oxidative addition of palladium had occurred. The sensitivity of Kosugi-Migita-Stille reactions to steric demands has been previously noted. ${ }^{135}$ This shortcoming, in addition to the difficulties discussed for isomer $\mathbf{2 6 7}$, resulted in this route's abandonment.

Based on the issues encountered in the previously mentioned examples, a route was sought which did not require coupling of a group positioned between two nitro groups. The goal was to find examples of condensation reactions of toluene or benzaldehyde derivatives in order to overcome the issue of dehalogenation while also avoiding potential selectivity issues experienced when using sequential coupling reactions.

In a unique aromatic substitution reaction, Kawakami ${ }^{136}$ reported the regioselective methylation of 2,4-dinitroaniline (306) through reaction with dimethyl sulfoxide (DMSO) and excess potassium tert-butoxide, affording 3-methyl-2,4dinitroaniline (307) (Scheme 116). The mechanism involves deprotonation of the amine followed by formation of an imino-aromatic system, which is then attacked by the methanesulfonyl anion. Interestingly, the authors state that the analogous reaction using 2,4-dinitrophenol affords none of the desired methylated product, though a similar mechanism can be envisioned.

\section{Scheme 116: Kawakami Methylation of 2,4-Dinitroaniline}

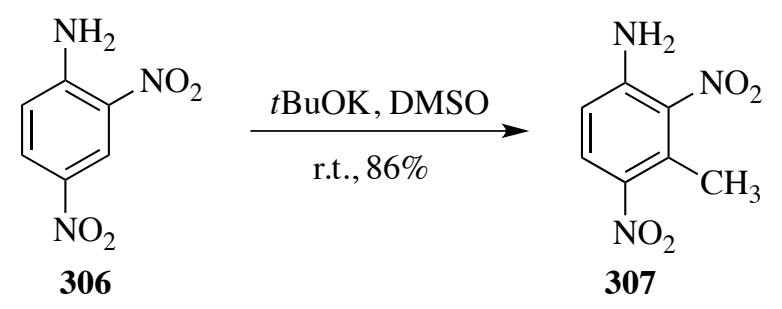


Aniline $\mathbf{3 0 7}$ was then converted to aryl iodide $\mathbf{3 0 8}$ using modified Sandmeyertype conditions ${ }^{137}$ (Scheme 117). The initially formed diazonium salt was displaced with potassium iodide in order to produce the more reactive aryl iodide for subsequent coupling reaction.

\section{Scheme 117: Preparation of Aryl Iodide}<smiles>Cc1c([N+](=O)[O-])ccc(N)c1[N+](=O)[O-]</smiles>

307

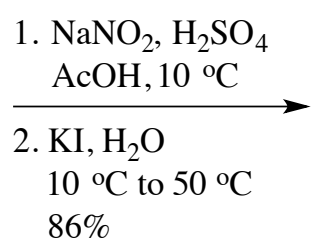
$86 \%$<smiles>Cc1c(I)ccc([N+](=O)[O-])c1[N+](=O)[O-]</smiles>

308

Condensation reactions of 2,6-dinitrotoluene (309) as well as the subsequent elimination to afford di-nitrostyrene have been reported by Mundla (Scheme 118). ${ }^{138}$ The initial condensation of $\mathbf{3 0 9}$ with para-formaldehyde in the presence of a catalytic amount of potassium hydroxide afforded alcohol 310. Conversion of alcohol $\mathbf{3 1 0}$ to mesylate $\mathbf{3 1 1}$ (which was not isolated) followed by subsequent elimination then afforded dinitrostyrene 311.

\section{Scheme 118: Mundla's Preparation of 2,6-Dinitrostyrene}<smiles>C=Cc1c([N+](=O)[O-])cccc1[N+](=O)[O-]</smiles>

Employing Mundla's methodology ${ }^{138}$ using toluene $\mathbf{3 0 8}$ afforded desired dinitrostyrene 313 in moderate yield over two steps (Scheme 119). 


\section{Scheme 119: Synthesis of Styrene}<smiles>Cc1c([N+](=O)[O-])ccc(I)c1[N+](=O)[O-]</smiles>

308<smiles>COC(=O)COC(=O)OCc1ccc(I)c([N+](=O)[O-])c1CCO</smiles>

312

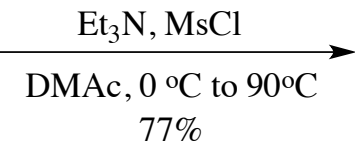<smiles>C=Cc1c([N+](=O)[O-])ccc(I)c1[N+](=O)[O-]</smiles>

313

Palladium-catalyzed Kosugi-Migita-Stille coupling between iodide $\mathbf{3 1 3}$ and isopropenyl stannane was achieved using typical conditions $\mathrm{A},{ }^{90}$ affording bis-alkenyl substrate 314 (Scheme 120).

Scheme 120: Kosugi-Migita-Stille Coupling of Aryl Iodide<smiles>C=Cc1c([N+](=O)[O-])ccc(I)c1[N+](=O)[O-]</smiles>

313

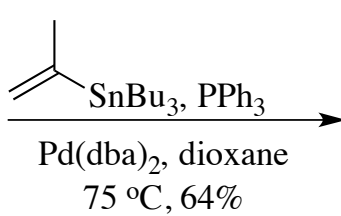

$75^{\circ} \mathrm{C}, 64 \%$<smiles>C=Cc1c([N+](=O)[O-])ccc(C(=C)C)c1[N+](=O)[O-]</smiles>

314

Attempts to broaden the scope of Mundla's ${ }^{138}$ methodology to prepare

dinitrostyrenes sought to employ different aldehydes. However, substitution of paraformaldehyde with hexanal were unsuccessful, likely due to steric hinderance from the nitro groups neighboring the anion formed from $\mathbf{3 0 8}$ and the more bulky aldehyde, preventing the formation of alcohol $\mathbf{3 1 5}$ (Scheme 121).

\section{Scheme 121: Attempted Condensation with Hexanal}<smiles>CCC(C)(O)C(C)(C)O</smiles>

308<smiles>CCCC(O)Cc1c([N+](=O)[O-])ccc(I)c1[N+](=O)[O-]</smiles>

315

Taking advantage of unique properties of dinitroarenes, a method was established to prepare non-symmetrical cyclization precursors in a straightforward manner. Initial 
issues involving failed coupling of halides situated between two nitro groups were overcome through use of a condensation-elimination sequence to install the alkene moiety. While the condensation step is currently limited to formation of an ethenyl group, further investigations may allow more broad elaboration.

\subsubsection{1,4-Dinitro-2,3-Dialkenyl Benzene Isomer}

While four of the five desired cyclization precursors were successfully prepared, the final isomer has proved elusive. This cyclization precursor $\mathbf{2 4 4}$ would feature two nitro groups oriented para to each other, as well as two neighboring alkenyl substituents (Figure 16).

\section{Figure 16: 1,4-Dinitro-2,3-Dialkenyl Benzene}

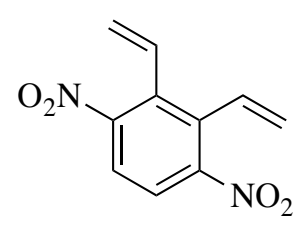

244

Retrosynthetically, dinitro-dialkenyl benzene 244 could result from KosugiMigita-Stille coupling between aryl triflate $\mathbf{3 1 6}$ and vinyl stannane (Scheme 122). Aryl triflate 316 could be achieved from dinitrophenol 317. Dinitrostyrene 317 could be envisioned from Kosugi-Migita-Stille coupling between iodophenol 318 and vinyl stannane. Dinitrophenol 318 could arise through either the nitration of 2-iodo-3nitrophenol (319) or the iodination of 2,5-dinitrophenol (283). 


\section{Scheme 122: Proposed Retrosynthetic Outline to Cyclization Precursor}<smiles>C=Cc1c([N+](=O)[O-])ccc([N+](=O)[O-])c1C=C</smiles>

244<smiles>C=COc1c([N+](=O)[O-])ccc([N+](=O)[O-])c1OCC</smiles>

316<smiles>C=CO[N+](=O)c1ccc([N+](=O)[O-])c(O)c1C=C</smiles>

317<smiles>C=C</smiles><smiles>C=C=C</smiles>
319<smiles>O=[N+]([O-])c1ccc([N+](=O)[O-])c(I)c1O</smiles>
318<smiles>O=[N+]([O-])c1ccc([N+](=O)[O-])c(O)c1</smiles>

It was initially envisioned that dinitrophenol $\mathbf{3 1 8}$ could be prepared through the nitration of 2-iodo-3-nitrophenol 319 (Scheme 123). In the event, it was found that rather forcing conditions were necessary for reaction to take place, as starting material was recovered in near quantitative yield in reactions using mixed acid at lower temperatures or under milder nitration conditions (such as $\mathrm{NaNO}_{3}, \mathrm{H}_{2} \mathrm{SO}_{4}$ ). Unfortunately, attempts to force the reaction along by heating phenol 319 in mixed acid resulted in the decomposition of starting material and afforded none of the desired dinitrophenol product 318.

\section{Scheme 123: Attempted Nitration of 2-Iodo-3-Nitrophenol}

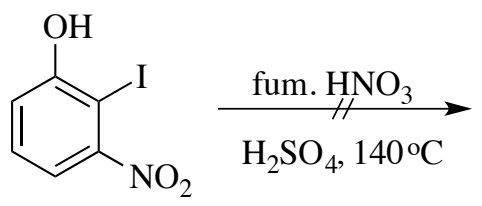

319<smiles>O=[N+]([O-])c1ccc([N+](=O)[O-])c(I)c1O</smiles>

318

Ortho directed functionalization reactions of phenols were then examined as potential routes to prepare dinitrophenol 318. It was proposed that the electron-rich hydroxy substituent could be used to direct nitration ${ }^{139}$ or iodination ${ }^{140}$ to the positions ortho to it (Scheme 124). 


\section{Scheme 124: Proposed ortho-Functionalization of Phenol}<smiles>O=[N+]([O-])c1cccc(O)c1I</smiles>

319<smiles>O=[N+]([O-])c1ccc([N+](=O)[O-])c(I)c1O</smiles>

318<smiles>O=[N+]([O-])c1ccc([N+](=O)[O-])c(O)c1</smiles>

283

Ortho-selective mono-nitration of substituted phenols using cerium (VI)

ammonium nitrate (CAN) has been reported by Sathunuru et al.$^{139}$ The regioselectivity of these reactions was attributed to the interaction of cerium with the hydroxyl oxygen of 320, leading to subsequent Fries-type rearrangement of intermediate $\mathbf{3 2 1}$ to afford the $o$ nitrophenol 323 upon re-aromatization of intermediate 322 (Scheme 125).

\section{Scheme 125: Mechanism of ortho-Selective Nitration of Phenol}

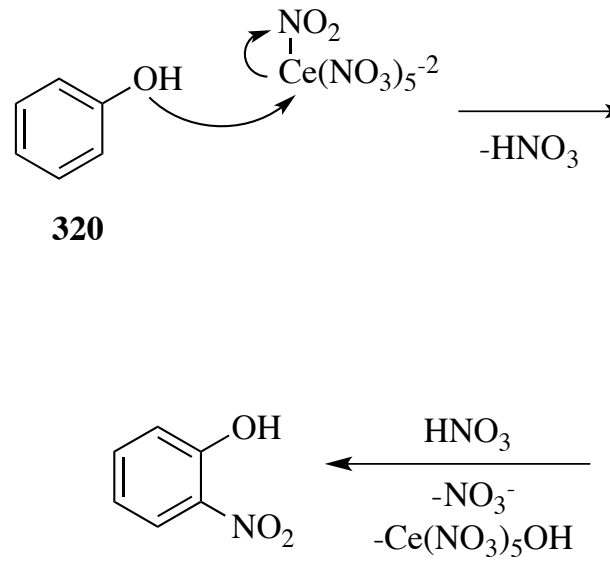

323

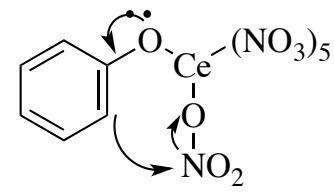

321<smiles>O=C(O[C@H](O)C(=O)O)C1C=CC=C[C@H]1[N+](=O)[O-]</smiles>

322

While Sathunuru's study ${ }^{139}$ reported the successful nitration of a number of electron-withdrawing substituted phenols, the authors reported that cyano- and nitrosubstituted phenols were unreactive, presumably due to the highly electron-deficient nature of arene. However, Sathunuru's methodology ${ }^{139}$ was applied in an attempt to nitrate phenol 319 (Scheme 126). In the event, the conditions appeared to be too mild and only unreacted starting material was recovered. 


\section{Scheme 126: Attempted ortho-Selective Nitration of Phenol}

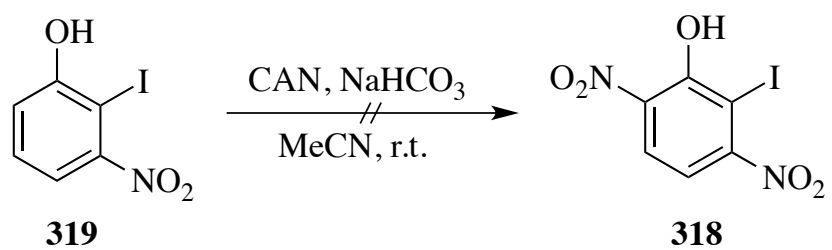

Similarly, ortho-selective halogenation of phenols was also explored. Previous work by Gaude et al..$^{40}$ explored the halogenation and nitration of phenols by either iodine nitrate or bromine nitrate (generated in situ using silver nitrate and either iodine or bromine) and reported that in the presence of pyridine or triethylamine, selective ortho halogenation of phenol (320) was achieved, affording 2-iodophenol (324) (Scheme 127). In the absence of amine, a mixture of halogenated and nitrated products was isolated.

\section{Scheme 127: Gaude's ortho-Iodination of Phenol}<smiles>O=[N+]([O-])c1cc(I)ccc1O</smiles>

195<smiles>O=[N+]([O-])c1ccc(O)cc1</smiles>

280

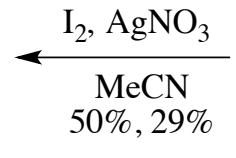<smiles>Oc1ccccc1</smiles><smiles>CCO[Mg]</smiles>

20<smiles>Oc1ccccc1I</smiles>

324

Gaude's methodology ${ }^{140}$ was applied to dinitrophenol 283 in hope of producing ortho-iodinated phenol 318 (Scheme 128). Unfortunately, no product was isolated, and starting material was recovered.

\section{Scheme 128: Attempted ortho-Iodination of Dinitrophenol}<smiles>O=[N+]([O-])c1ccc([N+](=O)[O-])c(O)c1</smiles>

283

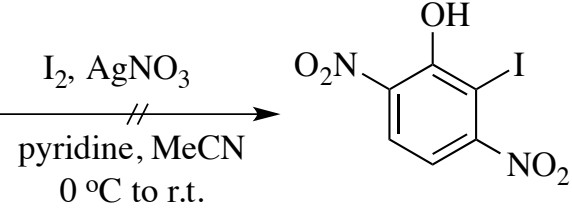

318

Ultimately, attempts to prepare 1,4-dinitro-2,3-dialkenyl benzene isomer $\mathbf{2 4 4}$ were unsuccessful. As apparent through the attempted routes, this isomer featuring 
"mismatched" electronic effects was highly elusive. The mismatched electronics rendered intermediate substrates highly unreactive and posed an obstacle to prepare the targeted isomer. Although this isomer was not pursued further, the importance of pyrrolo[3,2-e]indole frameworks as demonstrated by their use in anti-tumor compounds ${ }^{105}$ such as CC-1065, duocarmycin, and yatakemycin makes this isomer still highly sought after.

\subsubsection{Pyrroloindole Synthesis via Reductive Double $N$-Heteroannulation}

With a number of isomeric substrates in hand, focus was turned to the palladiumcatalyzed reductive double $N$-heterocyclizations to prepare each isomeric pyrroloindole. Using conditions previously established by Banini $\left(\mathrm{PPh}_{3}, \mathrm{Pd}(\mathrm{dba})_{2}, \mathrm{CO}\right.$ (6 atm), DMF, $120^{\circ} \mathrm{C}$ ), pyrroloindoles were prepared, although mono-cyclized products were also isolated even after extended reaction times ( 5 days). These results are outlined in Table 9.

Table 9: Double Cyclization Using Banini’s Conditions

Entry Substrate Product(s)<smiles>C=C(C)c1cc(C(=C)C)c([N+](=O)[O-])cc1[N+](=O)[O-]</smiles><smiles>C=C(C)c1cc2c(C)c[nH]c2cc1[N+](=O)[O-]</smiles><smiles>Cc1c[nH]c2cc3[nH]cc(C)c3cc12</smiles>

1 260 $325(32 \%)$<smiles>C=C(C)c1cc(/C=C/C)c([N+](=O)[O-])cc1[N+](=O)[O-]</smiles><smiles>C=C(C)c1cc2cc(C)[nH]c2cc1[N+](=O)[O-]</smiles>
$326(59 \%)$

2 274 $327(34 \%)$<smiles>C/C=C/c1cc2c(C)c[nH]c2cc1[N+](=O)[O-]</smiles>
$328(28 \%)$

Conditions: $\mathrm{PPh}_{3}, \mathrm{Pd}(\mathrm{dba})_{2}, \mathrm{CO}(6 \mathrm{~atm}), 120{ }^{\circ} \mathrm{C}, 120$ hours 
While these initial cyclizations were not completely successful in producing the desired pyrroloindoles, useful insight was still gained. Substrate $\mathbf{2 6 0}$ (Table 9, entry 1) was converted to pyrroloindole $\mathbf{3 2 6}$ in moderate yield although a fair amount of indole 325 resulting from mono-cyclization was also isolated. The incomplete conversion of 260 to pyrroloindole 326 suggested that the second cyclization step was significantly slower than the initial cyclization, since no unreacted starting material was recovered. Substrate 274 (table 9, entry 2) provided insight into how substituents affect the rate of cyclization. Indole 327, formed through the cyclization of the propenyl moiety of $\mathbf{2 7 4}$, was isolated in slightly higher yield than indole $\mathbf{3 2 8}$, which was the result of cyclization of the isopropenyl substituent. Though the isolated yields were not drastically different, this result suggests that the propenyl substituent may provide less hinderance for coordination of palladium to induce the cyclization, meaning the propenyl substituent cyclizes faster than the isopropenyl group.

With the hope of shortening reaction times while at the same time improving the yield of pyrroloindole products, other ligand systems were also explored. Alternative Soderberg annulation conditions using a two ligand system comprised of 1,10 phenanthroline (1,10-phen) and 1,3-(bis)-diphenylphosphinopropane (dppp) as opposed to simply triphenylphosphine were employed in order to overcome potential catalyst degradation due to the longer time scale of these reactions. Under these modified conditions, diisopropenylarene 260 was converted to pyrroloindole $\mathbf{3 2 6}$ in good yield with no evidence of mono-cyclization (Scheme 129). In addition, the reaction time was reduced to 48 hours. 


\section{Scheme 129: Double Cyclization Using 1,10-phenanthroline/dppp}<smiles>C=C(C)c1cc(C(=C)C)c([N+](=O)[O-])cc1[N+](=O)[O-]</smiles>

260

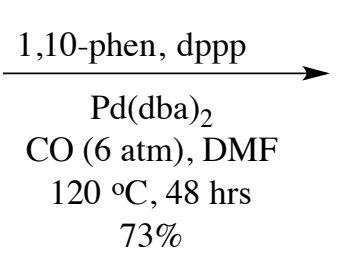

$73 \%$

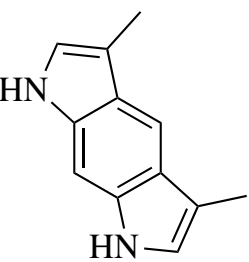

326

These modified Soderberg cyclization conditions were then applied to each of the substrates prepared in Chapter 5.4. The results of these reactions are summarized in Table 10 .

Table 10: Reductive Double $N$-Heterocyclizations ${ }^{\mathrm{a}}$

\section{$\begin{array}{lll}\text { Entry } & \text { Substrate } & \text { Product(s) }\end{array}$}<smiles>C=Cc1cc([N+](=O)[O-])c(C=C)cc1[N+](=O)[O-]</smiles><smiles>C=Cc1c([N+](=O)[O-])ccc(C(=C)C)c1[N+](=O)[O-]</smiles>

2

314<smiles>C=C(C)c1ccc(/C(=C\C)C(C)=O)c([N+](=O)[O-])c1[N+](=O)[O-]</smiles><smiles>c1cc2cc3[nH]ccc3cc2[nH]1</smiles>

239 (78\%)<smiles>C=C(C)c1ccc2[nH]ccc2c1[N+](=O)[O-]</smiles>

$329(47 \%)$

$330(30 \%)$<smiles>CC(=O)c1c(C)[nH]c2c1ccc1c(C)c[nH]c12</smiles>

331 (77\%) 
Table 10: Reductive Double $N$-Heterocyclizations ${ }^{\text {a }}$

Entry Substrate Product(s)<smiles>C=C(C)c1cc(/C=C/C)c([N+](=O)[O-])cc1[N+](=O)[O-]</smiles><smiles>Cc1cc2cc3c(C)c[nH]c3cc2[nH]1</smiles>

4 274 $332(23 \%)$<smiles>C=C(C)c1cc(/C(=C/C)c2ccccc2)c([N+](=O)[O-])cc1[N+](=O)[O-]</smiles><smiles>Cc1[nH]cc2cc3[nH]cc(C)c3cc12</smiles>

5 276

a) Conditions: 1,10-phenanthroline, dppp, $\mathrm{Pd}(\mathrm{dba})_{2}, \mathrm{CO}(6 \mathrm{~atm}), \mathrm{DMF}, 120^{\circ} \mathrm{C}, 120 \mathrm{hrs}$

b) Reaction run for 87 hours

Pyrroloindole 239 was obtained in moderate yield after 87 hours (Table 10, entry

1). It was anticipated that di-vinyl substrate $\mathbf{2 4 0}$ would cyclize faster than the other examples, which featured various substituents on the double bonds that could interfere with the coordination of palladium to the alkene. It is possible that the cyclization was complete in a shorter amount of time, however, difficulties in monitoring the reaction via TLC limited the certainty with which progress could be monitored. The modified catalyst conditions also improved on Banini's preparation of 239 (which used $\mathrm{PPh}_{3}$ and $\mathrm{Pd}(\mathrm{dba})_{2}$ in DMF under $\left.\mathrm{CO}(6 \mathrm{~atm})\right)$ to obtain the pyrroloindole 239 in $84 \%$ yield after 144 hours). Although the yield was slightly lower in our hands, reducing the reaction time by nearly three days was a modest improvement.

In the case of vinyl-isopropenyl substrate $\mathbf{3 1 4}$ (Table 10, entry 2), it appeared as though the added steric bulk of the methyl group slowed down the cyclization of the 
isopropenyl moiety as evidenced by the moderate amount of indole $\mathbf{3 2 9}$ isolated.

However, desired pyrroloindole $\mathbf{3 3 0}$ was also isolated, albeit in poor yield on account of the isolation of indole 329. It is notable that the vinyl group of $\mathbf{3 1 4}$ reacts regioselectively with the 4-nitro group and not with the other neighboring nitro substituent. The structure of indole $\mathbf{3 2 9}$ was confirmed through observed NOE between the $\mathrm{C} 7$ proton of the indole core and the indole $\mathrm{N}-\mathrm{H}$. This inherent regioselectivity could potentially be attributed to the less hindered nitro group being more accessible to interaction with the palladium catalyst.

The cyclization of 2,3-dinitro substrate $\mathbf{3 0 2}$ (Table 10, entry 3) afforded pyrroloindole $\mathbf{3 3 1}$ in good yield with no detected mono-cyclization. In comparison to the cyclization of di-isopropenyl substrate 257 which afforded mono-cyclized indole 259 as the major product along with pyrroloindole $\mathbf{2 5 8}$ (Section 5.3.3), substrate $\mathbf{3 0 2}$ featuring more substituted double bond cyclized at a faster rate with no mono-cyclized product isolated. The rate enhancement could be attributed to the altered catalyst conditions with the two ligand system employed herein producing a more active catalyst.

Issues arose in the cyclization of propenyl-isopropenyl substrate $\mathbf{2 7 4}$ (Table 10, entry 4). While no starting material was detected or recovered at the end of three different trials, only trace amounts of pyrroloindole $\mathbf{3 3 2}$ was isolated. It is believed that the unsubstituted 3-position of the propenyl-cyclized portion of the product could render the pyrroloindole product prone to oligomerization, as evidenced by a substantial amount of insoluble brown material upon crude work-up. Studies on the acid-induced dimerization and oligomerization of indoles reported that the 3-position of 2methylindole (17) is more nucleophilic than that of indole (1) on account of the added 
electron-donating methyl group. ${ }^{141}$ Also reported is the reaction of 2-methylindole (17)

and skatole (3-methylindole) (334) to afford mixed dimer 335 (Scheme 130).

\section{Scheme 130: Formation of Mixed Indole Dimer}

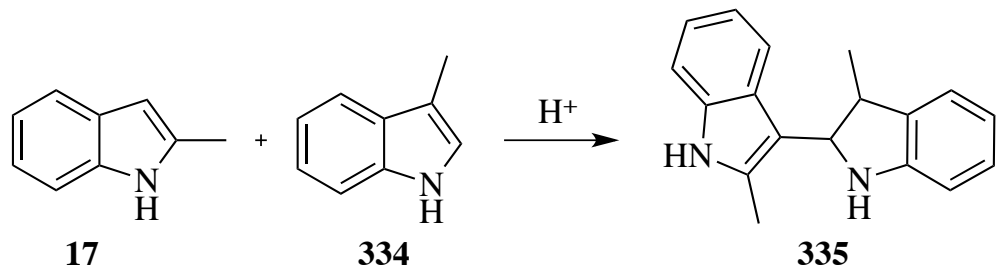

A similar transformation could be envisioned for pyrroloindole 332. In the event that pyrroloindole $\mathbf{3 3 2}$ is formed, intermolecular nucleophilic addition of the 2methylindole portion of the molecule to the 3-methylindole portion of a second molecule would afford dimer 336 (Scheme 131). However, the inability to characterize the insoluble residue that was obtained prevented the confirmation of this dimerization.

\section{Scheme 131: Intermolecular Dimerization of Pyrroloindole}<smiles>Cc1cc2cc3c(C)c[nH]c3cc2[nH]1</smiles>

332

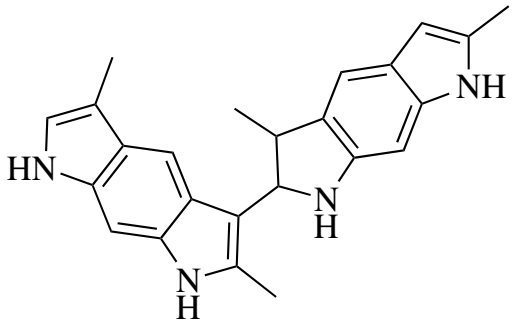

336

The last substrate examined $\mathbf{2 7 6}$ (Table 10, entry 5) cyclized to afford pyrroloindole 333 in moderate yield. The added bulk on account of the phenyl and methyl substituents on the alkenyl group of $\mathbf{2 7 6}$ appeared to have little effect on the overall rate of the double annulation, as no mono-cyclized indole products were obtained.

\subsection{Conclusions}

Novel non-symmetrical pyrroloindoles were prepared from dialkenyl-dinitro benzenes. Four of the five highly functionalized isomeric cyclization precursors were 
prepared through use of aromatic substitution along with Kosugi-Migita-Stille coupling reactions. Using Soderberg's palladium-catalyzed reductive double $N$-heteroannulation methodology, the structurally complex cyclization precursors were converted to the corresponding pyrroloindoles. The generally mild catalyst conditions offer significant improvements over previously reported methods using harsh ${ }^{108,109}$ conditions. Catalyst degradation due to extended reaction times was also prevented through use of a two ligand system, protecting the integrity of the catalyst while also reducing the time needed for the double annulations to reach completion. This methodology provides synthetic routes to previously elusive highly functionalized non-symmetrical pyrroloindoles, which are of interest in a number of applications. 


\section{Chapter 6}

\section{Synthesis of Functionalized Aromatics}

$\begin{array}{ll}\text { 6.1 Brominations of 2-Nitrobenzaldehyde } & 118\end{array}$

$\begin{array}{ll}\text { 6.1.1 Introduction } & 118\end{array}$

$\begin{array}{ll}\text { 6.1.2 Results and Discussion } & 119\end{array}$

$\begin{array}{ll}\text { 6.1.3 Conclusions } & 125\end{array}$

6.2 Synthesis of Functionalized Indoles $\quad 126$

$\begin{array}{ll}\text { 6.2.1 Synthesis of 6-Methyl-Nitroindoles } & 126\end{array}$

$\begin{array}{ll}\text { 6.2.2 Synthesis of 5-Methoxy-Nitroindoles } & 127\end{array}$

$\begin{array}{lr}\text { 6.2.3 Conclusions } & 130\end{array}$ 


\subsection{Brominations of 2-Nitrobenzaldehyde}

In the search for alternate routes to prepare non-symmetrical dinitro-dialkenyl aromatic compounds en route to the preparation of pyrroloindoles, the halogenation of nitrobenzaldehyde was explored. It was envisioned that the selectivity issues (discussed in Chapter 4) could be circumvented through use of a route that only required one coupling step. Therefore, halobenzaldehydes were sought as potential intermediates to prepare the desired cyclization precursors though Wittig condensation of the aldehyde and cross-coupling of the halide (Scheme 132).

\section{Scheme 132: Proposed Substrate Preparation From Halobenzaldehyde}

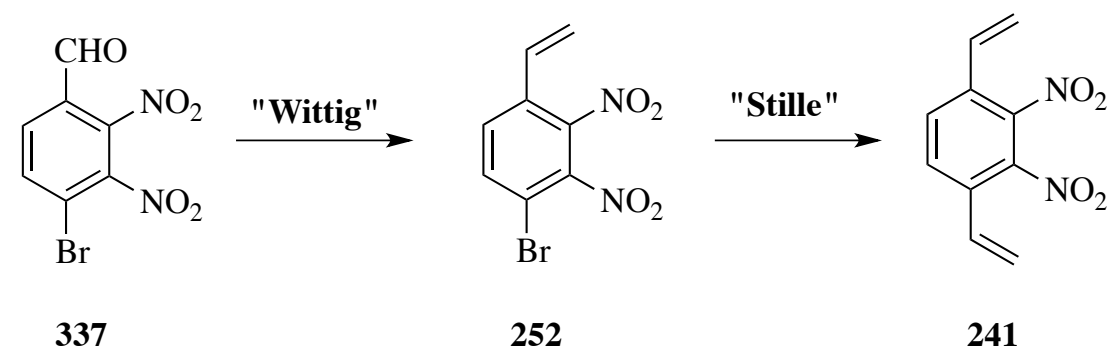

\subsubsection{Introduction}

A mild method for the bromination of deactivated aromatic compounds ${ }^{142}$ using $N$-bromosuccinimide (NBS) in sulfuric acid was reported by Saiganesh et al in $2007 . .^{143}$ Nine different substrates were examined and 60-92\% yields of single products were reported. For eight of the starting materials, the directing effect of the functional groups present on the aromatic ring for electrophilic aromatic bromination coincided in the same position. Thus, the regioselectivity of the incoming electrophile was not an issue. The last substrate examined by the authors, 2-nitrobenzaldehyde (338), did not have this luxury. Nevertheless, 4-bromo-2-nitrobenzaldehyde (339) was reported as the only 
product in over $60 \%$ yield (Scheme 133). In addition to the reaction of $\mathbf{3 3 8}$ with NBS in sulfuric acid, a related regioselective bromination using sodium bromide and sodium periodate in sulfuric acid - water to afford $\mathbf{3 3 9}$ in $88 \%$ isolated yield was reported by Kumar et al in 2012 (Scheme 133). ${ }^{144}$

\section{Scheme 133: Regioselective Bromination of 2-Nitrobenzaldehyde}

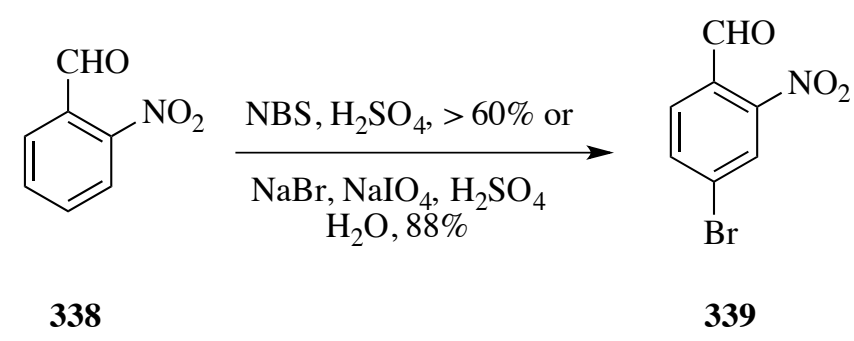

The experimental procedures, as described in the literature, were repeated a number of times. However, in our hands, the results reported could not be duplicated. The reactions with NBS gave a complex mixture of up to seven brominated products along with unreacted starting material. Reactions attempted using $\mathrm{NaBr}-\mathrm{NaIO}_{4}$ resulted only in quantitative recovery of starting material. The complex reaction mixtures obtained in the experiments using NBS were examined in order to assign structures to each product. It should be noted that each of the brominated products obtained are potentially useful building blocks in organic chemistry on account of their high degree of functionality, although each was obtained in relatively low yield.

\section{1 .2 Results and Discussion}

In an attempt to deconvolute the complex reaction mixtures obtained, 2nitrobenzaldehyde (338) was reacted with NBS in sulfuric acid at both the originally reported reaction temperature $\left(25^{\circ} \mathrm{C}\right)$ and at $60{ }^{\circ} \mathrm{C}$. In addition, experiments employing different amounts of NBS were performed. Complex mixtures containing the four 
possible monobrominated compounds (339-342) and three dibrominated products (343345) were observed in the crude reaction mixtures in all cases (Scheme 134).

\section{Scheme 134: Bromination of 2-Nitrobenzaldehyde}<smiles>O=Cc1cccc([N+](=O)[O-])c1[N+](=O)[O-]</smiles>

The molar ratios of products in each trial were extrapolated from integration of the crude ${ }^{1} \mathrm{H}$ NMR spectra and are presented in Table 11. Purification of the crude mixtures afforded pure products along with two or three component mixtures. The isolated yields of each product in each trial are reported in Table 12 .

Table 11: Molar Ratios of Brominated Products ${ }^{\mathrm{a}}$

\begin{tabular}{|c|c|c|c|c|c|c|c|c|}
\hline Equivalents NBS & $\mathbf{3 3 8}$ & $\mathbf{3 3 9}$ & $\mathbf{3 4 0}$ & $\mathbf{3 4 1}$ & $\mathbf{3 4 2}$ & $\mathbf{3 4 3}$ & $\mathbf{3 4 4}$ & $\mathbf{3 4 5}$ \\
\hline 0.75 & 45.1 & 17.8 & 2.9 & 10.6 & 19.3 & 1.6 & 1.2 & 1.6 \\
\hline 1.25 & 18.4 & 24.9 & 4.0 & 14.4 & 24.9 & 5.0 & 3.5 & 5.0 \\
\hline $1.25^{\mathrm{b}}$ & 17.6 & 25.9 & 3.2 & 12.1 & 20.9 & 5.2 & 10.3 & 4.8 \\
\hline 2.5 & 2.6 & 27.0 & 3.1 & 12.0 & 17.1 & 14.0 & 9.7 & 13.8 \\
\hline 5.0 & - & 13.5 & 0.6 & 3.4 & 1.1 & 28.7 & 26.7 & 26.1 \\
\hline
\end{tabular}

a) Reactions were performed at ambient temperature. Ratios were calculated from ${ }^{1} \mathrm{H}$ NMR spectra of the crude reaction mixtures. b) Reaction at $60^{\circ} \mathrm{C}$. 
Table 12: Isolated Yields of Brominated Products ${ }^{\mathrm{a}}$

\begin{tabular}{|c|c|c|c|c|c|c|c|c|c|}
\hline Equivalents NBS & $\mathbf{3 3 8}$ & $\mathbf{3 3 9}$ & $\mathbf{3 4 0}$ & $\mathbf{3 4 1}$ & $\mathbf{3 4 2}$ & $\mathbf{3 4 3}$ & $\mathbf{3 4 4}$ & $\mathbf{3 4 5}$ & $\begin{array}{c}\text { Total } \\
\text { Brominated }\end{array}$ \\
\hline 0.75 & 38 & 19 & 4 & 12 & 24 & - & 1 & 2 & $62 \%$ \\
\hline 1.25 & 17 & 21 & 4 & 9 & 20 & 5 & 1 & 3 & $63 \%$ \\
\hline $1.25^{\mathrm{b}}$ & 4 & 10 & 3 & 3 & 8 & 9 & 2 & 1 & $36 \%$ \\
\hline 2.5 & 3 & 8 & 8 & 20 & 14 & 12 & 9 & 13 & $84 \%$ \\
\hline 5.0 & - & 4 & - & - & - & 24 & 19 & 17 & $64 \%$ \\
\hline
\end{tabular}

Starting material (338) was detected in all reactions aside from the reaction using 5 equivalents of NBS. Chromatographic separation using silica gel was partially successful in separating the isomers. Monobrominated isomer $\mathbf{3 4 0}$ was isolated from the mixture when five equivalents of NBS was added. Pure 344 was isolated regardless of the amount of NBS used, and pure 343 and 345 were separated from the crude mixture when five equivalents of NBS was used. Monobrominated compounds 339, 341, and 342 were always isolated as inseparable mixtures. The identities of compounds $\mathbf{3 4 0 , 3 4 2}$, 344, and 345 were verified through comparison with literature NMR and/or melting point data.

In order to correctly identify each isomeric compound as well as to aide in the separation of the mixtures, all pure compounds and some of the mixtures were reduced to the corresponding benzylalcohols using sodium borohydride.

Reduction of $\mathbf{3 4 0}$ using sodium borohydride afforded known benzylalcohol 346 (Scheme 135). The aromatic ${ }^{1} \mathrm{H}$ NMR splitting pattern consiting of two doublets and a triplet along with a nuclear Overhauser effect (nOe) between the methylene protons and one of the aromatic doublets confirmed the structure of $\mathbf{3 4 6}$ and thus of isomer $\mathbf{3 4 0}$. This 
transformation also verified the structure of the other monobrominated product with the same doublet-doublet-triplet ${ }^{1} \mathrm{H}$ NMR splitting pattern assigned as $\mathbf{3 4 2}$.

\section{Scheme 135: Sodium Borohydride Reduction of 340}

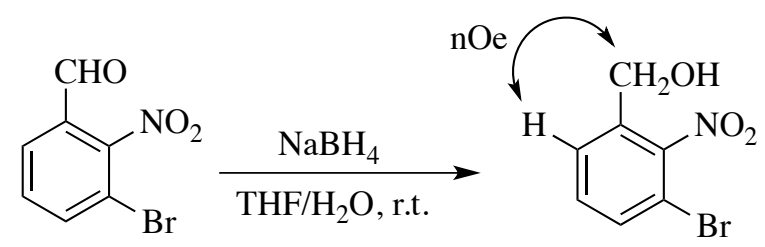

340

346

Aldehydes 339 and $\mathbf{3 4 1}$ were inseparable, however, treatment of the mixture with sodium borohydride provided a separable mixture of known benzylalcohols 347 and 348 . These benzylalcohols also corroborated the structure of $\mathbf{3 3 9}$ and $\mathbf{3 4 1}$ by nOe experiments (Scheme 136).

\section{Scheme 136: Reduction of Inseparable Mixture}

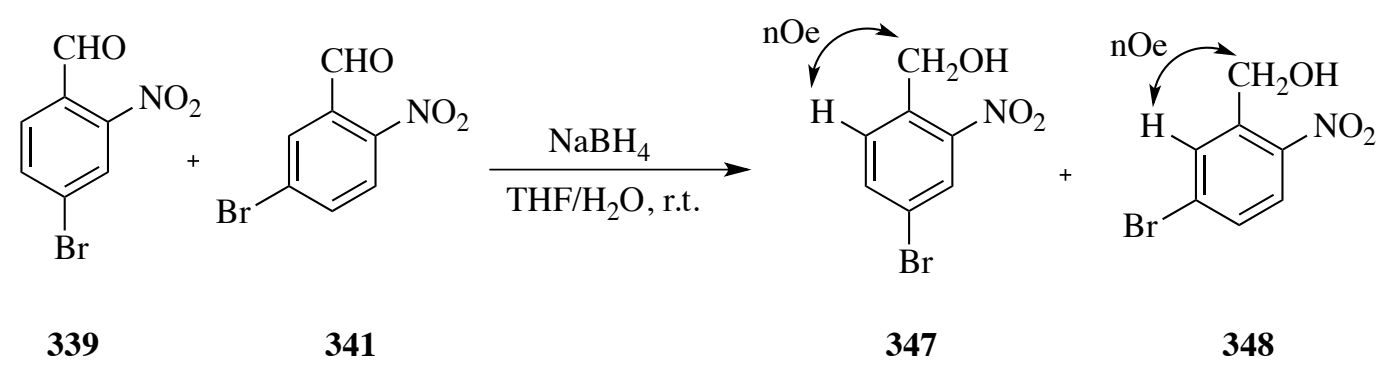

Similarly, reduction of aldehyde 343 afforded benzylalcohol 349 (Scheme 137).

The structure of $\mathbf{3 4 3}$ was then confirmed by and observed nOe between the benzyl protons and the ortho proton on the benzene ring of $\mathbf{3 4 9}$. 


\section{Scheme 137: Reduction of Aldehyde to Confirm Structure}

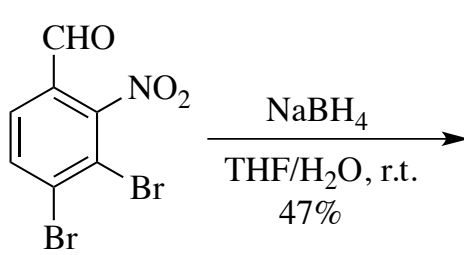

343<smiles>O=C(O)CCc1ccc(Br)c(Br)c1[N+](=O)[O-]</smiles>

349

Aldehyde 344 was also reduced using sodium borohydride (Scheme 138). The structure of $\mathbf{3 5 0}$ and subsequently 344 were again confirmed through nOe experiments.

\section{Scheme 138: Reduction of Aldehyde 344}

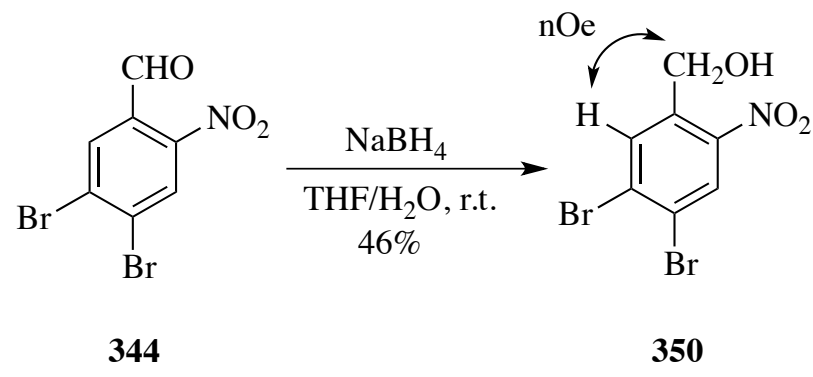

Similar reduction of aldehyde 345 afforded benzylalcohol 351 (Scheme 139). As expected, no nOe was observed between the methylene of $\mathbf{3 5 1}$ and any of the aromatic protons.

\section{Scheme 139: Reduction of Aldehyde 345}

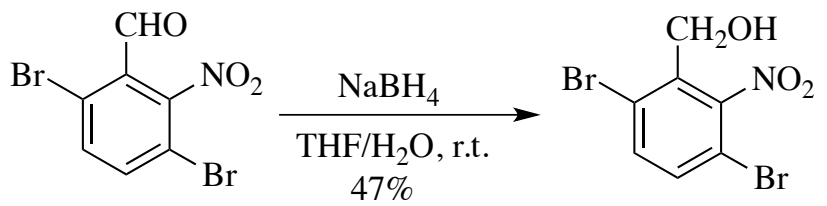

As anticipated, predominately monobrominated products were obtained from reactions using lower amounts of NBS and, not surprisingly, dibrominated products were 
the major components when a large excess of NBS was used. Interestingly, the total yield of brominated products was roughly the same regardless of the amount of NBS used. A significantly lower yield was obtained from the reaction using 2.5 equivalents of NBS at $60{ }^{\circ} \mathrm{C}$, even though little starting material was present in the crude mixture. The combination of elevated temperature and the acidic reaction medium may have caused decomposition of the starting material. Because only a small amount of dibrominated products were formed when less than one equivalent of NBS was used, the ratios of 339, 340, and 341 provided a measure of the reactivity of the different positions in $\mathbf{3 3 8}$ toward electrophilic aromatic bromination. The reactivity was normalized to compound $\mathbf{3 4 0}$, the minor monobrominated product, and was calculated to be $6.1: 1: 3.7: 6.7$ (in order from 339 to 342). This order of reactivity likely reflects the electron-withdrawing ability of the nitro group as compared to the aldehyde. Presumably the nitro group more effectively deactivates the meta positions (relative to the nitro group), in turn rendering the ortho and para positions (relative to the nitro group) more reactive. The results from the reactions using 0.75 and 5.0 equivalents of NBS also suggest that compounds $\mathbf{3 4 0}$, 341, and $\mathbf{3 4 2}$ undergo a second bromination at a much faster rate compared to $\mathbf{3 3 9}$, since a significant amount of the latter remains even at 5.0 equivalents of NBS. A rough order of reactivity for the second bromination of each monobrominated product was calculated by comparing the ratios of monobrominated products at 0.75 and 5.0 equivalents of NBS. The resulting order of $\mathbf{3 4 2}>\mathbf{3 4 0}>\mathbf{3 4 1}>\mathbf{3 3 9}$ potentially reflects the minimized steric congestion of $\mathbf{3 4 2}$ and $\mathbf{3 4 0}$ relative to $\mathbf{3 3 9}$ and $\mathbf{3 4 0 .}$ 


\subsubsection{Conclusions}

In summary, the reported results for the selective bromination of 2-

nitrobenzaldehyde (338) using NBS in sulfuric acid to give 4-bromo-2-nitrobenzaldehyde (339) reported in the literature were unable to be duplicated. However, the complex mixture of four monobrominated and three dibrominated products obtained in the attempts were isolated and identified. Pure products were achieved through chromatographic separation along with reduction of the aldehydes to the corresponding benzylalcohols, also aiding in the structural elucidation of each isomer. Based on the ratios achieved in each trial, the relative reactivity of each position of 2nitrobenzaldehyde towards electrophilic bromination was inferred and found to reflect the greater deactivating ability of the nitro group in comparision to the aldehyde. Each mono and dibrominated product possessed a high degree of functionality with the potential to be synthetically useful building blocks. 


\subsection{Synthesis of Functionalized Indoles}

Through extensive efforts to prepare non-symmetrical cyclization precursors in the synthesis of pyrroloindoles, a number number of highly functionalized nitroaromatic compounds were prepared. While these compounds were ultimately not used in the established routes to prepare pyrroloindoles, it was envisioned that nitro-substituted indoles could be prepared from some of the intermediate compounds that were synthesized. These functionalized indoles would provide a diverse array of starting materials for a number of further transformations.

\subsubsection{Synthesis of 6-Methyl-Nitroindoles}

The first functionalized indoles that were prepared arose from the dinitration of $p$ bromotoluene (245) (Scheme 140). Due to the harsh conditions used in this reaction, all four possible dinitrated isomers 246 and 352-354 were observed. Although separation of isomers 246 and $\mathbf{3 5 3}$ proved to be very difficult, $\mathbf{3 5 2}$ and $\mathbf{3 5 4}$ were separable using column chromatography.

\section{Scheme 140: Nitration of $p$-Bromotoluene}<smiles>Cc1ccc(Br)cc1</smiles>

245

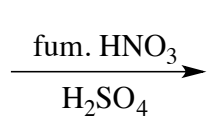<smiles>Cc1ccc(Br)c([N+](=O)[O-])c1[N+](=O)[O-]</smiles>

352<smiles>Cc1cc([N+](=O)[O-])c(Br)cc1[N+](=O)[O-]</smiles>

246<smiles>Cc1c([N+](=O)[O-])cc(Br)cc1[N+](=O)[O-]</smiles>

353<smiles>Cc1cc([N+](=O)[O-])c(Br)c([N+](=O)[O-])c1</smiles>

354

Under Kosugi-Migita-Stille cross-coupling conditions, aryl bromide 352 was coupled with vinyl stannane to afford dinitrostyrene 355 (Scheme 141).

\section{Scheme 141: Kosugi-Migita-Stille Coupling of Aryl Bromide}<smiles>Cc1ccc(Br)c([N+](=O)[O-])c1[N+](=O)[O-]</smiles>

352<smiles>C=Cc1ccc(C)c([N+](=O)[O-])c1[N+](=O)[O-]</smiles>

355 
Using modified Söderberg conditions, reductive $N$-heterocyclization of $o$ nitrostyrene 355 was achieved through treatment with triphenylphosphine and palladium (II) acetate in acetonitrile while heating in a sealed tube pressurized with carbon monoxide, affording 6-methyl-7-nitroindole (356), albeit in poor yield (Scheme 142).

Scheme 142: Reductive $N$-Heterocyclization of Nitrostyrene 355

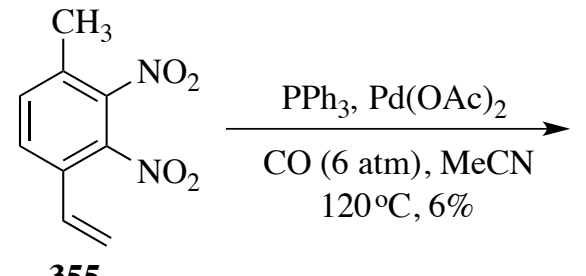

355<smiles>Cc1ccc2cc[nH]c2c1[N+](=O)[O-]</smiles>

356

Similar methodology was applied to 3,5-dinitrotoluene isomer 354 (Scheme 143).

Initial Kosugi-Migita-Stille coupling between aryl bromide $\mathbf{3 5 4}$ and vinyl stannane afforded styrene $\mathbf{3 5 7}$. Subsequent exposure of nitrostyrene $\mathbf{3 5 7}$ to the previously discussed reductive $N$-heterocyclization conditions afforded 6-methyl-4-nitroindole $\mathbf{3 5 8}$ in moderate yield over two steps.

\section{Scheme 143: Synthesis of 6-Methyl-4-Nitroindole}<smiles>Cc1cc([N+](=O)[O-])c(Br)c([N+](=O)[O-])c1</smiles>

354

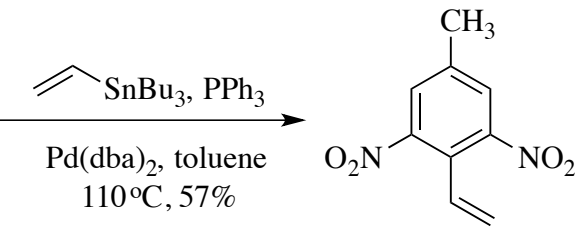

357

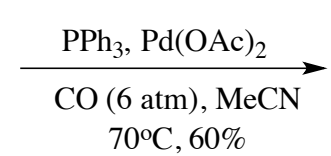

$70^{\circ} \mathrm{C}, 60 \%$<smiles>Cc1cc([N+](=O)[O-])c2cc[nH]c2c1</smiles>

358

\subsubsection{Synthesis of 5-Methoxy-Nitroindoles}

In the attempted synthesis of pyrroloindoles starting from either 3-bromoanisole (359) or 3-iodoanisole (362), dinitro-haloanisoles 360, 361, 363, and 364 were prepared through treatment of the corresponding anisole with fuming nitric acid (Scheme 144). Interestingly, none of the 2,6-dinitroanisole product was formed from either substrate. On account of this, it was presumed that the first nitro group likely added to the 4- 
position (relative to the methoxy group), then a second nitro group added to either of the positions ortho to the methoxy group. The substantial difference in the ratio of products 360:361 and 363:364 is also intriguing. While the iodine would be larger than the bromine and would pose a greater steric effect, the electronic effect in going from bromine to iodine likely influenced the observed differences in regioselectivity.

\section{Scheme 144: Dinitration of 3-Haloanisole}<smiles>[X]c1cccc(OC)c1</smiles>

$359(\mathrm{X}=\mathrm{Br})$ $362(X=I)$

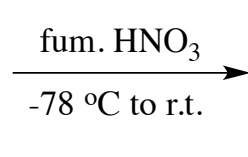
$\mathbf{3 6 3}(30 \%)$<smiles>[X]c1cc(OC)c([N+](=O)[O-])cc1[N+](=O)[O-]</smiles>

$\mathbf{3 6 0}(89 \%)$<smiles>[X]c1c([N+](=O)[O-])ccc(OC)c1[N+](=O)[O-]</smiles>

$361(6 \%)$

$364(35 \%)$

These isomers were then subjected to previously discussed Kosugi-Migita-Stille coupling conditions in the presence of vinyl stannane to afford dinitrostyrenes 365 and 367, which were then cyclized using Söderberg's palladium-catalyzed reductive $N$ heteroannulation conditions. This sequence afforded two isomeric methoxy-nitroindoles 366 and 368, albeit in low yield (Scheme 145). The structure of indole 368 was confirmed through the observed nOe between the $\mathrm{C} 7$ proton and the indole $\mathrm{H}$.

\section{Scheme 145: Preparation of 5-Methoxy-Nitroindoles}<smiles>COc1cc(Br)c([N+](=O)[O-])cc1[N+](=O)[O-]</smiles>

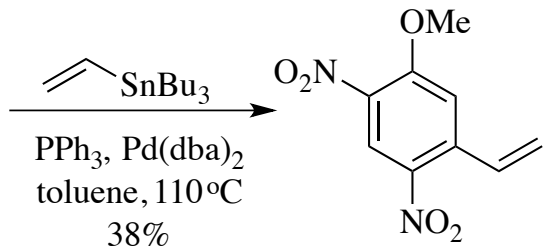<smiles>COc1cc2cc[nH]c2cc1[N+](=O)[O-]</smiles>

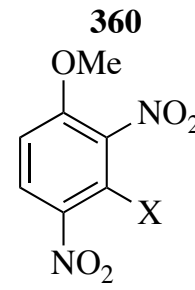

$361(\mathrm{X}=\mathrm{Br})$ $364(\mathrm{X}=\mathrm{I})$<smiles>C=Cc1c([N+](=O)[O-])ccc(OC)c1[N+](=O)[O-]</smiles>

1,10-phen, $\mathrm{Pd}(\mathrm{OAc})_{2}$

DMF, CO (6 atm)

$120{ }^{\circ} \mathrm{C}, 23 \%$

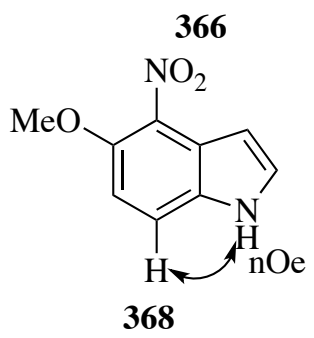


A similar route afforded 3-methylindoles $\mathbf{3 7 0}$ and $\mathbf{3 7 2}$ from intermediates $\mathbf{3 6 9}$ and 371, which resulted from Kosugi-Migita-Stille coupling between the requisite haloanisole and isopropenyl stannane (Scheme 146). The structure of indole 372 was again confirmed through the observed NOE between the $\mathrm{C} 7$ proton and the indole $\mathrm{N}-\mathrm{H}$.

Scheme 146: Preparation of 3-Methyl-5-Methoxy-Nitroindoles<smiles>[X]c1cc([N+](=O)[O-])c([N+](=O)[O-])cc1OC</smiles>

$360(\mathrm{X}=\mathrm{Br})$

$363(X=I)$

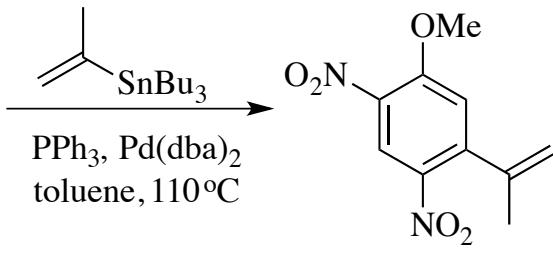

$369(46 \%)$ $369(37 \%)$

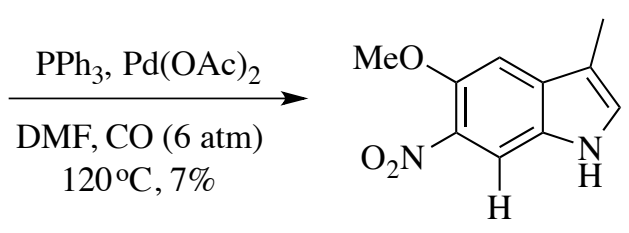

370<smiles>COc1ccc([N+](=O)[O-])c(Br)c1[N+](=O)[O-]</smiles>

361

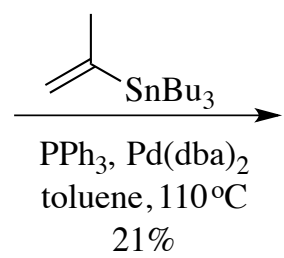

$21 \%$

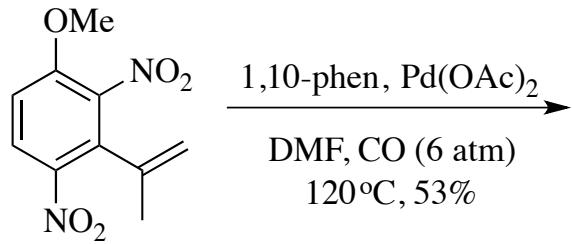

371<smiles>COC1=CC2=c3[nH]cc(C)c3=C([N+](=O)[O-])C(C)=CNC2=C1C(=O)O</smiles>

372

While the yields of the indole products were moderate at best, a number of significant observations were made. As observed in prior cases, ${ }^{52}$ the ortho alkenyl group is necessary for reduction of a nitro group; nitro groups lacking an ortho alkenyl substituent were inert to the reductive conditions. The regioselectivity observed in the cyclization of isomers $\mathbf{3 6 7}$ and $\mathbf{3 7 1}$ was also of interest. While cyclization of these could occur through either of the two ortho nitro groups relative to the alkenyl substituent, only one indole product (368 and 372) was isolated in each case. This infers that sterics influence the initial coordination-mediated nitro group reduction, as only the presumably more accessible nitro group reacted in these examples. 


\subsubsection{Conclusions}

A number of substituted indoles were prepared through palladium-catalyzed reductive $N$-heterocyclization of the requisite $o$-nitrostyrene precursor. These examples expand the already broad substrate scope of Söderberg's palladium-catalyzed reductive $\mathrm{N}$-heteroannulation methodology and exemplify the functional group compatibility of the

relatively mild reaction conditions. Insight was also gained regarding the regioselectivity of the initial nitro group reduction, confirming previously proposed principles that a neighboring alkenyl group mediates nitro group reduction. 


\section{Chapter 7}

\section{Supporting Information: Experimental Procedures}

7.1 Supporting Information for Chapter 2: Indole/Quinoline

7.2 Supporting Information for Chapter 3: Salviadione

7.3 Supporting Information for Chapter 4: Chemoselective Coupling

7.4 Supporting Information for Chapter 5: Pyrroloindoles

7.5 Supporting Information for Chapter 6.1: 2-Nitrobenzaldehyde Brominations 
General Procedures. NMR spectra were determined in $\mathrm{CDCl}_{3}$ at $600 \mathrm{MHz}\left({ }^{1} \mathrm{H} \mathrm{NMR}\right)$ and $150 \mathrm{MHz}\left({ }^{13} \mathrm{C} \mathrm{NMR}\right), 400 \mathrm{MHz}\left({ }^{1} \mathrm{H} \mathrm{NMR}\right)$ and $100 \mathrm{MHz}\left({ }^{13} \mathrm{C} \mathrm{NMR}\right)$, or at $270 \mathrm{MHz}$ $\left({ }^{1} \mathrm{H}\right.$ NMR) and $67.5 \mathrm{MHz}\left({ }^{13} \mathrm{C}\right.$ NMR). The chemical shifts are expressed in d values relative to $\mathrm{SiMe}_{4}\left(0.0 \mathrm{ppm},{ }^{1} \mathrm{H}\right.$ and $\left.{ }^{13} \mathrm{C}\right)$ or $\mathrm{CDCl}_{3}\left(77.0 \mathrm{ppm},{ }^{13} \mathrm{C}\right)$ internal standards. ${ }^{1} \mathrm{H}$ ${ }^{1} \mathrm{H}$ coupling constants are reported as calculated from spectra, thus a slight difference between $J_{\mathrm{a}, \mathrm{b}}$ and $J_{\mathrm{b}, \mathrm{a}}$ is usually observed. Tetrahydrofuran (THF) was distilled from sodium benzophenone ketyl prior to use. Hexanes and ethyl acetate were distilled from calcium hydride. Anhydrous acetonitrile, benzene, dichloromethane, 1,4-dioxane, $N, N$ dimethylformamide, and toluene were used as received. Chemicals prepared according to literature procedures have been footnoted the first time used; all other reagents were obtained from commercial sources and used as received. All reactions were performed under a nitrogen atmosphere in oven-dried glassware. Solvents were removed from reaction mixtures and products on a rotary evaporator at water aspirator pressure unless otherwise stated. Chromatography was performed on silica gel 60 (40-63 $\mu \mathrm{m}$, Sorbtech). Melting points (uncorrected) were recorded directly from products obtained by chromatography. 


\subsection{Supporting Information for Chapter 2: Indole/Quinoline}

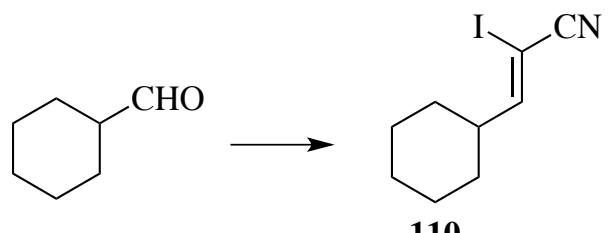

110

3-Cyclohexyl-2-iodo-2-propenenitrile (110): To a solution of triphenylarsonium ethanenitrile $(1.66 \mathrm{~g}, 3.90 \mathrm{mmol})$ in $\mathrm{MeCN}(15 \mathrm{~mL})$ at $10{ }^{\circ} \mathrm{C}$ was added potassium carbonate $(536 \mathrm{mg}, 3.88 \mathrm{mmol})$ and iodine $(997 \mathrm{mg}, 3.93 \mathrm{mmol})$. The solution was stirred under a nitrogen atmosphere (ambient temp., $30 \mathrm{~h}$ ) where after potassium carbonate (564 mg, $4.08 \mathrm{mmol})$, cyclohexylcarboxaldehyde (556 mg, $5.00 \mathrm{mmol}$ ) and $\mathrm{H}_{2} \mathrm{O}(0.5 \mathrm{~mL})$ were added. After an additional $48 \mathrm{~h}$, the solvents were removed under reduced pressure and the resulting crude product was purified by chromatography (hexanes/EtOAc, 95:5) to give $\mathbf{1 1 0}(171 \mathrm{mg}, 0.65 \mathrm{mmol}, 17 \%)$ as a brown oil. ${ }^{1} \mathrm{H}$ NMR $\delta$ $6.89(\mathrm{~d}, J=10.2 \mathrm{~Hz}, 1 \mathrm{H}), 2.54(\mathrm{qt}, J=10.8,3.6 \mathrm{~Hz}, 1 \mathrm{H}), 1.77-1.73(\mathrm{~m}, 6 \mathrm{H}), 1.25-1.14(\mathrm{~m}$, $4 \mathrm{H}) ;{ }^{13} \mathrm{C}$ NMR $\delta 167.3,116.1,49.7,45.7,45.0,31.4,25.3,25.0$; IR (ATR) 2935, 2851, $2211,1447,1148 \mathrm{~cm}^{-1}$; HRMS (ESI) calcd for $\mathrm{C}_{9} \mathrm{H}_{12} \mathrm{NaNI}\left(\mathrm{M}+\mathrm{Na}^{+}\right)$283.9912; found, 283.9906<smiles>N#C/C(I)=C/C1CCCCC1</smiles>

110<smiles>CCCSc1ccccc1[N+](=O)[O-]</smiles><smiles>C=CC</smiles>

3-Cyclohexyl-2-(2-nitrophenyl)-2-propenenitrile (111): Reaction of tributyl(2nitrophenyl) stannane (273 mg, $0.66 \mathrm{mmol}), 110(171 \mathrm{mg}, 0.65 \mathrm{mmol}), \mathrm{PdCl}_{2}\left(\mathrm{PhCN}_{2}\right.$ (16 mg, $0.04 \mathrm{mmol}), \mathrm{AsPh}_{3}(22 \mathrm{mg}, 0.07 \mathrm{mmol})$ and $\mathrm{CuI}(22 \mathrm{mg}, 0.12 \mathrm{mmol})$ in NMP (3 $\mathrm{mL})$, as described for $91\left(80{ }^{\circ} \mathrm{C}, 76 \mathrm{~h}\right)$, gave after chromatography (hexanes/EtOAc, 9:1) 111 (82 mg, $0.32 \mathrm{mmol}, 48 \%$, as a $14: 1$ mixture of isomers) as a pale brown oil. ${ }^{\mathrm{i}}{ }^{1} \mathrm{H}$ 
NMR $\delta 8.03(\mathrm{~d}, J=9.6 \mathrm{~Hz}, 1 \mathrm{H}), 7.65(\mathrm{t}, J=7.2 \mathrm{~Hz}, 1 \mathrm{H}), 7.55(\mathrm{t}, J=7.2 \mathrm{~Hz}, 1 \mathrm{H}), 7.41(\mathrm{~d}$, $J=7.8 \mathrm{~Hz}, 1 \mathrm{H}), 6.31(\mathrm{~d}, J=10.2 \mathrm{~Hz}, 1 \mathrm{H}), 2.78(\mathrm{tq}, J=4.2,0.8 \mathrm{~Hz}, 1 \mathrm{H}), 1.87(\mathrm{~d}, J=2.6 \mathrm{~Hz}$, 2H), 1.79 (dt, $J=13.8 \mathrm{~Hz}, 3.0 \mathrm{~Hz}, 2 \mathrm{H}), 1.72(\mathrm{dt}, J=13.2,3.6 \mathrm{~Hz}, 1 \mathrm{H}), 1.40$ (tq, $J=12.6$, $3.6 \mathrm{~Hz}, 2 \mathrm{H}), 1.23(\mathrm{~m}, 3 \mathrm{H}) ;{ }^{13} \mathrm{C}$ NMR $\delta 156.7,147.8,133.5,131.7,129.9,129.7,124.9$, 115.2, 110.6, 41.2, 31.6, 25.5, 25.1; IR (ATR) 2928, 2853, 2221, 1526, 1345, 854, 731 $\mathrm{cm}^{-1}$; HRMS (ESI) calcd for $\mathrm{C}_{15} \mathrm{H}_{16} \mathrm{NaN}_{2} \mathrm{O}_{2}\left(\mathrm{M}+\mathrm{Na}^{+}\right)$279.1110; found 279.1104.

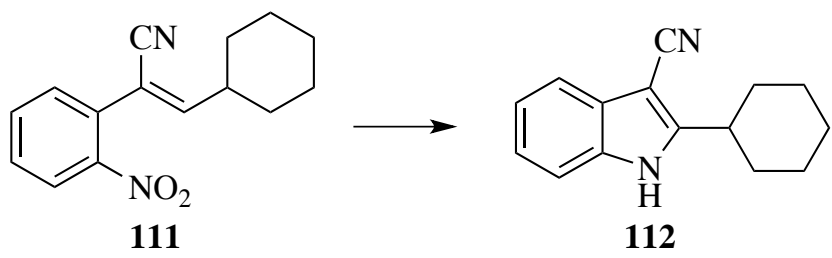

3-Cyano-2-cyclohexylindole (112): Reaction of 111 (73 mg, $0.31 \mathrm{mmol}), \mathrm{PPh}_{3}(26.2 \mathrm{mg}$, $0.10 \mathrm{mmol}), \mathrm{Pd}(\mathrm{OAc})(7.6 \mathrm{mg}, 0.03 \mathrm{mmol})$, and $\mathrm{CO}(6 \mathrm{~atm})$ in $\mathrm{DMF}(5 \mathrm{~mL})$, as described in for 77, gave after chromatography (hexanes/EtOAc, 7:3) 112 (34 mg, $0.15 \mathrm{mmol}$, $48 \%$ ) as a pale yellow solid. mp $171-173{ }^{\circ} \mathrm{C} ;{ }^{1} \mathrm{H}$ NMR $\delta 8.54$ (br s, $\left.1 \mathrm{H}\right), 7.67(\mathrm{~d}, J=4.8$ $\mathrm{Hz}, 1 \mathrm{H}), 7.38(\mathrm{~d}, J=7.2 \mathrm{~Hz}, 1 \mathrm{H}), 7.26-7.23(\mathrm{~m}, 2 \mathrm{H}), 3.05(\mathrm{tt}, J=8.4,3.6 \mathrm{~Hz}, 1 \mathrm{H}), 2.08(\mathrm{~d}$, $J=12.0 \mathrm{~Hz}, 2 \mathrm{H}), 1.90(\mathrm{dt}, J=13.8,3.0 \mathrm{~Hz}, 2 \mathrm{H}), 1.80(\mathrm{~d}, J=12.6 \mathrm{~Hz}, 1 \mathrm{H}), 1.61(\mathrm{dq}, J=12.6$, $3.6 \mathrm{~Hz}, 2 \mathrm{H}), 1.46(\mathrm{tq}, J=13.2,3.6 \mathrm{~Hz}, 2 \mathrm{H}), 1.31(\mathrm{tq}, J=12.6,3.6 \mathrm{~Hz}, 1 \mathrm{H}) ;{ }^{13} \mathrm{C}$ NMR $\delta$ $153.4,134.1,127.8,123.3,122.0,119.0,116.4,111.3,83.4,37.6,32.4,26.1,25.7 ;$ IR (ATR) 3227, 2919, 2849, 2215, 1439, $737 \mathrm{~cm}^{-1}$; HRMS (ESI) calcd for $\mathrm{C}_{15} \mathrm{H}_{16} \mathrm{NaN}_{2}$ $\left(\mathrm{M}+\mathrm{Na}^{+}\right)$247.1211, found 247.1199.<smiles>N#C/C(=C/C1CCCCC1)c1ccccc1[N+](=O)[O-]</smiles>

111

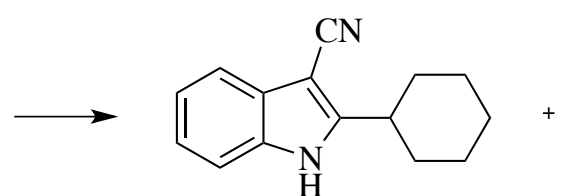

112

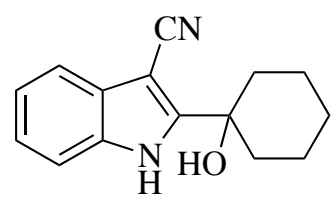

113 


\section{3-Cyano-2-cyclohexylindole (112) and 3-cyano-2-(1-hydroxycyclohexyl)indole (113).}

Reaction of 111 (82 mg, $0.32 \mathrm{mmol}$ ), $\mathrm{PPh}_{3}$ (42 mg, $0.16 \mathrm{mmol}$ ), DBU (53 mg, 0.36

$\mathrm{mmol})$, and $\mathrm{Pd}(\mathrm{OAc})_{2}(10 \mathrm{mg}, 0.05 \mathrm{mmol})$ in $\mathrm{DMF}(5 \mathrm{~mL})$ as described for $\mathbf{7 8}$ gave after chromatography (hexanes/EtOAc, 8:2) $\mathbf{1 1 2}(5 \mathrm{mg}, 0.02 \mathrm{mmol}, 7 \%)$ and $\mathbf{1 1 3}$ (24 mg, 0.10 mmol, 31\%) as a pale yellow oil. ${ }^{1} \mathrm{H}$ NMR $\delta 9.25$ (br s, $\left.1 \mathrm{H}\right), 7.68(\mathrm{~d}, J=6.6 \mathrm{~Hz}, 1 \mathrm{H}), 7.40$ (d, J=6.6 Hz, 1H), $7.25(\mathrm{~m}, 2 \mathrm{H}), 2.47$ (br s, 1H), 2.25 (dt, J=13.8, 4.8, 2H), 1.89 (d, $J=13.8 \mathrm{~Hz}, 2 \mathrm{H}), 1.82-1.64(\mathrm{~m}, J=16.2 \mathrm{~Hz}, 5 \mathrm{H}), 1.43(\mathrm{~m}, 1 \mathrm{H}) ;{ }^{13} \mathrm{C}$ NMR $\delta 154.6,133.1$, 129.2, 123.7, 122.3, 119.4, 116.8, 112.0, 80.7, 72.3, 37.4, 24.9, 21.6; IR (ATR) 3330, 2938, 2214, 1737, 1241, $1044 \mathrm{~cm}^{-1}$; HRMS (ESI) calcd for $\mathrm{C}_{15} \mathrm{H}_{16} \mathrm{NaN}_{2} \mathrm{O}\left(\mathrm{M}+\mathrm{Na}^{+}\right)$ 263.1160 , found 263.1155 . 


\subsection{Supporting Information for Chapter 3: Salviadione}

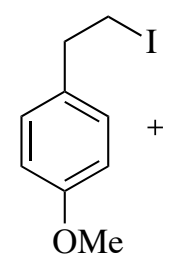

129

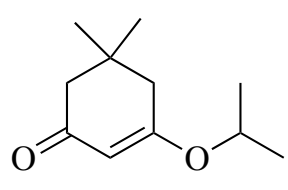

128

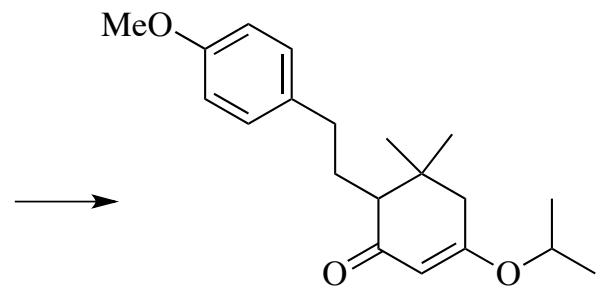

127

5,5-Dimethyl-6-(4-methoxyphenylethyl)-3-(1-methylethoxy)-cyclohex-2-enone (127):

5,5-Dimethyl-3-(1-methylethoxy)-2-cyclohexen-1-one (128) $)^{146}(4.133 \mathrm{~g}, 22.68 \mathrm{mmol})$ was dissolved in THF (25 mL) and cooled to $-78{ }^{\circ} \mathrm{C}$ under a positive flow of nitrogen. Lithium diisopropylamide $(2.0 \mathrm{M}, 13.6 \mathrm{~mL}, 27.21 \mathrm{mmol})$ was added drop wise via a cannula over $15 \mathrm{~min}$. The resulting yellow solution was stirred for $1 \mathrm{~h}$ at $-78{ }^{\circ} \mathrm{C}$ before slowly adding the solution drop wise via a cannula to a solution of 1-(2-iodoethyl)-4methoxybenzene $(\mathbf{1 2 9})^{145}(7.132 \mathrm{~g}, 27.21 \mathrm{mmol})$ in $\mathrm{THF}(25 \mathrm{~mL})$ cooled to $-78{ }^{\circ} \mathrm{C}$. The solution was allowed to stir up to ambient temperature (12 h) followed by addition of a solution of $\mathrm{NH}_{4} \mathrm{Cl}$ (sat. aqueous, $50 \mathrm{~mL}$ ) was carefully added and stirred for $5 \mathrm{~min}$. The phases were separated and the aqueous portion was extracted with EtOAc $(3 \times 200 \mathrm{~mL})$. The combined organic phases were dried $\left(\mathrm{MgSO}_{4}\right)$, filtered, and the solvents were removed under reduced pressure. The resulting orange oil was purified by chromatography (hexanes/EtOAc, 95:5 followed by 9:1) to afford 127 as a yellow oil (6.429 g, $20.32 \mathrm{mmol}, 90 \%) .{ }^{1} \mathrm{H}$ NMR (270 MHz) $\delta 7.13$ (d, J=8.5 Hz, 2H), 6.82 (d, $J=8.3 \mathrm{~Hz}, 2 \mathrm{H}), 5.34(\mathrm{~s}, 1 \mathrm{H}), 4.42(\mathrm{sept}, J=6.2 \mathrm{~Hz}, 1 \mathrm{H}), 3.78(\mathrm{~s}, 3 \mathrm{H}), 2.76(\mathrm{ddd}, J=13.4$, 9.7, 5.7 Hz, 1H), 2.54 (ddd, J=13.9, 9.7, 7.3, 1H), 2.26 (d, J=17.6 Hz, 1H), 2.18 (d, $J=17.6 \mathrm{~Hz}, 1 \mathrm{H}), 1.97(\mathrm{dd}, J=8.7,4.3 \mathrm{~Hz}, 1 \mathrm{H}), 1.83-1.69(\mathrm{~m}, 2 \mathrm{H}), 1.28(\mathrm{~d}, J=6.1 \mathrm{~Hz}, 6 \mathrm{H})$, $1.02(\mathrm{~s}, 3 \mathrm{H}), 0.94(\mathrm{~s}, 3 \mathrm{H}) ;{ }^{13} \mathrm{C}$ NMR $(67.5 \mathrm{MHz}) \delta 202.4(+), 173.1(+), 157.7(+), 134.6$ $(+), 129.4(-), 113.7(-), 100.9(-), 70.7(-), 56.2(-), 55.2(-), 42.2(+), 35.0(+), 34.0(+)$, 
$28.5(-), 28.4(+), 24.4(-), 21.5(-)$; IR (ATR) 2957, 2932, 1648, 1604, 1517, 1378, 1243 , $1107 \mathrm{~cm}^{-1}$.

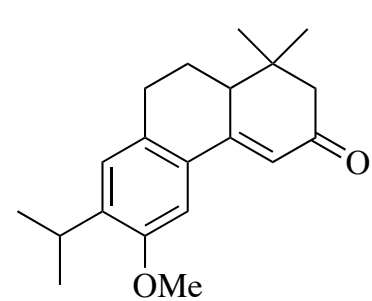

126

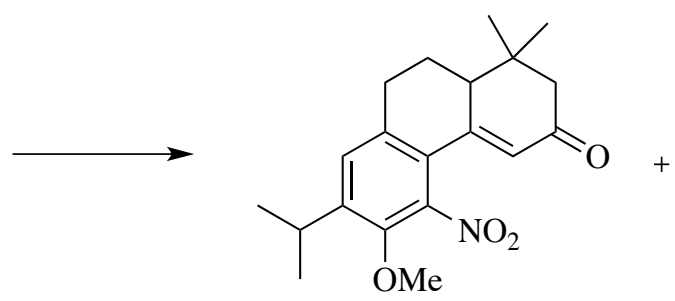

132<smiles>CC(C)c1cc2c(c([N+](=O)[O-])c1O)C1=CC(=O)CC(C)(C)C1CC2</smiles>

125

10,10a-Dihydro-1,1-dimethyl-6-methoxy-7-(1-methylethyl)-5-nitrophenanthren3(1H,2H,9H)-one (132) and 10,10a-Dihydro-1,1-dimethyl-6-hydroxy-7-(1methylethyl)-5-nitrophenanthren-3(1H,2H,9H)-one (125): Fuming nitric acid (5.0 mL, $144 \mathrm{mmol}$ ) was cooled to $-78^{\circ} \mathrm{C}$ and concentrated sulfuric acid (13 drops) was slowly added drop wise. After stirring for $20 \mathrm{~min}, 126$ (166 mg, $0.556 \mathrm{mmol}$ ) was added in one portion to the nitration solution. The resulting dark red solution was removed from the cold bath and allowed to stir for $10 \mathrm{~min}$. at ambient temperature. The mixture was poured into $200 \mathrm{~mL}$ of ice, neutralized with $\mathrm{Na}_{2} \mathrm{CO}_{3}(\mathrm{~s})$ and extracted with EtOAc $(3 \times 75 \mathrm{~mL})$. The combined organic phases were dried $\left(\mathrm{MgSO}_{4}\right)$, filtered, and the solvent was removed under reduced pressure. The resulting crude product was purified by chromatography (hexanes/acetone, 6:4) to afford in order of elution $132(47.9 \mathrm{mg}, 0.140 \mathrm{mmol}, 25 \%)$ as a colorless solid followed by 125 (59.6 $\mathrm{mg}, 0.181 \mathrm{mmol}, 32 \%)$ as a yellow solid. Analytical data for 132: mp 174-175 ${ }^{\circ} \mathrm{C} ;{ }^{1} \mathrm{H}$ NMR $(600 \mathrm{MHz}) \delta 7.15(\mathrm{~s}, 1 \mathrm{H}), 6.14(\mathrm{~d}$, $J=0.8 \mathrm{~Hz}, 1 \mathrm{H}), 3.83(\mathrm{~s}, 3 \mathrm{H}), 3.30$ (sept, $J=7.0 \mathrm{~Hz}, 1 \mathrm{H}), 2.81$ (dddd, $J=16.0,7.3,5.0,0.6$, 1H), 2.71 (dddd, $J=16.0,8.1,5.0,0.8 \mathrm{~Hz}, 1 \mathrm{H}) 2.61$ (dt, $J=7.1,2.4 \mathrm{~Hz}, 1 \mathrm{H}), 2.35$ (dd, $J=16.0,0.4 \mathrm{~Hz}, 1 \mathrm{H}), 2.27$ (dd. $J=16.0,0.8 \mathrm{~Hz}, 1 \mathrm{H}), 1.99$ (dddd, $J=13.5,7.3,7.1,7.1 \mathrm{~Hz}$, 1H), 1.87 (dddd, $J=13.5,8.1,5.0,5.0 \mathrm{~Hz}, 1 \mathrm{H}), 1.26(\mathrm{~d}, J=7.0 \mathrm{~Hz}, 3 \mathrm{H}), 1.24(J=7.0 \mathrm{~Hz}$, 3H), $1.16(\mathrm{~s}, 3 \mathrm{H}), 0.98(\mathrm{~s}, 3 \mathrm{H}) ;{ }^{13} \mathrm{C}$ NMR $(150 \mathrm{MHz}) \delta 198.5,151.8,147.5,145.5,144.4$, 
137.4, 127.6, 125.3, 124.8, 63.9, 51.6, 45.7, 37.1, 29.4, 28.9, 26.6, 23.4, 23.4, 23.2, 22.0;

IR (ATR) $2968,1664,1525,1283,1254 \mathrm{~cm}^{-1}$. HRMS (ESI) calcd for $\mathrm{C}_{20} \mathrm{H}_{26} \mathrm{NO}_{4}\left(\mathrm{M}+\mathrm{H}^{+}\right)$ 344.1862, found 344.1862. Analytical data for 125: mp 154-156 ${ }^{\circ} \mathrm{C}(\mathrm{dec}) ;{ }^{1} \mathrm{H}$ NMR (270 MHz) $\delta 9.20(\mathrm{br} \mathrm{s}, 1 \mathrm{H}), 7.17$ (s, 1H), $5.84(\mathrm{~d}, J=2.2 \mathrm{~Hz}, 1 \mathrm{H}), 3.37$ (sept, $J=6.9 \mathrm{~Hz}, 1 \mathrm{H})$, 2.82-2.57 (m, 4H), $2.40(\mathrm{~d}, J=16.0 \mathrm{~Hz}, 1 \mathrm{H}), 2.28(\mathrm{~d}, J=15.8 \mathrm{~Hz}, 1 \mathrm{H}), 2.04-1.97(\mathrm{~m}, 1 \mathrm{H})$, $1.26(\mathrm{~d}, J=6.7 \mathrm{~Hz}, 3 \mathrm{H}), 1.25(\mathrm{~d}, J=6.9 \mathrm{~Hz}, 3 \mathrm{H}), 1.23(\mathrm{~s}, 3 \mathrm{H}), 1.02(\mathrm{~s}, 3 \mathrm{H}) ;{ }^{13} \mathrm{C}$ NMR $(67.5$ MHz) $\delta 198.2(+), 154.2(+), 149.2(+), 139.7(+), 134.0(+), 133.6(+), 130.4(-), 129.0$ $(+), 125.3(-), 50.8(+), 45.3(-), 37.2(+), 28.7(-), 28.6(+), 27.2(-), 23.4(+), 22.7(-)$, 22.2 (-), 22.0 (-); IR (ATR) 3280, 2959, 1660, 1645, 1544, $726 \mathrm{~cm}^{-1}$; HRMS (ESI) calcd for $\mathrm{C}_{19} \mathrm{H}_{24} \mathrm{NO}_{4}\left(\mathrm{M}+\mathrm{H}^{+}\right) 330.1705$; found, 330.1705 .

Alternative procedure to 125 . Fuming $\mathrm{HNO}_{3}(500 \mathrm{~mL}, 12.0 \mathrm{mmol})$ was stirred at -20 ${ }^{\circ} \mathrm{C}$ for 5 min before concentrated $\mathrm{H}_{2} \mathrm{SO}_{4}(8$ drops) was slowly added drop wise. The solution was stirred for $30 \mathrm{~min}$ before $\mathbf{1 2 6}$ (79 $\mathrm{mg}, 0.26 \mathrm{mmol}$ ) was added. The resulting dark red solution was stirred for $90 \mathrm{~min}$ before quenching with ice $(30 \mathrm{~mL})$. The mixture was neutralized with $\mathrm{Na}_{2} \mathrm{CO}_{3}$ (aqueous, saturated) and extracted with EtOAc $(3 \times 50 \mathrm{~mL})$. The combined organic phases were dried $\left(\mathrm{MgSO}_{4}\right)$, filtered and the solvent was removed under reduced pressure. The resulting brown oil was immediately purified by chromatography (hexanes/EtOAc, 9:1) affording 125 (66 mg, $0.20 \mathrm{mmol}, 77 \%)$ as a yellow solid.

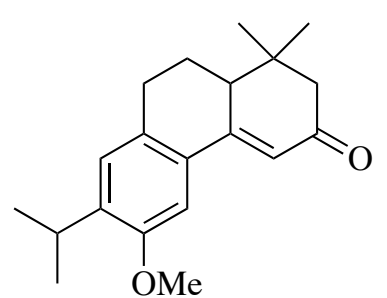

126

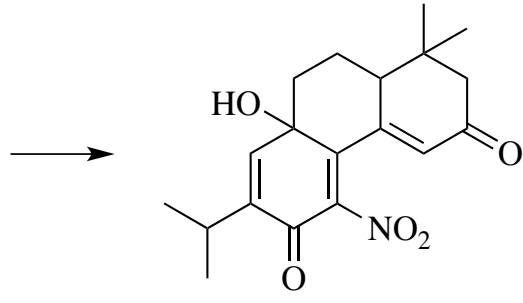

133 


\section{8a,9,10,10a-Tetrahydro-1,1-dimethyl-8a-hydroxy-7-(1-methylethyl)-5-}

nitrophenanthrene-3,6(1H,2H)-dione (133): Fuming $\mathrm{HNO}_{3}(6.9 \mathrm{~mL}, 164.0 \mathrm{mmol})$ was stirred at $0{ }^{\circ} \mathrm{C}$ for 5 min before concentrated $\mathrm{H}_{2} \mathrm{SO}_{4}$ (14 drops) was slowly added drop wise. After $30 \mathrm{~min}$ at $0{ }^{\circ} \mathrm{C}$, the solution was added drop wise via a pipette to $\mathbf{1 2 6}$ (1.078 $\mathrm{g}, 3.61 \mathrm{mmol})$ at ambient temperature. The resulting dark red solution was stirred for $4 \mathrm{~h}$ wherafter $\mathrm{H}_{2} \mathrm{O}(5 \mathrm{~mL})$ and $\mathrm{NH}_{4} \mathrm{Cl}$ (sat., aqueous, $40 \mathrm{~mL}$ ) was added sequentially. The precipitate was removed by filtration, dissolved in acetone $(50 \mathrm{~mL})$ and adhered to silica gel $(\approx 1.0 \mathrm{~g})$ and allowed to stand for $1 \mathrm{~h}$. The solvent was removed under reduced pressure and purification by chromatography (hexanes/acetone, 9:1 followed by 7:3) gave $133(0.832 \mathrm{~g}, 2.41 \mathrm{mmol}, 67 \%, 14: 1$ diastereomeric ratio) as an orange solid. mp 239-240 ${ }^{\circ} \mathrm{C}$; IR (ATR) 2952, 1663, 1530, 1266, $1253 \mathrm{~cm}^{-1}$; HRMS (ESI) calcd for $\mathrm{C}_{19} \mathrm{H}_{24} \mathrm{NO}_{5}$ $\left(\mathrm{M}+\mathrm{H}^{+}\right)$346.1654, found 346.1655. Spectral data of the major diastereomer 133 from the mixture: ${ }^{1} \mathrm{H}$ NMR $\left(600 \mathrm{MHz}, \mathrm{CDCl}_{3} / \mathrm{DMSO}_{6}\right) \delta 6.66(\mathrm{~d}, J=1.1 \mathrm{~Hz}, 1 \mathrm{H}), 6.02$ (d, J=2.4 Hz, 1H), 5.49 (d, J=1.5 Hz, 1H), 2.94 (dsept, J=6.8, 1.0 Hz, 1H), 2.41 (ddd, J=12.3, 5.7, $2.4 \mathrm{~Hz}, 1 \mathrm{H}), 2.32$ (ddd, $J=13.8,3.5,2.3 \mathrm{~Hz}, 1 \mathrm{H}), 2.30$ (d. $J=15.3 \mathrm{~Hz}, 1 \mathrm{H}), 2.26$ (d, J=15.3 $\mathrm{Hz}, 1 \mathrm{H}), 2.08(\mathrm{dq}, J=13.2,4.0 \mathrm{~Hz}, 1 \mathrm{H}), 1.97-1.92(\mathrm{~m}, 1 \mathrm{H}), 1.67$ (ddt, $J=13.2,4.2,1.2 \mathrm{~Hz}$, $1 \mathrm{H}), 1.12(\mathrm{~d}, J=7.2 \mathrm{~Hz}, 3 \mathrm{H}), 1.11(\mathrm{~d}, J=7.2 \mathrm{~Hz}, 3 \mathrm{H}), 1.11(\mathrm{~s}, 3 \mathrm{H}), 1.02(\mathrm{~s}, 3 \mathrm{H}) ;{ }^{13} \mathrm{C}$ NMR (150 MHz, $\mathrm{CDCl}_{3} / \mathrm{DMSO}_{6}$ ) $\delta$ 198.0, 175.5, 152.1, 147.9, 145.6, 143.4, 142.2, 126.5 , $68.9,50.9,49.0,37.4,35.6,28.4,26.4,23.0,21.3,21.0,20.5$. Partial spectral data for minor diastereomer 133 from the mixture: ${ }^{1} \mathrm{H}$ NMR $\left(600 \mathrm{MHz}, \mathrm{CDCl}_{3} / \mathrm{DMSO} \mathrm{d}_{6}\right) \delta 6.80$ $(\mathrm{d}, J=1.0 \mathrm{~Hz}, 1 \mathrm{H}), 6.22(\mathrm{~d}, J=2.8 \mathrm{~Hz}, 1 \mathrm{H}), 5.46(\mathrm{~s}, 1 \mathrm{H}) ;{ }^{13} \mathrm{C} \mathrm{NMR}(150 \mathrm{MHz}$, $\left.\mathrm{CDCl}_{3} / \mathrm{DMSO}_{-} \mathrm{d}_{6}\right) \delta 198.1,175.6,153.4,146.2,145.8,145.6,139.8,127.7,67.7,53.6$, $45.1,37.2,37.1,30.7,28.7,26.2,23.0,19.7,19.6$. 
<smiles>COc1c(C(C)C)cc2c(c1[N+](=O)[O-])C1=CC(=O)CC(C)(C)C1CC2</smiles>

132

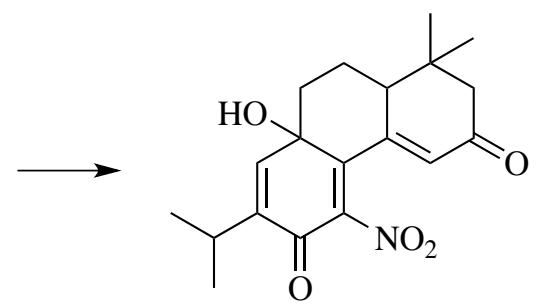

133

Alternative procedure to 133 . To a solution of $132(81 \mathrm{mg}, 0.236 \mathrm{mmol})$ in acetone (5 $\mathrm{mL})$ and acetic acid $(1 \mathrm{~mL})$ was added silica gel $(0.5 \mathrm{~g})$. The solvent was removed at reduced pressure and the yellow residue was allowed to stand open to air for $14 \mathrm{~h}$. A slight yellow tint was noticed after this time and the residue was purified by chromatography (hexanes/acetone, 6:4) to afford 133 (72.0 mg, $0.208 \mathrm{mmol}, 88 \%$ ).<smiles>COc1c(C(C)C)cc2c(c1[N+](=O)[O-])C1=CC(=O)CC(C)(C)C1CC2</smiles>

132

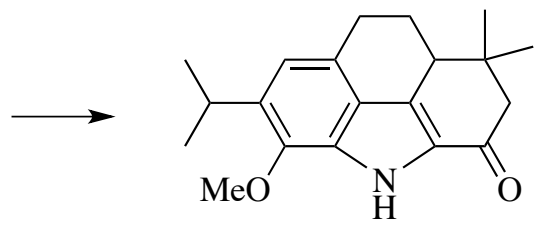

134

\section{1,8,9,9a-Dihydro-1,1-dimethyl-5-methoxy-6-(1-methylethyl)-4H-benzo[def]carbazol-}

3(2H)-one (134): To a solution of $132(22 \mathrm{mg}, 0.065 \mathrm{mmol})$ in anhydrous DMF $(1.0 \mathrm{~mL})$ in a threaded ACE glass pressure tube was added 1,10-phenanthroline monohydrate (1.6 mg, $0.008 \mathrm{mmol})$, 1,3-bis(diphenylphosphino)propane (1.7 mg, $0.004 \mathrm{mmol})$, and bis(dibenzylideneacetone)palladium $(2.3 \mathrm{mg}, 0.004 \mathrm{mmol})$. The tube was fitted with a pressure head, and the solution was saturated with carbon monoxide (4 cycles of $6 \mathrm{~atm}$ ). The reaction mixture was heated at $120{ }^{\circ} \mathrm{C}$ under carbon monoxide $(6 \mathrm{~atm})$ for $168 \mathrm{~h}$. The solvent was removed via bulb-to bulb distillation affording a brown residue. Water (5 $\mathrm{mL})$ was added to this residue and the mixture was extracted with ethyl acetate $(3 \times 30$ $\mathrm{mL})$. The combined organic phases were dried $\left(\mathrm{MgSO}_{4}\right)$, filtered and the solvent was removed under reduced pressure. The crude product was purified by chromatography 
(hexanes/acetone, 9:1) to give $\mathbf{1 3 4}(17.3 \mathrm{mg}, 0.057 \mathrm{mmol}, 87 \%)$ as a yellow solid. $\mathrm{mp}$ 228-230 ${ }^{\circ} \mathrm{C} ;{ }^{1} \mathrm{H}$ NMR $(600 \mathrm{MHz}) \delta 8.64(\mathrm{br} \mathrm{s}, 1 \mathrm{H}), 6.81(\mathrm{~s}, 1 \mathrm{H}), 3.94$ (s, 3H), 3.52 (sept, $J=7.0 \mathrm{~Hz}, 1 \mathrm{H}), 3.03(\mathrm{dd}, J=12.1,5.0 \mathrm{~Hz}, 1 \mathrm{H}), 3.02-2.95(\mathrm{~m}, 2 \mathrm{H}), 2.77(\mathrm{~d}, J=15.8 \mathrm{~Hz}$, $1 \mathrm{H}), 2.33(\mathrm{~d}, J=15.8 \mathrm{~Hz}, 1 \mathrm{H}), 2.21(\mathrm{~m}, 1 \mathrm{H}), 1.62(\mathrm{~m}, 1 \mathrm{H}), 1.27(\mathrm{~d}, J=7.0 \mathrm{~Hz}, 3 \mathrm{H}), 1.27$ (d, J=7.0 Hz, 3H), 1.24 (s, 3H), 0.85 (s, 3H); ${ }^{13} \mathrm{C}$ NMR $\delta 190.0,140.9,140.4,134.3$, $130.0,129.5,127.6,126.4,115.7,60.9,55.8,41.8,41.2,28.4,27.4,26.8,25.1,24.2,23.9$, 20.4; IR (ATR) 3264, 2958, 2930, 1653, $1620 \mathrm{~cm}^{-1}$; HRMS (ESI) calcd for $\mathrm{C}_{20} \mathrm{H}_{26} \mathrm{NO}_{3}$ $\left(\mathrm{M}+\mathrm{H}^{+}\right)$312.1964, found 312.1959.

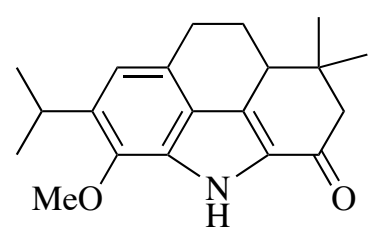

134

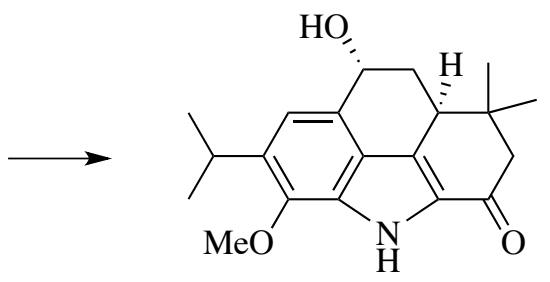

136

\section{1,8,9,9a-Tetrahydro-1,1-dimethyl-5-methoxy-6-(1-methylethyl)-8-hydroxy-4H-}

benzo[def]carbazol-3(2H)-one (136): To a solution of 134 (39.0 mg, $0.124 \mathrm{mmol})$ in THF (4 mL) was added DDQ (115 mg, $0.496 \mathrm{mmol})$ over a period of $5 \mathrm{~min}$. The resulting mixture was allowed to stir at ambient temperature for $4 \mathrm{~h}$ where after the solvent was removed under reduced pressure. The color of the residue changed from purple to orange over $30 \mathrm{~min}$. The crude product was purified by chromatography (hexanes/EtOAc, 7:3) to give $136\left(36.0 \mathrm{mg}, 87 \%\right.$ ) as a brown solid. $\mathrm{mp}=141-143{ }^{\circ} \mathrm{C} ;{ }^{1} \mathrm{H}$ NMR (600 MHz) $\delta 8.60($ br s, 1H), $7.03(\mathrm{~s}, 1 \mathrm{H}), 5.18(\mathrm{t}, J=2.7 \mathrm{~Hz}, 1 \mathrm{H}), 3.95(\mathrm{~s}, 3 \mathrm{H})$, 3.53 (sept, $J=7.2 \mathrm{~Hz}, 1 \mathrm{H}), 3.37$ (dd, $J=12.0,4.8 \mathrm{~Hz}, 1 \mathrm{H}), 2.81$ (d, $J=5.2,0.6,1 \mathrm{H}), 2.41$ (ddd, $J=13.8,5.4,3.0 \mathrm{~Hz}, 1 \mathrm{H}), 2.37(\mathrm{~d}, J=16.8,1 \mathrm{H}), 1.64(\mathrm{ddd}, J=16.8,12.0,3.0 \mathrm{~Hz}$, 1H), $1.60(\mathrm{br} \mathrm{s}, 1 \mathrm{H}), 1.28$ (d, J=7.2 Hz, 3H), 1.27 (d, J=7.2, 3H), 1.26 (s, 3H), 0.83 (s, $3 \mathrm{H}) ;{ }^{13} \mathrm{C}$ NMR (150 MHz) $\delta 189.2,143.0,140.3,132.8,129.9,128.9,128.0,125.5$, 
$116.8,67.7,60.7,55.9,41.6,35.7,32.5,28.3,27.0,24.1,23.8,20.7$; IR (ATR) 3279 (br), $2959,1650,1624 \mathrm{~cm}^{-1}$; HRMS (ESI) calcd for $\mathrm{C}_{20} \mathrm{H}_{26} \mathrm{NO}_{3}\left(\mathrm{M}+\mathrm{H}^{+}\right) 328.1913$, found 328.1909 .<smiles>CC(C)c1cc2c(c([N+](=O)[O-])c1O)C1=CC(=O)CC(C)(C)C1CC2</smiles>

125

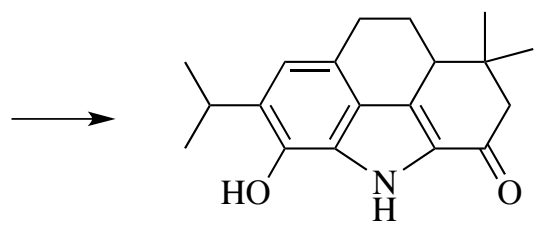

124

\section{1,8,9,9a-Tetrahydro-1,1-dimethyl-5-hydroxy-6-(1-methylethyl)-4H-}

benzo[def]carbazol-3(2H)-one (124): Reaction of $\mathbf{1 2 5}$ (140 mg, $0.566 \mathrm{mmol})$, bis(dibenzylideneacetone)palladium (20 mg, $0.034 \mathrm{mmol}), 1,3$-bis(diphenylphosphino)propane (14 mg, $0.034 \mathrm{mmol}$ ), and 1,10-phenanthroline (13 mg, $0.068 \mathrm{mmol})$ were dissolved in anhydrous DMF $(2 \mathrm{~mL})$ in a threaded ACE glass pressure tube. The tube was fitted with a pressure head, and the solution was saturated with carbon monoxide (four cycles of $6 \mathrm{~atm}$ of $\mathrm{CO}$ ). The reaction was heated at $120{ }^{\circ} \mathrm{C}$ under $\mathrm{CO}(6 \mathrm{~atm})$ for $32 \mathrm{~h}$. DMF was removed via vacuum distillation before water $(10 \mathrm{~mL})$ was added to the brown residue. The brown solution was extracted with ethyl acetate (3 x $40 \mathrm{~mL})$. The combined organic phases were dried $\left(\mathrm{MgSO}_{4}\right)$, filtered, and the solvent was removed. The resulting crude product was purified by chromatography (pentanes/acetone, $8: 2$ followed by 1:1) to afford $\mathbf{1 2 4}(91 \mathrm{mg}, 0.423 \mathrm{mmol}, 75 \%)$ as a light yellow solid. mp 234-236 ${ }^{\circ} \mathrm{C} ;{ }^{1} \mathrm{H}$ NMR $\left(600 \mathrm{MHz}, \mathrm{THF}-\mathrm{d}_{8}\right) \delta 9.94(\mathrm{~s}, 1 \mathrm{H}), 7.72$ (s, 1H), 6.69 (s, 1H), $3.50(\mathrm{sept}, J=7.5 \mathrm{~Hz}, 1 \mathrm{H}), 3.03(\mathrm{dd}, J=12.6,4.4 \mathrm{~Hz}, 1 \mathrm{H}), 2.96-2.88(\mathrm{~m}$, $2 \mathrm{H}), 2.7(\mathrm{~m}, 1 \mathrm{H}), 2.20-2.14(\mathrm{~m}, 2 \mathrm{H}), 1.53(\mathrm{dq}, J=11.9,5.4 \mathrm{~Hz}, 1 \mathrm{H}), 1.24(\mathrm{~d}, J=7.0 \mathrm{~Hz}$, $3 \mathrm{H}), 1.22(\mathrm{~d}, J=7.0 \mathrm{~Hz}, 3 \mathrm{H}), 1.19(\mathrm{~s}, 3 \mathrm{H}), 0.78(\mathrm{~s}, 3 \mathrm{H}) ;{ }^{13} \mathrm{C} \mathrm{NMR}\left(150 \mathrm{MHz}, \mathrm{THF}^{-} \mathrm{d}_{6}\right) \delta$ $187.9,138.9,133.6,133.2,129.5,128.6,126.8,125.8,115.7,56.8,42.4,42.1,28.7,28.3$, 
27.8, 26.5, 24.0, 23.9, 20.6; IR (ATR) 3394 (br), 3260, 2962, 2928, $1617 \mathrm{~cm}^{-1}$. HRMS

(ESI) calcd for $\mathrm{C}_{19} \mathrm{H}_{24} \mathrm{NO}_{2}\left(\mathrm{M}+\mathrm{H}^{+}\right)$298.1807, found 298.1802.

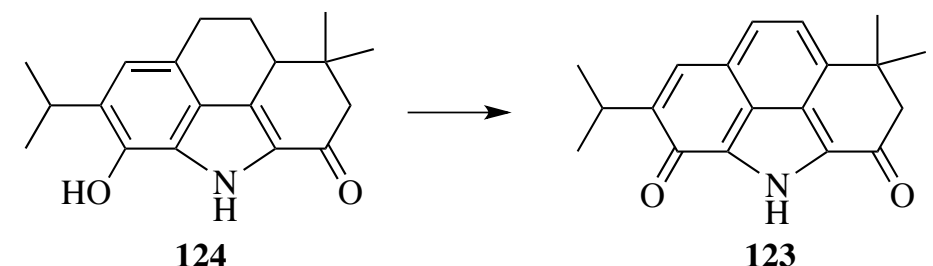

Salviadione (123): To a solution of $\mathbf{1 2 4}$ ( $35 \mathrm{mg}, 0.118 \mathrm{mmol}$ ) in THF (5 mL) was added 2,3-dichloro-5,6-dicyano-1,4-benzoquinone (107 $\mathrm{mg}, 0.471 \mathrm{mmol}$ ) slowly in 2 approximately equal portions with a 5 min interval. The reaction mixture was stirred for $4 \mathrm{~h}$ at ambient temperature. The solvent was removed under reduced pressure from the resulting dark red-purple solution. The residue was dissolved in acetone $(10 \mathrm{~mL})$ and silica gel $(\approx 1.0 \mathrm{~g})$ was added. The solvent was removed under reduced pressure and the resulting solid was allowed to stand open to the air (4 h). Purification by chromatography (hexanes/acetone, 9:1) gave salviadione (123) (28 $\mathrm{mg}, 0.095 \mathrm{mmol}, 81 \%)$ as an orange solid. Physical (mp) and spectroscopical data $\left({ }^{1} \mathrm{H},{ }^{13} \mathrm{C}, \mathrm{COSY}, \mathrm{HMBC}, \mathrm{HMQC}\right)$ were in accordance with literature values. HRMS (ESI) calcd for $\mathrm{C}_{19} \mathrm{H}_{20} \mathrm{NO}_{2}\left(\mathrm{M}+\mathrm{H}^{+}\right)$294.1494, found 294.1494 .<smiles>CC(C)C1=CC2(O)CCC3C(=CC(=O)CC3(C)C)C2=C([N+](=O)[O-])C1=O</smiles>

133

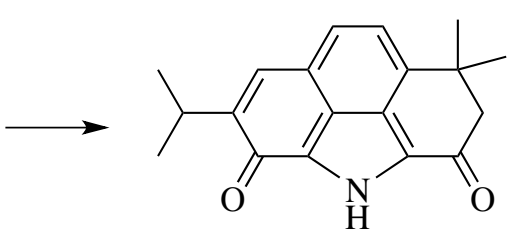

123

Alternative method to 123 To a solution of $\mathbf{1 3 3}(101 \mathrm{mg}, 0.29 \mathrm{mmol})$ in anhydrous DMF (7.0 mL) in a threaded ACE glass pressure tube was added dppp (7.7 mg, 0.019 $\mathrm{mmol})$, phen (19 $\mathrm{mg}, 0.095 \mathrm{mmol})$ and $\mathrm{Pd}(\mathrm{dba})_{2}(15 \mathrm{mg}, 0.027 \mathrm{mmol})$. The tube was 
fitted with a pressure head and the solution was saturated with carbon monoxide ( 3 cycles of 6 atm). The reaction mixture was heated at $120{ }^{\circ} \mathrm{C}$ under carbon monoxide for 168 hours. The solvent was removed via bulb-to-bulb distillation affording a brown oil. Water $(5 \mathrm{~mL})$ was added to this residue and the mixture was extracted with ethyl acetate (3 x $25 \mathrm{~mL})$. The combined organic phases were dried $\left(\mathrm{MgSO}_{4}\right)$, filtered, and solvent was removed under reduced pressure. The crude product was purified by chromatography (hexanes/ethyl acetate 7:3) to afford salviadione (123) (8.7 mg, 0.029 mmol, 10\%). 
7.3 Supporting Information for Chapter 4: Chemoselective Coupling<smiles>O=[N+]([O-])c1cc(O)ccc1Br</smiles>

2-Bromo-5-trifluoromethanesulfonyloxy-nitrobenzene (163): To an ice-cooled solution of $\mathbf{1 6 2}^{147}$ (269 $\left.\mathrm{mg}, 1.23 \mathrm{mmol}\right)$ in $\mathrm{CH}_{2} \mathrm{Cl}_{2}(5 \mathrm{~mL})$ was added pyridine (200 $\mu \mathrm{L}$, $2.48 \mathrm{mmol})$ and trifluoromethanesulfonic anhydride $\left(\mathrm{Tf}_{2} \mathrm{O}, 250 \mu \mathrm{L}, 1.48 \mathrm{mmol}\right)$. The mixture was removed from the cold bath and allowed to stir at ambient temperature for $30 \mathrm{~min}$. The resulting mixture was filtered through a small plug of silica gel and the solvent was removed under reduced pressure from the filtrate. Purification by chromatography (hexanes/EtOAc, 9:1) afforded $\mathbf{3}(391 \mathrm{mg}, 1.12 \mathrm{mmol}, 90 \%)$ as a yellow oil. ${ }^{1} \mathrm{H}$ NMR $\delta 7.89$ (d, $\left.J=9.0 \mathrm{~Hz}, 1 \mathrm{H}\right), 7.83(\mathrm{~d}, J=3.0 \mathrm{~Hz}, 1 \mathrm{H}), 7.42$ (dd, $J=9.0,3.0 \mathrm{~Hz}$, $1 \mathrm{H}) ;{ }^{13} \mathrm{C}$ NMR $\delta 150.1,147.9,136.8,126.3,119.3,118.6$ (q, $\left.J=319 \mathrm{~Hz}, \mathrm{C}-\mathrm{F}\right), 114.6$; IR (ATR) $3103,1541,1428,1208,1132 \mathrm{~cm}^{-1}$. HRMS (ESI) calcd for $\mathrm{C}_{7} \mathrm{H}_{3} \mathrm{BrNO}_{5} \mathrm{~F}_{3} \mathrm{~S}$ $\left(\mathrm{M}+\mathrm{Na}^{+}\right)$371.8759; found 371.8760.<smiles>CCOc1cccc(Br)c1[N+](=O)[O-]</smiles>

2-Bromo-6-trifluoromethanesulfonyloxy-nitrobenzene (166): Treatment of $\mathbf{1 6 5}^{116}$ (298 $\mathrm{mg}, 1.37 \mathrm{mmol})$ in $\mathrm{CH}_{2} \mathrm{Cl}_{2}(5 \mathrm{~mL})$ with pyridine $(250 \mu \mathrm{L}, 3.10 \mathrm{mmol})$ and $\mathrm{Tf}_{2} \mathrm{O}(300 \mu \mathrm{L}$, $1.77 \mathrm{mmol}$ ), as described for 163, gave after chromatography (hexanes/EtOAc, 8:2) 166 (394 mg, $1.13 \mathrm{mmol}, 80 \%$ ) as a red solid. mp 52-53 ${ }^{\circ} \mathrm{C} ;{ }^{1} \mathrm{H}$ NMR $\delta 7.73(\mathrm{dd}, J=6.6,3.0$ $\mathrm{Hz}, 1 \mathrm{H}), 7.49(\mathrm{~m}, 2 \mathrm{H}) ;{ }^{13} \mathrm{C} \mathrm{NMR} \delta 144.3,140.7,133.4,132.1,121.6,118.3$ (q, J=319 
Hz, C-F), 115.2; IR (ATR) 3099, 1538, 1434, 1360, 1219, $1132 \mathrm{~cm}^{-1}$; HRMS (ESI) calcd for $\mathrm{C}_{7} \mathrm{H}_{3} \mathrm{BrNO}_{5} \mathrm{~F}_{3} \mathrm{~S}\left(\mathrm{M}+\mathrm{Na}^{+}\right) 371.8759$; found 371.8767 .<smiles>O=[N+]([O-])c1cccc(Br)c1O</smiles>

149<smiles>CCOc1c(Br)cccc1[N+](=O)[O-]</smiles>

151

3-Bromo-2-trifluoromethanesulfonyloxy-nitrobenzene (151): Treatment of $\mathbf{1 4 9}^{93}$ (189 $\mathrm{mg}, 0.87 \mathrm{mmol})$ in $\mathrm{CH}_{2} \mathrm{Cl}_{2}(5 \mathrm{~mL})$ with pyridine $(150 \mu \mathrm{L}, 1.85 \mathrm{mmol})$ and $\mathrm{Tf}_{2} \mathrm{O}(200 \mu \mathrm{L}$, $1.18 \mathrm{mmol}$ ), as described for $\mathbf{1 6 3}$, gave after chromatography (hexanes/EtOAc, 7:3) 151 (299 mg, $0.85 \mathrm{mmol} 98 \%$ ) as a colorless oil. ${ }^{1} \mathrm{H}$ NMR $\delta 8.04(\mathrm{dd}, J=8.4,1.8 \mathrm{~Hz}, 1 \mathrm{H})$, $7.99(\mathrm{dd}, J=8.4,1.8 \mathrm{~Hz}, 1 \mathrm{H}), 7.46(\mathrm{t}, J=7.8 \mathrm{~Hz}, 1 \mathrm{H}) ;{ }^{13} \mathrm{C} \mathrm{NMR}$

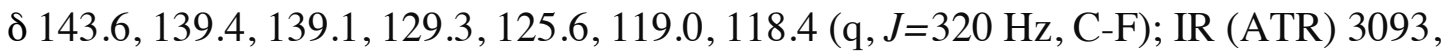
$1588,1540,1431,1347,1207 \mathrm{~cm}^{-1}$; $\mathrm{HRMS}(\mathrm{ESI})$ calcd for $\mathrm{C}_{7} \mathrm{H}_{3} \mathrm{BrNO}_{5} \mathrm{~F}_{3} \mathrm{~S}\left(\mathrm{M}+\mathrm{Na}^{+}\right)$ 371.8759; found 371.8761 .<smiles>O=[N+]([O-])c1cc(O)cc([18O]c2cc([18F])cc(Br)c2)c1</smiles>

3-Bromo-5-trifluoromethanesulfonyloxy-nitrobenzene (157): Treatment of $\mathbf{1 5 6}^{148}$ (329 $\mathrm{mg}, 1.51 \mathrm{mmol})$ in $\mathrm{CH}_{2} \mathrm{Cl}_{2}(10 \mathrm{~mL})$ with pyridine $(250 \mu \mathrm{L}, 3.10 \mathrm{mmol})$ and $\mathrm{Tf}_{2} \mathrm{O}(300$ $\mu \mathrm{L}, 1.78 \mathrm{mmol}$ ), as described for 163, gave after chromatography (hexanes/EtOAc, 7:3) 157 (316 mg, $0.90 \mathrm{mmol}, 60 \%)$ as a red oil. ${ }^{1} \mathrm{H}$ NMR $\delta 8.44(\mathrm{t}, J=1.8 \mathrm{~Hz}, 1 \mathrm{H}), 8.11(\mathrm{t}$, $J=1.8 \mathrm{~Hz}, 1 \mathrm{H}), 7.80(\mathrm{t}, J=1.8 \mathrm{~Hz}, 1 \mathrm{H}) ;{ }^{13} \mathrm{C} \mathrm{NMR}$ $\delta$ 149.1, 149.0, 130.7, 126.7, 123.8, 118.5 (q, J=319 Hz, C-F),116.0; IR (ATR) 3097, $1732,1542,1427,1344,1210,1134 \mathrm{~cm}^{-1}$; HRMS (ESI) calcd for $\mathrm{C}_{7} \mathrm{H}_{3} \mathrm{BrNO}_{5} \mathrm{~F}_{3} \mathrm{~S}$ $\left(\mathrm{M}+\mathrm{Na}^{+}\right)$371.8760; found 371.8764. 


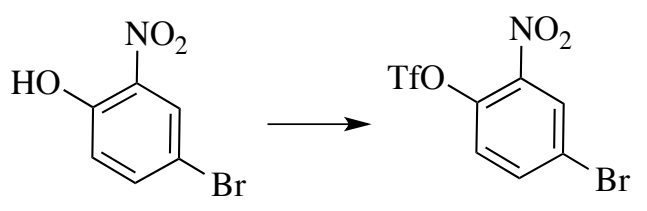

$153 \quad 154$

3-Bromo-6-trifluoromethanesulfonyloxy-nitrobenzene (154): Treatment of $\mathbf{1 5 3}^{149}$ (353 $\mathrm{mg}, 1.60 \mathrm{mmol})$ in $\mathrm{CH}_{2} \mathrm{Cl}_{2}(5 \mathrm{~mL})$ with pyridine $(260 \mu \mathrm{L}, 3.22 \mathrm{mmol})$ and $\mathrm{Tf}_{2} \mathrm{O}(330 \mu \mathrm{L}$, $1.95 \mathrm{mmol}$ ), as described for 163, gave after chromatography (hexanes/EtOAc, 7:3) 154 (540 mg, $1.54 \mathrm{mmol}, 97 \%)$ as a yellow oil. ${ }^{1} \mathrm{H}$ NMR $\delta 8.30(\mathrm{~d}, J=2.4 \mathrm{~Hz}, 1 \mathrm{H}), 7.88$ (dd, $J=9.0,2.4 \mathrm{~Hz}, 1 \mathrm{H}), 7.36(\mathrm{~d}, J=9.0 \mathrm{~Hz}, 1 \mathrm{H}) ;{ }^{13} \mathrm{C}$ NMR $\delta 141.9,140.5,138.2,129.7,125.6$, 122.3, 118.5 (q, J=319 Hz, C-F); IR 3105, 1540, 1431, 1207, $1131 \mathrm{~cm}^{-1}$;<smiles>CCOc1cc([N+](=O)[O-])ccc1Br</smiles>

4-Bromo-3-trifluoromethanesulfonyloxy-nitrobenzene (159): Treatment of $\mathbf{1 5 8}^{150}$ (119 $\mathrm{mg}, 0.55 \mathrm{mmol})$ in $\mathrm{CH}_{2} \mathrm{Cl}_{2}(5 \mathrm{~mL})$ with pyridine $(90.0 \mu \mathrm{L}, 1.12 \mathrm{mmol})$ and $\mathrm{Tf}_{2} \mathrm{O}(120 \mu \mathrm{L}$, $0.71 \mathrm{mmol}$ ), as described for $\mathbf{1 6 3}$, gave without further purification 159 (188 $\mathrm{mg}, 0.54$ mmol, 98\%) as a brown oil. ${ }^{1} \mathrm{H}$ NMR $\delta 8.22(\mathrm{~d}, J=2.4 \mathrm{~Hz}, 1 \mathrm{H}), 8.16(\mathrm{dd}, J=8.4,2.4 \mathrm{~Hz}$, $1 \mathrm{H}), 7.93(\mathrm{~d}, J=8.4 \mathrm{~Hz}, 1 \mathrm{H}) ;{ }^{13} \mathrm{C}$ NMR $\delta 147.7,147.0,135.2,124.1,123.9,118.5(\mathrm{q}$, $J=319 \mathrm{~Hz}, \mathrm{C}-\mathrm{F}$ ), 118.4; IR (ATR) 3104, 1534, 1431, 1348, 1211, $1134 \mathrm{~cm}^{-1}$; HRMS (ESI) calcd for $\mathrm{C}_{7} \mathrm{H}_{3} \mathrm{BrNO}_{5} \mathrm{~F}_{3} \mathrm{~S}\left(\mathrm{M}+\mathrm{Na}^{+}\right)$373.8739; found 373.8740 .<smiles>CCOc1cccc([N+](=O)[O-])c1Br</smiles>

140<smiles>C=Cc1c(OCC)cccc1[N+](=O)[O-]</smiles>

141 
2-Ethenyl-3-trifluoromethanesulfonyloxy-nitrobenzene (141): ${ }^{92}$ To a solution of $\mathrm{PPh}_{3}$ $(6.5 \mathrm{mg}, 0.03 \mathrm{mmol})$ and $\mathrm{Pd}(\mathrm{dba})_{2}(3.7 \mathrm{mg}, 0.006 \mathrm{mmol})$ in dioxane $(1.5 \mathrm{~mL})$, stirred for 5 min under an atmosphere $\mathrm{N}_{2}$, was added 140 (105 mg, $\left.0.30 \mathrm{mmol}\right)$ and ethenyl(tributyl)stannane (119 $\mathrm{mg}, 0.38 \mathrm{mmol})$. The solution was heated at reflux for 24 h. The solvent was removed under reduced pressure. The resulting crude oil was dissolved in EtOAc $(10 \mathrm{~mL})$ and washed with $\mathrm{NH}_{4} \mathrm{OH}(10 \%$ aq., 3 x $20 \mathrm{~mL}), \mathrm{H}_{2} \mathrm{O}(20$ $\mathrm{mL})$, and brine $(20 \mathrm{~mL})$. The organic phase was dried $\left(\mathrm{MgSO}_{4}\right)$, filtered, and solvents were removed under reduced pressure. Purification using chromatography (hexanes/EtOAc, 97:3) gave in order of elution, $141(39.3 \mathrm{mg}, 0.13 \mathrm{mmol}, 36 \%)$ as a yellow oil, a mixture of 140 and dba (9.8 mg), and $\mathbf{1 6 9}(40.8 \mathrm{mg}, 0.19 \mathrm{mmol}, 62 \%)$. Spectral data were in accordance with literature values.<smiles>CCOc1cccc([N+](=O)[O-])c1Br</smiles>

140

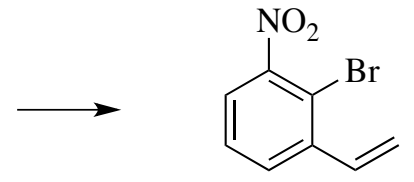

170

2-Bromo-3-ethenyl-nitrobenzene (170): To a solution of $\mathrm{LiCl}(40.9 \mathrm{mg}, 0.96 \mathrm{mmol})$ and $\mathrm{Pd}\left(\mathrm{PPh}_{3}\right)_{2} \mathrm{Cl}_{2}(4.8 \mathrm{mg}, 0.007 \mathrm{mmol})$ in $\mathrm{DMF}(1.5 \mathrm{~mL})$ under an atmosphere of $\mathrm{N}_{2}$ was added $140(110 \mathrm{mg}, 0.32 \mathrm{mmol})$ and ethenyl(tributyl)stannane $(123 \mathrm{mg}, 0.39 \mathrm{mmol})$. The resulting solution was stirred at ambient temperature for $24 \mathrm{~h}$. The solution was diluted with EtOAc $(10 \mathrm{~mL})$ and washed with $\mathrm{NH}_{4} \mathrm{OH}(10 \%$ aq., 3 x $20 \mathrm{~mL}), \mathrm{H}_{2} \mathrm{O}(20 \mathrm{~mL})$, and brine $(20 \mathrm{~mL})$. The organic phase was dried $\left(\mathrm{MgSO}_{4}\right)$, filtered, and solvents were removed under reduced pressure. The crude product was purified by chromatography (hexanes/EtOAc, 97:3) affording in order of elution, 11 (18.5 mg, $0.06 \mathrm{mmol}, 20 \%), \mathbf{1 7 0}$ (27.6 mg, $0.12 \mathrm{mmol}, 38 \%)$ as a colorless oil. ${ }^{1} \mathrm{H}$ NMR $\delta 7.70(\mathrm{~d}, J=7.8 \mathrm{~Hz}, 1 \mathrm{H}), 7.58(\mathrm{~d}$, $J=7.8 \mathrm{~Hz}, 1 \mathrm{H}), 7.42(\mathrm{t}, J=7.8 \mathrm{~Hz}, 1 \mathrm{H}), 7.10(\mathrm{dd}, \mathrm{J}=16.8,11.4 \mathrm{~Hz}, 1 \mathrm{H}), 5.76(\mathrm{~d}, J=17.4$ 
$\mathrm{Hz}, 1 \mathrm{H}), 5.52(\mathrm{dd}, J=10.8 \mathrm{~Hz}, 1 \mathrm{H}) ;{ }^{13} \mathrm{C}$ NMR $\delta 147.2,128.9,128.3,126.2,125.7,124.8$, 123.8, 119.5; IR (ATR) 3110, 1533, 1423, 1358, 1210, $1135 \mathrm{~cm}^{-1}$; HRMS (ESI) calcd for $\mathrm{C}_{8} \mathrm{H}_{6} \mathrm{NO}_{2} \mathrm{Br}\left(\mathrm{M}+\mathrm{Na}^{+}\right)$249.9474; found 249.9473.

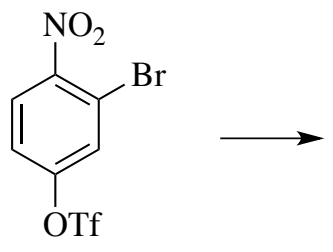

167<smiles>C=Cc1cc(OCC)ccc1[N+](=O)[O-]</smiles>

171

2-Ethenyl-4-trifluoromethanesulfonyloxy-nitrobenzene (171): Treatment of $\mathbf{1 6 7}$ (67.6 $\mathrm{mg}, 0.19 \mathrm{mmol}$ ) with ethenyl(tributyl)stannane (72.8 mg, $0.23 \mathrm{mmol}), \mathrm{PPh}_{3}(4.5 \mathrm{mg}, 0.02$ $\mathrm{mmol})$ and $\mathrm{Pd}(\mathrm{dba})_{2}(2.2 \mathrm{mg}, 0.004 \mathrm{mmol})$ in dioxane $(1.0 \mathrm{~mL})$, as described for 141, gave after chromatography (hexanes/EtOAc, 97:3) 171 as a colorless oil (47.3 mg, 0.16 mmol, 82\%). ${ }^{1} \mathrm{H}$ NMR $\delta 8.06(\mathrm{~d}, J=9.0 \mathrm{~Hz}, 1 \mathrm{H}), 7.52(\mathrm{~d}, J=2.4 \mathrm{~Hz}, 1 \mathrm{H}), 7.34(\mathrm{dd}, J=$ 9.0,3.0 Hz, 1H), $7.18(\mathrm{dd}, J=16.8,10.8 \mathrm{~Hz}, 1 \mathrm{H}), 5.81(\mathrm{~d}, J=17.4 \mathrm{~Hz}, 1 \mathrm{H}), 5.63(\mathrm{~d}, J=$ $10.8 \mathrm{~Hz}, 1 \mathrm{H}) ;{ }^{13} \mathrm{C}$ NMR $\delta 151.7,146.6,136.4,131.1,127.0,121.4,121.3,121.1,118.7$ (q, $J=319 \mathrm{~Hz}, \mathrm{C}-\mathrm{F})$; IR (ATR) 3118, 1530, 1424, 1350, 1207, $1131 \mathrm{~cm}^{-1}$; HRMS (ESI) calcd for $\mathrm{C}_{9} \mathrm{H}_{6} \mathrm{NO}_{5} \mathrm{~F}_{3} \mathrm{~S}\left(\mathrm{M}+\mathrm{Na}^{+}\right) 319.9811$; found 319.9809 .<smiles>C=Cc1ccc([N+](=O)[O-])c(Br)c1</smiles>

2-Bromo-4-ethenyl-nitrobenzene (172): Treatment of 167 (75.2 $\mathrm{mg}, 0.22 \mathrm{mmol})$ with ethenyl(tributyl)stannane $(85.5 \mathrm{mg}, 0.27 \mathrm{mmol}), \mathrm{LiCl}(28.1 \mathrm{mg}, 0.66 \mathrm{mmol})$ and 
$\mathrm{Pd}\left(\mathrm{PPh}_{3}\right)_{2} \mathrm{Cl}_{2}(3.6 \mathrm{mg}, 0.005 \mathrm{mmol})$ in $\mathrm{DMF}(1.0 \mathrm{~mL})$, as described for 170, gave after chromatography (hexanes/EtOAc, 97:3) a mixture of $\mathbf{1 7 2}$ and $\mathbf{1 7 1}$ (calculated from ${ }^{1} \mathrm{H}$ NMR spectrum: $18.0 \mathrm{mg}$ of $\mathbf{1 7 2}, 0.08 \mathrm{mmol}, 37 \%, 5.8 \mathrm{mg}$ of $\mathbf{1 7 1}, 9 \%$ ) as a yellow oil. ${ }^{1} \mathrm{H}$ NMR $\delta 7.86(\mathrm{~d}, J=7.8 \mathrm{~Hz}, 1 \mathrm{H}), 7.74(\mathrm{~d}, J=1.2 \mathrm{~Hz}, 1 \mathrm{H}), 7.45(\mathrm{dd}, J=8.4,1.8 \mathrm{~Hz}, 1 \mathrm{H})$, $6.69(\mathrm{dd}, J=18.0,11.4 \mathrm{~Hz}, 1 \mathrm{H}), 5.90,(\mathrm{~d}, J=17.4 \mathrm{~Hz}, 1 \mathrm{H}), 5.52(\mathrm{~d}, J=10.8 \mathrm{~Hz}, 1 \mathrm{H}) ;{ }^{13} \mathrm{C}$ NMR $\delta$ 142.9, 136.4, 133.7, 132.6, 126.1, 125.5, 119.3, 115.1; IR (ATR) 3095, 1573, $1526,1346,1217,1139 \mathrm{~cm}^{1}$; HRMS (ESI) calcd for $\mathrm{C}_{8} \mathrm{H}_{6} \mathrm{NO}_{2} \mathrm{Br}\left(\mathrm{M}+\mathrm{Na}^{+}\right)$249.9474; found 249.9474 .<smiles>C=Cc1ccc(OCC)cc1[N+](=O)[O-]</smiles>

2-Ethenyl-5-trifluoromethanesulfonyloxy-nitrobenzene (173): Treatment of 163 (110 $\mathrm{mg}, 0.32 \mathrm{mmol}$ ) with ethenyl(tributyl)stannane (134 mg, $0.42 \mathrm{mmol}), \mathrm{PPh}_{3}(6.9 \mathrm{mg}, 0.03$ $\mathrm{mmol})$ and $\mathrm{Pd}(\mathrm{dba})_{2}(3.6 \mathrm{mg}, 0.006 \mathrm{mmol})$ in dioxane $(1.5 \mathrm{~mL})$, as described for 11, gave after chromatography (hexanes/EtOAc, 97:3) $15(63.3 \mathrm{mg}, 0.21 \mathrm{mmol}, 68 \%)$ as a colorless oil. ${ }^{1} \mathrm{H}$ NMR $\delta 7.90(\mathrm{~d}, J=2.4 \mathrm{~Hz}, 1 \mathrm{H}), 7.74(\mathrm{~d}, J=9.0 \mathrm{~Hz}, 1 \mathrm{H}), 7.53(\mathrm{dd}, J=$ 9.0, 2.4 Hz, 1H), $7.18(\mathrm{dd}, J=17.4,10.8 \mathrm{~Hz}, 1 \mathrm{H}), 5.79(\mathrm{~d}, J=16.8 \mathrm{~Hz}, 1 \mathrm{H}), 5.61(\mathrm{~d}, J=$ $10.8 \mathrm{~Hz}, 1 \mathrm{H}) ;{ }^{13} \mathrm{C}$ NMR $\delta 148,147.7,133.8,131.1,130.5,126.2,121,118.6$ (q, $J=319$ Hz, C-F), 118; IR (ATR) 3110, 1533, 1426, 1351, 1208, $1133 \mathrm{~cm}^{-1}$; HRMS (ESI) calcd for $\mathrm{C}_{9} \mathrm{H}_{6} \mathrm{NO}_{5} \mathrm{~F}_{3} \mathrm{~S}\left(\mathrm{M}+\mathrm{Na}^{+}\right) 319.9811$; found 319.9810 .<smiles>C=Cc1ccc(Br)c([N+](=O)[O-])c1</smiles> 
2-Bromo-5-ethenyl-nitrobenzene (174): ${ }^{151}$ Treatment of $\mathbf{1 6 3}$ (120 mg, $\left.0.34 \mathrm{mmol}\right)$ with ethenyl(tributyl)stannane (135 mg, $0.43 \mathrm{mmol}), \mathrm{LiCl}(48.2 \mathrm{mg}, 1.13 \mathrm{mmol})$ and $\mathrm{Pd}\left(\mathrm{PPh}_{3}\right)_{2} \mathrm{Cl}_{2}(4.8 \mathrm{mg}, 0.007 \mathrm{mmol})$ in DMF $(1.5 \mathrm{~mL})$, as described for 170, gave after chromatography (hexanes/EtOAc, 97:3) in order of elution, 174 (27.8 mg, $0.12 \mathrm{mmol}$, $36 \%$ ) as a colorless oil and a mixture of $\mathbf{1 7 3}$ and $\mathbf{1 6 3}$ (calculated from ${ }^{1} \mathrm{H}$ NMR spectrum $22 \mathrm{mg}, 23 \%$ and $20 \mathrm{mg}, 17 \%$ ). Spectral data were in accordance with literature values.

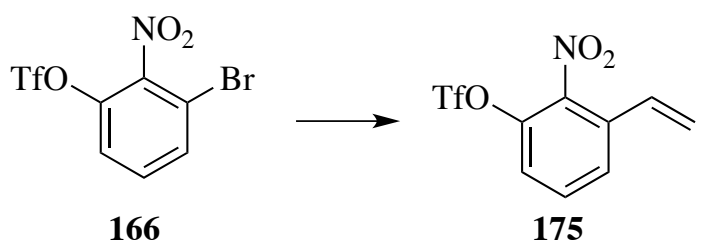

2-Ethenyl-6-trifluoromethanesulfonyloxy-nitrobenzene (175): Treatment of 166 (104 $\mathrm{mg}, 0.30 \mathrm{mmol})$ with ethenyl(tributyl)stannane (121 mg, $0.38 \mathrm{mmol}), \mathrm{PPh}_{3}(6.7 \mathrm{mg}, 0.03$ $\mathrm{mmol})$ and $\mathrm{Pd}(\mathrm{dba})_{2}(3.7 \mathrm{mg}, 0.006 \mathrm{mmol})$ in dioxane $(1.5 \mathrm{~mL})$ as described for $\mathbf{1 4 1}$, gave after chromatography (hexanes/EtOAc, 97:3) in order of elution, 176 (2.3 mg, 0.01 mmol, 3\%), and $\mathbf{1 7 5}(52.9 \mathrm{mg}, 0.18 \mathrm{mmol}, 60 \%)$ as a yellow solid. Spectral data for 175: $\mathrm{mp}=38-39{ }^{\circ} \mathrm{C} ;{ }^{1} \mathrm{H}$ NMR $\delta 7.67(\mathrm{~d}, J=8.4 \mathrm{~Hz}, 1 \mathrm{H}), 7.57(\mathrm{t}, J=8.4 \mathrm{~Hz}, 1 \mathrm{H}), 7.41(\mathrm{dd}, J=$ 8.4, 1.2 Hz, 1H), $6.68(\mathrm{dd}, J=17.4,10.8 \mathrm{~Hz}, 1 \mathrm{H}), 5.91(\mathrm{~d}, J=17.4 \mathrm{~Hz}, 1 \mathrm{H}), 5.61(\mathrm{~d}, J=$ 11.4 Hz, 1H); ${ }^{13} \mathrm{C}$ NMR $\delta 142.0,140.2,133.2,131.6,128.6,126.4,121.9,121.4,118.4$ (q, $J=319 \mathrm{~Hz}, \mathrm{C}-\mathrm{F}) ;$ IR (ATR) 3090, 1533, 1427, 1361, 1211, $1138 \mathrm{~cm}^{-1}$. HRMS (ESI) calcd for $\mathrm{C}_{9} \mathrm{H}_{6} \mathrm{NO}_{5} \mathrm{~F}_{3} \mathrm{~S}\left(\mathrm{M}+\mathrm{Na}^{+}\right)$319.9811; found 319.9809 .<smiles>CCC=Cc1cccc(Br)c1[N+](=O)[O-]</smiles>

2-Bromo-6-ethenyl-nitrobenzene (176): Treatment of $166(119 \mathrm{mg}, 0.34 \mathrm{mmol})$ with ethenyl(tributyl)stannane (138 mg, $0.44 \mathrm{mmol}), \mathrm{LiCl}(45.6 \mathrm{mg}, 1.08 \mathrm{mmol})$ and 
$\mathrm{Pd}\left(\mathrm{PPh}_{3}\right)_{2} \mathrm{Cl}_{2}(5.0 \mathrm{mg}, 0.007 \mathrm{mmol})$ in DMF $(1.5 \mathrm{~mL})$ as described for $\mathbf{1 7 0}$ gave after chromatography (hexanes/EtOAc, 97:3) $\mathbf{1 7 6}$ as an off-white solid (47.1 mg, $0.21 \mathrm{mmol}$, $61 \%)\left(\mathrm{mp}=42-44^{\circ} \mathrm{C}\right) .{ }^{1} \mathrm{H}$ NMR $\delta 7.57(\mathrm{~d}, J=7.8 \mathrm{~Hz}, 1 \mathrm{H}), 7.56(\mathrm{~d}, J=7.8 \mathrm{~Hz}, 1 \mathrm{H}), 7.33(\mathrm{t}$, $J=7.8 \mathrm{~Hz}, 1 \mathrm{H}), 6.57(\mathrm{dd}, J=16.8,11.4 \mathrm{~Hz}, 1 \mathrm{H}), 5.85(\mathrm{~d}, J=16.8 \mathrm{~Hz}, 1 \mathrm{H}), 5.51(\mathrm{~d}, J=10.8$ $\mathrm{Hz}, 1 \mathrm{H}) ;{ }^{13} \mathrm{C}$ NMR $\delta 150.1,132.6,131.6,130.9,129,125.6,120.8,112.9 ;$ IR (ATR) $3077,1557,1521,1460,1365,1187 \mathrm{~cm}^{1}$. HRMS (ESI) calcd for $\mathrm{C}_{8} \mathrm{H}_{6} \mathrm{NO}_{2} \mathrm{Br}\left(\mathrm{M}+\mathrm{Na}^{+}\right)$ 251.9454; found 251.9454 .<smiles>C=Cc1cccc([N+](=O)[O-])c1OCC</smiles>

3-Ethenyl-2-trifluoromethanesulfonyloxy-nitrobenzene (177): Treatment of 151 (135 $\mathrm{mg}, 0.39 \mathrm{mmol})$ with ethenyl(tributyl)stannane (129 mg, $0.41 \mathrm{mmol}), \mathrm{PPh}_{3}(8.2 \mathrm{mg}, 0.03$ $\mathrm{mmol})$ and $\mathrm{Pd}(\mathrm{dba})_{2}(4.5 \mathrm{mg}, 0.008 \mathrm{mmol})$ in dioxane $(2 \mathrm{~mL})$ before adding as described for 141 gave after chromatography (hexanes/EtOAc, 97:3) in order of elution, $\mathbf{4 6}$ and dicoupled (8.5 mg, 1:1 mixture, $5 \mathrm{mg}$ Tf-coupled, $3.5 \mathrm{mg}$ dicoupled), $\mathbf{1 4 9}^{92}$ (22.7 mg mixed with dba), and a mixture of 151 and $177(95.1 \mathrm{mg})$. The latter fraction was rechromatographed (hexanes/EtOAc, 97:3) to give in order of elution, 177 as a colorless oil (27.8 mg, $0.09 \mathrm{mmol}, 24 \%)$ and $151(12.3 \mathrm{mg}, 0.04 \mathrm{mmol}, 9 \%)$. Analytical data for 177: ${ }^{1} \mathrm{H}$ NMR $\delta 7.98(\mathrm{dd}, J=8.4,1.8 \mathrm{~Hz}, 1 \mathrm{H}), 7.91(\mathrm{dd}, J=8.4,1.8 \mathrm{~Hz}, 1 \mathrm{H}), 7.48(\mathrm{t}, J=8.4$ $\mathrm{Hz}, 1 \mathrm{H}), 6.99(\mathrm{dd}, J=17.4,10.8 \mathrm{~Hz}, 1 \mathrm{H}), 5.95(\mathrm{~d}, J=17.4 \mathrm{~Hz}, 1 \mathrm{H}), 5.67$ (d, $J=10.8 \mathrm{~Hz}$, $1 \mathrm{H}) ;{ }^{13} \mathrm{C}$ NMR $\delta 143.0,137.8,134.4,132,128.5,128.0,125.5,121.4,118.3$ (q, $J=319$ Hz, C-F); IR (ATR) 3103, 1539, 1429, 1351, 1210, $1131 \mathrm{~cm}^{-1}$; HRMS (ESI) calcd for $\mathrm{C}_{9} \mathrm{H}_{6} \mathrm{NO}_{5} \mathrm{~F}_{3} \mathrm{~S}\left(\mathrm{M}+\mathrm{Na}^{+}\right) 319.9811$; found 319.9808 . 


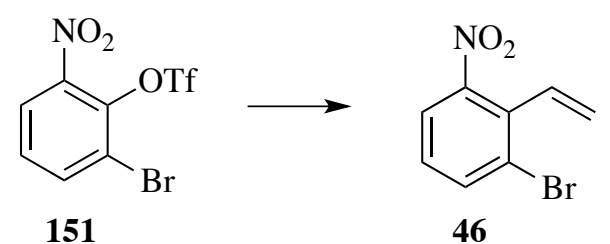

3-Bromo-2-ethenyl-nitrobenzene (46): ${ }^{36}$ Treatment of $\mathbf{1 5 1}(99.8 \mathrm{mg}, 0.29 \mathrm{mmol})$ with ethenyl(tributyl)stannane (97.5 $\mathrm{mg}, 0.31 \mathrm{mmol}), \mathrm{LiCl}(36.6 \mathrm{mg}, 0.86 \mathrm{mmol})$ and $\mathrm{Pd}\left(\mathrm{PPh}_{3}\right)_{2} \mathrm{Cl}_{2}(4.1 \mathrm{mg}, 0.006 \mathrm{mmol})$ in DMF $(1.5 \mathrm{~mL})$ as described for $\mathbf{1 7 0}$ afforded after chromatography (hexanes/EtOAc, 9:1) in order of elution, $\mathbf{4 6}$ as a yellow oil (28.3 mg, $0.12 \mathrm{mmol}, 43 \%), \mathbf{1 4 9} 9^{92}$ (7.4 mg, $\left.0.03 \mathrm{mmol}, 12 \%\right)$, and 151 (5.0 mg, $\left.0.01 \mathrm{mmol}, 5 \%\right)$. Spectral data were in accordance with literature values.

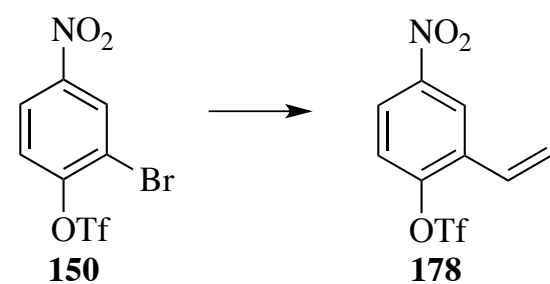

3-Ethenyl-4-trifluoromethanesulfonyloxy-nitrobenzene (178): Treatment of $\mathbf{1 5 0}$ (102 $\mathrm{mg}, 0.29 \mathrm{mmol}$ ) with ethenyl(tributyl)stannane (116 mg, $0.37 \mathrm{mmol}), \mathrm{PPh}_{3}(6.5 \mathrm{mg}, 0.03$ $\mathrm{mmol})$ and $\mathrm{Pd}(\mathrm{dba})_{2}(4.1 \mathrm{mg}, 0.007 \mathrm{mmol})$ in dioxane $(1.5 \mathrm{~mL})$ as described for $\mathbf{1 4 1}$ gave after chromatography (hexanes/EtOAc, 97:3) order of elution, 178 (19.3 mg, $0.06 \mathrm{mmol}$, $22 \%)$ as a light pink oil, $\mathbf{1 5 0}(7.0 \mathrm{mg}, 0.02 \mathrm{mmol}, 7 \%)$, and $148^{93}(5.2 \mathrm{mg}, 0.02 \mathrm{mmol}$, 7\%). Analytical data for 178: ${ }^{1} \mathrm{H}$ NMR $\delta 8.53(\mathrm{~d}, J=3.0 \mathrm{~Hz}, 1 \mathrm{H}), 8.21(\mathrm{dd}, J=9.0,2.4$ $\mathrm{Hz}, 1 \mathrm{H}), 7.48(\mathrm{~d}, J=9.0 \mathrm{~Hz}, 1 \mathrm{H}), 6.94(\mathrm{dd}, J=17.4,11.4 \mathrm{~Hz}, 1 \mathrm{H}), 6.04(\mathrm{~d}, J=17.4 \mathrm{~Hz}$, $1 \mathrm{H}), 5.70(\mathrm{~d}, J=11.4 \mathrm{~Hz}, 1 \mathrm{H}) ;{ }^{13} \mathrm{C} \delta 149.9,147.2,132.7,127.3,124.0,122.9,122.7$, 121.7, 118.5 (q, $J=319 \mathrm{~Hz}, \mathrm{C}-\mathrm{F})$; IR (ATR) 3107, 1536, 1424, 1347, 1209, $1134 \mathrm{~cm}^{-1}$; HRMS (ESI) calcd for $\mathrm{C}_{9} \mathrm{H}_{6} \mathrm{NO}_{5} \mathrm{~F}_{3} \mathrm{~S}\left(\mathrm{M}+\mathrm{Na}^{+}\right)$319.9811; found 319.9809 . 


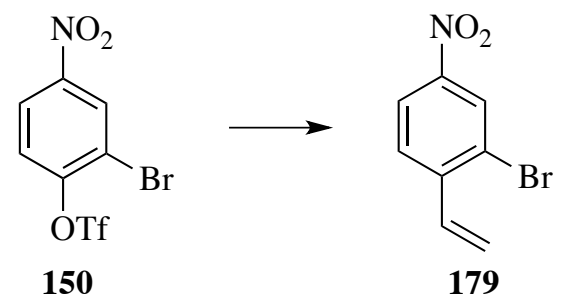

3-Bromo-4-ethenyl-nitrobenzene (179): Treatment of $\mathbf{1 5 0}(110 \mathrm{mg}, 0.32 \mathrm{mmol})$ with ethenyl(tributyl)stannane (138 mg, $0.43 \mathrm{mmol}), \mathrm{LiCl}(42.6 \mathrm{mg}, 1.0 \mathrm{mmol})$ and $\mathrm{Pd}\left(\mathrm{PPh}_{3}\right)_{2} \mathrm{Cl}_{2}(4.5 \mathrm{mg}, 0.006 \mathrm{mmol})$ in DMF $(1.5 \mathrm{~mL})$ as described for $\mathbf{1 7 0}$ gave after chromatography (hexanes/EtOAc, 97:3) in order of elution, 179 (40.7 mg, $0.18 \mathrm{mmol}$, 57\%) as a yellow oil, 150 (8.7 mg, $0.02 \mathrm{mmol}, 8 \%)$, and 148 (11.1 mg, $0.05 \mathrm{mmol}, 16 \%)$.

Analytical data for 179: ${ }^{1} \mathrm{H}$ NMR $\delta 8.43(\mathrm{~d}, J=2.4 \mathrm{~Hz}, 1 \mathrm{H}), 8.14(\mathrm{ddd}, J=8.4,2.4,0.6 \mathrm{~Hz}$, 1H), $7.69(\mathrm{~d}, J=9.0 \mathrm{~Hz}, 1 \mathrm{H}), 7.09(\mathrm{dd}, J=17.4,11.4 \mathrm{~Hz}, 1 \mathrm{H}), 5.88(\mathrm{~d}, J=18.0 \mathrm{~Hz}, 1 \mathrm{H})$, $5.60(\mathrm{dd}, J=10.8,0.6 \mathrm{~Hz}, 1 \mathrm{H}) ;{ }^{13} \mathrm{C}$ NMR $\delta 147.2,143.7,134.3,128.2,127.1,123.4$, 122.4, 120.9; IR (ATR) 3099, 1520, 1342, 1116, $1035 \mathrm{~cm}^{-1}$; HRMS (ESI) calcd for $\mathrm{C}_{8} \mathrm{H}_{6} \mathrm{NO}_{2} \mathrm{Br}\left(\mathrm{M}+\mathrm{Na}^{+}\right)$251.9454; found 251.9456.<smiles>O=[N+]([O-])c1cc(O)cc(Br)c1</smiles>

157

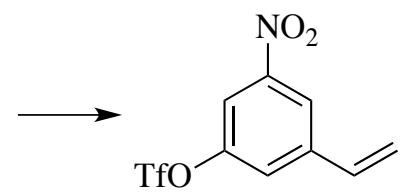

180

3-Ethenyl-5-trifluoromethanesulfonyloxy-nitrobenzene (180): Treatment of 157 (106 $\mathrm{mg}, 0.30 \mathrm{mmol}$ ) with ethenyl(tributyl)stannane (130 mg, $0.41 \mathrm{mmol}), \mathrm{PPh}_{3}(6.4 \mathrm{mg}, 0.02$ $\mathrm{mmol})$ and $\mathrm{Pd}(\mathrm{dba})_{2}(3.4 \mathrm{mg}, 0.006 \mathrm{mmol})$ in dioxane $(1.5 \mathrm{~mL})$ as described for 141 gave after chromatography (hexanes/EtOAc, 97:3) in order of elution, 157 (4.1 mg, 0.01 mmol, 4\%) and $180(70.0 \mathrm{mg}, 0.24 \mathrm{mmol}, 78 \%)$ as a colorless oil. ${ }^{1} \mathrm{H}$ NMR $\delta 8.30(\mathrm{t}, J=$ $1.8 \mathrm{~Hz}, 1 \mathrm{H}), 8.02(\mathrm{t}, J=1.8 \mathrm{~Hz}, 1 \mathrm{H}), 7.61(\mathrm{t}, J=1.8 \mathrm{~Hz}, 1 \mathrm{H}), 6.78(\mathrm{dd}, J=17.4,10.8 \mathrm{~Hz}$, $1 \mathrm{H}), 5.98(\mathrm{~d}, J=17.4 \mathrm{~Hz}, 1 \mathrm{H}), 5.61(\mathrm{~d}, J=10.8 \mathrm{~Hz}, 1 \mathrm{H}) ;{ }^{13} \mathrm{C}$ 
NMR $\delta$ 149.4, 149.2, 141.7, 133.2, 124.6, 120.6, 119.8, 118.6 (q, $J=319 \mathrm{~Hz}, \mathrm{C}-$

F), 115.6; IR (ATR) 3103, 1540, 1425, 1348, 1213, $1132 \mathrm{~cm}^{-1}$; HRMS (ESI) calcd for $\mathrm{C}_{9} \mathrm{H}_{6} \mathrm{NO}_{5} \mathrm{~F}_{3} \mathrm{~S}\left(\mathrm{M}+\mathrm{Na}^{+}\right)$319.9811; found 319.9813 .

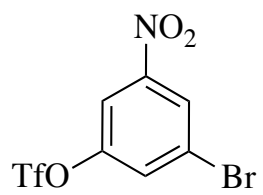

157

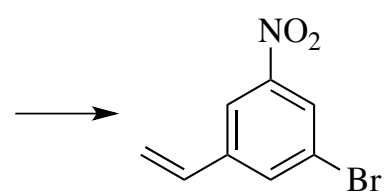

181

3-Bromo-5-ethenyl-nitrobenzene (181): Treatment of 157 (109 mg, $0.31 \mathrm{mmol})$ with ethenyl(tributyl)stannane (139 mg, $0.44 \mathrm{mmol}), \mathrm{LiCl}(45.0 \mathrm{mg}, 1.1 \mathrm{mmol})$ and $\mathrm{Pd}\left(\mathrm{PPh}_{3}\right)_{2} \mathrm{Cl}_{2}(4.7 \mathrm{mg}, 0.007 \mathrm{mmol})$ in DMF $(1.5 \mathrm{~mL})$ as described for $\mathbf{1 7 0}$ gave after chromatography (hexanes/EtOAc, 97:3) $\mathbf{1 8 1}(47.1 \mathrm{mg}, 0.21 \mathrm{mmol}, 66 \%)$ as an off-white solid. mp=37-39 ${ }^{\circ} \mathrm{C} ;{ }^{1} \mathrm{H}$ NMR $\delta 8.23(\mathrm{t}, J=1.8 \mathrm{~Hz}, 1 \mathrm{H}), 8.17(\mathrm{t}, J=1.8 \mathrm{~Hz}, 1 \mathrm{H}), 7.83(\mathrm{t}$, $J=1.8 \mathrm{~Hz}, 1 \mathrm{H}), 6.71(\mathrm{dd}, J=17.4,10.8 \mathrm{~Hz}, 1 \mathrm{H}), 5.91(\mathrm{~d}, J=17.4 \mathrm{~Hz}, 1 \mathrm{H}), 5.50(\mathrm{~d}, J=11.4$ $\mathrm{Hz}, 1 \mathrm{H}) ;{ }^{13} \mathrm{C}$ NMR $\delta 149,140.8,134.8,133.6,125.3,122.9,119.6,118.5 ;$ IR (ATR) $3079,1531,1339,1301,1214 \mathrm{~cm}^{1}$; HRMS (ESI) calcd for $\mathrm{C}_{8} \mathrm{H}_{6} \mathrm{NO}_{2} \mathrm{Br}\left(\mathrm{M}+\mathrm{Na}^{+}\right)$ 251.9454 ; found 251.9452 .

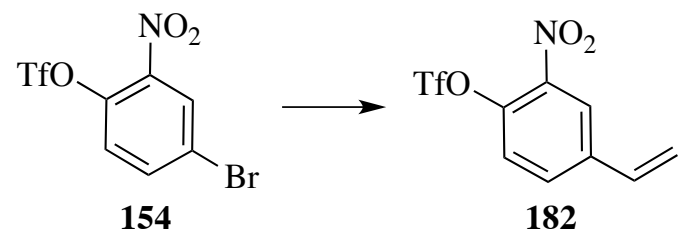

3-Ethenyl-6-trifluoromethanesulfonyloxy-nitrobenzene (182): Treatment of 154 (103 $\mathrm{mg}, 0.29 \mathrm{mmol})$ with ethenyl(tributyl)stannane (112.4 mg, $0.35 \mathrm{mmol}), \mathrm{PPh}_{3}(6.8 \mathrm{mg}$, $0.03 \mathrm{mmol})$ and $\mathrm{Pd}(\mathrm{dba})_{2}(3.4 \mathrm{mg}, 0.006 \mathrm{mmol})$ in dioxane $(1.5 \mathrm{~mL})$ as described for $\mathbf{1 4 1}$ gave after chromatography (hexanes/EtOAc, 9:1) in order of elution, 154 (10\%) followed by $\mathbf{1 8 2}$ as a yellow oil mixed with dibenzylideneacetone ( $34.6 \mathrm{mg}, 0.12 \mathrm{mmol}, 44 \%$ based on ${ }^{1} \mathrm{H}$ NMR integration). Spectral data from the mixture of $\mathbf{1 8 2}$ and dba: ${ }^{1} \mathrm{H}$ NMR $\delta$ 
$8.15(\mathrm{~d}, J=2.4 \mathrm{~Hz}, 1 \mathrm{H}), 7.72(\mathrm{dd}, J=9.0,2.4 \mathrm{~Hz}, 1 \mathrm{H}), 7.41(\mathrm{~d}, J=9.0 \mathrm{~Hz}, 1 \mathrm{H}), 6.75(\mathrm{dd}$, $J=17.4,10.8 \mathrm{~Hz}, 1 \mathrm{H}), 5.92(\mathrm{~d}, J=17.4 \mathrm{~Hz}, 1 \mathrm{H}), 5.55(\mathrm{~d}, J=10.8 \mathrm{~Hz}, 1 \mathrm{H}) ;{ }^{13} \mathrm{C}$ NMR $\delta$ 141.7, 140.3, 139.2, 133.1, 132.2, 124.3, 123.8, 119.2, 118.5 (q, $J=319 \mathrm{~Hz}, \mathrm{C}-\mathrm{F})$; IR (ATR) $3094,1541,1429,1346,1205,1134 \mathrm{~cm}^{-1}$; HRMS (ESI) calcd for $\mathrm{C}_{9} \mathrm{H}_{6} \mathrm{NO}_{5} \mathrm{~F}_{3} \mathrm{~S}$ $\left(\mathrm{M}+\mathrm{Na}^{+}\right)$319.9811; found 319.9812.

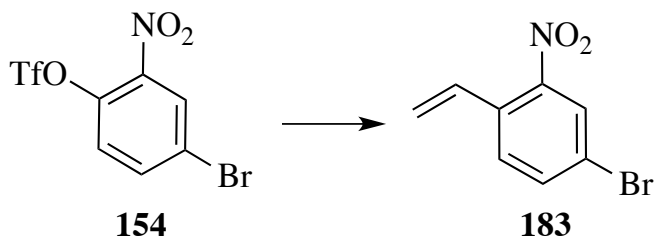

5-Bromo-2-ethenyl-nitrobenzene (183): Treatment of $154(111 \mathrm{mg}, 0.32 \mathrm{mmol})$ with ethenyl(tributyl)stannane (131 mg, $0.41 \mathrm{mmol}), \mathrm{LiCl}(44.5 \mathrm{mg}, 0.86 \mathrm{mmol})$ and $\mathrm{Pd}\left(\mathrm{PPh}_{3}\right)_{2} \mathrm{Cl}_{2}(4.2 \mathrm{mg}, 0.006 \mathrm{mmol})$ in DMF $(1.5 \mathrm{~mL})$ as described for $\mathbf{1 7 0}$ gave after chromatography (hexanes/EtOAc, 9:1) $\mathbf{1 8 3}$ as a yellow solid (56.3 $\mathrm{mg}, 0.25 \mathrm{mmol}, 78 \%$ ). $\mathrm{mp}=40-41^{\circ} \mathrm{C} ;{ }^{1} \mathrm{H}$ NMR $\delta 8.07(\mathrm{~d}, J=2.4 \mathrm{~Hz}, 1 \mathrm{H}), 7.70(\mathrm{dd}, J=8.4,2.4 \mathrm{~Hz}, 1 \mathrm{H}), 7.51(\mathrm{~d}$, $J=8.4 \mathrm{~Hz}, 1 \mathrm{H}), 7.11(\mathrm{dd}, J=17.4,11.4 \mathrm{~Hz}, 1 \mathrm{H}), 5.76(\mathrm{~d}, J=17.4 \mathrm{~Hz}, 1 \mathrm{H}), 5.55(\mathrm{~d}, J=11.4$ $\mathrm{Hz}, 1 \mathrm{H}) ;{ }^{13} \mathrm{C}$ NMR $\delta 148.0,136.1,132.2,131.5,129.7,127.3,121.4,119.7$; IR (ATR) $3097,1552,1514,1341,1149 \mathrm{~cm}^{-1}$; HRMS (ESI) calcd for $\mathrm{C}_{8} \mathrm{H}_{6} \mathrm{NO}_{2} \mathrm{Br}\left(\mathrm{M}+\mathrm{Na}^{+}\right)$ 251.9454; found 251.9455.<smiles>C=Cc1ccc([N+](=O)[O-])c(OCC)c1</smiles>
4-Ethenyl-2-trifluoromethanesulfonyloxy-nitrobenzene (184): Treatment of $\mathbf{1 6 8}^{52}$ (104 $\mathrm{mg}, 0.29 \mathrm{mmol}$ ) with ethenyl(tributyl)stannane (121 mg, $0.38 \mathrm{mmol}), \mathrm{PPh}_{3}(6.8 \mathrm{mg}, 0.03$ $\mathrm{mmol})$ and $\mathrm{Pd}(\mathrm{dba})_{2}(3.4 \mathrm{mg}, 0.006 \mathrm{mmol})$ in dioxane $(1.5 \mathrm{~mL})$ as described for 141 gave 
after chromatography (hexanes/EtOAc, 97:3) a mixture of $\mathbf{1 8 4}$ and dba (62.3 mg, calculated $55.9 \mathrm{mg}, 64 \% 184$ and $6.6 \mathrm{mg}$ dba) as a yellow oil. Analytical data for $184:{ }^{1} \mathrm{H}$ NMR $\delta 8.16(\mathrm{~d}, J=8.4 \mathrm{~Hz}, 1 \mathrm{H}), 7.55(\mathrm{dd}, J=8.4,1.8 \mathrm{~Hz}, 1 \mathrm{H}), 7.41(\mathrm{~d}, J=1.8 \mathrm{~Hz}, 1 \mathrm{H})$, $6.76(\mathrm{dd}, J=17.4,10.8 \mathrm{~Hz}, 1 \mathrm{H}), 5.97(\mathrm{~d}, J=17.4 \mathrm{~Hz}, 1 \mathrm{H}), 5.63(\mathrm{~d}, J=10.8 \mathrm{~Hz}, 1 \mathrm{H}) ;{ }^{13} \mathrm{C}$ NMR $\delta 145.2,141.9,140.1,133.4,127.1,126.2,121.4,120.9,118.6(q, J=319$ Hz, CF); IR (ATR) 3114, 1587, 1529, 1429, 1341, $1209 \mathrm{~cm}^{-1}$; HRMS (ESI) calcd for $\mathrm{C}_{9} \mathrm{H}_{6} \mathrm{NO}_{5} \mathrm{~F}_{3} \mathrm{~S}\left(\mathrm{M}+\mathrm{Na}^{+}\right) 319.9811$; found 319.9808 .

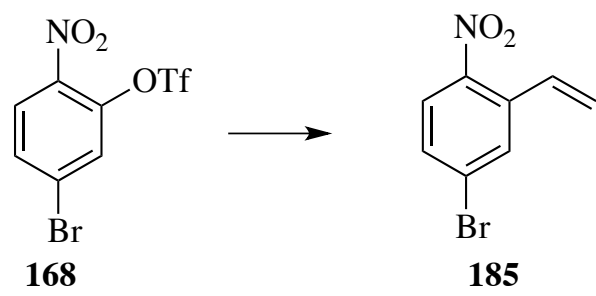

4-Bromo-2-ethenyl-nitrobenzene (185): ${ }^{152}$ Treatment of $\mathbf{1 6 8}(99.5 \mathrm{mg}, 0.29 \mathrm{mmol})$ with ethenyl(tributyl)stannane $(98.1 \mathrm{mg}, 0.31 \mathrm{mmol}), \mathrm{LiCl}(37.0 \mathrm{mg}, 0.87 \mathrm{mmol})$ and $\mathrm{Pd}\left(\mathrm{PPh}_{3}\right)_{2} \mathrm{Cl}_{2}(4.0 \mathrm{mg}, 0.006 \mathrm{mmol})$ in $\mathrm{DMF}(1.5 \mathrm{~mL})$ as described for $\mathbf{1 7 0}$ gave after chromatography (hexanes/EtOAc, 9:1) in order of elution, 185 (11.0 mg, $0.05 \mathrm{mmol}$, 17\%) as an off-white solid, 168 (22.9 mg, $0.07 \mathrm{mmol}, 22 \%)$ and 184 (19.4 mg, 0.07 mmol, 23\%). Spectral data for $\mathbf{1 8 5}$ were in accordance with literature values.<smiles>CC=Cc1ccc([N+](=O)[O-])cc1OCCC</smiles>

4-Ethenyl-3-trifluoromethanesulfonyloxy-nitrobenzene (186): Treatment of 159 (74.7 $\mathrm{mg}, 0.21 \mathrm{mmol}$ ) with ethenyl(tributyl)stannane (83.2 mg, $0.26 \mathrm{mmol}), \mathrm{PPh}_{3}(5.0 \mathrm{mg}, 0.02$ $\mathrm{mmol})$ and $\mathrm{Pd}(\mathrm{dba})_{2}(2.7 \mathrm{mg}, 0.005 \mathrm{mmol})$ in dioxane $(1.5 \mathrm{~mL})$ as described for $\mathbf{1 4 1}$ gave after chromatography (hexanes/EtOAc, 97:3) in order of elution, 159 (14.8 mg, 0.04 
mmol, 20\%) and $\mathbf{1 8 6}(30.2 \mathrm{mg}, 0.10 \mathrm{mmol}, 48 \%)$ as a yellow oil. Analytical data for 186: ${ }^{1} \mathrm{H}$ NMR $\delta 8.25(\mathrm{dd}, J=8.4,1.8 \mathrm{~Hz}, 1 \mathrm{H}), 8.17(\mathrm{~d}, J=1.8 \mathrm{~Hz}, 1 \mathrm{H}), 7.83(\mathrm{~d}, J=8.4 \mathrm{~Hz}$, 1H), $6.98(\mathrm{dd}, J=17.4,10.8 \mathrm{~Hz}, 1 \mathrm{H}), 6.05(\mathrm{~d}, J=17.4 \mathrm{~Hz}, 1 \mathrm{H}), 5.75(\mathrm{~d}, J=10.8 \mathrm{~Hz}, 1 \mathrm{H})$; ${ }^{13} \mathrm{C}$ NMR $\delta 147.4,146,137.5,127.9,127.6,123.3,122.9,118.5$ (q, J C-F=319 Hz, C-F), 117.8; IR (ATR) 3118, 1528, 1425, 1346, 1210, $1132 \mathrm{~cm}^{-1}$; HRMS (ESI) calcd for $\mathrm{C}_{9} \mathrm{H}_{6} \mathrm{NO}_{5} \mathrm{~F}_{3} \mathrm{~S}\left(\mathrm{M}+\mathrm{Na}^{+}\right)$319.9811; found 319.9809 .

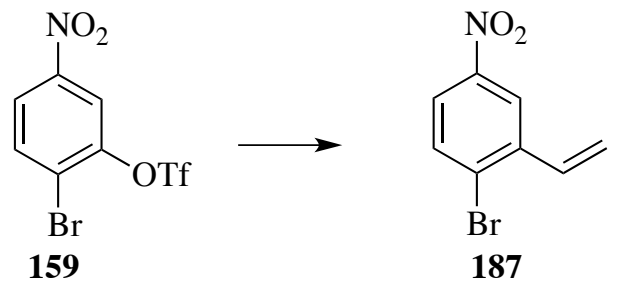

4-Bromo-3-ethenyl-nitrobenzene (187): Treatment of $\mathbf{1 5 9}(85.9 \mathrm{mg}, 0.25 \mathrm{mmol})$ with ethenyl(tributyl)stannane ( $98.2 \mathrm{mg}, 0.31 \mathrm{mmol}), \mathrm{LiCl}(32.2 \mathrm{mg}, 0.76 \mathrm{mmol})$ and $\mathrm{Pd}\left(\mathrm{PPh}_{3}\right)_{2} \mathrm{Cl}_{2}(3.6 \mathrm{mg}, 0.005 \mathrm{mmol})$ in DMF $(1.5 \mathrm{~mL})$ as described for $\mathbf{1 7 0}$ gave after chromatography (hexanes/EtOAc, 97:3) in order of elution, 187 (3.8 mg, $0.02 \mathrm{mmol}, 7 \%$ ) as an off-white solid, $\mathbf{1 5 9}(27.0 \mathrm{mg}, 0.08 \mathrm{mmol}, 31 \%)$ and $\mathbf{1 8 6}(22.4 \mathrm{mg}, 0.08 \mathrm{mmol}$, 31\%). Spectral data for 186: $\mathrm{mp}=38-40{ }^{\circ} \mathrm{C} ;{ }^{1} \mathrm{H}$ NMR $\delta 8.39(\mathrm{~d}, J=2.4 \mathrm{~Hz}, 1 \mathrm{H}), 7.97(\mathrm{dd}$, $J=8.4,2.4 \mathrm{~Hz}, 1 \mathrm{H}), 7.74(\mathrm{~d}, J=9.0 \mathrm{~Hz}, 1 \mathrm{H}), 7.06(\mathrm{dd}, J=17.4,10.8 \mathrm{~Hz}, 1 \mathrm{H}), 5.99$ (d, $J=17.4 \mathrm{~Hz}, 1 \mathrm{H}), 5.56(\mathrm{~d}, J=10.8 \mathrm{~Hz}, 1 \mathrm{H}) ;{ }^{13} \mathrm{C}$ NMR $\delta 143.5,139.1,134.2,133.9,131.4$, 123.1, 121.5, 119.7; IR (ATR) 3099, 2926, 1525, 1341, $1030 \mathrm{~cm}^{1}$; HRMS (ESI) calcd for $\mathrm{C}_{8} \mathrm{H}_{6} \mathrm{NO}_{2} \mathrm{Br}\left(\mathrm{M}+\mathrm{Na}^{+}\right)$251.9454; found 251.9452 .

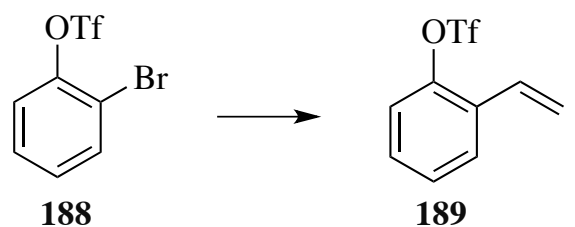


2-ethenyl-trifluoromethanesulfonyloxybenzene (189): Treatment of $\mathbf{1 8 8}$ (96.5 mg, 0.32 mmol) with ethenyl(tributyl)stannane (124 mg, $0.39 \mathrm{mmol}), \mathrm{PPh}_{3}(7.2 \mathrm{mg}, 0.03 \mathrm{mmol})$ and $\mathrm{Pd}(\mathrm{dba})_{2}(3.5 \mathrm{mg}, 0.006 \mathrm{mmol})$ in dioxane $(1 \mathrm{~mL})$ as described for 141 afforded after chromatography (hexanes/EtOAc, 97:3) a mixture of $\mathbf{1 8 9}$ and unreacted $\mathbf{1 8 8}(32.0 \mathrm{mg}$ ) (calculated from ${ }^{1} \mathrm{H}$ NMR spectrum $8.0 \mathrm{mg}, 10 \%$ and $24.0 \mathrm{mg}, 24 \%$ ) as a colorless oil. ${ }^{1} \mathrm{H}$ NMR d 7.65 (dd, $J=7.2,2.4 \mathrm{~Hz}, 1 \mathrm{H}), 7 .(\mathrm{dd}, J=8.4,1.8 \mathrm{~Hz}, 1 \mathrm{H}), 7.48(\mathrm{t}, J=8.4 \mathrm{~Hz}, 1 \mathrm{H})$, $6.93(\mathrm{dd}, J=17.4,11.4 \mathrm{~Hz}, 1 \mathrm{H}), 5.85(\mathrm{dd}, J=17.4,0.6 \mathrm{~Hz}, 1 \mathrm{H}), 5.49$ (d, $J=10.8,0.6 \mathrm{~Hz}$, 1H); ${ }^{13} \mathrm{C}$ NMR d 146.9, 131.1, 129.3, 128.9, 128.4, 127.3, 121.6, 118.6, 118.5 (q, $J=319.1 \mathrm{~Hz}, \mathrm{C}-\mathrm{F})$; IR (ATR) 2963, 1469, 1258, $1135,875 \mathrm{~cm}^{-1}$.<smiles>Brc1ccccc1Br</smiles>

188

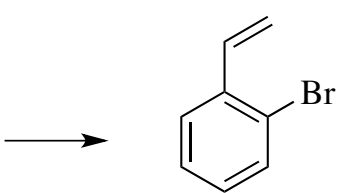

190

2-ethenyl-bromobenzene (190): Treatment of $\mathbf{1 8 8}(88.3 \mathrm{mg}, 0.25 \mathrm{mmol})$ with vinyl(tributyl)stannane (98.0 mg, $0.31 \mathrm{mmol}), \mathrm{LiCl}(32.2 \mathrm{mg}, 0.76 \mathrm{mmol})$ and $\mathrm{Pd}\left(\mathrm{PPh}_{3}\right)_{2} \mathrm{Cl}_{2}(3.8 \mathrm{mg}, 0.005 \mathrm{mmol})$ in $\mathrm{DMF}(1 \mathrm{~mL})$ as described for $\mathbf{1 7 0}$ afforded crude yellow oil (108 mg) which was purified by column chromatography (hexanes/ethyl acetate, 9:1), however, the product reacted on the silica gel so no product was isolated. Crude spectral data were in accordance with literature values with a 6:1 ratio of triflatecoupled 190 to bromine-coupled 189.<smiles>CCOc1cccc(Br)c1</smiles>

143<smiles>C=Cc1cccc(OCC)c1</smiles>

191

3-ethenyl-trifluoromethanesulfonyloxybenzene (191): Treatment of $\mathbf{1 4 3}$ (105 mg, 0.34 mmol) with vinyl(tributyl)stannane (127 mg, $0.40 \mathrm{mmol}), \mathrm{PPh}_{3}(7.6 \mathrm{mg}, 0.03 \mathrm{mmol})$, and 
$\operatorname{Pd}(\mathrm{dba})_{2}(4.0 \mathrm{mg}, 0.007 \mathrm{mmol})$ in dioxane $(1.5 \mathrm{~mL})$ as described for 141 afforded after chromatography (hexanes/ethyl acetate, 97:3) a mixture of 191 and unreacted 143 (49.2 $\mathrm{mg}$ ) (calculated from ${ }^{1} \mathrm{H}$ NMR spectrum $28.8 \mathrm{mg}, 33 \%$ and $20.2 \mathrm{mg}, 19 \%$ ) as a colorless oil. ${ }^{1} \mathrm{H}$ NMR d $7.40(\mathrm{~m}, 2 \mathrm{H}), 7.29(\mathrm{~d}, J=0.6 \mathrm{~Hz}, 1 \mathrm{H}), 7.15(\mathrm{dt}, J=7.2,1.8 \mathrm{~Hz}, 1 \mathrm{H}), 6.60$ (dd, $J=17.4,10.8 \mathrm{~Hz}, 1 \mathrm{H}), 5.80(\mathrm{~d}, J=18.0 \mathrm{~Hz}, 1 \mathrm{H}), 5.38(\mathrm{~d}, J=10.8 \mathrm{~Hz}, 1 \mathrm{H}) ;{ }^{13} \mathrm{C}$ NMR d 149.9, 140.3, 135.0, 130.3, 126.1, 120.2, 118.8, 118.7 (q, J=319.1 Hz, C-F), 116.5; IR (ATR) $3103,1539,1429,1351,1210,1131 \mathrm{~cm}^{-1}$.

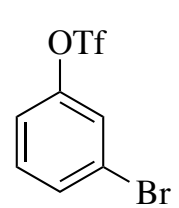

143

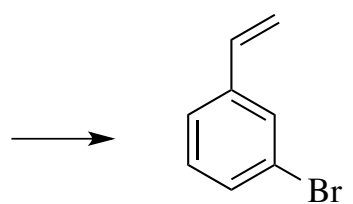

192

3-bromostyrene (192): Treatment of $\mathbf{1 4 3}$ (108 $\mathrm{mg}, 0.36 \mathrm{mmol})$ with vinyl(tributyl)stannane (141 mg, $0.44 \mathrm{mmol}), \mathrm{LiCl}(45.6 \mathrm{mg}, 1.08 \mathrm{mmol})$ and $\mathrm{Pd}\left(\mathrm{PPh}_{3}\right)_{2} \mathrm{Cl}_{2}(5.4 \mathrm{mg}, 0.008 \mathrm{mmol})$ in DMF $(1.5 \mathrm{~mL})$ as described for $\mathbf{1 7 0}$ afforded crude yellow oil (175 mg) which was purified by column chromatography (hexanes/ethyl acetate, 9:1), however, the product reacted on the silica gel so no product was isolated. Crude spectral data were in accordance with literature values with a 6:1 ratio of triflatecoupled 192 to bromine-coupled 191.

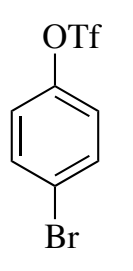

137

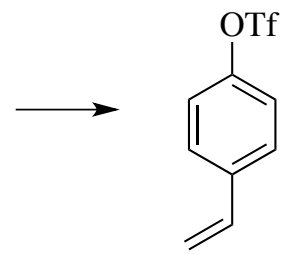

138

4-ethenyl-trifluoromethanesulfonyloxybenzene $(\mathbf{1 3 8})^{\mathbf{9 0}}$ : Treatment of $\mathbf{1 3 7}(102 \mathrm{mg}$, $0.34 \mathrm{mmol})$ with vinyl(tributyl)stannane (128 mg, $0.40 \mathrm{mmol}), \mathrm{PPh}_{3}(7.5 \mathrm{mg}, 0.03$ $\mathrm{mmol})$, and $\mathrm{Pd}(\mathrm{dba})_{2}(4.3 \mathrm{mg}, 0.007 \mathrm{mmol})$ in dioxane $(1.5 \mathrm{~mL})$ as described for $\mathbf{1 4 1}$ 
afforded after chromatography (hexanes/ethyl acetate, 97:3) 138 (25.2 mg, $0.10 \mathrm{mmol}$, $30 \%$ ) as a colorless oil. Spectral data were in accordance with literature values.

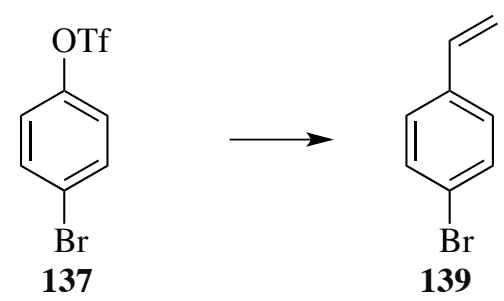

4-bromostyrene (139) ${ }^{\mathbf{9 0}}$ : Treatment of 137 (108 $\left.\mathrm{mg}, 0.35 \mathrm{mmol}\right)$ with vinyl(tributyl)stannane (141 mg, $0.44 \mathrm{mmol}), \mathrm{LiCl}(45.5 \mathrm{mg}, 1.07 \mathrm{mmol})$ and $\mathrm{Pd}\left(\mathrm{PPh}_{3}\right)_{2} \mathrm{Cl}_{2}(4.7 \mathrm{mg}, 0.007 \mathrm{mmol})$ in $\mathrm{DMF}(1.5 \mathrm{~mL})$ as described for $\mathbf{1 7 0}$ afforded crude yellow oil which was purified by column chromatography (hexanes/ethyl acetate, 9:1), however, the product reacted on the silica gel so no product was isolated. Crude spectral data were in accordance with literature values with a 6:1 ratio of triflate-coupled 139 to bromine-coupled 138.

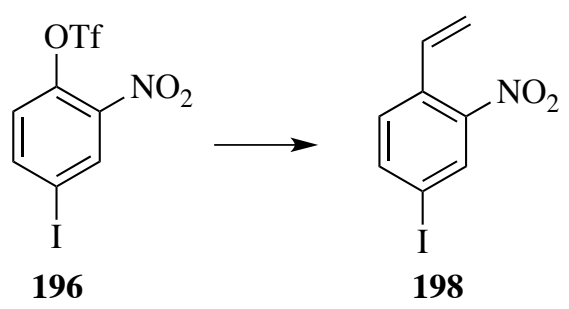

3-Iodo-6-ethenyl-nitrobenzene (198): Treatment of 196 (116 mg, $0.29 \mathrm{mmol})$ with ethenyl(tributyl)stannane (120 mg, $0.38 \mathrm{mmol}), \mathrm{LiCl}(39.7 \mathrm{mg}, 0.94 \mathrm{mmol})$ and $\mathrm{Pd}\left(\mathrm{PPh}_{3}\right)_{2} \mathrm{Cl}_{2}(4.1 \mathrm{mg}, 0.006 \mathrm{mmol})$ in $\mathrm{DMF}(1.5 \mathrm{~mL})$ as described for $\mathbf{1 7 0}$ gave after chromatography (hexanes/EtOAc, 97:3) in order of elution, 198 (3.2 mg, $0.01 \mathrm{mmol}, 4 \%)$ as a brown oil, $195(8.7 \mathrm{mg}, 0.03 \mathrm{mmol}, 14 \%)$, and a mixture of 196 and 197 (67.1 mg). Spectral data for 198: ${ }^{1} \mathrm{H}$ NMR $\delta 8.25(\mathrm{~d}, J=1.8 \mathrm{~Hz}, 1 \mathrm{H}), 7.89(\mathrm{dd}, J=7.8,1.8 \mathrm{~Hz}, 1 \mathrm{H})$, $7.35(\mathrm{~d}, J=7.8 \mathrm{~Hz}, 1 \mathrm{H}), 7.10(\mathrm{dd}, J=16.8,10.8 \mathrm{~Hz}, 1 \mathrm{H}), 5.77(\mathrm{~d}, J=16.8 \mathrm{~Hz}, 1 \mathrm{H}), 5.52$ (d, 
$J=10.8 \mathrm{~Hz}, 1 \mathrm{H}) ;{ }^{13} \mathrm{C}$ NMR $\delta 148.0,142.0,133.0,132.8,131.6,129.8,119.7,91.8 ; \mathrm{IR}$ (ATR) 3094, 2925, 1519, 1341, 1261, $1086 \mathrm{~cm}^{-1}$; HRMS (ESI) calcd for $\mathrm{C}_{8} \mathrm{H}_{6} \mathrm{NO}_{2} \mathrm{I}$ $\left(\mathrm{M}+\mathrm{Na}^{+}\right)$297.9335; found 297.9333.

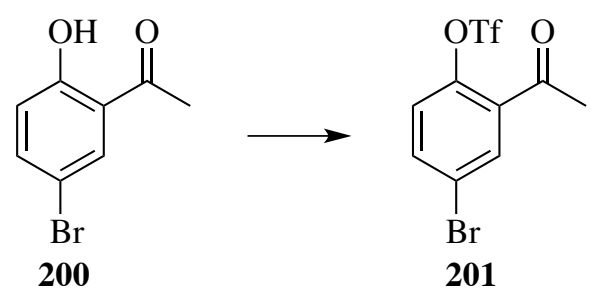

3-Bromo-6-trifluoromethanesulfonyloxy acetophenone (201): Treatment of 200 (381 $\mathrm{mg}, 1.77 \mathrm{mmol})$ in $\mathrm{CH}_{2} \mathrm{Cl}_{2}(10 \mathrm{~mL})$ with pyridine $(275 \mu \mathrm{L}, 3.41 \mathrm{mmol})$ and $\mathrm{Tf}_{2} \mathrm{O}(325$ $\mu \mathrm{L}, 1.92 \mathrm{mmol}$ ) as described for 163, gave without further purification 201 (590 mg, 1.70 mmol, 96\%) as an orange oil. ${ }^{1} \mathrm{H}$ NMR $\delta 7.92(\mathrm{~d}, J=2.4 \mathrm{~Hz}, 1 \mathrm{H}), 7.72(\mathrm{dd}, J=8.4,2.4$ $\mathrm{Hz}, 1 \mathrm{H}), 7.23(\mathrm{~d}, J=8.4 \mathrm{~Hz}, 1 \mathrm{H}), 2.63(\mathrm{~s}, 3 \mathrm{H}) ;{ }^{13} \mathrm{C}$ NMR d 195.1, 145.5, 136.4, 133.5, 133.4, 124.3, 122.0, 118.4 (q, J=320 Hz, C-F), 29.2; IR (ATR) 3101, 1701, 1424, 1202, $1133 \mathrm{~cm}^{-1}$.

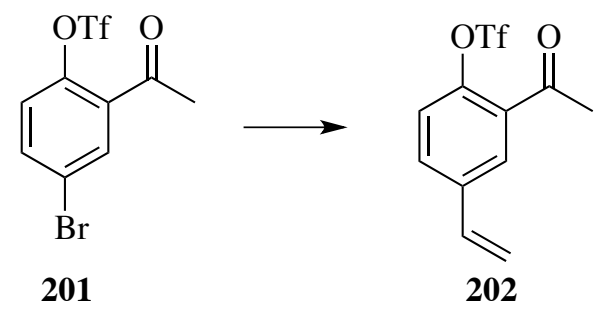

3-Ethenyl-6-trifluoromethanesulfonyloxy acetophenone (202): Treatment of 201 (101 $\mathrm{mg}, 0.29 \mathrm{mmol}$ ) with ethenyl(tributyl)stannane (116 mg, $0.37 \mathrm{mmol}), \mathrm{PPh}_{3}(6.4 \mathrm{mg}, 0.02$ $\mathrm{mmol})$ and $\mathrm{Pd}(\mathrm{dba})_{2}(3.8 \mathrm{mg}, 0.007 \mathrm{mmol})$ in dioxane $(2 \mathrm{~mL})$ as described for $\mathbf{1 4 1}$ gave after chromatography (hexanes/EtOAc, 9:1) $202(11.6 \mathrm{mg}, 0.04 \mathrm{mmol}, 14 \%)$ as a colorless oil. ${ }^{1} \mathrm{H}$ NMR $\delta 7.78(\mathrm{~d}, J=2.4 \mathrm{~Hz}, 1 \mathrm{H}), 7.61(\mathrm{dd}, J=8.4,2.4 \mathrm{~Hz}, 1 \mathrm{H}), 7.30$ (d, $J=8.4 \mathrm{~Hz}, 1 \mathrm{H}), 6.73(\mathrm{dd}, J=17.4,10.8 \mathrm{~Hz}, 1 \mathrm{H}), 5.83(\mathrm{~d}, J=17.4 \mathrm{~Hz}, 1 \mathrm{H}), 5.44(\mathrm{~d}, J=$ 
$10.8 \mathrm{~Hz}, 1 \mathrm{H}), 2.65$ (s, 3H); ${ }^{13} \mathrm{C}$ NMR $\delta 196.6,145.9,138.1,134.3,132.3,130.7,128.3$, 122.9, 118.5 (q, $J=319 \mathrm{~Hz}, \mathrm{C}-\mathrm{F}), 117.3,29.6$; IR (ATR) 3096, 1698, 1421, 1202, 1134 $\mathrm{cm}^{-1}$

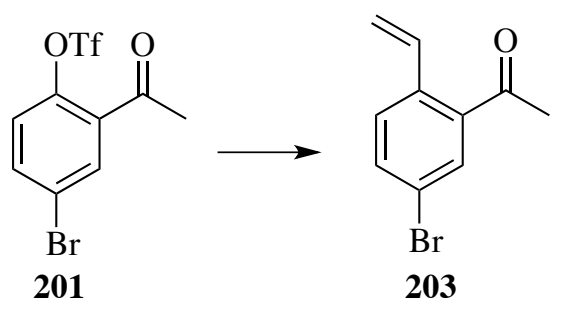

6-Ethenyl-3-bromoacetophenone (203): Treatment of 201 (112 $\mathrm{mg}, 0.32 \mathrm{mmol})$ with ethenyl(tributyl)stannane (129 mg, $0.41 \mathrm{mmol}), \mathrm{LiCl}(43.5 \mathrm{mg}, 1.03 \mathrm{mmol})$ and $\mathrm{Pd}\left(\mathrm{PPh}_{3}\right)_{2} \mathrm{Cl}_{2}(4.8 \mathrm{mg}, 0.007 \mathrm{mmol})$ in $\mathrm{DMF}(2 \mathrm{~mL})$ as described for $\mathbf{1 7 0}$ gave after chromatography (hexanes/EtOAc, 8:2) 203 (42.6 $\mathrm{mg}, 0.19 \mathrm{mmol}, 58 \%$ ) as a colorless oil. ${ }^{1} \mathrm{H}$ NMR $\delta 7.73(\mathrm{~d}, J=1.8 \mathrm{~Hz}, 1 \mathrm{H}), 7.57$ (dd, $\left.J=8.4,1.8 \mathrm{~Hz}, 1 \mathrm{H}\right), 7.43(\mathrm{~d}, J=8.4 \mathrm{~Hz}, 1 \mathrm{H})$, 7.09 (dd, $J=17.4,10.8 \mathrm{~Hz}, 1 \mathrm{H}), 5.64(\mathrm{dd}, J=17.4,1.2 \mathrm{~Hz}, 1 \mathrm{H}), 5.37$ (dd, $J=11.4,1.2 \mathrm{~Hz}$, 1H), $2.56(\mathrm{~s}, 3 \mathrm{H}) ;{ }^{13} \mathrm{C}$ NMR $\delta 200.5,138.9,136.4,134.7,134.4,131.3,129.1,121.1$, 117.4, 29.8; IR (ATR) 3088, 1686, 1472, 1355, 1235, $830 \mathrm{~cm}^{-1}$;

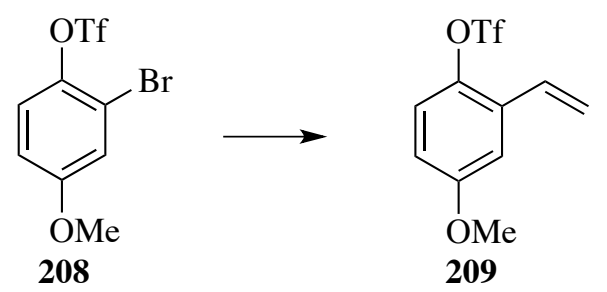

3-Ethenyl-4-trifluoromethanesulfonyloxy anisole (209): Treatment of $\mathbf{2 0 8}^{153}$ (106 mg, $0.32 \mathrm{mmol}$ ) with vinyl(tributyl)stannane (126 mg, $0.40 \mathrm{mmol}), \mathrm{PPh}_{3}(7.0 \mathrm{mg}, 0.03 \mathrm{mmol})$ and $\operatorname{Pd}(\mathrm{dba})_{2}(3.7 \mathrm{mg}, 0.006 \mathrm{mmol})$ in dioxane $(2 \mathrm{~mL})$ as described for 141 gave after chromatography (hexanes/EtOAc, 9:1) $\mathbf{2 0 9}$ (29.6 mg, $0.10 \mathrm{mmol}, 33 \%$ ) as a colorless oil. ${ }^{1} \mathrm{H}$ NMR $\delta 7.18(\mathrm{~d}, J=9.0 \mathrm{~Hz}, 1 \mathrm{H}), 7.09(\mathrm{~d}, J=3.0 \mathrm{~Hz}, 1 \mathrm{H}), 6.89(\mathrm{dd}, J=17.4,11.4 \mathrm{~Hz}$, $1 \mathrm{H}), 6.84(\mathrm{dd}, J=9.0,3.0 \mathrm{~Hz}, 1 \mathrm{H}), 5.83(\mathrm{~d}, J=18.0 \mathrm{~Hz}, 1 \mathrm{H}), 5.49(\mathrm{~d}, J=11.4 \mathrm{~Hz}, 1 \mathrm{H})$, 
$3.84(\mathrm{~s}, 1 \mathrm{H}) ;{ }^{13} \mathrm{C}$ NMR $\delta 158.9,140.5,132.1,129.1,122.6,118.6,118.3(\mathrm{q}, J=319 \mathrm{~Hz}$, C-F), 114.5, 111.6, 55.7; IR (ATR) 2968, 1485, 1419, 1206, 1137, $868 \mathrm{~cm}^{-1}$;

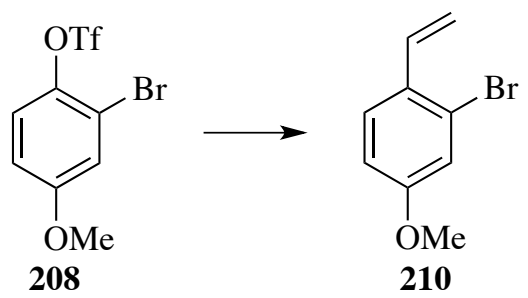

3-Bromo-4-ethenylanisole (210): ${ }^{154}$ Treatment of 208 (103 mg, $\left.0.31 \mathrm{mmol}\right)$ with ethenyl(tributyl)stannane (119 mg, $0.38 \mathrm{mmol}), \mathrm{LiCl}(38.9 \mathrm{mg}, 0.92 \mathrm{mmol})$ and $\mathrm{Pd}\left(\mathrm{PPh}_{3}\right)_{2} \mathrm{Cl}_{2}(4.5 \mathrm{mg}, 0.006 \mathrm{mmol})$ in $\mathrm{DMF}(2 \mathrm{~mL})$ as described for $\mathbf{1 7 0}$ gave after chromatography (hexanes/EtOAc, 9:1) in order of elution, 210 (26.3 mg, $0.12 \mathrm{mmol}$, $43 \%)$ as an orange oil and $\mathbf{2 0 9}(3.3 \mathrm{mg}, 0.01 \mathrm{mmol}, 4 \%)$. Spectral data were in accordance with literature values. 


\section{4: Supporting Information for Chapter 5: Pyrroloindoles}

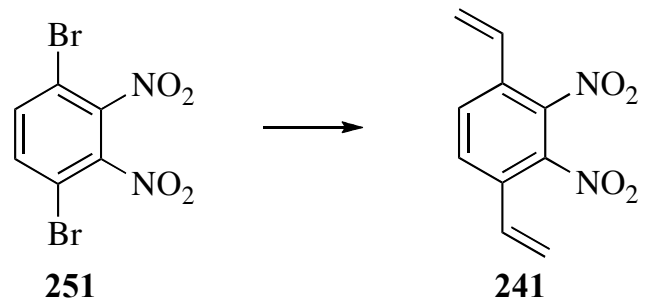

2,3-dinitro-1,4-ethenylbenzene (241): To a solution of $\mathrm{PPh}_{3}(42.3 \mathrm{mg}, 0.16 \mathrm{mmol})$ and $\operatorname{Pd}(\mathrm{dba})_{2}(14.8 \mathrm{mg}, 0.03 \mathrm{mmol})$ in toluene $(5 \mathrm{~mL})$ were added $251(99.2 \mathrm{mg}, 0.30 \mathrm{mmol})$ and a solution of tributyl(vinyl) stannane $(274.8 \mathrm{mg}, 0.87 \mathrm{mmol})$ in toluene $(2 \mathrm{~mL})$. Mixture heated at $110^{\circ} \mathrm{C}$ for 68 hours. Resulting brown solution filtered through Celite, diluted with EtOAc $(20 \mathrm{~mL})$, washed with $\mathrm{NH}_{4} \mathrm{OH}(10 \% \mathrm{aq}, 3 \times 30 \mathrm{~mL}), \mathrm{H}_{2} \mathrm{O}(30 \mathrm{~mL})$, then brine $(30 \mathrm{~mL})$. Organic layer dried $\left(\mathrm{MgSO}_{4}\right)$, filtered, then solvent removed under reduced pressure. The resulting crude brown oil was purified using column chromatography (hexanes:EtOAc, 8:2) to afford 241 (32.5 mg, $0.15 \mathrm{mmol}, 49 \%)$ as a yellow solid. ${ }^{1} \mathrm{H} \mathrm{NMR}\left(\mathrm{CDCl}_{3}\right) \delta 7.78(\mathrm{~s}, 2 \mathrm{H}), 6.74(\mathrm{dd}, 2 \mathrm{H}, J=17.5,11.1 \mathrm{~Hz}), 5.93(\mathrm{~d}$, $2 \mathrm{H}, J=17.2 \mathrm{~Hz}$ ), $5.64(\mathrm{~d}, 2 \mathrm{H}, J=11.1 \mathrm{~Hz}) ; \mathrm{HRMS}(\mathrm{ESI})$ calcd for $\mathrm{C}_{10} \mathrm{H}_{8} \mathrm{~N}_{2} \mathrm{O}_{4} \mathrm{Na}$ $\left(\mathrm{M}+\mathrm{Na}^{+}\right)$243.0376, found 243.0375.

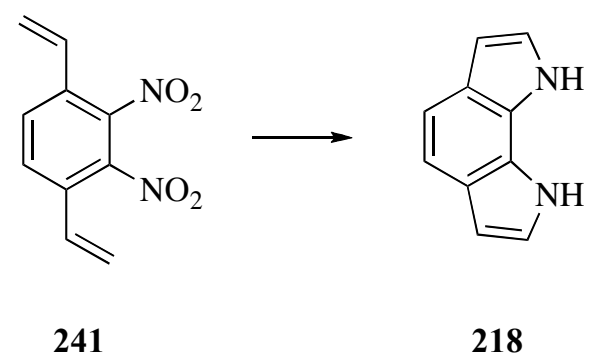

1,8-dihydropyrrolo[3,2-g]indole (218) ${ }^{103}$ : In an oven-dried ACE glass pressure tube was mixed 241 (31.5 mg, $0.14 \mathrm{mmol}), \operatorname{Pd}(\mathrm{OAc})_{2}(3.2 \mathrm{mg}, 0.01 \mathrm{mmol}), 1,10-$ phenanthroline (1.8 $\mathrm{mg}, 0.01 \mathrm{mmol})$, and anhydrous DMF $(3 \mathrm{~mL})$. The tube was fitted with a pressure head, and the solution was saturated with $\mathrm{CO}$ (four cycles, $6 \mathrm{~atm}$ ). The 
tube was then allowed to heat at $120{ }^{\circ} \mathrm{C}$ for $143 \mathrm{~h}$. The solvent was then removed by a bulb-to-bulb resulting in a dark brown crude material which was subsequently diluted with $\mathrm{H}_{2} \mathrm{O}(15 \mathrm{~mL})$ and extracted with EtOAc $(3 \times 25 \mathrm{~mL})$. Combined organic layers dried $\left(\mathrm{MgSO}_{4}\right)$, filtered, then solvent removed under reduced pressure. Resulting crude brown oil purified by chromatography (hexanes: EtOAc, 85:15, then 7:3) to give 218 (12.3 mg, $0.08 \mathrm{mmol}, 56 \%)$ as a dark grey solid. HRMS (ESI) calcd for $\mathrm{C}_{10} \mathrm{H}_{8} \mathrm{~N}_{2} \mathrm{H}\left(\mathrm{M}+\mathrm{H}^{+}\right)$ 157.0760, found 157.0758 .

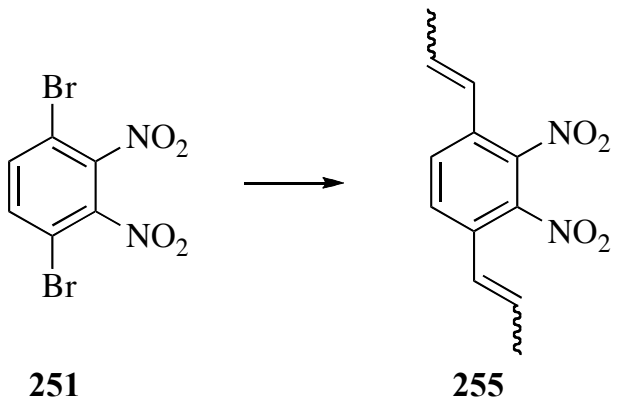

2,3-dinitro-1,4-di(prop-1-enyl)benzene (255): To a solution of $\mathrm{PPh}_{3}(56.7 \mathrm{mg}, 0.22$ $\mathrm{mmol})$ and $\mathrm{Pd}(\mathrm{dba})_{2}(20.9 \mathrm{mg}, 0.04 \mathrm{mmol})$ in toluene $(5 \mathrm{~mL})$ were added $251(154 \mathrm{mg}$, $0.47 \mathrm{mmol}$ ) and a solution of tributyl(prop-1-enyl)stannane (430 $\mathrm{mg}, 1.3 \mathrm{mmol})$ in toluene $(5 \mathrm{~mL})$. Mixture heated at $110^{\circ} \mathrm{C}$ for 72 hours. Resulting brown solution filtered through Celite, diluted with EtOAc $(20 \mathrm{~mL})$, washed with $\mathrm{NH}_{4} \mathrm{OH}(10 \%$ aq, $3 \times 30 \mathrm{~mL})$, $\mathrm{H}_{2} \mathrm{O}(30 \mathrm{~mL})$, then brine $(30 \mathrm{~mL})$. Organic layer dried $\left(\mathrm{MgSO}_{4}\right)$, filtered, then solvent removed under reduced pressure. The resulting crude brown oil was purified using column chromatography (hexanes:EtOAc, 9:1) to afford 255 (75.8 mg, $0.31 \mathrm{mmol}, 65 \%)$ as a yellow solid. ${ }^{1} \mathrm{H} \mathrm{NMR}\left(\mathrm{CDCl}_{3}\right) \delta 7.50(\mathrm{~s}, 2 \mathrm{H}), 6.42(\mathrm{dd}, J=11.4,1.8 \mathrm{~Hz}, 2 \mathrm{H}), 6.08$ $(\mathrm{dq}, 2 \mathrm{H}, J=11.4,6.6 \mathrm{~Hz}), 1.78(\mathrm{dd}, 6 \mathrm{H}, J=7.2,1.8 \mathrm{~Hz}) ;{ }^{13} \mathrm{C} \mathrm{NMR} \delta 133.5,132.9,131.0$, 122.4, 14.8; IR (ATR) 3041, 2954, 1649, 1528, 1358, 859, $820 \mathrm{~cm}^{-1}$; HRMS (ESI) calcd for $\mathrm{C}_{12} \mathrm{H}_{12} \mathrm{~N}_{2} \mathrm{O}_{4} \mathrm{Na}\left(\mathrm{M}+\mathrm{Na}^{+}\right)$271.0689, found 271.0688. 


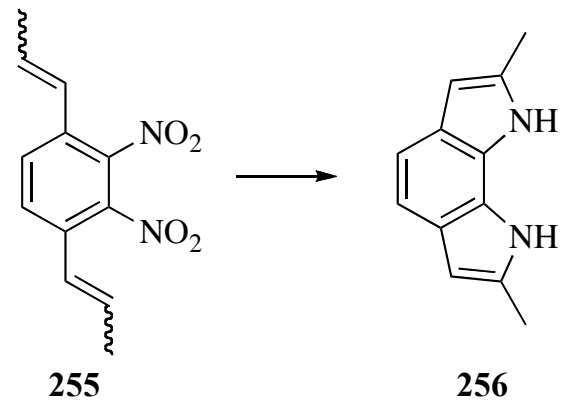

2,7-dimethyl-1,8-dihydropyrrolo[3,2-g]indole $(\mathbf{2 5 6})^{103}$ : In an oven-dried ACE glass pressure tube was mixed $255(29.9 \mathrm{mg}, 0.12 \mathrm{mmol}), \mathrm{Pd}(\mathrm{OAc})_{2}(3.1 \mathrm{mg}, 0.01 \mathrm{mmol})$, 1,10-phenanthroline (2.9 mg, $0.01 \mathrm{mmol})$, and anhydrous DMF ( $3 \mathrm{~mL})$. The tube was fitted with a pressure head, and the solution was saturated with CO (four cycles, 6 atm). The tube was then allowed to heat at $120{ }^{\circ} \mathrm{C}$ for $20 \mathrm{~h}$ until all starting material was consumed as judged by TLC. The solvent was then removed by a bulb-to-bulb resulting in a dark brown crude which was subsequently diluted with $\mathrm{H}_{2} \mathrm{O}(15 \mathrm{~mL})$ and extracted with EtOAc $(3 \times 25 \mathrm{~mL})$. Combined organic layers dried $\left(\mathrm{MgSO}_{4}\right)$, filtered, then solvent removed under reduced pressure. Resulting crude brown oil purified by chromatography (hexanes: EtOAc, 8:2) to give $\mathbf{2 5 6}$ (9.5 $\mathrm{mg}, 0.05 \mathrm{mmol}, 43.0 \%$ ) as a dark grey solid. HRMS (ESI) calcd for $\mathrm{C}_{12} \mathrm{H}_{12} \mathrm{~N}_{2} \mathrm{H}\left(\mathrm{M}+\mathrm{H}^{+}\right)$185.1073, found 185.1071.

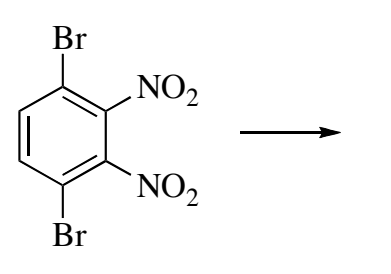

251<smiles>C=C(C)c1ccc(C(=C)C)c([N+](=O)[O-])c1[N+](=O)[O-]</smiles>

257

2,3-dinitro-1,4-di(prop-1-en-2-yl)benzene (257): To a solution of $\mathrm{PPh}_{3}(64.9 \mathrm{mg}, 0.25$ $\mathrm{mmol})$ and $\mathrm{Pd}(\mathrm{dba})_{2}(28.2 \mathrm{mg}, 0.05 \mathrm{mmol})$ in toluene $(10 \mathrm{~mL})$ were added $251(198 \mathrm{mg}$, $0.61 \mathrm{mmol}$ ) and a solution of tributyl(prop-1-en-2-yl)stannane (487 mg, $1.47 \mathrm{mmol})$. Mixture heated at $110^{\circ} \mathrm{C}$ for 45 hours. Resulting brown solution filtered through Celite, 
diluted with EtOAc $(30 \mathrm{~mL})$, washed with $\mathrm{NH}_{4} \mathrm{OH}(10 \%$ aq, $3 \times 30 \mathrm{~mL}), \mathrm{H}_{2} \mathrm{O}(30 \mathrm{~mL})$, then brine $(30 \mathrm{~mL})$. Organic layer dried $\left(\mathrm{MgSO}_{4}\right)$, filtered, then solvent removed under reduced pressure. The resulting crude green residue was purified using column chromatography (hexanes:EtOAc, 7:3) to afford 257 (93.0 $\mathrm{mg}, 0.37 \mathrm{mmol}, 64 \%)$ as a yellow solid $\left(\mathrm{mp}=135-137{ }^{\circ} \mathrm{C}\right) .{ }^{1} \mathrm{H}$ NMR $\left(\mathrm{CDCl}_{3}\right) \delta 7.44(\mathrm{~s}, 2 \mathrm{H}), 5.27(\mathrm{~m}, 2 \mathrm{H}), 5.03(\mathrm{~m}$, 2H), $2.11(\mathrm{~m}, 6 \mathrm{H}) ;{ }^{13} \mathrm{C}$ NMR $\delta 142.1,139.2,137.4,131.5,118.1,23.1$; IR (ATR) 3094, $2973,1643,1543,1526,1355,911,847 \mathrm{~cm}^{-1}$

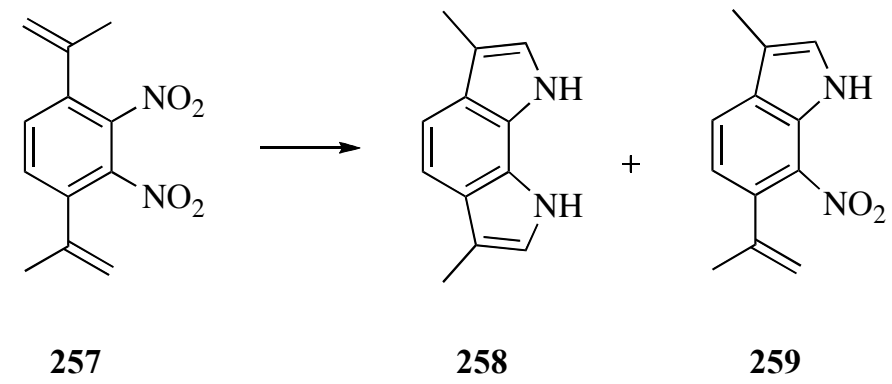

3,6-dimethyl-1,8-dihydropyrrolo[3,2-g]indole (258) ${ }^{103}$ : In an oven-dried ACE glass pressure tube was mixed $257(26.3 \mathrm{mg}, 0.11 \mathrm{mmol}), \mathrm{Pd}(\mathrm{dba})_{2}(4.8 \mathrm{mg}, 0.008 \mathrm{mmol})$, $\mathrm{PPh}_{3}(12.1 \mathrm{mg}, 0.05 \mathrm{mmol})$, and anhydrous DMF $(3 \mathrm{~mL})$. The tube was fitted with a pressure head, and the solution was saturated with $\mathrm{CO}$ (four cycles, 6 atm). The tube was heated at $120{ }^{\circ} \mathrm{C}$ for 96 hours until all starting material was consumed as judged by TLC. The solvent was then removed by a bulb-to-bulb resulting in a dark brown crude material which was subsequently diluted with $\mathrm{H}_{2} \mathrm{O}(15 \mathrm{~mL})$ and extracted with EtOAc $(3 \times 25 \mathrm{~mL})$. Combined organic layers dried $\left(\mathrm{MgSO}_{4}\right)$, filtered, then solvent removed under reduced pressure. Resulting crude brown oil was purified by chromatography (hexanes: EtOAc, 8:2) to afford in order of elution $258(5.1 \mathrm{mg}, 0.03 \mathrm{mmol}, 25 \%)$ as a dark grey solid and $259(12.9 \mathrm{mg}, 0.06 \mathrm{mmol}, 54 \%)$ as a brown solid $\left(\mathrm{mp}=72-74{ }^{\circ} \mathrm{C}\right)$. HRMS of $258(\mathrm{ESI})$ calcd for $\mathrm{C}_{12} \mathrm{H}_{12} \mathrm{~N}_{2} \mathrm{H}\left(\mathrm{M}+\mathrm{H}^{+}\right)$185.1073, found 185.1071 . 


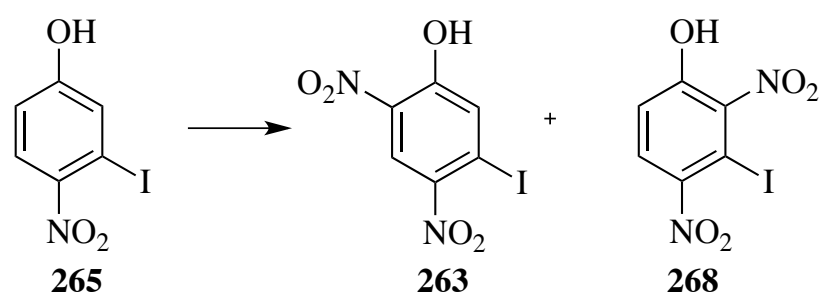

\section{5-iodo-2,4-dinitrophenol (263) and 3-iodo-2,4-dinitrophenol (268)}

To an ice-cooled solution of $\mathbf{2 6 5}(256 \mathrm{mg}, 1.45 \mathrm{mmol})$ in $\mathrm{H}_{2} \mathrm{SO}_{4}(4 \mathrm{~mL})$ was added $\mathrm{NaNO}_{3}(215 \mathrm{mg}, 2.53 \mathrm{mmol})$. The resulting brown solution was stirred at ambient temperature (4 hours). Solution quenched with ice, then extracted with EtOAc $(3 \times 30$ $\mathrm{mL})$. The combined organic layers were dried $\left(\mathrm{MgSO}_{4}\right)$, filtered, then the solvents were removed under reduced pressure. Purification using column chromatography (Hexanes/EtOAc, 8:2) afforded in order of elution 263 (307 mg, $0.99 \mathrm{mmol}, 69 \%$ ) and 268 (103 mg, $0.33 \mathrm{mmol}, 23 \%)$. Spectral data for 263: ${ }^{1} \mathrm{H}$ NMR (600 MHz) $\delta 10.74$ (s, 1H), $8.78(\mathrm{~s}, 1 \mathrm{H}), 7.97(\mathrm{~s}, 1 \mathrm{H}) ;{ }^{13} \mathrm{C}$ NMR $(150 \mathrm{MHz}) \delta 155.9,134.1,133.7,122.7,122.5$, 97.2; IR (ATR) 3268, 3098, 1607, 1567, 1520, $1315 \mathrm{~cm}^{-1}$; HRMS (ESI) calcd for $\mathrm{C}_{6} \mathrm{H}_{3} \mathrm{~N}_{2} \mathrm{O}_{4} \mathrm{I}(\mathrm{M}-\mathrm{H})$ 308.90084, found 308.90105.Spectral data for $\mathbf{2 6 8}{ }^{1} \mathrm{H}$ NMR (600 MHz) $\delta 7.83(\mathrm{~d}, J=9.0 \mathrm{~Hz}, 1 \mathrm{H}), 7.24(\mathrm{~d}, J=9.0 \mathrm{~Hz}, 1 \mathrm{H}) ;{ }^{13} \mathrm{C}$ NMR $(150 \mathrm{MHz}) \delta 152.4$, 145.2, 127.9, 127.2, 117.3, 81.5; IR (ATR) 3232, 3097, 1525, 1336, $1296 \mathrm{~cm}^{-1}$;

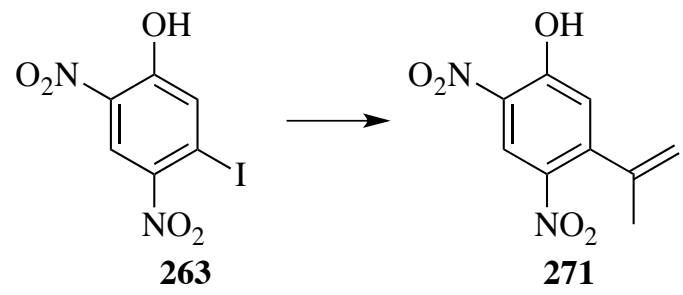

2,4-dinitro-5-(prop-1-en-2-yl)phenol (271): To a solution of 263 (363 mg, 1.17 mmol) in dioxane $(4 \mathrm{~mL})$ was added $\mathrm{PPh}_{3}(24.7 \mathrm{mg}, 0.09 \mathrm{mmol}), \mathrm{Pd}(\mathrm{dba})_{2}(13.3 \mathrm{mg}, 0.02$ mmol), CuI (225 mg, $1.18 \mathrm{mmol})$, BHT (26.8 mg, $0.12 \mathrm{mmol})$, and tributyl(prop-1-en-2yl)stannane (396 mg, $1.2 \mathrm{mmol})$. The resulting solution was heated at $105^{\circ} \mathrm{C}$ (16 hours). 
Solvents were removed under reduced pressure, then the residue was purified using column chromatography (hexanes/EtOAc, $1: 1$ with $5 \% \mathrm{Et}_{3} \mathrm{~N}$ followed by hexanes/EtOAc, 9:1 with 5\% AcOH) to afford $271(44.7 \mathrm{mg}, 0.20 \mathrm{mmol}, 17 \%)$ as a brown solid. ${ }^{1} \mathrm{H}$ NMR (600 MHz) $\delta 10.83(\mathrm{~s}, 1 \mathrm{H}), 8.83(\mathrm{~s}, 1 \mathrm{H}), 7.11(\mathrm{~s}, 1 \mathrm{H}), 5.26(\mathrm{~s}$, 1H), $5.02(\mathrm{~s}, 1 \mathrm{H}), 2.08(\mathrm{~s}, 3 \mathrm{H}) ;{ }^{13} \mathrm{C}$ NMR $(150 \mathrm{MHz}) \delta 157.2,148.8,141.5,140.1,131.4$, 122.8, 121.9, 116.6, 22.7. IR (ATR) 3115, 2960, 1582, $1330 \mathrm{~cm}^{-1}$; HRMS (ESI) calcd for $\mathrm{C}_{9} \mathrm{H}_{8} \mathrm{~N}_{2} \mathrm{O}_{5}$ (M-H) 223.03550, found 223.03562.

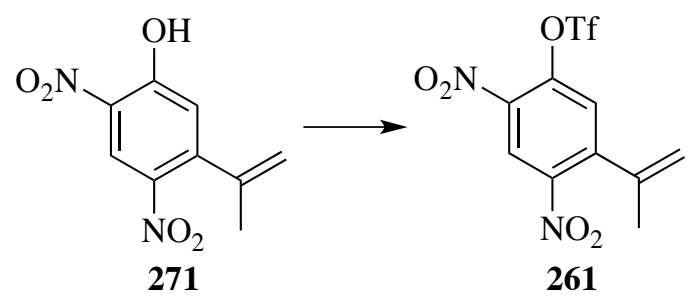

2,4-dinitro-5-(prop-1-en-2-yl)phenyl trifluoromethanesulfonate (261): To an icecooled solution of 271 (118 $\mathrm{mg}, 0.52 \mathrm{mmol})$ in $\mathrm{CH}_{2} \mathrm{Cl}_{2}(5 \mathrm{~mL})$ was added pyridine (90.0 $\mu \mathrm{L}, 1.11 \mathrm{mmol})$, and $\mathrm{Tf}_{2} \mathrm{O}(100 \mu \mathrm{L}, 0.59 \mathrm{mmol})$ and the solution stirred while warming to ambient temperature over 30 minutes. Solvents were removed under reduced pressure and the crude product purified using column chromatography (hexanes/EtOAc, 85:15) to afford $261(160 \mathrm{mg}, 45 \mathrm{mmol}, 86 \%)$ as a yellow oil. ${ }^{1} \mathrm{H}$ NMR $(600 \mathrm{MHz}) \delta 8.71(\mathrm{~s}, 1 \mathrm{H})$, $7.45(\mathrm{~s}, 1 \mathrm{H}), 5.39(\mathrm{~d}, J=1.8 \mathrm{~Hz}, 1 \mathrm{H}), 5.11(\mathrm{~s}, 1 \mathrm{H}), 2.13(\mathrm{~s}, 3 \mathrm{H}) ;{ }^{13} \mathrm{C}(150 \mathrm{MHz}) \delta 146.3$, $146.2,143.1,139.8,139.6,126.7,123.2,118.9,118.5(\mathrm{q}, J=319 \mathrm{~Hz}), 22.5$; IR (ATR) $3144,2928,1590,1538,1435,1340 \mathrm{~cm}^{-1}$ 


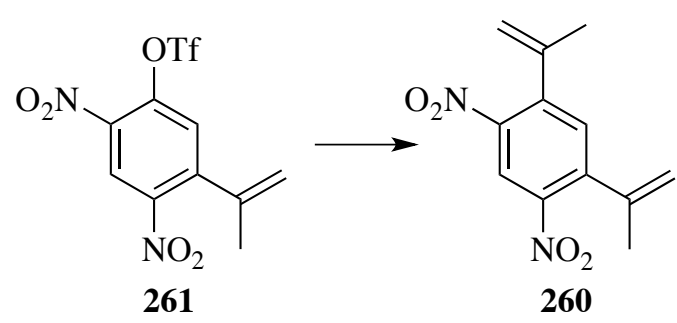

1,5-dinitro-2,4-di(prop-1-en-2-yl)benzene (260): To a solution of 261 (149 mg, 0.34 mmol) in DMF (2 mL) was added $\mathrm{PdCl}_{2}\left(\mathrm{PPh}_{3}\right)_{2}(5.3 \mathrm{mg}, 0.007 \mathrm{mmol}), \mathrm{LiCl}(50.3 \mathrm{mg}$, $1.19 \mathrm{mmol})$, BHT (7.0 mg, $0.03 \mathrm{mmol})$, and tributyl(prop-1-en-2-yl)stannane (293 mg, $0.88 \mathrm{mmol})$. The resulting solution was heated at $60{ }^{\circ} \mathrm{C}$ ( 16 hours $)$. The resulting brown solution was quenched with $\mathrm{NH}_{4} \mathrm{OH}(10 \%$ aq., $15 \mathrm{~mL})$, then extracted with EtOAc (20 $\mathrm{mL})$. The organic layer was washed with $\mathrm{NH}_{4} \mathrm{OH}(10 \%$ aq., $20 \mathrm{~mL}), \mathrm{H}_{2} \mathrm{O}(20 \mathrm{~mL})$, and brine $(20 \mathrm{~mL})$, then dried $\left(\mathrm{MgSO}_{4}\right)$, filtered, then solvents were removed under reduced pressure. Purification using column chromatography (hexanes/EtOAc, 85:15) afforded $260(73.3 \mathrm{mg}, 0.29 \mathrm{mmol}, 87 \%)$ as a white solid $\left(\mathrm{mp}=77-78^{\circ} \mathrm{C}\right) .{ }^{1} \mathrm{H} \mathrm{NMR}(600 \mathrm{MHz}) \delta$ $8.45(\mathrm{~s}, 1 \mathrm{H}), 7.33(\mathrm{~s}, 1 \mathrm{H}), 5.29(\mathrm{~d}, J=1.2 \mathrm{~Hz}, 2 \mathrm{H}), 5.03(\mathrm{~d}, J=1.2 \mathrm{~Hz}, 2 \mathrm{H}), 2.11(\mathrm{~d}, J=$ $1.2 \mathrm{~Hz}, 6 \mathrm{H}) ;{ }^{13} \mathrm{C}$ NMR $(150 \mathrm{MHz}) \delta 146.3,143.3,141.1,133.0,120.6,117.0,22.9$; IR (ATR) $3101,2983,1584,1525,1345 \mathrm{~cm}^{-1}$;

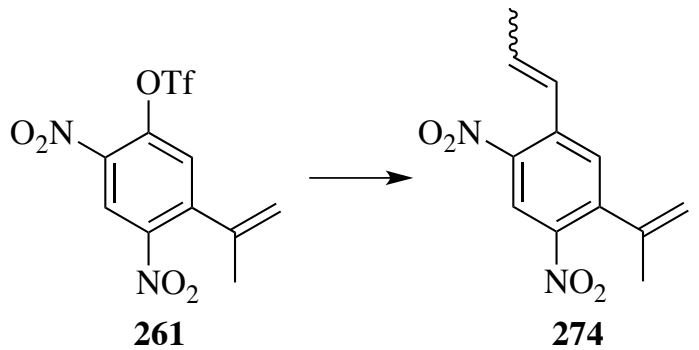

1,5-dinitro-2-(prop-1-en-2-yl)-4-(prop-1-enyl)benzene (274): Reaction of 261 (86.3 $\mathrm{mg}, 0.24 \mathrm{mmol})$, tributyl(prop-1-enyl)stannane (117 mg, $0.35 \mathrm{mmol}), \mathrm{PdCl}_{2}\left(\mathrm{PPh}_{3}\right)_{2}(4.3$ $\mathrm{mg}, 0.006 \mathrm{mmol}), \mathrm{LiCl}(38.6 \mathrm{mg}, 0.91 \mathrm{mmol})$ in DMF $(2 \mathrm{~mL})$ as described for $\mathbf{2 6 0}$ afforded after purification using column chromatography (hexanes/EtOAc, 9:1) 274 (52.1 
$\mathrm{mg}, 0.21 \mathrm{mmol}, 87 \%)$ as a yellow oil ( 2:1 ratio of Z/E isomers). ${ }^{1} \mathrm{H}$ NMR for $Z$-isomer (400 MHz) $\delta 8.61(\mathrm{~s}, 1 \mathrm{H}), 7.38(\mathrm{~s}, 1 \mathrm{H}), 6.76(\mathrm{dq}, J=11.6,2.0 \mathrm{~Hz}, 1 \mathrm{H}), 6.11(\mathrm{dq}, J=11.6$, $7.2 \mathrm{~Hz}, 1 \mathrm{H}), 5.29(\mathrm{t}, J=1.6 \mathrm{~Hz}, 1 \mathrm{H}), 5.03(\mathrm{t}, J=1.2 \mathrm{~Hz}, 1 \mathrm{H}), 2.11(\mathrm{t}, J=1.2 \mathrm{~Hz}, 3 \mathrm{H}), 1.80$ (dd, $J=7.6,2.0 \mathrm{~Hz}, 3 \mathrm{H}) ;{ }^{1} \mathrm{H}$ NMR for $E$-isomer $(400 \mathrm{MHz}) \delta 8.52(\mathrm{~s}, 1 \mathrm{H}), 7.55(\mathrm{~s}, 1 \mathrm{H})$, $6.94(\mathrm{dq}, J=15.6,1.6 \mathrm{~Hz}, 1 \mathrm{H}), 6.47(\mathrm{dq}, J=15.6,6.8 \mathrm{~Hz}, 1 \mathrm{H}), 5.28(\mathrm{t}, J=1.2 \mathrm{~Hz}, 1 \mathrm{H})$, $5.02(\mathrm{t}, J=1.2 \mathrm{~Hz}, 1 \mathrm{H}), 2.11(\mathrm{t}, J=0.8 \mathrm{~Hz}, 3 \mathrm{H}), 2.01(\mathrm{dd}, J=7.2,2.0 \mathrm{~Hz}, 3 \mathrm{H}) ;{ }^{13} \mathrm{C} \mathrm{NMR}$ (combined isomers, $100 \mathrm{MHz}$ ) $\delta 146.2,145.8,145.4,145.2,143.4,143.1,141.6,141.3$, 137.6, 137.1, 136.0, 134.3, 131.7, 130.7, 124.7, 124.5, 121.3, 121.2, 116.9, 116.6, 23.0, 22.9, 19.1, 14.6; IR (ATR) 3105, 2978, 1581, 1520, $1341 \mathrm{~cm}^{-1}$;

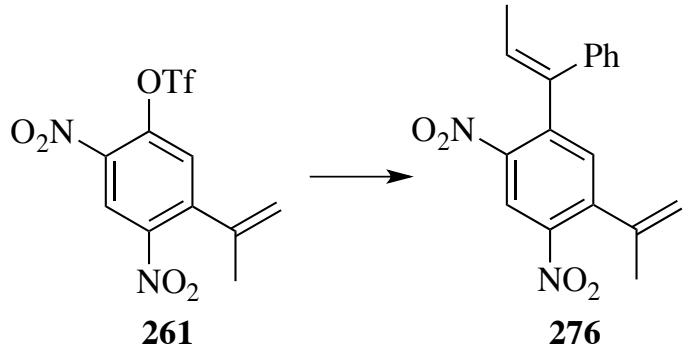

(E)-1,5-dinitro-2-(1-phenylprop-1-enyl)-4-(prop-1-en-2-yl)benzene (276): Reaction of 261 (83.5 mg, $0.23 \mathrm{mmol})$, (E)-tributyl(1-phenylprop-1-enyl)stannane (117 mg, 0.29 $\mathrm{mmol}), \mathrm{PPh}_{3}(13.4 \mathrm{mg}, 0.05 \mathrm{mmol})$, and $\mathrm{Pd}(\mathrm{dba})_{2}(7.3 \mathrm{mg}, 0.01 \mathrm{mmol})$ in $\mathrm{DMF}(2 \mathrm{~mL})$ at $50{ }^{\circ} \mathrm{C}$ as described for $\mathbf{2 6 0}$ afforded after purification using column chromatography (hexanes/EtOAc, 85:15) $276(34.9 \mathrm{mg}, 0.11 \mathrm{mmol}, 46 \%)$ as a yellow solid ( $\mathrm{mp}=92-94$ $\left.{ }^{\circ} \mathrm{C}\right) .{ }^{1} \mathrm{H}$ NMR $(400 \mathrm{MHz}) \delta 8.33(\mathrm{~s}, 1 \mathrm{H}), 7.39(\mathrm{~s}, 1 \mathrm{H})$, 7.34-7.29 (m, 3H), 7.17-7.15 (m, 2H), $6.01(\mathrm{q}, J=7.6 \mathrm{~Hz}, 1 \mathrm{H}), 5.27(\mathrm{~m}, 1 \mathrm{H}), 5.03(\mathrm{~m}, 1 \mathrm{H}), 2.11(\mathrm{~m}, 3 \mathrm{H}), 1.93(\mathrm{~d}, J=7.6$ $\mathrm{Hz}, 3 \mathrm{H}) ;{ }^{13} \mathrm{C}$ NMR (100 MHz) $\delta 147.3,146.0,143.1,142.8,141.2,137.2,136.4,134.5$, $129.8,129.7,128.2,127.9,120.7,117.0,23.0,15.7$; IR (ATR) 2954, 1583, 1525, 1346 $\mathrm{cm}^{-1}$ 


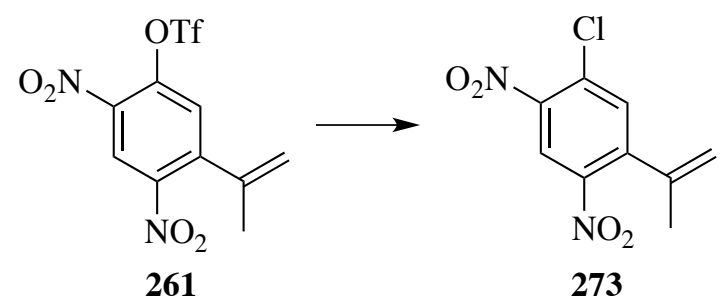

1-chloro-2,4-dinitro-5-(prop-1-en-2-yl)benzene (273): To a solution of 261 (198 mg, $0.55 \mathrm{mmol})$ in DMF (1 mL) was added $\mathrm{LiCl}(79.0 \mathrm{mg}, 1.86 \mathrm{mmol})$ and the solution stirred at $60{ }^{\circ} \mathrm{C}$ (8 hours). The resulting solution was diluted with EtOAc $(15 \mathrm{~mL})$ and washed with $\mathrm{H}_{2} \mathrm{O}(5 \times 20 \mathrm{~mL})$. The organic phase was dried $\left(\mathrm{MgSO}_{4}\right)$, filtered, and solvents were removed. Purification using column chromatography (hexanes/EtOAc, 7:3) afforded $273(64.2 \mathrm{mg}, 0.26 \mathrm{mmol}, 48 \%)$ as a white solid $\left(\mathrm{mp}=128-130{ }^{\circ} \mathrm{C}\right) .{ }^{1} \mathrm{H}$ NMR $(400 \mathrm{MHz}) \delta 8.50(\mathrm{~s}, 1 \mathrm{H}), 7.59(\mathrm{~s}, 1 \mathrm{H}), 5.33(\mathrm{~s}, 1 \mathrm{H}), 5.07(\mathrm{~s}, 1 \mathrm{H}), 2.11(\mathrm{~s}, 3 \mathrm{H}) ;{ }^{13} \mathrm{C}$ NMR (100 MHz) $\delta 145.8,145.7,143.9,140.1,134.2,131.7,122.1,117.8,22.6$; IR (ATR) 3091, 1593, 1568, 1507, $1338 \mathrm{~cm}^{-1}$;

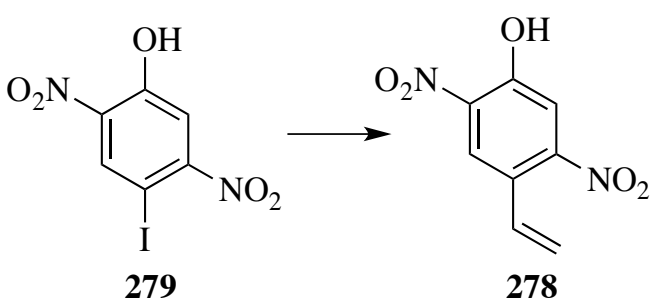

2,5-dinitro-4-ethenylphenol (278): Reaction of $\mathbf{2 7 9}^{129}$ (122 mg, 0.39 mmol), $\mathrm{PPh}_{3}(10.3$ mg, $0.04 \mathrm{mmol}), \mathrm{Pd}(\mathrm{dba})_{2}(5.2 \mathrm{mg}, 0.01 \mathrm{mmol}), \mathrm{CuI}(74.3 \mathrm{mg}, 0.39 \mathrm{mmol})$, BHT (9.7 $\mathrm{mg}, 0.04 \mathrm{mmol})$, and tri-n-butyl(ethenyl)stannane $(168 \mathrm{mg}, 0.64 \mathrm{mmol}$ in dioxane $(3 \mathrm{~mL})$ $105{ }^{\circ} \mathrm{C}$ (5 hours) as described for $\mathbf{2 7 1}$ afforded after purification using column chromatography (hexanes/EtOAc, 95:5 w/ 5\% AcOH) 278 (55.8 $\mathrm{mg}, 0.27 \mathrm{mmol}, 68 \%)$ as a yellow solid $\left(\mathrm{mp}=97-98{ }^{\circ} \mathrm{C}\right) .{ }^{1} \mathrm{H}$ NMR $(600 \mathrm{MHz}) \delta 10.44(\mathrm{~s}, 1 \mathrm{H}), 8.40(\mathrm{~s}, 1 \mathrm{H}), 7.64$ (s, 1H), $6.93(\mathrm{dd}, J=17.4,10.8 \mathrm{~Hz}, 1 \mathrm{H}), 5.80(\mathrm{~d}, J=16.8 \mathrm{~Hz}, 1 \mathrm{H}), 5.55(\mathrm{~d}, J=11.4 \mathrm{~Hz}$, 
1H); ${ }^{13} \mathrm{C}$ NMR $(150 \mathrm{MHz}) \delta 153.7,152.3,135.3,129.4,125.1,125.0,120.0,115.9 ; \mathrm{IR}$ (ATR) 2245, 2080, 1630, 1525, $1263 \mathrm{~cm}^{-1}$;

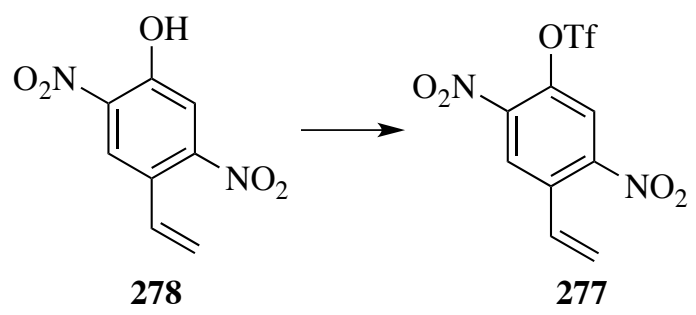

2,5-dinitro-4-vinylphenyl trifluoromethanesulfonate (277): Reaction of 278 (56.3 mg, $0.27 \mathrm{mmol}), \mathrm{Et}_{3} \mathrm{~N}(100 \mu \mathrm{L}, 0.72 \mathrm{mmol})$, and $\mathrm{Tf}_{2} \mathrm{O}(60 \mu \mathrm{L}, 0.36 \mathrm{mmol})$ in $\mathrm{CH}_{2} \mathrm{Cl}_{2}(5 \mathrm{~mL})$ as described for $\mathbf{2 6 1}$ afforded after purification using column chromatography (hexanes/EtOAc, 7:3) 277 (90.8 mg, $0.26 \mathrm{mmol}, 98 \%)$ as an orange oil. ${ }^{1} \mathrm{H}$ NMR (400 MHz) $\delta 8.40(\mathrm{~s}, 1 \mathrm{H}), 8.02(\mathrm{~s}, 1 \mathrm{H}), 7.16(\mathrm{dd}, J=17.2,11.2 \mathrm{~Hz}, 1 \mathrm{H}), 5.99(\mathrm{~d}, J=17.2 \mathrm{~Hz}$, 1H), $5.80(\mathrm{~d}, J=11.2 \mathrm{~Hz}, 1 \mathrm{H}) ;{ }^{13} \mathrm{C}$ NMR $(100 \mathrm{MHz}) \delta 148.8,143.4,139.5,126.7,123.8$, 121.6, 120.6, 118.5, 118.2 (q, J=319. Hz, C-F); IR (ATR) 3117, 1557, 1436, 1344, 1225 $\mathrm{cm}^{-1} ;$ HRMS

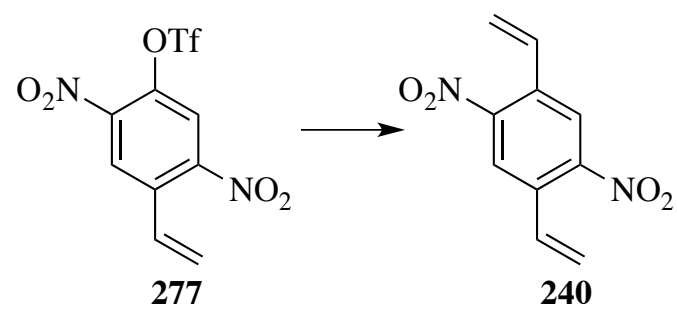

2,5-dinitro-1,4-diethenylbenzene (240): Reaction of $\mathbf{2 7 7}$ (92.9 mg, $0.27 \mathrm{mmol}$ ), tri-nbutyl(ethenyl)stannane (105 mg, $0.33 \mathrm{mmol}), \mathrm{PdCl}_{2}\left(\mathrm{PPh}_{3}\right)_{2}(4.2 \mathrm{mg}, 0.006 \mathrm{mmol})$, and $\mathrm{LiCl}$ (39.1 $\mathrm{mg}, 0.92 \mathrm{mmol})$ in DMF (3 mL) at ambient temperature (18 hours) as described for $\mathbf{2 6 0}$ afforded after purification using column chromatography (hexanes/EtOAc, 9:1) 240 (38.8 $\mathrm{mg}, 0.18 \mathrm{mmol}, 65 \%)$ as a yellow solid ( $\mathrm{mp}=140-141$ $\left.{ }^{\circ} \mathrm{C}\right) .{ }^{1} \mathrm{H}$ NMR $(600 \mathrm{MHz}) \delta 8.14(\mathrm{~s}, 2 \mathrm{H}), 7.10(\mathrm{dd}, J=17.4,10.8 \mathrm{~Hz}, 2 \mathrm{H}), 5.91(\mathrm{~d}, J=17.4$ 
$\mathrm{Hz}, 2 \mathrm{H}), 5.65(\mathrm{~d}, J=10.8 \mathrm{~Hz}, 2 \mathrm{H}) ;{ }^{13} \mathrm{C}$ NMR $(150 \mathrm{MHz}) \delta 149.3,132.9,129.9,124.2$, 121.5; IR (ATR) 3112, 3075, 1517, 1348, $1283 \mathrm{~cm}^{-1}$;<smiles>CC(C)(C)c1ccc(O)c([N+](=O)[O-])c1[N+](=O)[O-]</smiles>

4-bromo-2,3-dinitrophenol (292): To a solution of $\mathbf{2 5 1}^{155}$ (730 mg, $\left.2.24 \mathrm{mmol}\right)$ in THF ( $8 \mathrm{~mL})$ and $\mathrm{H}_{2} \mathrm{O}(0.5 \mathrm{~mL})$ was added $\mathrm{NaOH}(1.04 \mathrm{~g}, 26.1 \mathrm{mmol})$. Solution heated at 80 ${ }^{\circ} \mathrm{C}$ in sealed pressure tube for 18 hours. Resulting brown solution diluted with $\mathrm{H}_{2} \mathrm{O}$ (25 $\mathrm{mL})$ and acidified with $\mathrm{HCl}$ then extracted with EtOAc $(3 \times 30 \mathrm{~mL})$. Combined organic layers dried $\left(\mathrm{MgSO}_{4}\right)$, filtered, and solvents removed under reduced pressure. Crude brown oil was purified using column chromatography (hexanes/EtOAc, 7:3 w/ 5\% AcOH) to afford $292(554 \mathrm{mg}, 2.11 \mathrm{mmol}, 94 \%)$ as a yellow solid $\left(\mathrm{mp}=59-61{ }^{\circ} \mathrm{C}\right) .{ }^{1} \mathrm{H}$ NMR (400 MHz) $\delta 7.55(\mathrm{~d}, J=8.4 \mathrm{~Hz}, 1 \mathrm{H}), 7.20(\mathrm{~d}, J=8.8 \mathrm{~Hz}, 1 \mathrm{H}) ;{ }^{13} \mathrm{C}$ NMR $(100$ MHz) $\delta 149.0,138.3,136.1,126.5,114.5,111.5 ;$ IR (ATR) 3391, 1566, 1535, 1456, 1374 $\mathrm{cm}^{-1}$; HRMS (ESI) calcd for $\mathrm{C}_{6} \mathrm{H}_{3} \mathrm{~N}_{2} \mathrm{O}_{5} \mathrm{Br}(\mathrm{M}-\mathrm{H})$ 260.91471, found 260.91484.<smiles>C/C=C(\C(C)=O)c1ccc(Br)c([N+](=O)[O-])c1[N+](=O)[O-]</smiles>

\section{(Z)-methyl 2-(4-bromo-2,3-dinitrophenyl)but-2-enoate (301)}

To a solution of $\mathbf{2 5 1}^{\mathrm{a}}$ (309 $\mathrm{mg}, 0.95 \mathrm{mmol}$ ) in THF (5 mL) was added (E)-methyl 2 (tributylstannyl)but-2-enoate (599 mg, $1.54 \mathrm{mmol}), \mathrm{AsPh}_{3}(115 \mathrm{mg}, 0.38 \mathrm{mmol})$, $\operatorname{Pd}(\mathrm{dba})_{2}(46.2 \mathrm{mg}, 0.08 \mathrm{mg})$, and $\mathrm{CuI}(187 \mathrm{mg}, 0.98 \mathrm{mmol})$. The resulting solution was 
heated at $80{ }^{\circ} \mathrm{C}$ (42 hours). The resulting brown solution was diluted with $\mathrm{Et}_{2} \mathrm{O}(25 \mathrm{~mL})$ and washed with $10 \%$ aq. $\mathrm{NH}_{4} \mathrm{OH}(3 \times 20 \mathrm{~mL}), \mathrm{H}_{2} \mathrm{O}(20 \mathrm{~mL})$, and brine $(20 \mathrm{~mL})$. The organic phase was dried $\left(\mathrm{MgSO}_{4}\right)$, filtered, and solvents were removed under reduced pressure. The resulting brown oil was purified using column chromatography (hexanes/EtOAc, $7: 3)$ to afford $\mathbf{3 0 1}(101 \mathrm{mg}, 0.29 \mathrm{mmol}, 31 \%)$ as a yellow solid $(\mathrm{mp}=$ 132-134 $\left.{ }^{\circ} \mathrm{C}\right) .{ }^{1} \mathrm{HNMR}(400 \mathrm{MHz}) 7.86(\mathrm{~d}, J=8.4 \mathrm{~Hz}, 1 \mathrm{H}), 7.37(\mathrm{~d}, J=8.4 \mathrm{~Hz}, 1 \mathrm{H}), 6.50$ $(\mathrm{q}, J=7.6 \mathrm{~Hz}, 1 \mathrm{H}), 3.69(\mathrm{~s}, 3 \mathrm{H}), 2.30(\mathrm{~d}, J=7.6 \mathrm{~Hz}, 3 \mathrm{H}) ;{ }^{13} \mathrm{C} 164.0,147.0,136.8,136.6$, 134.8, 134.2, 131.7, 128.3, 121.5, 114.3, 51.8, 16.4; IR (ATR) 3089, 2956, 1726, 1534, $1350,1215 \mathrm{~cm}^{-1}$

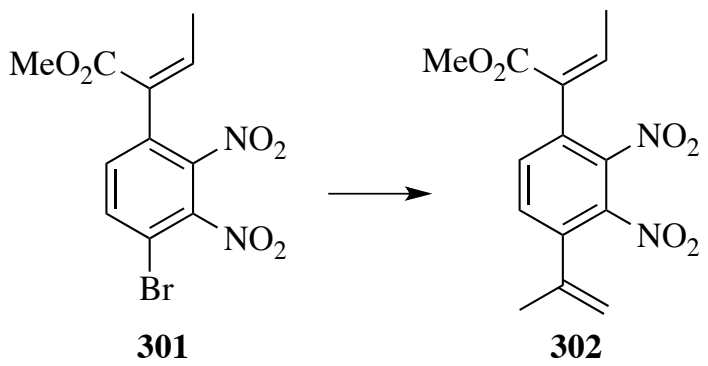

\section{(Z)-methyl 2-(2,3-dinitro-4-(prop-1-en-2-yl)phenyl)but-2-enoate (302)}

Reaction of $\mathbf{3 0 1}$ (99.0 mg, $0.29 \mathrm{mmol})$, tributyl(prop-1-en-2-yl)stannane (120 mg, 0.36 $\mathrm{mmol}), \mathrm{PPh}_{3}(15.8 \mathrm{mg}, 0.06 \mathrm{mmol})$, and $\mathrm{Pd}(\mathrm{dba})_{2}(8.8 \mathrm{mg}, 0.15 \mathrm{mmol})$ in toluene $(3 \mathrm{~mL})$ at $110{ }^{\circ} \mathrm{C}$ (24 hours) as described for 271 afforded after purification using column chromatography (hexanes/EtOAc, 8:2) $302(57.6 \mathrm{mg}, 0.19 \mathrm{mmol}, 66 \%)$ as a yellow oil. ${ }^{1} \mathrm{H}$ NMR $(400 \mathrm{MHz}) \delta 7.54(\mathrm{~d}, J=8.0 \mathrm{~Hz}, 1 \mathrm{H}), 7.36(\mathrm{~d}, J=8.0 \mathrm{~Hz}, 1 \mathrm{H}), 7.34(\mathrm{q}, J=7.2$ $\mathrm{Hz}, 1 \mathrm{H}), 5.30(\mathrm{~s}, 1 \mathrm{H}), 5.08(\mathrm{~s}, 1 \mathrm{H}), 3.73(\mathrm{~s}, 3 \mathrm{H}), 2.14(\mathrm{~s}, 3 \mathrm{H}), 1.77(\mathrm{~d}, J=6.8 \mathrm{~Hz}, 3 \mathrm{H}) ;{ }^{13} \mathrm{C}$ NMR (100 MHz) $\delta 164.9,146.4,144.1,142.7,139.0,138.3,133.7,132.0,129.9,128.8$, 118.4, 52.3, 23.1, 15.7; IR (ATR) 2952, 1726, 1552, 1354, $1205 \mathrm{~cm}^{-1}$; 


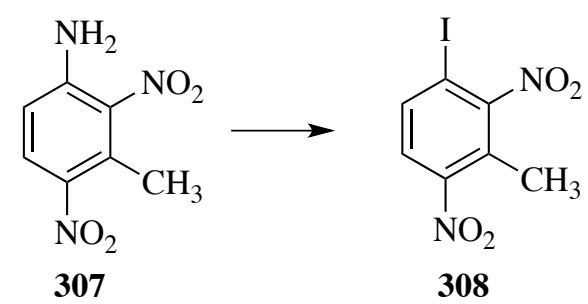

1-iodo-3-methyl-2,4-dinitrobenzene (308): To a solution of 2,4-dinitro-3-methylaniline $307^{156}(492 \mathrm{mg}, 2.5 \mathrm{mmol})$ in $\mathrm{AcOH}(8 \mathrm{~mL})$ cooled to $10{ }^{\circ} \mathrm{C}$, slowly added a solution of $\mathrm{NaNO}_{2}$ (226 mg, $3.3 \mathrm{mmol}$ ) in $\mathrm{H}_{2} \mathrm{SO}_{4}$ (conc., $1.5 \mathrm{~mL}$ ). Resulting red/brown solution stirred at $10{ }^{\circ} \mathrm{C}$ for 1 hour before adding a solution of $\mathrm{KI}(600 \mathrm{mg}, 3.6 \mathrm{mmol})$ in $\mathrm{H}_{2} \mathrm{O}(5$ $\mathrm{mL}$ ). Resulting brown suspension heated at $50{ }^{\circ} \mathrm{C}$ for 15 minutes. Suspension neutralized with $\mathrm{NaHCO}_{3}$ (sat. aq.) and extracted with EtOAc $(3 \times 30 \mathrm{~mL})$. Combined organic layers were washed with $\mathrm{NaHSO}_{3}$ (sat. aq., $50 \mathrm{~mL}$ ) and brine $(50 \mathrm{~mL})$, then organic layers dried $\left(\mathrm{MgSO}_{4}\right)$, filtered, and solvents removed under reduced pressure. The resulting red solid was purified using column chromatography (hexanes/EtOAc, 8:2) to afford $308(647 \mathrm{mg}, 2.10 \mathrm{mmol}, 86 \%)$ as an orange solid $\left(\mathrm{mp}=91-92{ }^{\circ} \mathrm{C}\right)\left(\mathrm{Lit} .90^{\circ} \mathrm{C}\right)^{157}$ ${ }^{1} \mathrm{HNMR}(600 \mathrm{MHZ}) \delta 7.93(\mathrm{~d}, J=9.0 \mathrm{~Hz}, 1 \mathrm{H}), 7.71(\mathrm{~d}, J=9.0 \mathrm{~Hz}, 1 \mathrm{H}), 2.47(\mathrm{~s}, 3 \mathrm{H})$;

${ }^{13} \mathrm{CNMR} \delta 157.1,149.9,138.5,126.4,126.2,91.1,14.9$; IR (ATR) 1594, 1529, 1344, 902 $\mathrm{cm}^{-1}$<smiles>Cc1c(I)ccc([N+](=O)[O-])c1[N+](=O)[O-]</smiles>

2-(3-iodo-2,6-dinitrophenyl)ethanol (312): To a solution of $\mathbf{3 0 8}(221 \mathrm{mg}, 0.71 \mathrm{mmol})$ in $N, N$-dimethylacetamide $(5 \mathrm{~mL})$ was added para-formaldehyde $(30.0 \mathrm{mg}, 1.00 \mathrm{mmol})$ and potassium hydroxide $(10.2 \mathrm{mg}, 0.18 \mathrm{mmol})$. The resulting brown solution was stirred at 
ambient temperature (5 hours). The resulting brown solution was diluted with EtOAc (20 $\mathrm{mL})$ and washed with $\mathrm{H}_{2} \mathrm{O}(4 \times 25 \mathrm{~mL})$ and brine $(25 \mathrm{~mL})$. The organic phase was dried $\left(\mathrm{MgSO}_{4}\right)$, filtered, and solvents were removed under reduced pressure. The resulting orange solid was purified using column chromatography (hexanes/EtOAc, 7:3) to afford 312 (176 mg, $0.52 \mathrm{mmol}, 72 \%)$ as a light yellow solid $\left(\mathrm{mp}=137-140{ }^{\circ} \mathrm{C}\right) .{ }^{1} \mathrm{H}$ NMR (400 MHz) $\delta 7.98(\mathrm{~d}, J=8.4 \mathrm{~Hz}, 1 \mathrm{H}), 7.72(\mathrm{~d}, J=8.4 \mathrm{~Hz}, 1 \mathrm{H}), 3.87(\mathrm{t}, J=6.4 \mathrm{~Hz}, 2 \mathrm{H}), 3.17(\mathrm{t}$, $J=6.4 \mathrm{~Hz}, 2 \mathrm{H}), 2.10(\mathrm{~s}, 1 \mathrm{H}) ;{ }^{13} \mathrm{C} \mathrm{NMR}(100 \mathrm{MHz}) \delta 157.1,150.7,139.2,127.1,126.5$, 91.4, 61.8, 31.2; IR (ATR) 3391, 3083, 1531, 1357, $1031 \mathrm{~cm}^{-1}$;<smiles>C=Cc1c([N+](=O)[O-])cc(I)c([N+](=O)[O-])c1I</smiles>
1-iodo-2,4-dinitro-3-ethenylbenzene (313): To an ice-cooled solution of $\mathbf{3 1 2}$ (164 mg, $0.48 \mathrm{mmol})$ in $\mathrm{N}, \mathrm{N}$-dimethylacetamide $(5 \mathrm{~mL})$ was added methanesulfonyl chloride $(75$ $\mu \mathrm{L}, 0.97 \mathrm{mmol})$ and triethylamine $(200 \mu \mathrm{L}, 1.43 \mathrm{mmol})$. The resulting cloudy solution was stirred while warming to ambient temperature (2 hours), then heated at $90{ }^{\circ} \mathrm{C}(22$ hours). The resulting light brown solution was diluted with EtOAc $(15 \mathrm{~mL})$ and washed with $\mathrm{H}_{2} \mathrm{O}(5 \times 20 \mathrm{~mL})$ and brine $(20 \mathrm{~mL})$. The organic phase was dried $\left(\mathrm{MgSO}_{4}\right)$, filtered, and solvents were removed under reduced pressure. The resulting brown oil was purified using column chromatography (hexanes/EtOAc, 7:3) to afford $\mathbf{3 1 3}$ (118 mg, 0.37 mmol, 77\%) as a yellow solid $\left(\mathrm{mp}=86-88{ }^{\circ} \mathrm{C}\right) .{ }^{1} \mathrm{H}$ NMR $(400 \mathrm{MHz}) \delta 8.02(\mathrm{~d}, J=8.8 \mathrm{~Hz}$, $1 \mathrm{H}), 7.80(\mathrm{~d}, J=8.4 \mathrm{~Hz}, 1 \mathrm{H}), 6.85(\mathrm{dd}, J=18.4,11.6 \mathrm{~Hz}, 1 \mathrm{H}), 5.59(\mathrm{~d}, J=12 \mathrm{~Hz}, 1 \mathrm{H})$, 
$5.49(\mathrm{~d}, J=18.4 \mathrm{~Hz}, 1 \mathrm{H}) ;{ }^{13} \mathrm{C}$ NMR $(100 \mathrm{MHz}) \delta 148.2,140.1,139.7,127.9,126.8$,

125.8, 123.4, 91.6; IR (ATR) 30.87, 2891, 1522, 1337, $837 \mathrm{~cm}^{-1}$;<smiles>C=Cc1c([N+](=O)[O-])ccc(C(=C)C)c1[N+](=O)[O-]</smiles>

1,3-dinitro-4-(prop-1-en-2-yl)-2-ethenylbenzene (314): Reaction of 313 (109 mg, 0.34 mmol), tributyl(prop-1-en-2-yl)stannane (132 mg, $0.40 \mathrm{mmol}), \mathrm{PPh}_{3}(18.2 \mathrm{mg}, 0.07$ mmol $), \operatorname{Pd}(\mathrm{dba})_{2}(9.9 \mathrm{mg}, 0.02 \mathrm{mmol})$, and BHT (2 pieces) in dioxane $(3 \mathrm{~mL})$ at $75^{\circ} \mathrm{C}$ (23 hours) as described for 271 afforded after purification using column chromatography (hexanes/EtOAc, 85:15) $314(51.2 \mathrm{mg}, 0.22 \mathrm{mmol}, 64 \%)$ as a yellow solid ( $\mathrm{mp}=48-50$ $\left.{ }^{\circ} \mathrm{C}\right) .{ }^{1} \mathrm{H}$ NMR $(400 \mathrm{MHz}) \delta 8.04(\mathrm{~d}, J=8.4 \mathrm{~Hz}, 1 \mathrm{H}), 7.42(\mathrm{~d}, J=8.8 \mathrm{~Hz}, 1 \mathrm{H}), 6.90(\mathrm{dd}, J=$ $18.0,12.0 \mathrm{~Hz}, 1 \mathrm{H}), 5.55(\mathrm{~d}, J=12.0 \mathrm{~Hz}, 1 \mathrm{H}), 5.45(\mathrm{dd}, J=17.6,0.8 \mathrm{~Hz}, 1 \mathrm{H}), 5.31(\mathrm{~m}$, 1H), $5.07(\mathrm{~m}, 1 \mathrm{H}), 2.10(\mathrm{~s}, 3 \mathrm{H}) ;{ }^{13} \mathrm{C} \mathrm{NMR}(100 \mathrm{MHz}) \delta 149.8,147.0,140.9,138.9,128.7$, 127.3, 127.2, 125.2, 122.4, 118.8, 23.3; IR (ATR) 3096, 2981, 1524, 1345, $918 \mathrm{~cm}^{-1}$;<smiles>C=C(C)c1cc(C(=C)C)c([N+](=O)[O-])cc1[N+](=O)[O-]</smiles>

260

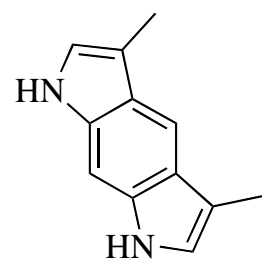

326

3,5-dimethyl-1,7-dihydropyrrolo[3,2-f]indole (326) In an oven-dried ACE glass

pressure tube was mixed 260 (47.5 mg, $0.19 \mathrm{mmol}), 1,10$-phenanthroline $(6.4 \mathrm{mg}, 0.032$ mmol), dppp (6.2 mg, $0.015 \mathrm{mmol})$, and Pd(dba) 2 (7.8 mg, $0.013 \mathrm{mmol})$ and dry DMF (2 $\mathrm{mL}$ ). The tube was fitted with a pressure head, and the vessel was saturated with $\mathrm{CO}$ (three cycles, $6 \mathrm{~atm}$ ). The tube was heated at $120{ }^{\circ} \mathrm{C}$ for 48 hours, then the solvent was 
removed by bulb-to-bulb and the resulting dark brown crude mixture afforded after column chromatography (hexanes/EtOAc, 1:1) $326(25.2 \mathrm{mg}, 0.14 \mathrm{mmol}, 73 \%)$ as a brown solid $\left(\mathrm{mp}=195-200{ }^{\circ} \mathrm{C}\right) .{ }^{1} \mathrm{H} \mathrm{NMR}(600 \mathrm{MHz}) \delta 7.63(\mathrm{~s}, 1 \mathrm{H}), 7.56(\mathrm{~s}, 2 \mathrm{H}), 7.18(\mathrm{~s}$, 1H), $6.91(\mathrm{~s}, 2 \mathrm{H}), 2.39(\mathrm{~s}, 3 \mathrm{H}) ;{ }^{13} \mathrm{C}$ NMR $\delta 135.3,124.7,121.1,111.0,106.7,90.9,10.1$; IR (ATR) 3275, 2920, 1668, 1631, 1507, $1255 \mathrm{~cm}^{-1}$;

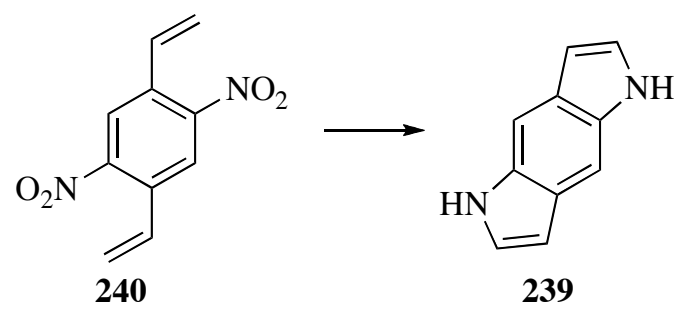

1,5-dihydropyrrolo[2,3-f]indole (239) Reaction of $\mathbf{2 4 0}$ (29.6 mg, $0.13 \mathrm{mmol}$ ), 1,10phenanthroline (3.4 mg, $0.017 \mathrm{mmol})$, dppp (3.5 mg, $0.008 \mathrm{mmol})$, and $\mathrm{Pd}(\mathrm{dba})_{2}(5.0 \mathrm{mg}$, $0.008 \mathrm{mmol}$ ) in DMF ( $2 \mathrm{~mL}$ ) under carbon monoxide (3 cycles, 6 atm) for 87 hours as described for $\mathbf{3 2 6}$ afforded after column chromatography (hexanes/EtOAc, 85:15) 239 $(15.9 \mathrm{mg}, 0.10 \mathrm{mmol}, 78 \%)$ as an off white solid. Spectral data were in accordance with literature values. ${ }^{106}$<smiles>C=C(C)c1ccc([N+](=O)[O-])c(/C=C\O)c1[N+](=O)[O-]</smiles>

314

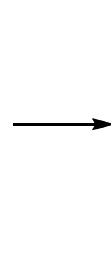

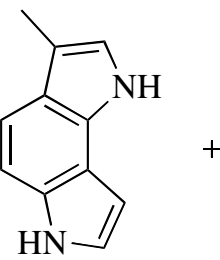

330<smiles>C=C(C)c1ccc2[nH]ccc2c1[N+](=O)[O-]</smiles>

329

3-methyl-2,6-dihydropyrrolo[2,3-g]indole (330) Reaction of $\mathbf{3 1 4}$ (87.9 mg, $0.37 \mathrm{mmol})$, 1,10-phenanthroline ( $9.3 \mathrm{mg}, 0.047 \mathrm{mmol})$, dppp (9.2 mg, $0.022 \mathrm{mmol})$, and $\mathrm{Pd}(\mathrm{dba})_{2}$ (13.8 mg, $0.024 \mathrm{mmol}$ ) in DMF (3 mL) under carbon monoxide (3 cycles, $6 \mathrm{~atm}$ ) for 110 hours as described for $\mathbf{3 2 6}$ afforded after column chromatography (hexanes/EtOAc, 1:1) in order of elution $\mathbf{3 3 0}(19.4 \mathrm{mg}, 0.11 \mathrm{mmol}, 30 \%)$ as brown solid $\left(\mathrm{mp}=89-91^{\circ} \mathrm{C}\right)$ and 
329 (35.5 mg, $0.17 \mathrm{mmol}, 47 \%$ ) as a brown solid. Spectral data for $330{ }^{1} \mathrm{H}$ NMR (400 MHz) $\delta 8.24($ br s, 1H), $8.11($ br s, 1H), $7.39(\mathrm{~d}, J=8.4 \mathrm{~Hz}, 1 \mathrm{H}), 7.18(\mathrm{dd}, J=8.8 \mathrm{~Hz}, 0.8$ $\mathrm{Hz}, 1 \mathrm{H}), 7.14(\mathrm{t}, J=2.8 \mathrm{~Hz}, 1 \mathrm{H}), 6.87(\mathrm{~m}, 1 \mathrm{H}), 6.63(\mathrm{~m}, 1 \mathrm{H}), 2.38(\mathrm{~s}, 3 \mathrm{H}),{ }^{13} \mathrm{C} \mathrm{NMR}(100$ MHz) $\delta 133.0,128.9,128.4,121.9,121.0,117.8,113.9,112.7,104.5,98.5,10.1 ;$ IR (ATR) $3398,2924,1632,1402,724 \mathrm{~cm}^{-1}$;

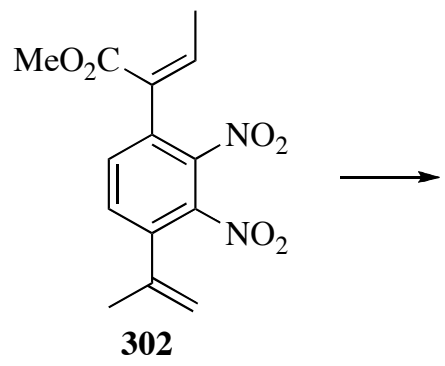

Methyl 2,6-dimethyl-1,8-dihydropyrrolo[3,2-g]indole-3-carboxylate (331): Reaction of 302 (46.9 mg, $0.15 \mathrm{mmol}$ ), 1,10-phenanthroline (3.8 mg, $0.019 \mathrm{mmol}$ ), dppp (4.1 mg, $0.010 \mathrm{mmol})$, and $\mathrm{Pd}(\mathrm{dba})_{2}(5.6 \mathrm{mg}, 0.010 \mathrm{mmol})$ in $\mathrm{DMF}(2 \mathrm{~mL})$ under carbon monoxide ( 3 cycles, 6 atm) for 110 hours as described for $\mathbf{3 2 6}$ afforded after column chromatography (hexanes/EtOAc, 1:1) 331 (28.6 mg, $0.12 \mathrm{mmol}, 77 \%$ ) as an off-white solid that turned green/brown upon dissolving in $\mathrm{CDCl}_{3} .{ }^{1} \mathrm{H}$ NMR (400 MHz, DMSO) $\delta$ $10.75(\mathrm{~s}, 1 \mathrm{H}), 9.57(\mathrm{~s}, 1 \mathrm{H}), 7.76(\mathrm{~d}, J=8.4 \mathrm{~Hz}, 1 \mathrm{H}), 7.32(\mathrm{~d}, J=8.4 \mathrm{~Hz}, 1 \mathrm{H}), 3.92(\mathrm{~s}, 3 \mathrm{H})$, $2.75(\mathrm{~s}, 3 \mathrm{H}), 2.36(\mathrm{~s}, 3 \mathrm{H}) ;{ }^{13} \mathrm{C}$ NMR $(100 \mathrm{MHz}) \delta 166.6,139.9,123.5,122.1,121.8$, 120.9, 118.3, 112.4, 112.2, 111.6, 104.3, 50.1, 113.9, 9.6; IR (ATR) 3381, 1655, 1446, $1419,1198,1109 \mathrm{~cm}^{-1}$ 


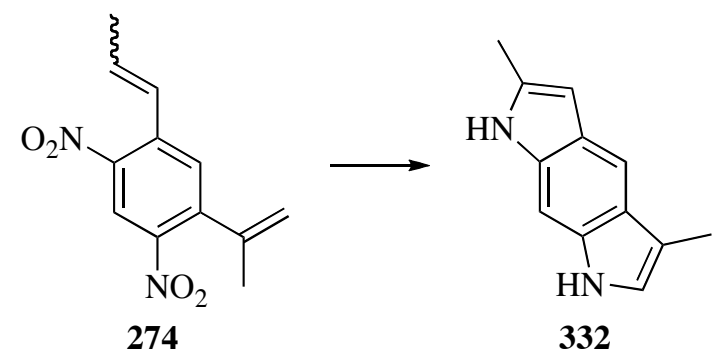

2,5-dimethyl-1,7-dihydropyrrolo[3,2-f]indole (332): Reaction of $\mathbf{2 7 4}$ (67.8 mg, 0.27 mmol), 1,10-phenanthroline (6.5 mg, $0.032 \mathrm{mmol})$, dppp (6.8 mg, $0.016 \mathrm{mmol})$, and $\mathrm{Pd}(\mathrm{dba})_{2}(10.0 \mathrm{mg}, 0.017 \mathrm{mmol})$ in DMF $(2.5 \mathrm{~mL})$ under carbon monoxide (3 cycles, 6 atm) for 110 hours as described for $\mathbf{3 2 6}$ afforded after column chromatography (hexanes/EtOAc, 1:1) $332(12.3 \mathrm{mg}, 0.07 \mathrm{mmol}, 24 \%)$ as a brown solid (mp= 183-185 $\left.{ }^{\circ} \mathrm{C}\right) .{ }^{1} \mathrm{H}$ NMR $(600 \mathrm{MHz}) \delta 11.9(\mathrm{br} \mathrm{s}, 1 \mathrm{H}), 8.76(\mathrm{~s}, 1 \mathrm{H}), 8.50(\mathrm{~d}, J=1.8 \mathrm{~Hz}, 1 \mathrm{H}), 8.28(\mathrm{br}$ $\mathrm{s}, 1 \mathrm{H}), 8.12(\mathrm{~s}, 1 \mathrm{H}), 6.25(\mathrm{~d}, J=1.2 \mathrm{~Hz}, 1 \mathrm{H}), 2.72(\mathrm{~s}, 3 \mathrm{H}), 2.45(\mathrm{~s}, 3 \mathrm{H}) ;{ }^{13} \mathrm{C}$ NMR $(150$ MHz) $\delta 159.8,139.5,137.5,134.5,128.9,124.8,124.4,116.7,102.8,101.4,14.1,13.7$; IR (ATR) 3197, 2924, 2857, 1677, 1625, $1504 \mathrm{~cm}^{-1}$;

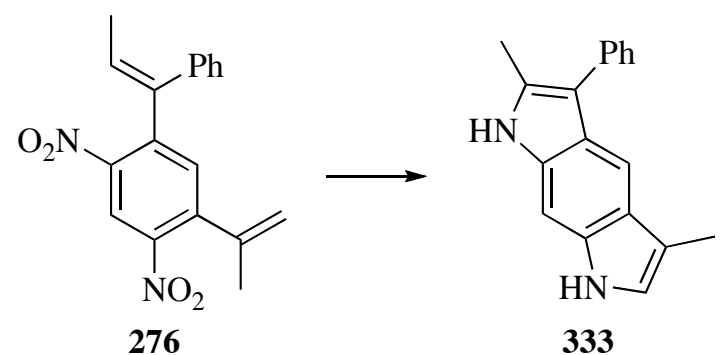

2,5-dimethyl-3-phenyl-1,7-dihydropyrrolo[3,2-f]indole (333): Reaction of 276 (25.6 $\mathrm{mg}, 0.079 \mathrm{mmol}), \mathrm{Pd}(\mathrm{dba})_{2}(3.2 \mathrm{mg}, 0.005 \mathrm{mmol}), 1,10$-phenanthroline (2.5 mg, 0.012 mmol), dppp (2.4 mg, $0.006 \mathrm{mmol})$, in DMF (1.5 mL) under carbon monoxide (3 cycles, $6 \mathrm{~atm}$ ) for 120 hours as described for $\mathbf{3 2 6}$ afforded after column chromatography (hexanes: EtOAc, 1:1) $\mathbf{3 3 3}$ (4.8 $\mathrm{mg}, 0.02 \mathrm{mmol}, 17 \%)$ as a brown residue. ${ }^{1} \mathrm{H}$ NMR (600 MHz) $\delta 11.95(\mathrm{~s}, 1 \mathrm{H}), 8.78(\mathrm{~s}, 1 \mathrm{H}), 8.49(\mathrm{~s}, 1 \mathrm{H}), 8.40(\mathrm{~s}, 1 \mathrm{H}), 8.20(\mathrm{~s}, 1 \mathrm{H}), 7.53-7.45(\mathrm{~m}$, 
5H), $3.28(\mathrm{~s}, 3 \mathrm{H}), 2.67(\mathrm{~s}, 3 \mathrm{H}) ;{ }^{13} \mathrm{C}$ NMR $(150 \mathrm{MHz}) \delta 159.8,134.3,131.2,130.0,129.8$, 129.3, 128.8, 128.5, 127.6, 127.1, 126.4, 125.3, 124.0, 122.1, 15.5, 12.5; IR (ATR) 3383, $2918,2850,1706,1602,1495 \mathrm{~cm}^{-1}$ 
7.5 Supporting Information Chapter 6.1: 2-Nitrobenzaldehyde Brominations<smiles>[13CH2]c1ccc(Br)c([N+](=O)[O-])c1Br</smiles>

\section{Bromination of 2-nitrobenzaldehyde (338) using 0.75 equivalents of NBS. To a}

solution of 338 ( $986 \mathrm{mg}, 6.53 \mathrm{mmol}$ ) in $\mathrm{H}_{2} \mathrm{SO}_{4}$ (conc. $5.0 \mathrm{~mL}$ ) was added $\mathrm{N}$ bromosuccinimide (NBS) $(878 \mathrm{mg}, 4.93 \mathrm{mmol})$. The resulting mixture was stirred at ambient temperature $(3 \mathrm{~h})$. The reaction was quenched with ice and extracted with ethyl acetate $(3 \times 20 \mathrm{~mL})$. The combined organic phases were washed with saturated $\mathrm{NaCl}$ (aqueous, $30 \mathrm{~mL}$ ), dried $\left(\mathrm{MgSO}_{4}\right)$, filtered through a silica gel plug, and the solvents were evaporated under reduced pressure. The resulting brown oil was purified by column chromatography (hexanes/EtOAc, 8:2) to afford the following fractions in order of elution: (I) 4-bromo-2-nitrobenzaldehyde (339) ${ }^{158}$ (216 mg, 19\%), 5-bromo-2nitrobenzaldehyde (341) $)^{159}$ (134 mg, 12\%), and 4,5-dibromo-2-nitrobenzaldehyde (344) (18 mg, 1\%) as a yellow solid; (II) 6-bromo-2-nitrobenzaldehyde (342) (268 mg, 24\%), ${ }^{160}$ 3,6-dibromo-2-nitrobenzaldehyde (345) (25 mg, 2\%), ${ }^{161}$ and $\mathbf{3 3 8}(409 \mathrm{mg}, 41 \%)$ as a yellow oil; (III) 3-bromo-2-nitrobenzaldehyde (340) $)^{162}$ as a yellow solid (60 mg, 0.26 mmol, $4 \%)$. 340: $\mathrm{mp}=73-74{ }^{\circ} \mathrm{C}\left(\right.$ Lit. $\left.\mathrm{mp}=75-77^{\circ} \mathrm{C}\right)$.

Bromination of 338 using 1.25 equivalents of NBS. Reaction of $\mathbf{3 3 8}(1.01 \mathrm{~g}, 6.71$ mmol) and NBS (1.48 g, $8.32 \mathrm{mmol})$ in $\mathrm{H}_{2} \mathrm{SO}_{4}(5.0 \mathrm{~mL})$ as described above afforded the 
following fractions in order of elution: (I) 344 (28 $\mathrm{mg}, 0.09 \mathrm{mmol}, 1 \%)$ as an off-white solid; (II) 339 (328 mg, 21\%), 341 (140 mg, 9\%), and 3,4-dibromo-2-nitrobenzaldehyde (343) (94 mg, 5\%); (III) 342 (310 mg, 20\%), 345 (65 mg, 3\%), 338 (168 mg, 17\%); (IV) 340 (54 mg, $0.23 \mathrm{mmol}, 4 \%)$.

Data for 343: $\mathrm{mp}=105-106{ }^{\circ} \mathrm{C}\left(\right.$ Lit. $\left.\mathrm{mp}=105-107{ }^{\circ} \mathrm{C}\right) ;{ }^{1631} \mathrm{H}$ NMR $\delta 10.39(\mathrm{~s}, 1 \mathrm{H}), 8.39$ (s, 1H), $8.19(\mathrm{~s}, 1 \mathrm{H}) ;{ }^{13} \mathrm{C}$ NMR $\delta$ 185.9, 147.7, 134.3, 132.5, 130.8, 130.4, 129.6; IR (ATR) $3088,2890,1687,1525,1338,1178 \mathrm{~cm}^{-1}$

Bromination of 338 using 1.25 equivalents of NBS at $60^{\circ} \mathrm{C}$. Reaction of $338(459 \mathrm{mg}$, $3.03 \mathrm{mmol})$ and NBS $(677 \mathrm{mg}, 3.80 \mathrm{mmol})$ in $\mathrm{H}_{2} \mathrm{SO}_{4}(3.0 \mathrm{~mL})$ at $60{ }^{\circ} \mathrm{C}$ as described above afforded the following fractions in order of elution: (I) 344 (21 $\mathrm{mg}, 0.07 \mathrm{mmol}$, 2\%); (II) 339 (66 mg, 9\%) and $\mathbf{3 4 1}$ (24 mg, 3\%); (III): 339 (5 mg, 0.7\%) and $\mathbf{3 4 3}$ (85 mg, 9\%); (IV) 342 (59 mg, 8\%), 345 (13 mg, 1\%), ${ }^{162}$ and 338 (18 mg, 4\%); (V) 340 (20 mg, $0.09 \mathrm{mmol}, 3 \%)$.

Bromination of 338 using 2.5 equivalents of NBS. Reaction of $\mathbf{3 3 8}$ (504 mg, 3.33 mmol) and NBS (1.48 g, $8.30 \mathrm{mmol})$ in $\mathrm{H}_{2} \mathrm{SO}_{4}(3.0 \mathrm{~mL})$ as described above provided after purification by chromatography (hexanes/EtOAc, $85: 15$ ) the following fractions in order of elution: (I) 344 (65 mg, $0.21 \mathrm{mmol}, 9 \%)$; (II) 339 (58 mg, 8\%), 341 (153 mg, 20\%), and $\mathbf{3 4 3}$ (128 mg, 12\%); (III) $\mathbf{3 4 2 ~ ( 1 0 8 ~ m g , ~ 1 4 \% ) , ~} 345$ (129 mg, 13\%), and 338 (17 mg, 3\%); (IV) 340 (57 mg, $0.25 \mathrm{mmol}, 8 \%)$.

Bromination of $\mathbf{3 3 8}$ using $\mathbf{5 . 0}$ equivalents of NBS. Reaction of $\mathbf{3 3 8}$ (504 mg, 3.33 mmol) and $N$-bromosuccinimide $(2.94 \mathrm{~g}, 16.5 \mathrm{mmol})$ in $\mathrm{H}_{2} \mathrm{SO}_{4}(3.0 \mathrm{~mL})$ as described above afforded the following in order of elution: (I) 344 (193 mg, $0.62 \mathrm{mmol}, 19 \%)$; (II) 343 (247 mg, 24\%) and 339 (32 mg, 4\%); (III) 345 (174 mg, $0.56 \mathrm{mmol}, 17 \%)$. 
Data for 343: $\mathrm{mp}=98-99{ }^{\circ} \mathrm{C}$ (white solid); ${ }^{1} \mathrm{H}$ NMR $\delta 10.21(\mathrm{~s}, 1 \mathrm{H}), 7.94(\mathrm{~d}, J=9.0 \mathrm{~Hz}$, 1H), $7.93(\mathrm{~d}, J=8.4 \mathrm{~Hz}, 1 \mathrm{H}) ;{ }^{13} \mathrm{C} \mathrm{NMR} \delta 187.8,146.2,135.9,135.5,133.6,123.9,123.8$;

IR (ATR) 3112, 3075, 1702, 1522, $1342 \mathrm{~cm}^{-1}$; HRMS (ESI) calcd for $\mathrm{C}_{7} \mathrm{H}_{3} \mathrm{Br}_{2} \mathrm{NO}_{3}$ $\left(\mathrm{M}+\mathrm{H}^{+}\right)$307.8552; found 307.8556.

3-Bromo-2-nitrobenzylalcohol (346). ${ }^{164}$ To a solution of $\mathbf{3 4 0}(105 \mathrm{mg}, 0.46 \mathrm{mmol})$ in THF ( $3 \mathrm{~mL}$ ) and $\mathrm{H}_{2} \mathrm{O}(1 \mathrm{~mL})$ was added sodium borohydride $(37 \mathrm{mg}, 0.98 \mathrm{mmol})$. The resulting solution was stirred at ambient temperature (20 min), water was added $(20 \mathrm{~mL})$ and the mixture was extracted with EtOAc $(3 \times 20 \mathrm{~mL})$. The combined organic phases were dried $\left(\mathrm{MgSO}_{4}\right)$, filtered, and the solvents were evaporated under reduced pressure to give 346 ( $87 \mathrm{mg}, 0.37 \mathrm{mmol}, 81 \%$ ) as a pale yellow oil. No further purification was required. Spectral data are in accordance with literature data.

\section{4-Bromo-2-nitrobenzylalcohol (347) ${ }^{165}$ and 5-bromo-2-nitrobenzylalcohol (348). ${ }^{164}$}

Reaction of a mixture of $\mathbf{3 3 9}$ and $\mathbf{3 4 1}$ (1.40 g, 64:36 ratio, $6.09 \mathrm{mmol})$ in dry THF (15 $\mathrm{mL}$ ) and $\mathrm{H}_{2} \mathrm{O}(5 \mathrm{~mL})$ was added sodium borohydride (405 $\left.\mathrm{mg}, 10.7 \mathrm{mmol}\right)$, as described for 346, afforded after work up and purification by chromatography (hexanes/EtOAc, 8:2) in order of elution 5-bromo-2-nitrobenzylalcohol 348 (476 mg, $2.04 \mathrm{mmol})$ and 4bromo-2-nitrobenzylalcohol 347 (713 mg, $3.07 \mathrm{mmol}$ ) both as faint yellow solids. Spectral data were in accordance with literature data.

\section{Fraction II:}

To a mixture of 6-bromo-2-nitrobenzaldehyde (342), 3,6-dibromo-2-nitrobenzaldehyde (345), and 2-nitrobenzaldehyde (338) (150 mg, $0.68: 0.09: 0.23)$ in THF (5 mL) and $\mathrm{H}_{2} \mathrm{O}(3 \mathrm{~mL})$ was added sodium borohydride $(49.8 \mathrm{mg}, 1.32 \mathrm{mmol})$ as described for $\mathbf{3 4 6}$ afforded after purification by chromatography (hexanes/EtOAc, 8:2) in order of elution: a 
mixture of 6-bromo-2-nitrobenzyl alcohol ${ }^{166}$ and 3,6-dibromo-2-nitrobenzyl alcohol (148 $\mathrm{mg}$ ) followed by 2-nitrobenzyl alcohol ${ }^{167}(36 \mathrm{mg}, 0.23 \mathrm{mmol})$.

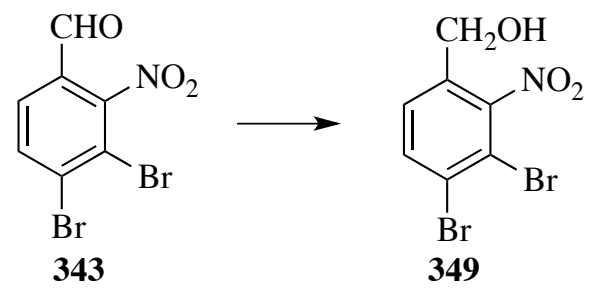

3,4-Dibromo-2-nitrobenzylalcohol (349). Reaction of 343 (101 mg, $0.33 \mathrm{mmol})$ with sodium borohydride (42.6 mg, $1.13 \mathrm{mmol})$ in THF $(3 \mathrm{~mL})$ and $\mathrm{H}_{2} \mathrm{O}(1 \mathrm{~mL})$ as described for 346 afforded after purification by chromatography (hexanes/EtOAc, 7:3) 349 (48 mg, $0.16 \mathrm{mmol}, 47 \%)$ as an off-white solid. $\mathrm{mp} 76-78{ }^{\circ} \mathrm{C}{ }^{1} \mathrm{H} \mathrm{NMR} \delta 7.78(\mathrm{~d}, J=9.0 \mathrm{~Hz}, 1 \mathrm{H})$, $7.69(\mathrm{~d}, J=8.4 \mathrm{~Hz}, 1 \mathrm{H}), 4.99(\mathrm{~s}, 2 \mathrm{H}), 2.72$ (br s, $1 \mathrm{H}) ;{ }^{13} \mathrm{C}$ NMR $\delta 149.8,136.2,133.6$, 132.2, 130.1, 124.0, 62.8; IR (ATR) 3554, 3073, 1518, 1339, $1028 \mathrm{~cm}^{1}$; HRMS (ESI) calcd for $\mathrm{C}_{7} \mathrm{H}_{5} \mathrm{NO}_{3} \mathrm{Br}\left(\mathrm{M}+\mathrm{Na}^{+}\right)$333.8508; found 333.8508.<smiles>O=Cc1cc(Br)c(Br)cc1[N+](=O)[O-]</smiles>

4,5-Dibromo-2-nitrobenzylalcohol (350). Reaction of 344 (292 mg, $0.94 \mathrm{mmol})$ with sodium borohydride $(71.8 \mathrm{mg}, 1.90 \mathrm{mmol})$ in THF $(3 \mathrm{~mL})$ and $\mathrm{H}_{2} \mathrm{O}(1 \mathrm{~mL})$ as described for 346 afforded after purification by chromatography (hexanes/EtOAc, 8:2) 350 (136 mg, $0.44 \mathrm{mmol}, 46 \%$ ) as an off-white solid. mp $112-113{ }^{\circ} \mathrm{C} ;{ }^{1} \mathrm{H}$ NMR $\delta 8.36(\mathrm{~s}, 1 \mathrm{H}), 8.11$ (s, 1H), 4.98 (s, 2H), 2.41 (br s, $1 \mathrm{H}) ;{ }^{13} \mathrm{C}$ NMR $\delta 145.8,137.3,134.1,132.2,129.7,124.1$, 
61.5; IR (ATR) 3225, 3089, 1505, 1336, $1035 \mathrm{~cm}^{1}$; HRMS (ESI) calcd for $\mathrm{C}_{7} \mathrm{H}_{5} \mathrm{NO}_{3} \mathrm{Br}$ $\left(\mathrm{M}+\mathrm{Na}^{+}\right)$333.8508; found 333.8509.

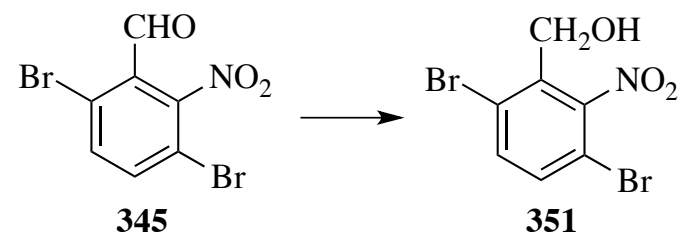

3,6-Dibromo-2-nitrobenzylalcohol (351). Reaction 345 (130 mg, $0.42 \mathrm{mmol})$ and sodium borohydride (30 $\mathrm{mg}, 0.79 \mathrm{mmol})$ in THF ( $3 \mathrm{~mL})$ and $\mathrm{H}_{2} \mathrm{O}(1 \mathrm{~mL})$ as described for 346 afforded after purification by chromatography (hexanes/EtOAc, 7:3) 351 (99 mg, $0.32 \mathrm{mmol}, 76 \%)$ as a white solid. $\mathrm{mp} 75-76{ }^{\circ} \mathrm{C} ;{ }^{1} \mathrm{H}$ NMR $\delta 7.62(\mathrm{~d}, J=9.0 \mathrm{~Hz}, 1 \mathrm{H}), 7.51$ $(\mathrm{d}, J=9.0 \mathrm{~Hz}, 1 \mathrm{H}), 4.76(\mathrm{~d}, J=6.6 \mathrm{~Hz}, 2 \mathrm{H}), 2.23(\mathrm{t}, J=7.2 \mathrm{~Hz}, 1 \mathrm{H}) ;{ }^{13} \mathrm{C}$ NMR $\delta 152.0$, 135.7, 134.3, 133.4, 124.5, 112.5, 61.6; IR (ATR) 3194, 1539, 1356, 1076, $1043 \mathrm{~cm}^{1}$; HRMS (ESI) calcd for $\mathrm{C}_{7} \mathrm{H}_{5} \mathrm{NO}_{3} \mathrm{Br}\left(\mathrm{M}+\mathrm{Na}^{+}\right)$333.8508; found 333.8510 . 
7.6 Supporing Information for Chapter 6.2: Functionalized Indoles<smiles>Cc1ccc(Br)c([N+](=O)[O-])c1CSc1ccc(Br)c([N+](=O)[O-])c1[N+](=O)[O-]</smiles>

2,3-dinitro-4-ethenyltoluene (355): To a solution of $352(155 \mathrm{mg}, 0.60 \mathrm{mmol})$ in toluene (5 mL) was added (ethenyl)tri-n-butylstannane (203 mg, $0.64 \mathrm{mmol}), \mathrm{PPh}_{3}(62.9 \mathrm{mg}$, $0.24 \mathrm{mmol}), \mathrm{Pd}(\mathrm{dba})_{2}(29.3 \mathrm{mg}, 0.05 \mathrm{mmol})$ and the solution was heated at $110^{\circ} \mathrm{C}$ for 66 hours. The resulting brown solution was filtered through Celite, diluted with ethyl acetate $(20 \mathrm{~mL})$, washed with $\mathrm{NH}_{4} \mathrm{OH}(10 \%$ aq, $3 \times 30 \mathrm{~mL}), \mathrm{H}_{2} \mathrm{O}(30 \mathrm{~mL})$, and brine (30 $\mathrm{mL})$. The organic layer was dried $\left(\mathrm{MgSO}_{4}\right)$, filtered, then solvents removed under reduced pressure. Purification using column chromatography (hexanes/EtOAc, 8:2) afforded $355(82.3 \mathrm{mg}, 0.40 \mathrm{mmol}, 66 \%)$ as a brown solid $\left(\mathrm{mp}=68-71{ }^{\circ} \mathrm{C}\right){ }^{1} \mathrm{H}$ NMR $(600$ MHz) $\delta 7.70(\mathrm{~d}, J=8.4 \mathrm{~Hz}, 1 \mathrm{H}), 7.46(\mathrm{~d}, J=8.4 \mathrm{~Hz}, 1 \mathrm{H}), 6.73(\mathrm{dd}, J=17.4,11.4 \mathrm{~Hz}, 1 \mathrm{H})$, $5.87(\mathrm{~d}, J=16.8 \mathrm{~Hz}, 1 \mathrm{H}), 5.60(\mathrm{~d}, J=11.4 \mathrm{~Hz}, 1 \mathrm{H}), 2.46(\mathrm{~s}, 3 \mathrm{H}) ;{ }^{13} \mathrm{C} \mathrm{NMR}(150 \mathrm{MHz}) \delta$ 143.5, 142.1, 134.1, 132.2, 130.3, 129.1, 128.5, 121.5, 18.1; IR (ATR) 3031, 2963, 1612, $1558,1341 \mathrm{~cm}^{-1}$<smiles>Cc1ccc(C=S)c([N+](=O)[O-])c1[N+](=O)[O-]</smiles>

6-methyl-7-nitroindole (356): Reaction of $\mathbf{3 5 5}$ ( $82.3 \mathrm{mg}, 0.40 \mathrm{mmol}), \mathrm{PPh}_{3}(26.3 \mathrm{mg}$, $0.10 \mathrm{mmol}), \mathrm{Pd}(\mathrm{OAc})_{2}(5.7 \mathrm{mg}, 0.02 \mathrm{mmol})$ in $\mathrm{MeCN}(2 \mathrm{~mL})$ under pressurized $\mathrm{CO}(\mathrm{g})$ 
(3 cycles, 90 psi) at $70{ }^{\circ} \mathrm{C}$ for 168 hours afforded after column chromatography (hexanes/EtOAc, 9:1) $\mathbf{3 5 6}$ (3.9 $\mathrm{mg}, 0.02 \mathrm{mmol}, 6 \%$ ) as a yellow solid.

HNMR (270 MHz) $\delta 9.97($ br s, $1 \mathrm{H}), 7.80(\mathrm{~d}, J=7.9 \mathrm{~Hz}, 1 \mathrm{H}), 7.33(\mathrm{t}, J=2.8 \mathrm{~Hz}, 1 \mathrm{H})$, $7.07(\mathrm{~d}, J=7.9 \mathrm{~Hz}, 1 \mathrm{H}), 6.65(\mathrm{t}, J=2.8 \mathrm{~Hz}, 1 \mathrm{H}), 2.83$ (s, 3H); IR (ATR) 3376, 2927, $1475,1312,1267 \mathrm{~cm}^{-1}$<smiles>C=Cc1c([N+](=O)[O-])cc(C)cc1[N+](=O)[O-]</smiles>

3,5-dinitro-4-ethenyltoluene (357): Reaction of 354 (148 mg, $0.57 \mathrm{mmol}$ ), (ethenyl)trin-butylstannane (206 mg, $0.65 \mathrm{mmol}), \mathrm{PPh}_{3}(60.9 \mathrm{mg}, 0.23 \mathrm{mmol}), \mathrm{Pd}(\mathrm{dba})_{2}(26.0 \mathrm{mg}$, $0.04 \mathrm{mmol})$ in toluene $(5 \mathrm{~mL})$ at $110{ }^{\circ} \mathrm{C}$ for 48 hours as described for 355 afforded after column chromatography (hexanes/EtOAc, 8:2) $357(67.2 \mathrm{mg}, 0.32 \mathrm{mmol}, 57 \%)$ as a brown solid (mp= 59-61 $\left.{ }^{\circ} \mathrm{C}\right){ }^{1} \mathrm{H}$ NMR $(600 \mathrm{MHz}) \delta 7.84(\mathrm{~s}, 2 \mathrm{H}), 7.00(\mathrm{dd}, J=17.4,11.4$ $\mathrm{Hz}, 1 \mathrm{H}), 5.49(\mathrm{dd}, J=11.4,0.6 \mathrm{~Hz}, 1 \mathrm{H}), 5.31(\mathrm{dd}, J=18.0,0.6 \mathrm{~Hz}, 1 \mathrm{H}), 2.52(\mathrm{~s}, 3 \mathrm{H}) ;{ }^{13} \mathrm{C}$ NMR (150 MHz) $\delta 149.9,140.1,128.1,127.6,125.8,121.1,20.9$; IR (ATR) 3015, 2971, $1629,1538,1352 \mathrm{~cm}^{-1}$<smiles>C=Cc1c([N+](=O)[O-])cc(C)cc1[N+](=O)[O-]</smiles>

357<smiles>Cc1cc([N+](=O)[O-])c2cc[nH]c2c1</smiles>
358

6-methyl-4-nitroindole (358): Reaction of $\mathbf{3 5 7}(67.2 \mathrm{mg}, 0.32 \mathrm{mmol}), \mathrm{PPh}_{3}(21.0 \mathrm{mg}$, $0.08 \mathrm{mmol}), \mathrm{Pd}(\mathrm{OAc})_{2}(4.4 \mathrm{mg}, 0.02 \mathrm{mmol})$ in $\mathrm{MeCN}(2 \mathrm{~mL})$ under pressurized $\mathrm{CO}(\mathrm{g})$ (3 cycles, $90 \mathrm{psi}$ ) at $70{ }^{\circ} \mathrm{C}$ for 18 hours afforded after column chromatography 
(hexanes/EtOAc, 9:1) 358 (34.0 mg, $0.19 \mathrm{mmol}, 60 \%)$ as an orange solid (mp=169-171 $\left.{ }^{\circ} \mathrm{C}\right)$; HNMR $8.52(\mathrm{br} \mathrm{s}, 1 \mathrm{H}), 8.00(\mathrm{~s}, 1 \mathrm{H}), 7.52(\mathrm{~s}, 1 \mathrm{H}), 7.40(\mathrm{t}, J=2.4 \mathrm{~Hz}, 1 \mathrm{H}), 7.24(\mathrm{t}, J=$ $2.4 \mathrm{~Hz}, 1 \mathrm{H}), 2.54$ (s, 3H); CNMR 140.1, 138.2, 131.3, 127.8, 120.2, 119.0, 118.1, 103.2, 21.3; IR (ATR) 3364, 3115, 2915, 1504, 1346, $1277 \mathrm{~cm}^{-1}$;<smiles>COc1cccc(Br)c1</smiles>

359 360<smiles>COc1ccc([N+](=O)[O-])c(Br)c1[N+](=O)[O-]</smiles>

361

5-bromo-2,4-dinitroanisole (360) ${ }^{168}$ and 3-bromo-2,4-dinitroanisole (361): To fuming nitric acid (5 mL) cooled to $-78^{\circ} \mathrm{C}$ was added $\mathbf{3 5 9}(309 \mathrm{mg}, 1.7 \mathrm{mmol})$. Solution removed from cold bath upon addition of starting material and allowed to warm to ambient temperature over 30 minutes. Resulting yellow solution poured over $100 \mathrm{~mL}$ ice, causing off-white solid to form. Aqueous mixture extracted with ethyl acetate (3 x $40 \mathrm{~mL}$ ), then combined organic layers washed with sodium carbonate (sat. aq, $50 \mathrm{~mL}$ ), dried over $\mathrm{MgSO}_{4}$, filtered, and solvents removed to give yellow solid. Purification via column chromatography (hexanes/EtOAc, 85:15) afforded in order of elution 361 (white solid, $26.1 \mathrm{mg}, 6 \%$ ) and $\mathbf{3 6 0}$ (off-white solid, $407 \mathrm{mg}, 89 \%)^{168}$. Spectral data for $\mathbf{3 6 1}$ : ${ }^{1}$ HNMR $\delta 7.97(\mathrm{~d}, J=8.9 \mathrm{~Hz}, 1 \mathrm{H}), 7.57(\mathrm{~d}, J=8.9 \mathrm{~Hz}, 1 \mathrm{H}), 4.04(\mathrm{~s}, 3 \mathrm{H})$;<smiles>COc1cccc(I)c1</smiles>

5-iodo-2,4-dinitroanisole (363) 3-iodo-2,4-dinitroanisole (364): To a solution of fuming nitric acid $(10 \mathrm{~mL})$ cooled to $-78^{\circ} \mathrm{C}$ was added $\mathbf{3 6 2}(3.02 \mathrm{~g}, 12.9 \mathrm{mmol})$. Solution removed from cold bath upon addition of starting material and allowed to warm 
to ambient temperature over 30 minutes. Resulting brown solution poured over $100 \mathrm{~mL}$ ice, causing off-white solid to form. Aqueous mixture extracted with ethyl acetate (3 x $40 \mathrm{~mL}$ ), then combined organic layers washed with sodium carbonate (sat. aq, $50 \mathrm{~mL}$ ), dried over $\mathrm{MgSO}_{4}$, filtered, and solvents removed to give yellow solid. Purification via column chromatography (hexanes/EtOAc , 7:3) afforded in order of elution 364 (yellow solid, $454.6 \mathrm{mg}, 10.9 \%$ ) and $\mathbf{3 6 3}$ (yellow solid, $742.6 \mathrm{mg}, 17.7 \%$ )

Spectral data for 364: $\mathrm{mp}=154-157{ }^{\circ} \mathrm{C},{ }^{1} \mathrm{HNMR} \delta 8.08(\mathrm{~d}, J=9.0 \mathrm{~Hz}, 1 \mathrm{H}), 7.13(\mathrm{~d}, J=$ $9.6 \mathrm{~Hz}, 1 \mathrm{H}), 4.01(\mathrm{~s}, 3 \mathrm{H}) ;{ }^{13} \mathrm{CNMR} \delta 154.0,148.6,146.3,128.0,112.5,82.0,57.6$; IR (ATR) $1575,1520,1342,1283,1020 \mathrm{~cm}^{-1}$

Spectral data for 363: $\mathrm{mp}=113-115{ }^{\circ} \mathrm{C},{ }^{1} \mathrm{HNMR} \delta 8.58(s, 1 H), 7.76(s, 1 H), 4.09(s$, $3 H) ;{ }^{13} \mathrm{C}$ NMR $\delta$ 155.0, 144.2, 138.2, 127.3, 123.4, 94.3, 57.9; IR (ATR) 1594, 1526, 1339, 1247, $987 \mathrm{~cm}^{-1}$; HRMS (ESI) calc'd for $\mathrm{C}_{7} \mathrm{H}_{5} \mathrm{~N}_{2} \mathrm{O}_{5} \mathrm{I}(\mathrm{M}+\mathrm{Na})=346.9135$, found 346.9139.

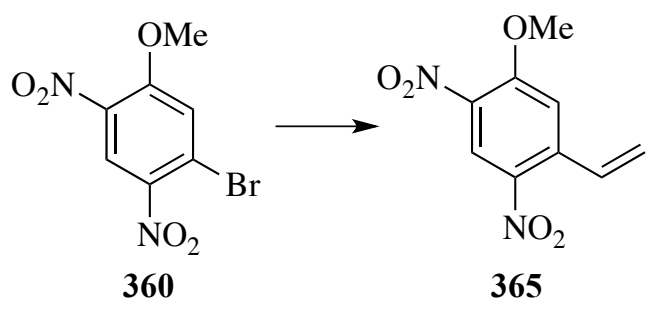

2,4-dinitro-5-ethenylanisole (365): Reaction of $\mathbf{3 6 0}$ (159 mg, $0.58 \mathrm{mmol}$ ), (ethenyl)trin-butylstannane (304 mg, $0.96 \mathrm{mmol}), \mathrm{PPh}_{3}(68.6 \mathrm{mg}, 0.26 \mathrm{mmol})$, and $\mathrm{Pd}(\mathrm{dba})_{2}(27.4$ $\mathrm{mg}, 0.05 \mathrm{mmol})$ in toluene $(6 \mathrm{~mL})$ for 48 hours as described for $\mathbf{3 5 5}$ afforded after purification using column chromatography (hexanes/EtOAc, 8:2) 365 (55.8 mg, 38\%) as a yellow solid $\left(\mathrm{mp}=72-74{ }^{\circ} \mathrm{C}\right) .{ }^{1} \mathrm{H}$ NMR $\delta 8.69(\mathrm{~s}, 1 \mathrm{H}), 7.36(\mathrm{dd}, J=17.2,10.8 \mathrm{~Hz}, 1 \mathrm{H})$, 
$7.20(\mathrm{~s}, 1 \mathrm{H}), 5.82(\mathrm{~d}, J=17.2 \mathrm{~Hz}, 1 \mathrm{H}), 5.68(\mathrm{~d}, J=10.9 \mathrm{~Hz}, 1 \mathrm{H}), 4.12(\mathrm{~s}, 3 \mathrm{H}) ;{ }^{13} \mathrm{C} \mathrm{NMR} \delta$

156.0, 140.7, 132.5, 124.0, 123.7, 121.8, 115.3, 112.9, 57.3; IR (ATR) 3108, 3060, 1607, $1579,1516,1338,1277,1249 \mathrm{~cm}^{-1}$;

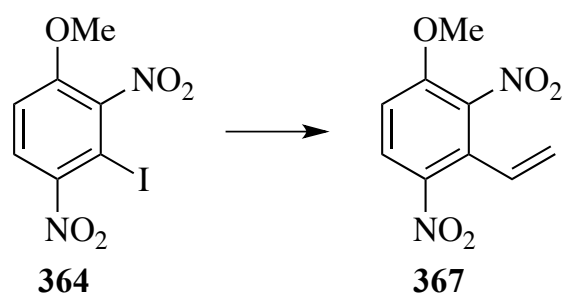

2,4-dinitro-3-ethenylanisole (367): To a solution of 364 (240.3 $\mathrm{mg}, 0.74 \mathrm{mmol})$ in toluene (10 mL) was added (ethenyl)tri-n-butylstannane (342.1 mg, $1.07 \mathrm{mmol}), \mathrm{PPh}_{3}$ (85.3 $\mathrm{mg}, 0.32 \mathrm{mmol})$, and $\mathrm{Pd}(\mathrm{dba})_{2}(35.2 \mathrm{mg}, 0.06 \mathrm{mmol})$ and 2,6-di-t-butyl-4methylphenol (18.7 mg, $0.08 \mathrm{mmol}$ ) for 48 hours as described for $\mathbf{3 5 5}$ afforded after purification using column chromatography (hexanes/EtOAc, 7:3) gave 367 (135.6 mg, 82\%) as a brown solid. ${ }^{1}$ HNMR $\delta 8.04(\mathrm{~d}, J=8.4 \mathrm{~Hz}, 1 \mathrm{H}), 7.48(\mathrm{~d}, J=8.4 \mathrm{~Hz}, 1 \mathrm{H}), 6.60$ (dd, $J=17.4,10.8 \mathrm{~Hz}, 1 \mathrm{H}), 6.00$ (d, $J=17.4 \mathrm{~Hz}, 1 \mathrm{H}), 5.69$ (d, $J=11.4 \mathrm{~Hz}, 1 \mathrm{H}), 4.02$ (s, $3 \mathrm{H}) ;{ }^{13} \mathrm{CNMR} \delta 146.9,136.0,129.2,128.6,128.2,127.1,123.8,121.5,65.0 ;$ IR (ATR) $3109,2958,1672,1577,1519,1340,1286 \mathrm{~cm}^{-1}$;

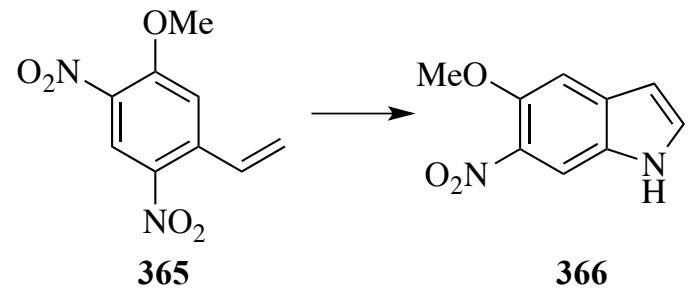

5-methoxy-6-nitroindole (366) ${ }^{169}$ : In an oven-dried ACE glass pressure tube dissolved 365 (41.5 mg, $0.19 \mathrm{mmol})$, , 1,10-phenanthroline ( $7.4 \mathrm{mg}, 0.04 \mathrm{mmol})$, and $\mathrm{Pd}(\mathrm{OAc})_{2}(4.1$ $\mathrm{mg}, 0.02 \mathrm{mmol}$ ) in N,N-dimethylformamide ( $3 \mathrm{~mL}$ ). Tube fitted with pressure head and 
pressurized with carbon monoxide ( 4 cycles, 6 atm), then placed in aluminum heating block at heated at $120^{\circ} \mathrm{C}$ for 28 hours. Brown solution diluted with $\mathrm{H}_{2} \mathrm{O}(25 \mathrm{~mL})$, then extracted with ethyl acetate $(4 \times 40 \mathrm{~mL})$. Combined organic layers dried over $\mathrm{MgSO}_{4}$, filtered, then solvents removed under reduced pressure. Purification of crude brown residue via column chromatography (hexanes/EtOAc, 1:1) afforded 366 (10.3 mg, 28\%) as a brown residue. Spectral data were in accordance with literature values.

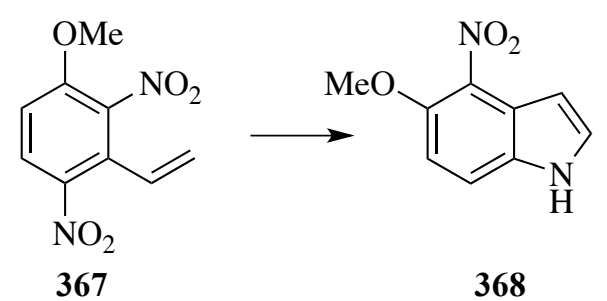

5-methoxy-4-nitroindole (368) ${ }^{169}$ : In an oven-dried ACE glass pressure tube dissolved 367 (55.7 mg, $0.25 \mathrm{mmol}), 1,10$-phenanthroline ( $9.8 \mathrm{mg}, 0.05 \mathrm{mmol})$, and $\mathrm{Pd}(\mathrm{OAc})_{2}(5.3$ $\mathrm{mg}, 0.024 \mathrm{mmol})$ in N,N-dimethylformamide $(3 \mathrm{~mL})$. Tube fitted with pressure head and pressurized with carbon monoxide ( 4 cycles, $6 \mathrm{~atm}$ ), then placed in aluminum heating block at heated at $120^{\circ} \mathrm{C}$ for 24 hours. Solvent removed via bulb-to-bulb distillation. Purification of crude brown residue via column chromatography (hexanes/EtOAc afforded 368 (10.9 mg, 23\%) as a yellow solid. Spectral data were in accordance with literature values.<smiles>C=C(C)c1cc(OC)c([N+](=O)[O-])cc1[N+](=O)[O-]</smiles>

363 369

2,4-dinitro-5-(prop-1-en-2-yl)anisole (369): Reaction of 363 (257 mg, 0.79 mmol), tributyl(prop-1-en-2-yl)stannane (312 mg, $0.94 \mathrm{mmol}), \mathrm{PPh}_{3}(57.0 \mathrm{mg}, 0.22 \mathrm{mmol})$, and $\operatorname{Pd}(\mathrm{dba})_{2}(35.4 \mathrm{mg}, 0.06 \mathrm{mmol})$ in toluene $(10 \mathrm{~mL})$ for 41 hours as described for 355 
afforded after purification using column chromatography (hexanes/EtOAc, 8:2) 369 (68.8 $\mathrm{mg}, 0.29 \mathrm{mmol}, 37 \%)$ as a yellow oil. ${ }^{1} \mathrm{HNMR} \delta 8.61(\mathrm{~s}, 1 \mathrm{H}), 6.97(\mathrm{~s}, 1 \mathrm{H}), 5.27(\mathrm{~s}, 1 \mathrm{H})$, $5.01(\mathrm{~s}, 1 \mathrm{H}), 4.08(\mathrm{~s}, 3 \mathrm{H}), 2.11(\mathrm{~s}, 3 \mathrm{H}),{ }^{13} \mathrm{CNMR} \delta 156.0,146.5,142.5,139.5,137.4$, 123.4, 226.4, 115.5, 57.5, 23.1; IR (ATR) 3114, 3045, 1615, 1579, 1516, $1338 \mathrm{~cm}^{-1}$<smiles>C=C(C)c1cc(OC)c([N+](=O)[O-])cc1[N+](=O)[O-]</smiles>

3-methyl-5-methoxy-6-nitroindole (370): Reaction of $\mathbf{3 6 9}$ (68.8 mg, $0.29 \mathrm{mmol}), \mathrm{PPh}_{3}$ (33.2 $\mathrm{mg}, 0.13 \mathrm{mmol})$, and $\mathrm{Pd}(\mathrm{OAc})_{2}(7.8 \mathrm{mg}, 0.03 \mathrm{mmol})$ in $\mathrm{MeCN}(3 \mathrm{~mL})$ under $\mathrm{CO}$ (g) (3 cycles, $90 \mathrm{psi})$ at $120^{\circ} \mathrm{C}(48 \mathrm{hrs})$ afforded after purification using column chromatography (hexanes/EtOAc, 8:2) $\mathbf{3 7 0}(4.3 \mathrm{mg}, 0.02 \mathrm{mmol}, 7 \%)$ as a yellow solid $\left(\mathrm{mp}=127-129^{\circ} \mathrm{C}\right) .{ }^{1} \mathrm{HNMR} \delta 8.11(\mathrm{br} \mathrm{s}, 1 \mathrm{H}), 8.00(\mathrm{~s}, 1 \mathrm{H}), 7.21(\mathrm{t}, J=1.2 \mathrm{~Hz}, 1 \mathrm{H}), 7.10$ (s, 1H), $4.01(\mathrm{~s}, 3 \mathrm{H}), 2.32(\mathrm{~d}, J=1.2 \mathrm{~Hz}, 3 \mathrm{H}) ;{ }^{13} \mathrm{CNMR} \delta 148.1,136.4,132.6,129.2$, $128.1,112.4,109.8,102.3,57.4,9.7$; IR (ATR) $3289,3098,2945,1524,1340,1271 \mathrm{~cm}^{=1}$ ; HMRS (ESI) calc'd for $\mathrm{C}_{10} \mathrm{H}_{10} \mathrm{~N}_{2} \mathrm{O}_{3}(\mathrm{M}+\mathrm{Na}) 229.0583$, found 229.0583.<smiles>C=C(C)c1c(N=[Zn])ccc(OC)c1[N+](=O)[O-]</smiles>

2,4-dinitro-3-(prop-1-en-2-yl)anisole (371): Reaction of 361 (204 mg, $0.74 \mathrm{mmol})$, tributyl(prop-1-en-2-yl)stannane (519 mg, $1.57 \mathrm{mmol}), \mathrm{PPh}_{3}(79.9 \mathrm{mg}, 0.30 \mathrm{mmol})$, and $\operatorname{Pd}(\mathrm{dba})_{2}(34.6 \mathrm{mg}, 0.06 \mathrm{mmol})$ in toluene $(20 \mathrm{~mL})$ for 47 hours as described for $\mathbf{3 5 5}$ afforded after purification using column chromatography (hexanes/EtOAc, 9:1) 371 (37.0 
$\mathrm{mg}, 0.16 \mathrm{mmol}, 21 \%)$ as a brown oil. ${ }^{1} \mathrm{HNMR} \delta 8.25(\mathrm{~d}, J=9.6 \mathrm{~Hz}, 1 \mathrm{H}), 7.08(\mathrm{~d}, J=9.6$

$\mathrm{Hz}, 1 \mathrm{H}), 5.30(\mathrm{~m}, 1 \mathrm{H}), 4.98(\mathrm{~m}, 1 \mathrm{H}), 4.01(\mathrm{~s}, 3 \mathrm{H}), 2.11(\mathrm{~s}, 3 \mathrm{H}) ;{ }^{13} \mathrm{CNMR} \delta 154.4,141.4$, 140.3, 137.2, 134.2, 128.2, 118.3, 111.1, 57.3, 23.3; IR (ATR) 3113, 2954, 1662, 1581, $1345,1275 \mathrm{~cm}^{-1}$;

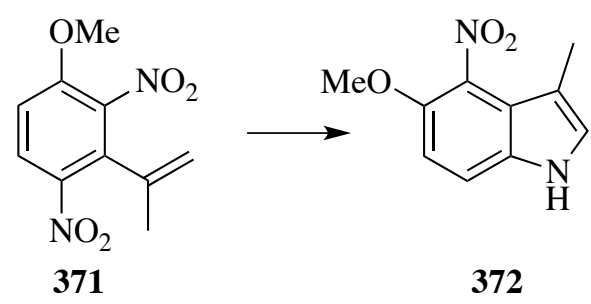

3-methyl-5-methoxy-4-nitroindole (372): Reaction of 371 (34.8 mg, $0.15 \mathrm{mmol}), 1,10$ phenanthroline $(6.6 \mathrm{mg}, 0.04 \mathrm{mmol})$, and $\mathrm{Pd}(\mathrm{OAc})_{2}(3.1 \mathrm{mg}, 0.01 \mathrm{mmol})$ in $\mathrm{DMF}(3 \mathrm{~mL})$ under $\mathrm{CO}(\mathrm{g})(3$ cycles, $90 \mathrm{psi})$ at $120{ }^{\circ} \mathrm{C}(43 \mathrm{hrs})$ afforded after purification using column chromatography (hexanes/EtOAc, 7:3) $372(16.5 \mathrm{mg}, 0.08 \mathrm{mmol}, 53 \%)$ as a brown solid $\left(\mathrm{mp}=152-154{ }^{\circ} \mathrm{C}\right) .{ }^{1} \mathrm{HNMR} \delta 8.07(\mathrm{br} \mathrm{s}, 1 \mathrm{H}), 7.39(\mathrm{~d}, J=9.0 \mathrm{~Hz}, 1 \mathrm{H}), 7.06(\mathrm{t}$, $J=0.6 \mathrm{~Hz}, 1 \mathrm{H}), 6.94(\mathrm{~d}, J=9.0 \mathrm{~Hz}, 1 \mathrm{H}), 3.93(\mathrm{~s}, 3 \mathrm{H}), 2.18(\mathrm{~d}, J=1.2 \mathrm{~Hz}, 3 \mathrm{H}) ;{ }^{13} \mathrm{CNMR} \delta$ 145.6, 133.5, 133.0, 126.2, 120.1, 114.1, 109.9, 108.9, 58.2, 10.1; IR (ATR) 3348, 2984, 1521, 1322, $1242 \mathrm{~cm}^{-1}$; HMRS (ESI) calc'd for $\mathrm{C}_{10} \mathrm{H}_{10} \mathrm{~N}_{2} \mathrm{O}_{3}(\mathrm{M}+\mathrm{Na}$ ) 229.0583, found 229.0583 . 


\section{References and Notes}

1. Sumpter, W. C.; Miller, F. M. The Chemistry of Heterocyclic Compounds, Indole and Carbazole Systems; Interscience: New York, 1954.

2. Van Order, R. B.; Lindwall, H. G. Chem. Rev. 1942, 30, 69.

3. Sundberg, R. J. The Chemistry of Indoles; Academic Press: New York, 1970.

4. Sundberg, R. J. Indoles; Academic Press: London, 1996.

5. Poulsen, T. B.; Jorgensen, K. A. Chem. Rev. 2008, 108, 2903.

6. Baeyer, A.; Knopp, C. A. Chemische Berichte. 1868, 1, 17.

7. Baeyer, A.; Emmerling, A. Chemische Berichte. 1869, 2, 679.

8. Barden, T. C. Top. Het. Chem. 2010, 26, 31.

9. Houlihan, W. J.; Remers, W. A.; Indoles; Wiley-Interscience: New York, 1971.

10. Willcock, E. G.; Hopkins, F. G. J. Physiology 1907, 35, 88.

11. Rapport, M. M.; Green, A. A. J. Biol. Chem. 1948, 176, 1243.

12. Wurtman, R.J.; Anton-Tay F. Recent Prog. Horm. Res. 1969, 25, 493.

13. Gul, W.; Hamann, M. T. Life Sciences 2005, 78, 442.

14. Kohmoto, S.; Kashman, Y.; McConnell, O.J.; Rinehart, K.L.; Wright, A.; Koehn, F. J. Org. Chem. 1988, 53, 3116.

15. Rinehart, K. L.; Kobayashi, J.; Harbour, G. C.; Hughes, R. G.; Mizsak, S. A.; Scahill, T. A. J.Am. Chem. Soc. 1984, 106, 1524.

16. Laycock, M. V., Wright, J. L. C.; Findlay, J. A.; Patil, A. D. Canadian Journal of Chemistry 1986, 64, 1312.

17. Dowle, M. D.; Coates, I. H., GB 2124 210, 1983.

18. Daugan, A.; Grondin, P.; Ruault, C.; Le Monnier de Gouville, A.; Coste, H.; Kirilovsky, J.; Hyafil, F.; Labaudinière, R. J. Med. Chem. 2003, 46, 4525. 
19. Chen, C.; Lieberman, D. R.1 Larsen, R. D.; Reamer, R. A.; Verhoeven, T. R.; Reider, P. J.; Cottrell, I. F.; Houghton, P. G. Tet. Lett. 1994, 35, 6981.

20. Zacharia, J. T.; Tanaka, T. J. Org. Chem. 2010, 75, 7514.

21. Inman, M.; Moody, C. J. Chem. Sci. 2013, 4, 29.

22. Feniuk, W.; Humphrey, P. P. A. Drug Dev. Res. 1992, 26, 235.

23. Brock, G.B.; McMahon, C. G.; Chen, K. K; Costigan, T.; Shen, W.; Watkins, V.; Anglin, G.; Whitaker, S. J. Urol. 2002, 168, 1332.

24. Jokubaitis, L. A. Br. J. Clin. Prac. Suppl. 1994, 77, 11.

25. Robinson, B. The Fischer Indole Synthesis; Wiley-Interscience: New York, 1982.

26. Fischer, E.; Jourdan, F. Ber. 1883, 16, 2241.

27. Fischer, E.; Hess, O. Ber. 1884, 17, 559.

28. Hirofumi, U.; Satoh'H.; Matsumoto, K.; Sugimoto, K.; Fukuyama, T.; Tokuyama, H. Angew. Chem. Int.Ed. 2009, 48, 7600-7603.

29. Madelung, W. Ber. 1912, 45, 1128.

30. Allen, C. F. H.; Van Allen, J. Org. Synth. Coll. Vol. III. 1955, 597.

31. Houlihan, W. J.; Parrino, V. A.; Uike, Y.J. Org. Chem. 1981, 46, 4511.

32. Fuhrer, W.; Gschwend, H. W. J. Org. Chem. 1979, 44, 1133.

33. Smith, A. B.; Kanoh, N.; Ishiyama, H.; Minakawa, N.; Rainier, J. D.; Hartz, R. A.; Cho, Y. S.; Cui, H.; Moser, W. H. J. Am. Chem. Soc. 2003, 125, 8228.

34. Hegedus, L. S.; Allen, G. F.; Waterman, E. L. J. Am. Chem. Soc. 1976, 98, 2674.

35. Akermark, B.1 Baechvall, J. E.; Hegedus, L. S.; Zetterberg, K.; Siirala-Hansen, K.; Sjoeberg, K. J. Organomet. Chem. 1974, 72, 127.

36. Harrington, P. J.; Hegedus, L. S. J. Org. Chem. 1984, 49, 2657-2662.

37. Larock, R. C.; Yum, E. K.; Refvik, M. D. J. Org. Chem. 1998, 63, 7652-7662. 
38. Humora, M. J.; Modi, S. P.; Srivastava, S. K.; Williams, A. D. U.S. Pat Appl. Publ. (1996) US 5,550,239.

39. Humphrey, G. R.; Kuethe, J. T. Chem. Rev. 2006, 106, 2875-2911.

40. Newhouse, T.; Baran, P. S. J. Am. Chem. Soc. 2008, 130, 10886-10887.

41. Wolfe, J. P.; Wagaw, S.; Marcoux, J. F.; Buchwald, S. L. Acc. Chem. Res. 1998, 31, 805.

42. Aoki, K.; Peat, A. J.; Buchwald, S. L. J. Am. Chem. Soc. 1998, 120, 3068.

43. Reissert, A.; Ber. 1897, 30, 1030.

44. Batcho, A. D.; Leimgruber, W. Org. Synth. 1985, 63, 214-220.

45. Cadogan, J. I. G.; Cameron-Wood, M.; Mackie, R. K.; Searle, R. J. G. J. Chem. Soc. 1965, 4831 .

46. Bunyan, P. J.; Cadogan, J. I. G.; J. Chem. Soc. 1963, 42.

47. Majgier-Baranowska, H.; Williams, J. D.; Li, B.; Peet, N. P.; Tet. Lett. 2012, 53, 4785.

48. Humphrey, G. R.; Kuethe, J. T. Chem. Rev. 2006, 106, 2875-2911.

49. Cacchi, S.; Fabrizi, G. Chem. Rev. 2005, 105, 2873-2920.

50. Akazome, M.; Kondo, T.; Watanabe, Y.J. Org Chem. 1994, 59, 3375.

51. Davies, I. W.; Smitrovich, J. H.; Sidler, R.; Qu, C.; Gresham, V.; Bazaral, C. Tetrahedron. 2005, 61, 6425-6437.

52. Söderberg, B. C.; Shriver, J. A. J. Org. Chem. 1997, 62, 5838-5845.

53. Clawson, R. W., Jr.; Deavers, R. E., III; Akhmedov, N. G.; Söderberg, B. C. G. Tetrahedron 2006, 62, 10829.

54. Sundberg, R. J. J. Org. Chem. 1965, 30, 3604.

55. Sundberg, R. J.; Yamazaki, T.; J. Org. Chem. 1967, 32, 290-294. 
56. Dacko, C. A.; Akhmedov, N. G.; Söderberg, B. C. G. Tetrahedron Asymm. 2008, 19, 2775-2783.

57. Gorugantula, S. P.; Carrero-Martinez, G. M.; Dantale, S. W.; Söderberg, B. C. G. Tetrahedron 2010, 66, 1800-1805.

58. Clawson Jr., R. W.; Dacko, C. A.; Deavers III, R. E.; Akhmedov, N. G.; Söderberg, B. C. G. Tetrahedron 2009, 65, 8786-8793.

59. Clawson Jr., R. W.; Söderberg, B. C. G. Tetrahedron Lett. 2007, 48, 6019-6021.

60. Scott, T. L.; Söderberg, B. C. G. Tetrahedron 2003, 59, 6323-6332.

61. Dantale, S. W.; Söderberg, B. C. G. Tetrahedron 2003, 59, 5507-5514.

62. Scott, T. L.; Yu, X.; Gorunatula, S. P.; Carrero-Martínez, G.; Söderberg, B. C. G. Tetrahedron 2006, 62, 10835-10842.

63. Söderberg, B. C.; Chisnell, A. C.; O'Neil, S. N.; Shriver, J. A. J. Org. Chem. 1999, 64, 9731-9734.

64. Soderberg, B. C. G.; Banini, S. R.; Turner, M. R.; Minter, A. R.; Arrington, A. K. Synthesis 2008, 903-912.

65. Makosza, M.; Tyrala, A. Synth. Commun. 1986, 16, 419-423.

66. Pearson, R. G.; Dillon, R. L. J. Am. Chem. Soc. 1953, 75, 2439-2443.

67. Wrobel, Z.; Makosza, M. Tetrahedron 1993, 49, 5315-5326.

68. Nyerges, M.; Somfai, B.; Toth, J.; Toke, L,; Dancso, A.; Blasko, G. Synthesis 2005, 2039-2045.

69. Nyerges, M.; Viranyi, A.; Zhang, W.; Groundwater, P. W.; Blasko, G.; Tokea, L. Tetrahedron 2004, 60, 9937-9944.

70. For a review, see Dalpozzo, R.; Bartoli, G. Curr. Org. Chem. 2005, 9, 163-178. 
71. For an extensive review on the biological activity of compounds isolated from Salvia miltiorhiza, see: Wang, X.; Morris-Natschke, S. L.; Lee, K. S. Med. Res. Rev. 2007, 27, 133-148.

72. Don, M. J.; Shen, C. C.; Lin, Y. L.; Syu, W. J.; Ding, Y. H.; Sun, C. M. J. Nat. Prod. 2005, 68, 1066-1070.

73. The parent compound has been isolated from for example coal tar pitch, in sediment samples, and in smoke from a variety of sources. For example, see: Kruber, O.; Grigoleit, G. Chem. Ber. 1954, 87, 1895-1905.

74. (a) Horaguchi, T.; Oyanagi, T.; Creencia, E. C.; Tanemura, K.; Suzuki, T. J. Heterocycl. Chem. 2004, 41, 1-6; (b) Kreher, R.; Kohler, W. Angew. Chem., Int. Ed. Engl. 1975, 14, 264-265; (c) Horaguchi, T.; Yamazaki, R.; Abe, T. Bull. Chem. Soc. Jpn. 1980, 53, 494-497.

75. Peng, X.; She, X.; Su, Y.; Wu, T.; Pan, X. Tetrahedron Lett. 2004, 45, 3283-3285.

76. Burnell, R. H.; Desfosses, S. Synth. Commun. 1997, 27, 355-360.

77. Prepared in $95 \%$ yield according to the procedure described by: Lanni, T. B., Jr.; Greene, K. L.; Kolz, C. N.; Para, K. S.; Visnick, M.; Mobley, J. L.; Dudley, D. T.; Baginski, T. J.; Liimatta, M. B. Bioorg. Med. Chem. Lett. 2007, 17, 756-760.

78. Following the procedure for a related compound: Liu, H.-J.; Tran, D. D.-P. Tetrahedron Lett. 1999, 40, 3827-3830.

79. The compound has been reported without any spectral data, see: Ref. 3 .

80. For regioselective nitration of related compounds, see: (a) Matsumoto, T.; Imai, S.; Yuki, S.; Mitsuki, M.; Miuchi, S.; Sunaoka, Y. Bull. Chem. Soc. Jpn. 1983, 56, 290294; (b) Akita, H.; Oishi, T. Chem. Pharm. Bull.Jpn. 1981, 29, 1567-1579; (c) Ohtsuka, Y.; Akita, H.; Tahara, A. Chem. Lett. 1973, 229-232. 
81. (a) Yin, S.; Su, Z.-S.; Zhou, Z.-W.; Dong, L.; Yue, J.-M. J. Nat. Prod. 2008, 71, 1414-1417; (b) Larsson, E. A.; Ulicny, J.; Laaksonen, A.; Widmalm, G. Org. Lett. 2002, 4, 1831-1834.

82. For some recent examples of benzylic oxidation to afford benzylic alcohols using DDQ, see: (a) Aubry, S.; Pellet-Rostaing, S.; Lemaire, M. Eur. J. Org. Chem. 2007, 5212-5225; (b) Es-Safi, N.-E.; Ducrot, P.-H. Lett. Org. Chem. 2006, 3, 231-234; (c) Zhou, H.; Liao, X.; Yin,W.; Ma, J.; Cook, J. M. J. Org. Chem. 2006, 71, 251-259.

83. For a few examples, see: (a) Rijo, P.; Simoes, M. F.; Fransisco, A. P.; Rojas, R.; Gilman, R. H.; Vaisberg, A. J.; Rodriguez, B.; Moiteiro, C. Chem. Biodiversity 2010, 7, 922-932; (b) Lin, S.; Zhang, Y.; Liu, M.; Yang, S.; Gan, M.; Zi, J.; Song, W.; Fan, X.; Wang, S.; Liu, Y.; Yang, Y.; Chen, X.; Guo, Y.; Wang, W.; Shi, J. J. Nat. Prod. 2010, 73, 1914-1921; (c) Yoshikawa, K.; Suzuki, K.; Umeyama, A.; Arihara, S. Chem. Pharm. Bull. 2006, 54, 574-578.

84. Kosugi, M.; Shimizu, Y.; Migita, T. Chem. Lett. 1977, 1423.

85. Kosugi, M.; Shimizu, Y .; Migita, T. J. Organomet. Chem. 1977, 129, C36.

86. Kosugi, M.; Sasazawa, K.; Shimizu, Y.; Migita, T. Chem. Lett. 1977, 301.

87. Milstein, D.; Stille, J. K. J. Am. Chem. Soc. 1978, 100, 3636.

88. Farina, V.; Krishnamurthy, V. Org. Reactions. 1997, 50.

89. Trost, B. M. Science 1983, 219, 245.

90. Echavarren, A. M.; Stille, J. K. J. Am. Chem. Soc. 1987, 109, 5478.

91. Scott, W. J.; Stille, J. K. J. Am. Chem. Soc. 1986, 108, 3033.

92. Krolski, M. E.; Renaldo, A. F.; Rudisill, D. E.; Stille, J. K. J. Org. Chem., 1988, 53, 1170. 
93. Habibi, D.; Zolfigol, M. A.; Shiri, M.; Sedaghat, A. S. Afr. J. Chem., 2006, 59, 93.

94. Lee, M. et al PCT Int. Appl., 2006125208, 23 Nov 2006

95. Lee, Y. M.; Moon, M. E.; Vajpayee, V.; Filimonov, V. D.; Chi, K. Tetrahedron, 2010, 66, 7418 .

96. Skaddan, M. B.; Bergman, R. G. J. Label. Compd. Radiopharm., 2006, 49, 623.

97. Stille, J. K. Angew. Chem. Int. Ed. Engl. 1986, 25, 508.

98. Saa, J. M.; Martorell, G.: Garcia-Raso, A. J. Org. Chem. 1992, 5, 678-685.

99. Amatore, C.; Azzabi, M.; Jutand, A. J. Organomet. Chem. 1989, 363, C41-C45.

100. Farina, V.; Krishnan, B. J. Am. Chem. Soc. 1991, 113, 9585-9595.

101. Farina, V.; Krishnan, B.; Marshall, D. R.; Roth, G. P. J. Org. Chem., 1993, 58, 5434.

102. Coste, A.; Toumi, M.; Wright, K.; Razafimahaleo, V.; Couty, F.; Marrot, J.; Evano, G. Org. Lett. 2008, 10, 3841 .

103. Berlin, A.; Pagani, G.; Schiavon, G.; Zotti, G. Mol. Cryst. Liq. Cryst. 1990, 187, 193.

104. Tsuji, R.; Nakagawa, M.; Nishida, A. Heterocycles 2002, 58, 587.

105. MacMillan, K. S.; Nguyen, T.; Hwang, I.; Boger, D. L. J. Am. Chem. Soc. 2009, $131,1187$.

106. Berlin, A.; Bradamante, S.; Ferraccioli, R.; Pagani, G.; Sannicolo, F. J. Chem. Soc., Chem. Comm. 1987, 1176.

107. Chunchatprasert, L.; Shannon, P. V. R. J. Chem. Soc., Perkin Trans. 1 1996, 1787.

108. Samsoniya, S. A.; Chikvaidze, I. S.; Kadzhrishvili, D. O.; Targamadze, N. L. Chem. Het. Comp. 2010, 46, 536. 
109. Samsoniya, S. A.; Chikvaidze, I. S.; Kadzhrishvili, D. O. Pharm. Chem. J. 2011, 45, 24.

110. Wei, Y.; Deb, I.; Yoshikai, N. J. Am. Chem. Soc. 2012, 134, 9098.

111. Suzuki, Y.; Ohta, Y.; Oishi, S.; Fujii, N.; Ohno, H. J. Org. Chem. 2009, 74, 4246.

112. Kleene, R. D. J. Am. Chem. Soc. 1949, 71, 2259.

113.Ponticello, G. S.; Baldwin, J. J. J. Org. Chem. 1979, 44, 4003.

114. Banwell, M. G.; Jones, M. T.; Loong, D. T. J.; Lupton, D. W.; Pinkerton, D. M.;

Ray, J. K.; Willis, A. C. Tetrahedron 2010, 66, 9252.

115.Hodgson, H. H.; Moore, F. H. J. Chem. Soc. Trans. 1925, 1599.

116. Hodgson, H. H.; Moore, F. H. J. Chem. Soc. 1926, 155.

117.Roger, J.; Doucet, H. Org. Biomol. Chem. 2008, 6, 169

118. Liesbeskind, L. S.; Fengl, R. W. J. Org. Chem. 1990, 55, 5359.

119. Allred, G. D.; Liebeskind, L. S. J. Am. Chem. Soc. 1996, 118, 2748.

120.Farina, V.; Kapadia, S.; Krishnan, B.; Wang, C.; Liebeskind, L. S. J. Org. Chem. 1994, 59, 5905 .

121.Hudgens, T. L.; Turnbull, K. D. Tet. Lett. 1999, 40, 2719

122.For examples, see (a) Farina, V.; Baker, S. R.; Benigni, D. A.; Hauck, S. I.; Sapino, C. J. Org. Chem. 1990, 55, 5833-5847. (b) Hettrick, C. M.; Kling, J. K.; Scott, W. J. J. Org. Chem. 1991, 56, 1489. (c) Robl, J. A. Synthesis 1991, 56.

123.Flatt, A. K.; Dirk, S. M.; Henderson, J. C.; Shen, D. E.; Su, J.; Reed, M. A.; Tour, J. M. Tetrahedron 2003, 59, 8555.

124.Zhao, H.; Dai, R.; Cai, M. Syn. Comm., 2009, 39, 4454.

125.Organic Reactions; Paquette, L. A., Ed.; Wiley-Interscience: New York, 1997. 126. Hodgson, H. H.; Nixon, J.; J. Chem. Soc. 1931, 2268. 
127. Smith, C. J.; Ali, A.; Chen, L.; Hammond, M. L.; Anderson, M. S.; Chen, Y.; Eveland, S. S.; Guo, Q.; Hyland, S. A.; Milot, D. P.; Sparrow, C. P.; Wright, S. D.; Sinclair, P. J. Bioorg. Med. Chem. Lett. 2010, 20, 346.

128. Hajipour, A. R.; Mallakpour, S. E.; Adibi, H. Chem. Lett. 2000, 460.

129. Hajipour, A. R.; Adibi, H. J. Chem. Res. 2004, 4, 294.

130.Olah, G. A.; Wang, Q.; Sandford, G.; Prakash, G. K. S. J. Org. Chem. 1993, 58, 3194.

131.Smith, C. J.; Ali, A.; Chen, L.; Hammond, M. L.; Anderson, M. S.; Chen, Y.; Eveland, S. S.; Guo, Q.; Hyland, S. A.; Milot, D. P.; Sparrow, C. P.; Wright, S. D.; Sinclair, P. J. Bioorg. Med. Chem. Lett. 2010, 20, 346.

132. Badone, D.; Cecchi, R.; Guzzi, U. J. Org. Chem. 1992, 57, 6321.

133. Naber, J. R.; Fors, B. P.; Wu, X.; Gunn, J. T.; Buchwald, S. L. Heterocycles 2010 , $80,1215$.

134. Suttle, N. A.; Williams, A. J. Chem. Soc. Perkin Trans. 2: Phys. Org. Chem. 1983, $10,1537$.

135.Han, X.; Stoltz, B. M.; Corey, E. J. J. Am. Chem. Soc. 1999, 121, 7600.

136.Kawakami, T.; Suzuki, H. Tet. Lett. 2000, 41, 7093.

137. Kawai, Y.; Hayashi, M.; Tokitoh, N. Tetrahedron 2005, 61, 5049.

138. Mundla, S. R. Tet. Lett. 2000, 41, 6319.

139. Sathunuru, R.; Rao, U. N.; Biehl, E. ARKIVOC, 2003, 15, 124.

140.Gaude, D.; Gellon, G.; le Goaller, R.; Pierre, J. L. Can. J. Chem. 1989, 67, 104.

141. Wayland, N. E.; Hammer, C. F. J. Org. Chem. 1960, 25, 1525

142. For a review of brominations of deactivated aromatic compounds, see: Andrievsky, A. M.; Gorelik, M. V.Russ. Chem. Rev. 2011, 80, 421-428. 
143.Rajesh, K.; Somasundaram, M.; Saiganesh, R.; Balasubramanian, K. K. J. Org . Chem. 2007, 72, 5867-5869.

144.Kumar. L.; Mahajan, T.; Agarwal, D. D. Ind. Eng. Chem. Res. 2012, 51, 1159311597.

145.DePuy, C. H.; Froemsdorf, D. H. J. Am. Chem. Soc. 1957, 79, 3710.

146.Das, B.; Laxminarayana, K.; Ravikanth, B. J. Mol. Cat. A: Chem. 2007, 271, 131.

147.Li, D.; Zhao, B.; Sim, S.; Li, T.; Liu, A.; Liu, L. F.; LaVoie, E. J. Bioorg. Med. Chem. 2003, 11, 521-528.

148.Lee, Y.; Kelly, M. J. Tet. Lett. 2006, 47, 4897-4901.

149. Ghorbani-Choghamarani, A.; Nikoorazm, M.; Goudarziafshar, H.; Naserifar, Z.; Zamani, P. Chinese. J. Chem. 2011, 29, 731-734.

150. Suresh, P.; Annalakshmi, S.; Pitchumani, K. Tetrahedron 2007, 63, 4959-4967.

151.Jarkas, N.; McConathy, J.; Voll, R. J.; Goodman, M. M. J. Med. Chem. 2005, 48, 4254-4265.

152. Oda, N.; Yoshida, Y.: Nagai, S.; Ueda, T.; Sakakibara, J. Chem. Pharm. Bull. 1987, $35,1796-1802$.

153. Dai, M.; Wang, Z.; Danishefsky, S. J. Tetrahedron Lett. 2008, 49, 6613-6616.

154. Grigg, R. D.; Van Hoveln, R.; Schomaker, J. M. J. Am. Chem. Soc. 2012, 134, 16131-16134.

155.Blaszczyk, A.; Chadim, M.; von Hanisch, C.; Mayor, M. Eur.J. Org. Chem. 2006, 17,3809 .

156.Szpakiewicz, B.; Grzegozek, M. Russian J. Org. Chem. 2004, 40, 869.

157. Korner, G.; Contardi, A. Atti Accad. Naz. Linc., Class. Sci.Fis., Mat., Nat., Rend. 1916, 25,339 . 
158. For a synthesis, mp, and spectral data, see: Seo, J. H.; Artman III, G. D.; Weinreb, S. M. J. Org. Chem. 2006, 71, 8891-8900.

159. For a synthesis, mp, and spectral data, see: Kenwright, J. L.; Galloway, W. R. J. D.; Blackwell, D. T.; Isidor-Llobet, A.; Hodgkinson, J.; Wortmann, L.; Bowen, S. D.; Welch, M.; Spring, D. R. Chem. Eur. J. 2011, 17, 2981-2986.

160. Ashburn, B. O.; Carter, R. G.; Zakharov, L. V. J. Am. Chem. Soc. 2007, 129, 91099116.

161.Rozhkov, E.; Piskunova, I.; Gol'd, M.; Kalvin'sh, I. Chem. Het. Compds. 1998, 34 , $77-91$.

162. Ashburn, B. O.; Rathbone, L. K.; Camp, E. H.; Carter, R. G. Tetrahedron, 2008, 64, 856

163. Nodiff. E. A.; Saggiomo, A. J.; Shinbo, M.; Chen, E. H.; Otomasu, H.; Kondo, Y,; Kikuchi, T.; Verma, B. L.; Matsuura, S.; Tanabe, K.; Tyagi, M. P.; Morosawa, S. J. Med. Chem. 1972, 15, 775-780.

164. Jacquart, A.; Tauc, P.; Pansu, R. B.; Ishow, E. Chem. Commun. 2010, 46, 43604362 .

165.Lemasson, F.; Tittmann, J.; Hennrich, F.; Sturzl, N.; Malik, S.; Kappes, M. M.; Mayor, M. Chem. Commun. 2011, 47, 7428-7430.

166. Sui, M.; Zhao, S. PCT Int. Appl. WO2005067933, 2005.

167.Le Stanc, Y.; Le Corre, M.; Can.J. Chem., 1985, 63, 2958-2960.

168.Carpenter, M. S.; Easter, W. M.; Wood, T. F. J. Org. Chem. 1951, 16, 586.

169. Roue, N.; Delahaigue, T.; Barret, R. Heterocycles 1996, 43, 263 


\section{Appendix}

\section{${ }^{1} \mathrm{H}$ and ${ }^{13} \mathrm{C}$ NMR Spectra}

- $\quad{ }^{1} \mathrm{H}$ and ${ }^{13} \mathrm{C}$ NMR for Chapter 2: Indole/Quinoline 209

- $\quad{ }^{1} \mathrm{H}$ and ${ }^{13} \mathrm{C}$ NMR for Chapter 3: Salviadione 218

- $\quad{ }^{1} \mathrm{H}$ and ${ }^{13} \mathrm{C}$ NMR for Chapter 4: Chemoselective Coupling 232

- $\quad{ }^{1} \mathrm{H}$ and ${ }^{13} \mathrm{C}$ NMR for Chapter 5: Pyrroloindoles 284

- $\quad{ }^{1} \mathrm{H}$ and ${ }^{13} \mathrm{C}$ NMR for Chapter 6.1: Bromination 330

- $\quad{ }^{1} \mathrm{H}$ and ${ }^{13} \mathrm{C}$ NMR for Chapter 6.2: Functionalized Indoles 340 
${ }^{1} \mathrm{H}$ and ${ }^{13} \mathrm{C}$ NMR for Chapter 2: Indole/Quinoline

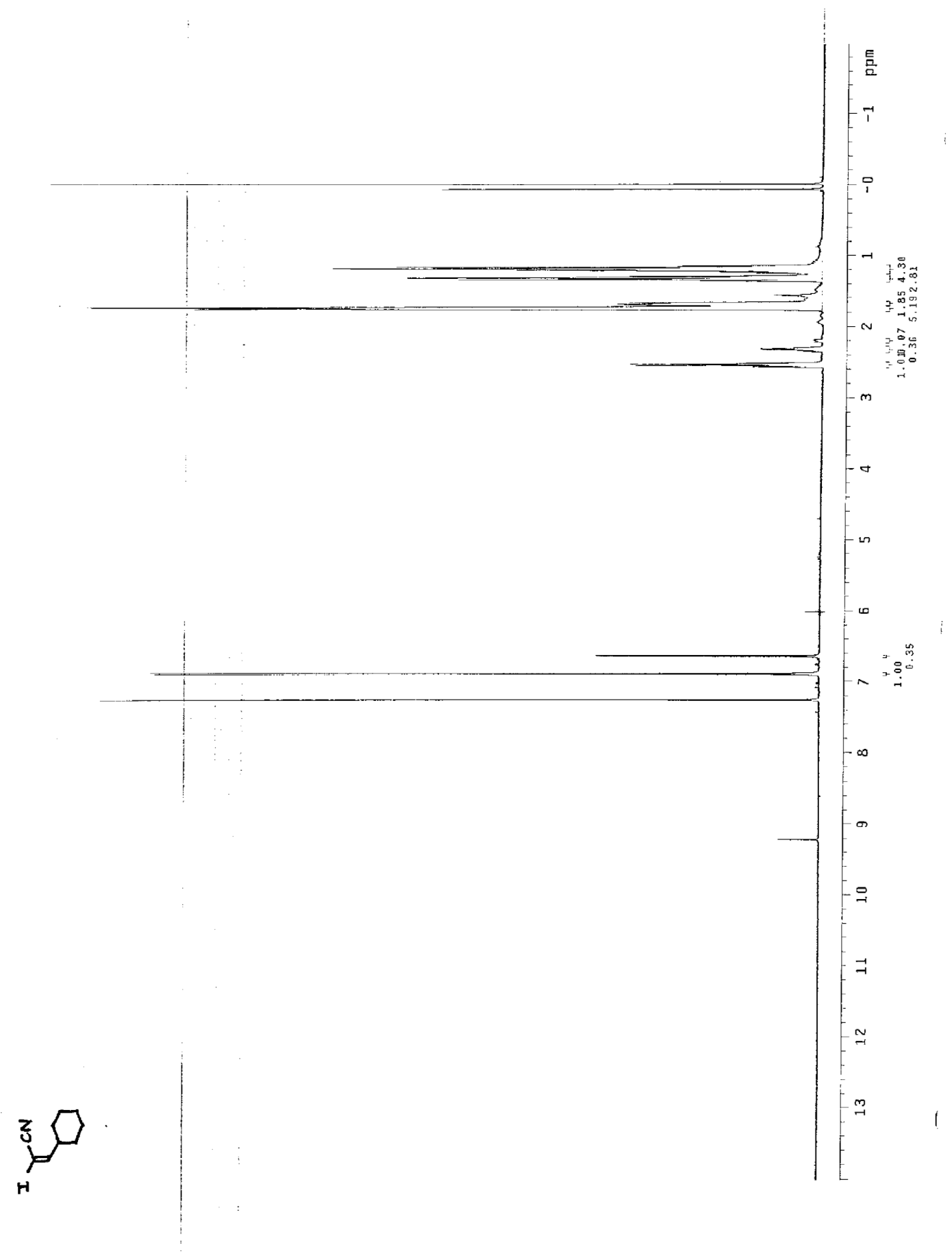

Figure 17: ${ }^{1} \mathrm{H}$ NMR of 3-Cyclohexyl-2-iodo-2-propenenitrile (110) 


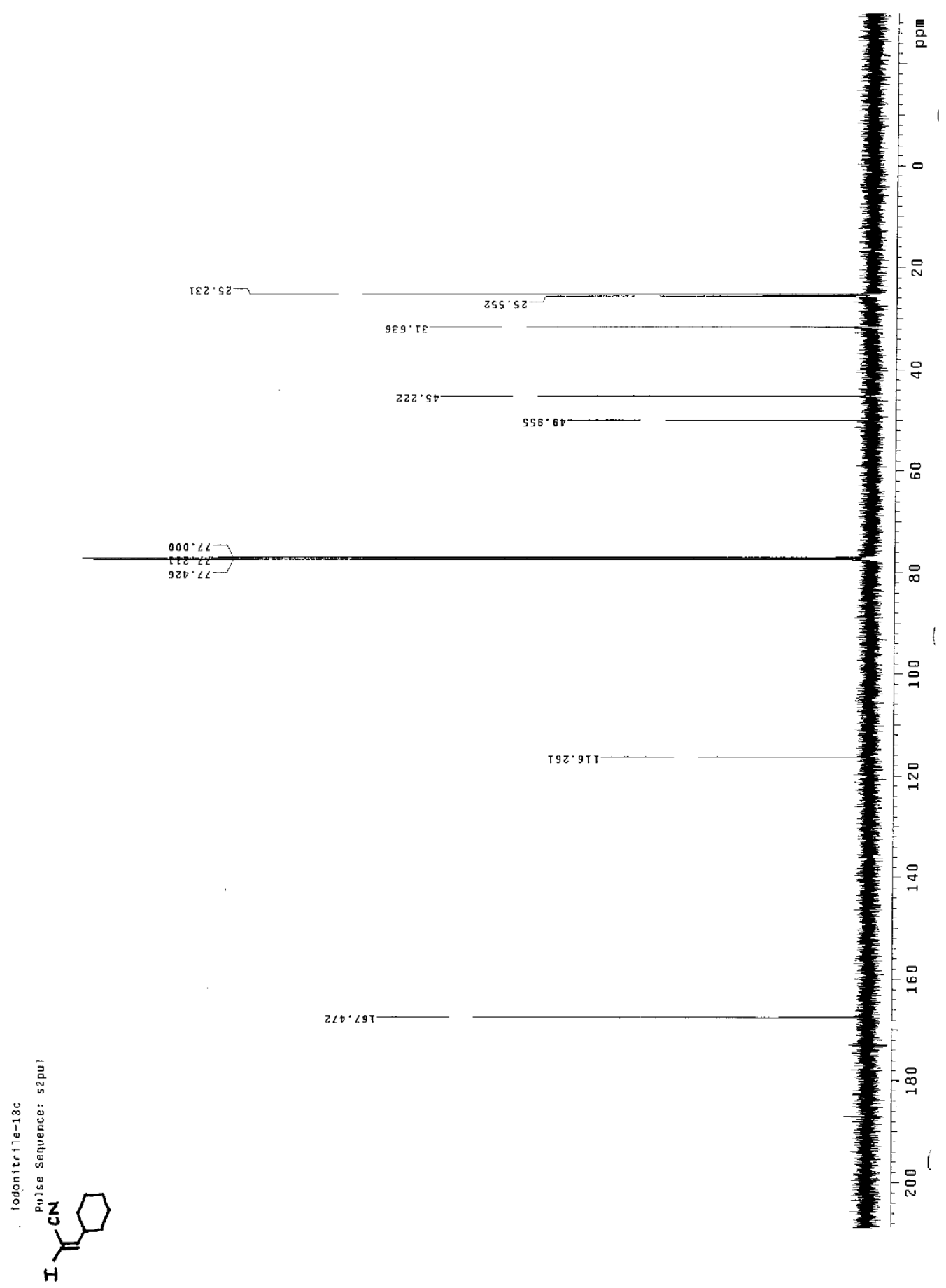

Figure 18: ${ }^{13} \mathrm{C}$ NMR of 3-Cyclohexyl-2-iodo-2-propenenitrile (110) 


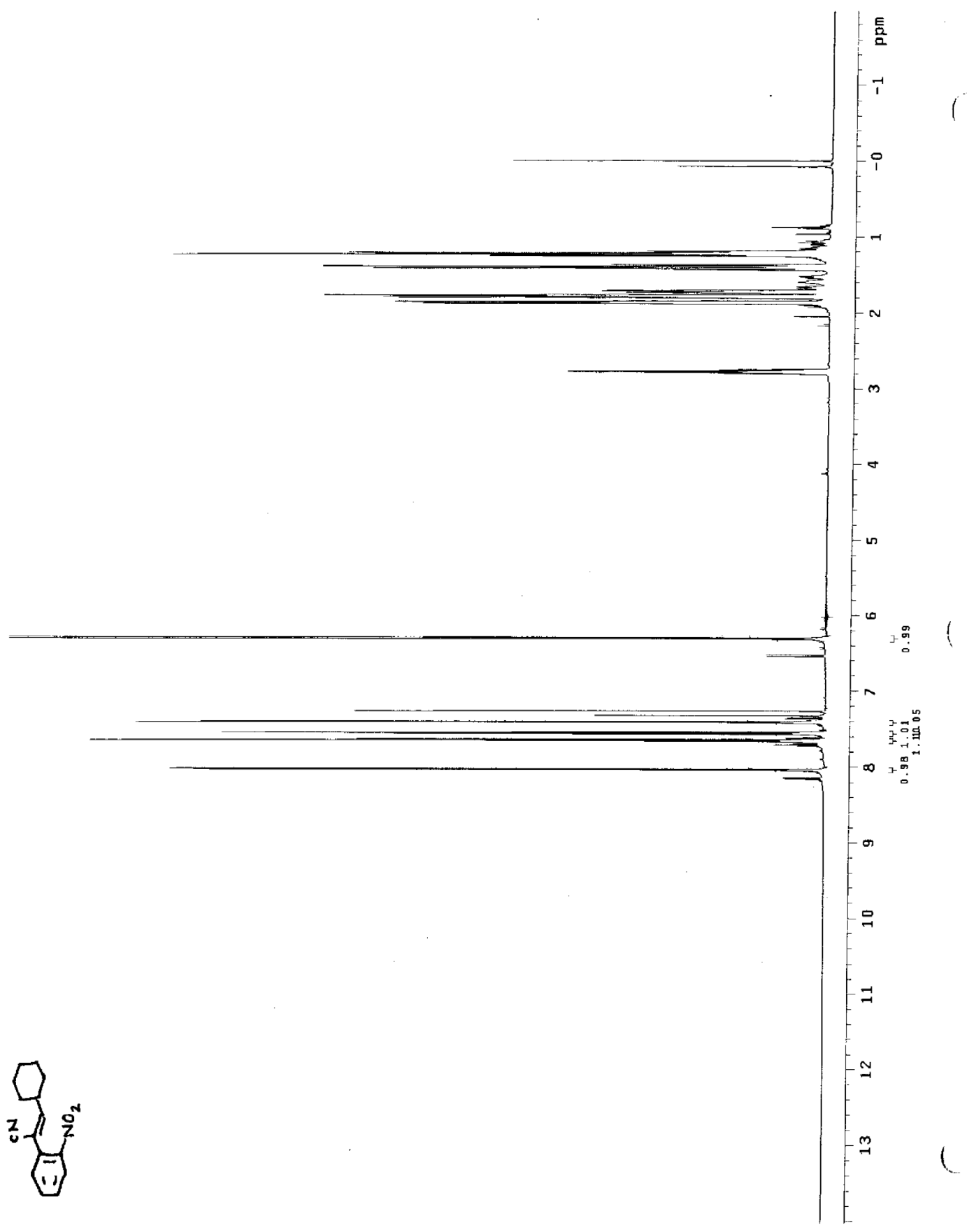

Figure 19: ${ }^{1} \mathrm{H}$ NMR of 3-Cyclohexyl-2-(2-nitrophenyl)-2-propenenitrile (111) 


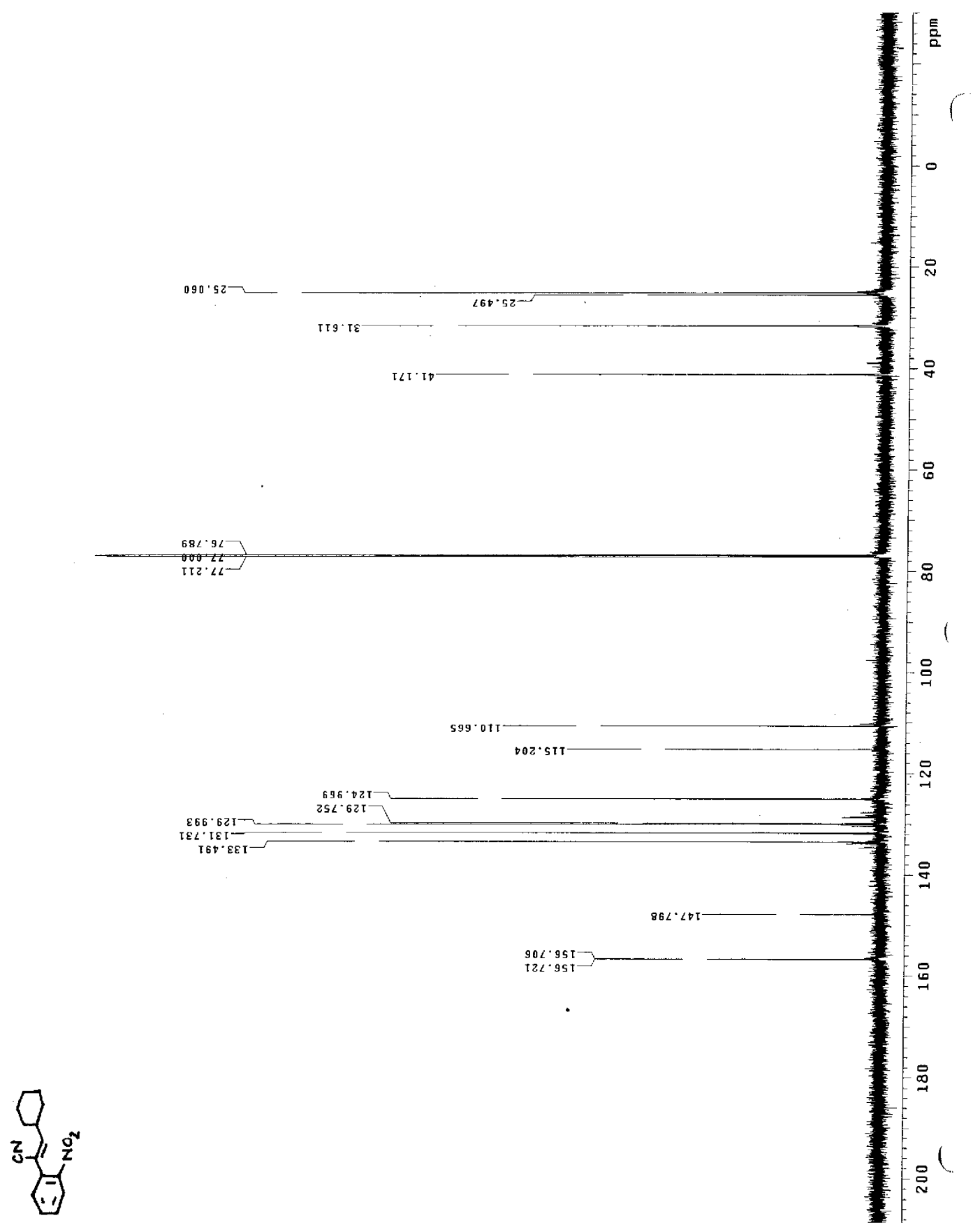

Figure 20: ${ }^{13} \mathrm{C}$ NMR of 3-Cyclohexyl-2-(2-nitrophenyl)-2-propenenitrile (111) 


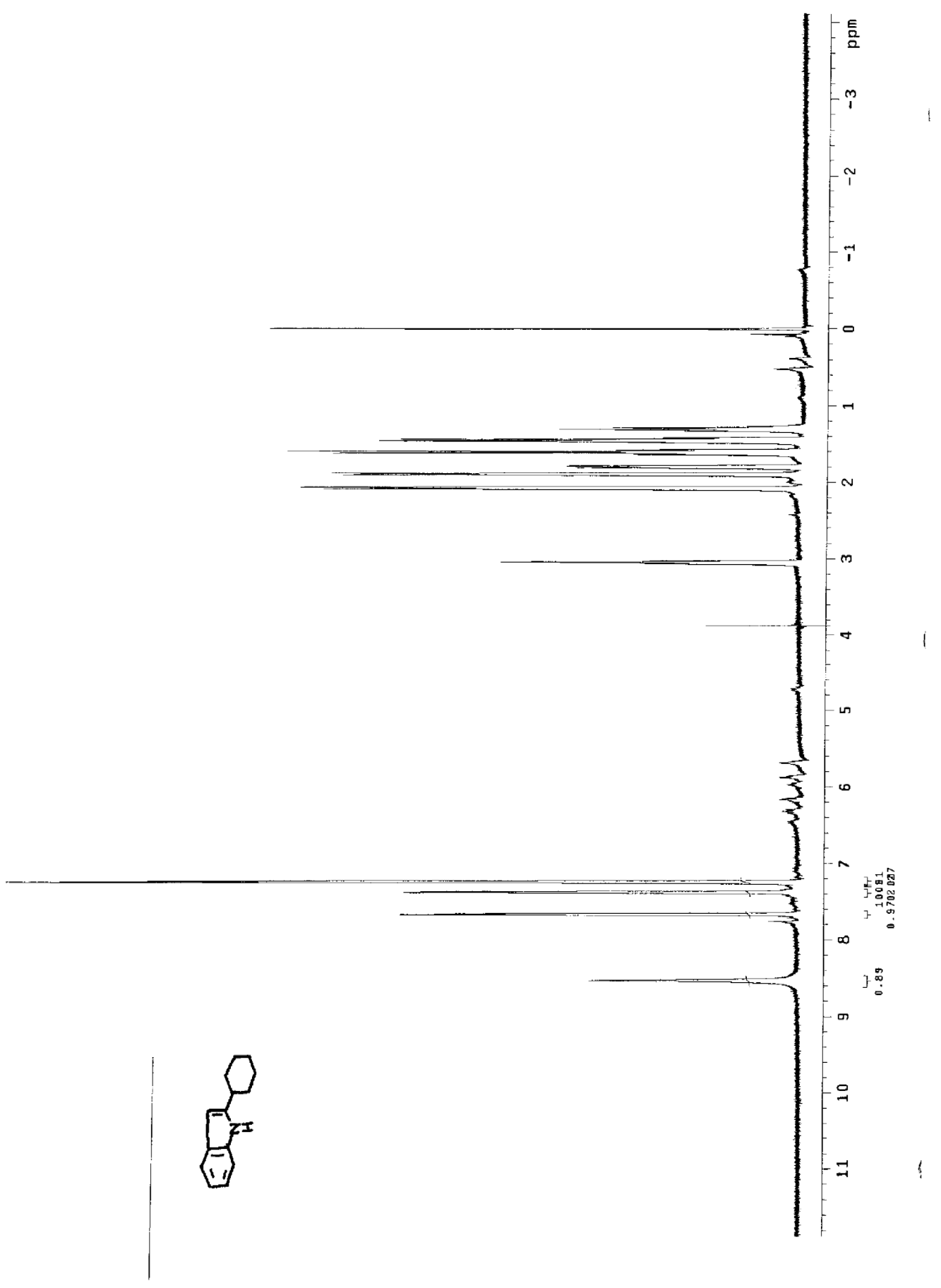

Figure 21: ${ }^{1} \mathrm{H}$ NMR of 3-Cyano-2-cyclohexylindole (112) 


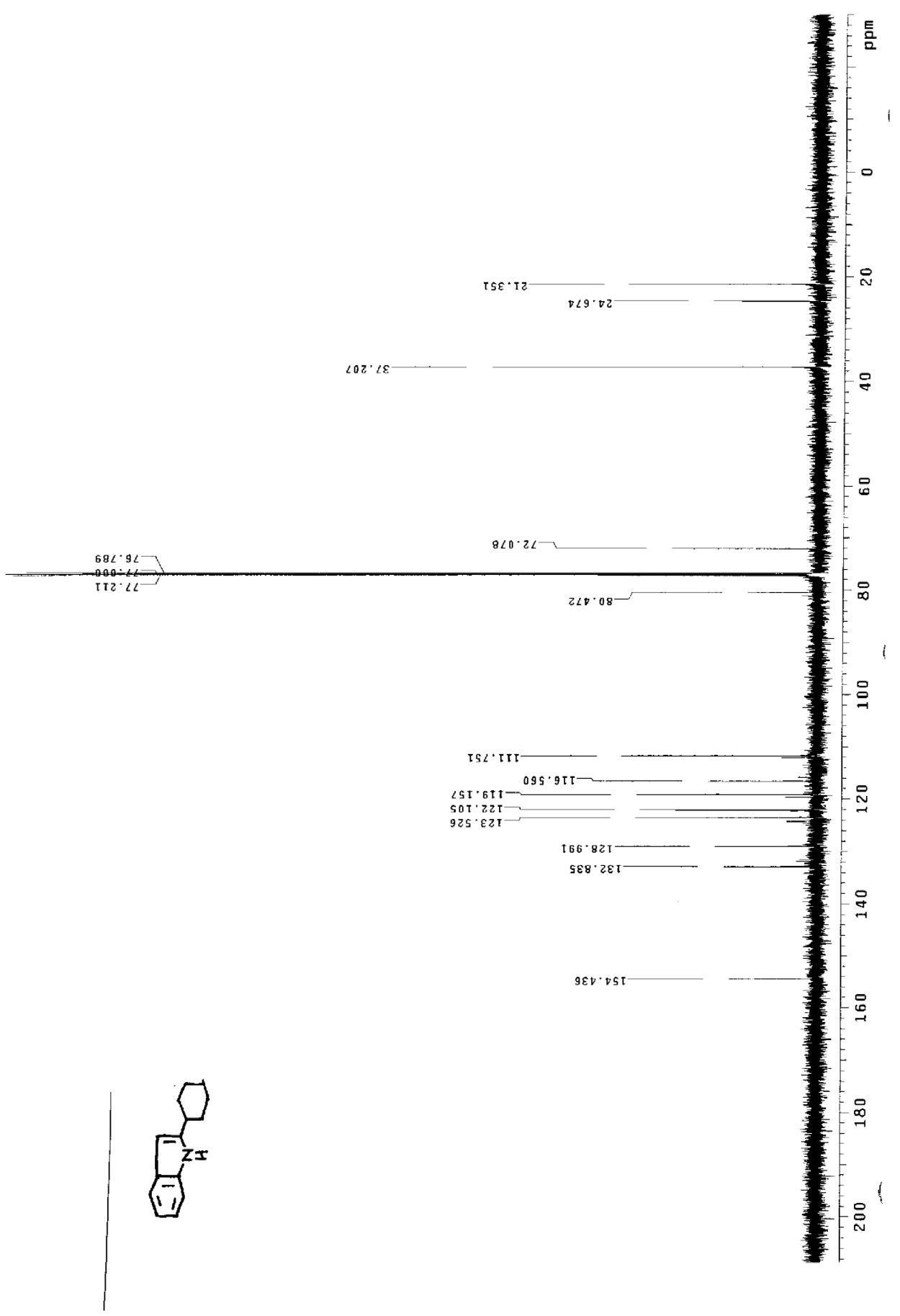

Figure 22: ${ }^{13} \mathrm{C}$ NMR of 3-Cyano-2-cyclohexylindole (112) 


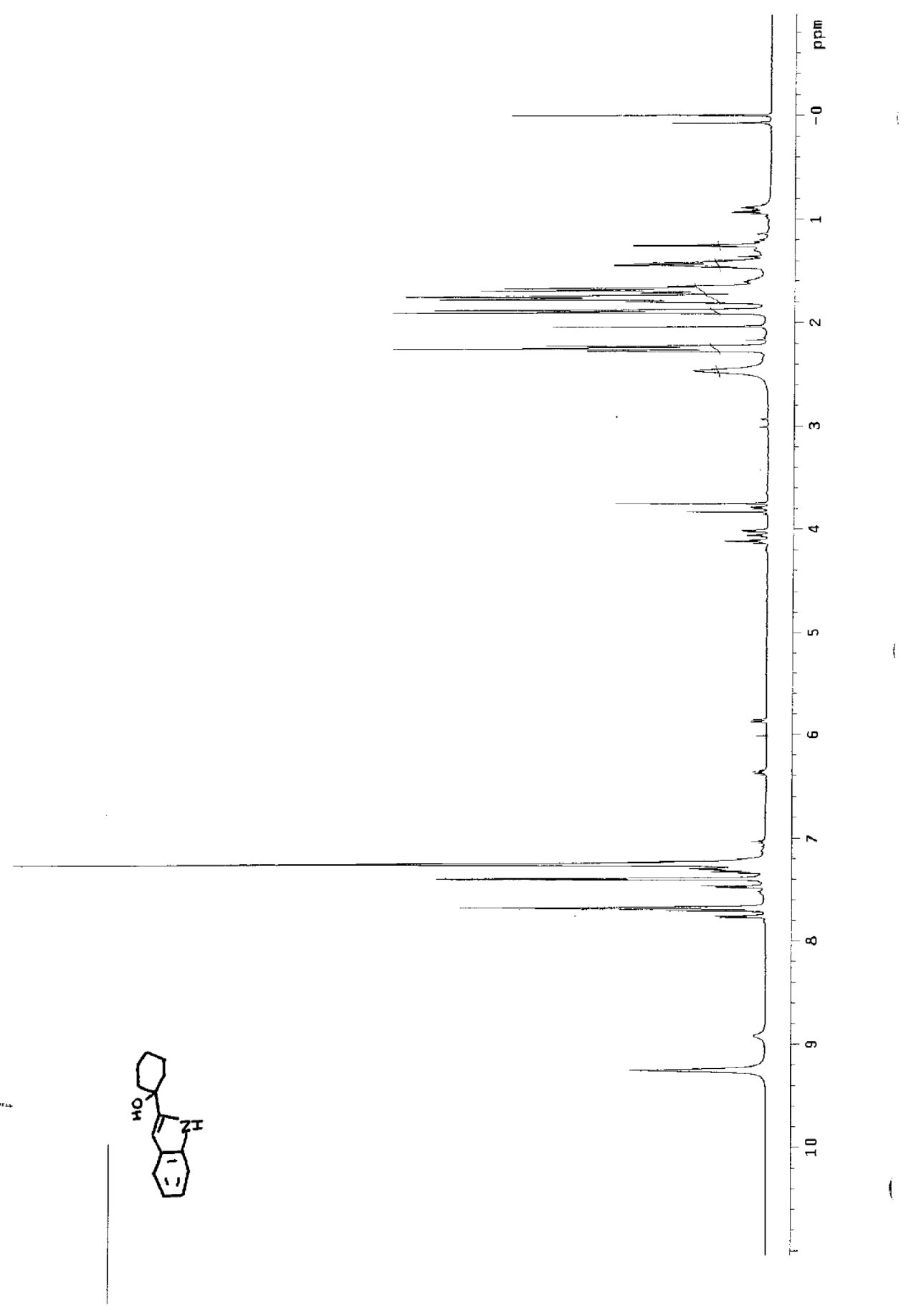

Figure 23: ${ }^{1} \mathrm{H}$ NMR of 3-cyano-2-(1-hydroxycyclohexyl)indole (113) 


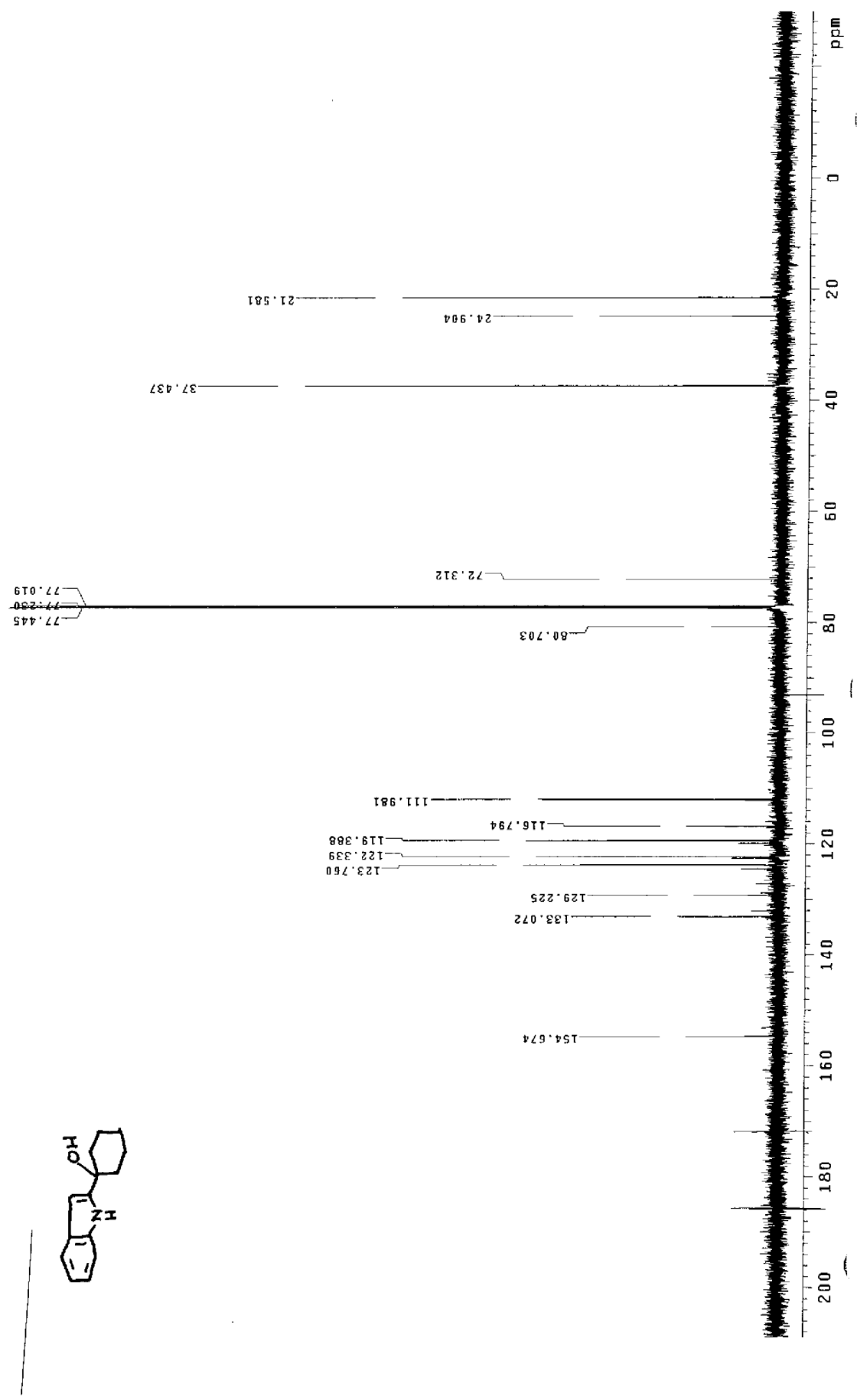

Figure 24: ${ }^{13} \mathrm{C}$ NMR of 3-cyano-2-(1-hydroxycyclohexyl)indole (113) 
${ }^{1} \mathrm{H}$ and ${ }^{13} \mathrm{C}$ NMR for Chapter 3: Salviadione

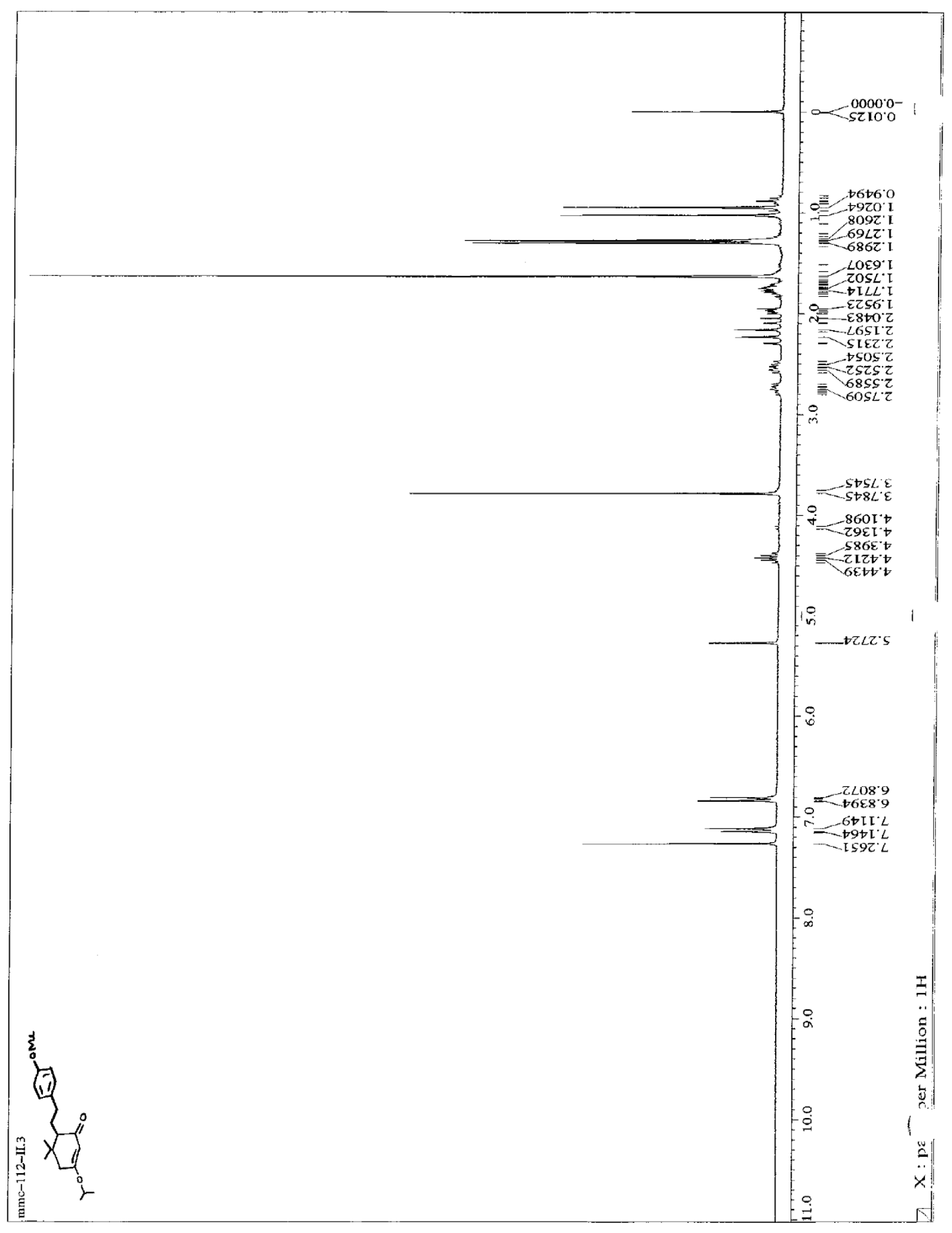

Figure 25: ${ }^{1} \mathrm{H}$ NMR of 5,5-Dimethyl-6-(4-methoxyphenylethyl)-3-(1-methylethoxy)cyclohex-2-enone (127) 


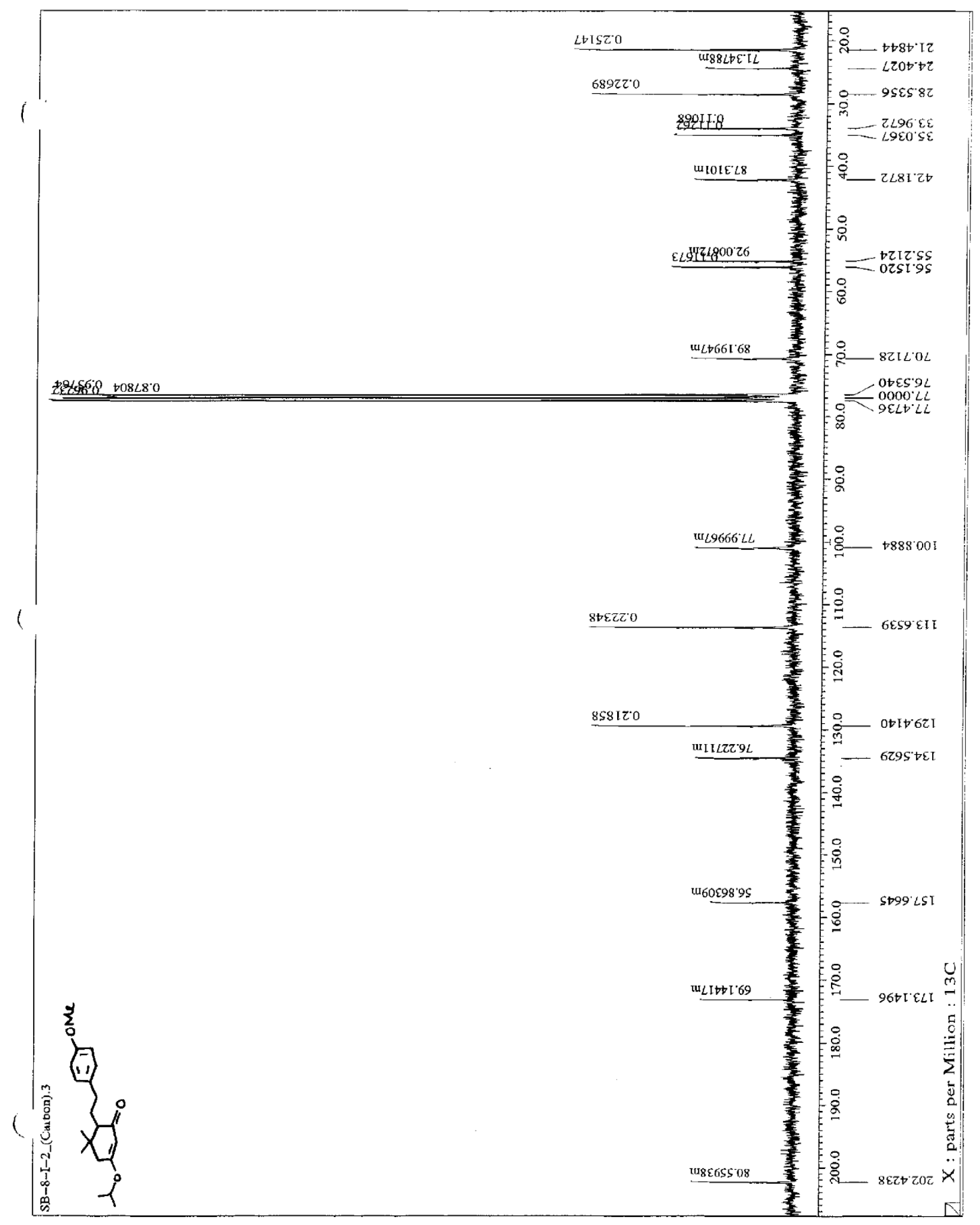

Figure 26: ${ }^{13} \mathrm{C}$ NMR of 5,5-Dimethyl-6-(4-methoxyphenylethyl)-3-(1-methylethoxy)cyclohex-2-enone (127) 


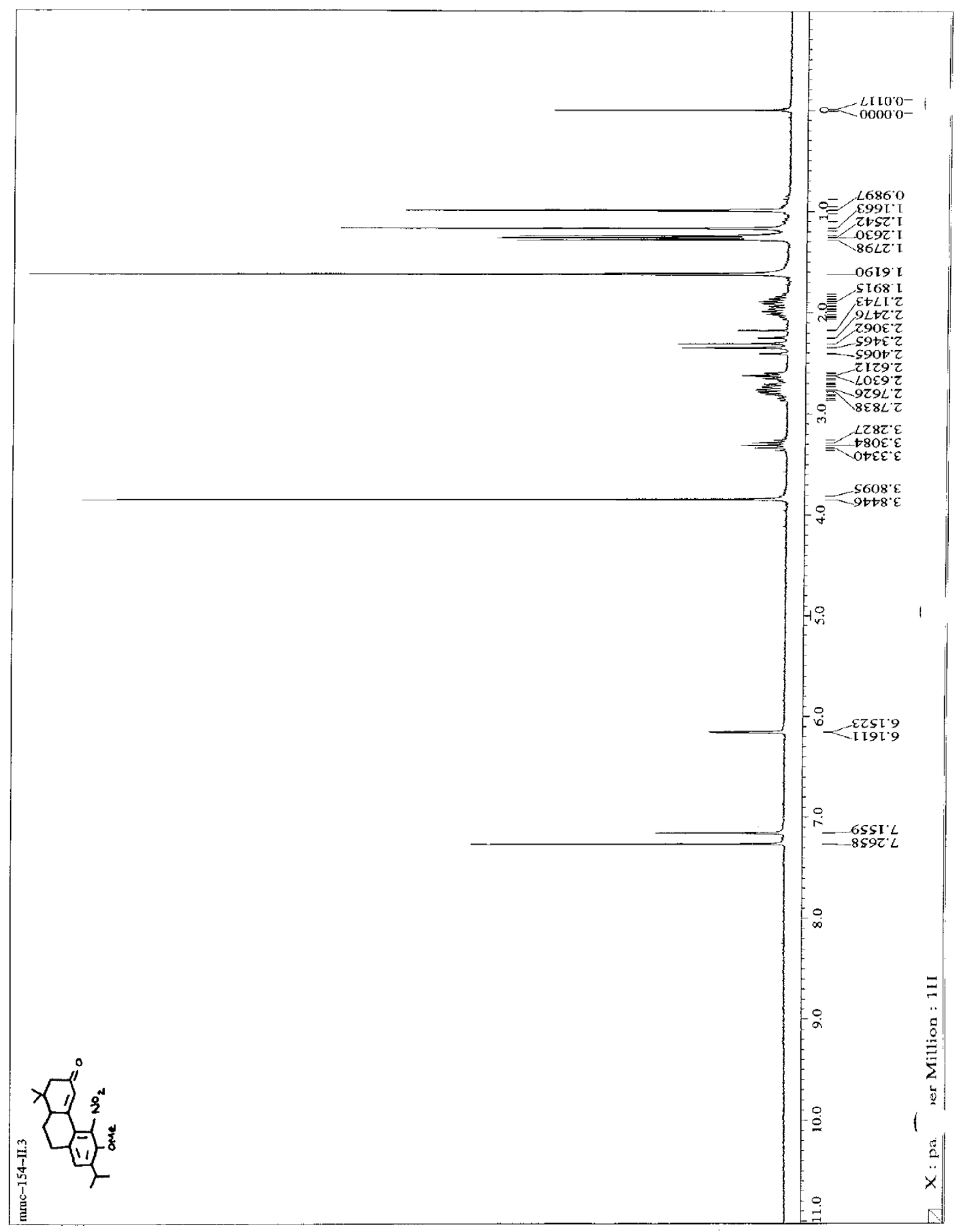

Figure 27: ${ }^{1} \mathrm{H}$ NMR of 10,10a-Dihydro-1,1-dimethyl-6-methoxy-7-(1-methylethyl)-5nitrophenanthren-3(1H,2H,9H)-one (132) 


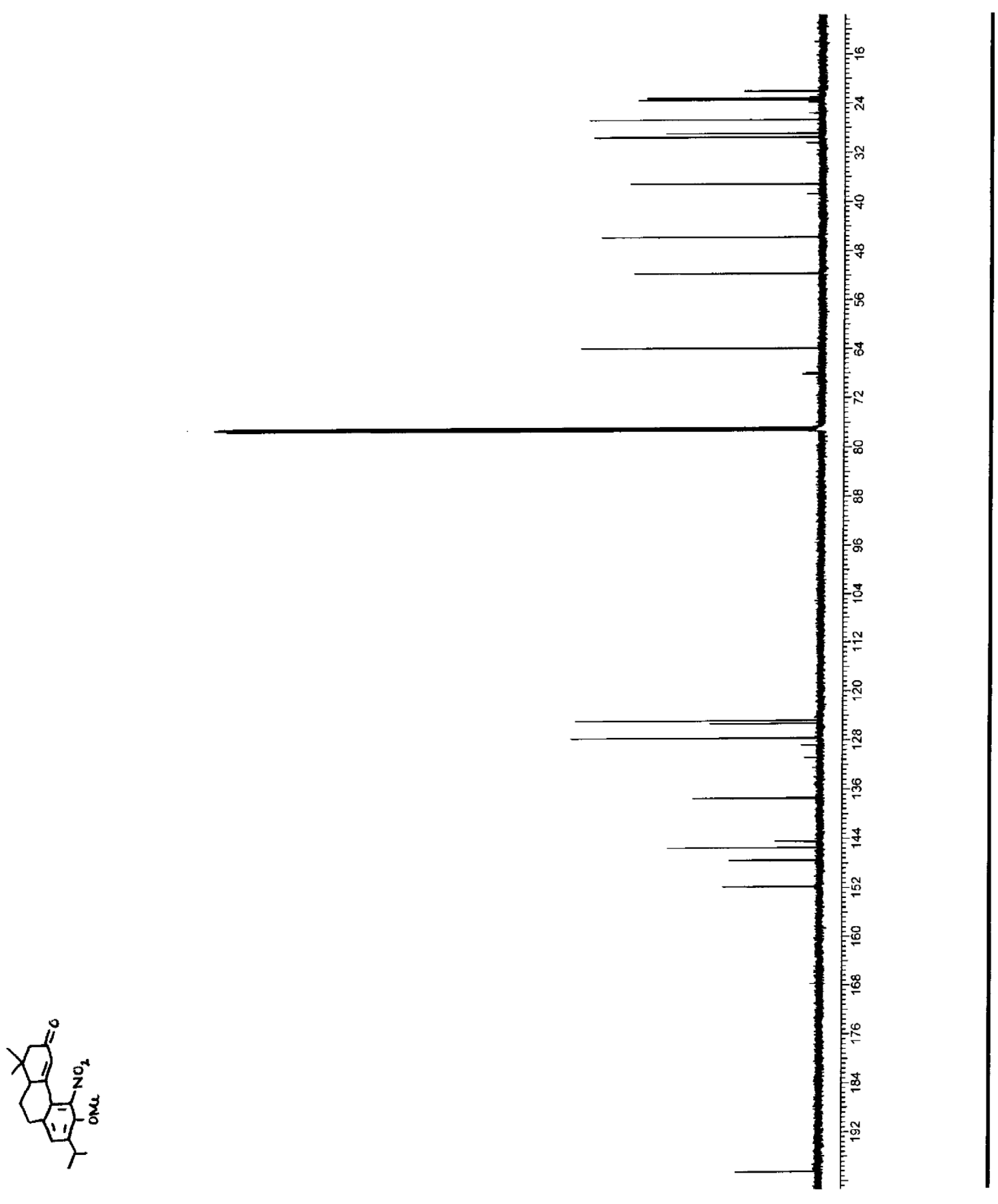

Figure $28:{ }^{13} \mathrm{C}$ NMR of 10,10a-Dihydro-1,1-dimethyl-6-methoxy-7-(1-methylethyl)-5nitrophenanthren-3(1H,2H,9H)-one (132) 


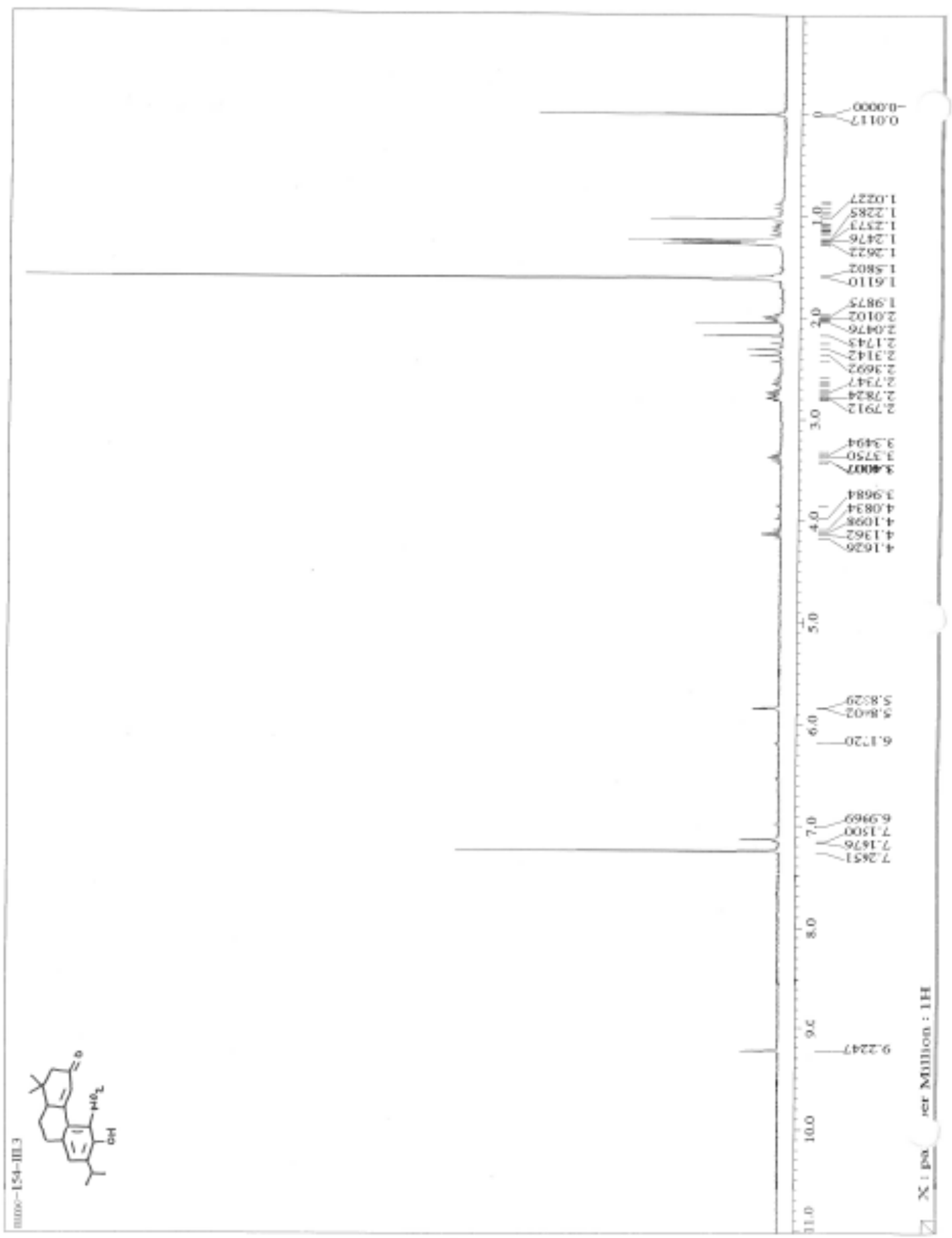

Figure 29: ${ }^{1} \mathrm{H}$ NMR of 10,10a-Dihydro-1,1-dimethyl-6-hydroxy-7-(1-methylethyl)-5nitrophenanthren-3(1H,2H,9H)-one (125) 


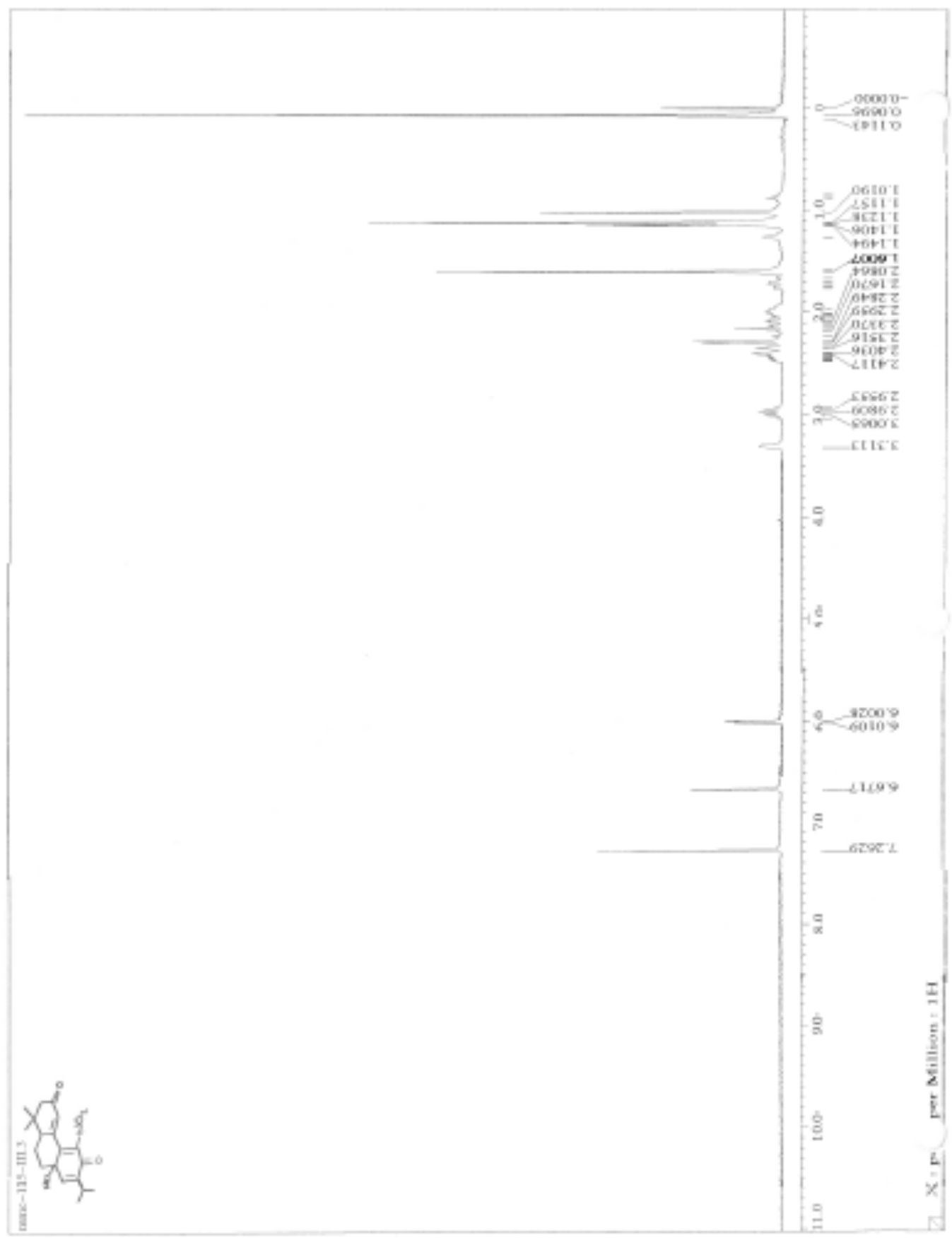

Figure 30: ${ }^{1} \mathrm{H}$ NMR of $8 a, 9,10,10 a$-Tetrahydro-1,1-dimethyl-8a-hydroxy-7-(1methylethyl)-5-nitrophenanthrene-3,6(1H,2H)-dione (133) 


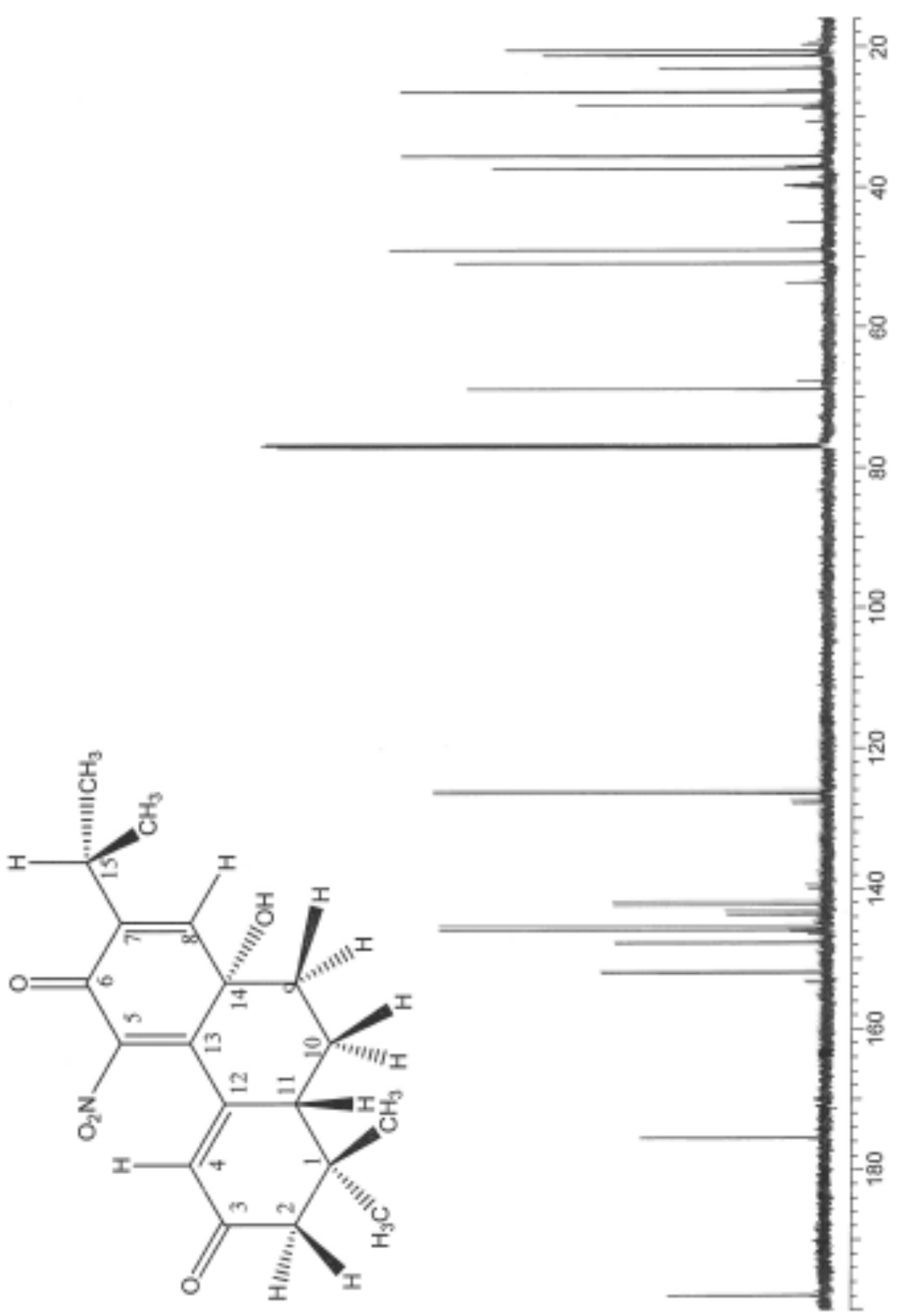

Figure $31:{ }^{13} \mathrm{C}$ NMR of $8 a, 9,10,10 a$-Tetrahydro-1,1-dimethyl-8a-hydroxy-7-(1methylethyl)-5-nitrophenanthrene-3,6(1H,2H)-dione (133) 


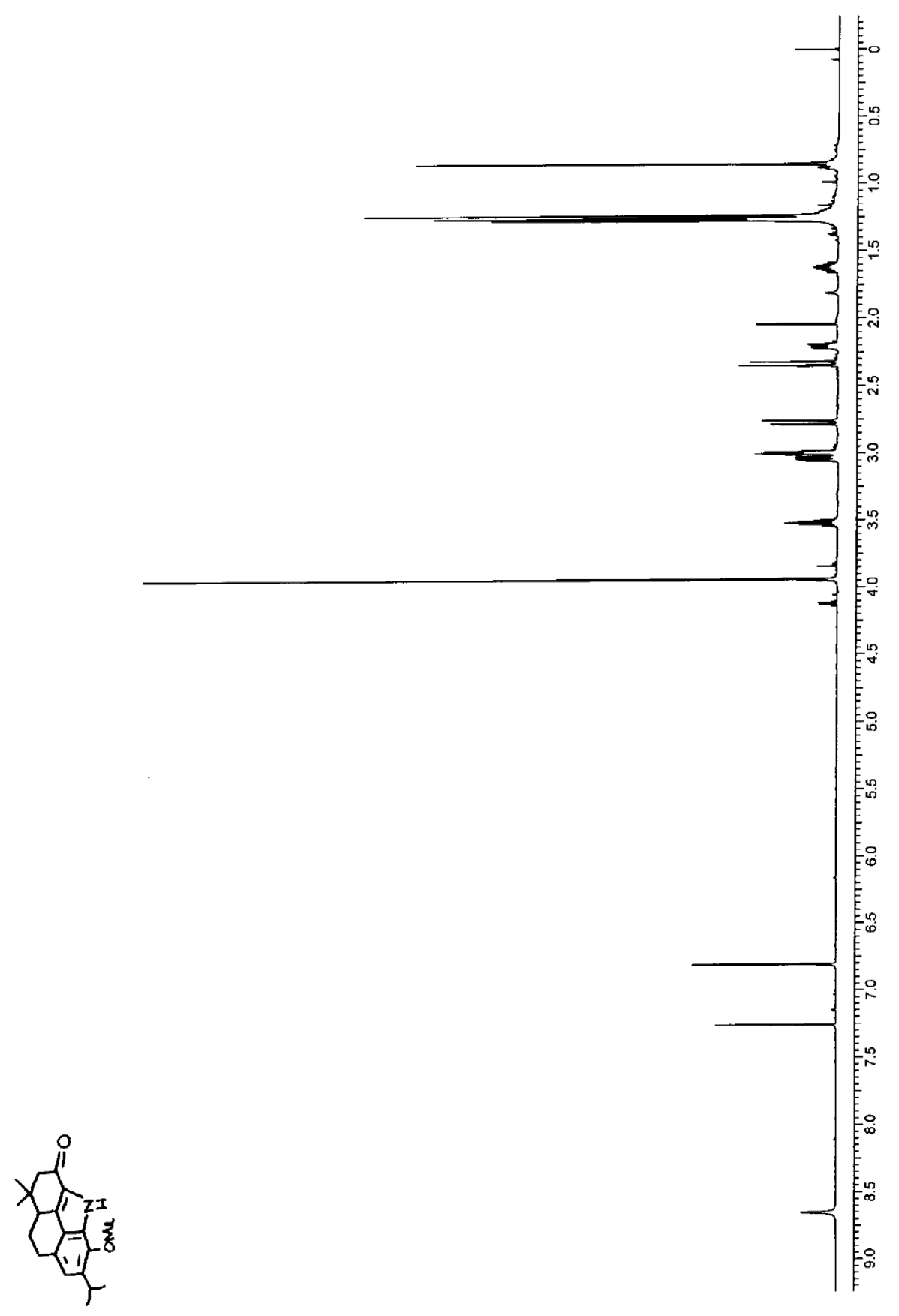

Figure 32: ${ }^{1} \mathrm{H}$ NMR of 1,8,9,9a-Dihydro-1,1-dimethyl-5-methoxy-6-(1-methylethyl)-4Hbenzo[def]carbazol-3(2H)-one (134) 


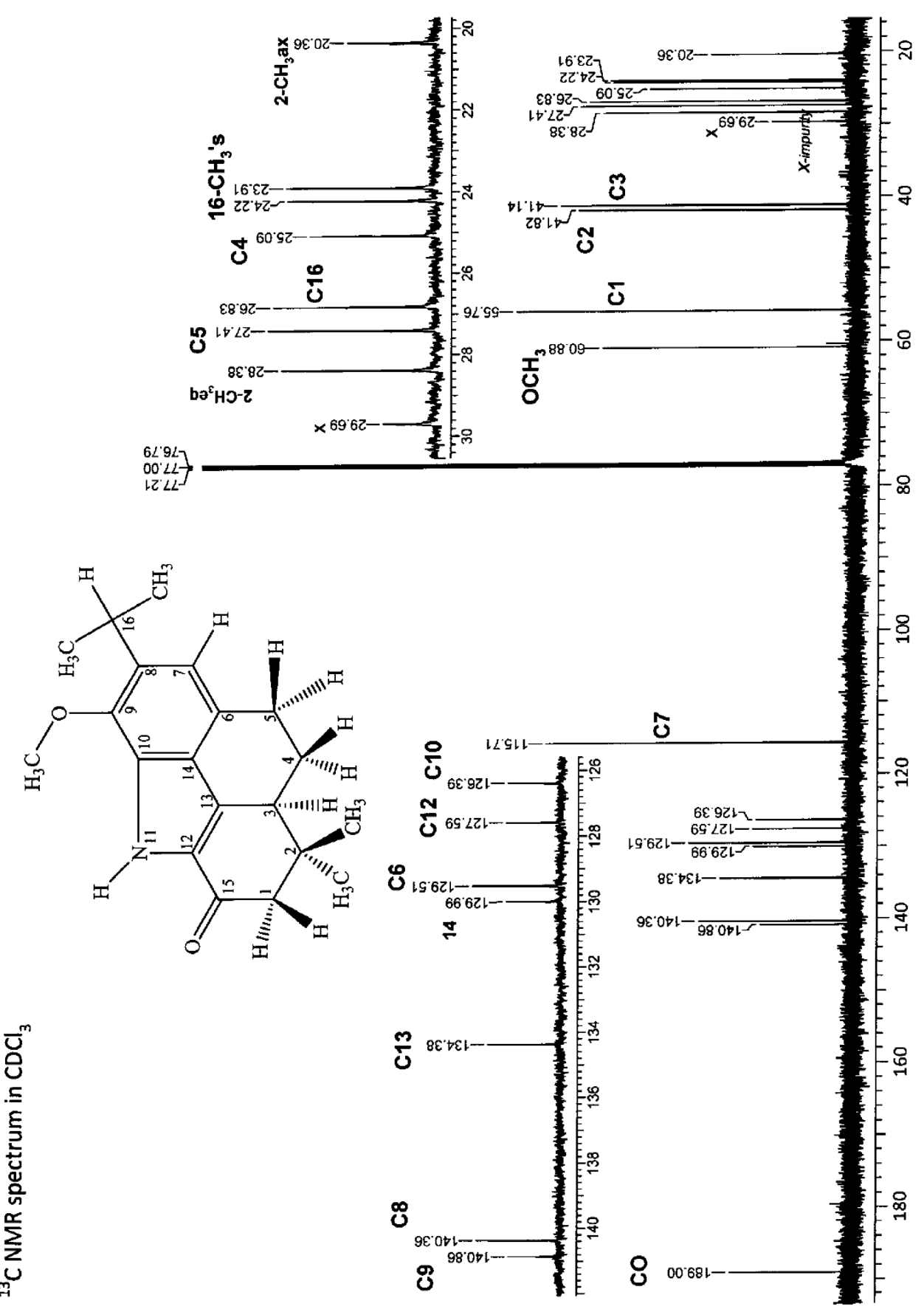

Figure 33: ${ }^{13} \mathrm{C}$ NMR of 1,8,9,9a-Dihydro-1,1-dimethyl-5-methoxy-6-(1-methylethyl)-4Hbenzo[def]carbazol-3(2H)-one (134) 


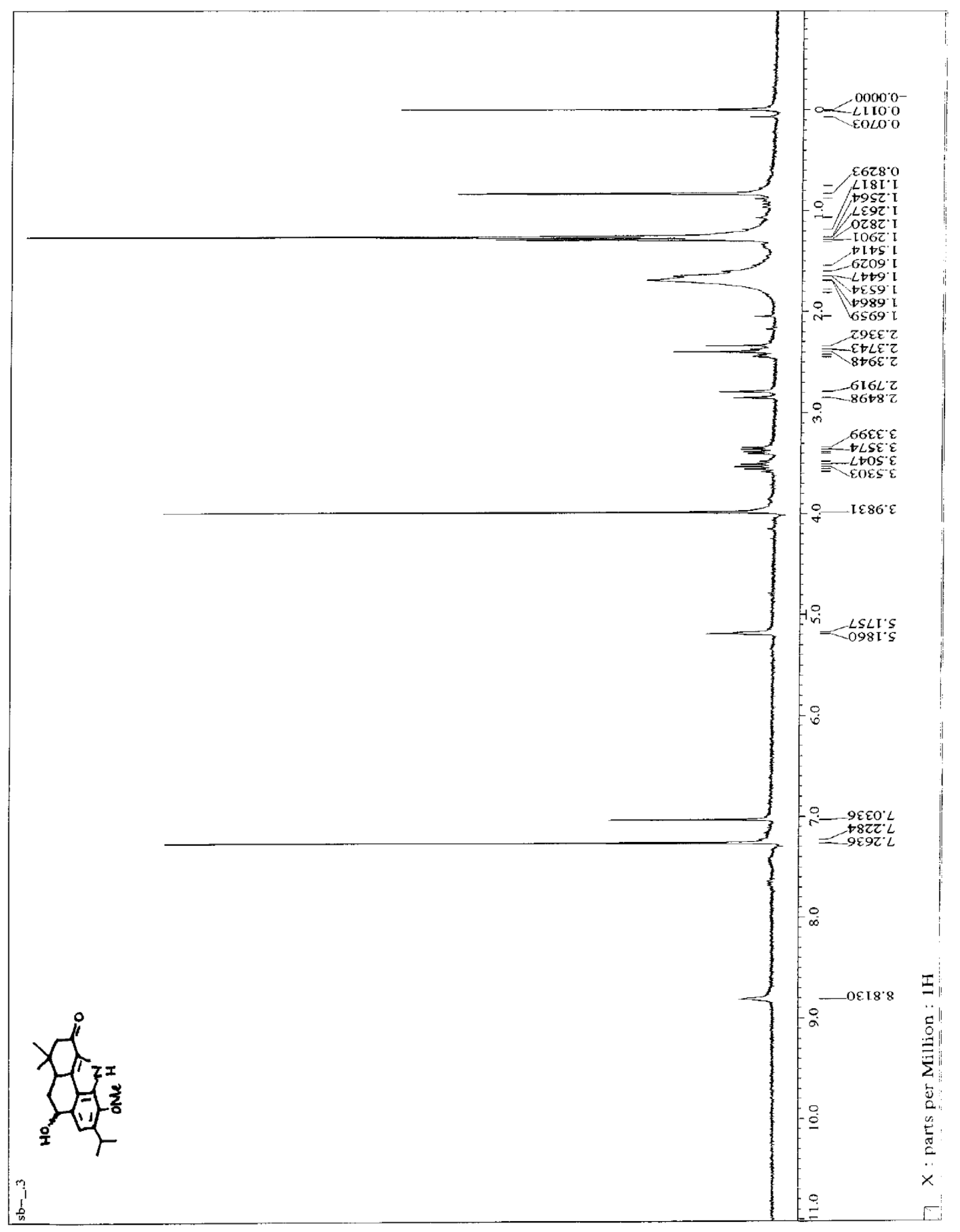

Figure 34: ${ }^{1} \mathrm{H}$ NMR of 1,8,9,9a-Tetrahydro-1,1-dimethyl-5-methoxy-6-(1-methylethyl)-8hydroxy-4H-benzo[def]carbazol-3(2H)-one (136) 


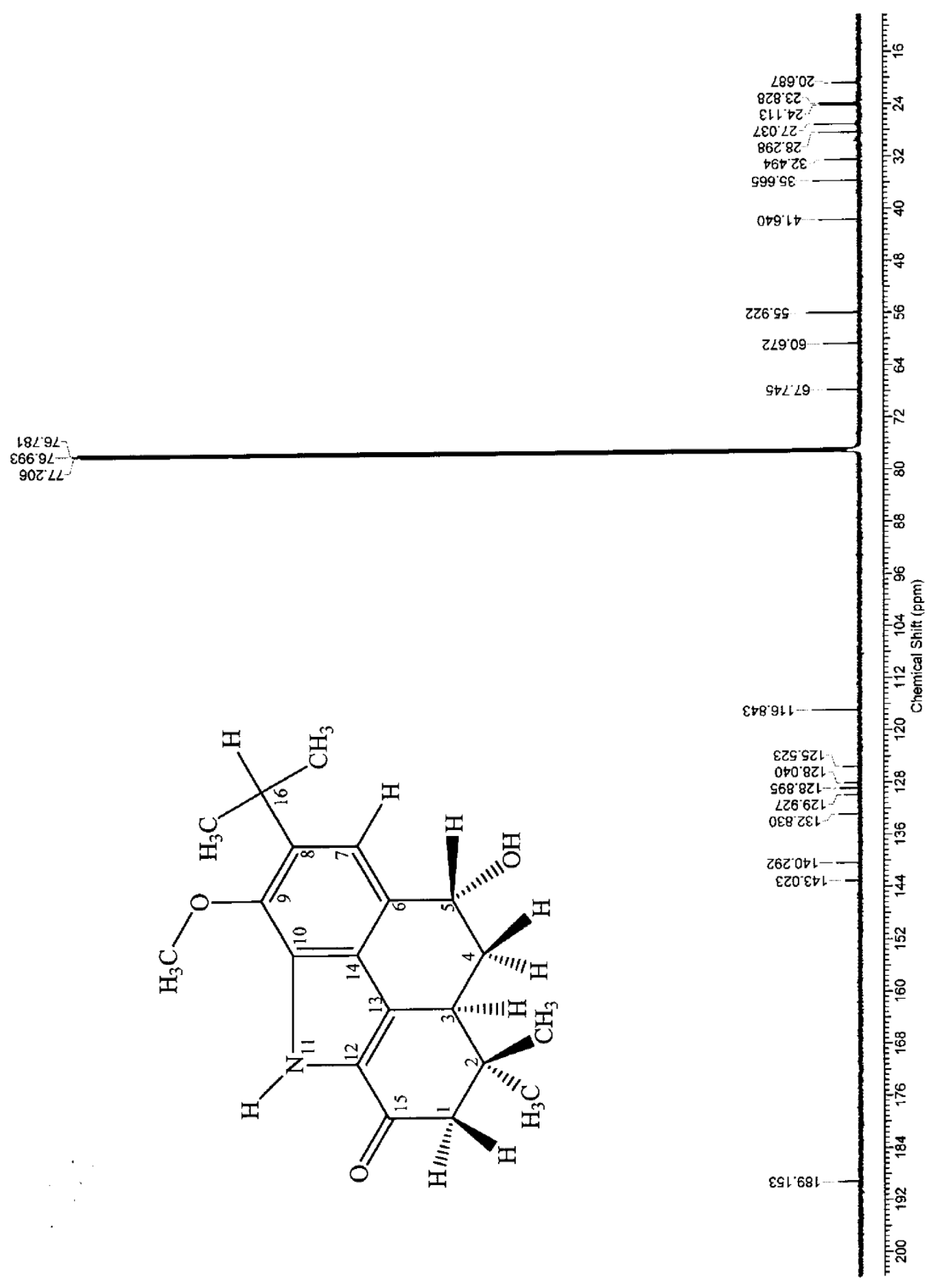

Figure $35:{ }^{13} \mathrm{C}$ NMR of 1,8,9,9a-Tetrahydro-1,1-dimethyl-5-methoxy-6-(1-methylethyl)-8hydroxy-4H-benzo[def]carbazol-3(2H)-one (136) 


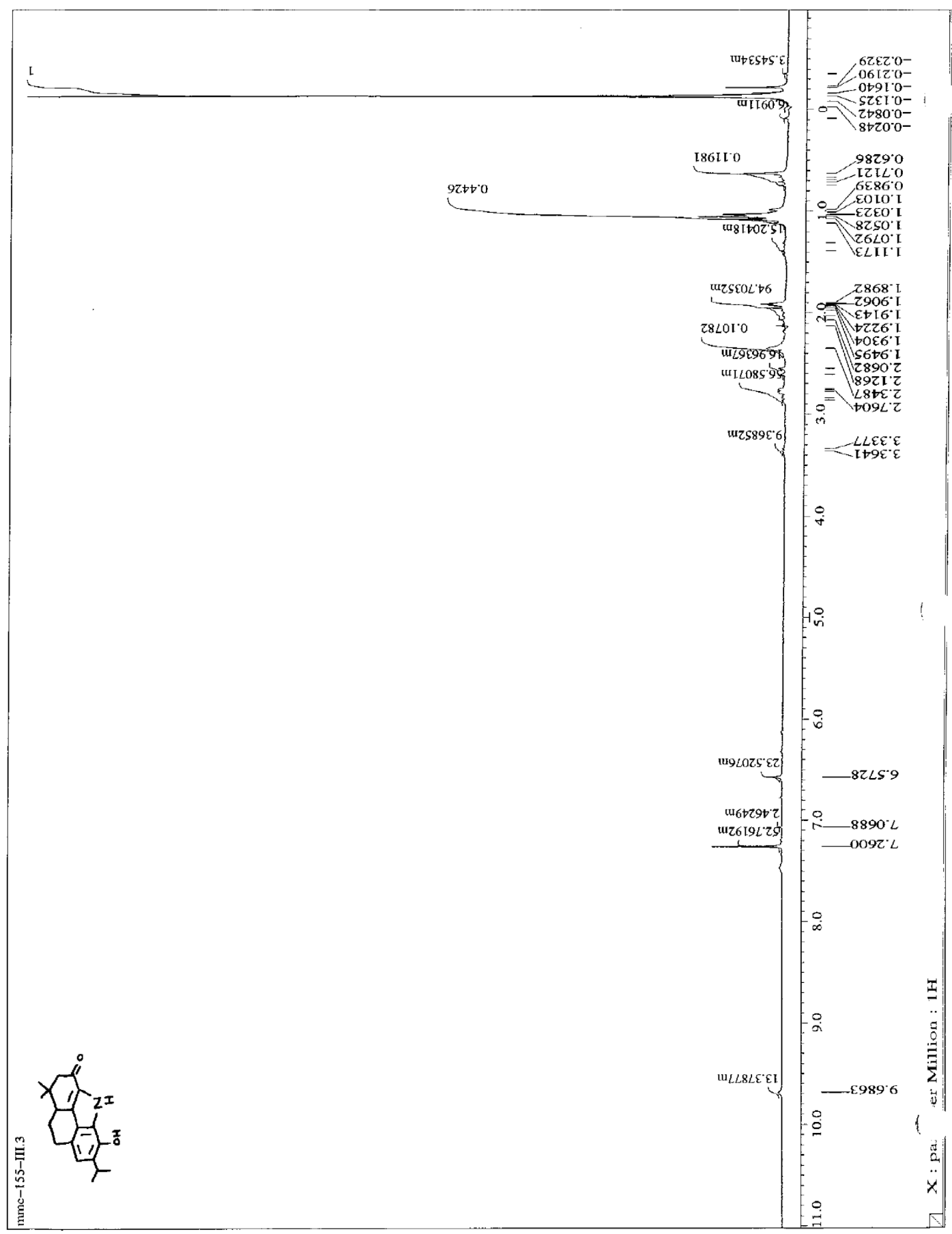

Figure 36: ${ }^{1} \mathrm{H}$ NMR of 1,8,9,9a-Tetrahydro-1,1-dimethyl-5-hydroxy-6-(1-methylethyl)4H-benzo[def]carbazol-3(2H)-one (124) 


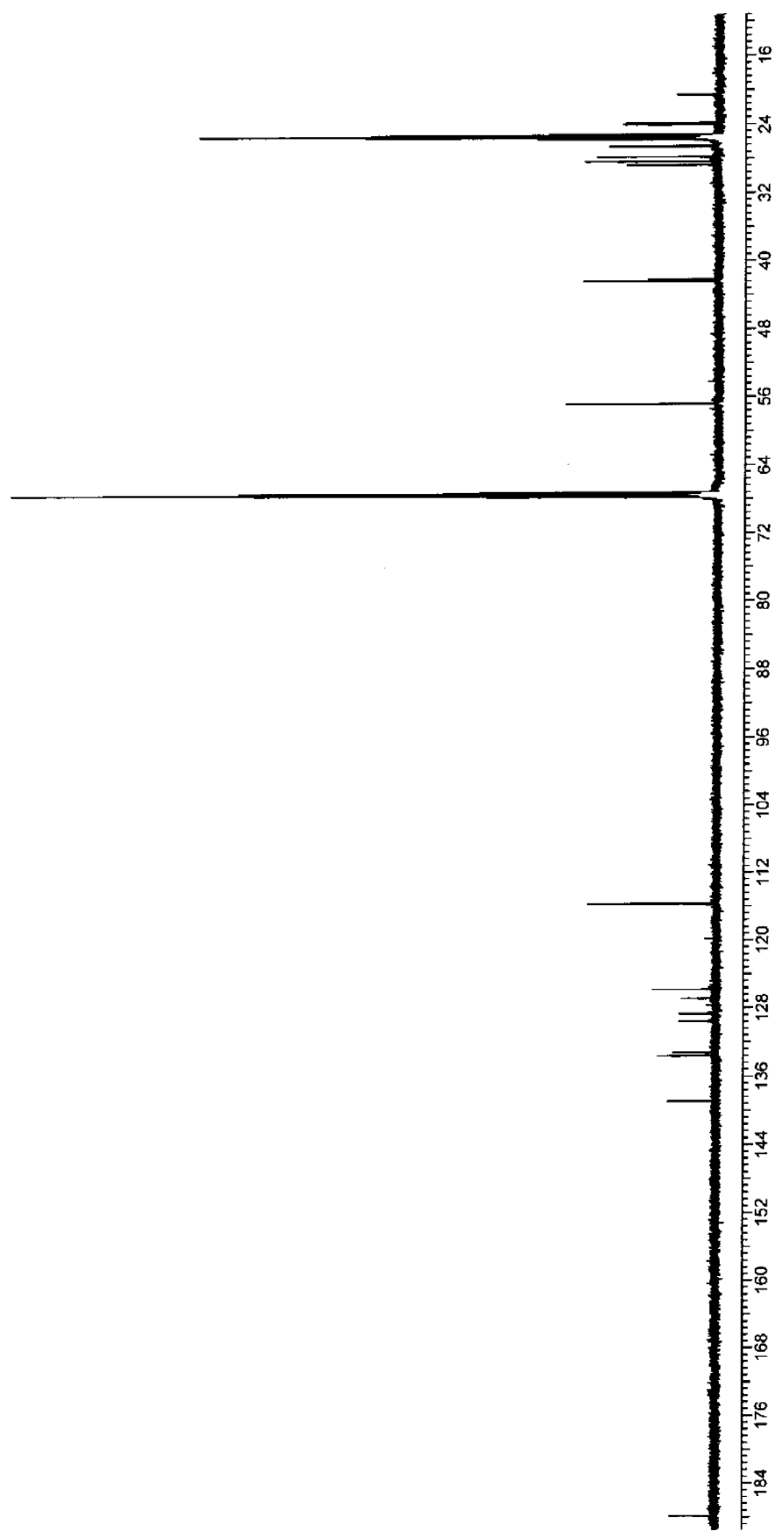

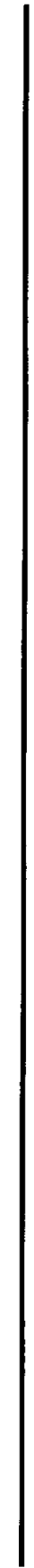

Figure $37:{ }^{13} \mathrm{C}$ NMR of 1,8,9,9a-Tetrahydro-1,1-dimethyl-5-hydroxy-6-(1-methylethyl)4H-benzo[def]carbazol-3(2H)-one (124) 


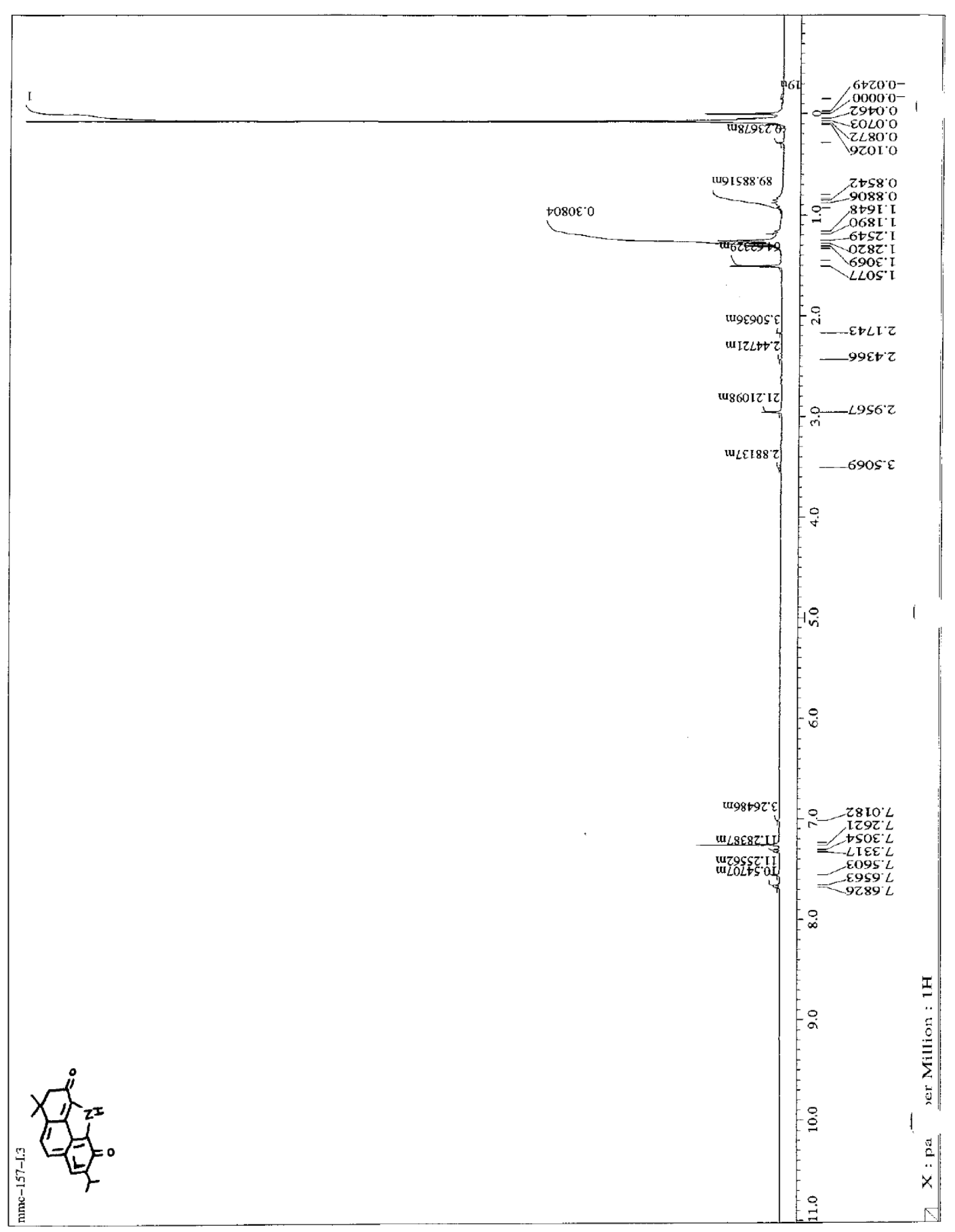

Figure 38: ${ }^{1} \mathrm{H}$ NMR of Salviadione (123) 


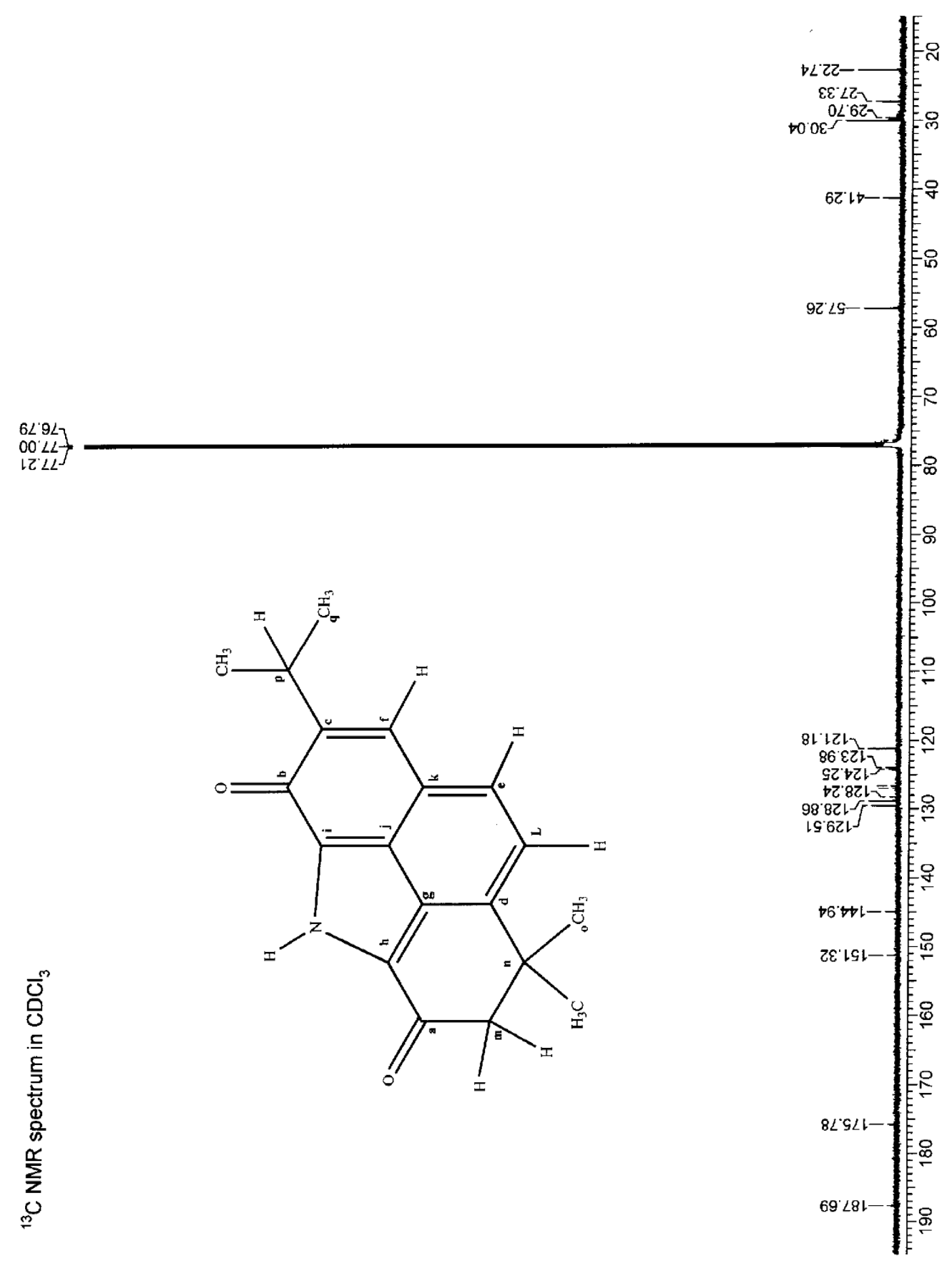

Figure 39: ${ }^{13} \mathrm{C}$ NMR of Salviadione (123) 
${ }^{1} \mathrm{H}$ and ${ }^{13} \mathrm{C}$ NMR for Chapter 4: Chemoselective Coupling

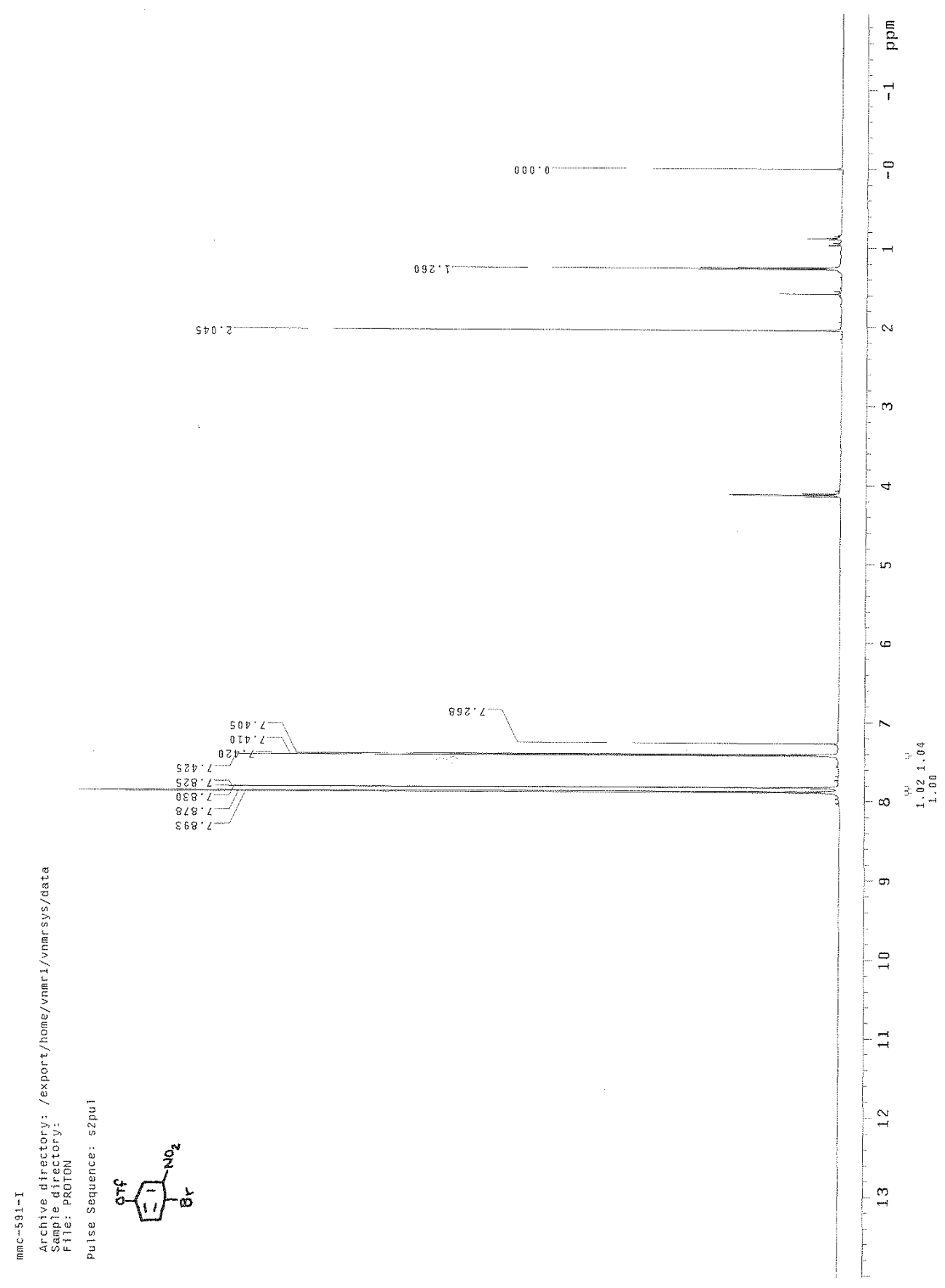

Figure 40: ${ }^{1} \mathrm{H}$ NMR of 2-Bromo-5-trifluoromethanesulfonyloxy-nitrobenzene (163) 


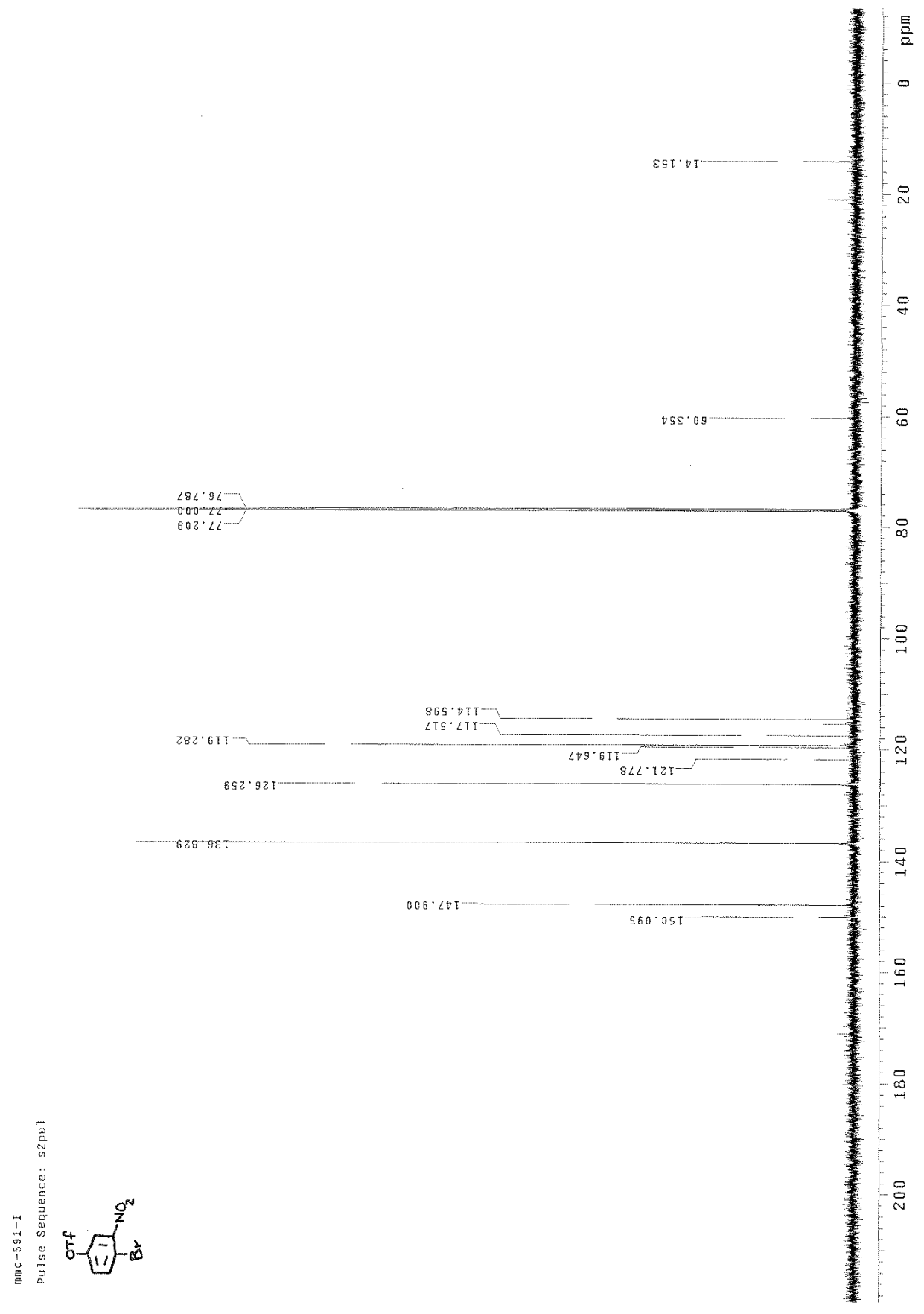

Figure 41: ${ }^{13} \mathrm{C}$ NMR of 2-Bromo-5-trifluoromethanesulfonyloxy-nitrobenzene (163) 


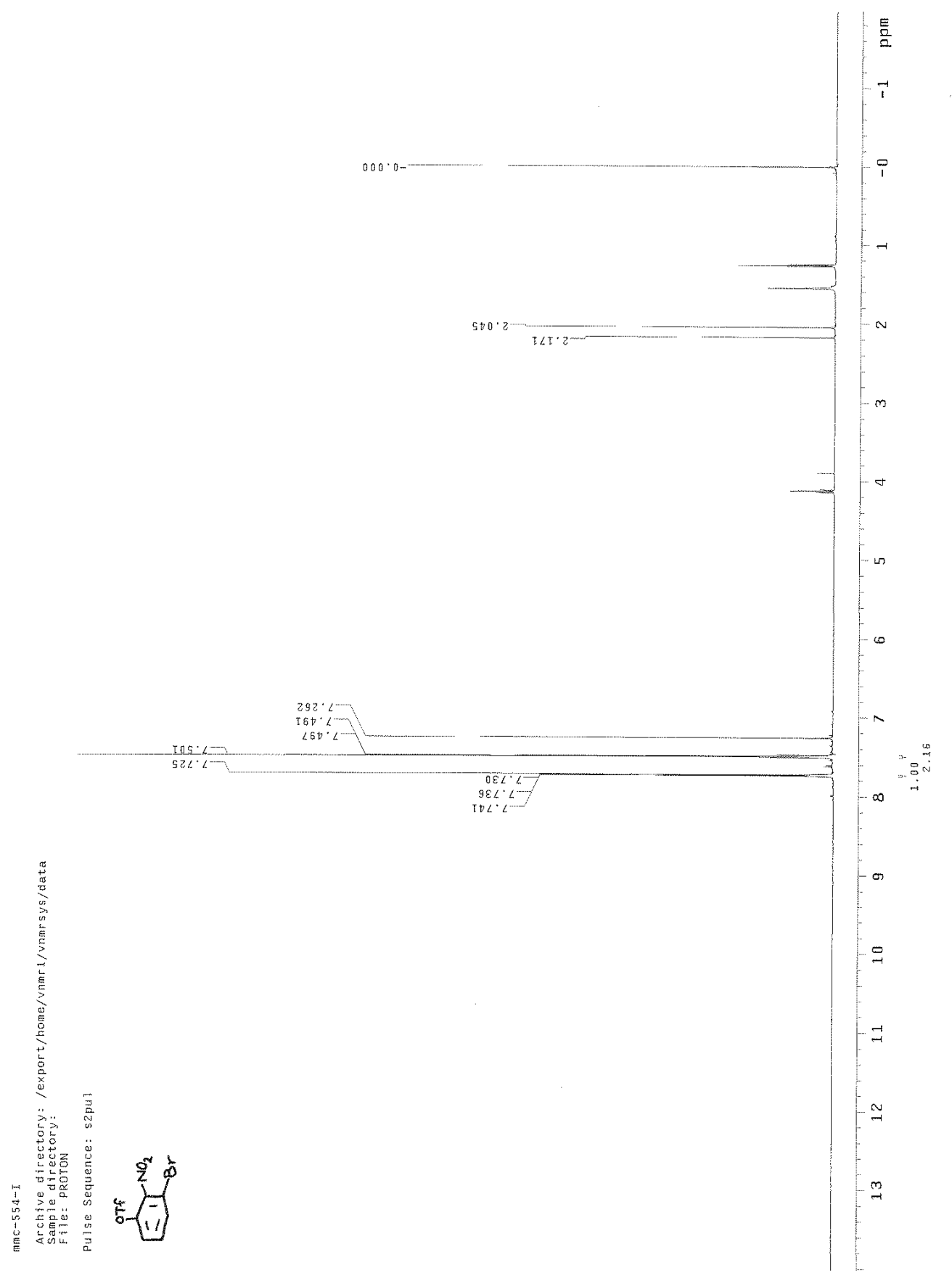

Figure 42: ${ }^{1} \mathrm{H}$ NMR of 2-Bromo-6-trifluoromethanesulfonyloxy-nitrobenzene (166) 


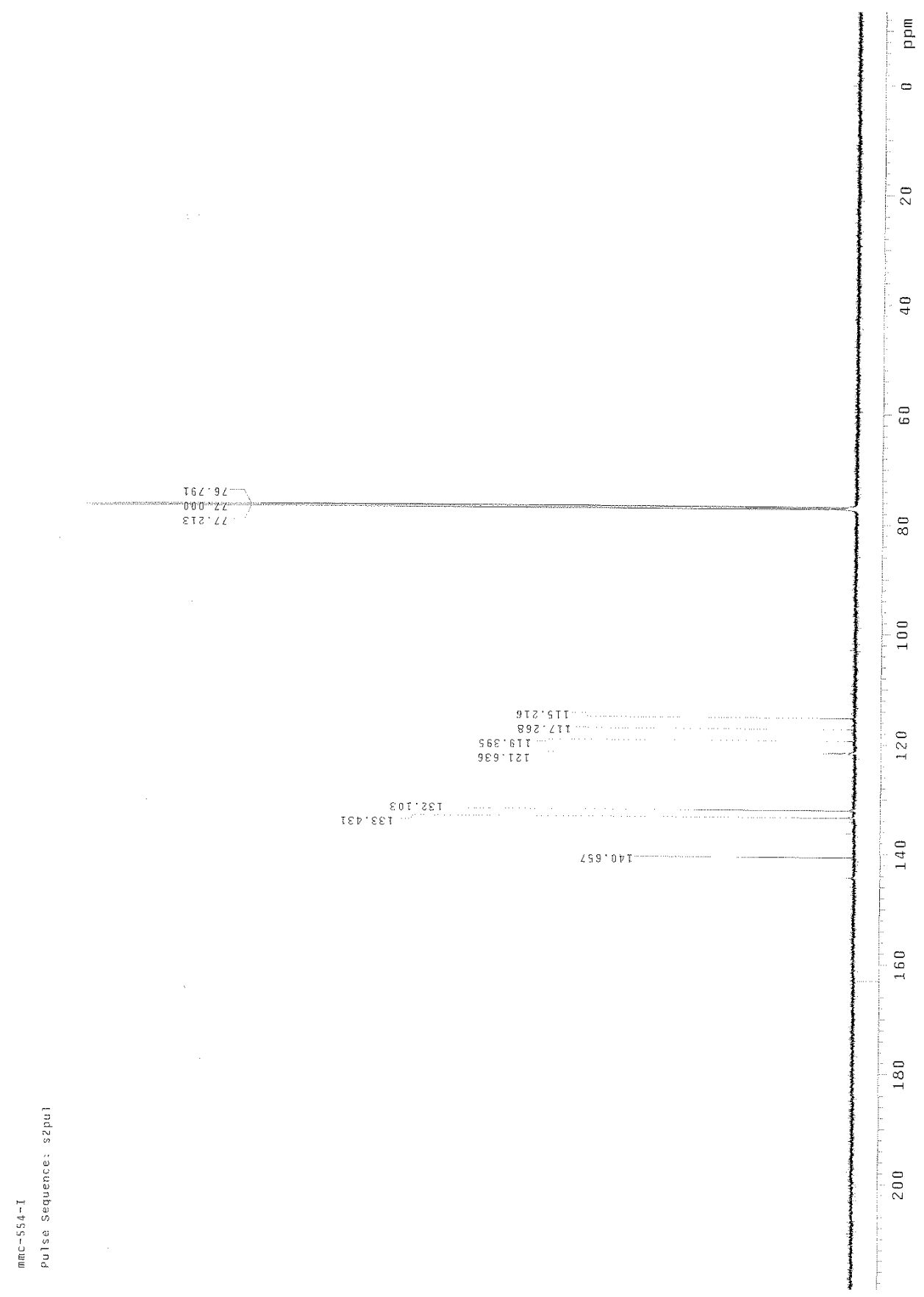

Figure 43: ${ }^{13} \mathrm{C}$ NMR of 2-Bromo-6-trifluoromethanesulfonyloxy-nitrobenzene (166) 


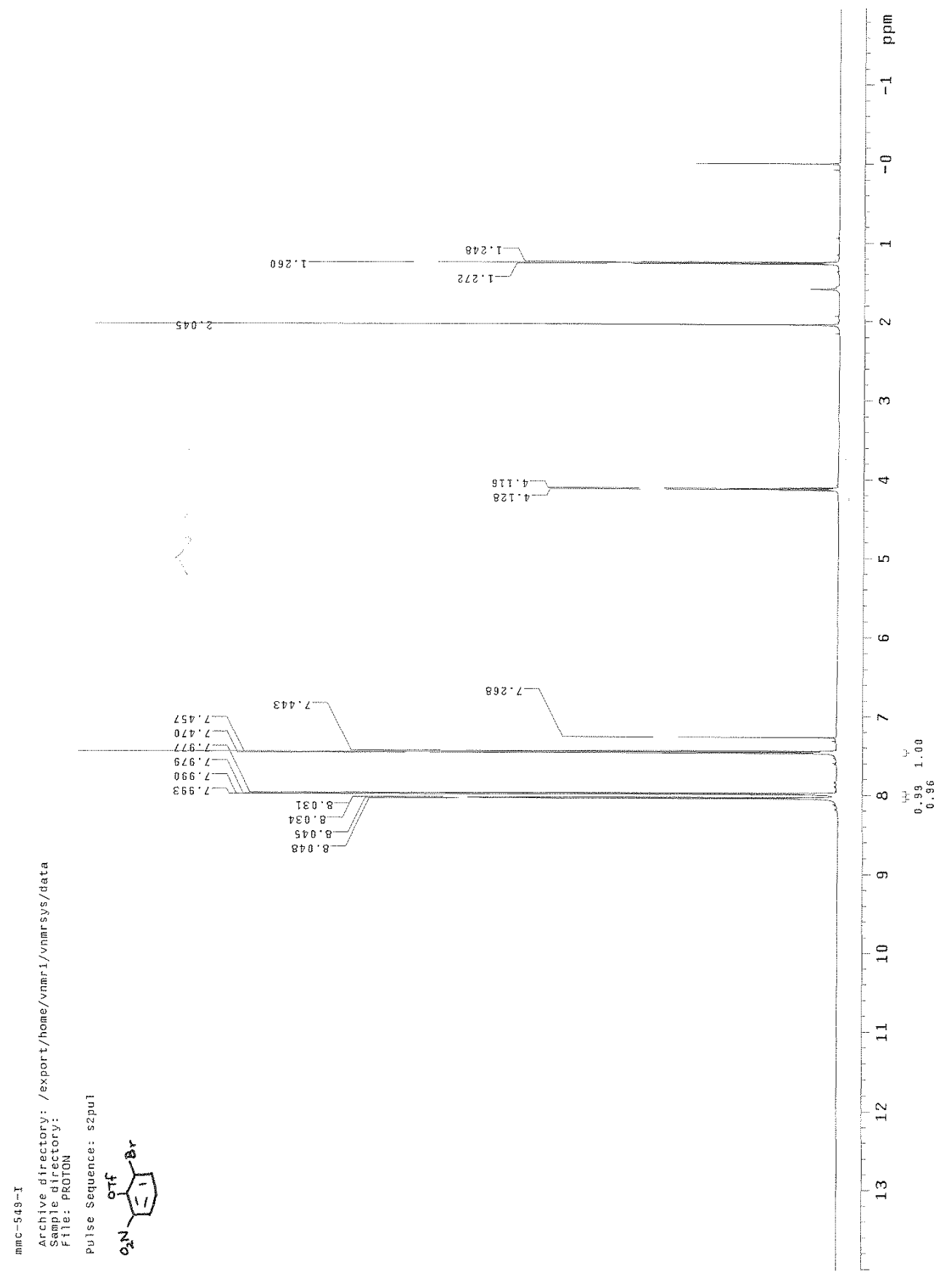

Figure 44: ${ }^{1} \mathrm{H}$ NMR of 3-Bromo-2-trifluoromethanesulfonyloxy-nitrobenzene (151) 


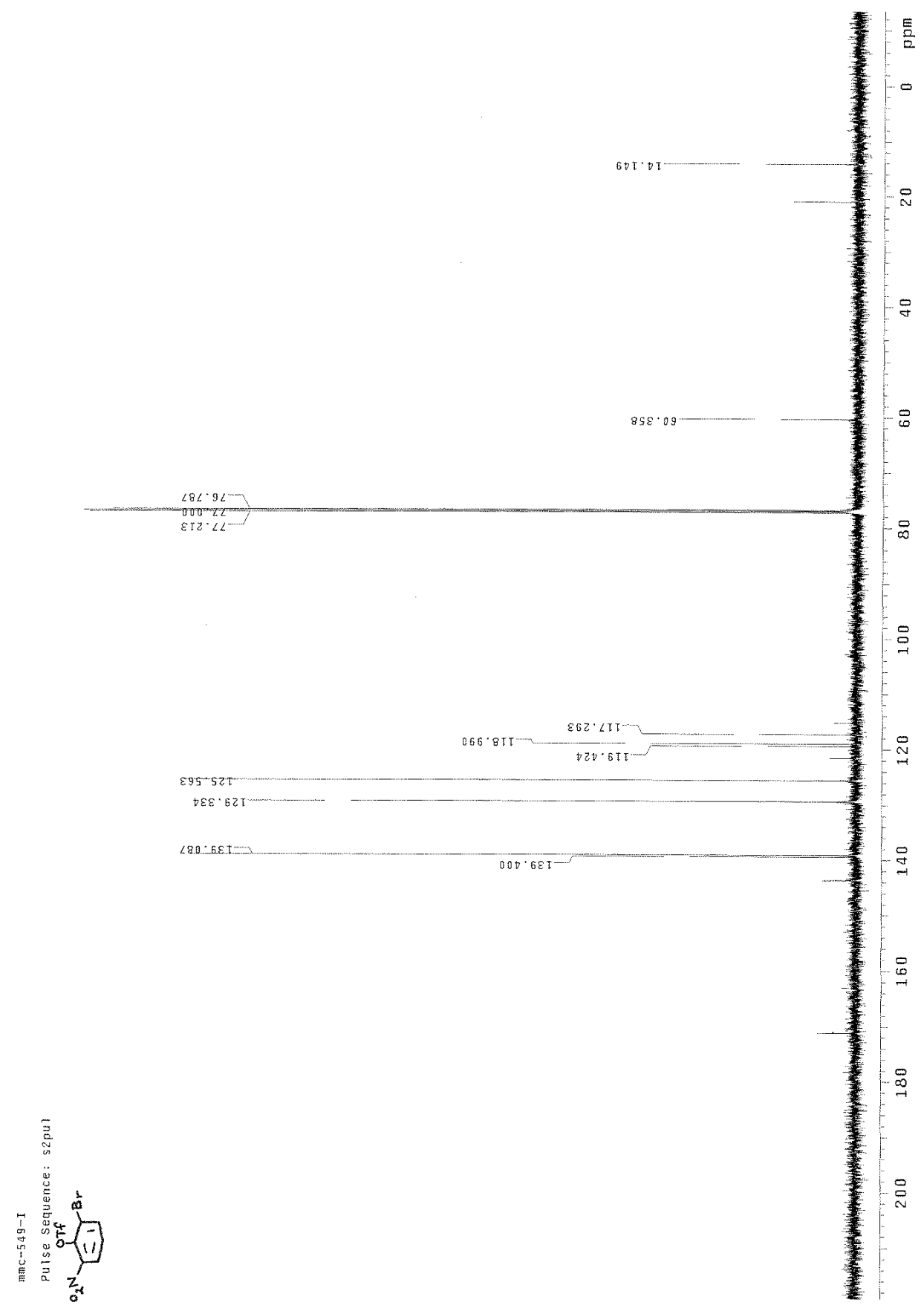

Figure 45: ${ }^{13} \mathrm{C}$ NMR of 3-bromo-2-trifluoromethanesulfonyloxy-nitrobenzene (151) 


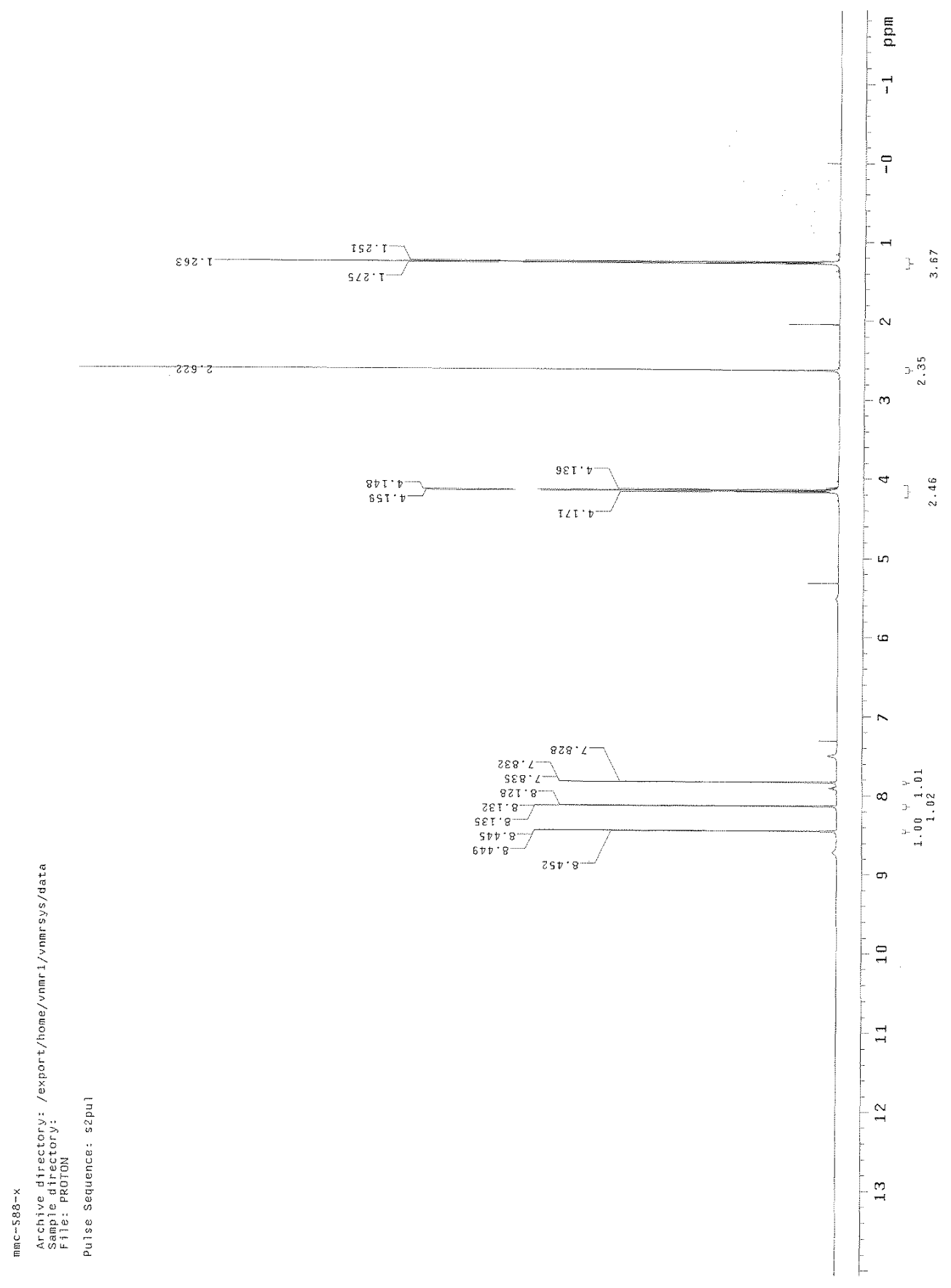

Figure 46: ${ }^{1} \mathrm{H}$ NMR of 3-Bromo-5-trifluoromethanesulfonyloxy-nitrobenzene (157) 


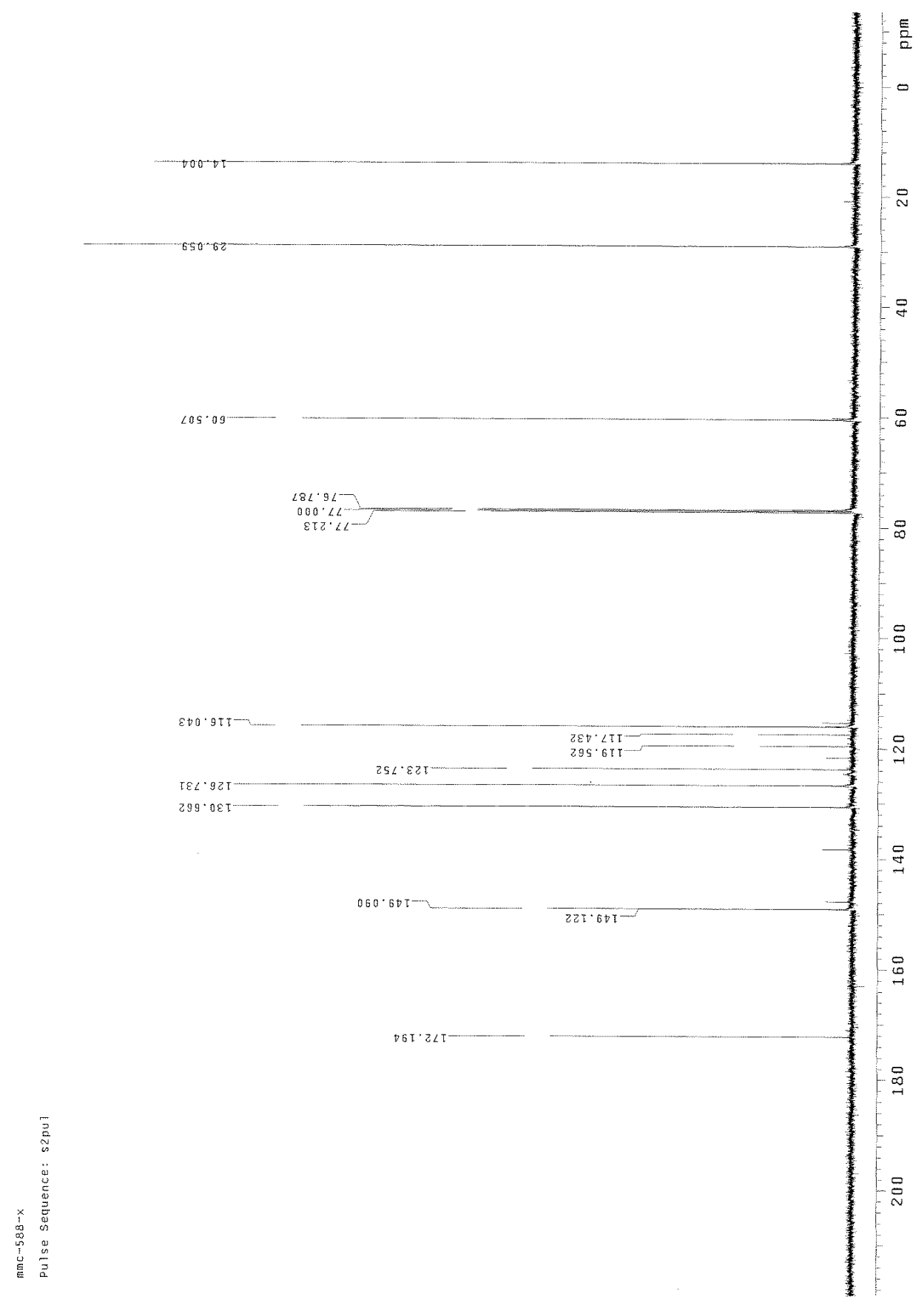

Figure 47: ${ }^{13} \mathrm{C}$ NMR of 3-Bromo-5-trifluoromethanesulfonyloxy-nitrobenzene (157) 


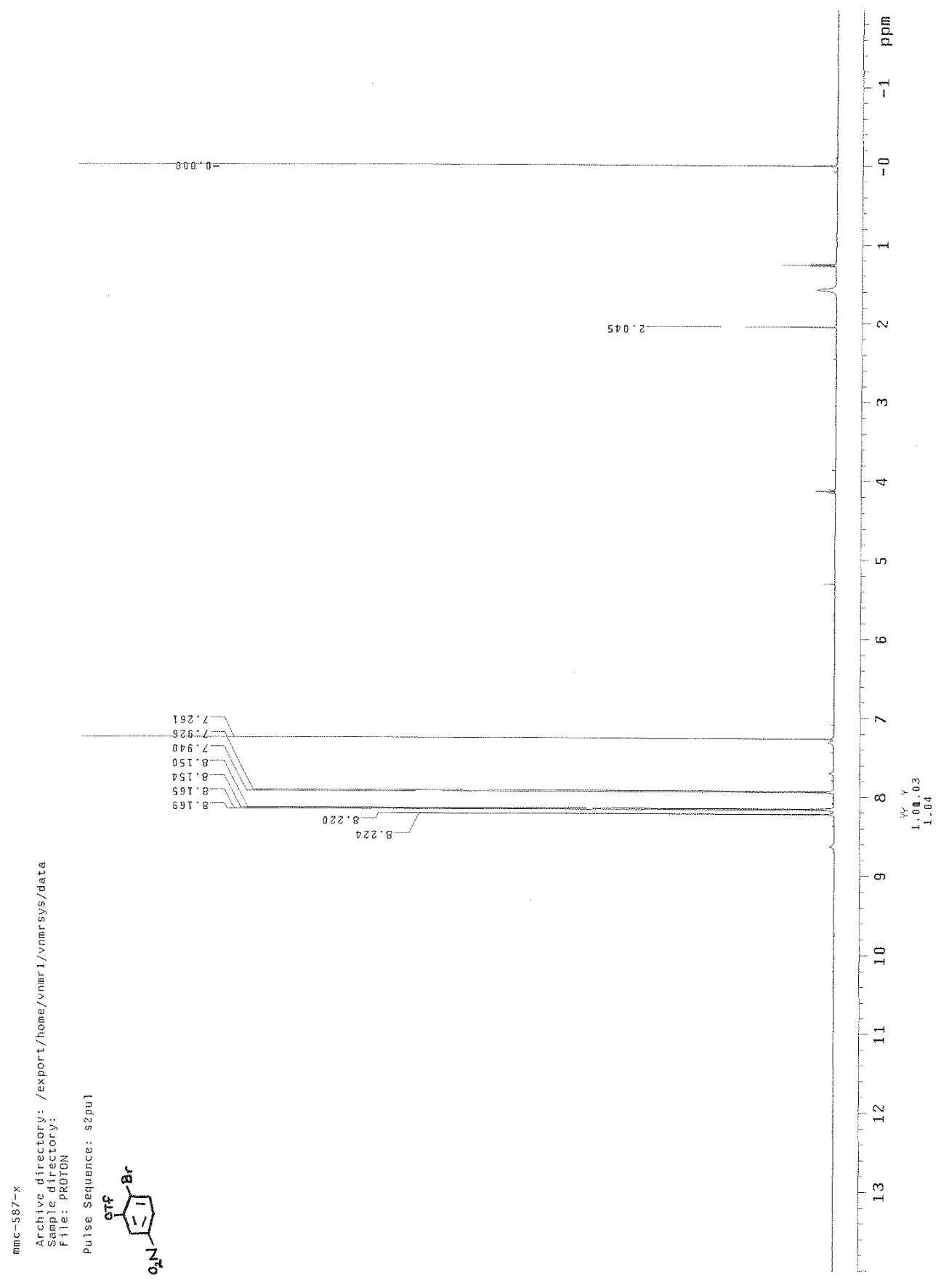

Figure 48: ${ }^{1} \mathrm{H}$ NMR of 4-Bromo-3-trifluoromethanesulfonyloxy-nitrobenzene (159) 


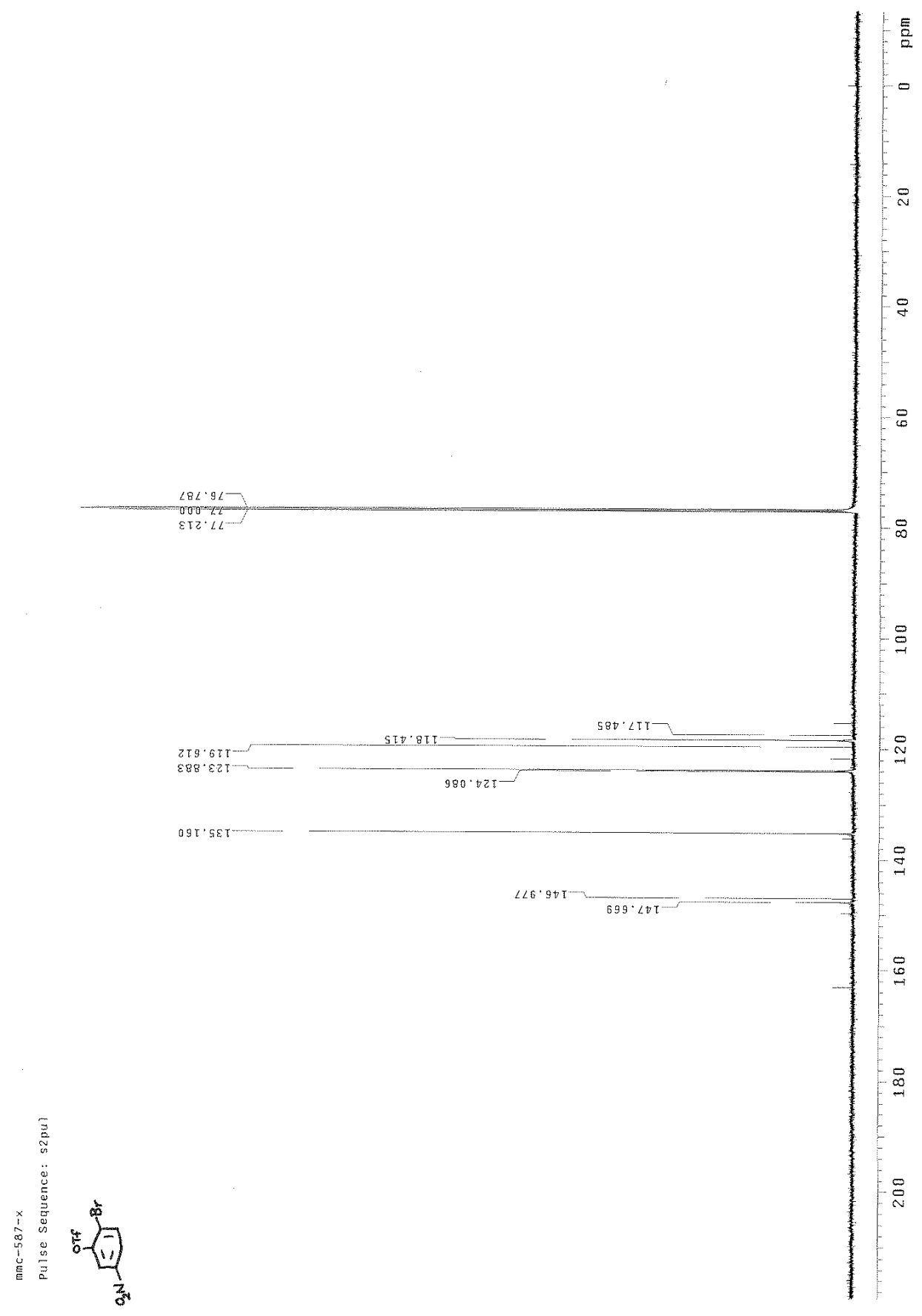

Figure 49: ${ }^{13} \mathrm{C}$ NMR of 4-Bromo-3-trifluoromethanesulfonyloxy-nitrobenzene (159) 


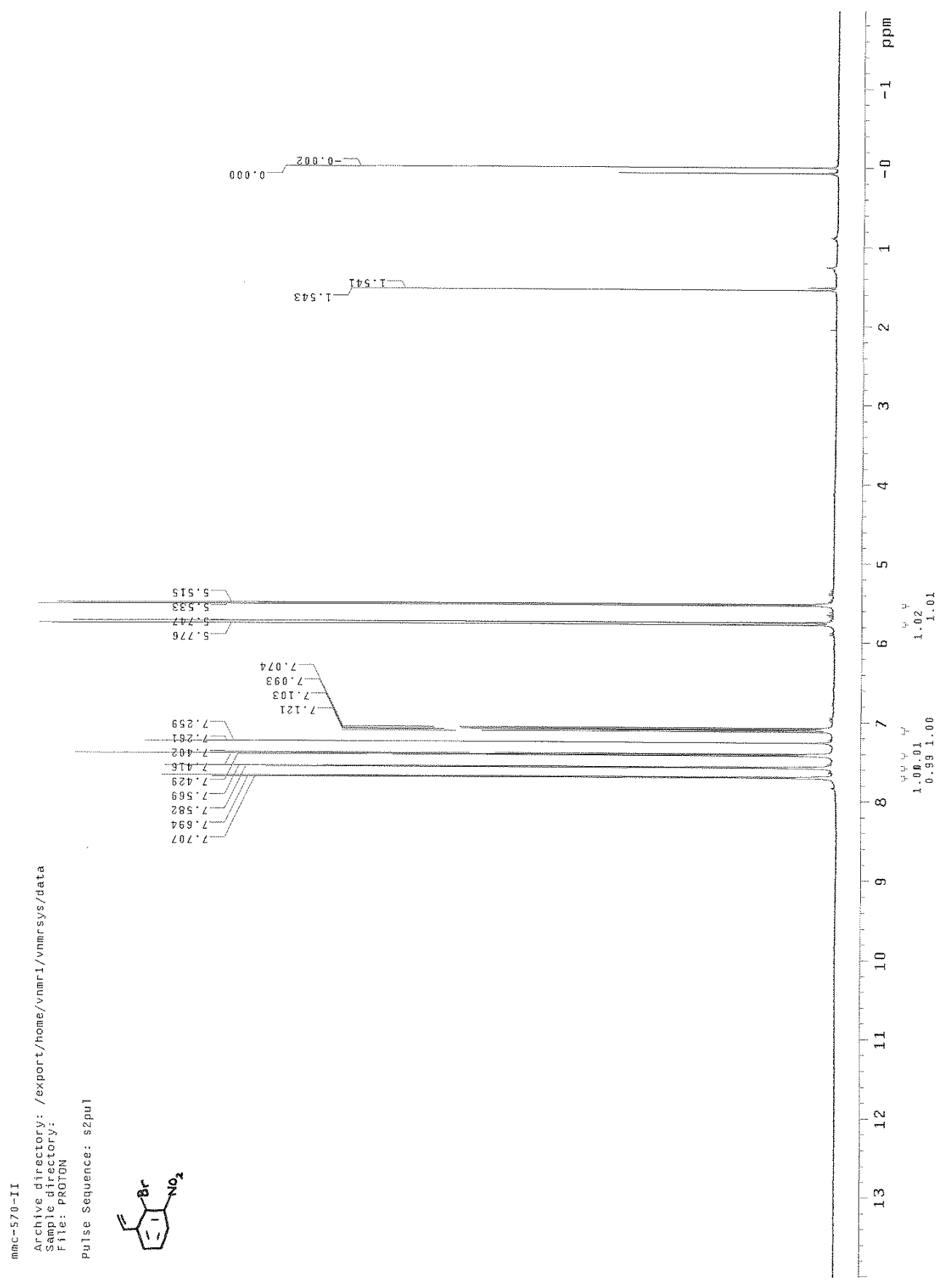

Figure 50: ${ }^{1} \mathrm{H}$ NMR of 2-Bromo-3-ethenyl-nitrobenzene (170) 


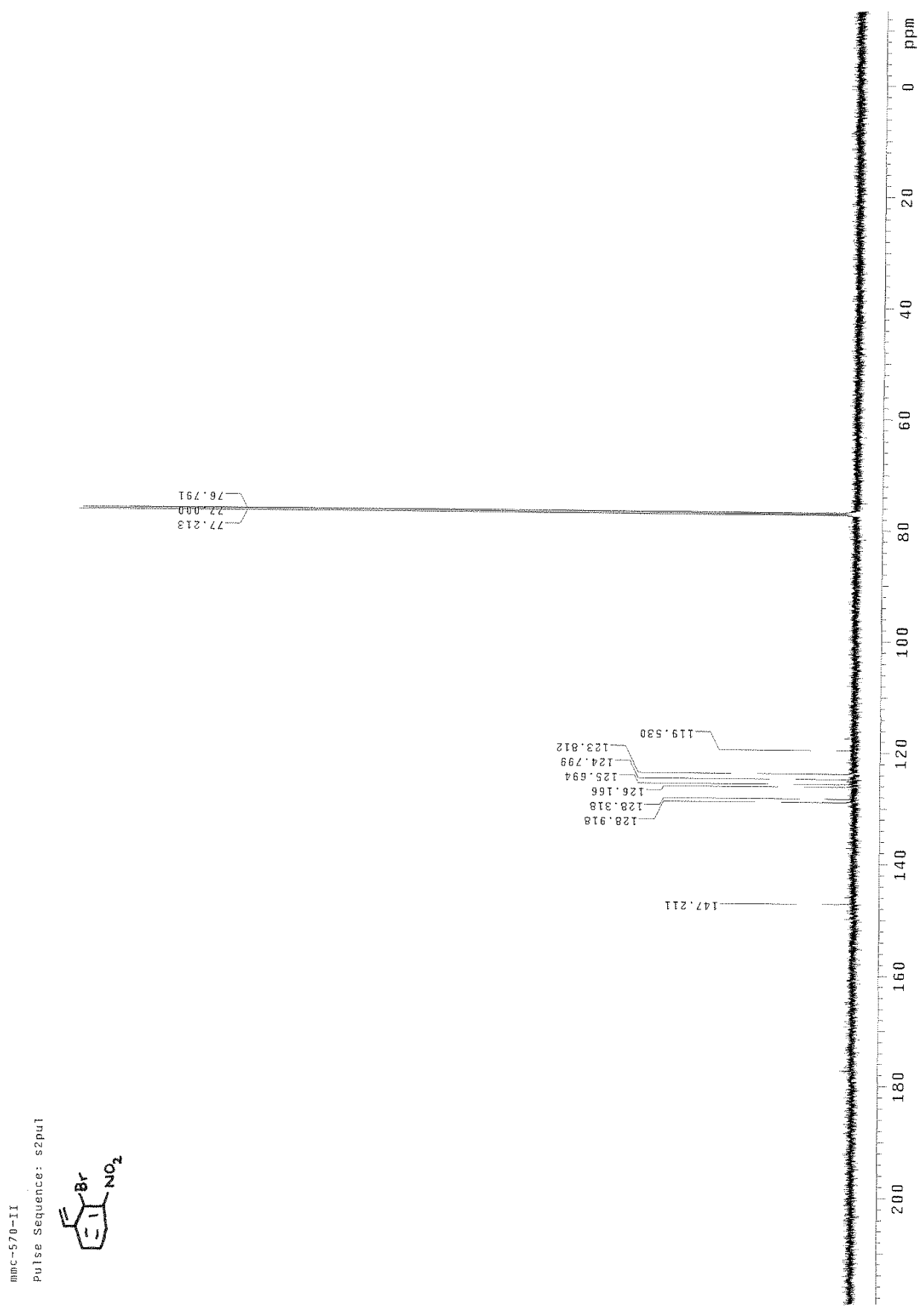

Figure 51: ${ }^{13} \mathrm{C}$ NMR of 2-Bromo-3-ethenyl-nitrobenzene (170) 


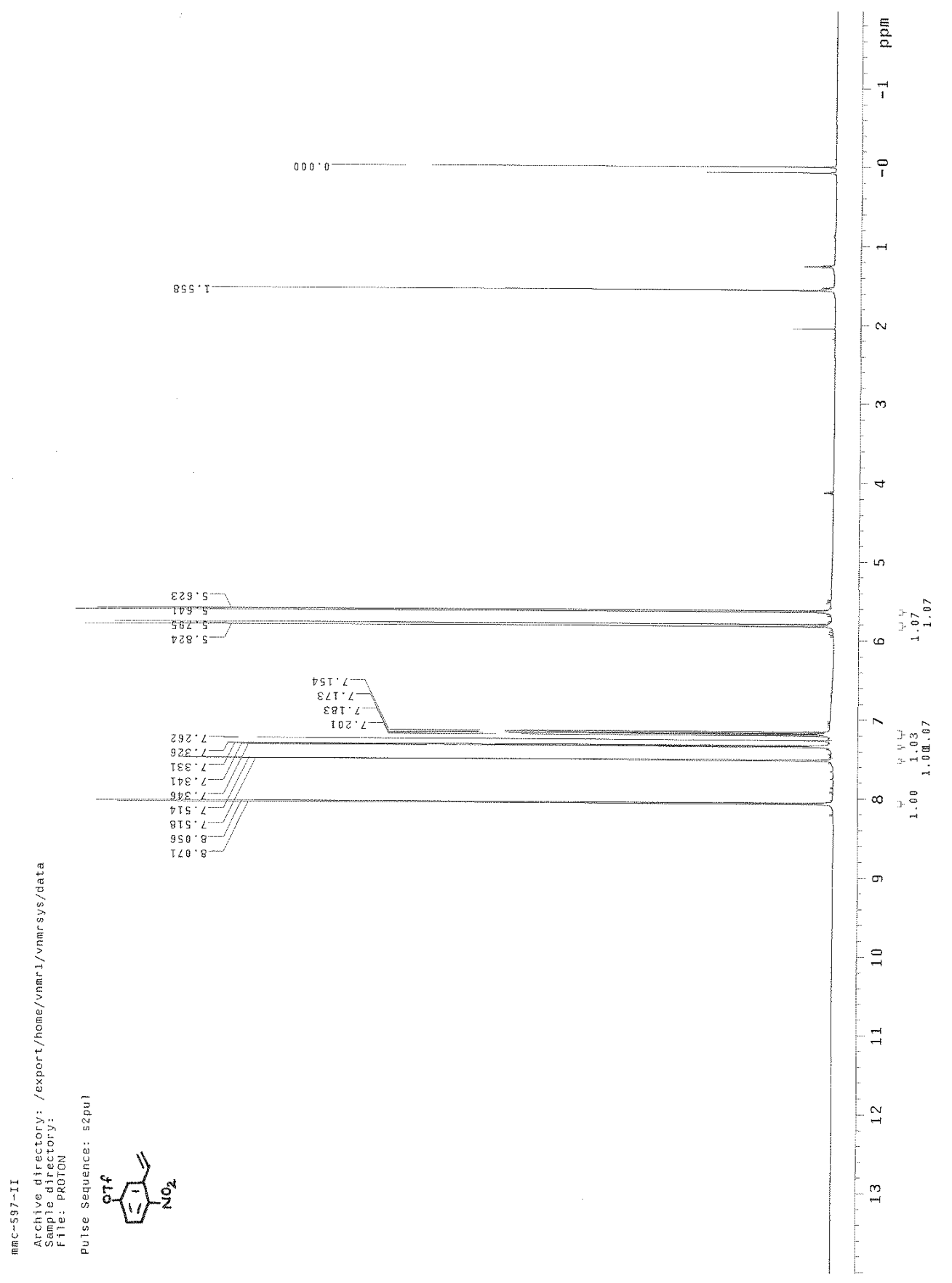

Figure 52: ${ }^{1} \mathrm{H}$ NMR of 2-Ethenyl-4-trifluoromethanesulfonyloxy-nitrobenzene (171) 


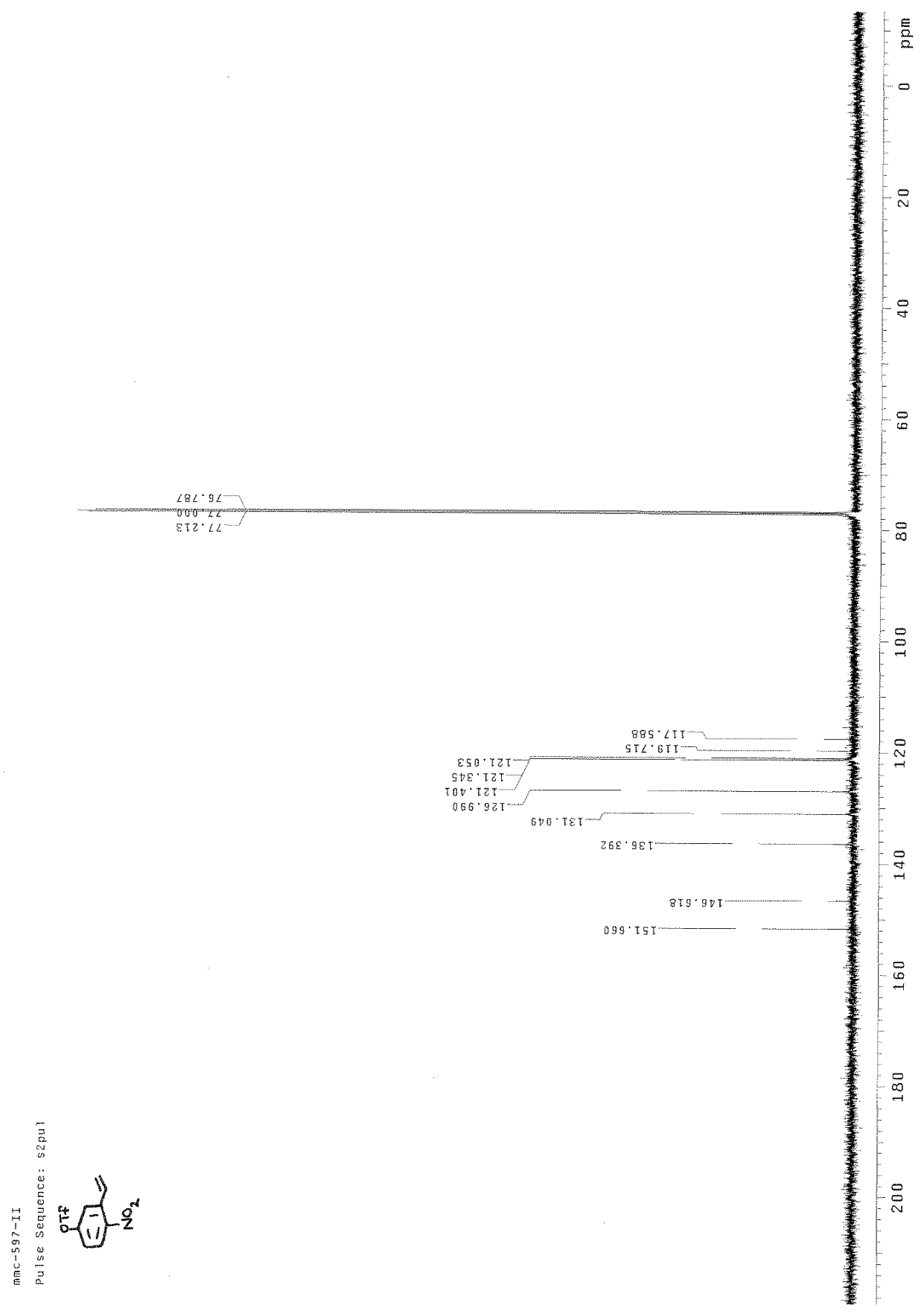

Figure 53: ${ }^{13} \mathrm{C}$ NMR of 2-Ethenyl-4-trifluoromethanesulfonyloxy-nitrobenzene (171) 


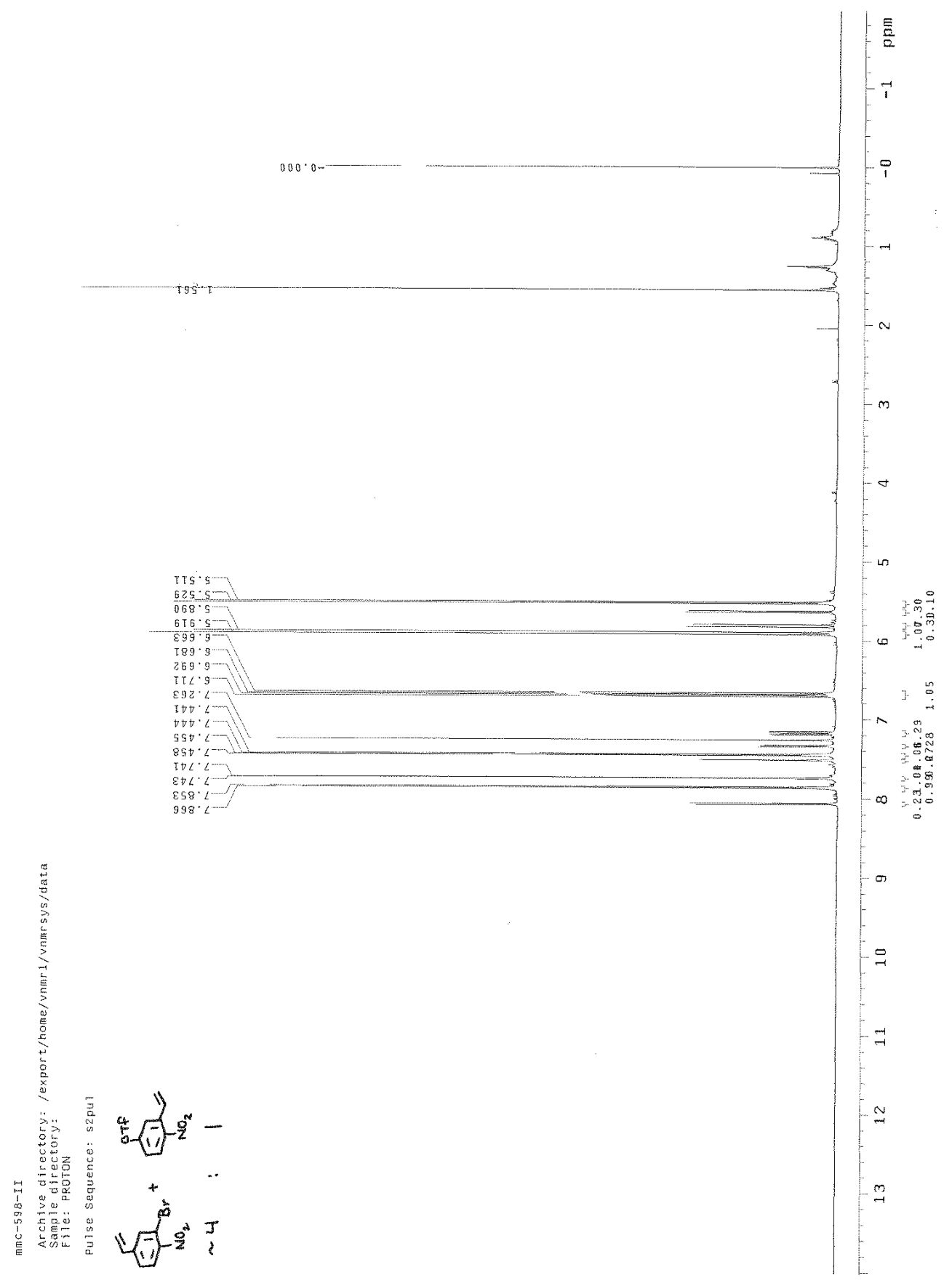

Figure 54: ${ }^{1} \mathrm{H}$ NMR of 2-Bromo-4-ethenyl-nitrobenzene (172) 


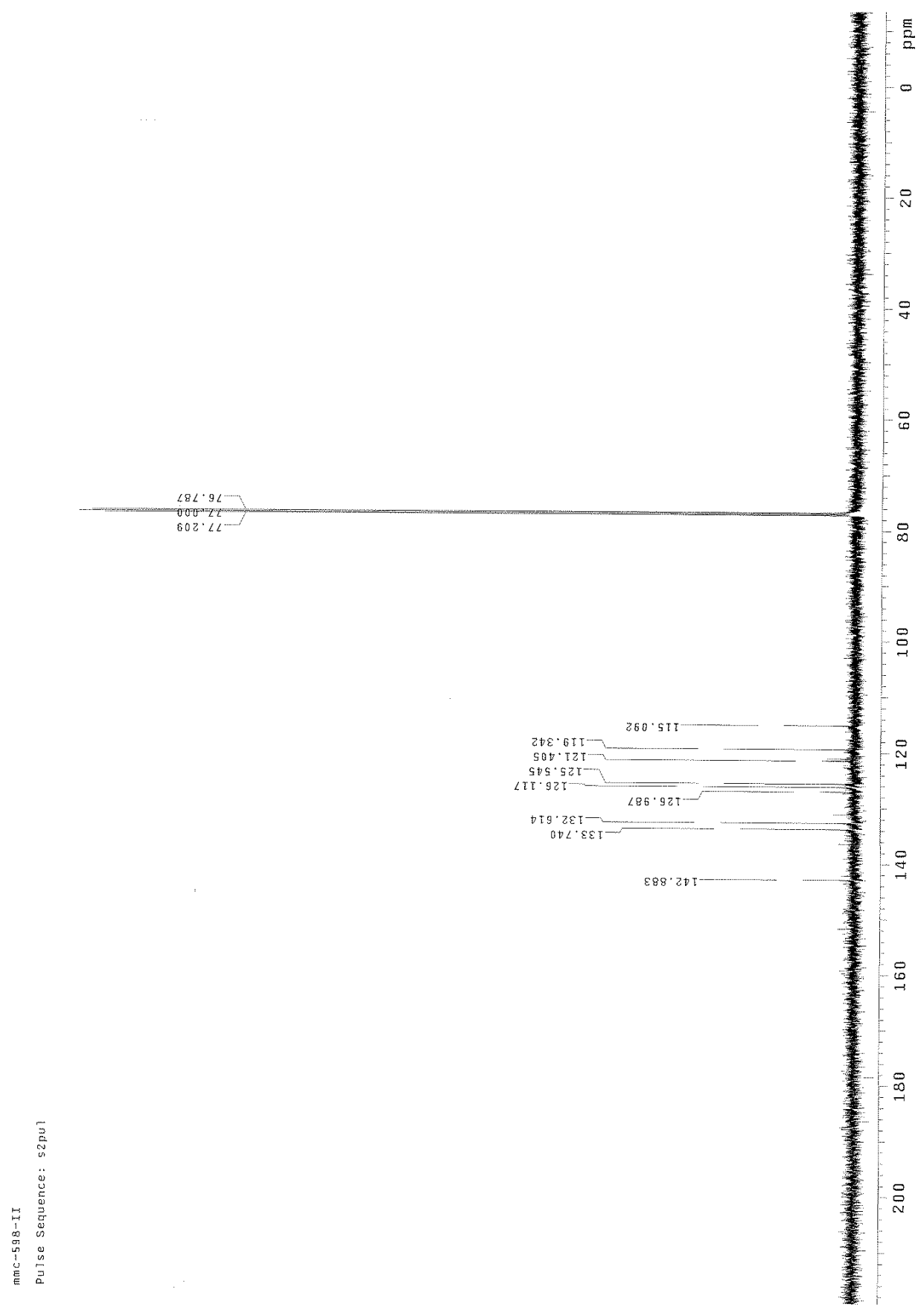

Figure 55: ${ }^{13} \mathrm{C}$ NMR of 2-Bromo-4-ethenyl-nitrobenzene (172) 


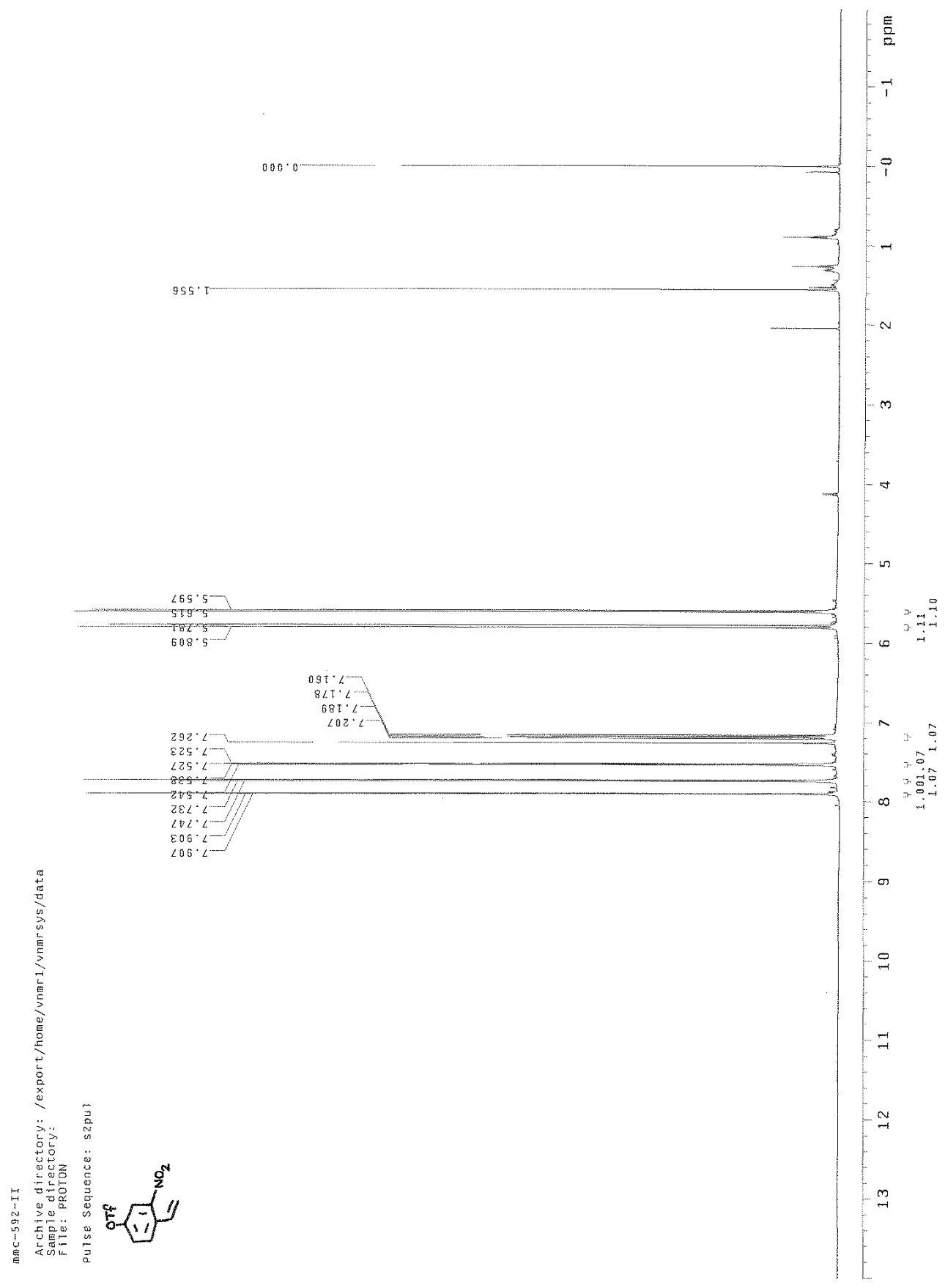

Figure 56: ${ }^{1} \mathrm{H}$ NMR of 2-Ethenyl-5-trifluoromethanesulfonyloxy-nitrobenzene (173) 


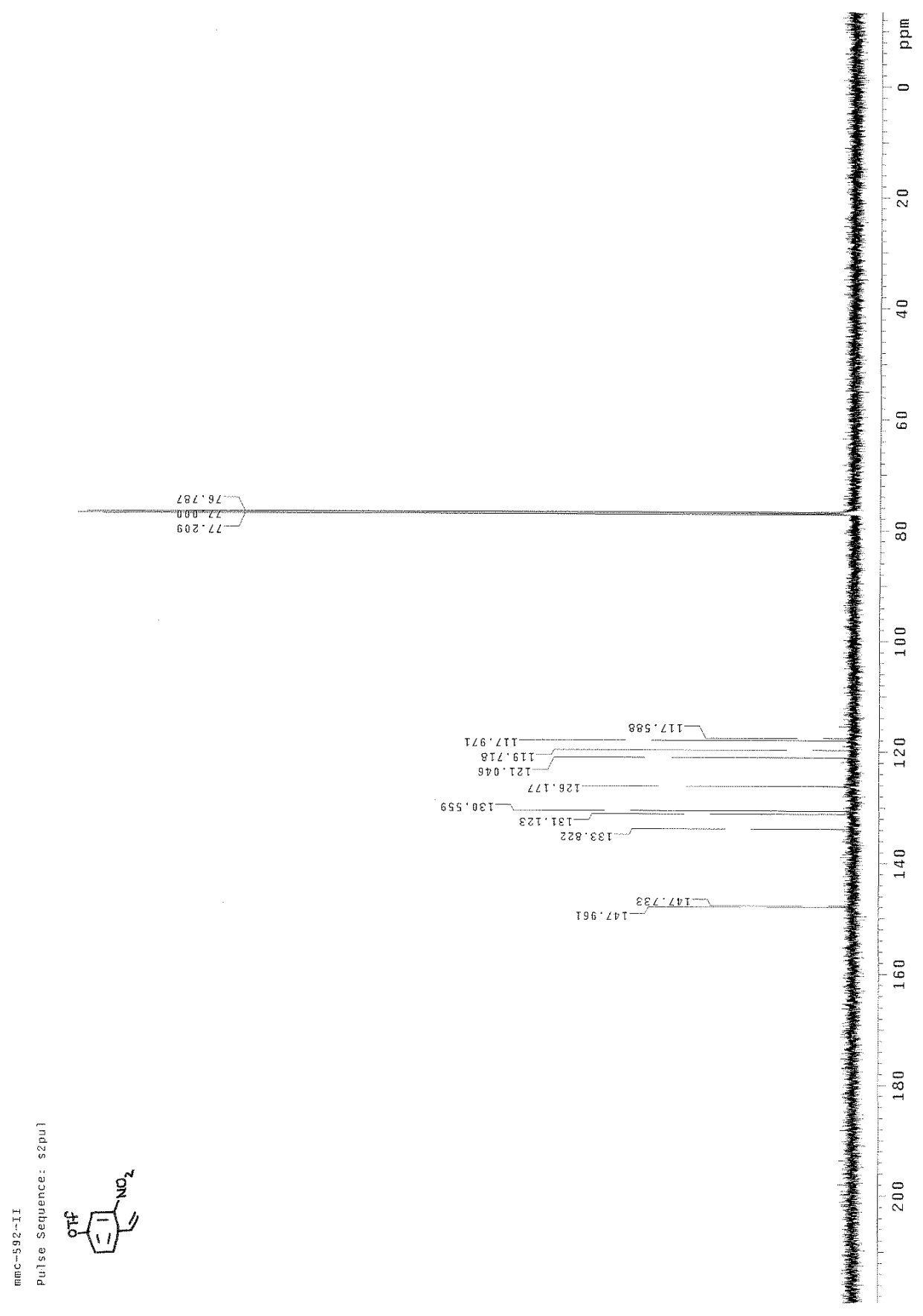

Figure 57: ${ }^{13} \mathrm{C}$ NMR of 2-Ethenyl-5-trifluoromethanesulfonyloxy-nitrobenzene (173) 


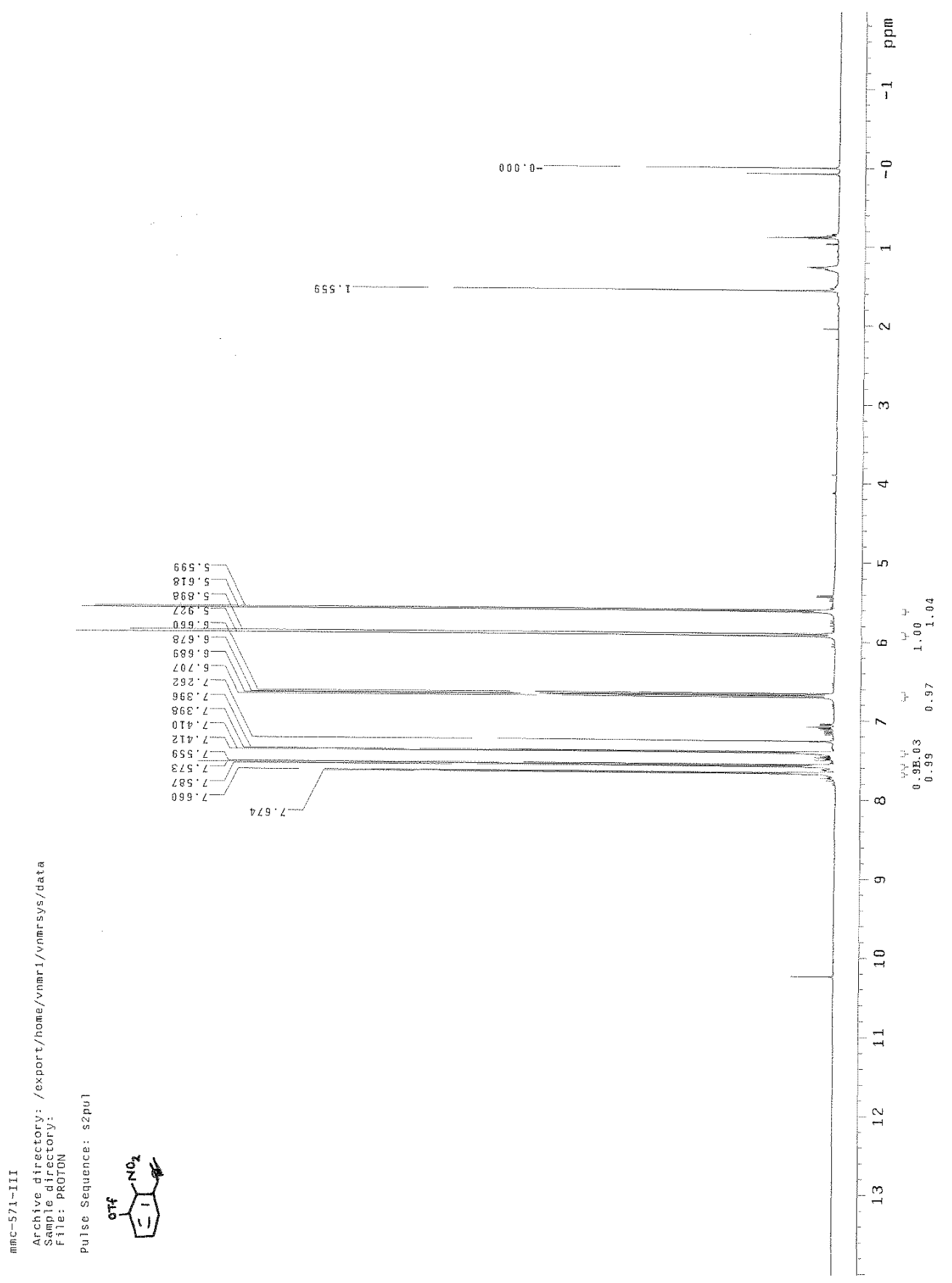

Figure 58: ${ }^{1} \mathrm{H}$ NMR of 2-Ethenyl-6-trifluoromethanesulfonyloxy-nitrobenzene (175) 


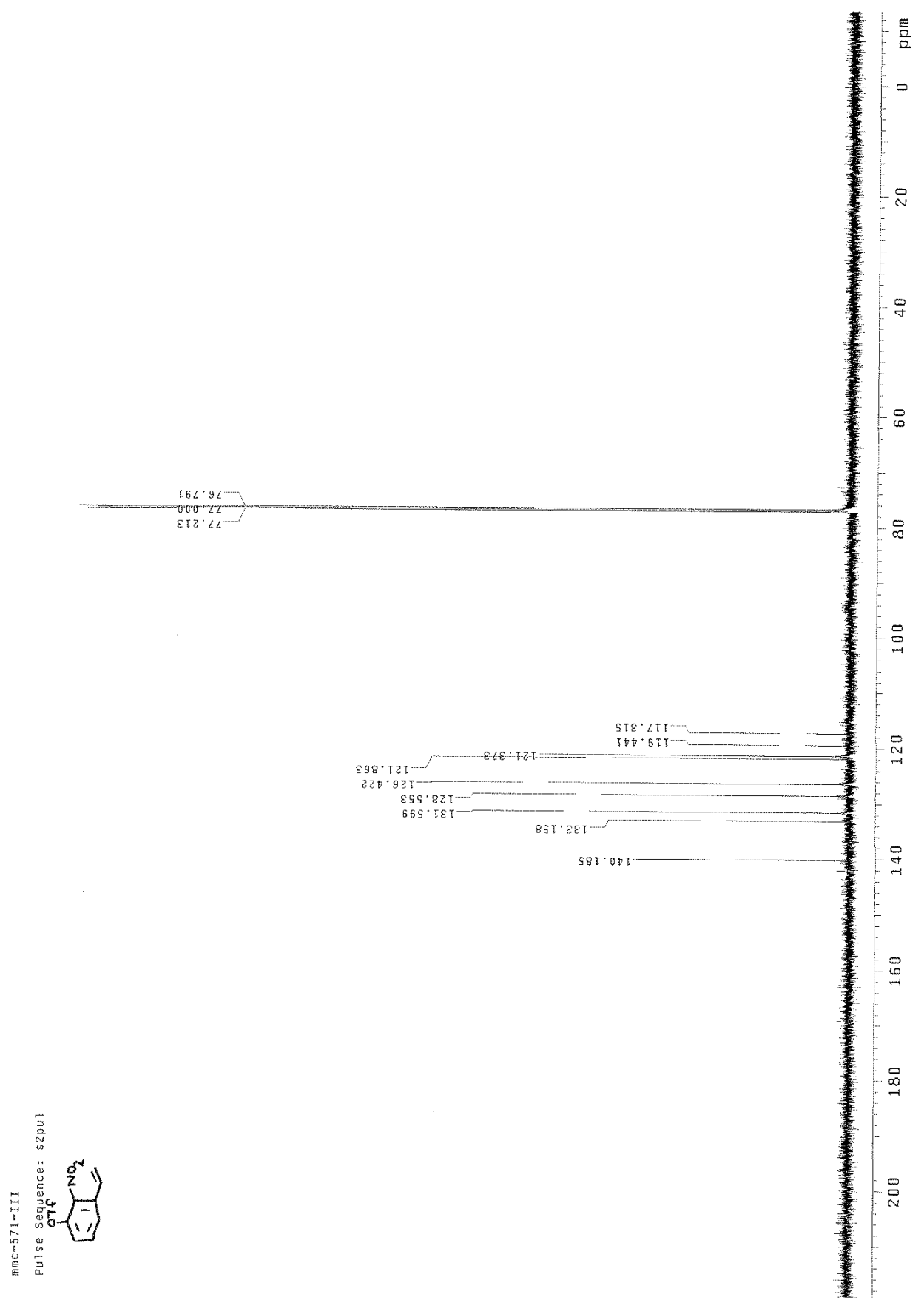

Figure 59: ${ }^{13} \mathrm{C}$ NMR of 2-Ethenyl-6-trifluoromethanesulfonyloxy-nitrobenzene (175) 


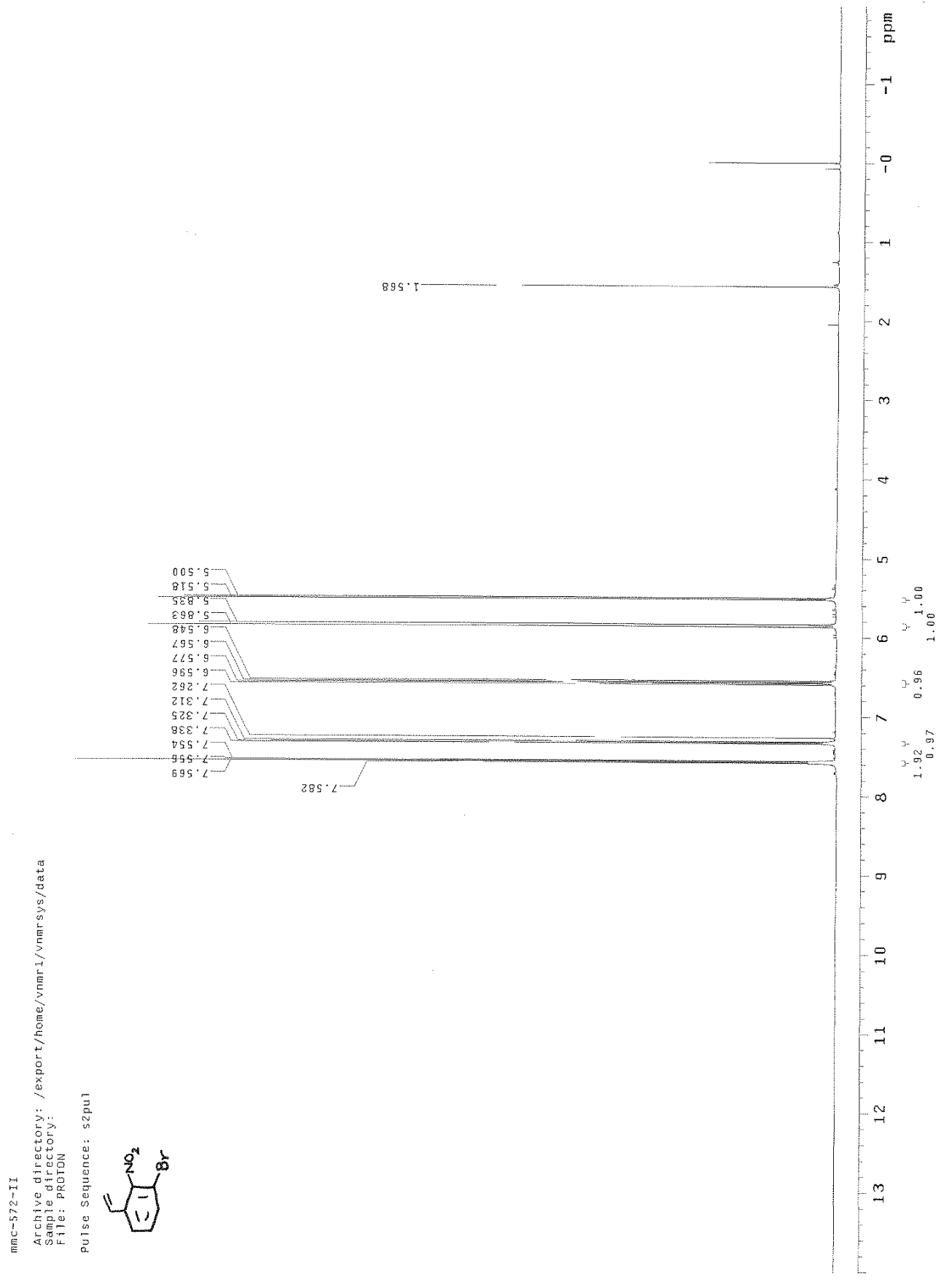

Figure 60: ${ }^{1} \mathrm{H}$ NMR of 2-Bromo-6-ethenyl-nitrobenzene (176) 


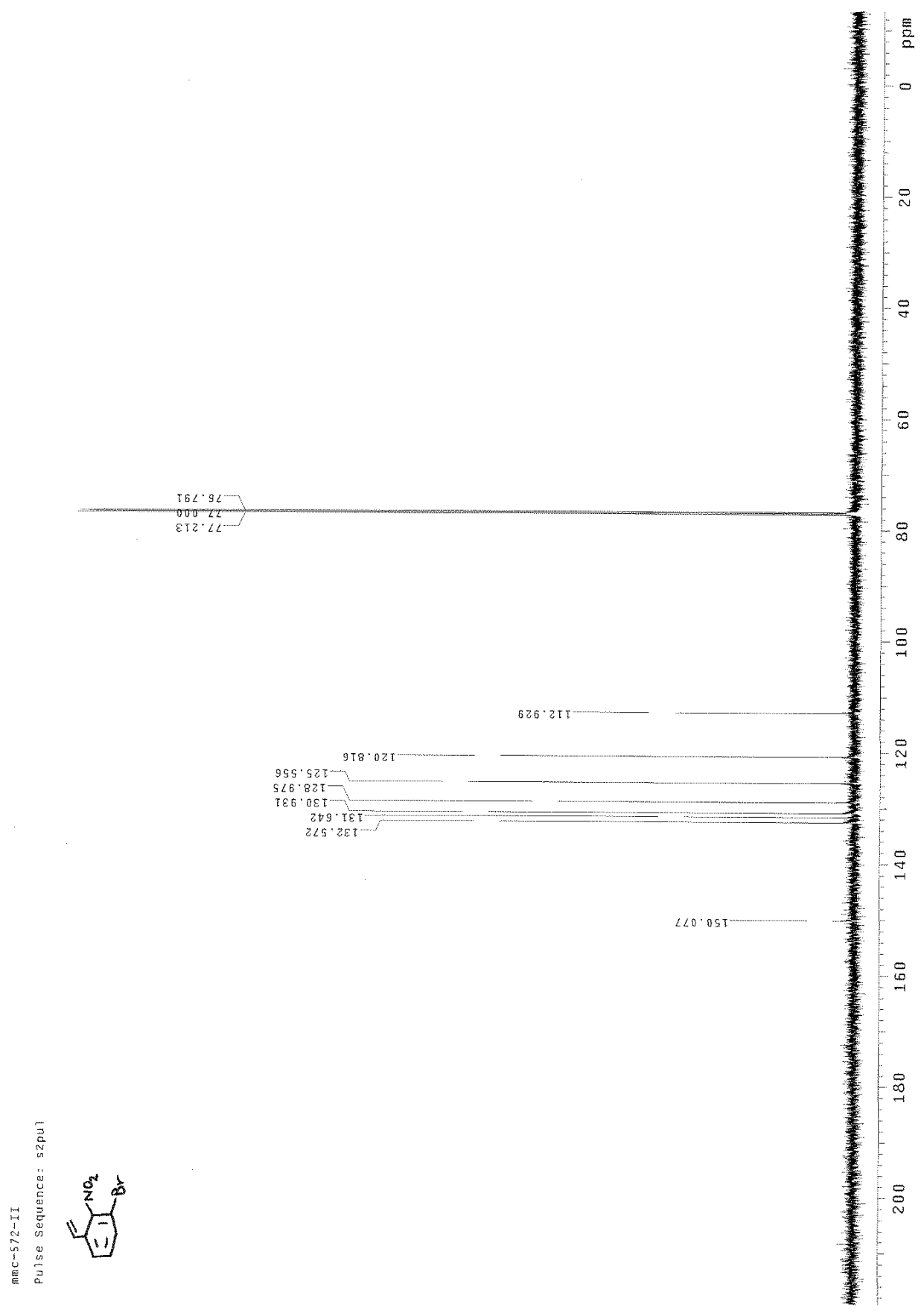

Figure 61: ${ }^{13} \mathrm{C}$ NMR of 2-Bromo-6-ethenyl-nitrobenzene (176) 


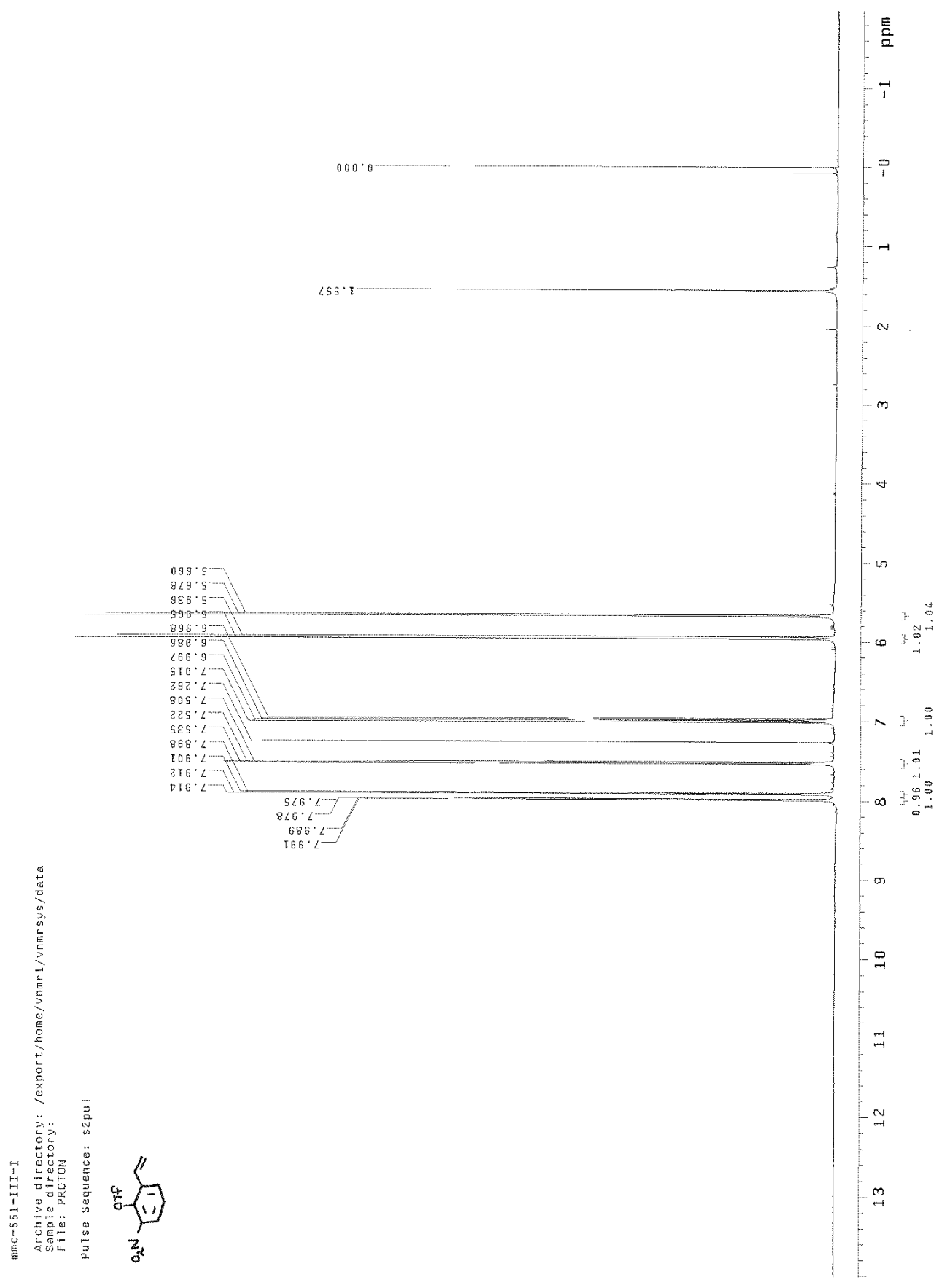

Figure 62: ${ }^{1} \mathrm{H}$ NMR of 3-Ethenyl-2-trifluoromethanesulfonyloxy-nitrobenzene (177) 


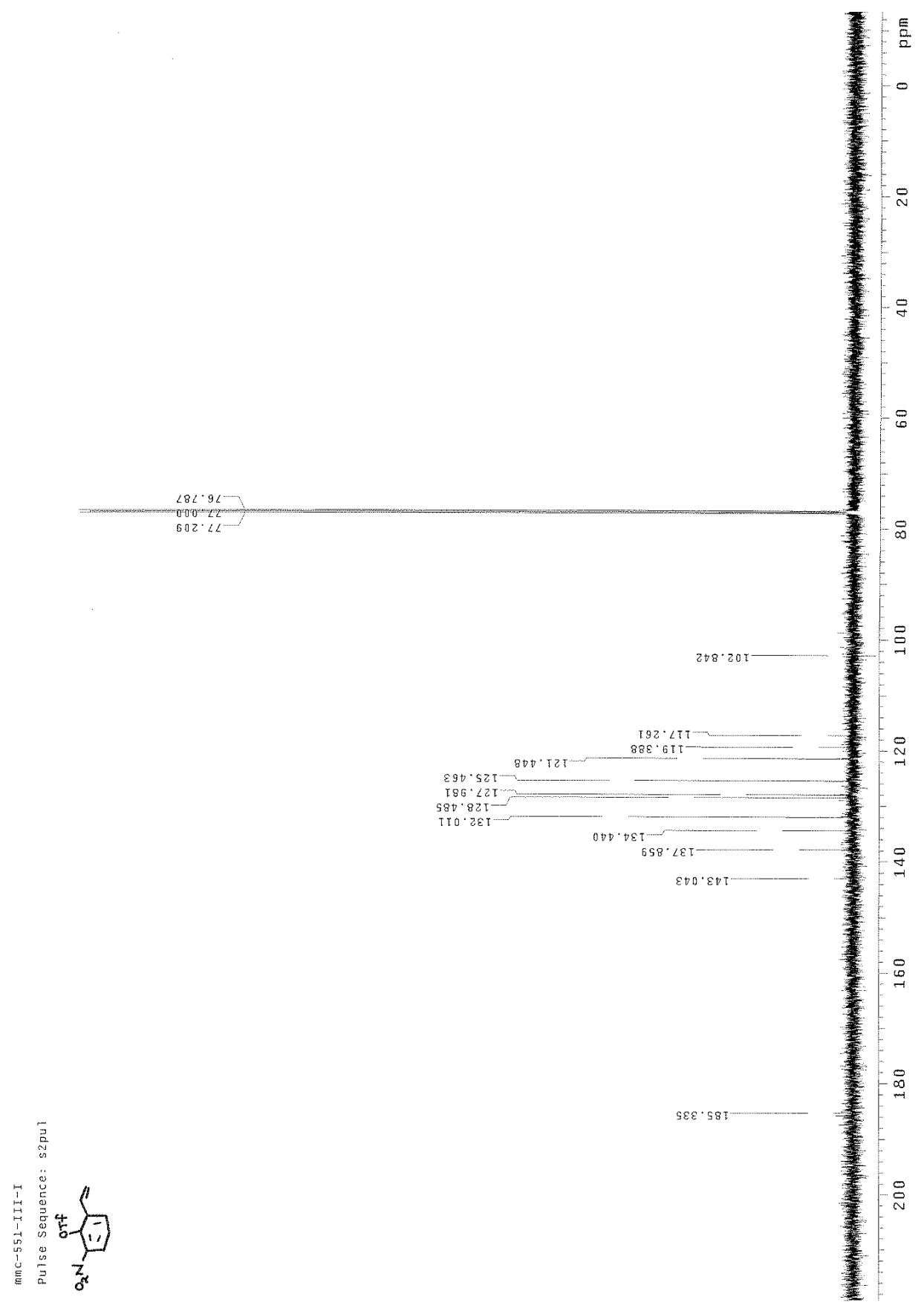

Figure 63: ${ }^{13} \mathrm{C}$ NMR of 3-Ethenyl-2-trifluoromethanesulfonyloxy-nitrobenzene (177) 


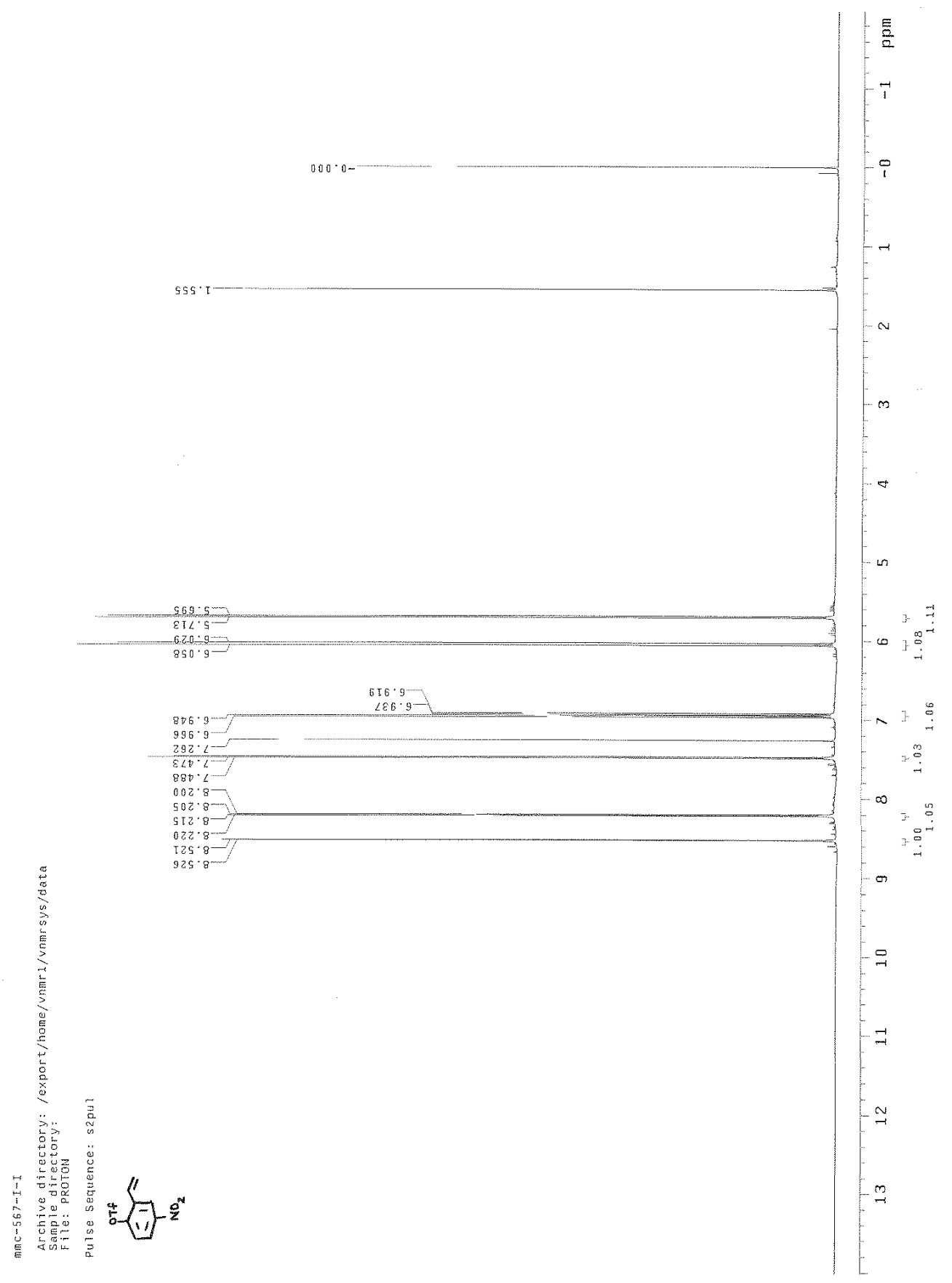

Figure 64: ${ }^{1} \mathrm{H}$ NMR of 3-Ethenyl-4-trifluoromethanesulfonyloxy-nitrobenzene (178) 


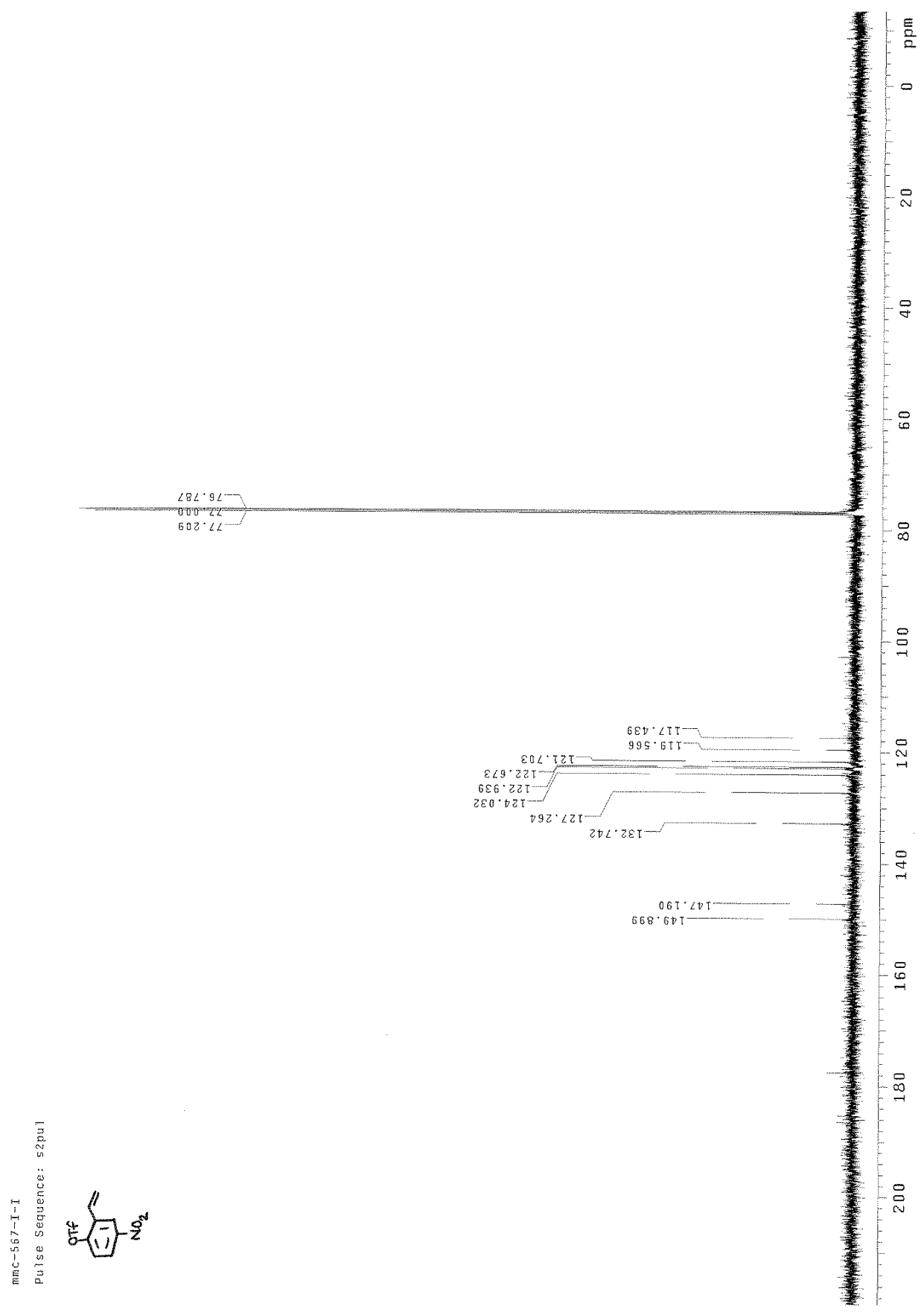

Figure $65:{ }^{13} \mathrm{C}$ NMR of 3-Ethenyl-4-trifluoromethanesulfonyloxy-nitrobenzene (178) 


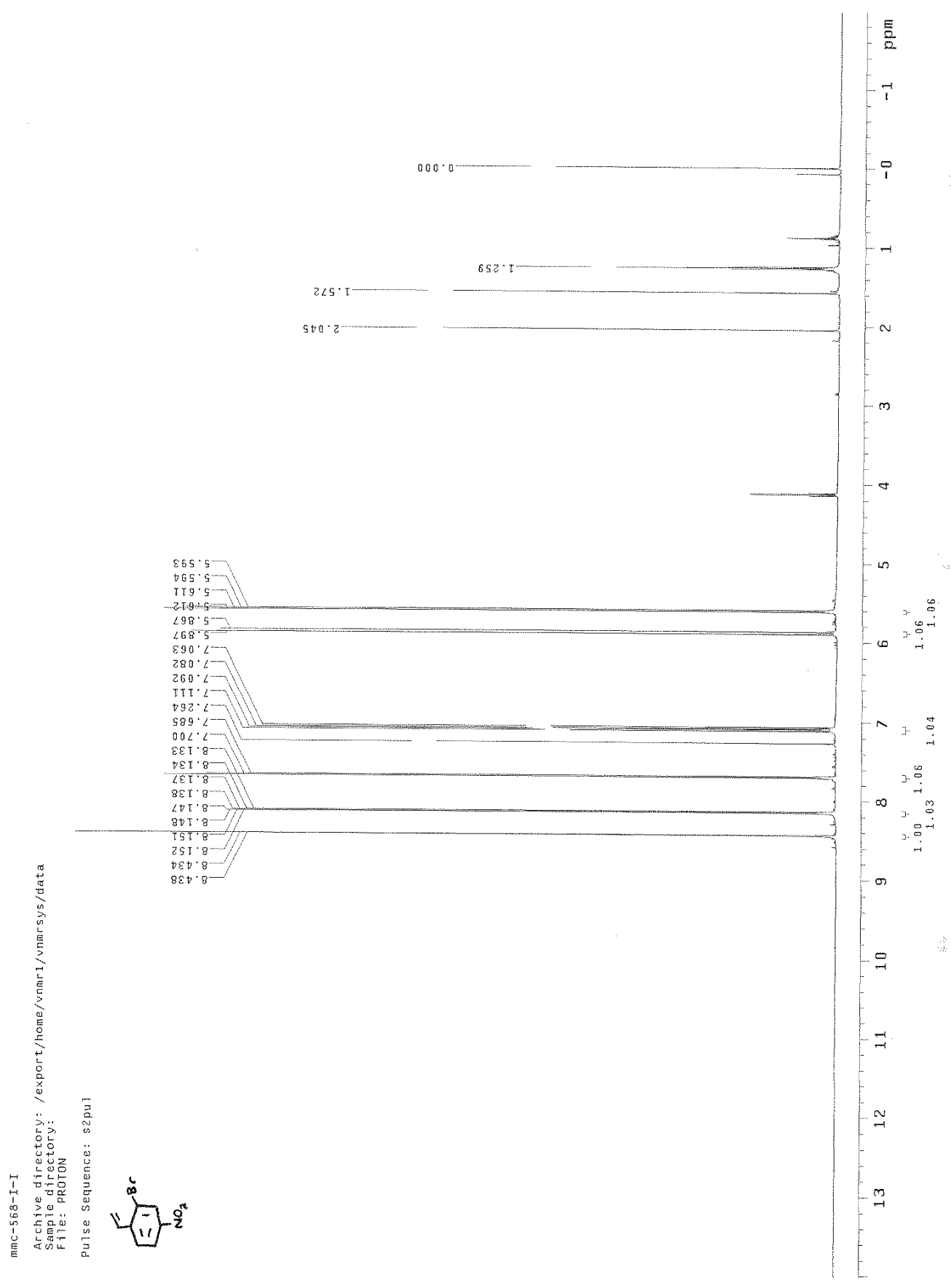

Figure 66: ${ }^{1} \mathrm{H}$ NMR of 3-Bromo-4-ethenyl-nitrobenzene (179) 


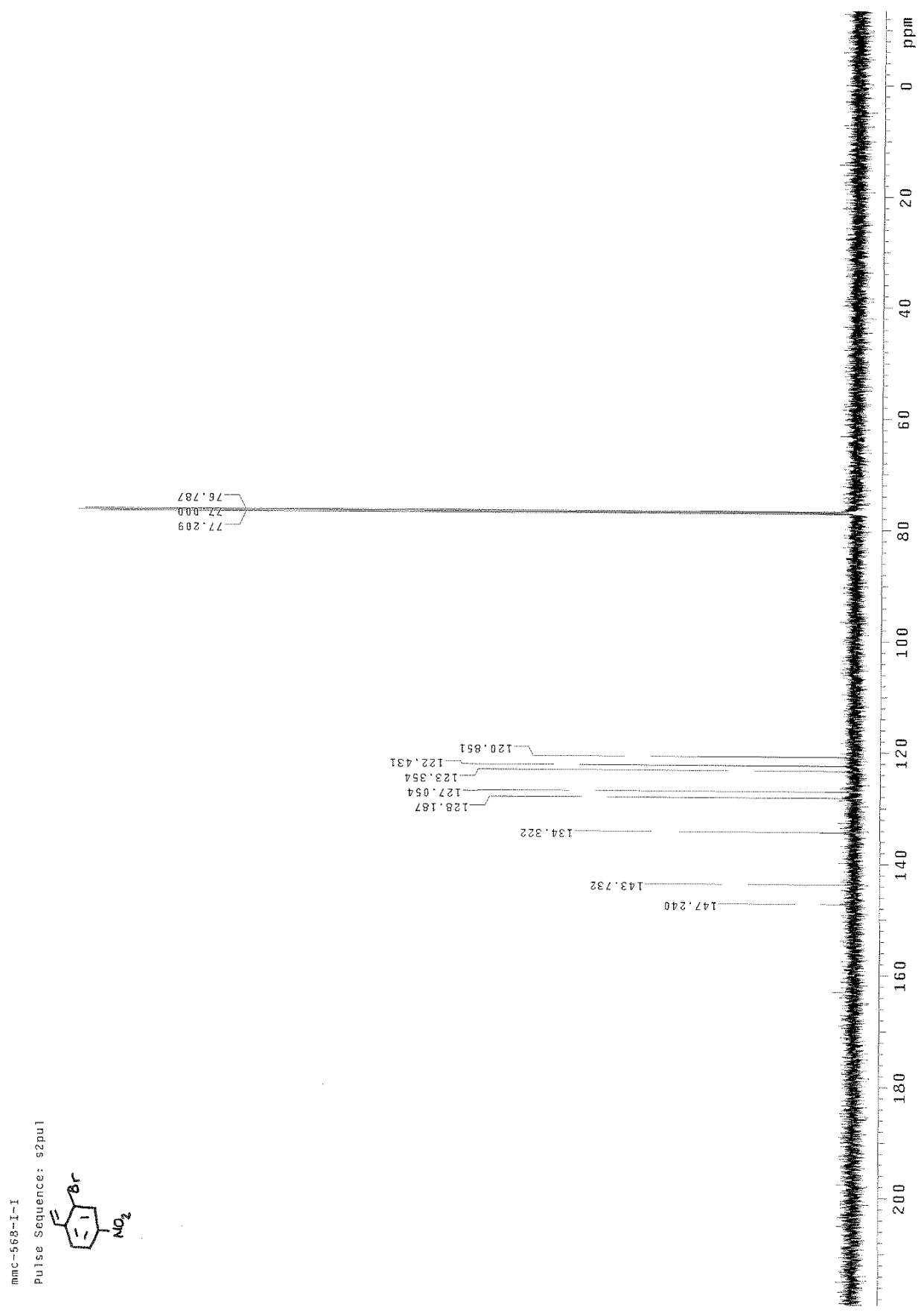

Figure 67: ${ }^{13} \mathrm{C}$ NMR of 3-Bromo-4-ethenyl-nitrobenzene (179) 


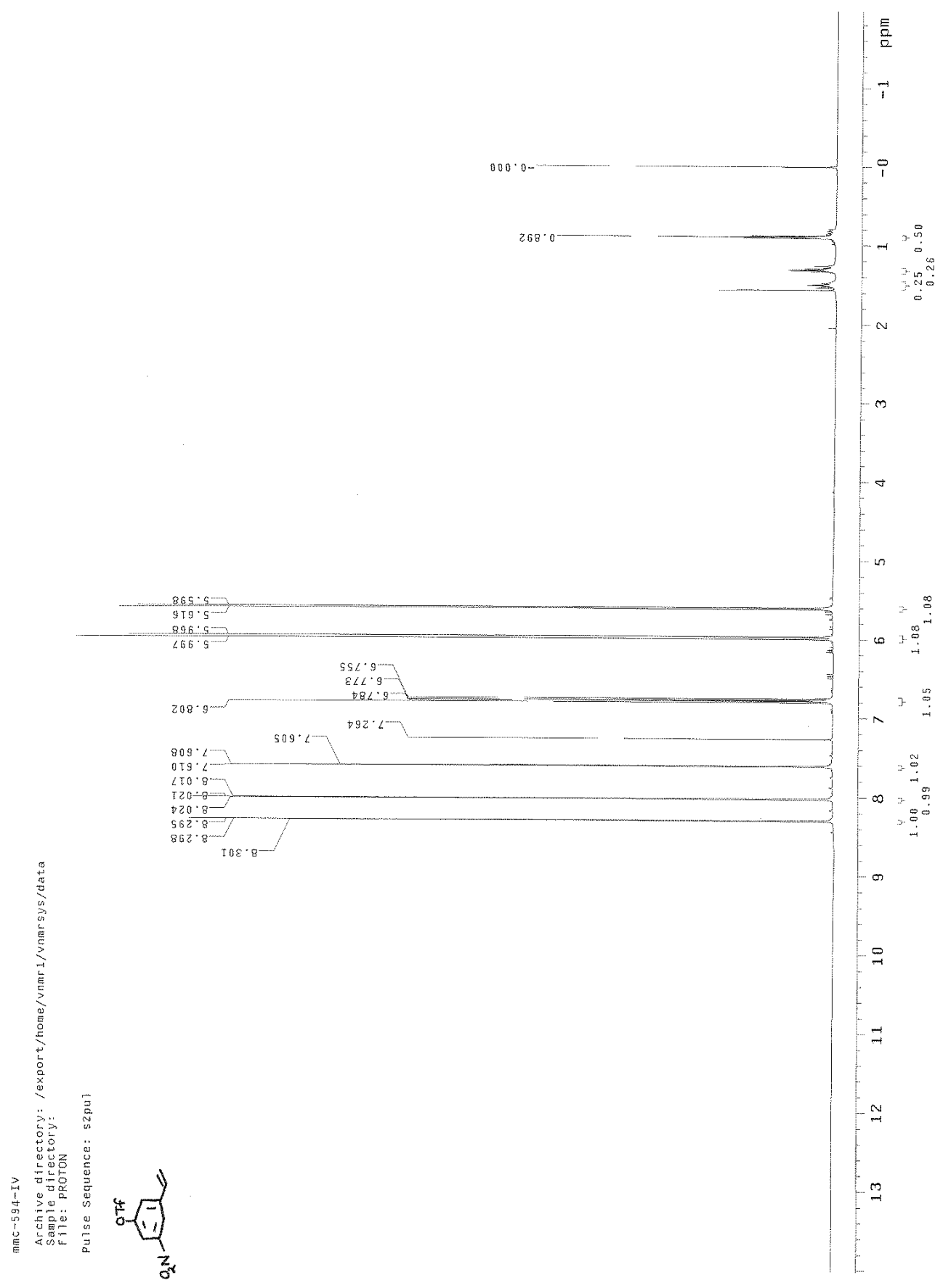

Figure 68: ${ }^{1} \mathrm{H}$ NMR of 3-Ethenyl-5-trifluoromethanesulfonyloxy-nitrobenzene (180) 


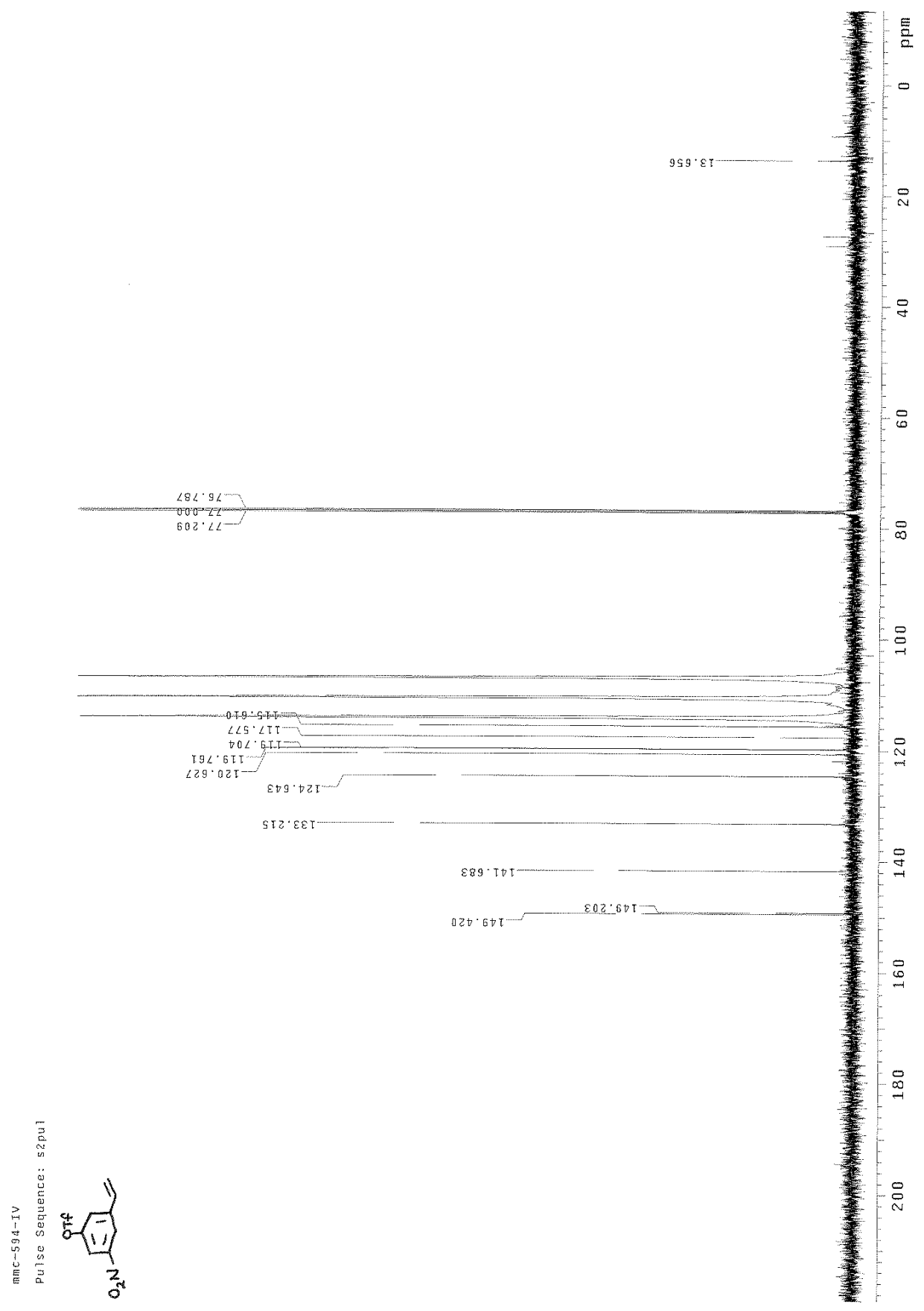

Figure 69: ${ }^{13} \mathrm{C}$ NMR of 3-Ethenyl-5-trifluoromethanesulfonyloxy-nitrobenzene (180) 


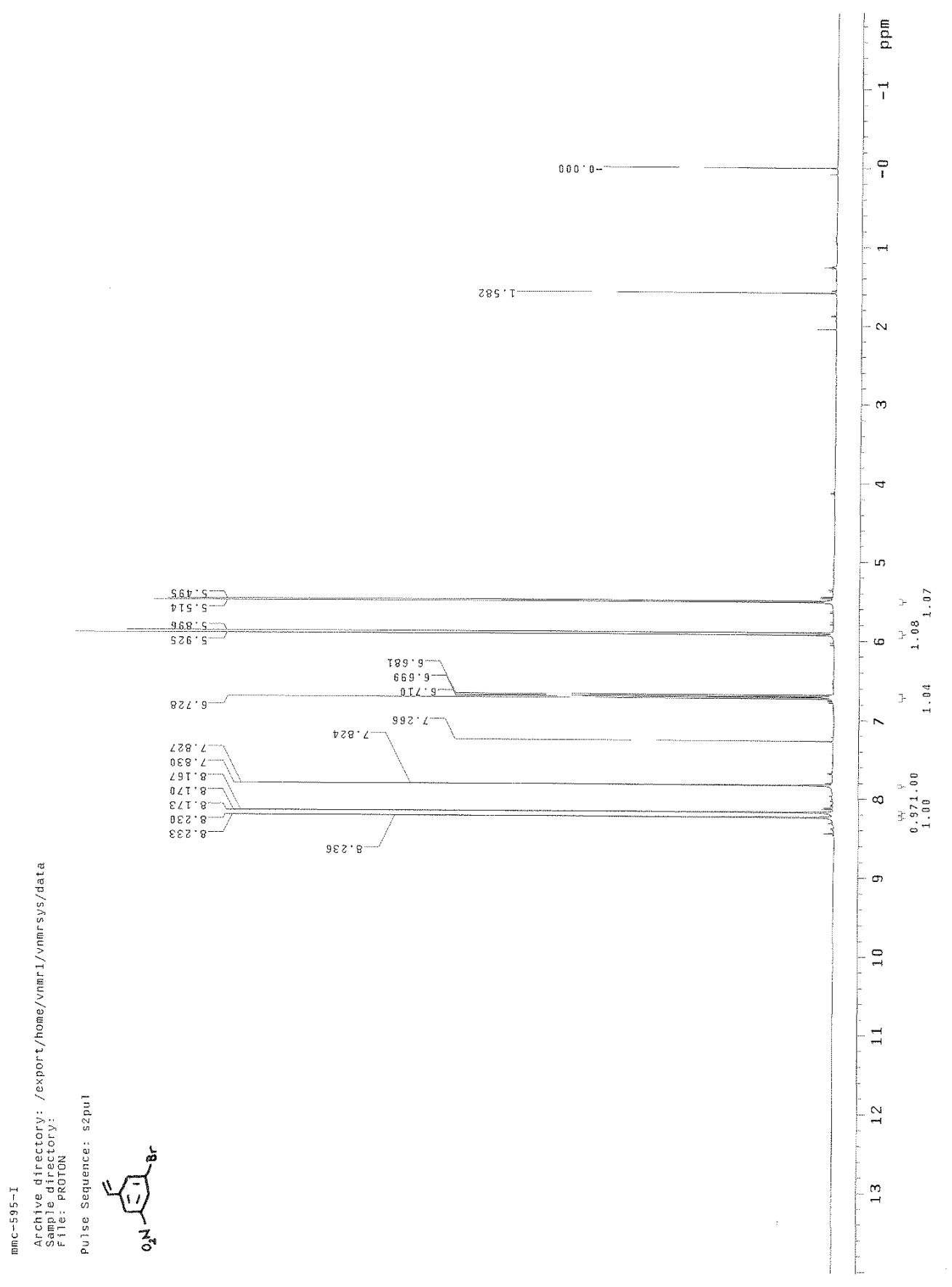

Figure 70: ${ }^{1} \mathrm{H}$ NMR of 3-Bromo-5-ethenyl-nitrobenzene (181) 


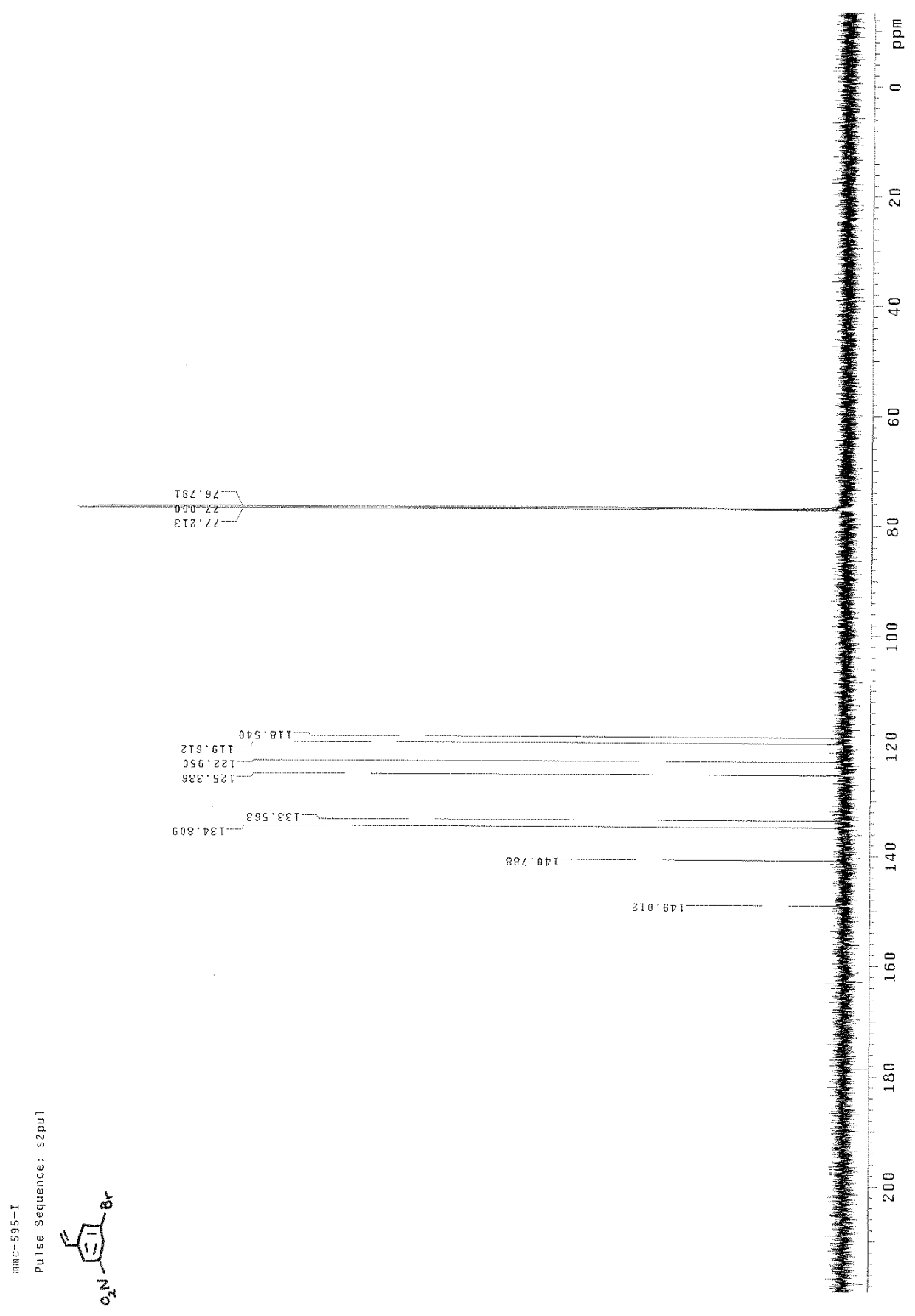

Figure 71: ${ }^{13} \mathrm{C}$ NMR of 3-Bromo-5-ethenyl-nitrobenzene (181) 


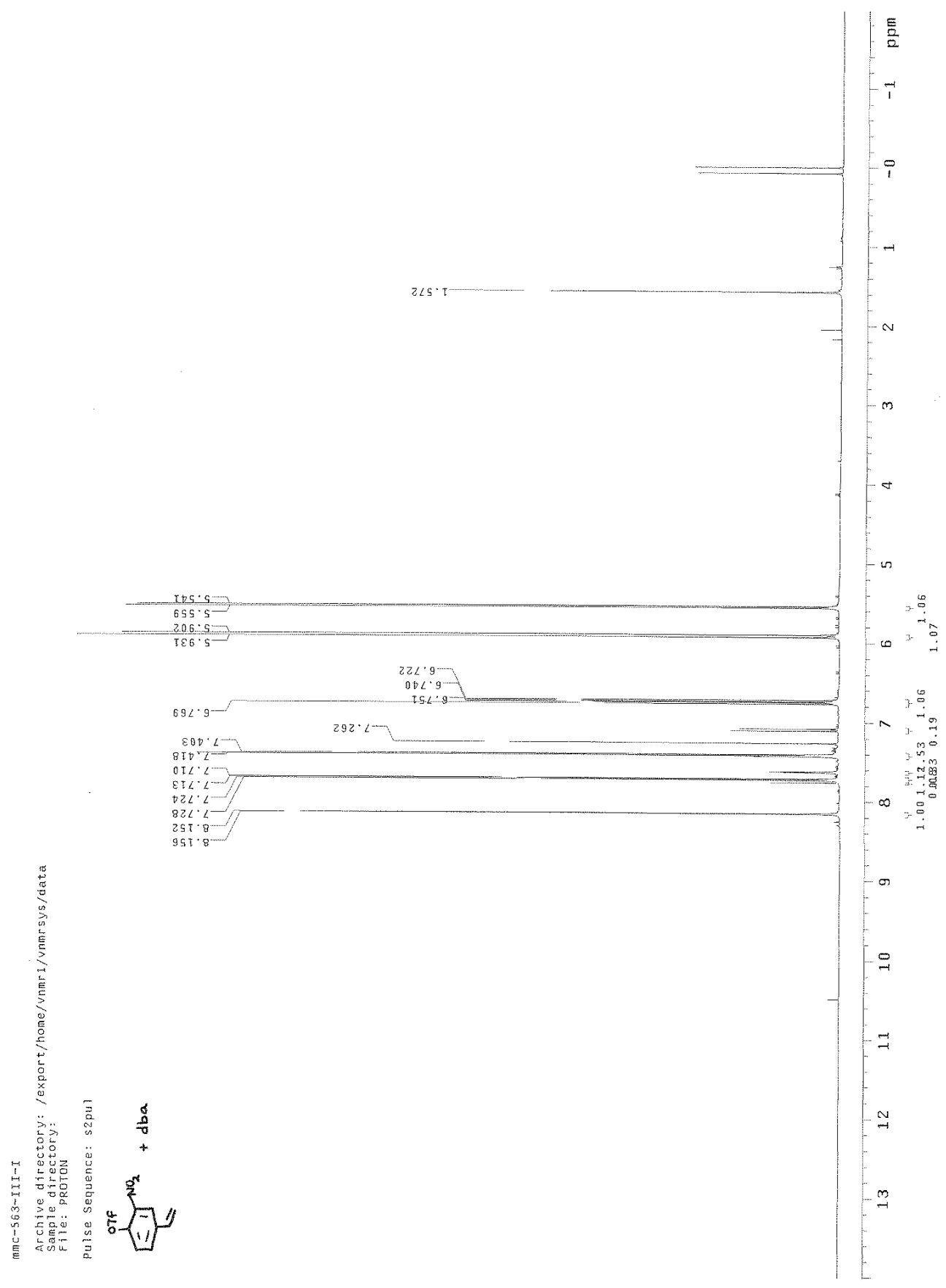

Figure 72: ${ }^{1} \mathrm{H}$ NMR of 3-Ethenyl-6-trifluoromethanesulfonyloxy-nitrobenzene (182) 


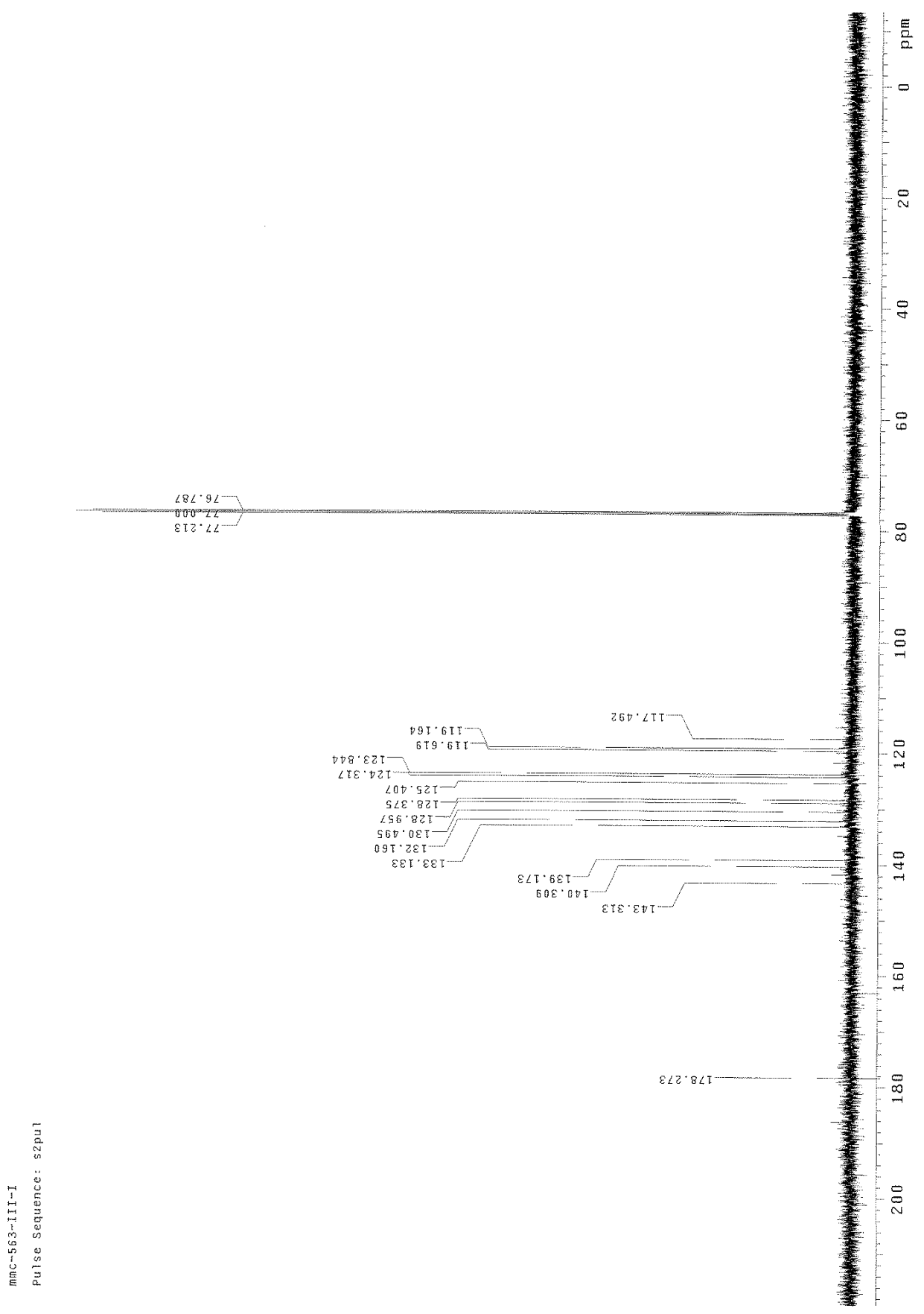

Figure 73: ${ }^{13} \mathrm{C}$ NMR of 3-Ethenyl-6-trifluoromethanesulfonyloxy-nitrobenzene (182) 


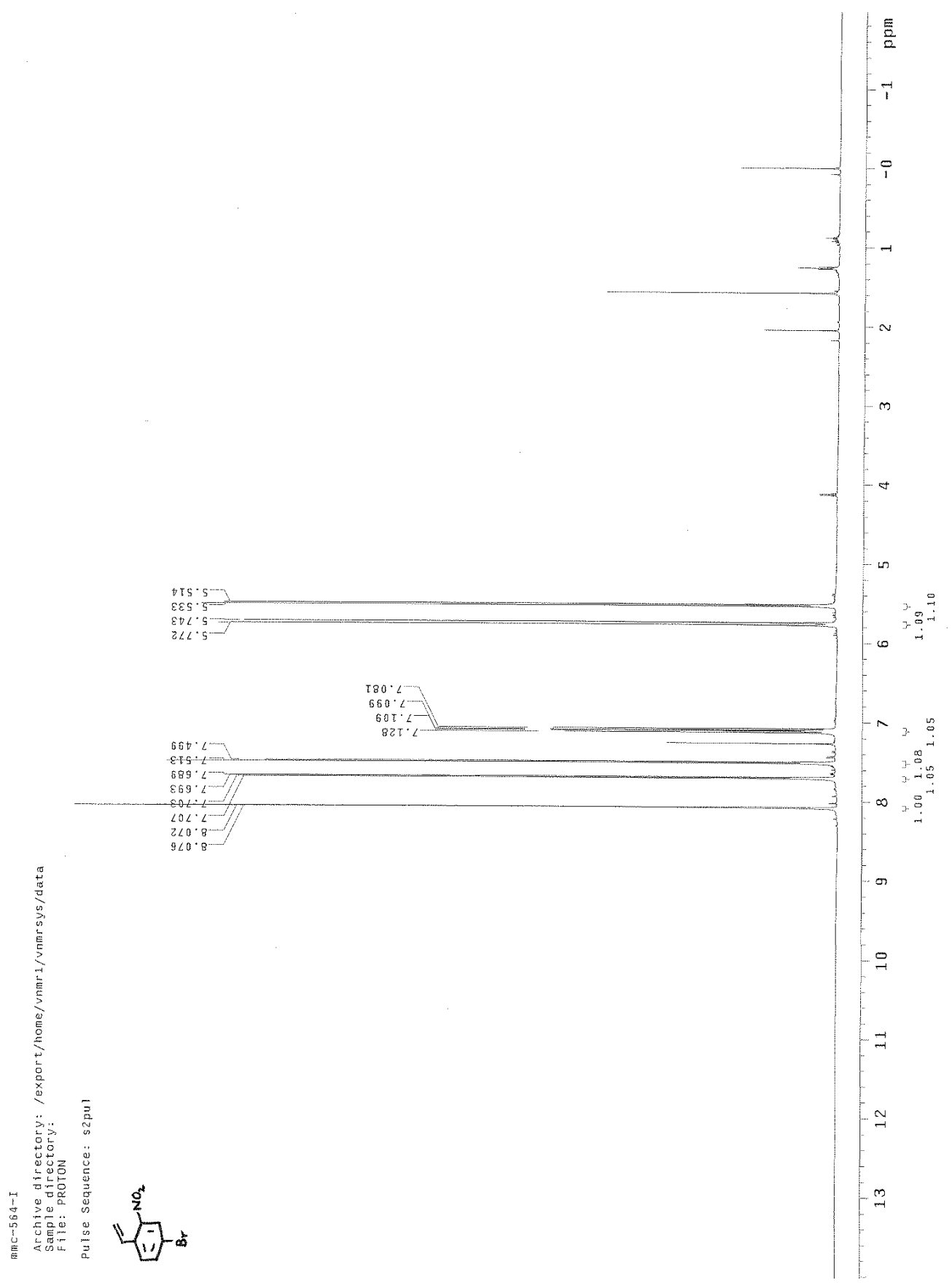

Figure 74: ${ }^{1} \mathrm{H}$ NMR of 5-Bromo-2-ethenyl-nitrobenzene (183) 


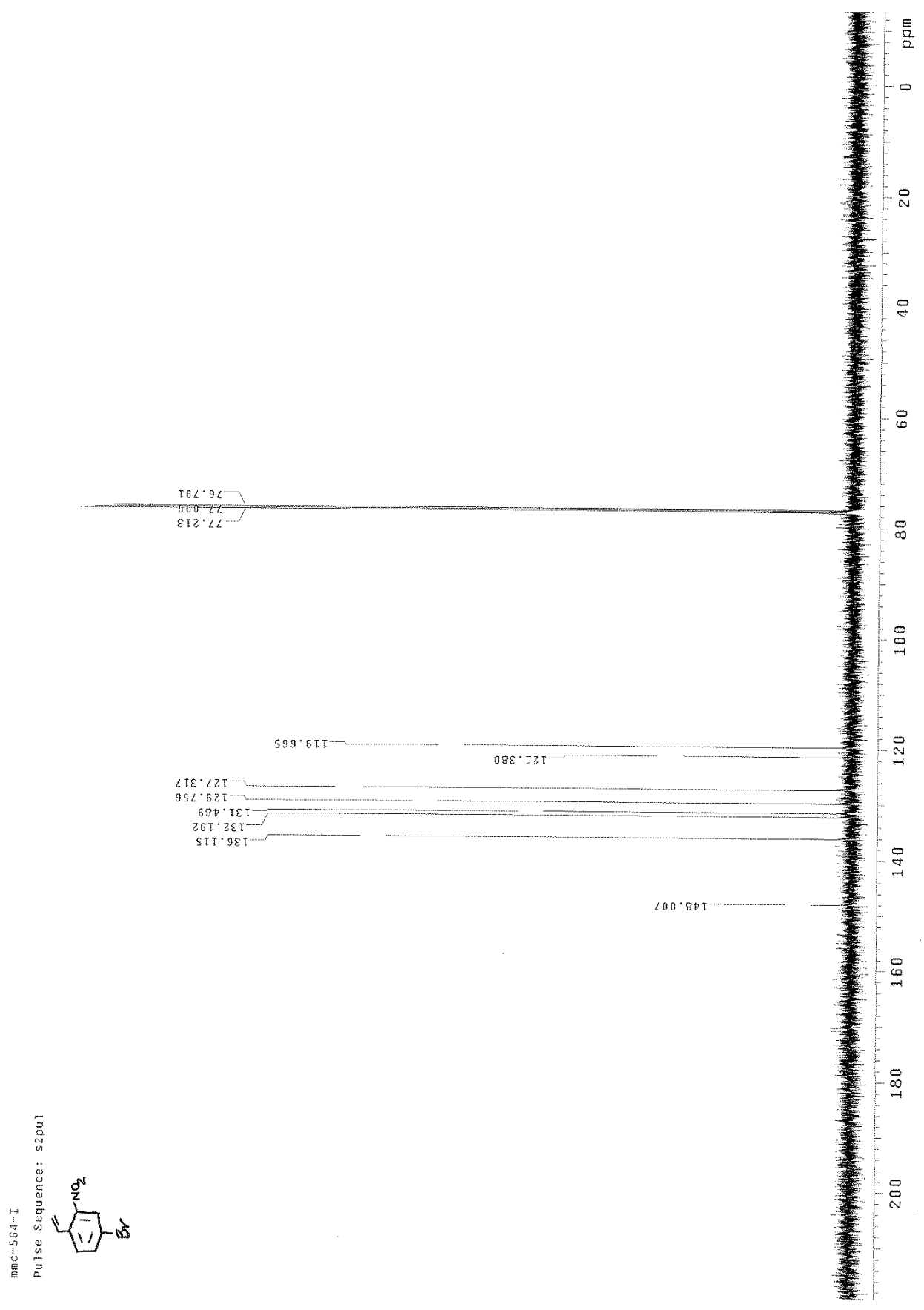

Figure $75:{ }^{13} \mathrm{C}$ NMR of 5-Bromo-2-ethenyl-nitrobenzene (183) 


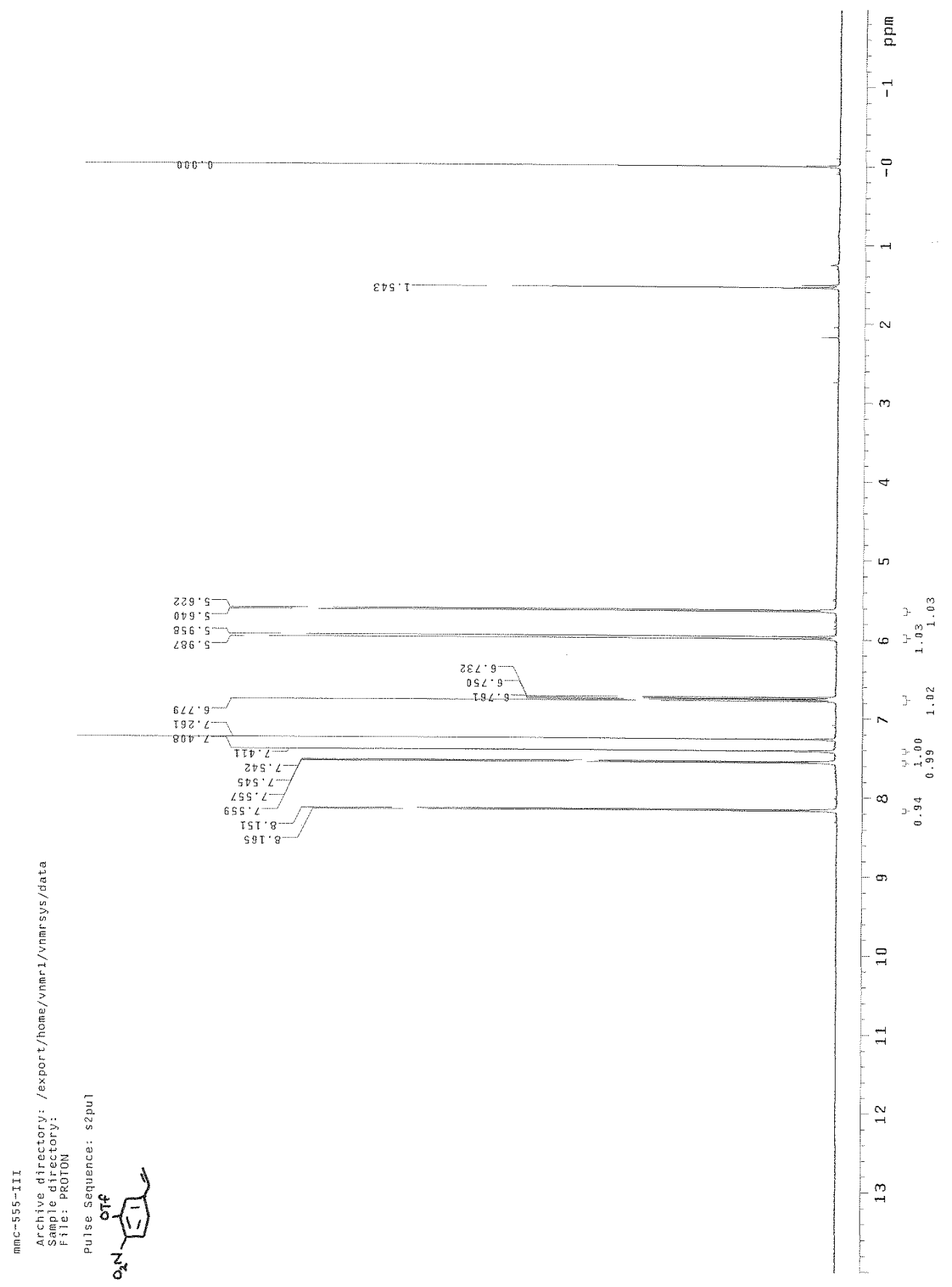

Figure 76: ${ }^{1} \mathrm{H}$ NMR of 4-Ethenyl-2-trifluoromethanesulfonyloxy-nitrobenzene (184) 


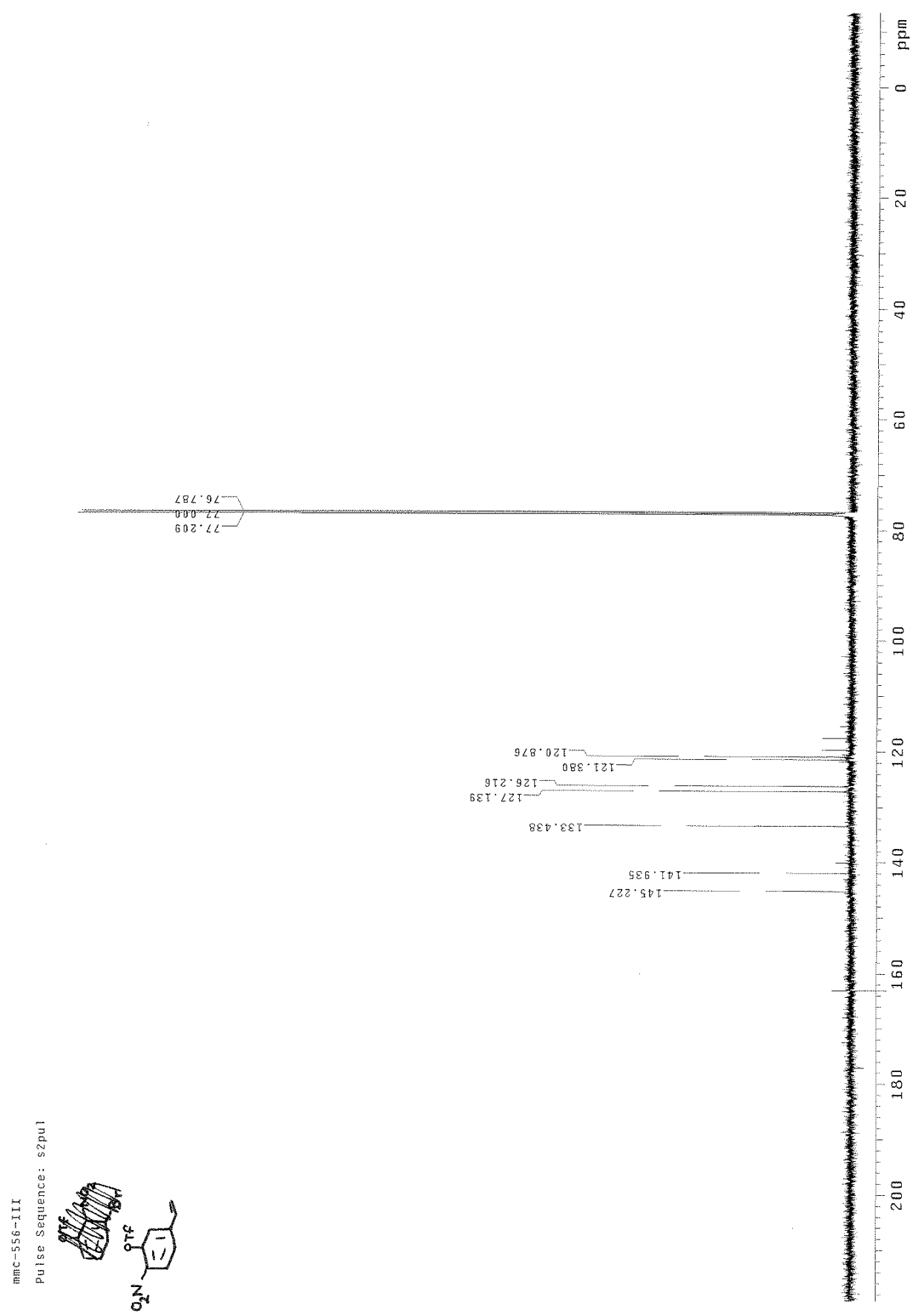

Figure 77: ${ }^{13} \mathrm{C}$ NMR of 4-Ethenyl-2-trifluoromethanesulfonyloxy-nitrobenzene (184) 


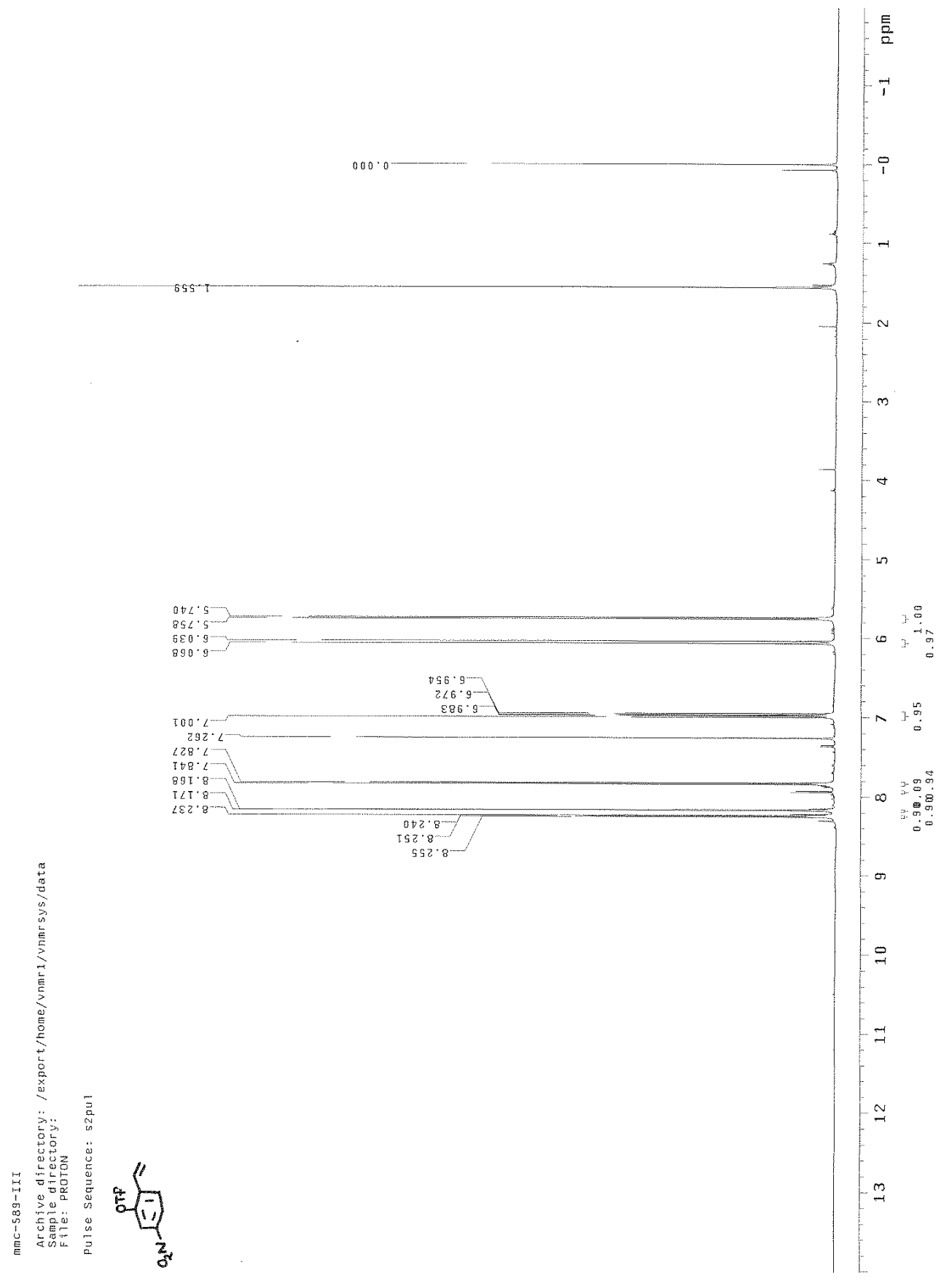

Figure 78: ${ }^{1} \mathrm{H}$ NMR of 4-Ethenyl-3-trifluoromethanesulfonyloxy-nitrobenzene (186) 


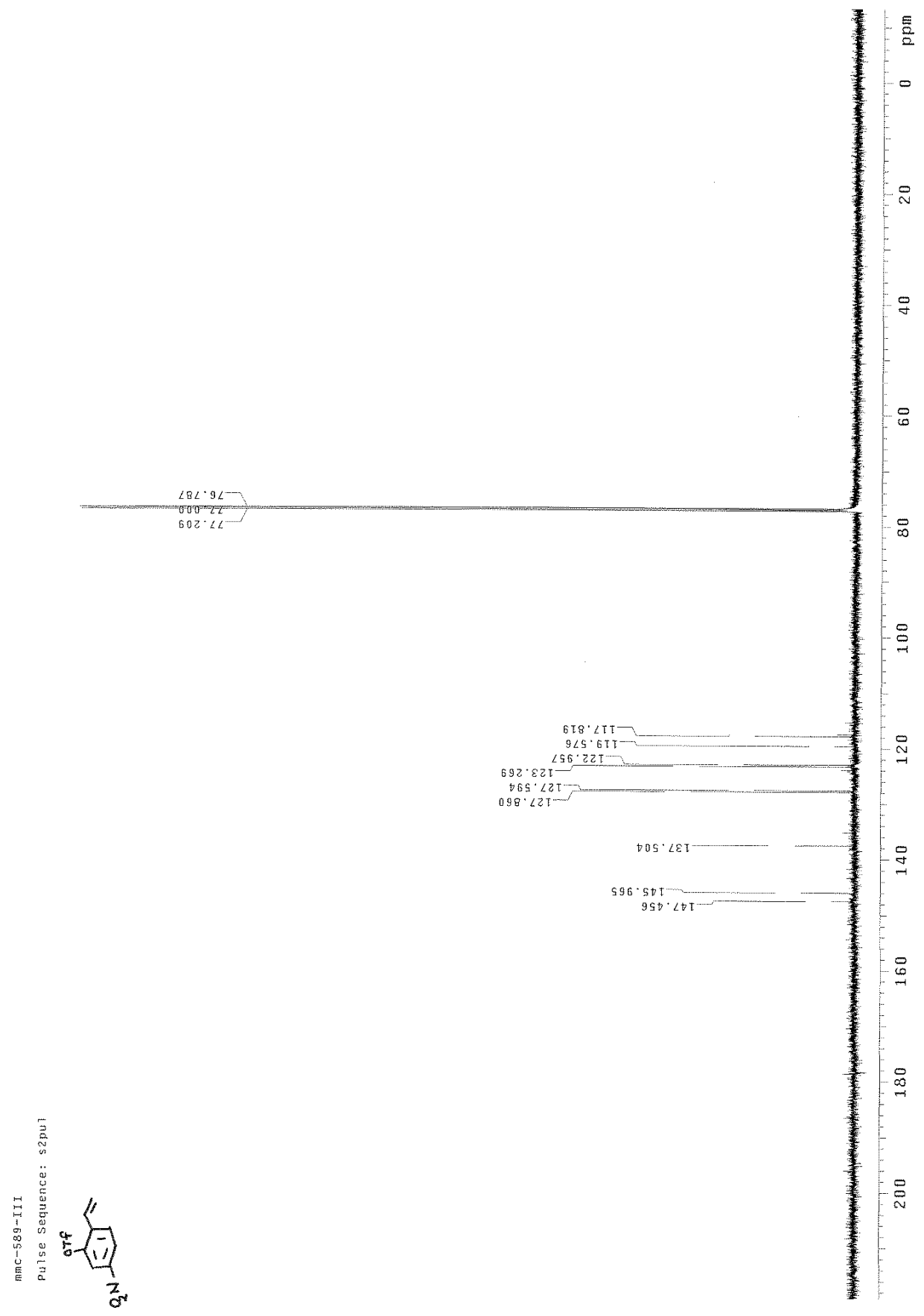

Figure 79: ${ }^{13} \mathrm{C}$ NMR of 4-Ethenyl-3-trifluoromethanesulfonyloxy-nitrobenzene (186) 


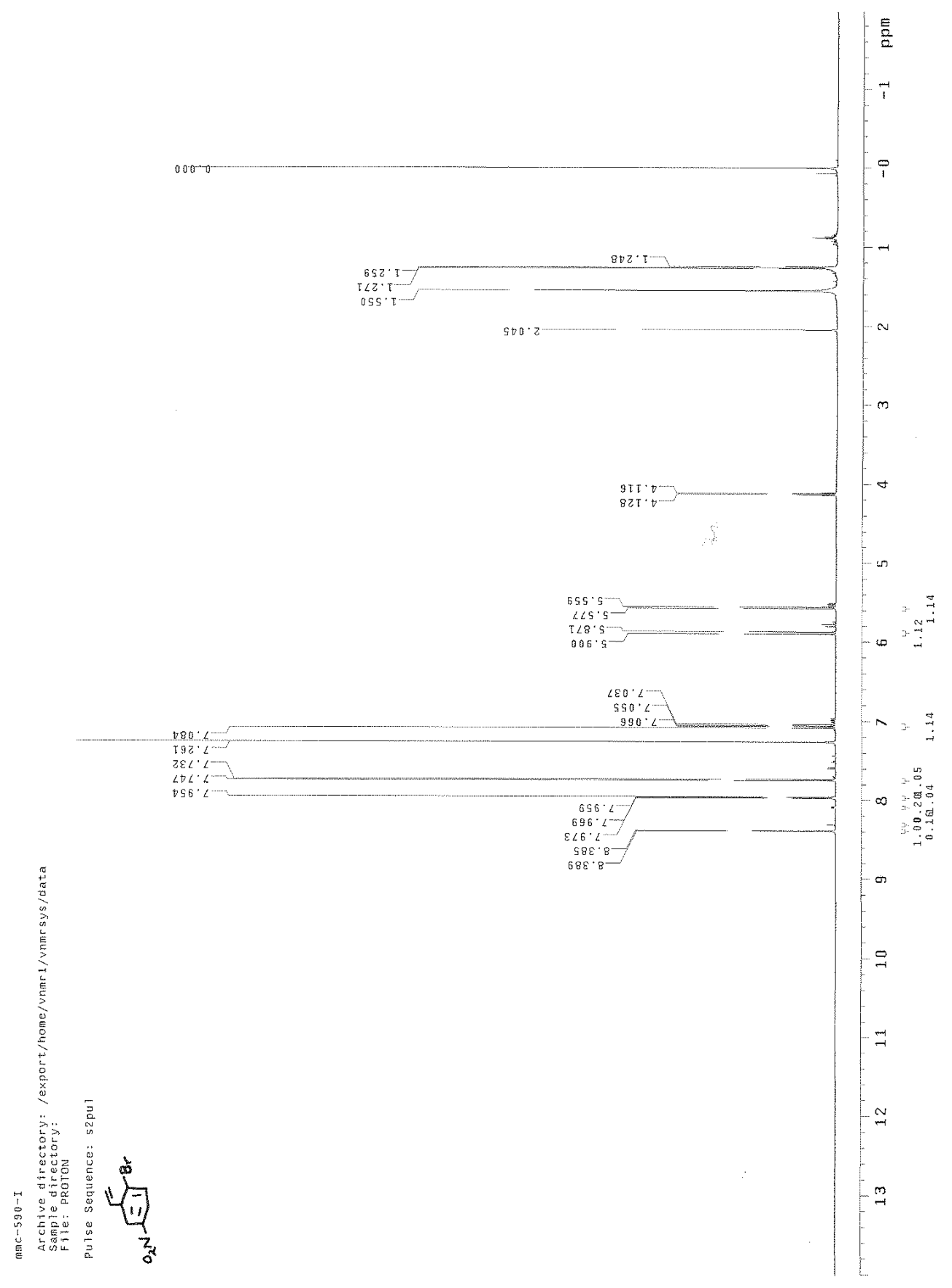

Figure 80: ${ }^{1} \mathrm{H}$ NMR of 4-Bromo-3-ethenyl-nitrobenzene (187) 


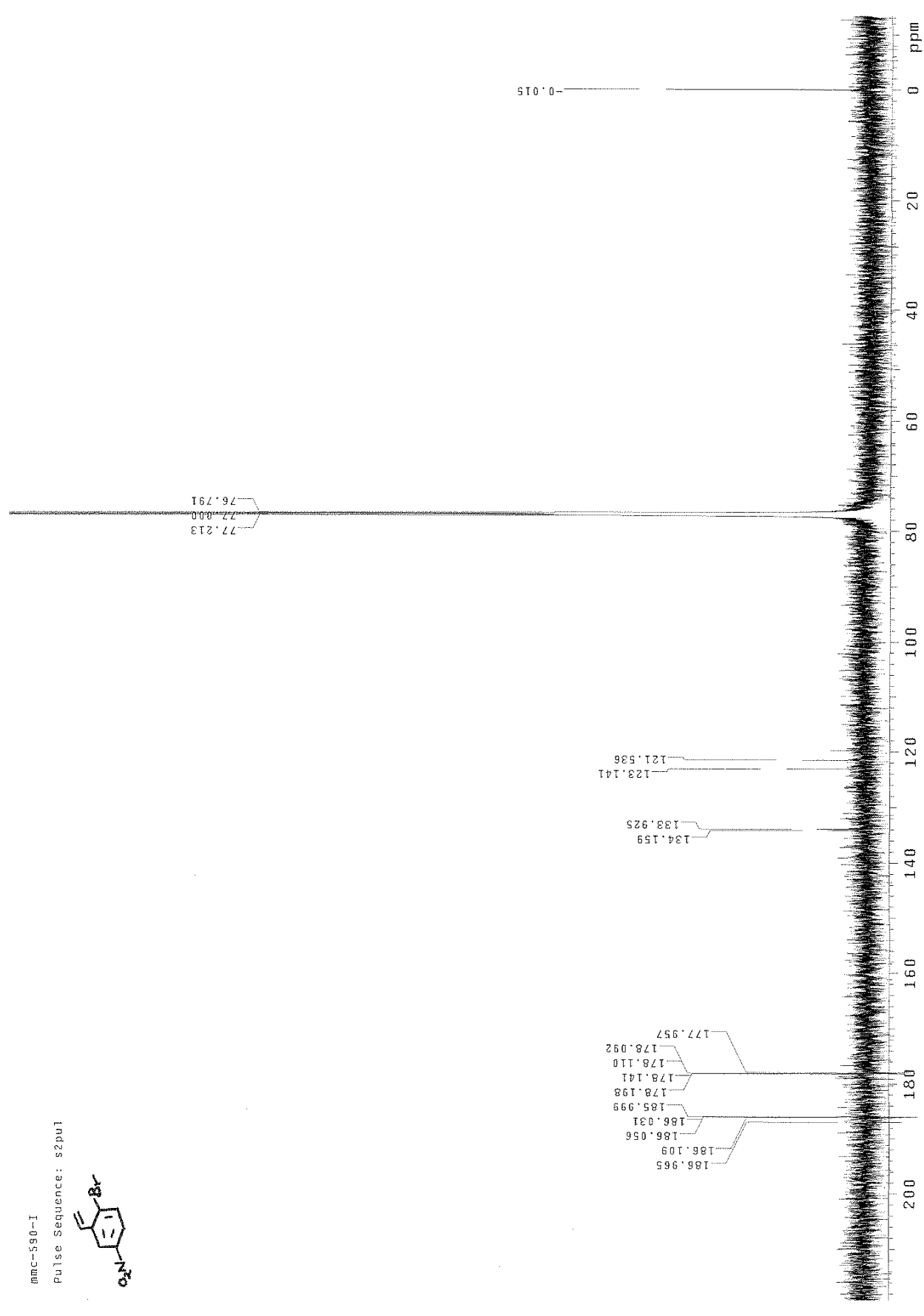

Figure 81: ${ }^{13} \mathrm{C}$ NMR of 4-Bromo-3-ethenyl-nitrobenzene (187) 


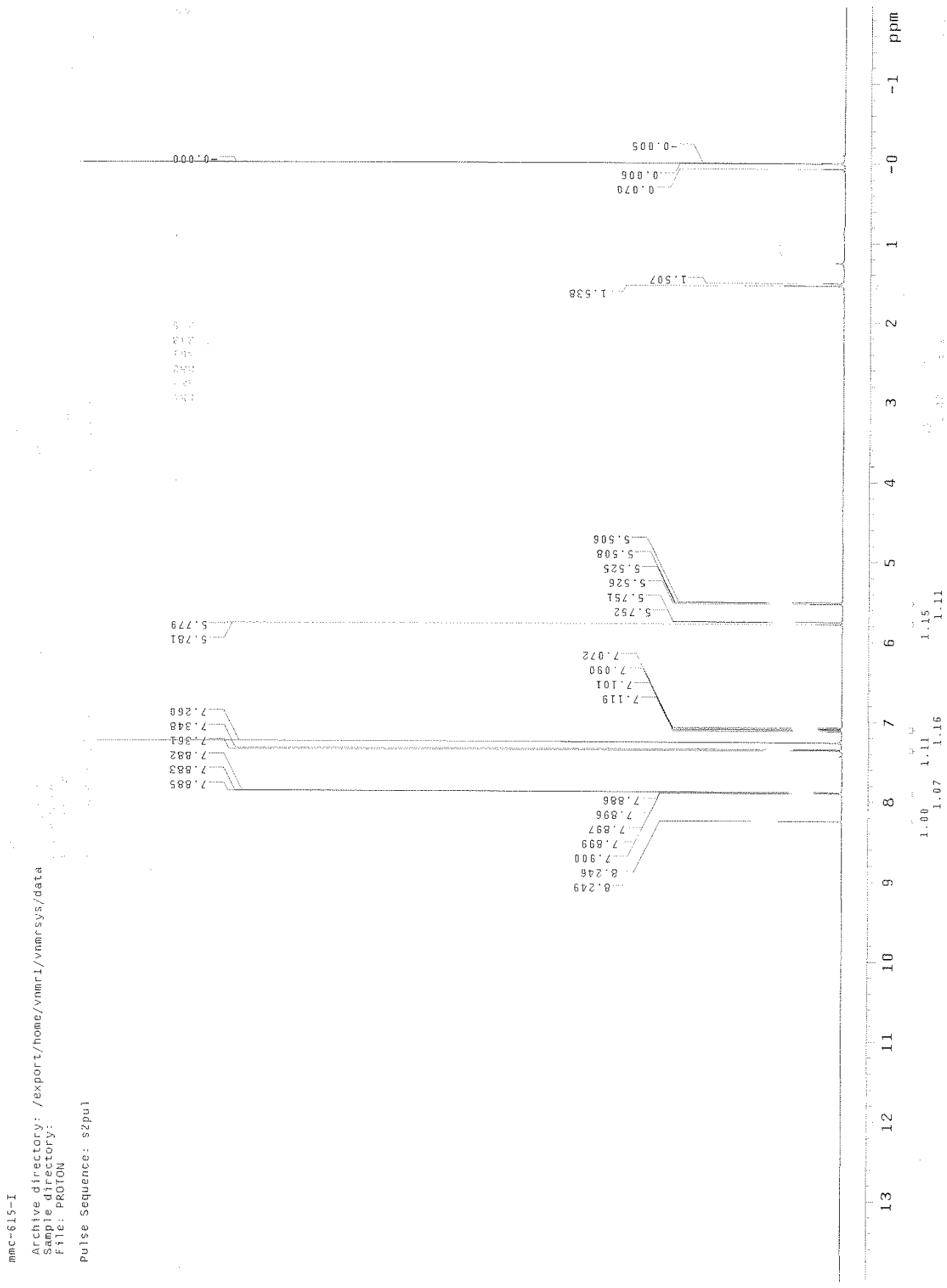

Figure 82: ${ }^{1} \mathrm{H}$ NMR of 3-Iodo-6-ethenyl-nitrobenzene (198) 


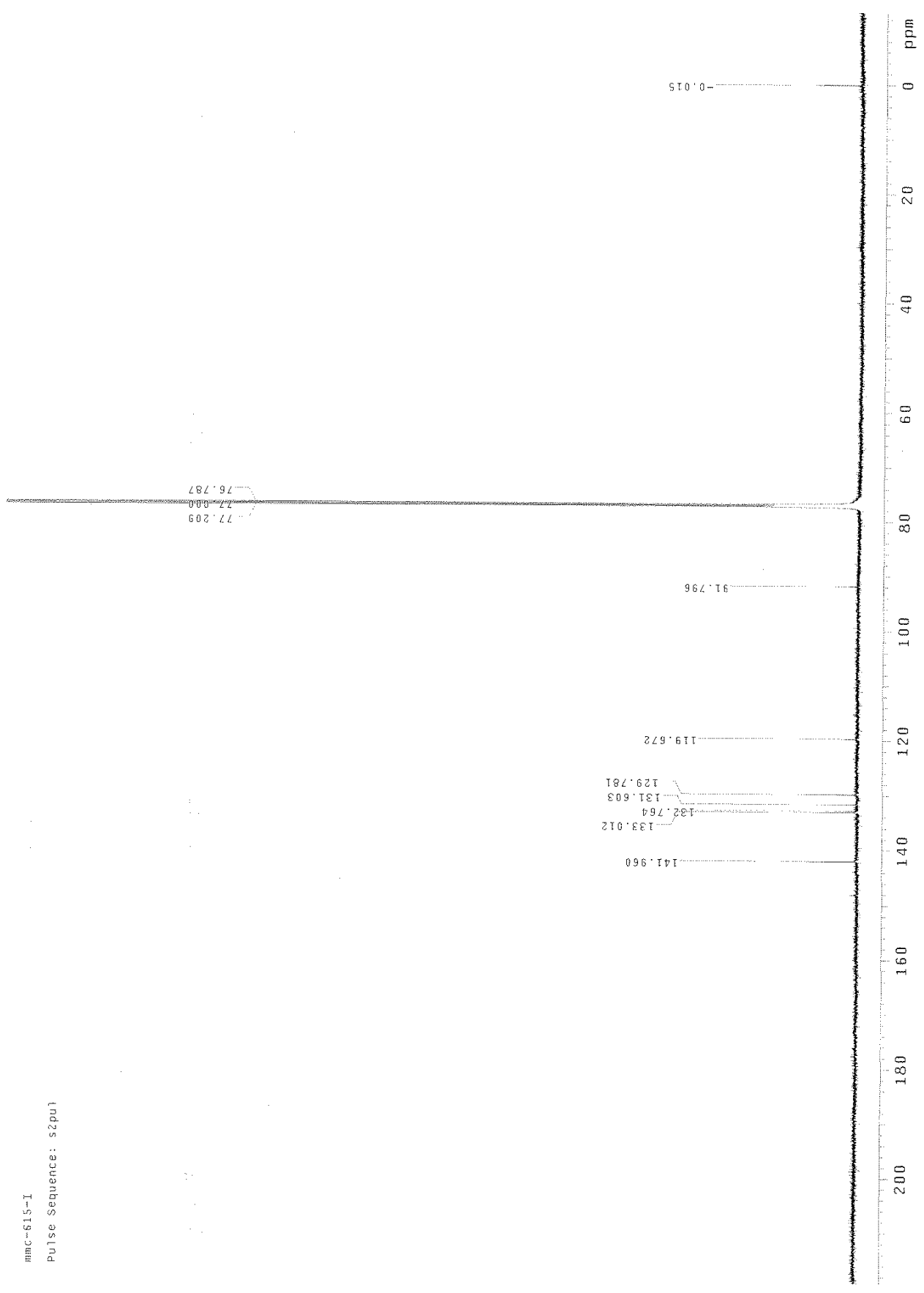

Figure 83: ${ }^{13} \mathrm{C}$ NMR of 3-Iodo-6-ethenyl-nitrobenzene (198) 


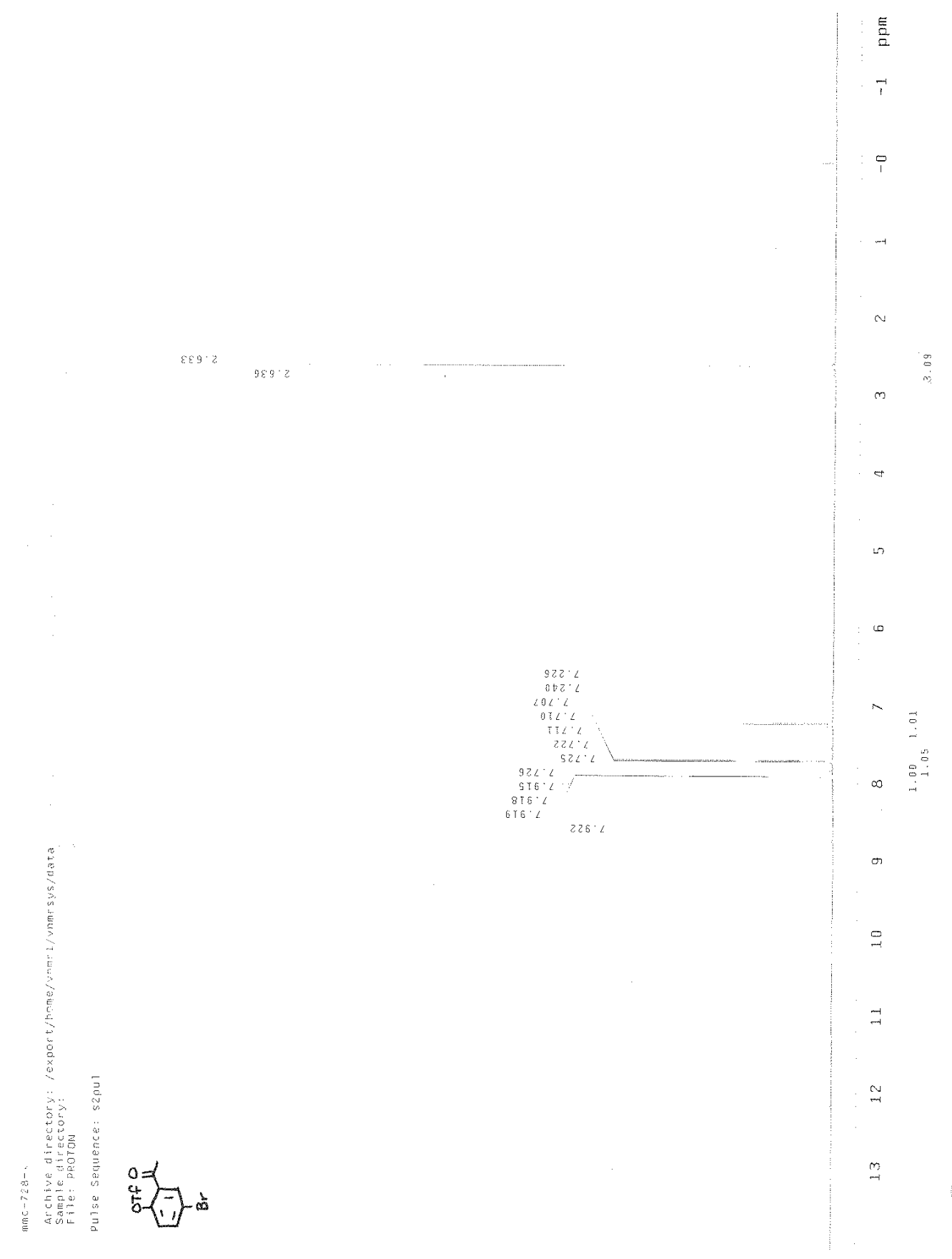

Figure 84: ${ }^{1} \mathrm{H}$ NMR of 3-Bromo-6-trifluoromethanesulfonyloxy acetophenone (201) 


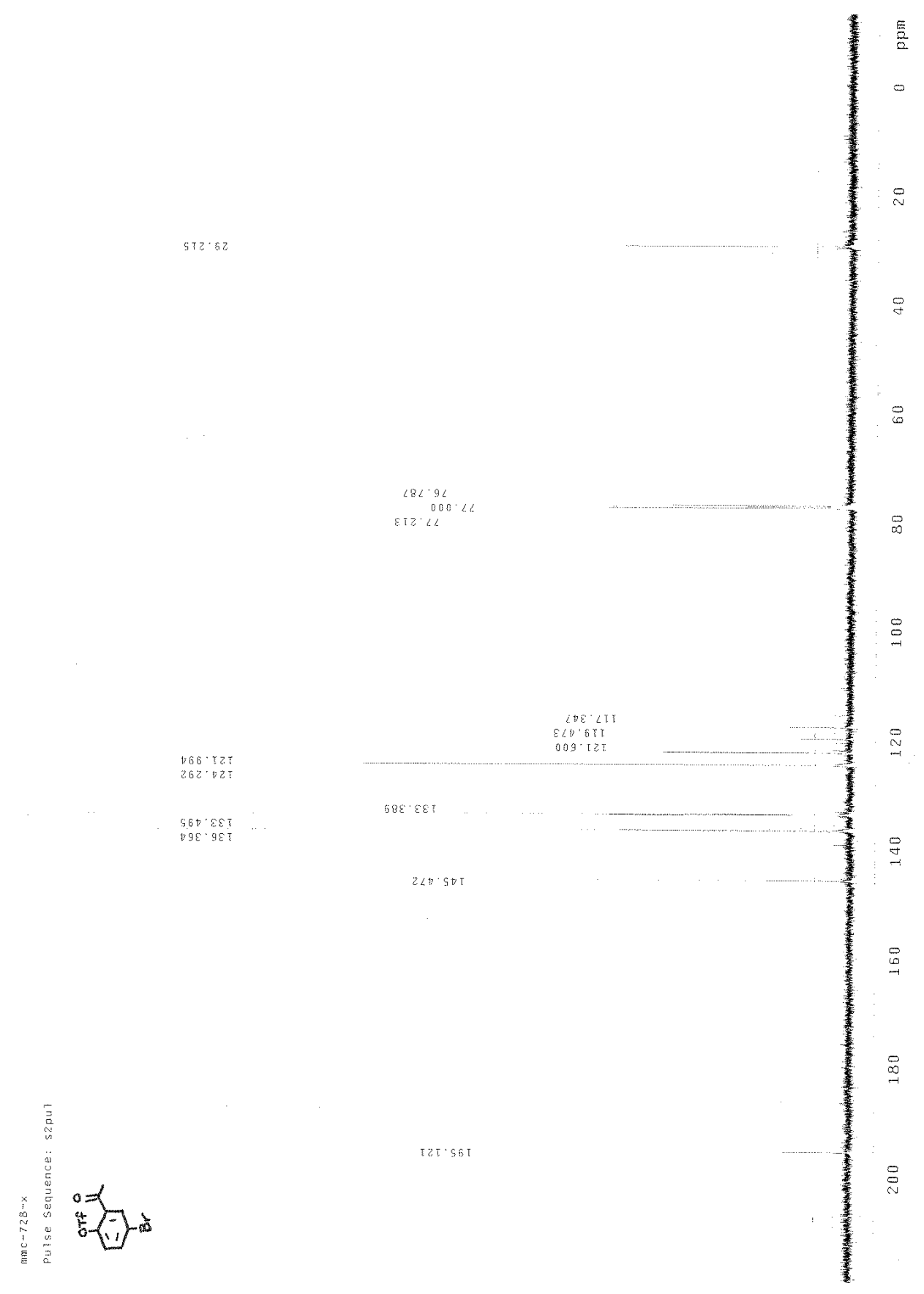

Figure 85: ${ }^{13} \mathrm{C}$ NMR of 3-Bromo-6-trifluoromethanesulfonyloxy acetophenone (201) 


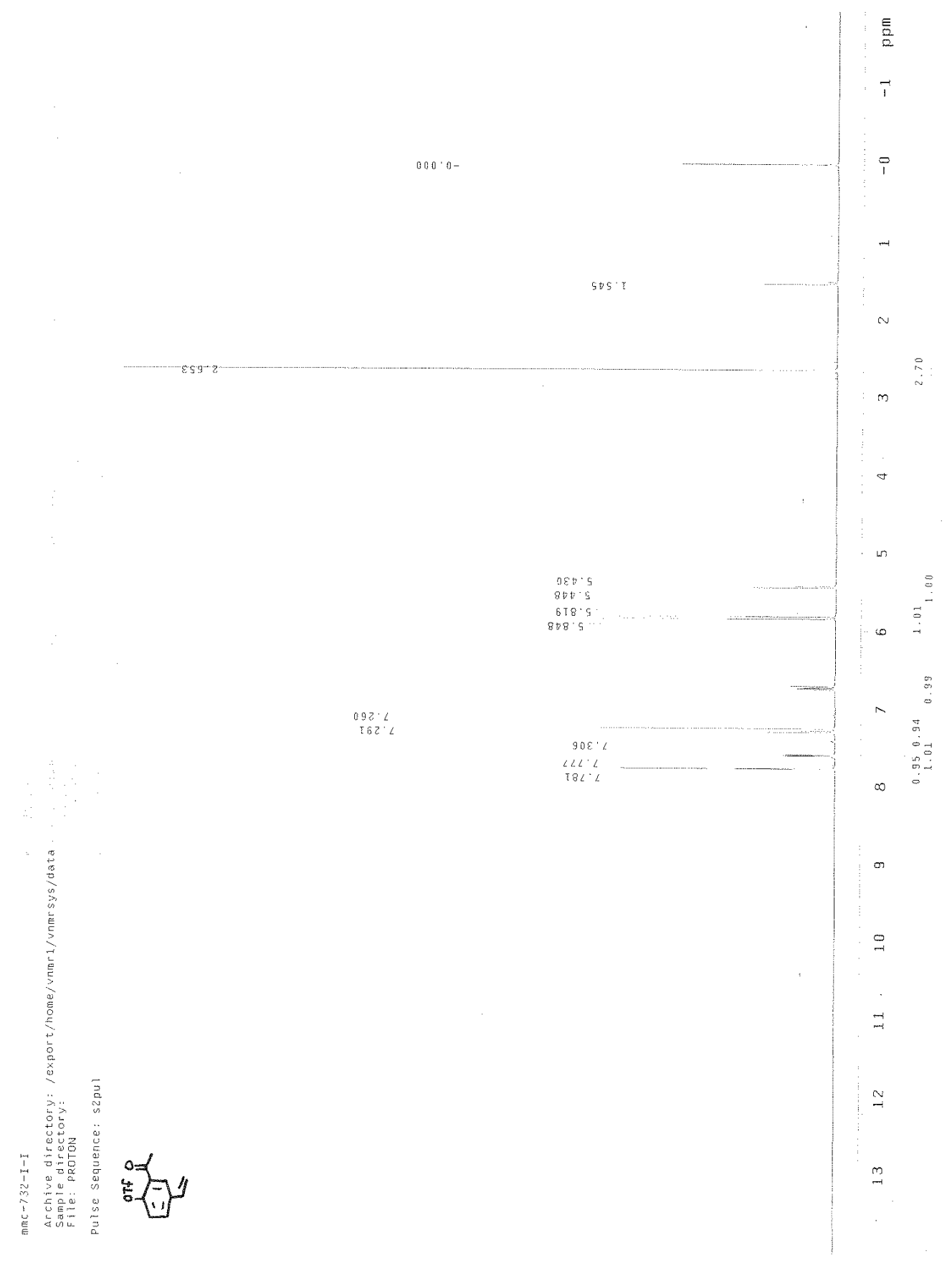

Figure 86: ${ }^{1} \mathrm{H}$ NMR of 3-Ethenyl-6-trifluoromethanesulfonyloxy acetophenone (202) 


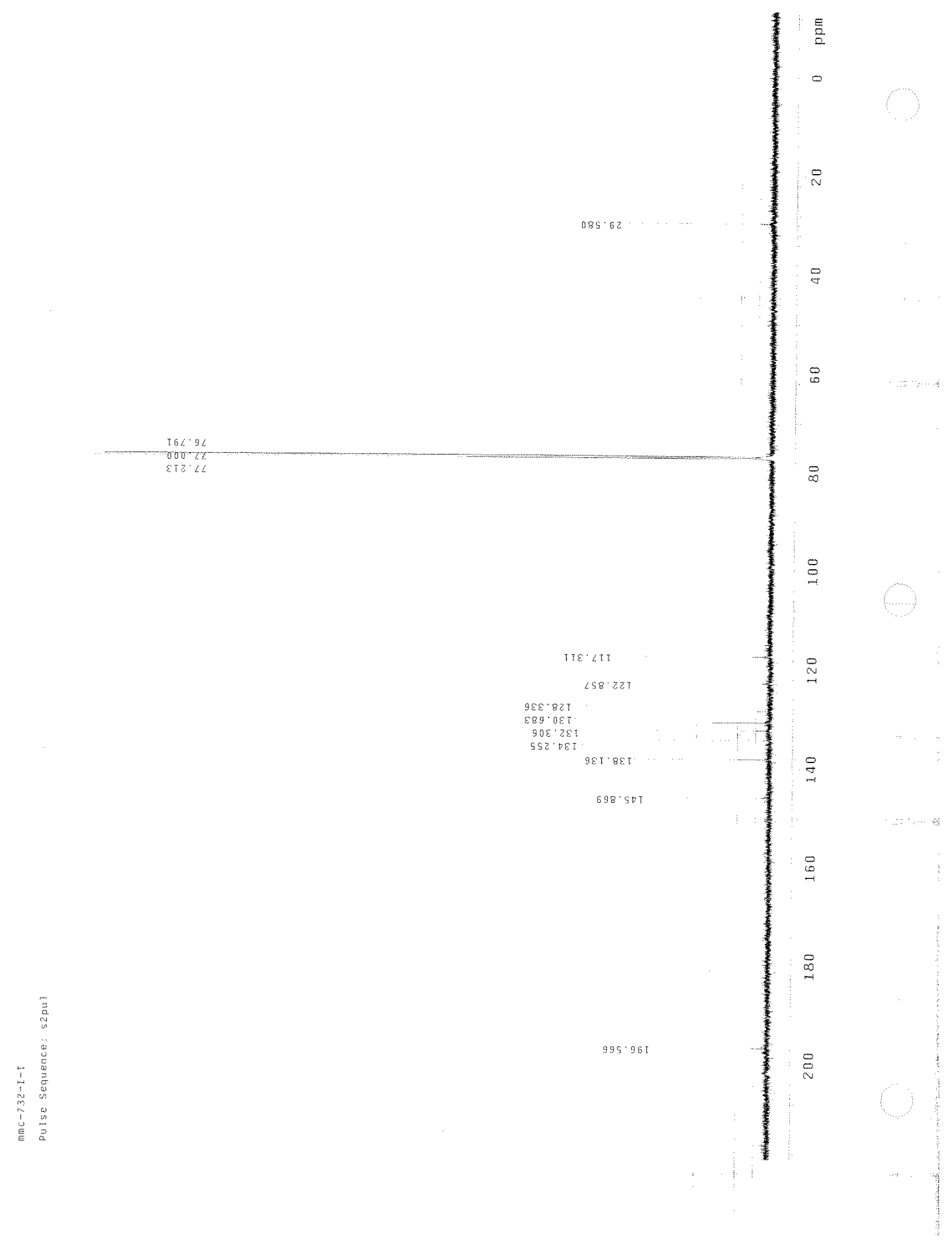

Figure 87: ${ }^{13} \mathrm{C}$ NMR of 3-Ethenyl-6-trifluoromethanesulfonyloxy acetophenone (202) 


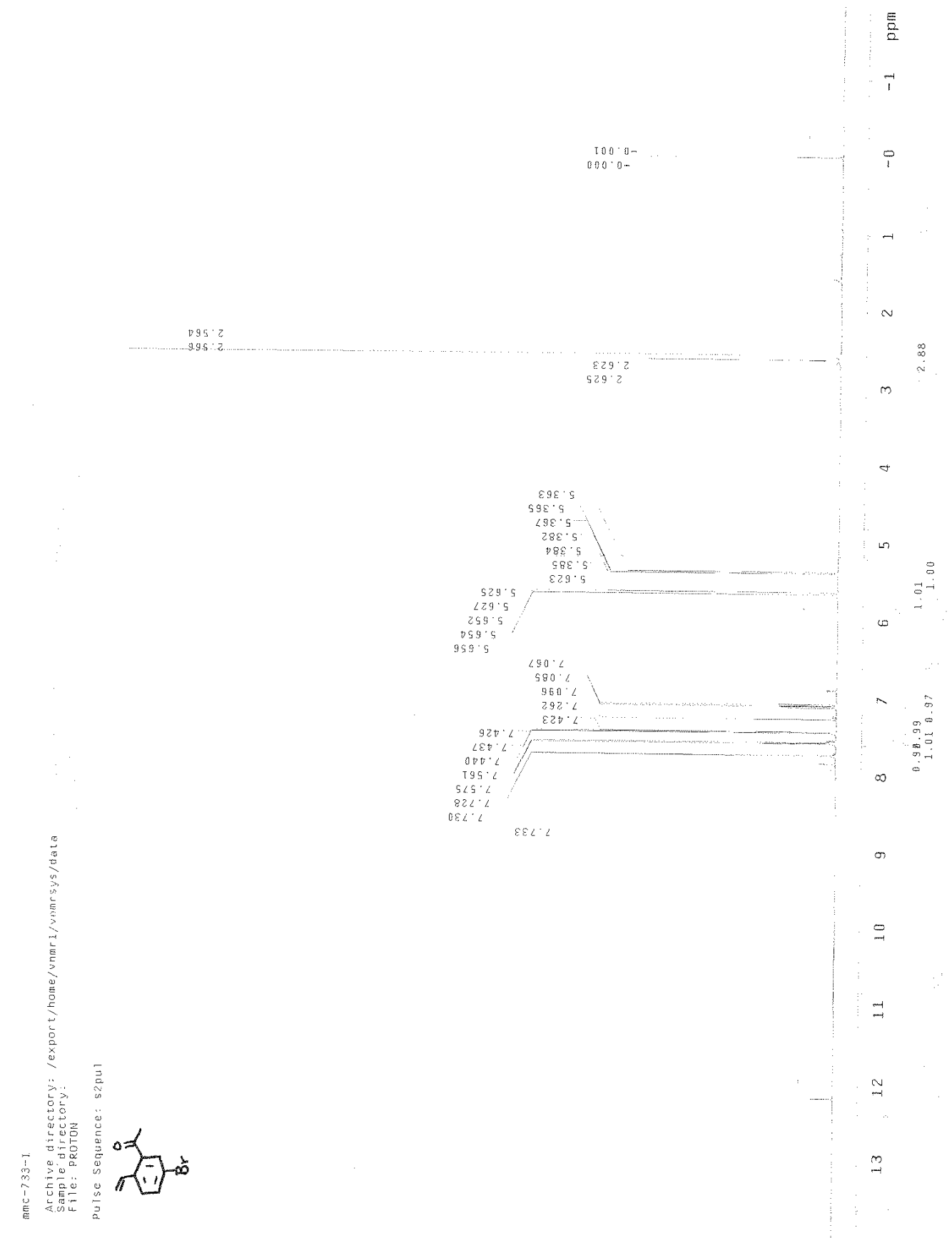

Figure 88: ${ }^{1} \mathrm{H}$ NMR of 6-Ethenyl-3-bromoacetophenone (203) 


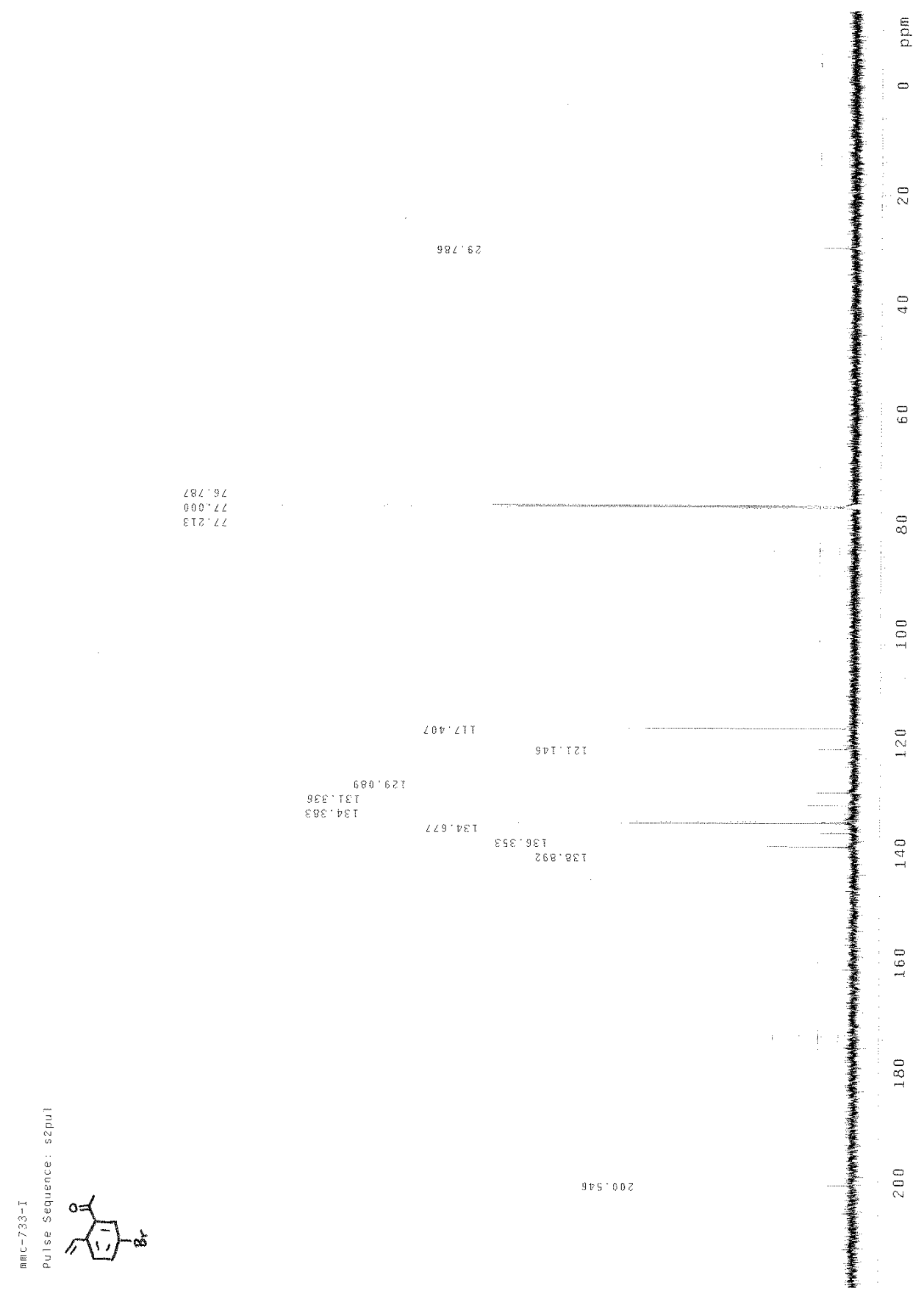

Figure 89: ${ }^{13} \mathrm{C}$ NMR of 6-Ethenyl-3-bromoacetophenone (203) 


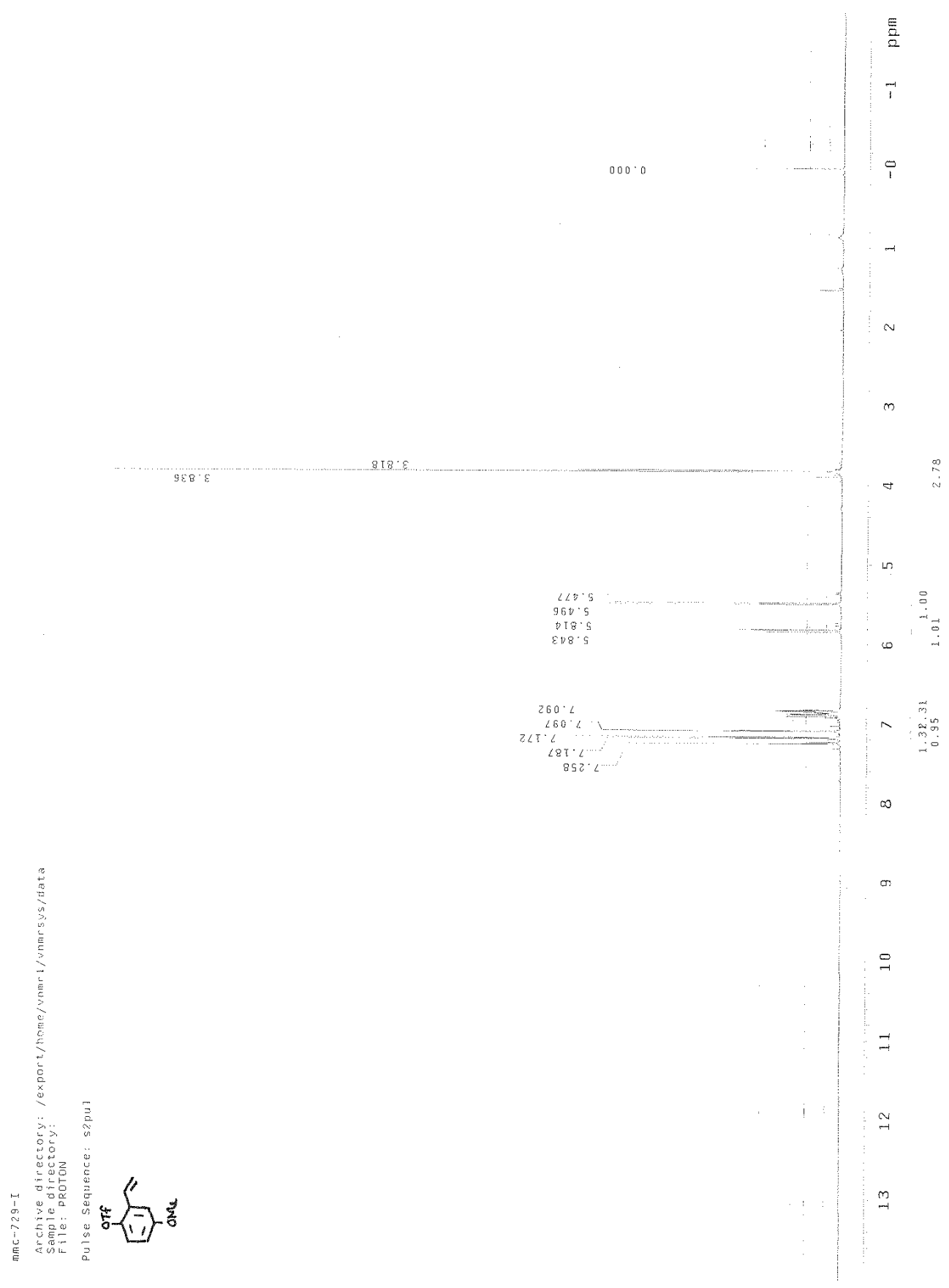

Figure 90: ${ }^{1} \mathrm{H}$ NMR of 3-Ethenyl-4-trifluoromethanesulfonyloxy anisole (209) 


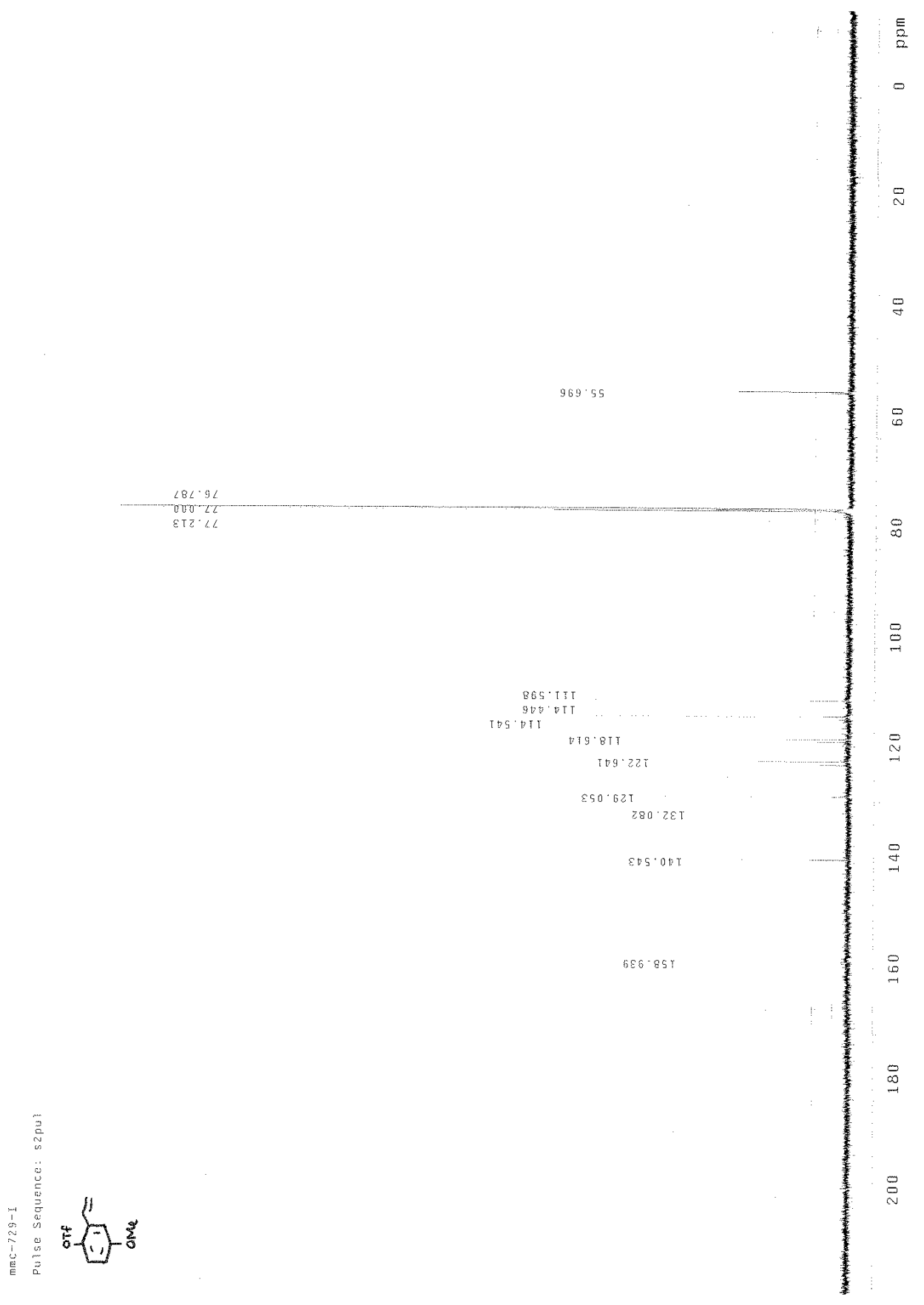

Figure 91: ${ }^{13} \mathrm{C}$ NMR of 3-Ethenyl-4-trifluoromethanesulfonyloxy anisole (209) 
${ }^{1} \mathrm{H}$ and ${ }^{13} \mathrm{C}$ NMR for Chapter 5: Pyrroloindoles

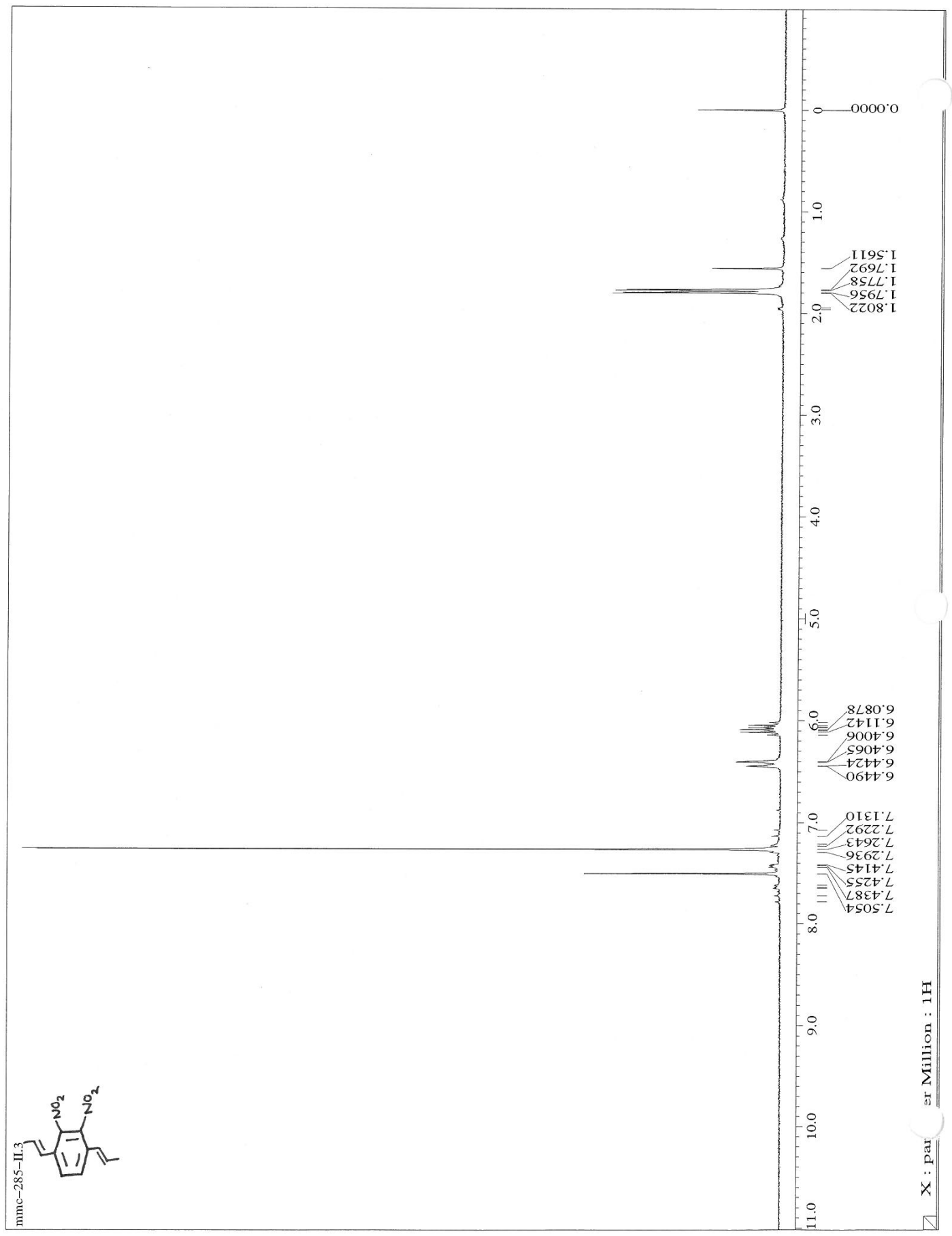

Figure 92: ${ }^{1} \mathrm{H}$ NMR of 2,3-dinitro-1,4-ethenylbenzene (241) 


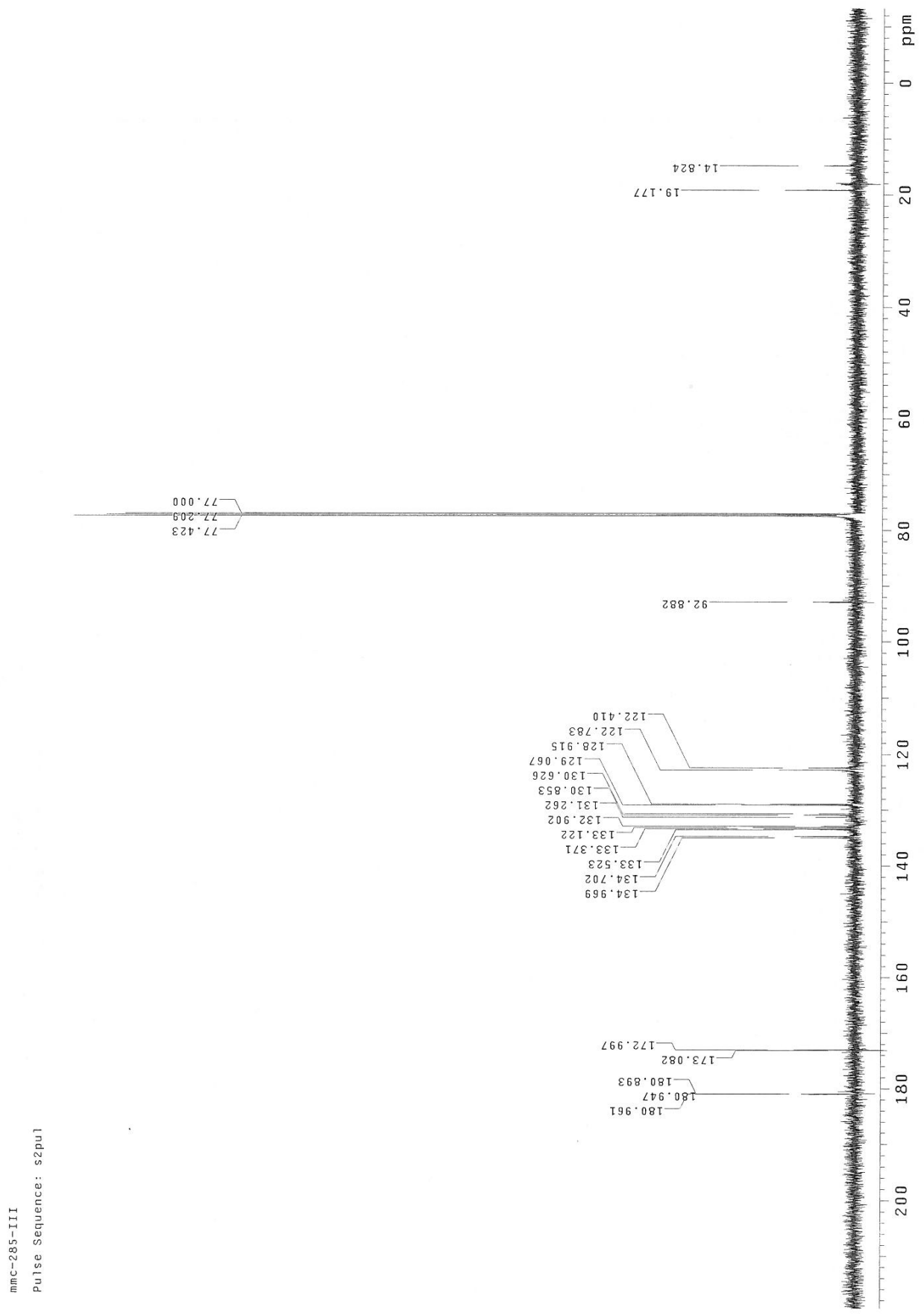

Figure 93: ${ }^{13} \mathrm{C}$ NMR of 2,3-dinitro-1,4-ethenylbenzene (241) 


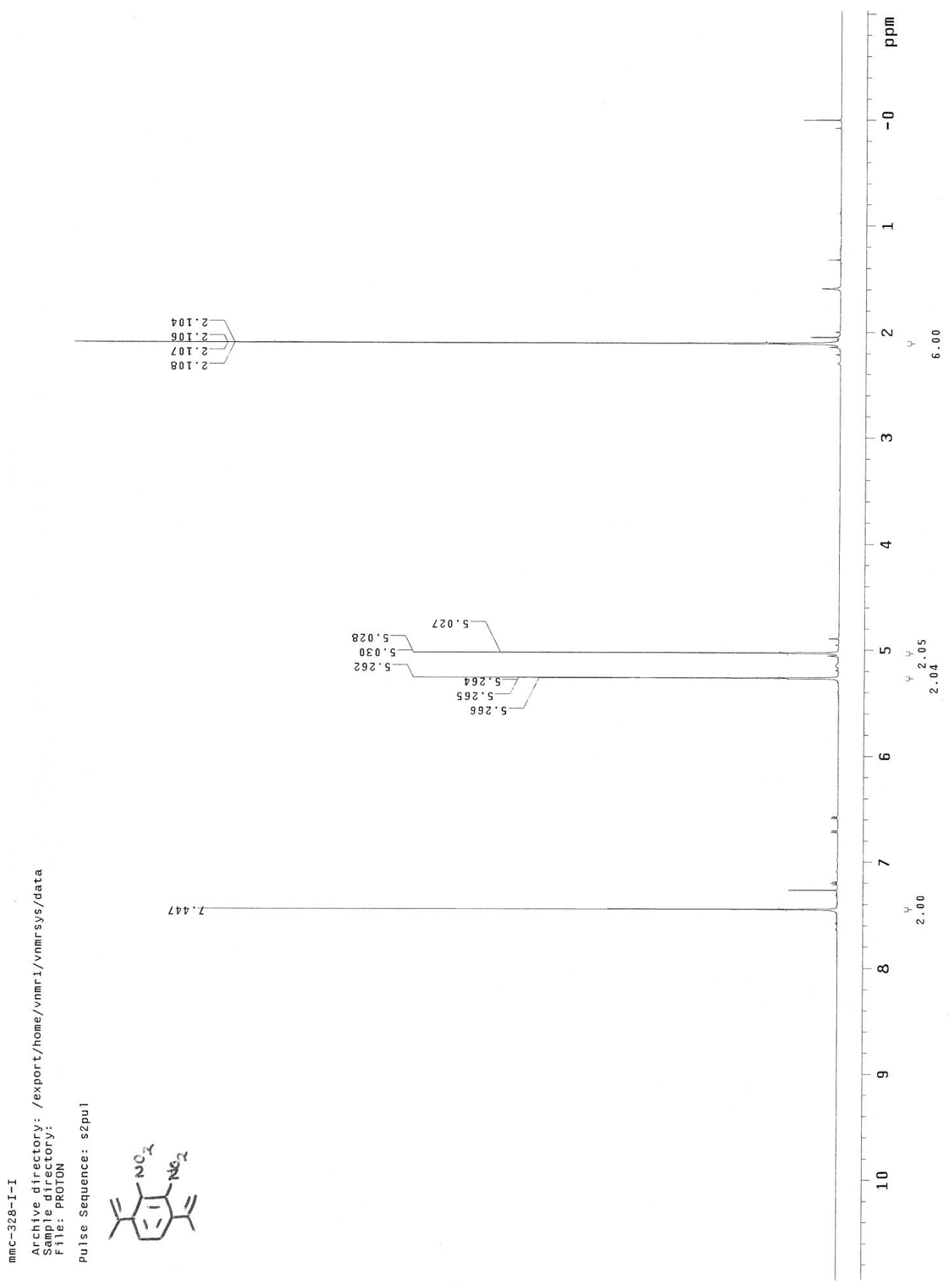

Figure 94: ${ }^{1} \mathrm{H}$ NMR of 2,3-dinitro-1,4-di(prop-1-en-2-yl)benzene (257) 


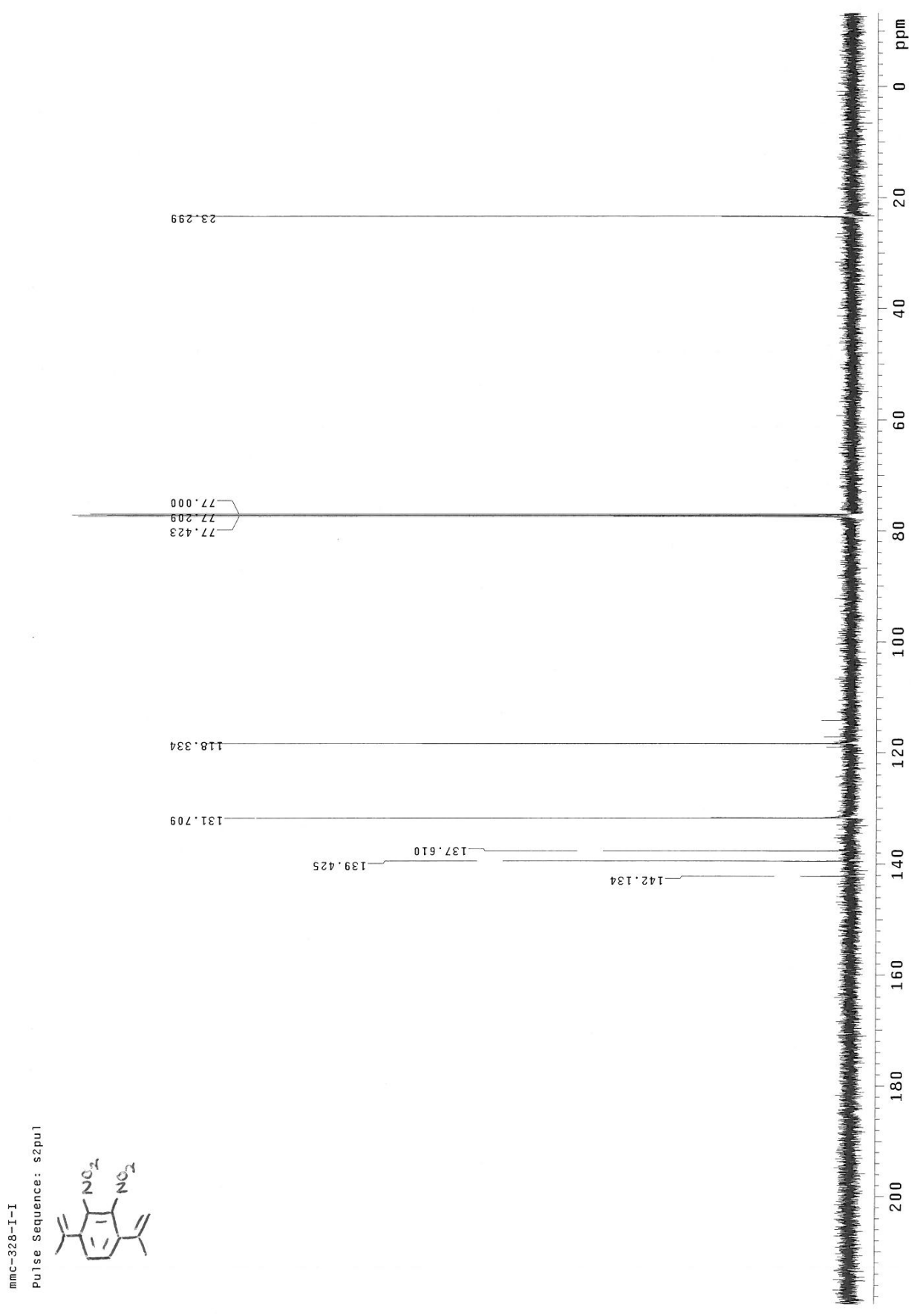

Figure 95: ${ }^{13} \mathrm{C}$ NMR of 2,3-dinitro-1,4-di(prop-1-en-2-yl)benzene (257) 


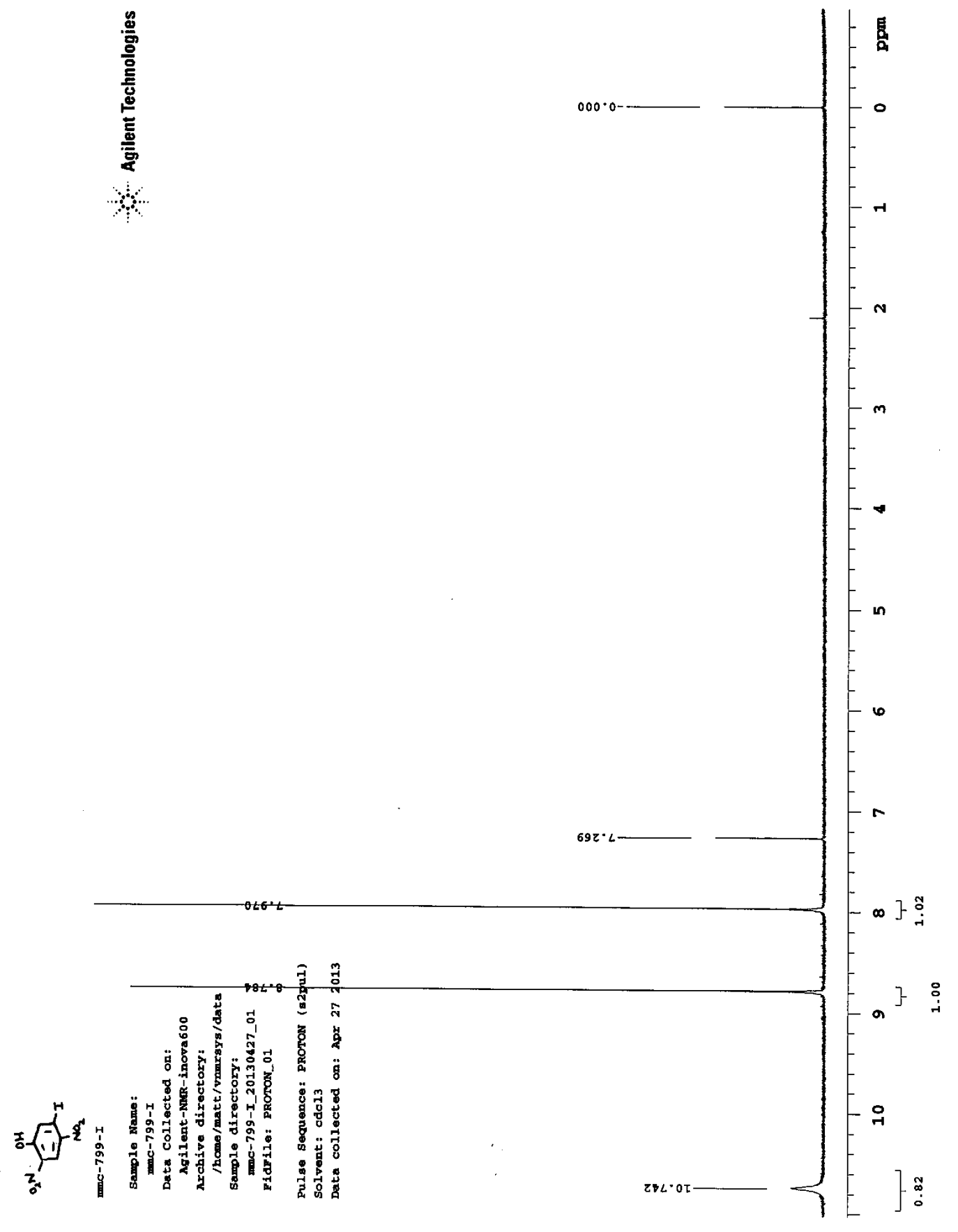

Figure 96: ${ }^{1} \mathrm{H}$ NMR of 5-iodo-2,4-dinitrophenol (263) 


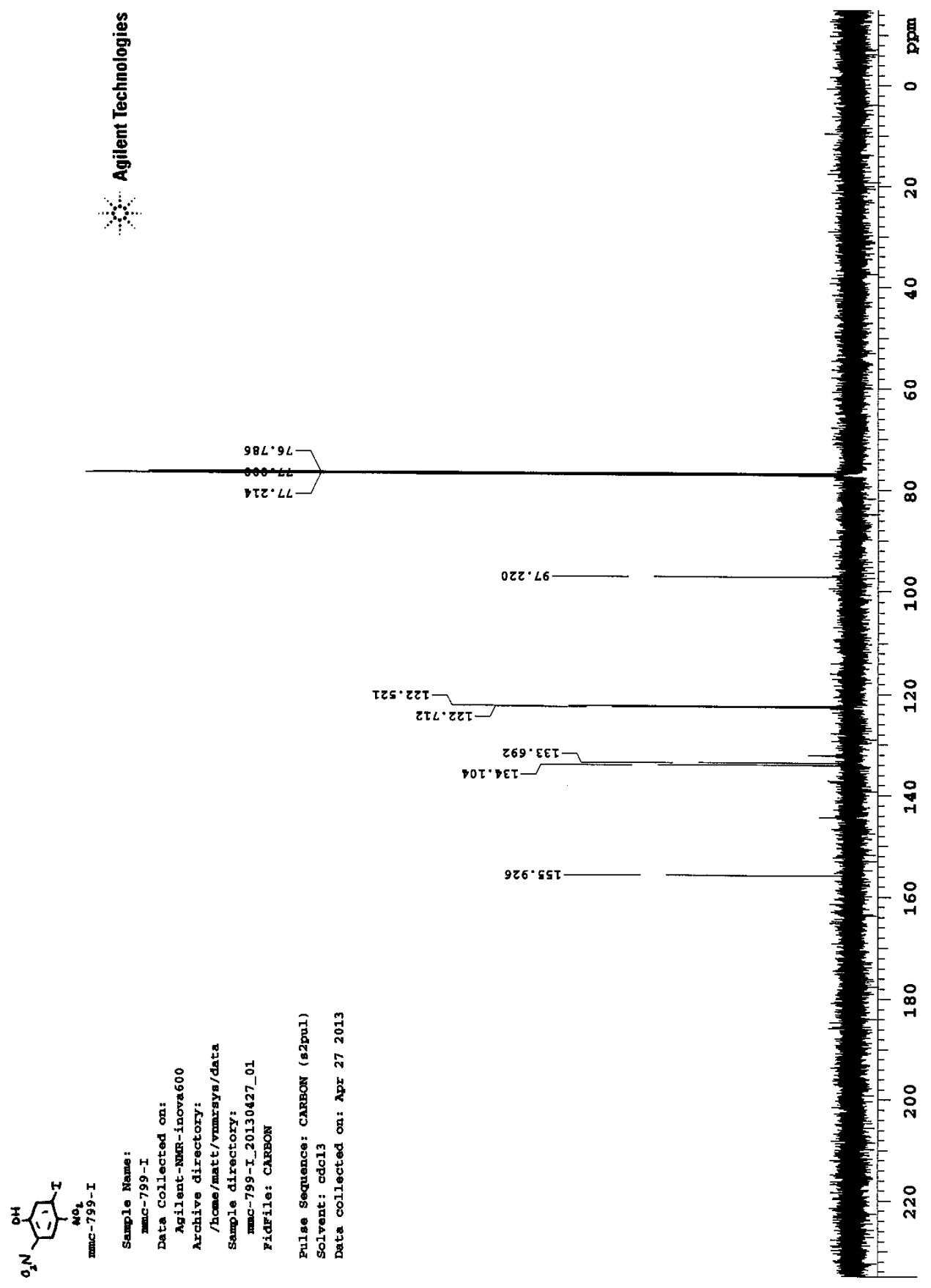

Figure 97: ${ }^{13} \mathrm{C}$ NMR of 5-iodo-2,4-dinitrophenol (263) 


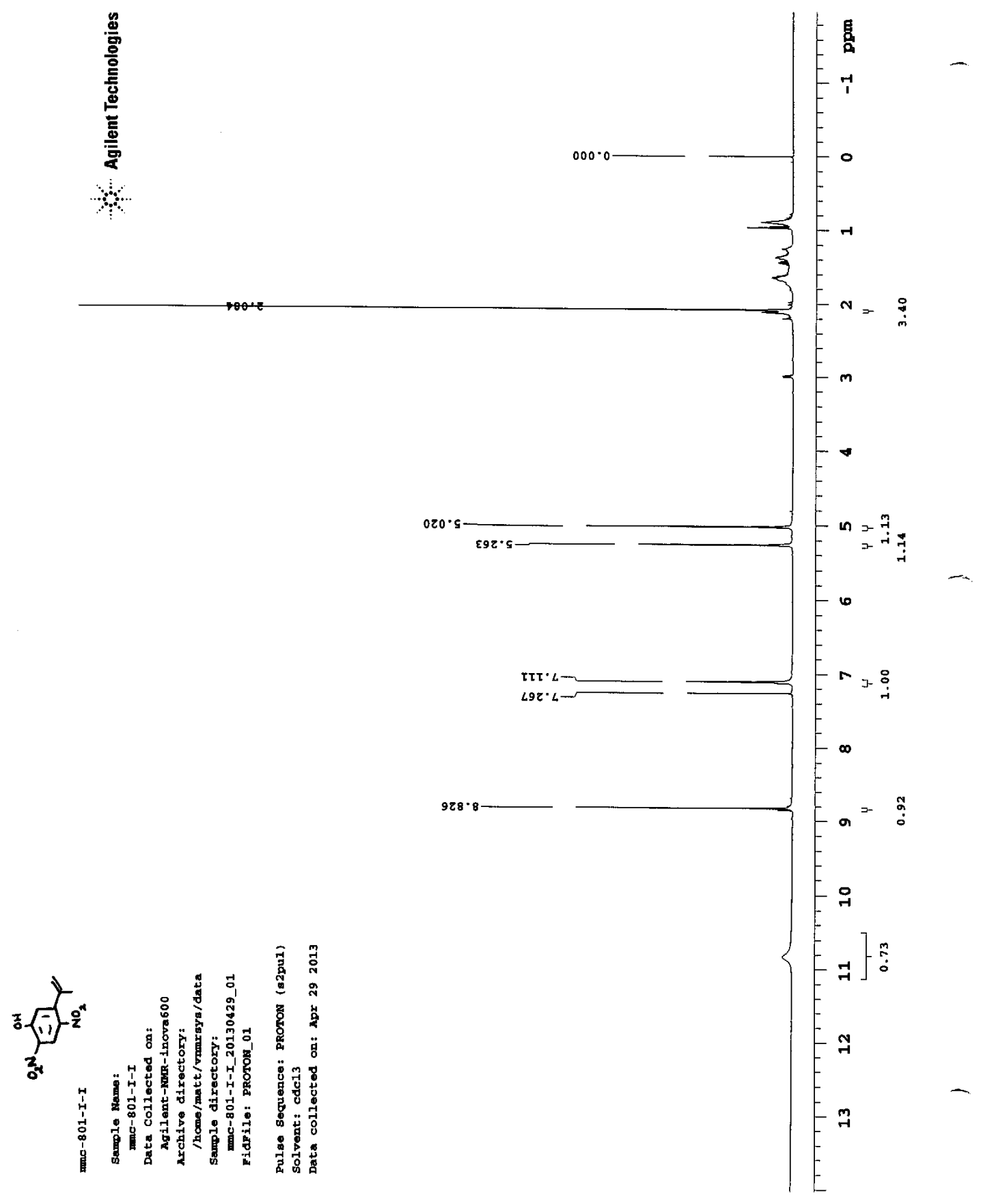

Figure 98: ${ }^{1} \mathrm{H}$ NMR of 2,4-dinitro-5-(prop-1-en-2-yl)phenol (271) 


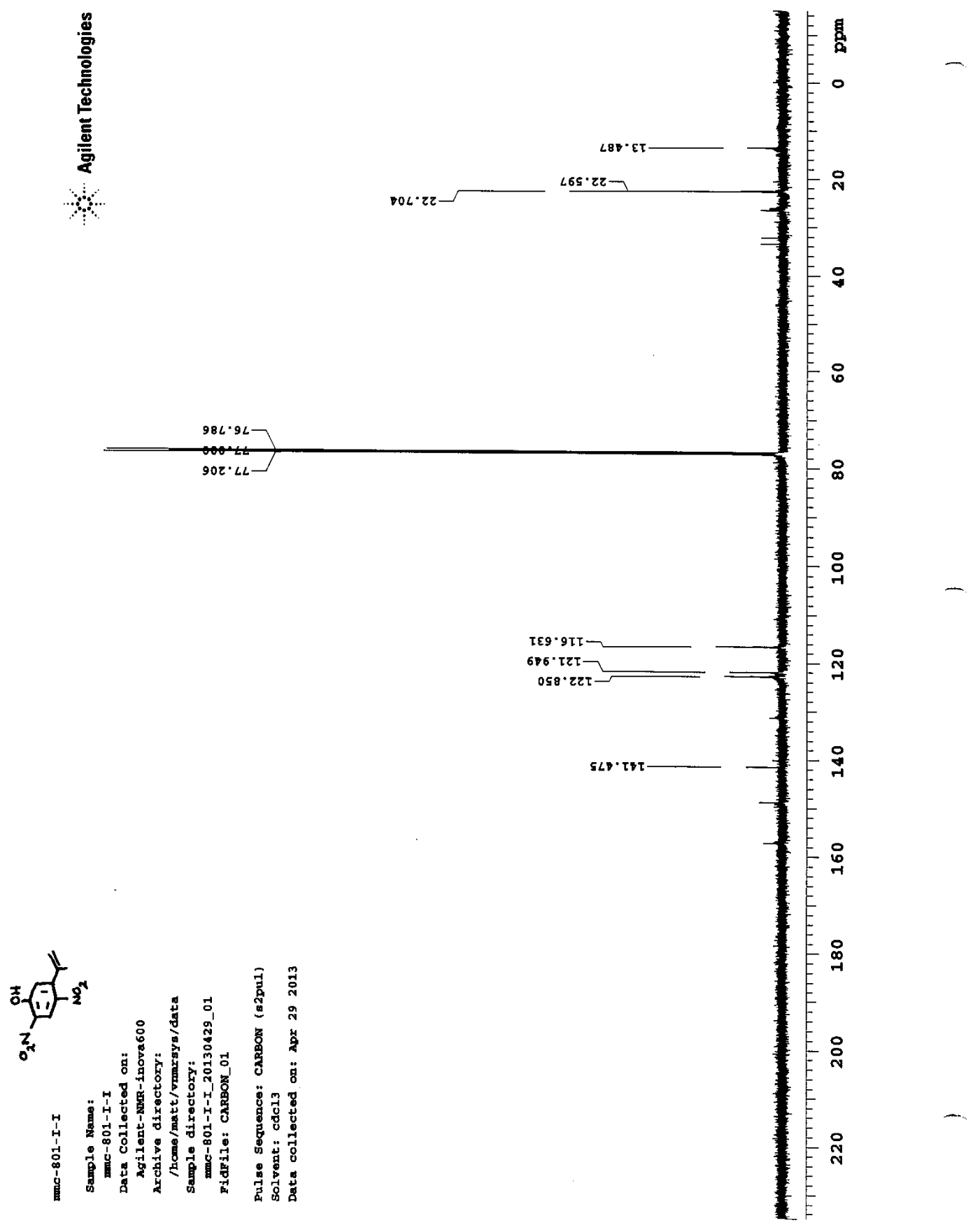

Figure 99: ${ }^{13} \mathrm{C}$ NMR of 2,4-dinitro-5-(prop-1-en-2-yl)phenol (271) 


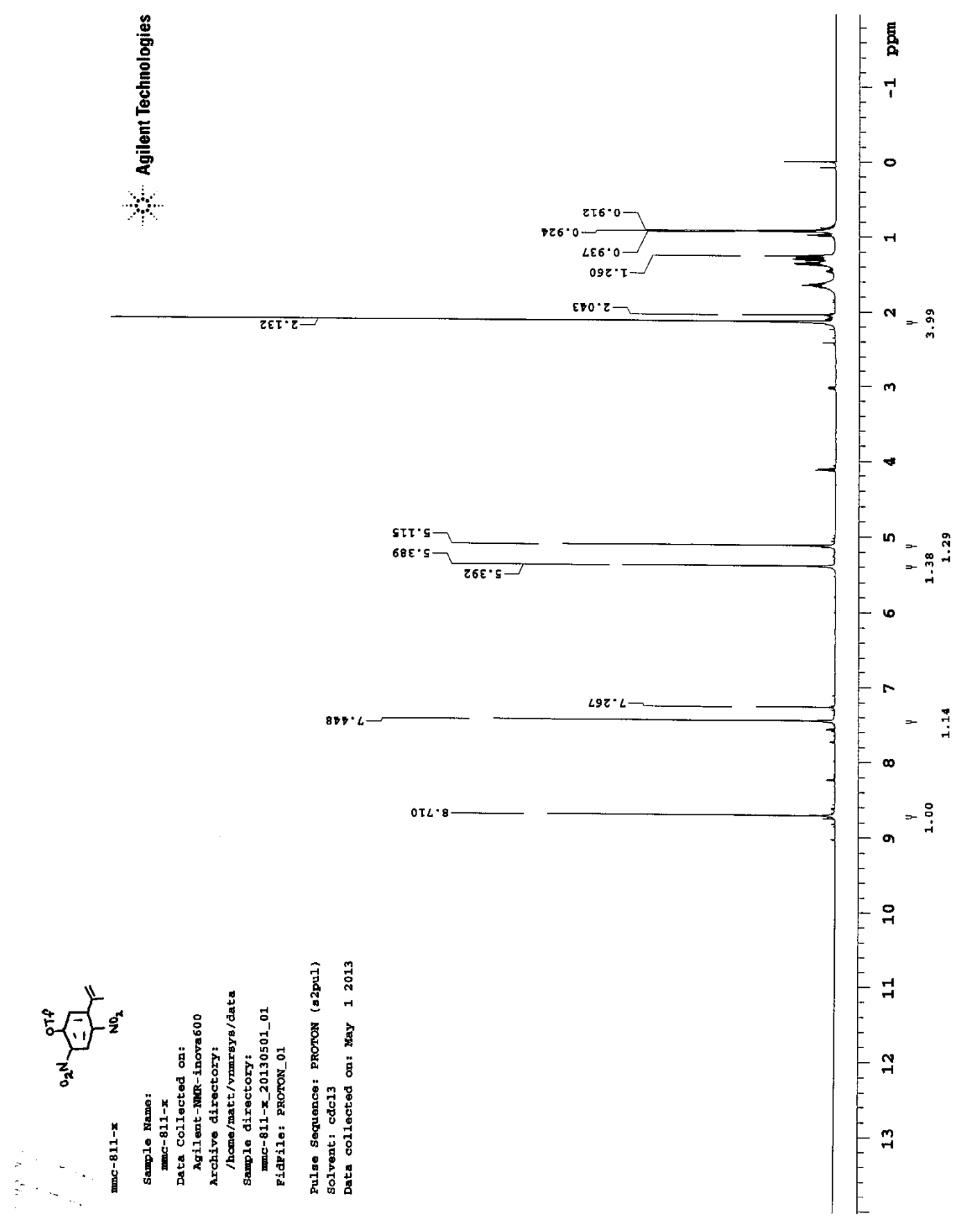

Figure 100: ${ }^{1} \mathrm{H}$ NMR of 2,4-dinitro-5-(prop-1-en-2-yl)phenyl trifluoromethanesulfonate (261) 


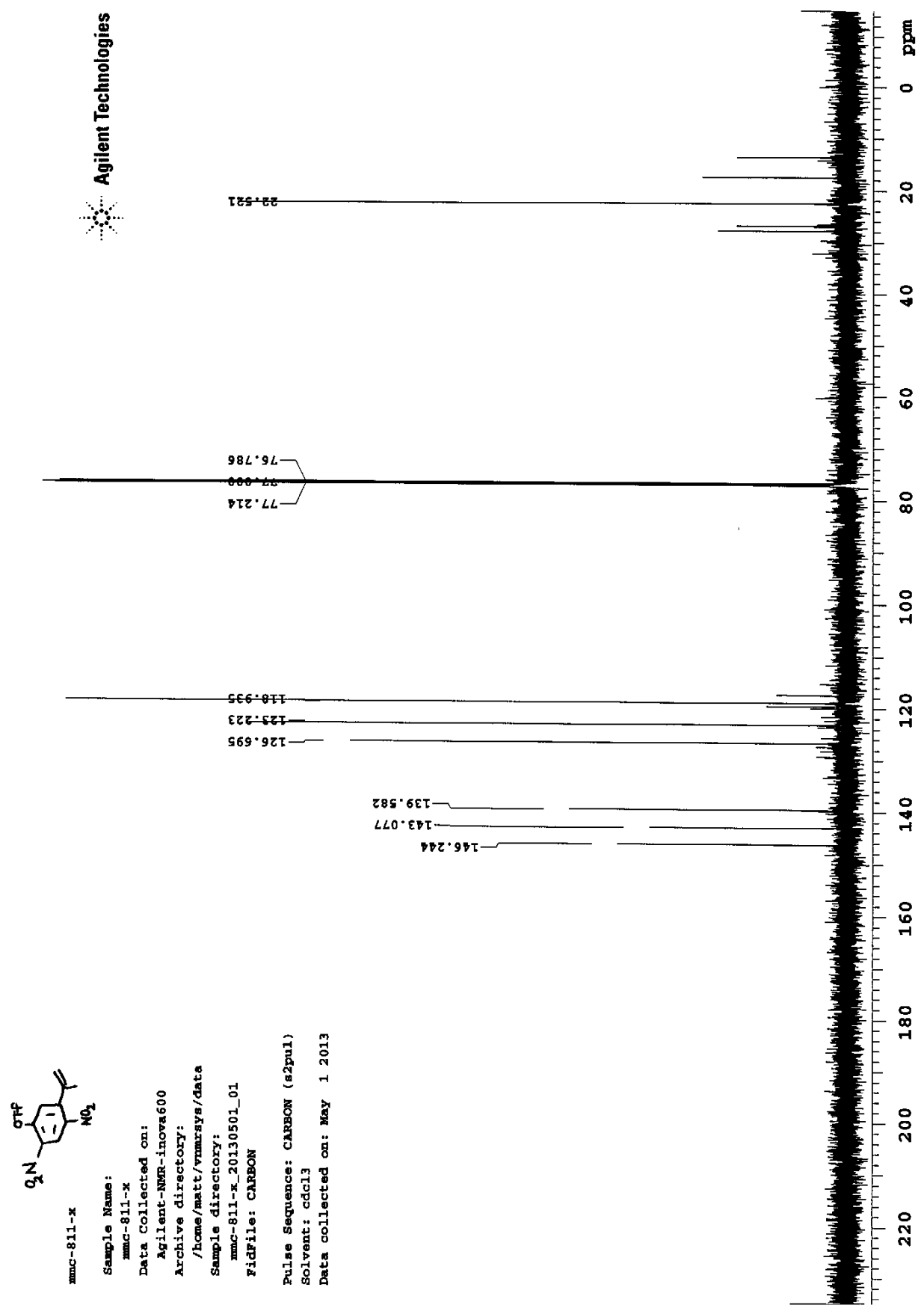

Figure 101: ${ }^{13} \mathrm{C}$ NMR of 2,4-dinitro-5-(prop-1-en-2-yl)phenyl trifluoromethanesulfonate (261) 


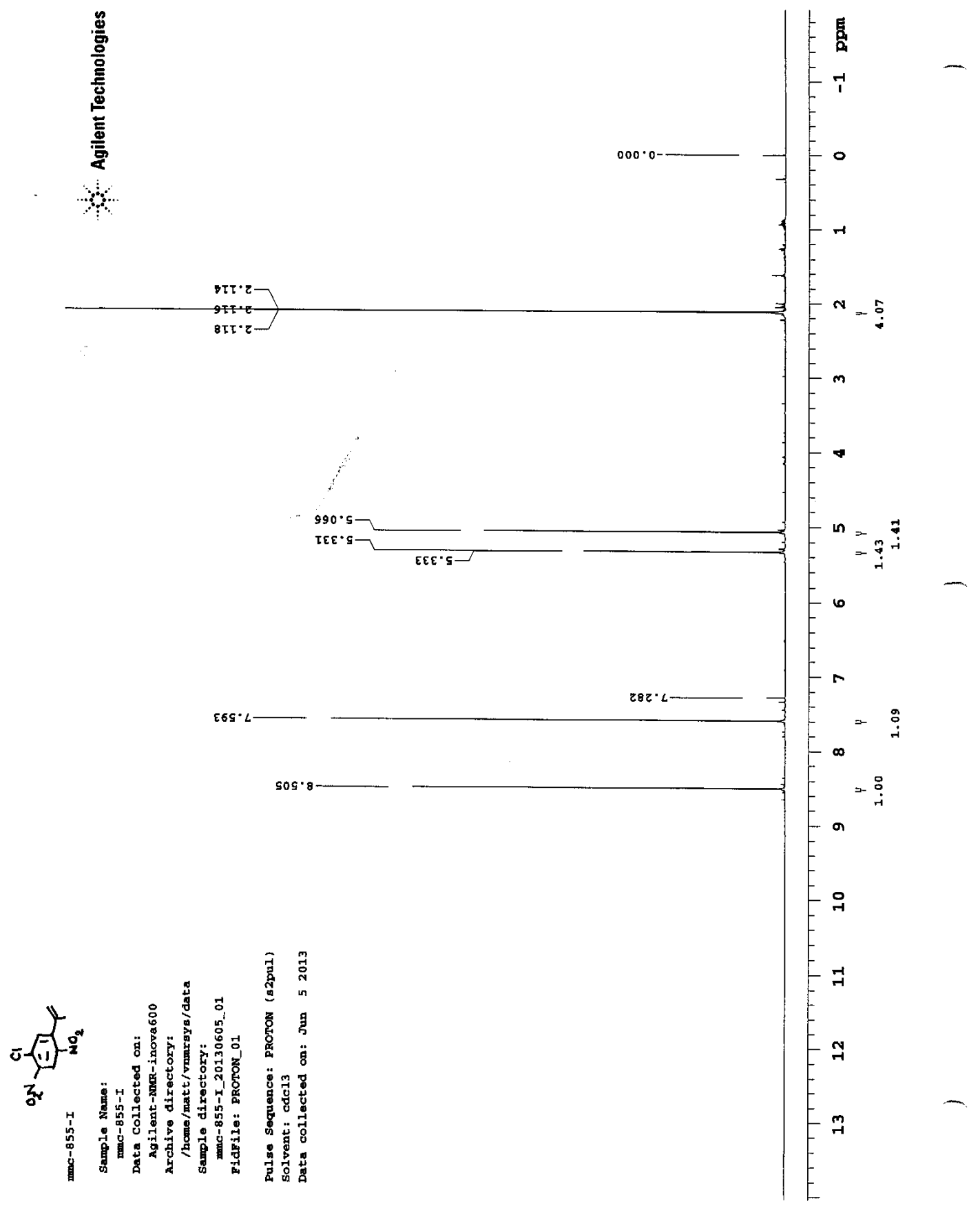

Figure 102: ${ }^{1} \mathrm{H}$ NMR of 1-chloro-2,4-dinitro-5-(prop-1-en-2-yl)benzene (273) 


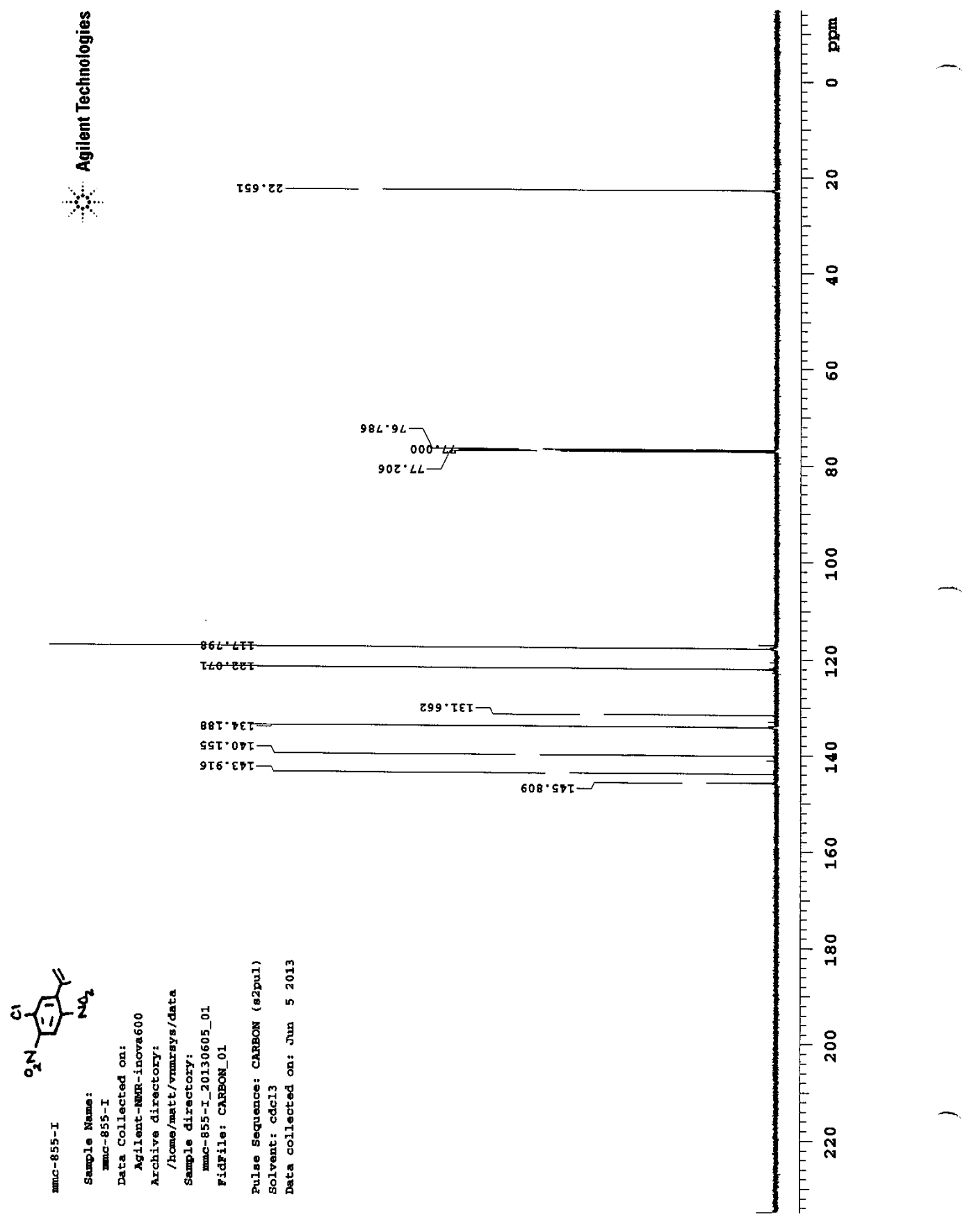

Figure 103: ${ }^{13} \mathrm{C}$ NMR of 1-chloro-2,4-dinitro-5-(prop-1-en-2-yl)benzene (273) 


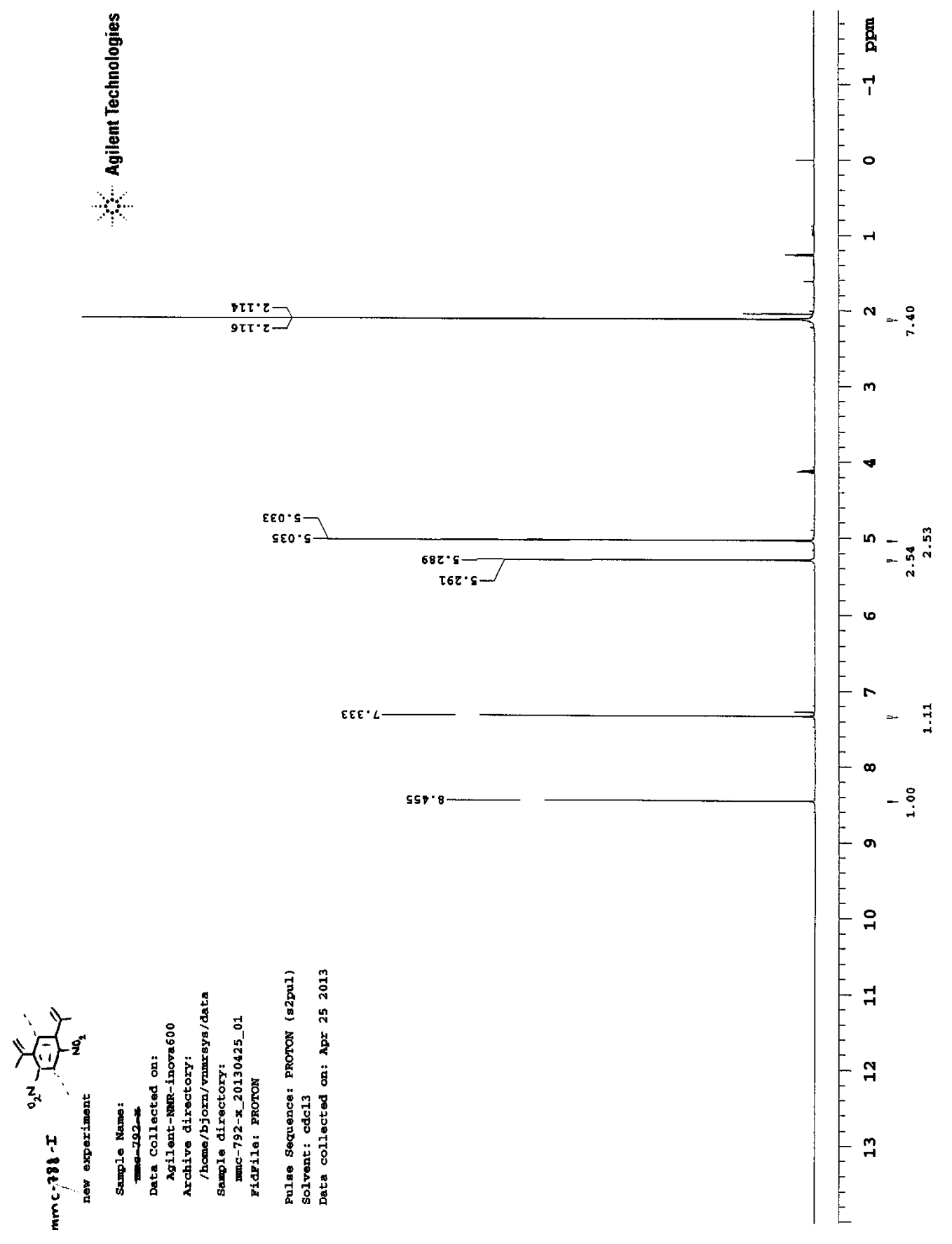

Figure 104: ${ }^{1} \mathrm{H}$ NMR of 1,5-dinitro-2,4-di(prop-1-en-2-yl)benzene (260) 


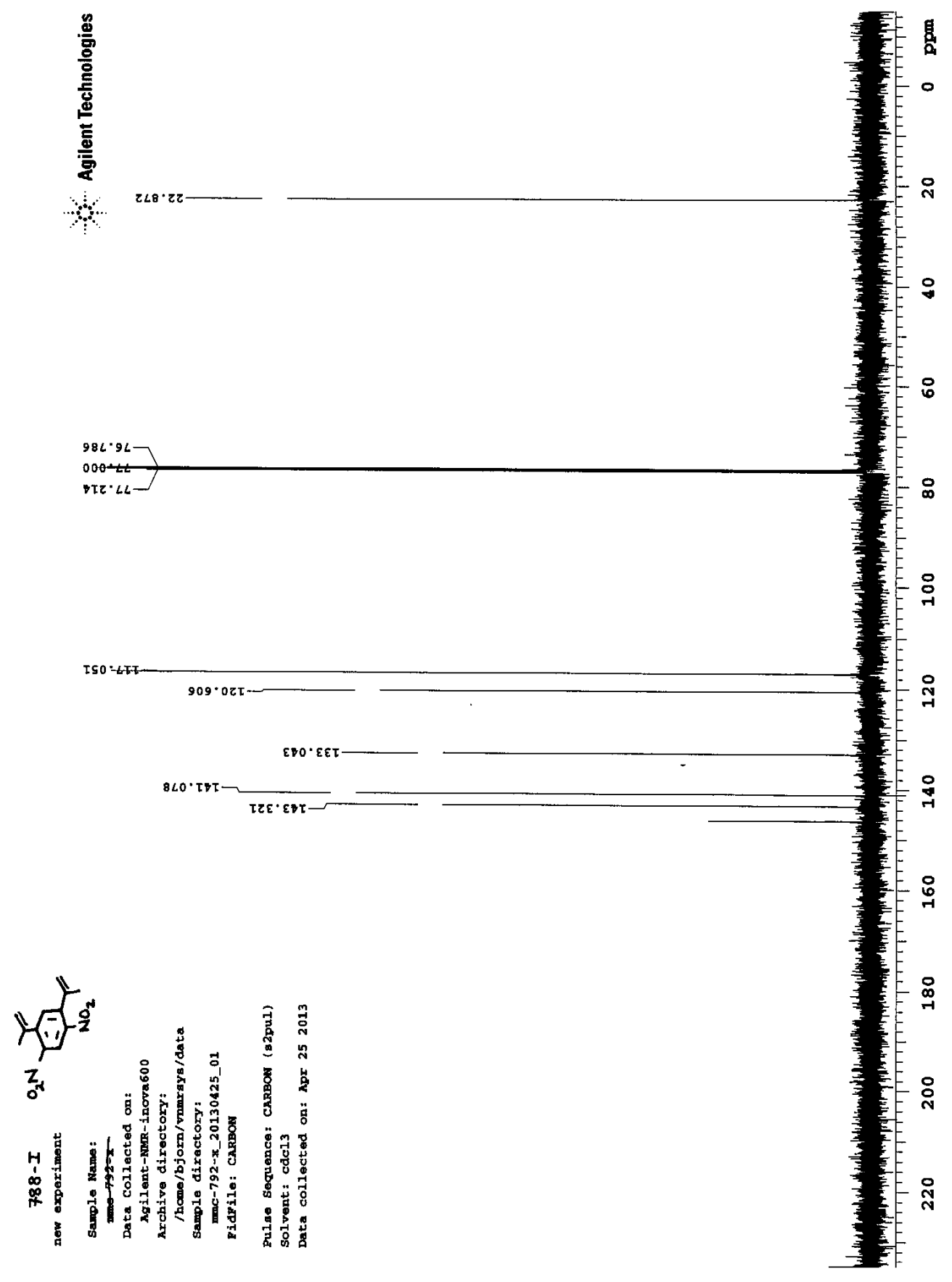

Figure 105: ${ }^{13} \mathrm{C}$ NMR of 1,5-dinitro-2,4-di(prop-1-en-2-yl)benzene (260) 


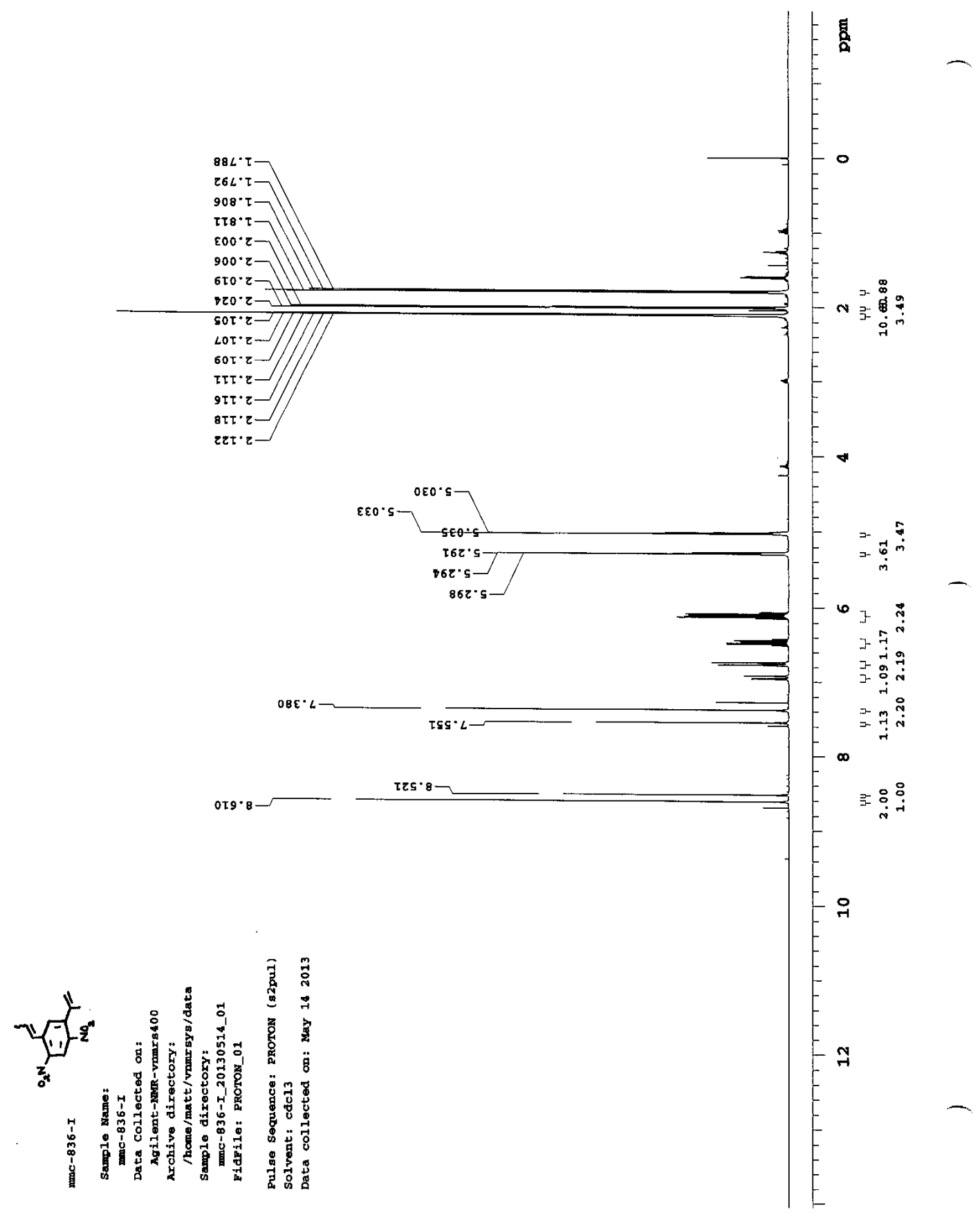

Figure 106: ${ }^{1} \mathrm{H}$ NMR of 1,5-dinitro-2-(prop-1-en-2-yl)-4-(prop-1-enyl)benzene (274) 


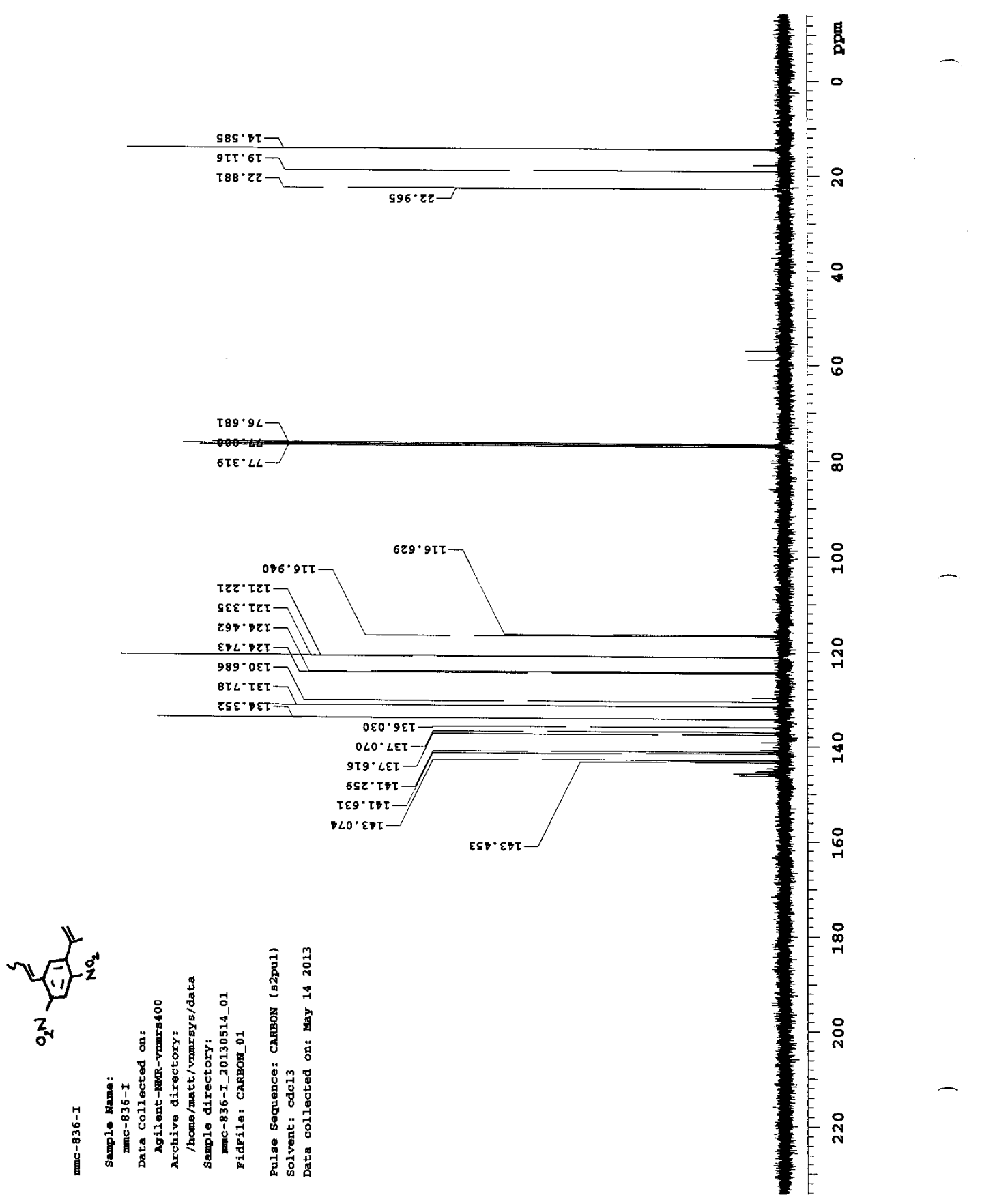

Figure 107: ${ }^{13} \mathrm{C}$ NMR of 1,5-dinitro-2-(prop-1-en-2-yl)-4-(prop-1-enyl)benzene (274) 


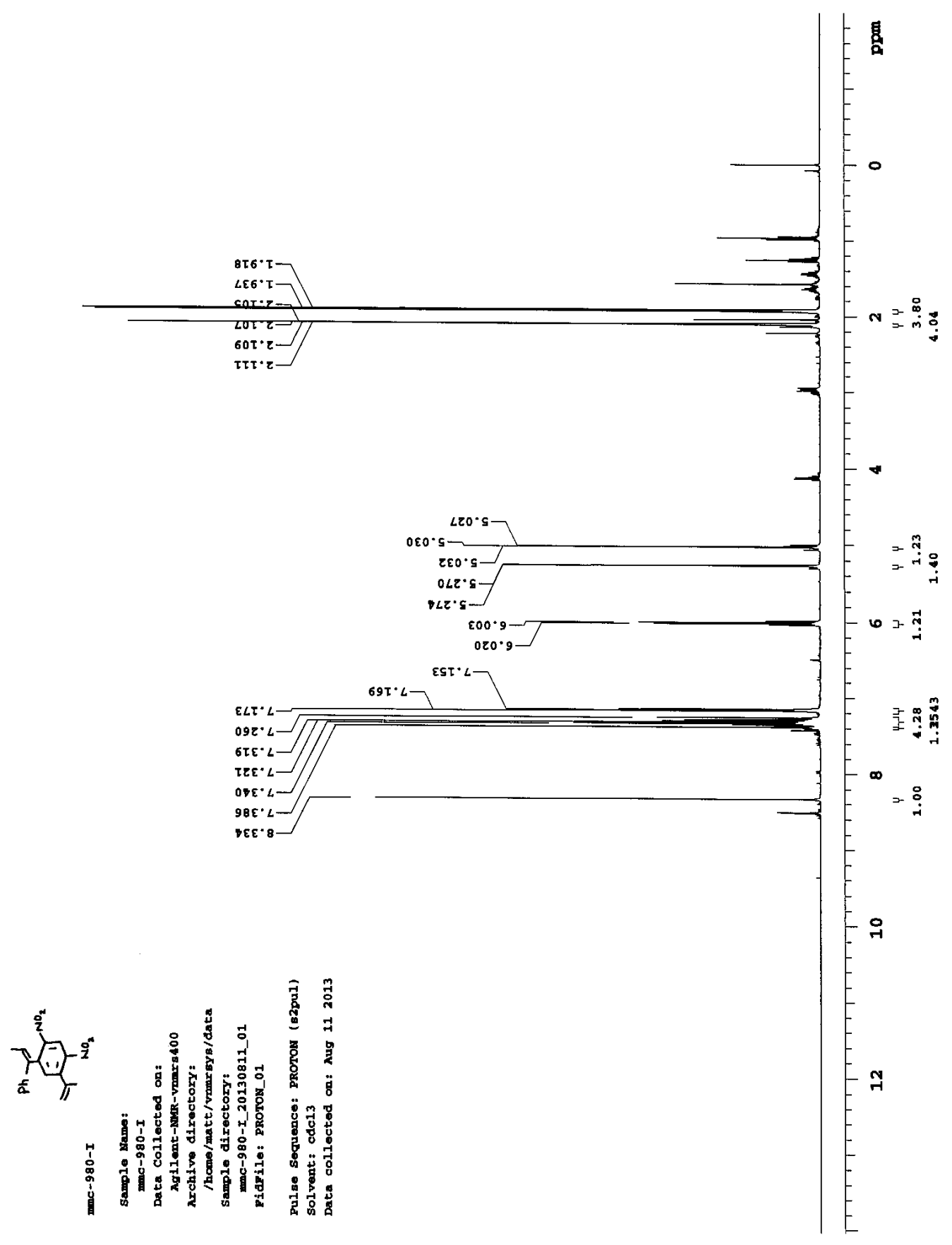

Figure 108: ${ }^{1} \mathrm{H}$ NMR of $(E)-1,5$-dinitro-2-(1-phenylprop-1-enyl)-4-(prop-1-en-2yl)benzene (276) 


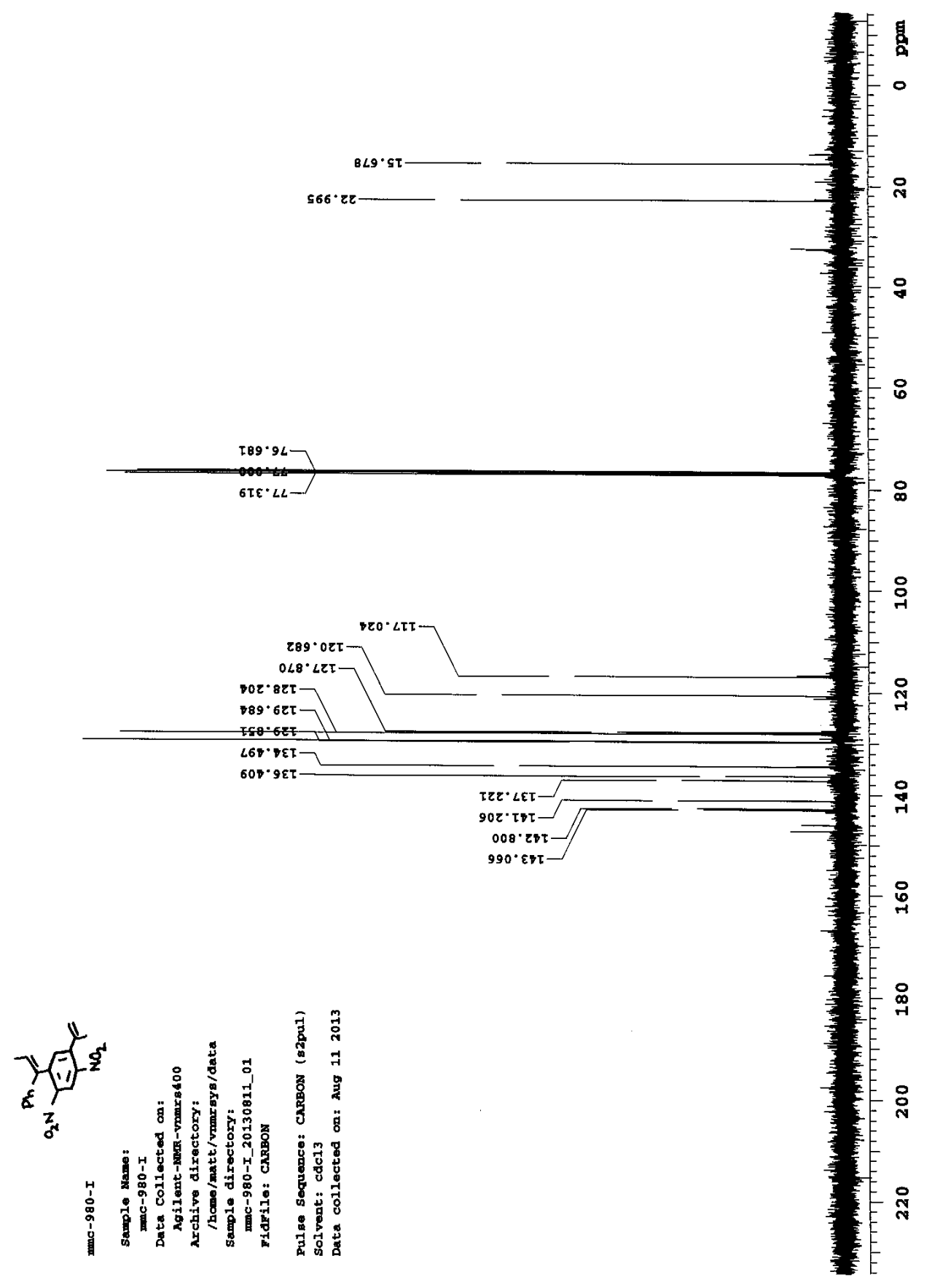

Figure 109: ${ }^{13} \mathrm{C}$ NMR of $(E)-1,5$-dinitro-2-(1-phenylprop-1-enyl)-4-(prop-1-en-2yl)benzene (276) 


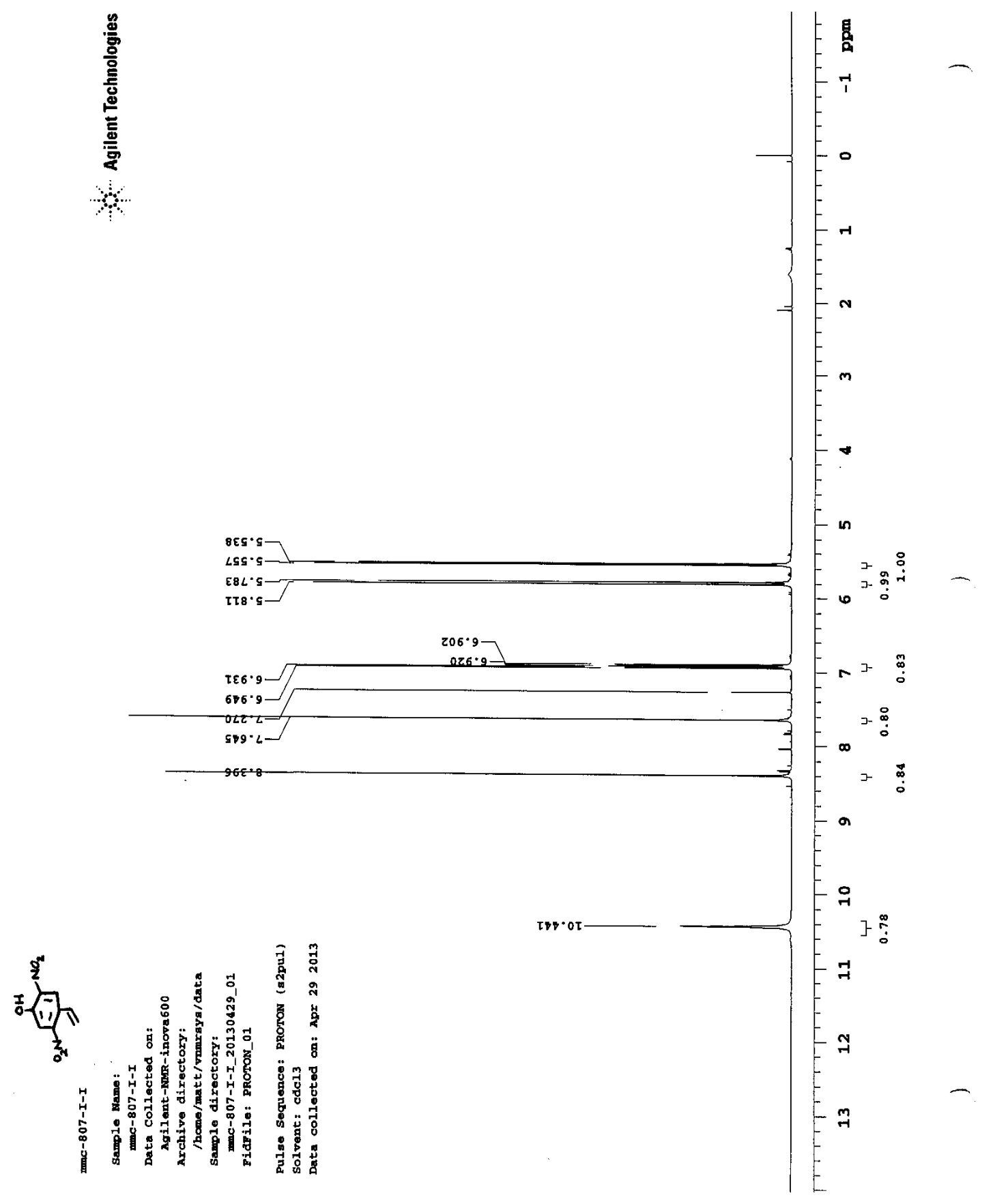

Figure 110: ${ }^{1} \mathrm{H}$ NMR of 2,5-dinitro-4-ethenylphenol (278) 


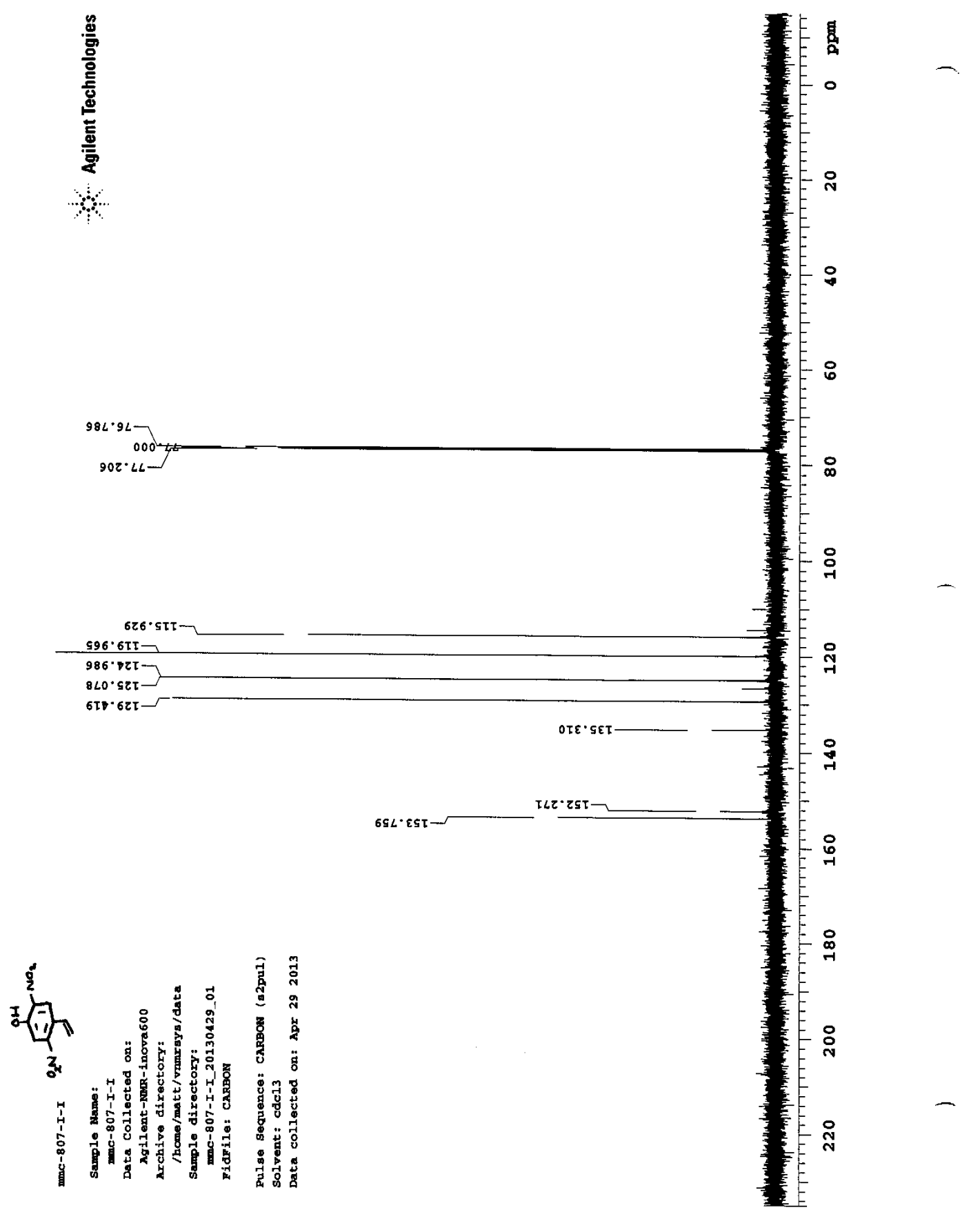

Figure 111: ${ }^{13} \mathrm{C}$ NMR of 2,5-dinitro-4-ethenylphenol (278) 


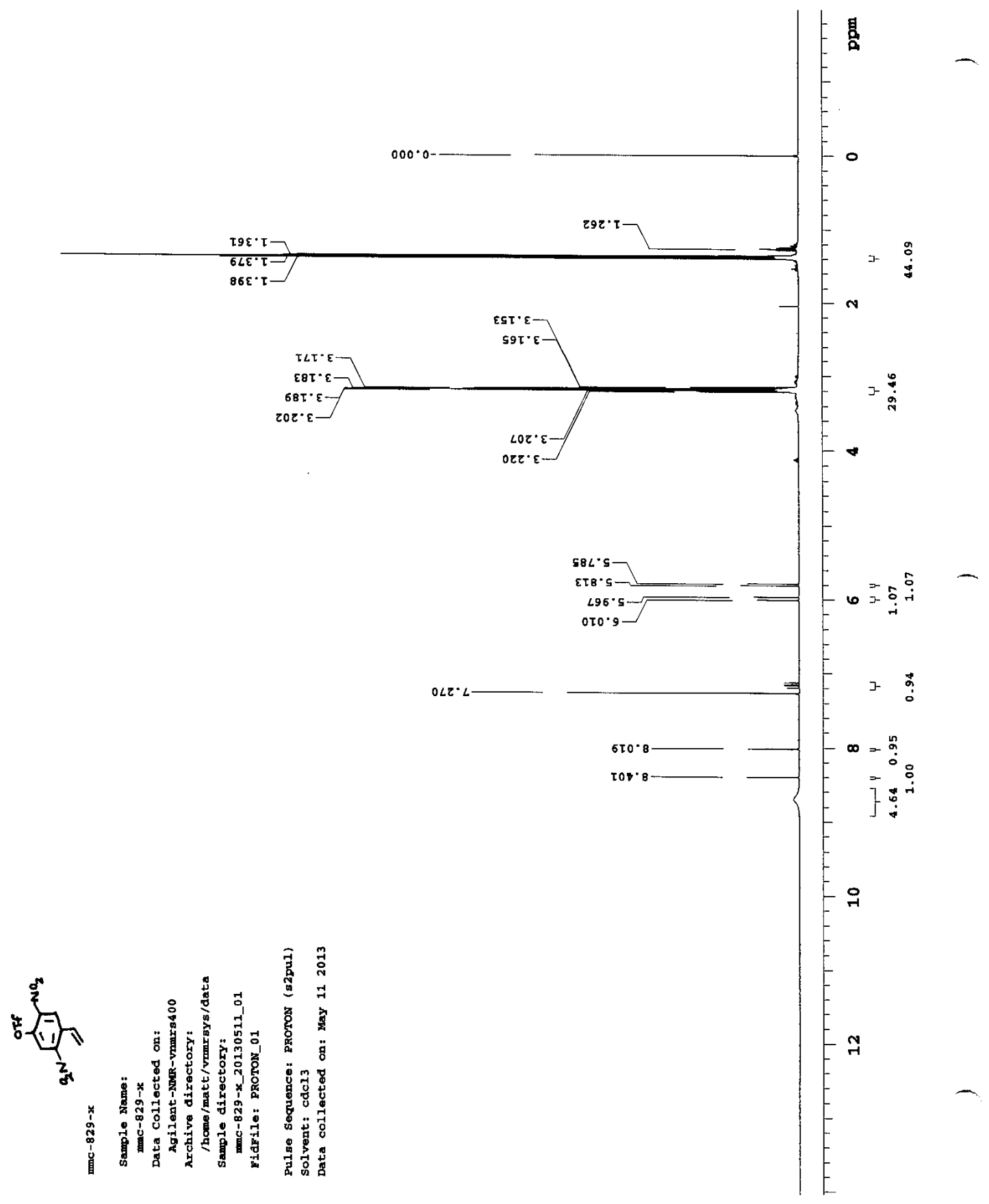

Figure 112: ${ }^{1} \mathrm{H}$ NMR of 2,5-dinitro-4-ethenylphenyl trifluoromethanesulfonate (277) 


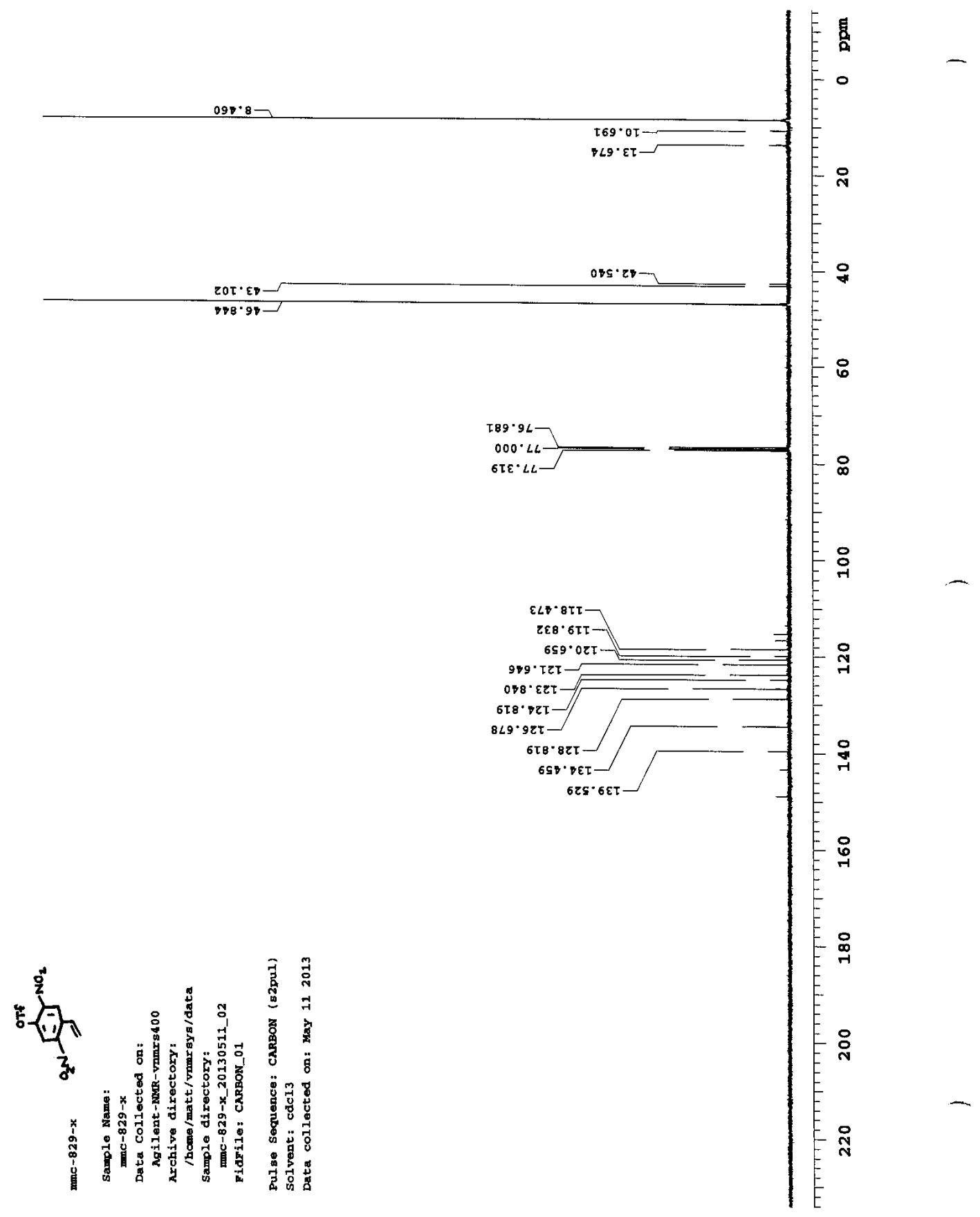

Figure 113: ${ }^{13} \mathrm{C}$ NMR of 2,5-dinitro-4-ethenylphenyl trifluoromethanesulfonate (277) 


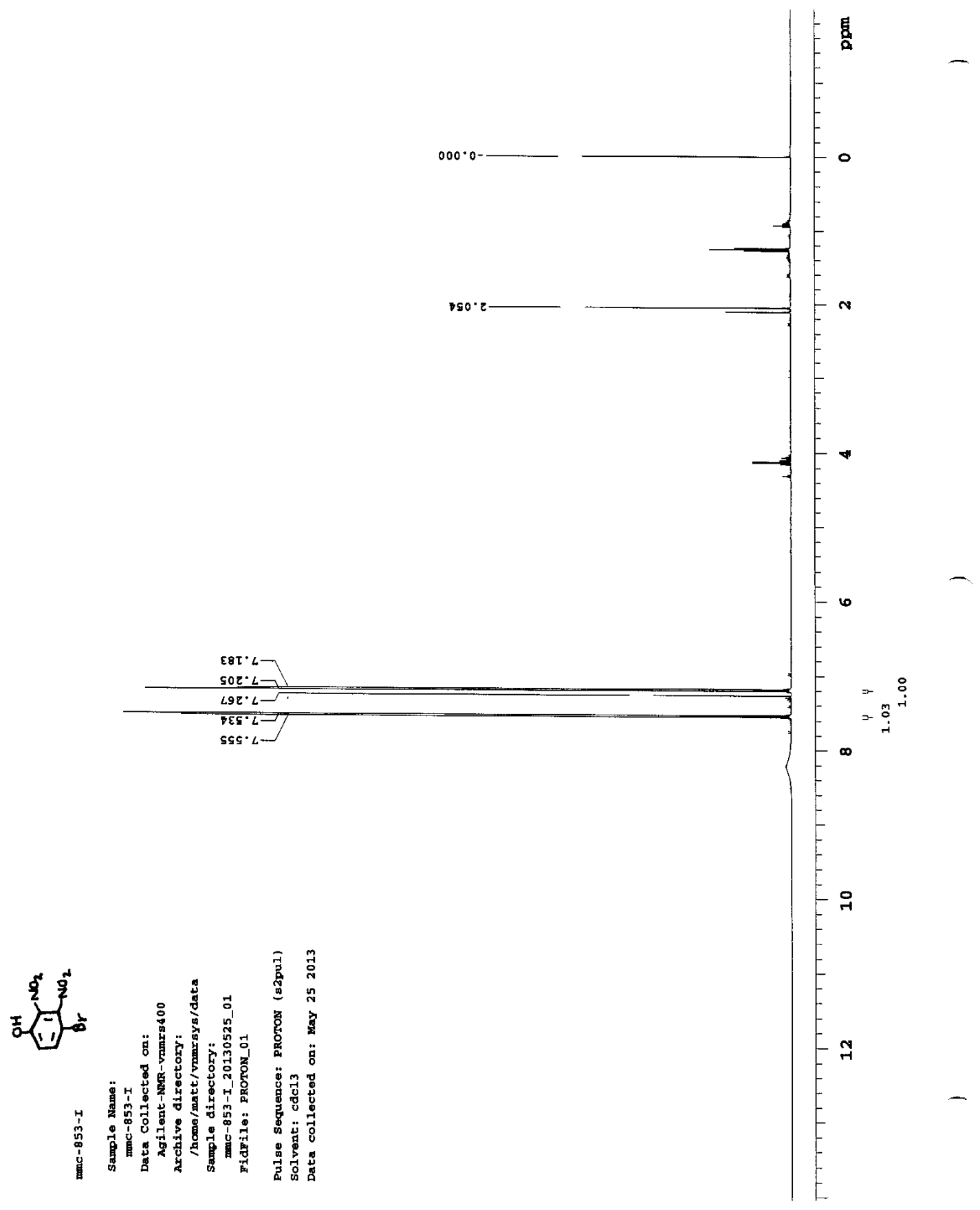

Figure 114: ${ }^{1} \mathrm{H}$ NMR of 4-bromo-2,3-dinitrophenol (292) 


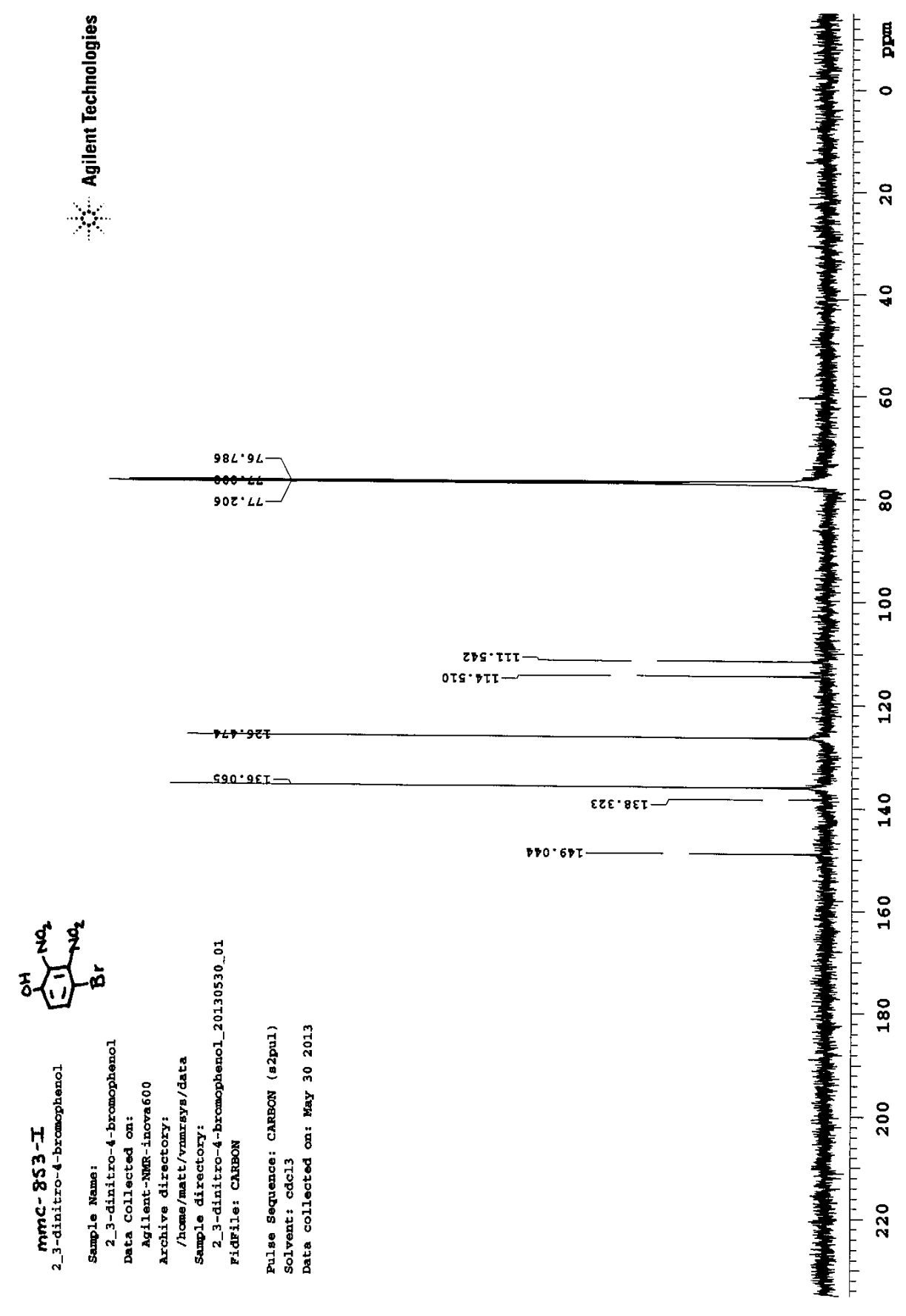

Figure 115: ${ }^{13} \mathrm{C}$ NMR of 4-bromo-2,3-dinitrophenol (292) 


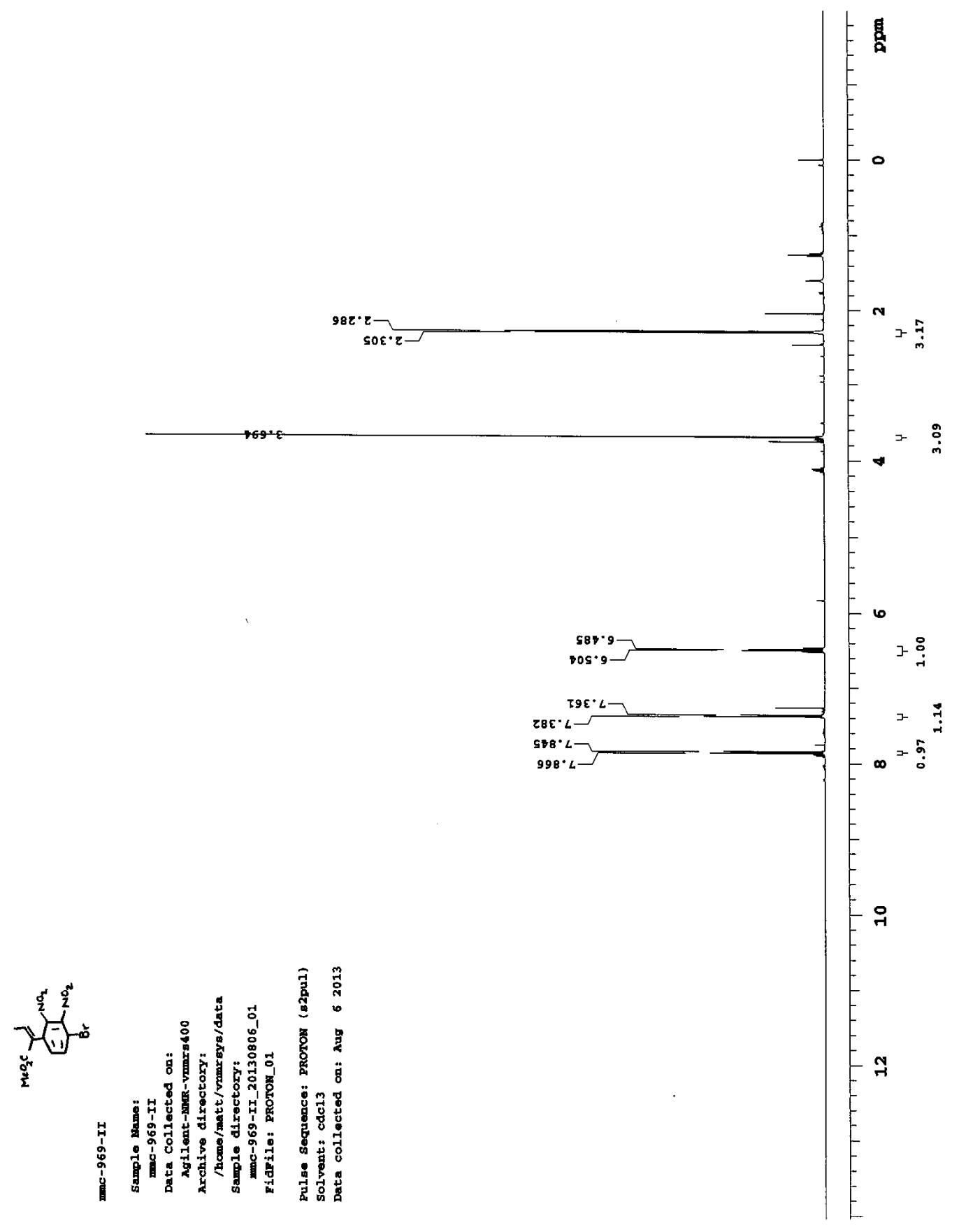

Figure 116: ${ }^{1} \mathrm{H}$ NMR of (Z)-methyl 2-(4-bromo-2,3-dinitrophenyl)but-2-enoate (301) 


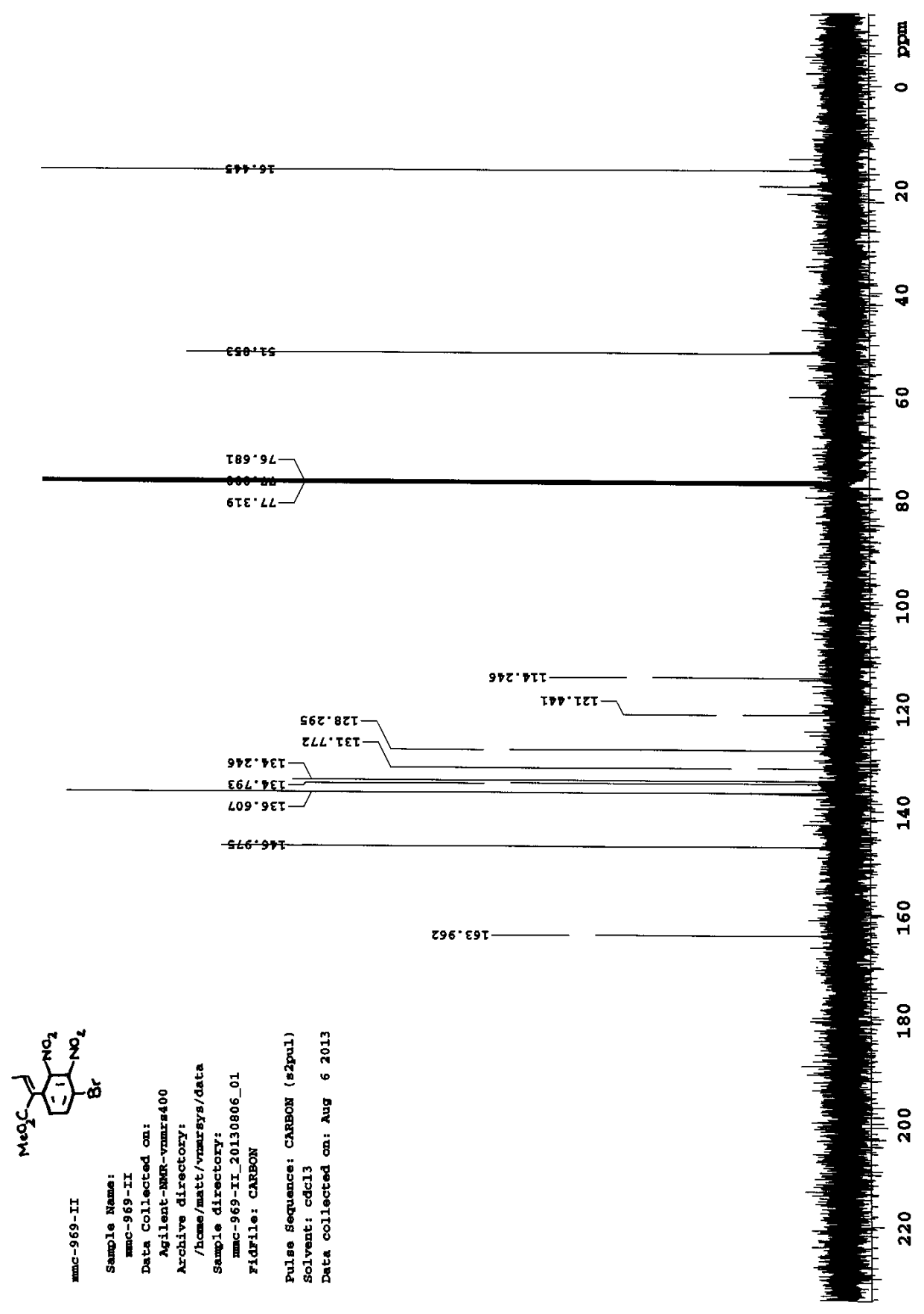

Figure 117: ${ }^{13} \mathrm{C}$ NMR of (Z)-methyl 2-(4-bromo-2,3-dinitrophenyl)but-2-enoate (301) 


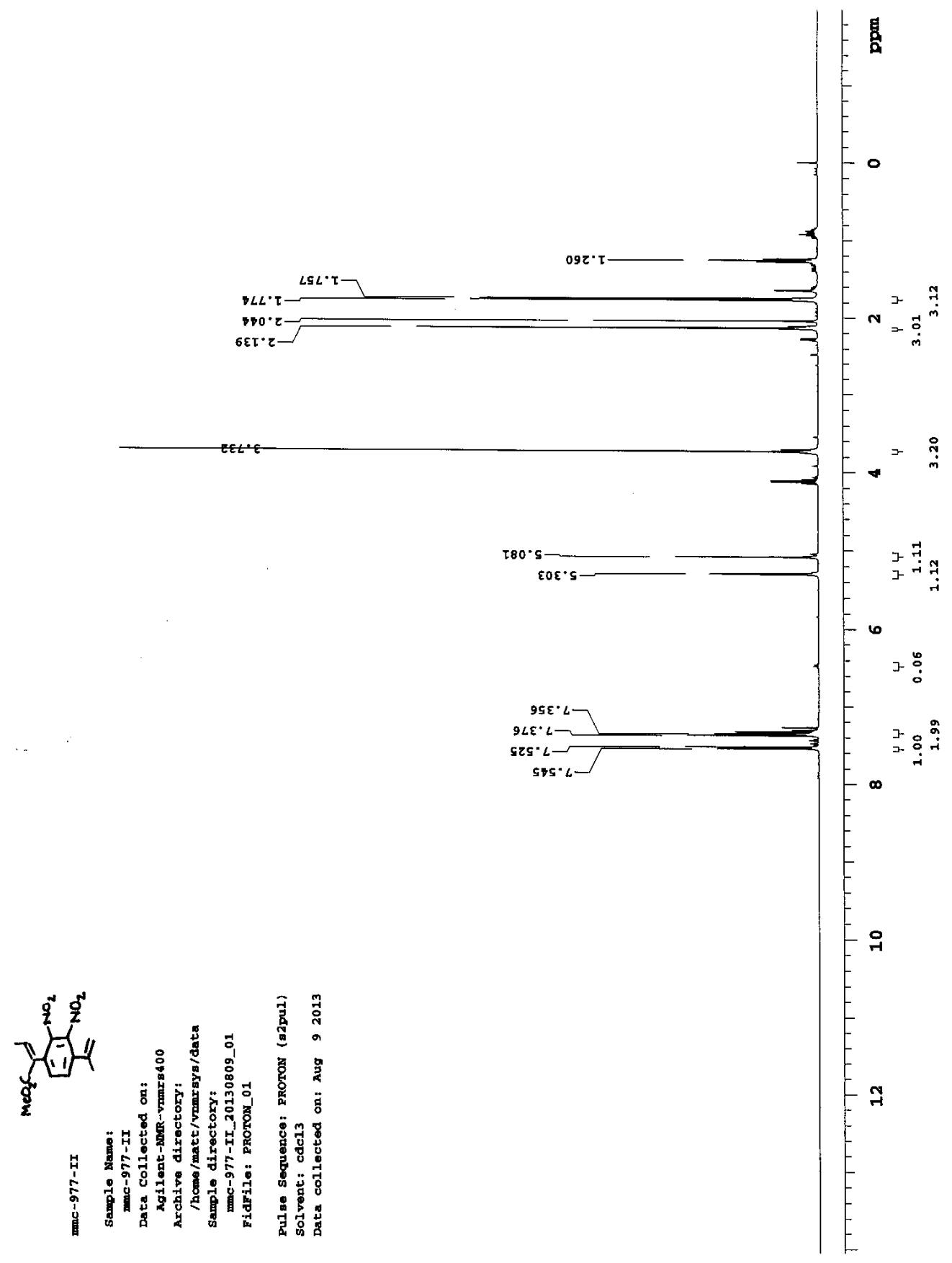

Figure 118: ${ }^{1} \mathrm{H}$ NMR of (Z)-methyl 2-(2,3-dinitro-4-(prop-1-en-2-yl)phenyl)but-2-enoate (302) 


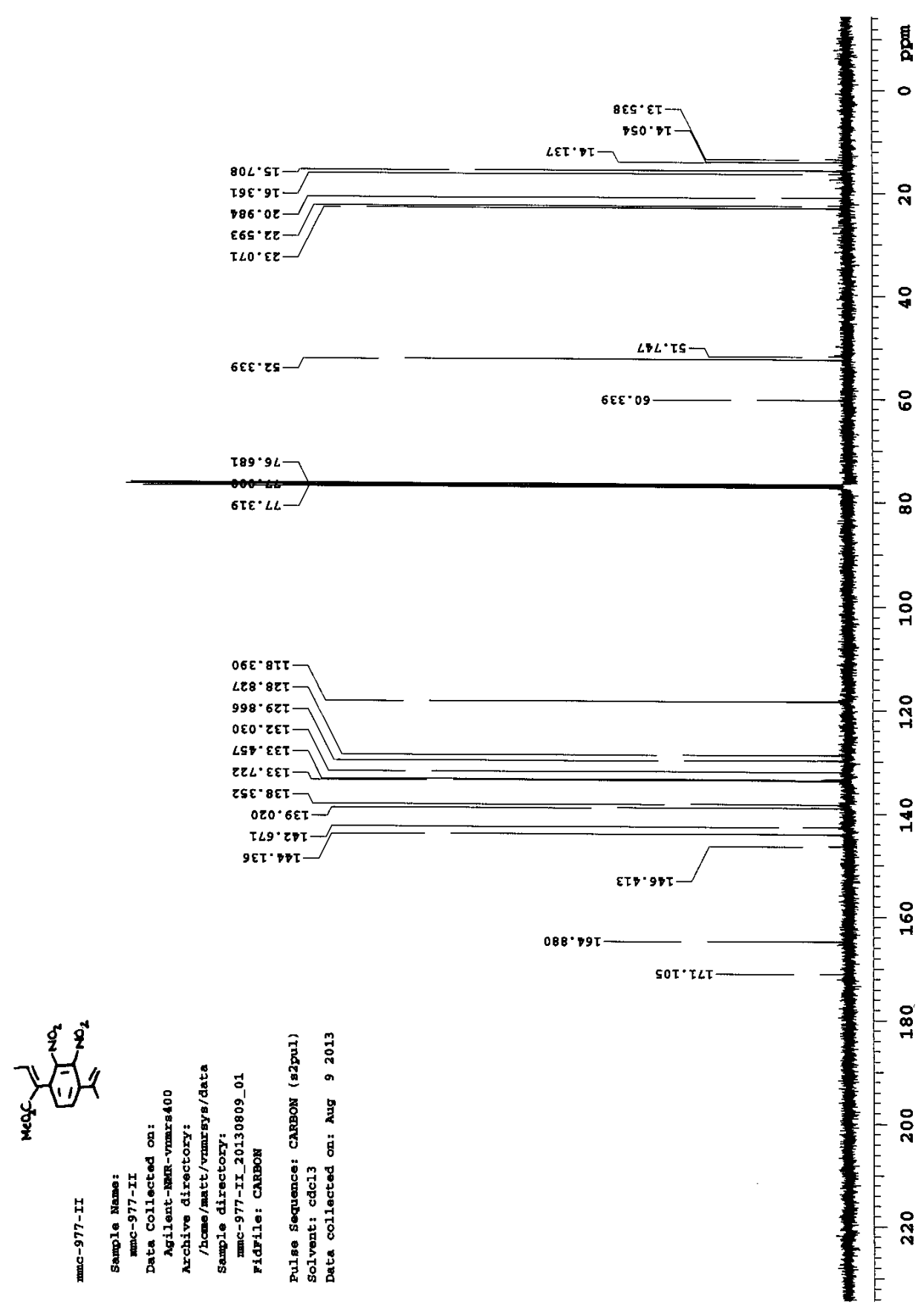

Figure 119: ${ }^{13} \mathrm{C}$ NMR of $(Z)$-methyl 2-(2,3-dinitro-4-(prop-1-en-2-yl)phenyl)but-2enoate (302) 


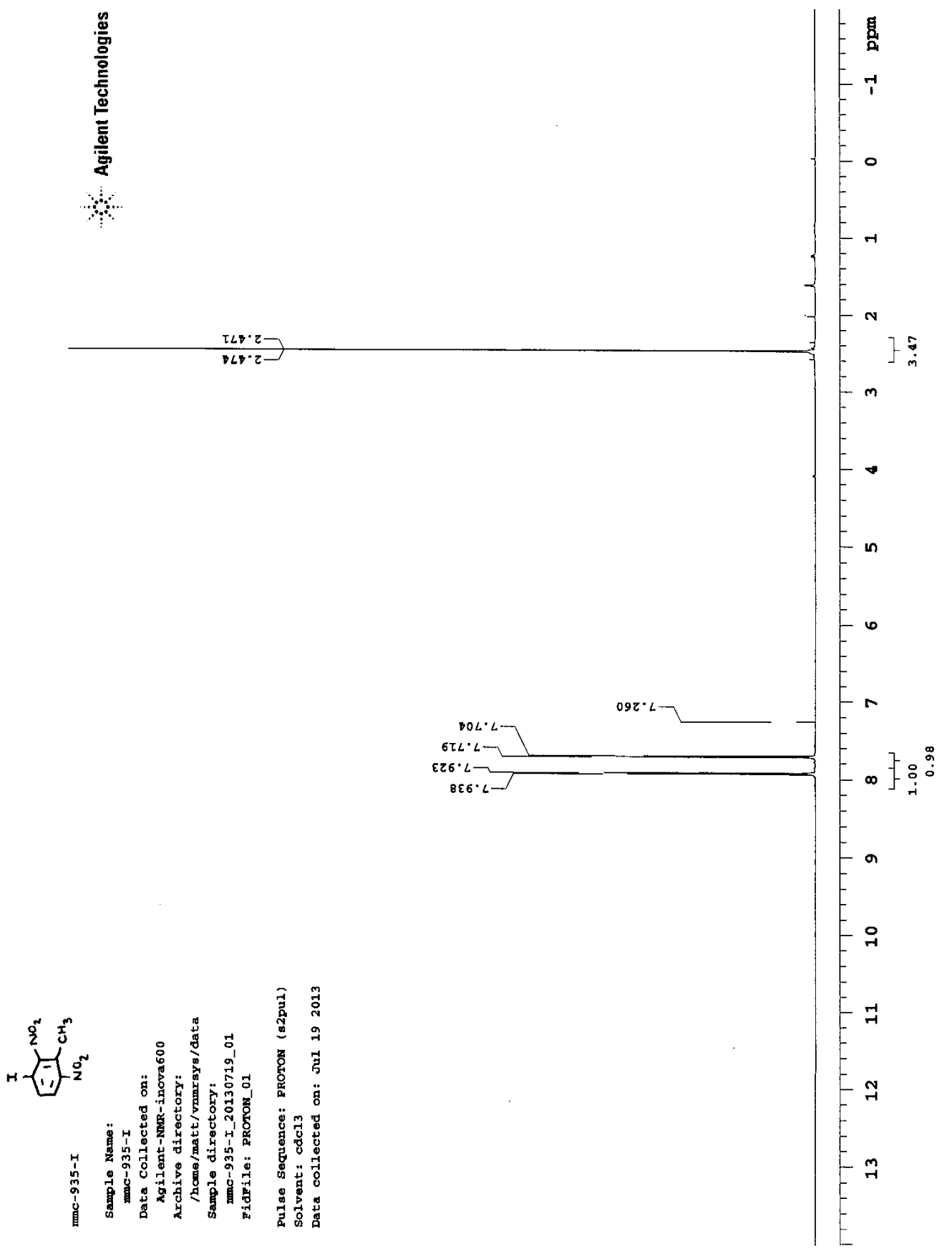

Figure 120: ${ }^{1} \mathrm{H}$ NMR of 1-iodo-3-methyl-2,4-dinitrobenzene (308) 


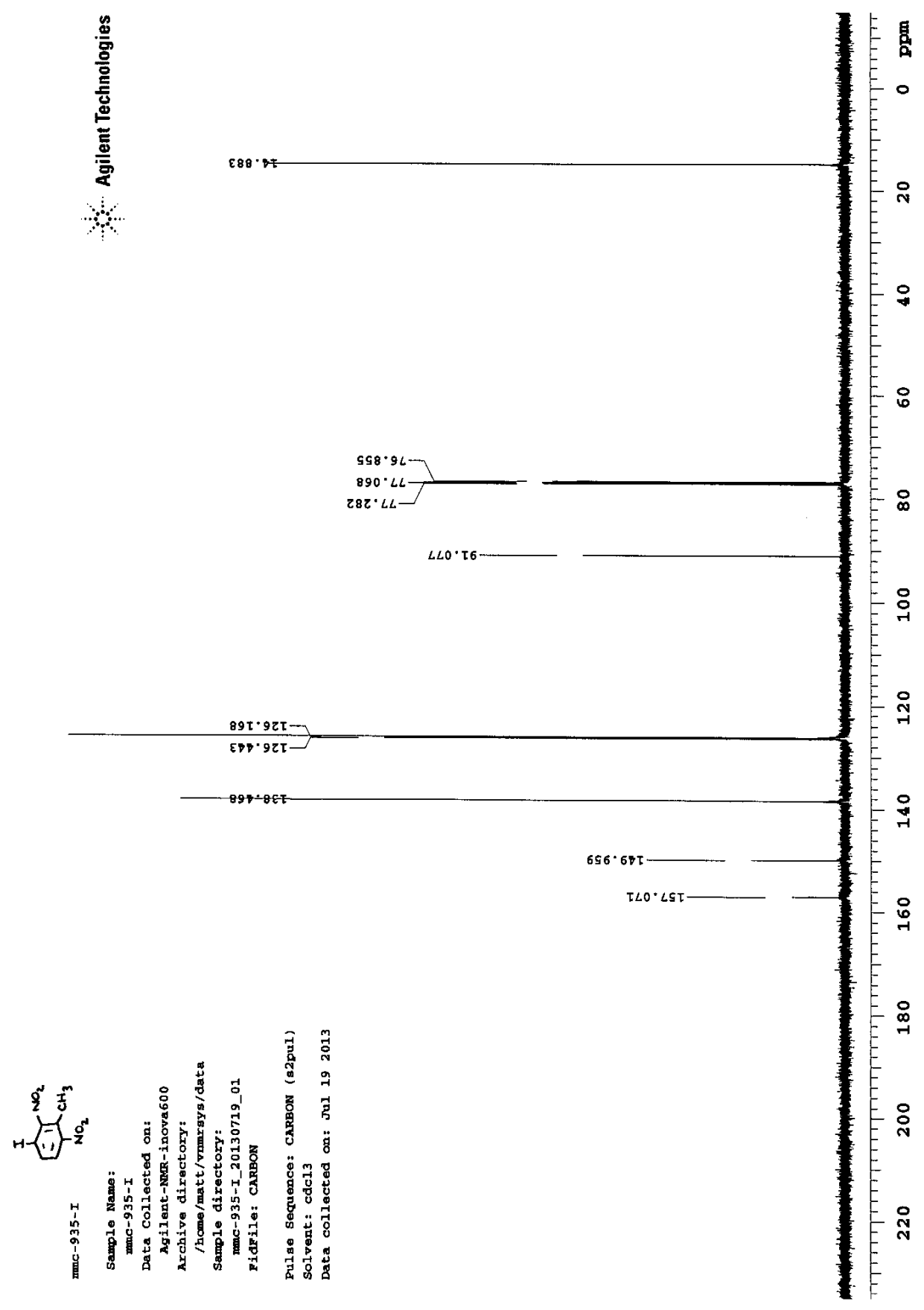

Figure 121: ${ }^{13} \mathrm{C}$ NMR of 1-iodo-3-methyl-2,4-dinitrobenzene (308) 


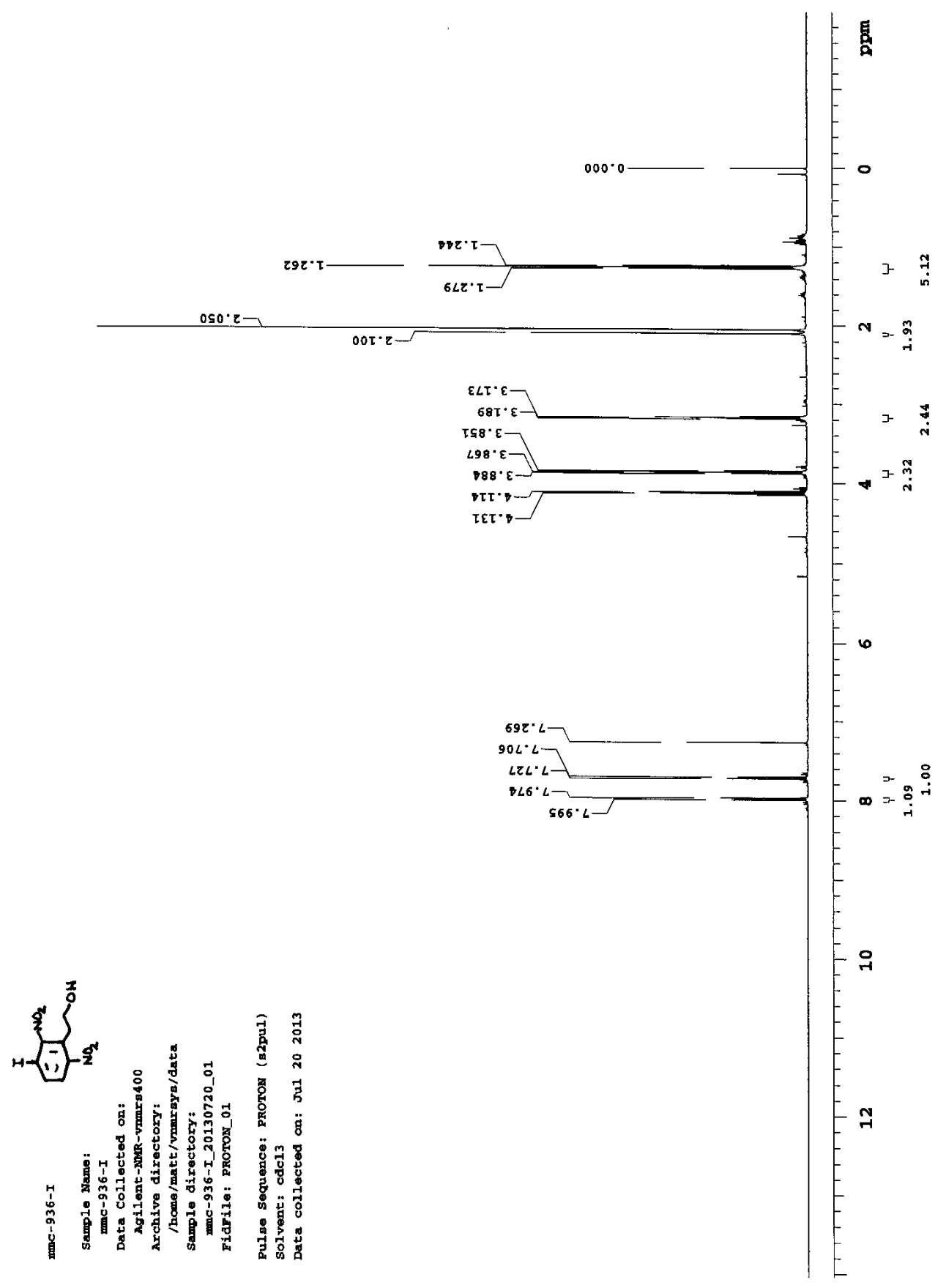

Figure 122: ${ }^{1} \mathrm{H}$ NMR of 2-(3-iodo-2,6-dinitrophenyl)ethanol (312) 


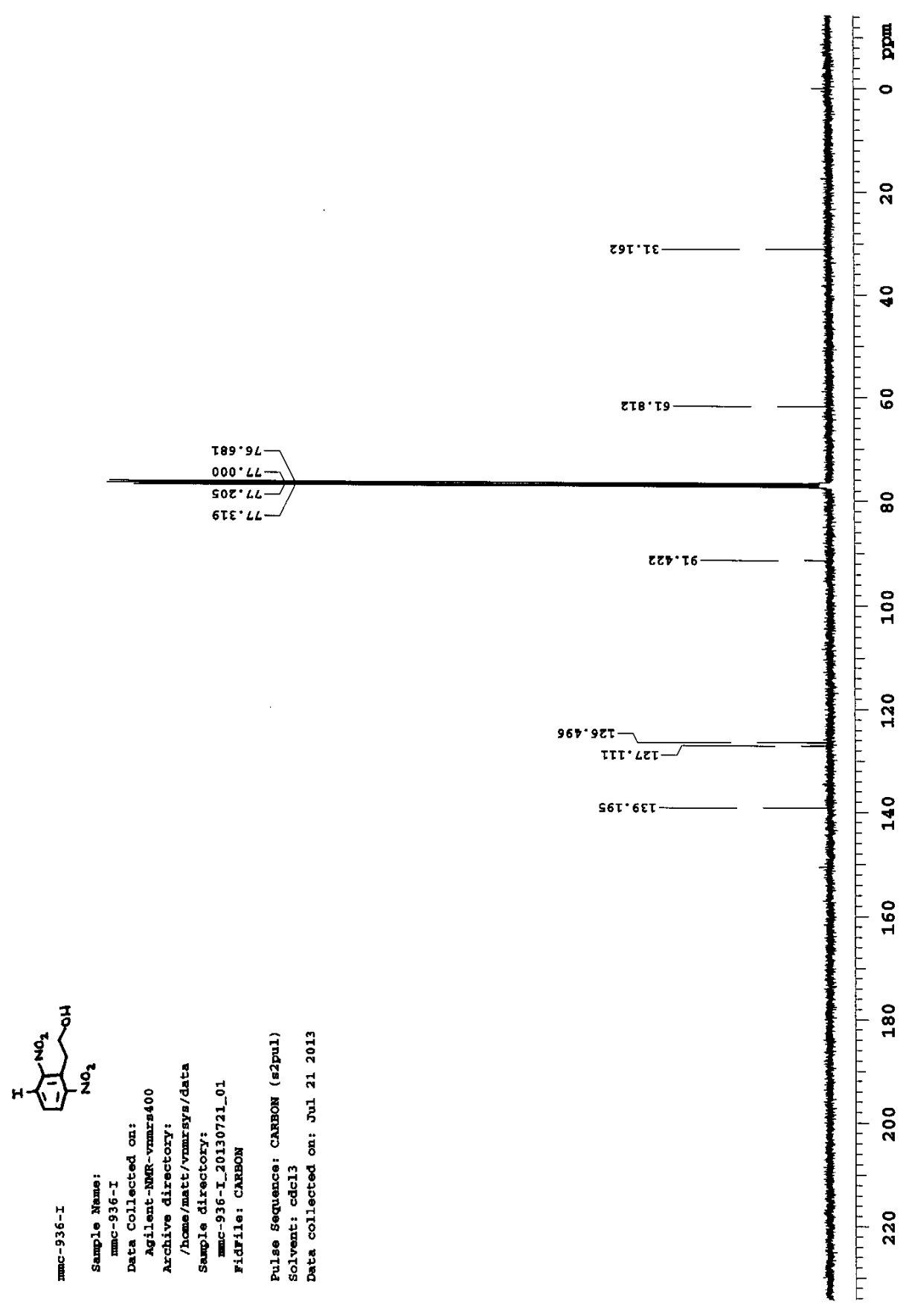

Figure 123: ${ }^{13} \mathrm{C}$ NMR of 2-(3-iodo-2,6-dinitrophenyl)ethanol (312) 


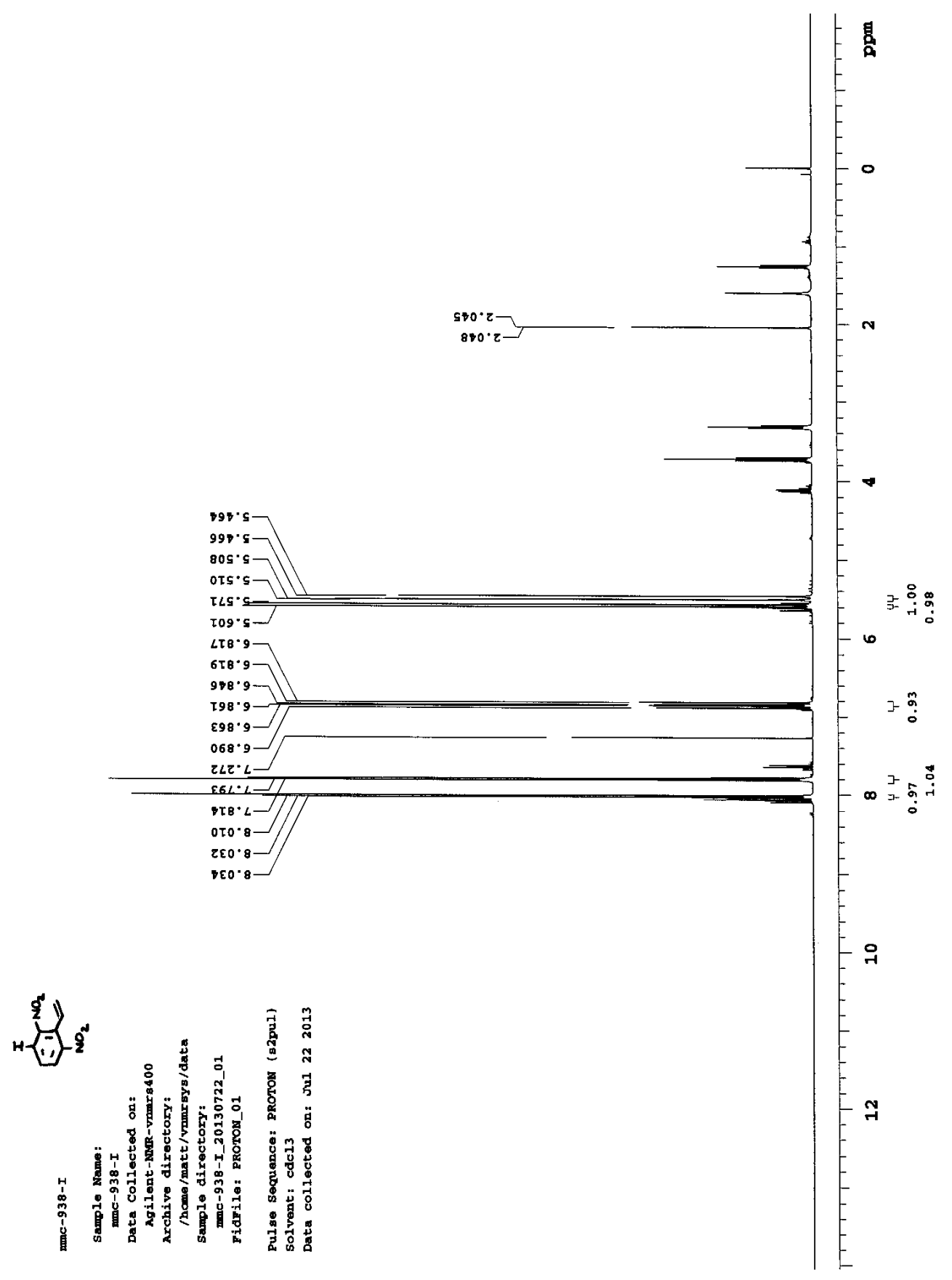

Figure 124: ${ }^{1} \mathrm{H}$ NMR of 1-iodo-2,4-dinitro-3-ethenylbenzene (313) 


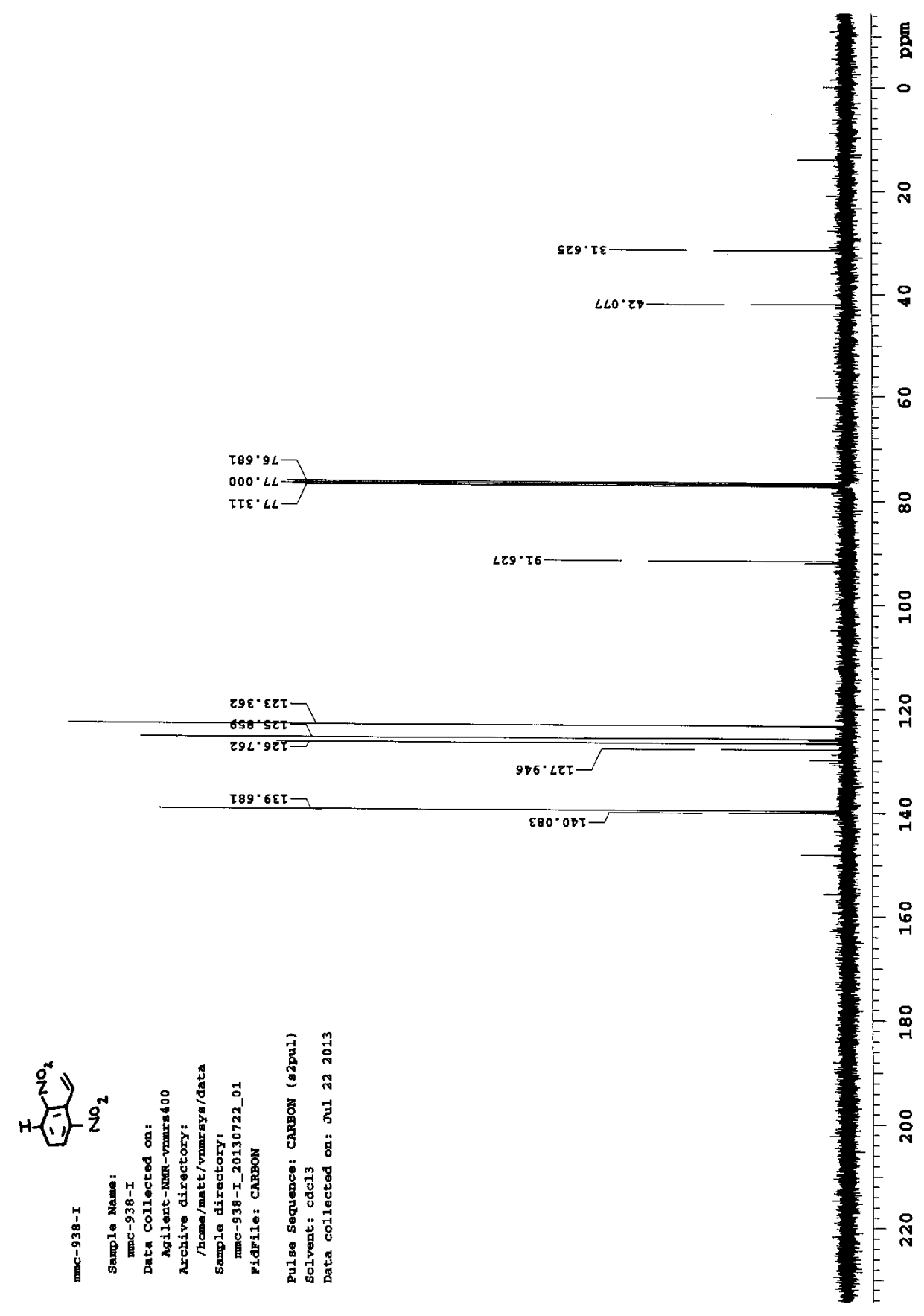

Figure 125: ${ }^{13} \mathrm{C}$ NMR of 1-iodo-2,4-dinitro-3-ethenylbenzene (313) 


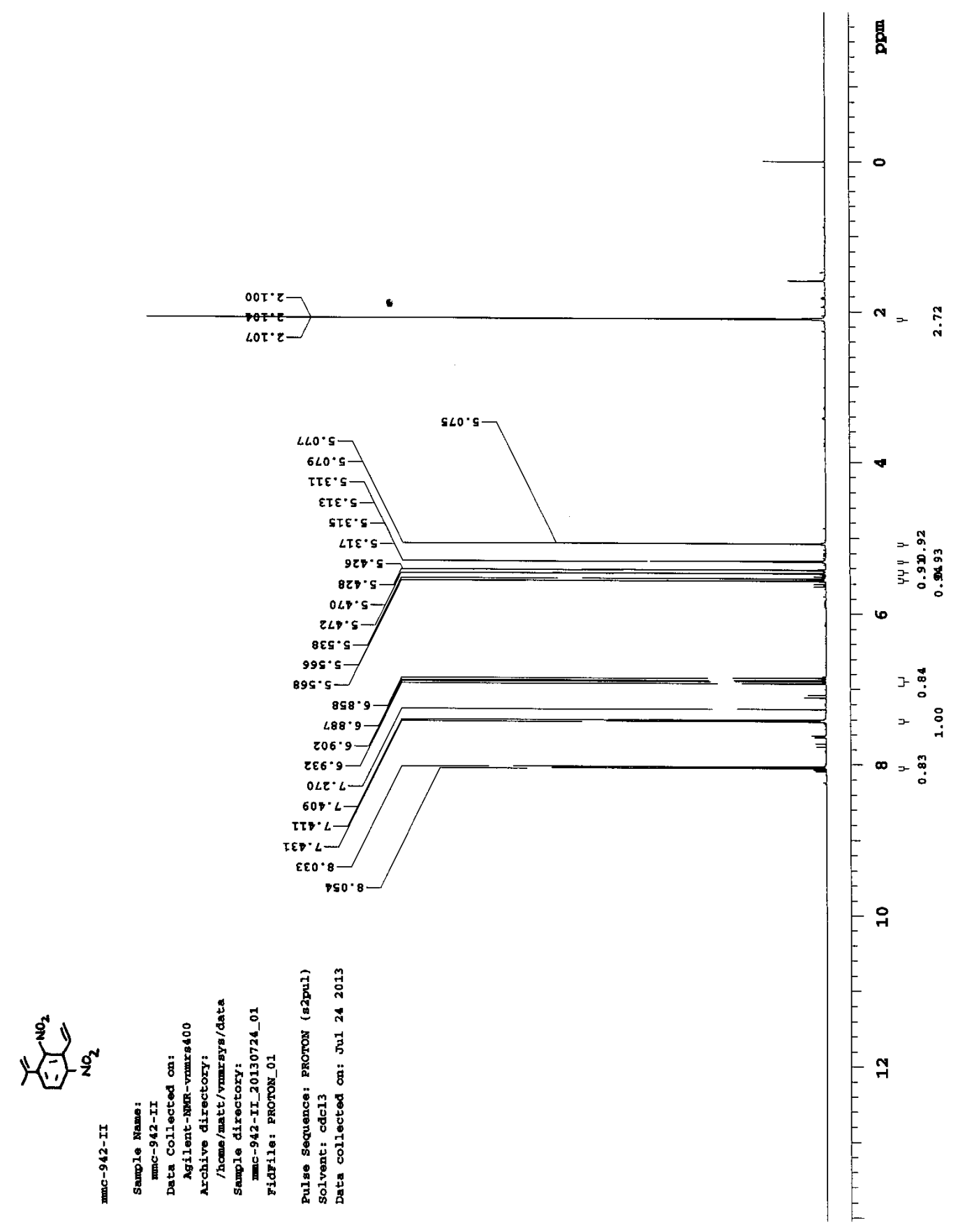

Figure 126: ${ }^{1} \mathrm{H}$ NMR of 1,3-dinitro-4-(prop-1-en-2-yl)-2-ethenylbenzene (314) 


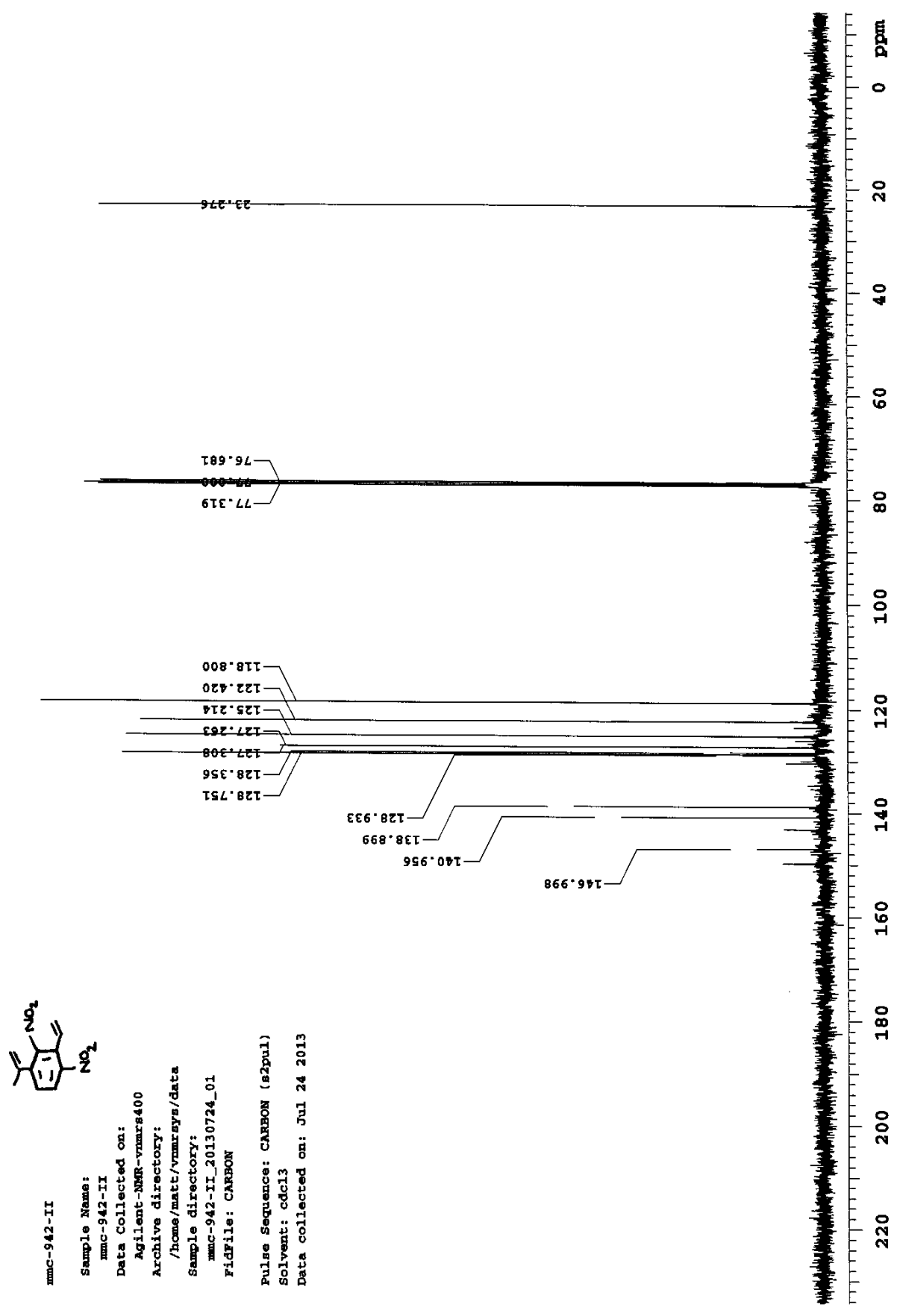

Figure 127: ${ }^{13} \mathrm{C}$ NMR of 1,3-dinitro-4-(prop-1-en-2-yl)-2-ethenylbenzene (314) 


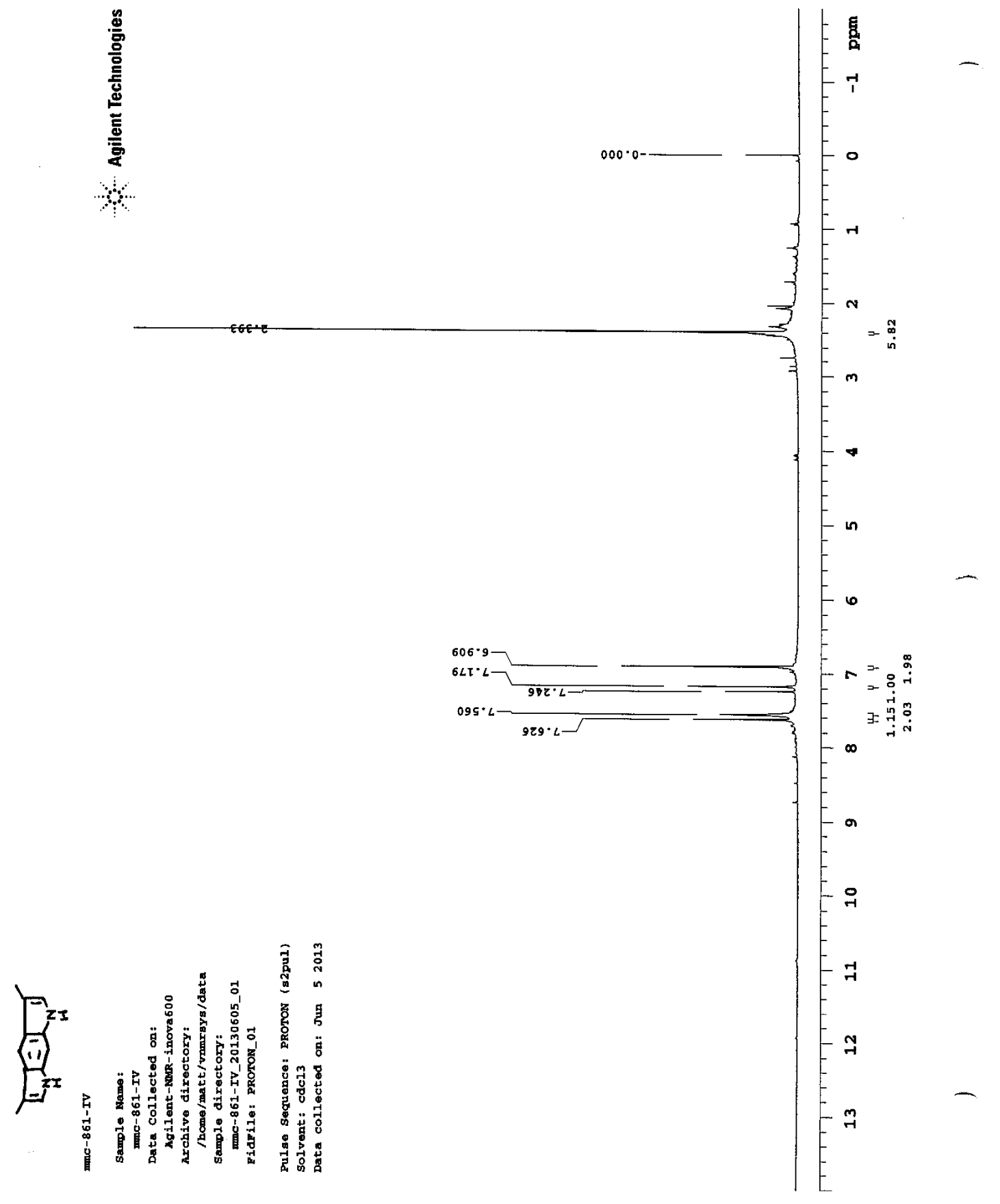

Figure 128: ${ }^{1} \mathrm{H}$ NMR of 3,5-dimethyl-1,7-dihydropyrrolo[3,2-f]indole (326) 


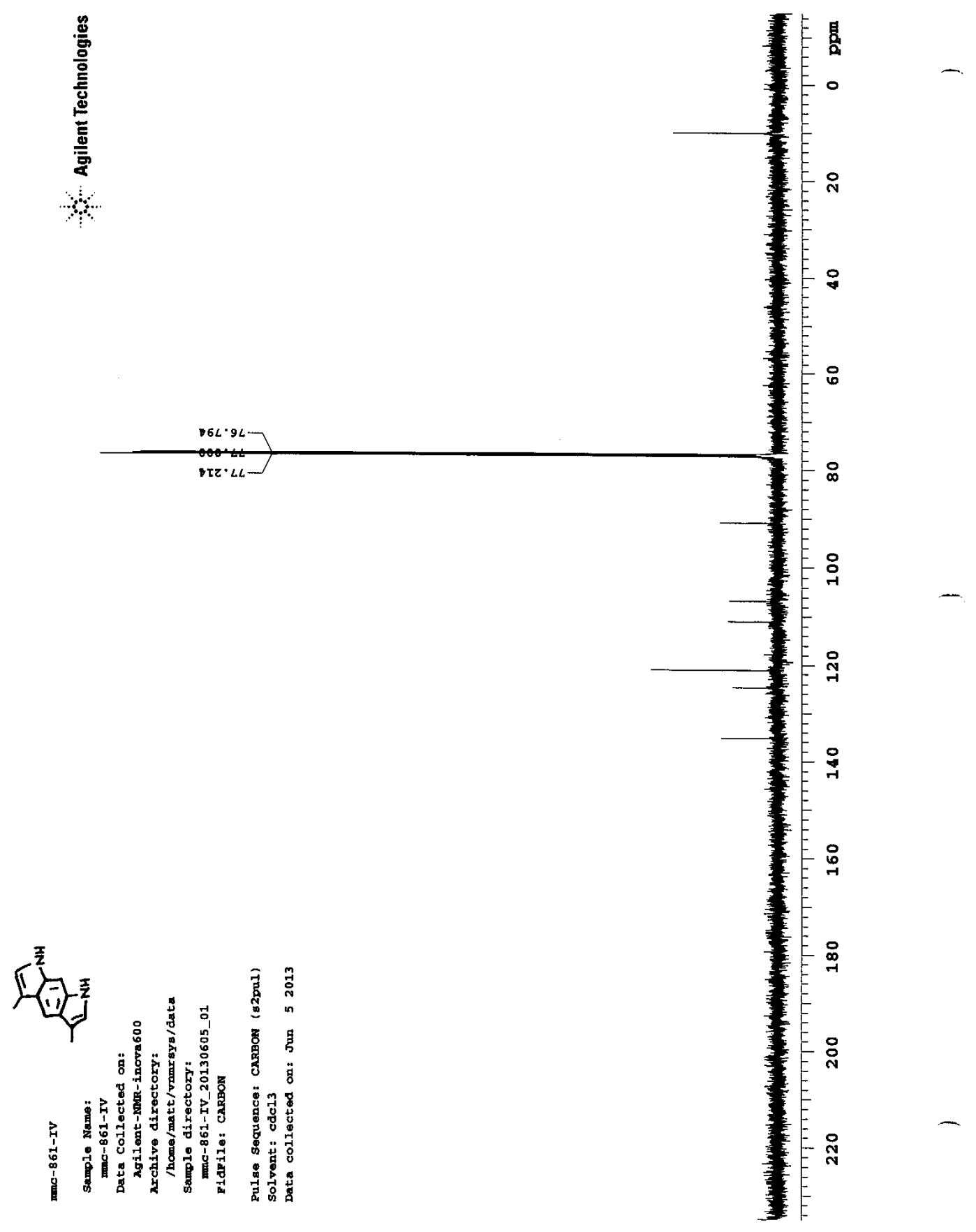

Figure 129: ${ }^{13} \mathrm{C}$ NMR of 3,5-dimethyl-1,7-dihydropyrrolo[3,2-f]indole (326) 


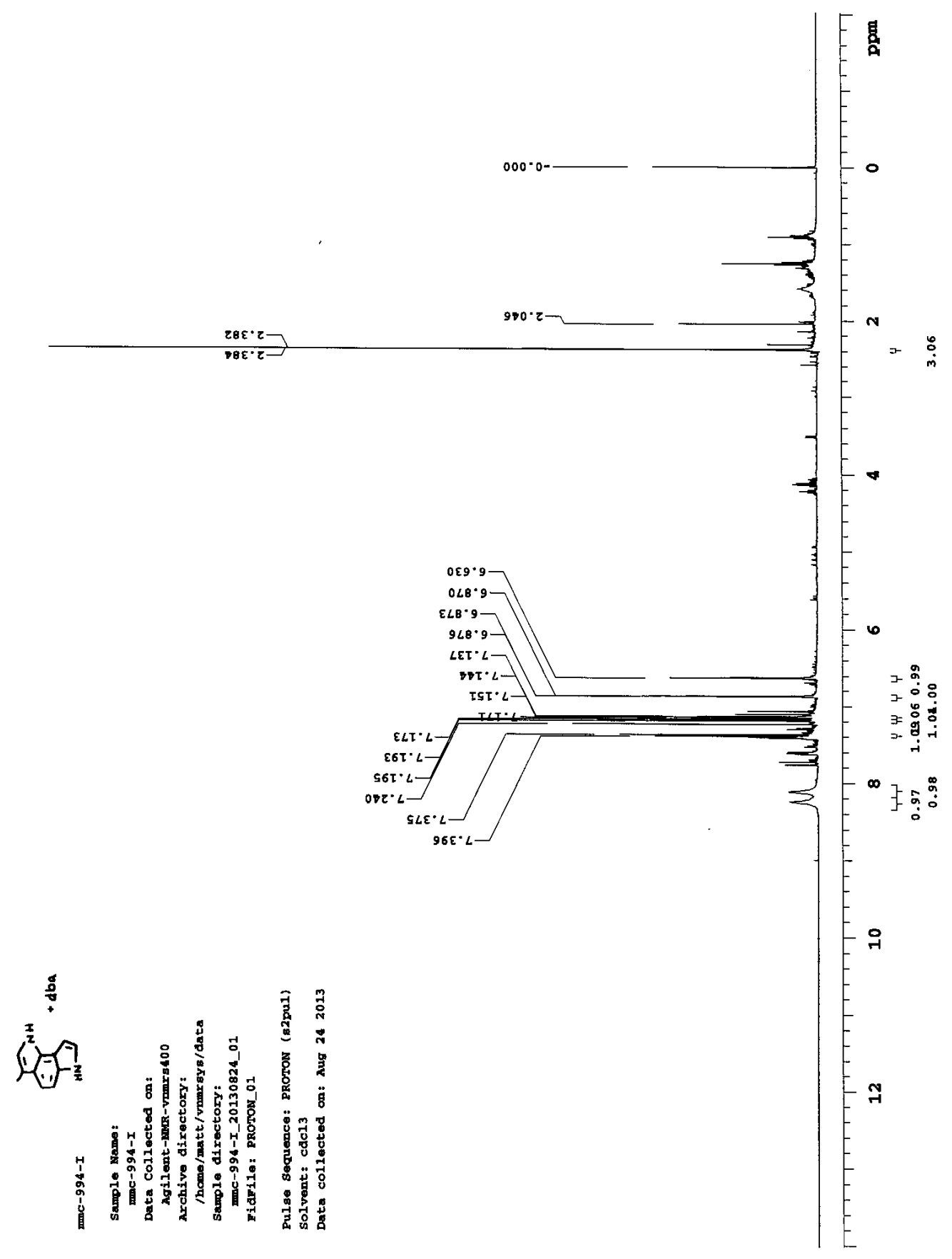

Figure 130: ${ }^{1} \mathrm{H}$ NMR of 3-methyl-2,6-dihydropyrrolo[2,3-g]indole (330) 


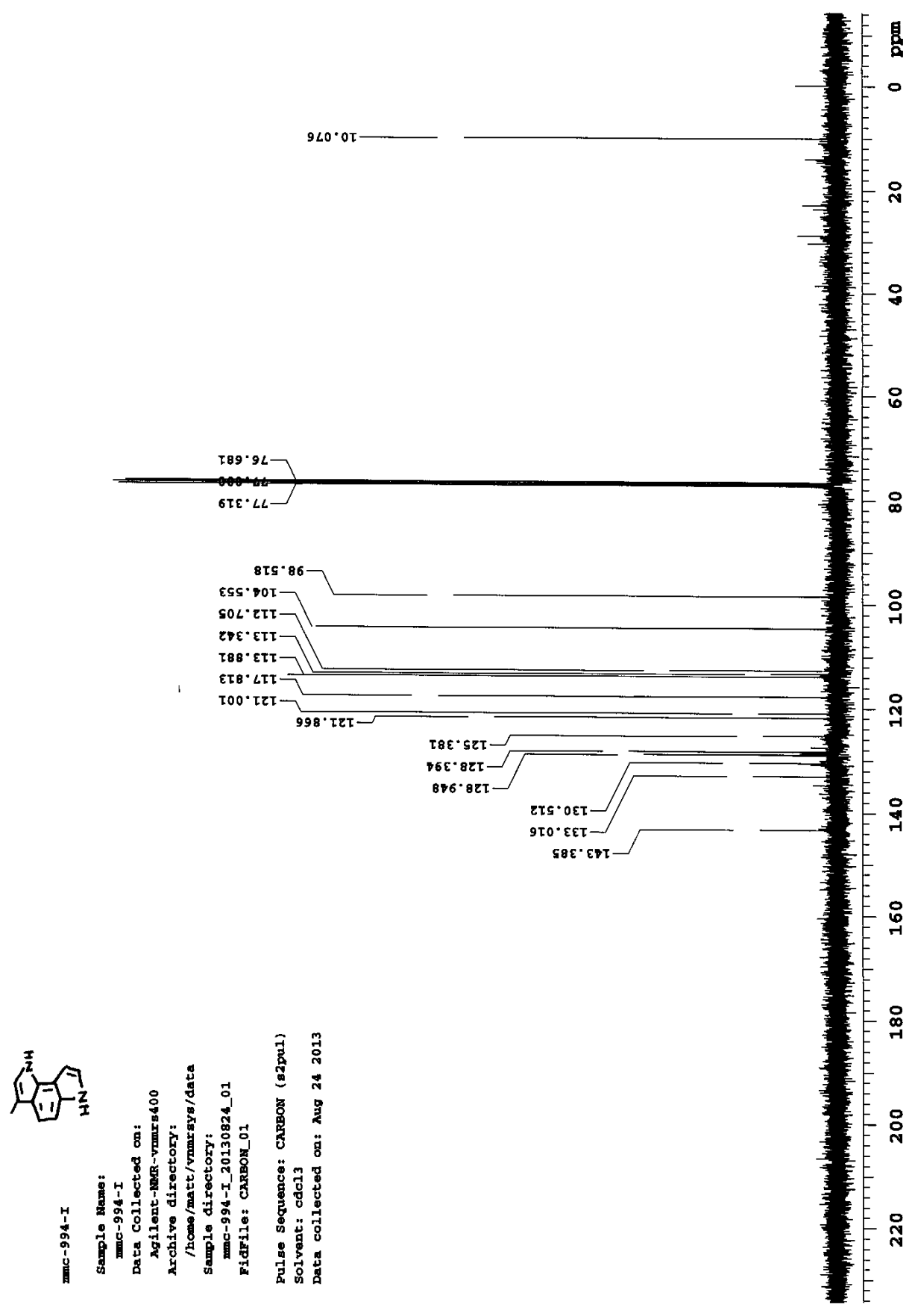

Figure 131: ${ }^{13} \mathrm{C}$ NMR of 3-methyl-2,6-dihydropyrrolo[2,3-g]indole (330) 


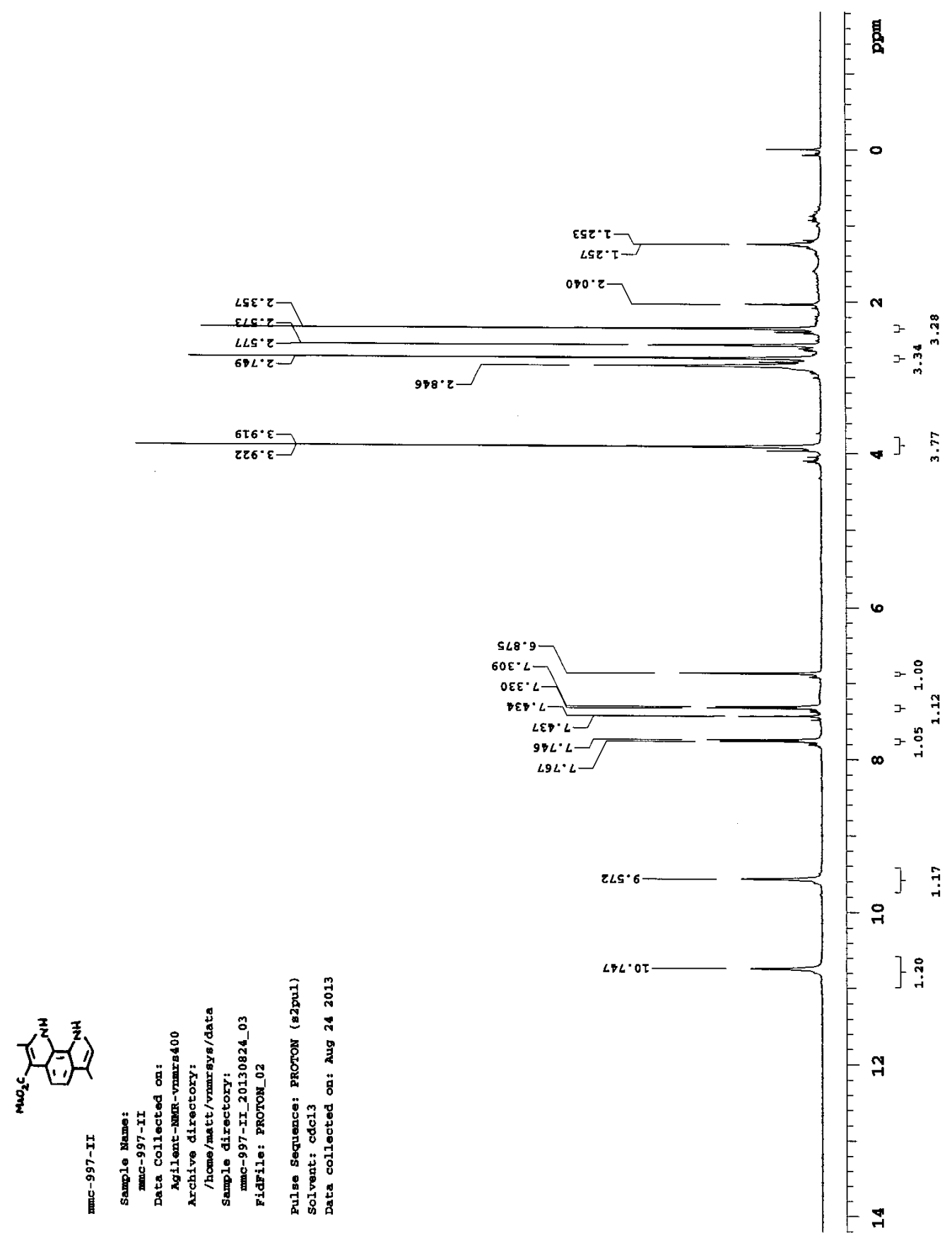

Figure 132: ${ }^{1} \mathrm{H}$ NMR of Methyl 2,6-dimethyl-1,8-dihydropyrrolo[3,2-g]indole-3carboxylate (331): 


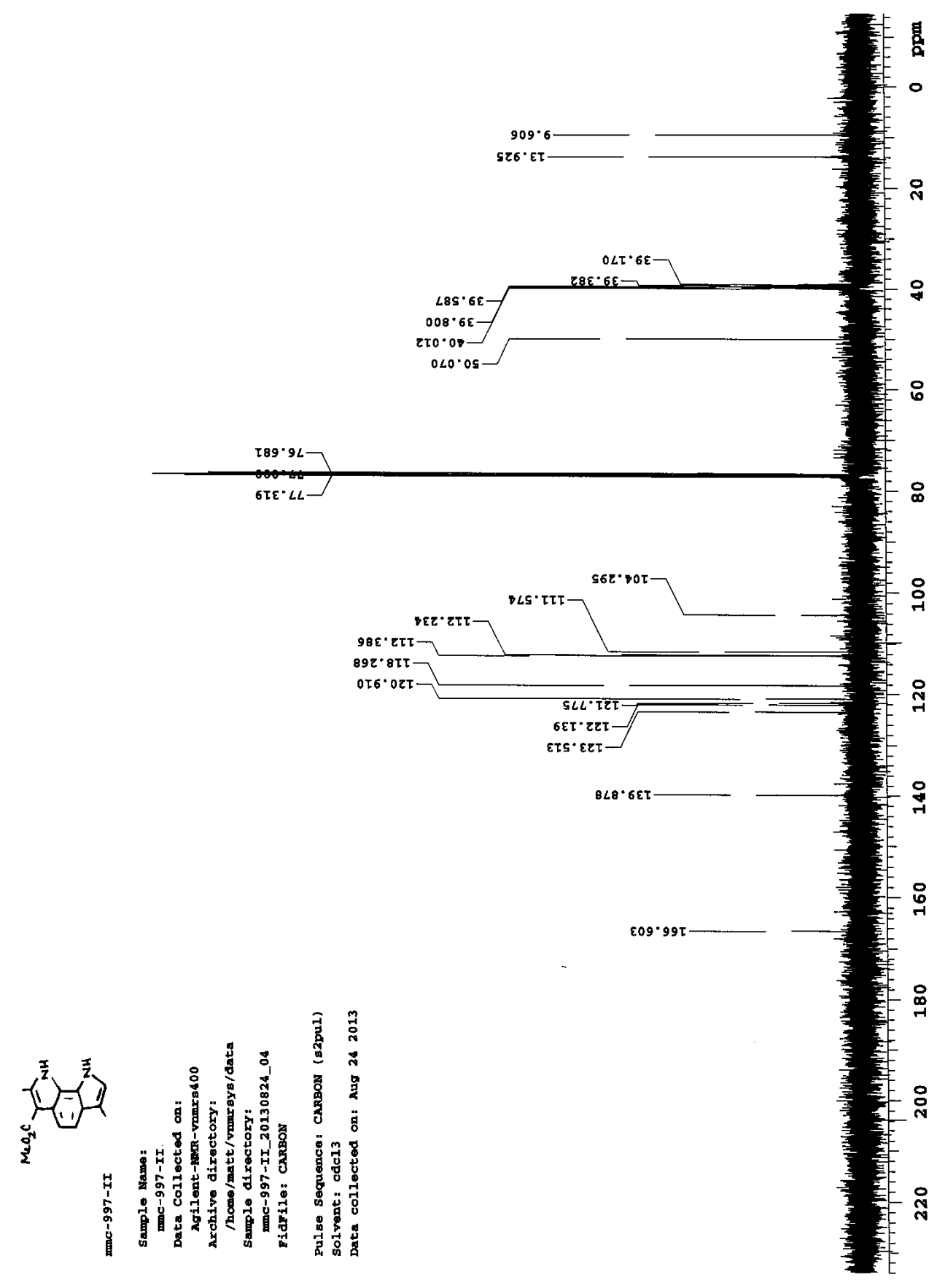

Figure 133: ${ }^{13} \mathrm{C}$ NMR of Methyl 2,6-dimethyl-1,8-dihydropyrrolo[3,2-g]indole-3carboxylate (331): 


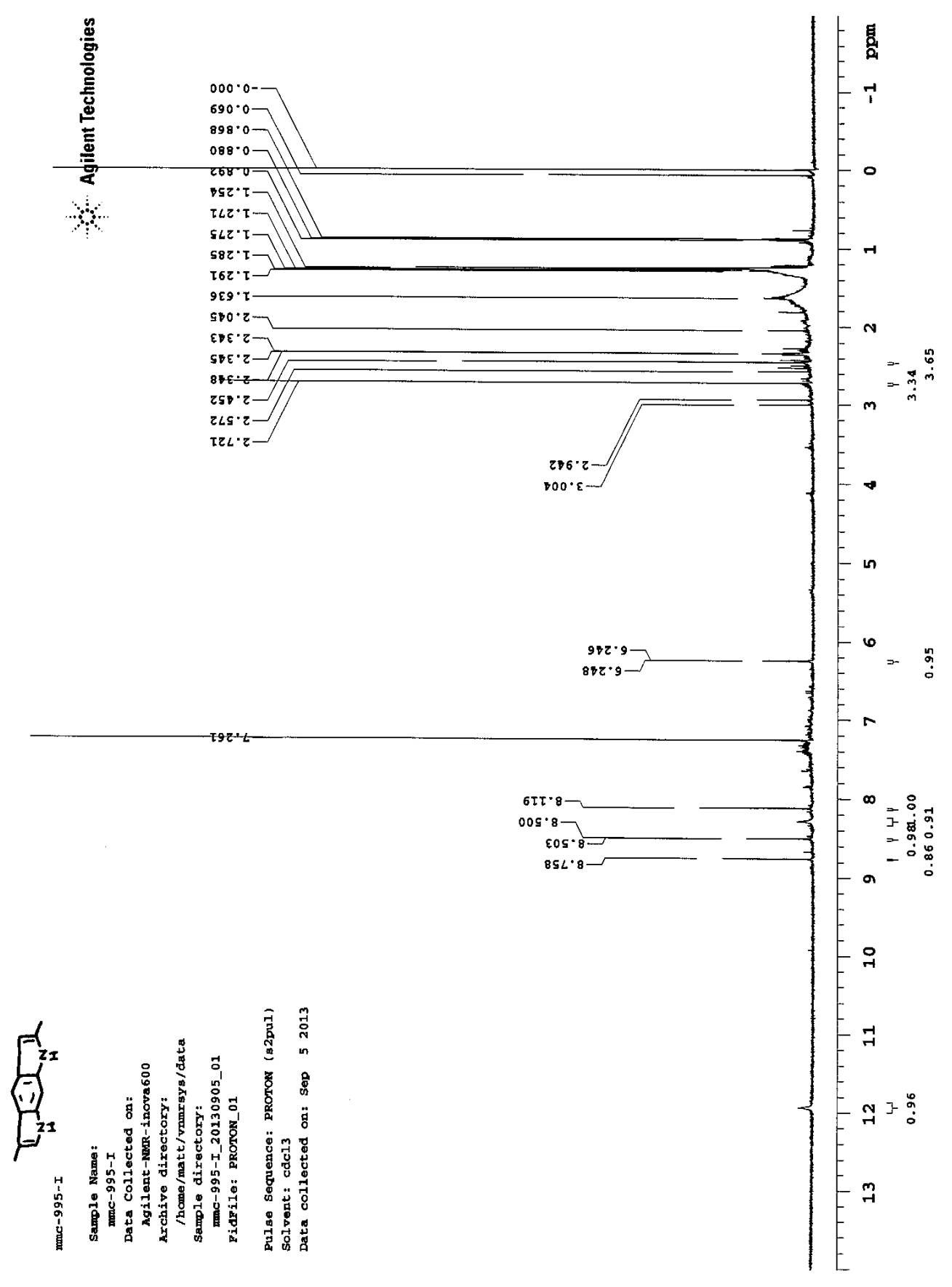

Figure 134: ${ }^{1} \mathrm{H}$ NMR of 2,5-dimethyl-1,7-dihydropyrrolo[3,2-f]indole (332) 


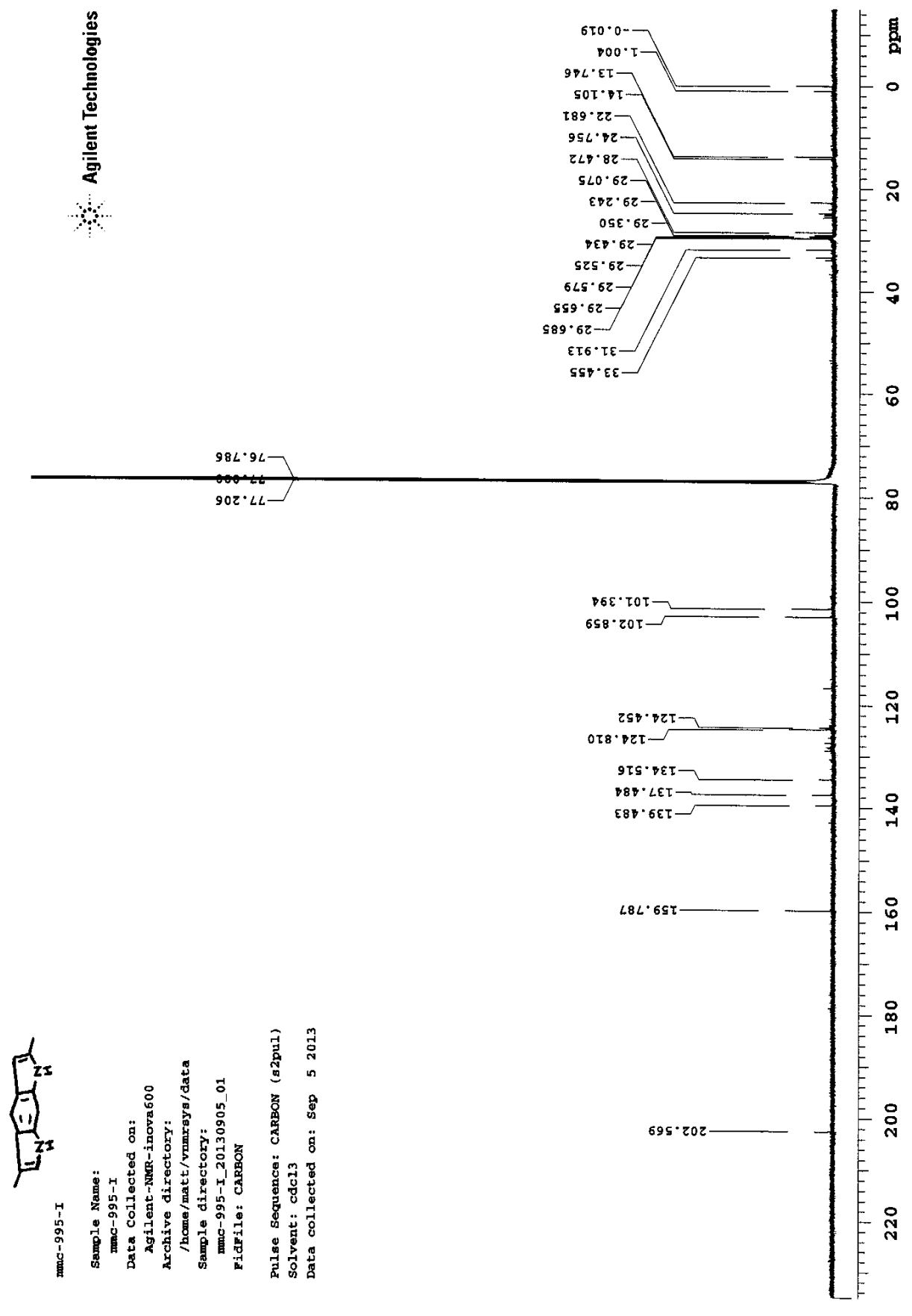

Figure 135: ${ }^{13} \mathrm{C}$ NMR of 2,5-dimethyl-1,7-dihydropyrrolo[3,2-f]indole (332) 


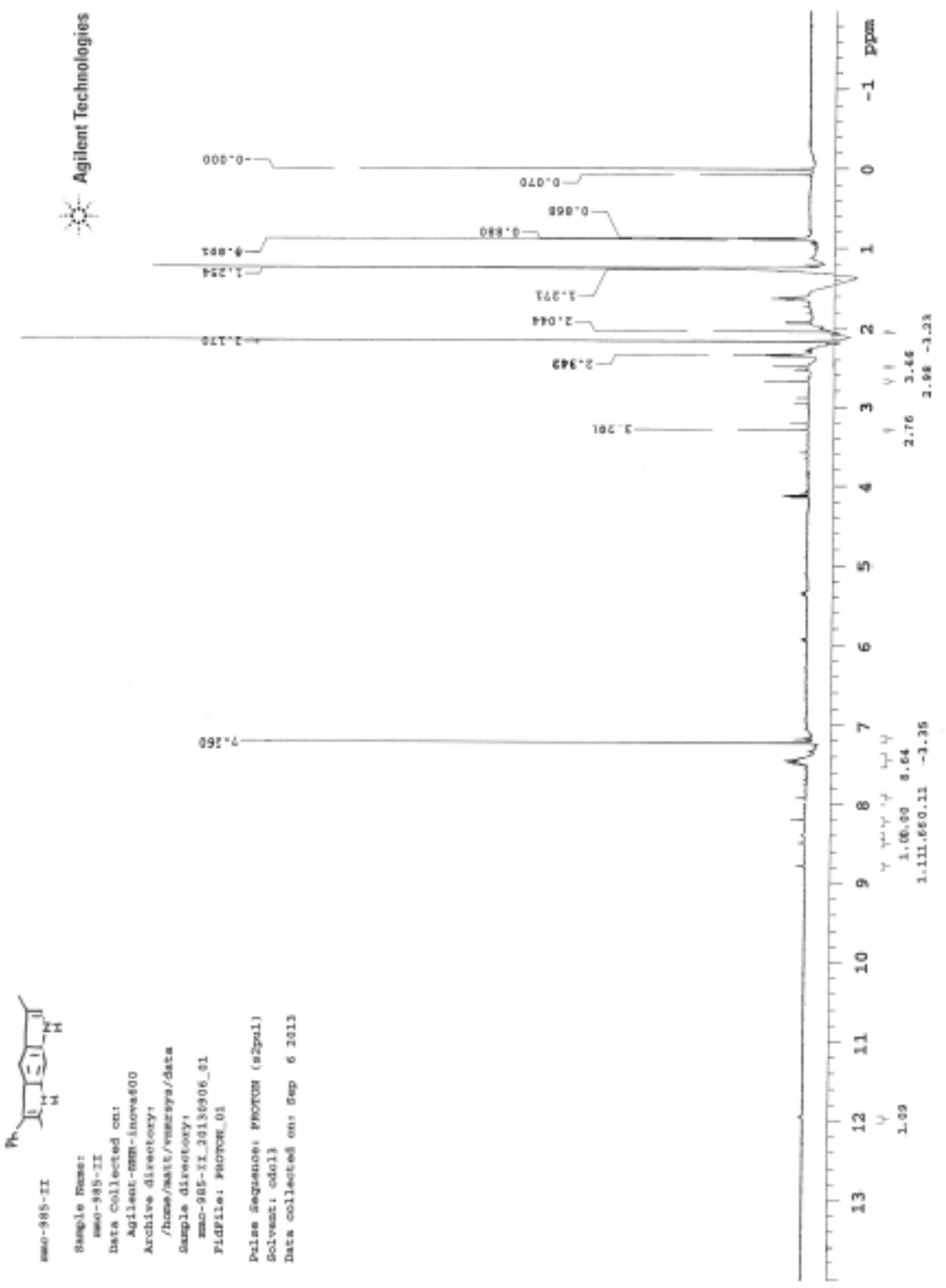

Figure 136: ${ }^{1} \mathrm{H}$ NMR of 2,5-dimethyl-3-phenyl-1,7-dihydropyrrolo[3,2-f]indole (333) 


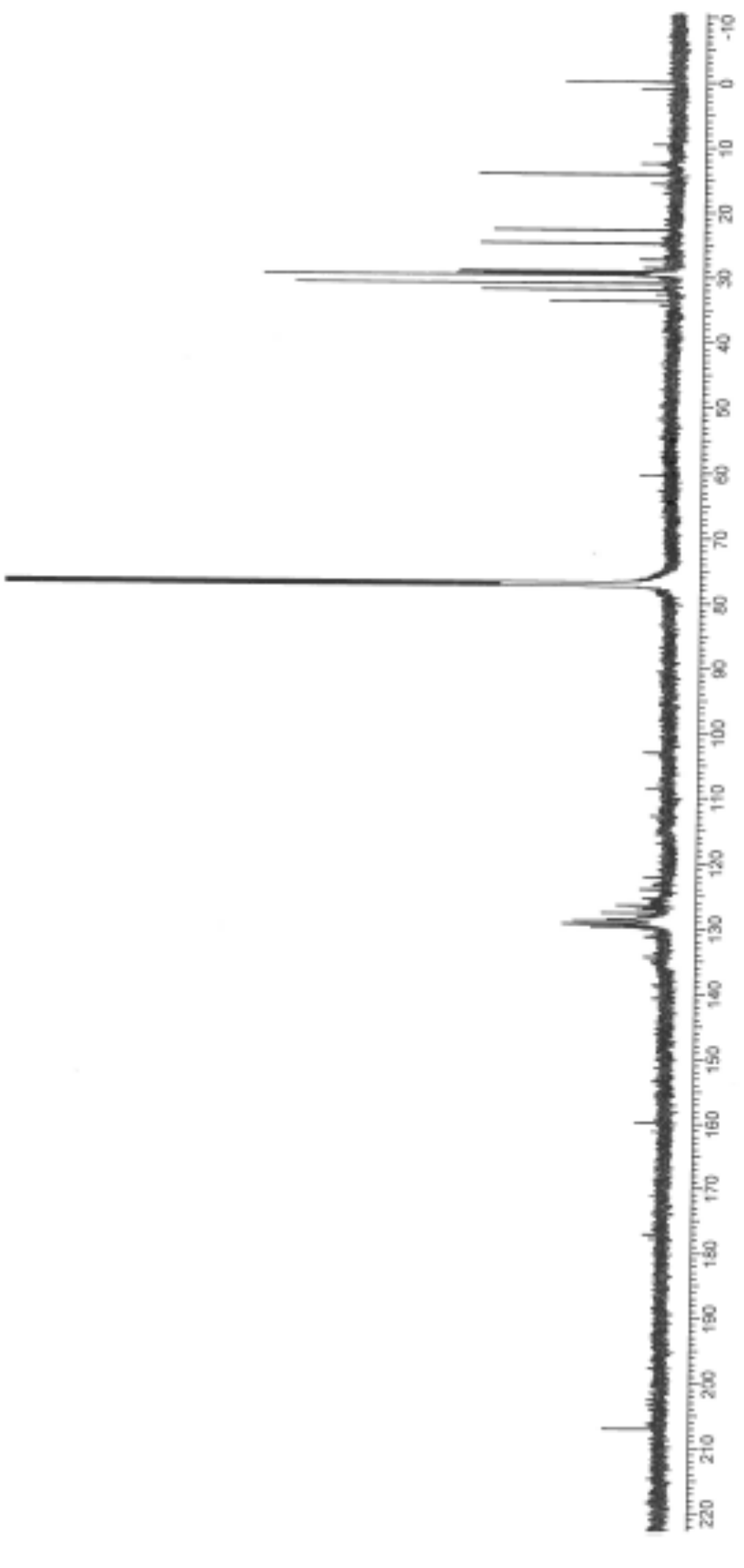

Figure 137: ${ }^{13} \mathrm{C}$ NMR of 2,5-dimethyl-3-phenyl-1,7-dihydropyrrolo[3,2-f]indole (333) 
${ }^{1} \mathrm{H}$ and ${ }^{13} \mathrm{C}$ NMR for Chapter 6.1: Bromination

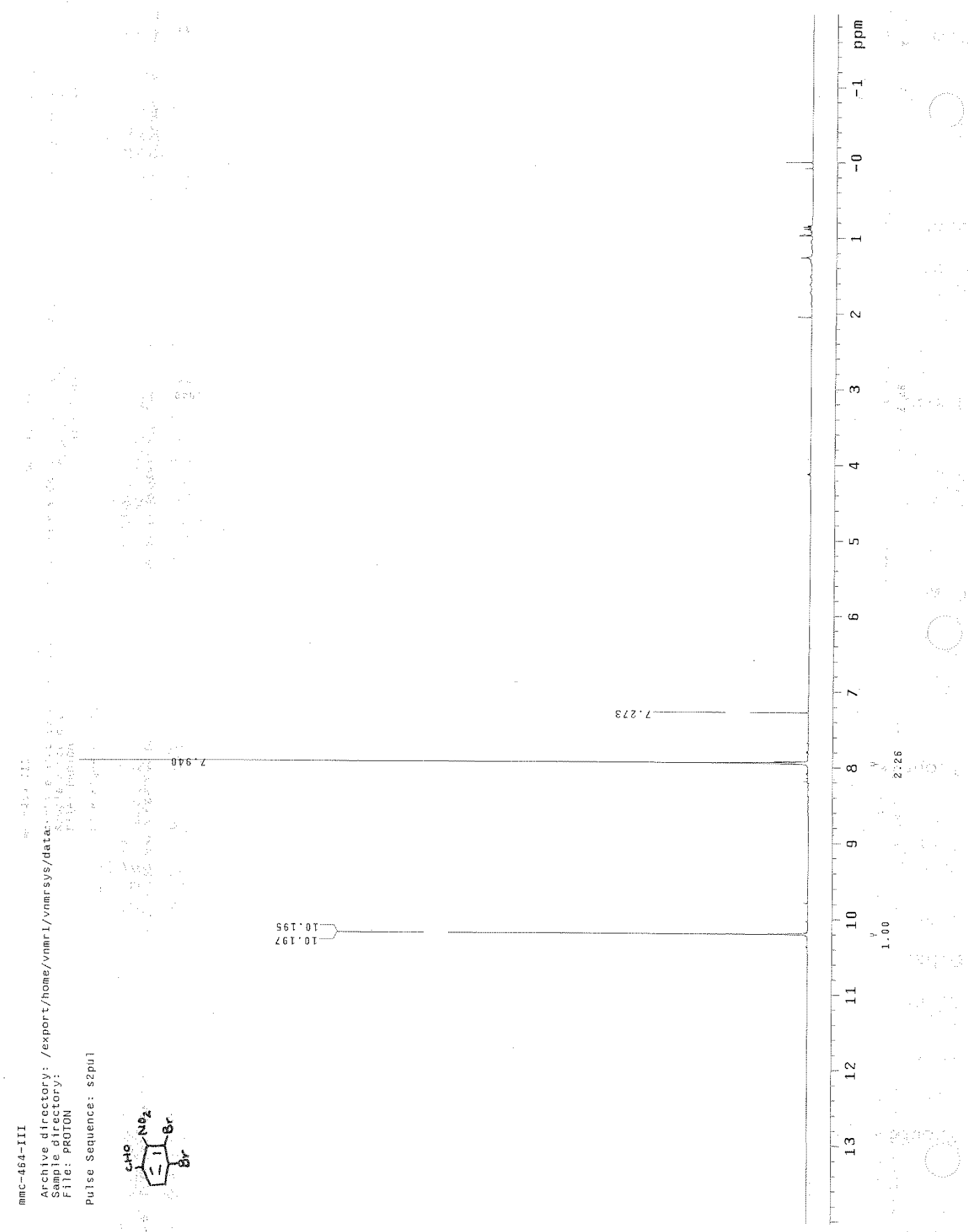

Figure 138: ${ }^{1} \mathrm{H}$ NMR of 3,4-dibromo-2-nitrobenzaldehyde (343) 


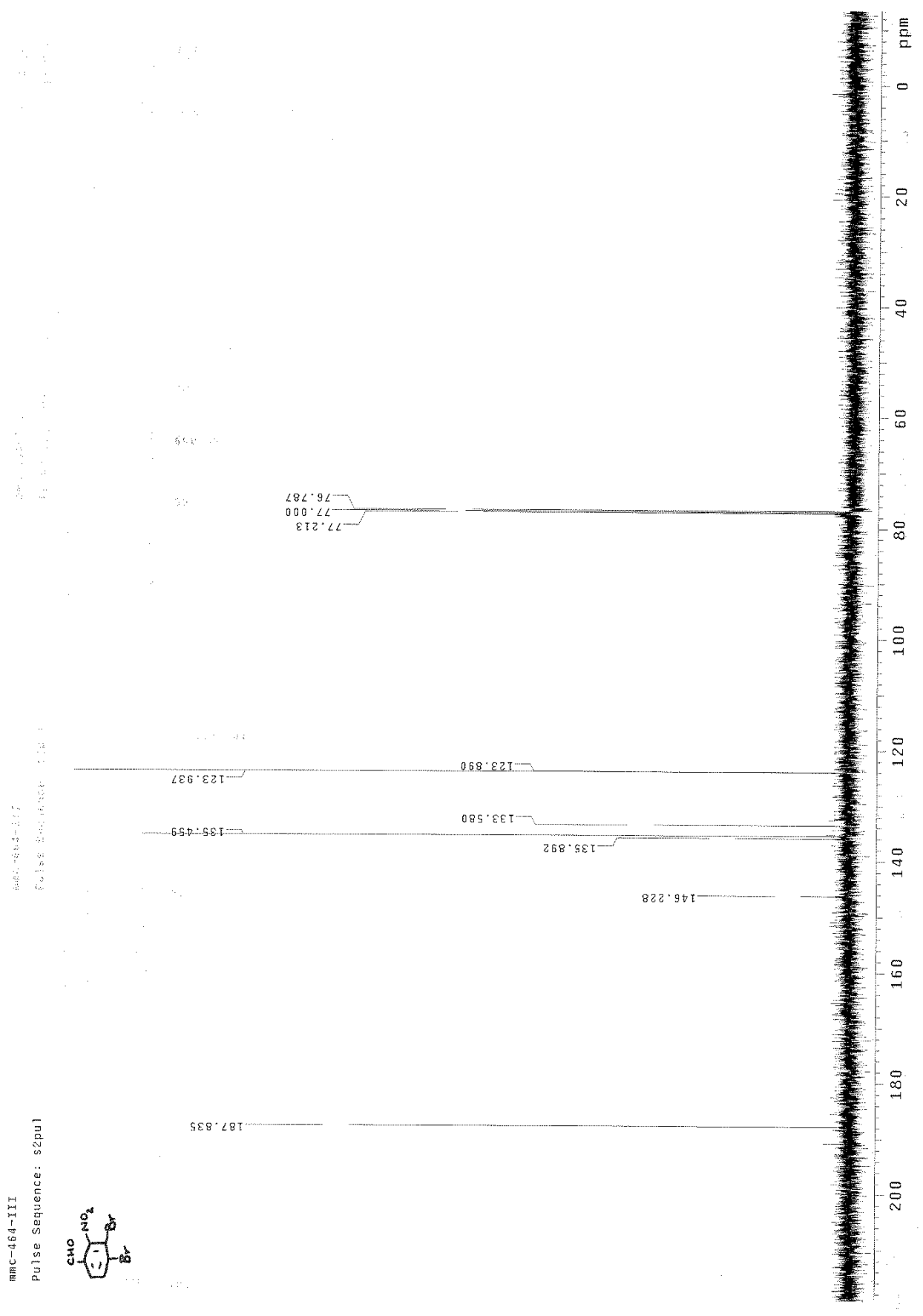

Figure 139: ${ }^{13} \mathrm{C}$ NMR of 3,4-dibromo-2-nitrobenzaldehyde (343) 


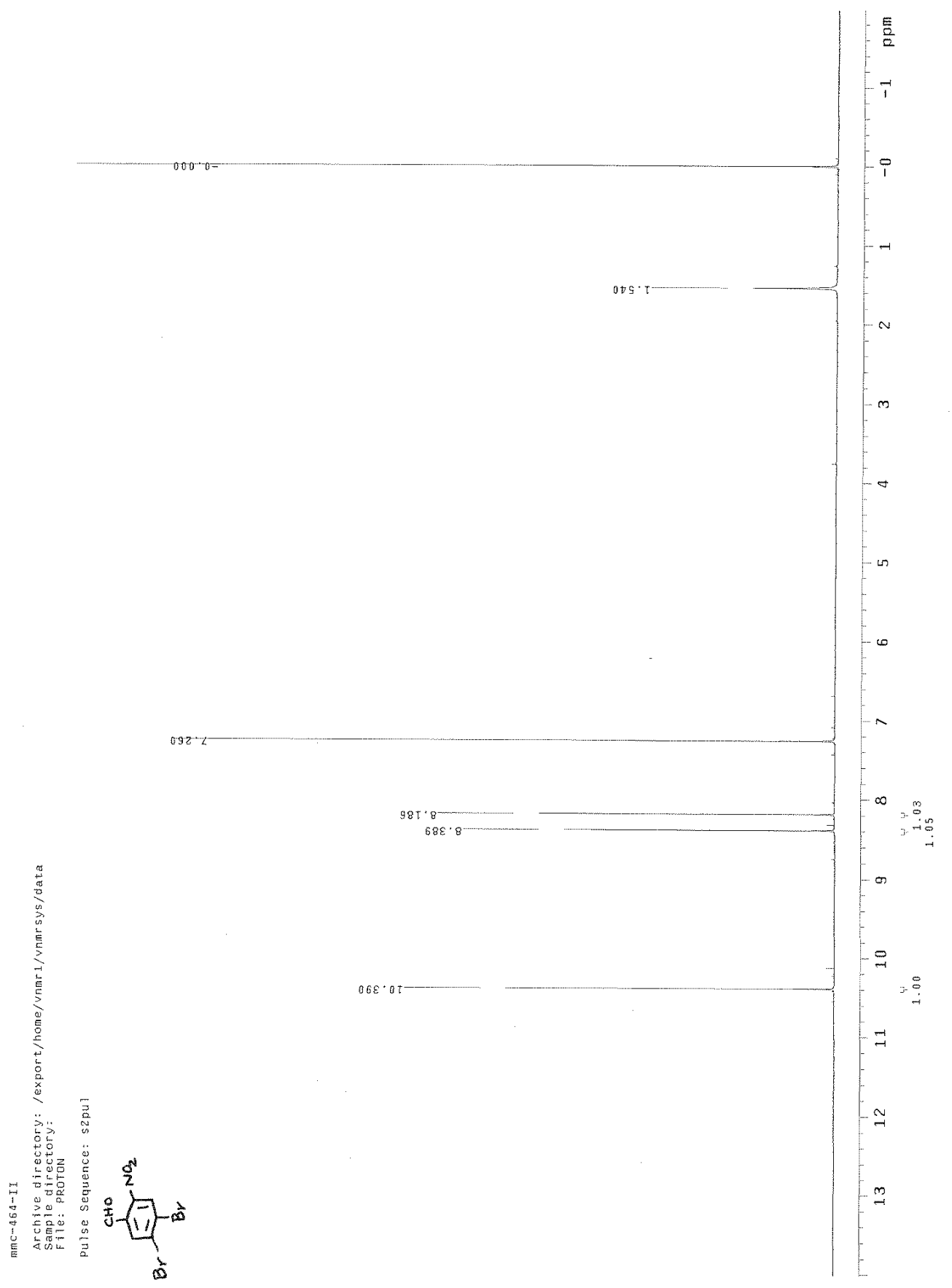

Figure 140: ${ }^{1} \mathrm{H}$ NMR of 4,5-dibromo-2-nitrobenzaldehyde (344) 


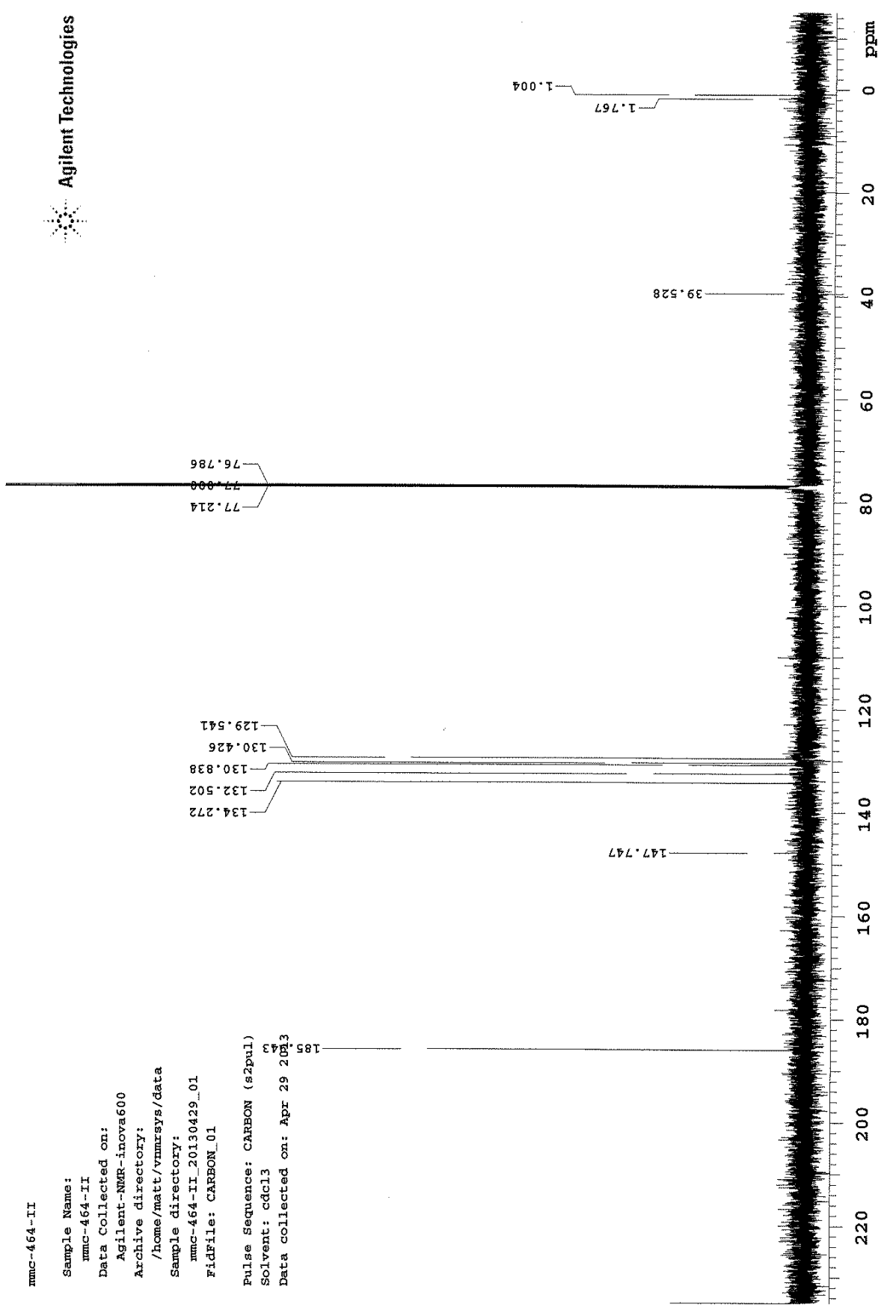

Figure 141: ${ }^{13} \mathrm{C}$ NMR of 4,5-dibromo-2-nitrobenzaldehyde (344) 


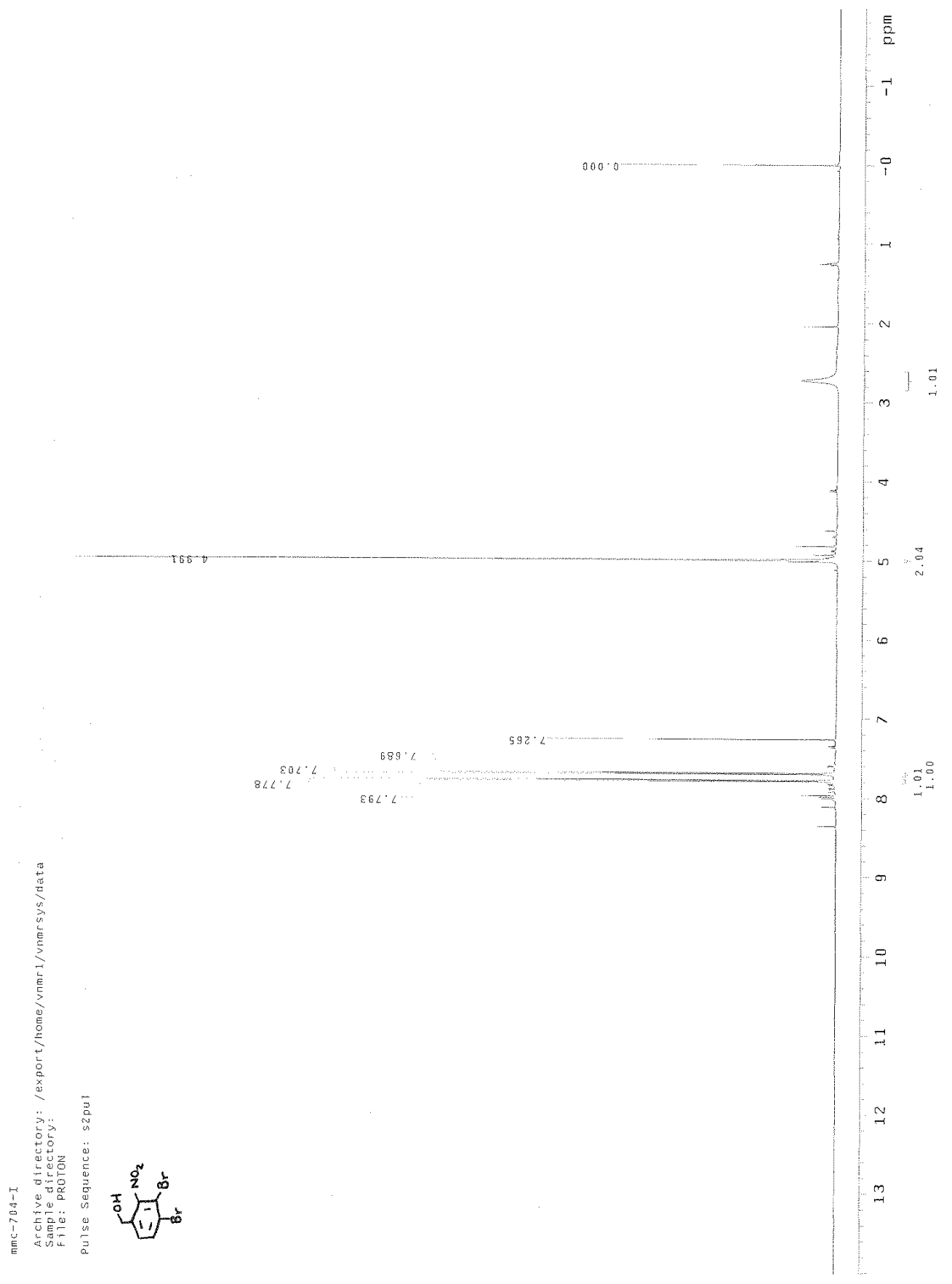

Figure 142: ${ }^{1} \mathrm{H}$ NMR of 3,4-dibromo-2-nitrobenzylalcohol (349) 


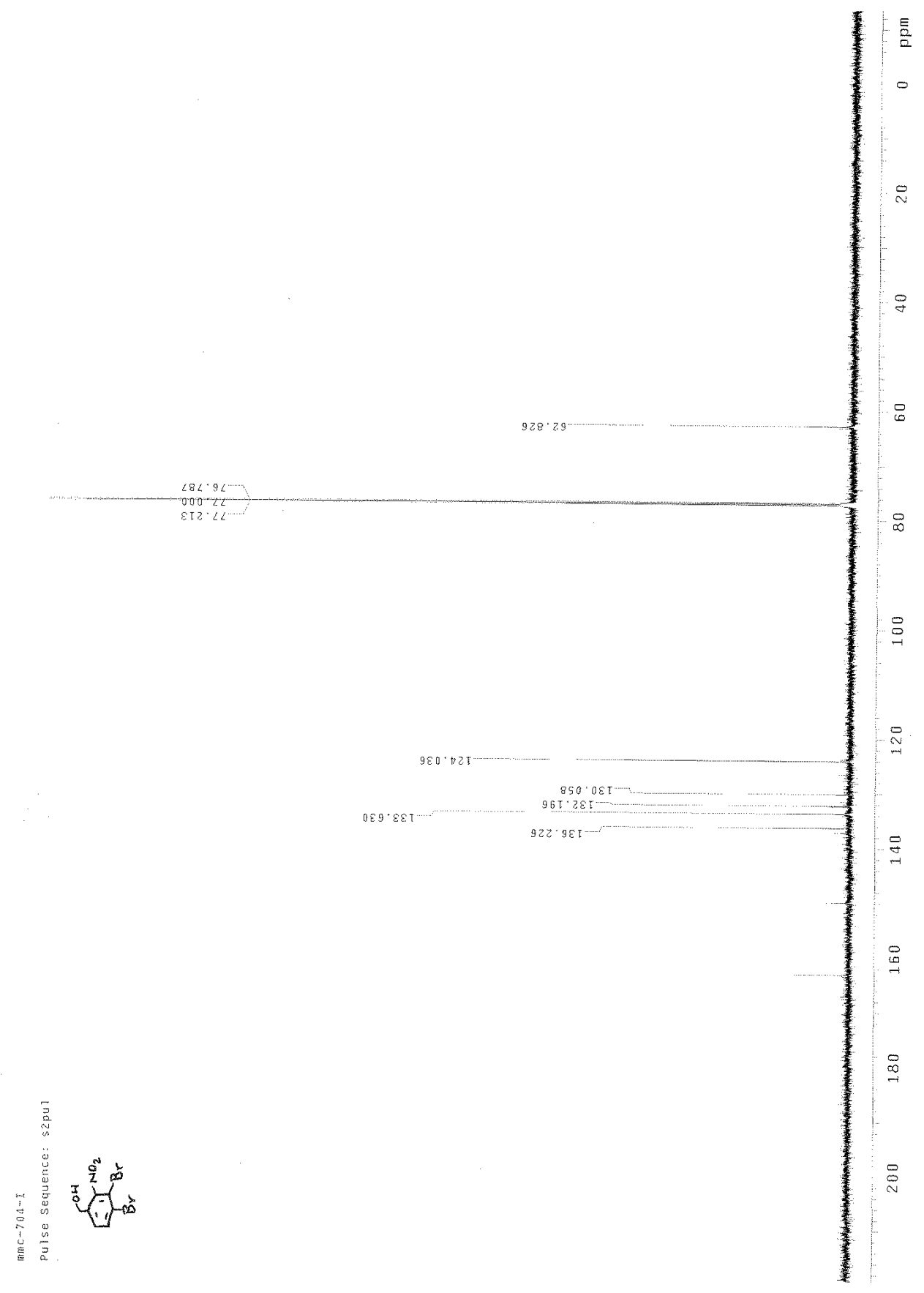

Figure 143: ${ }^{13} \mathrm{C}$ NMR of 3,4-dibromo-2-nitrobenzylalcohol (349) 


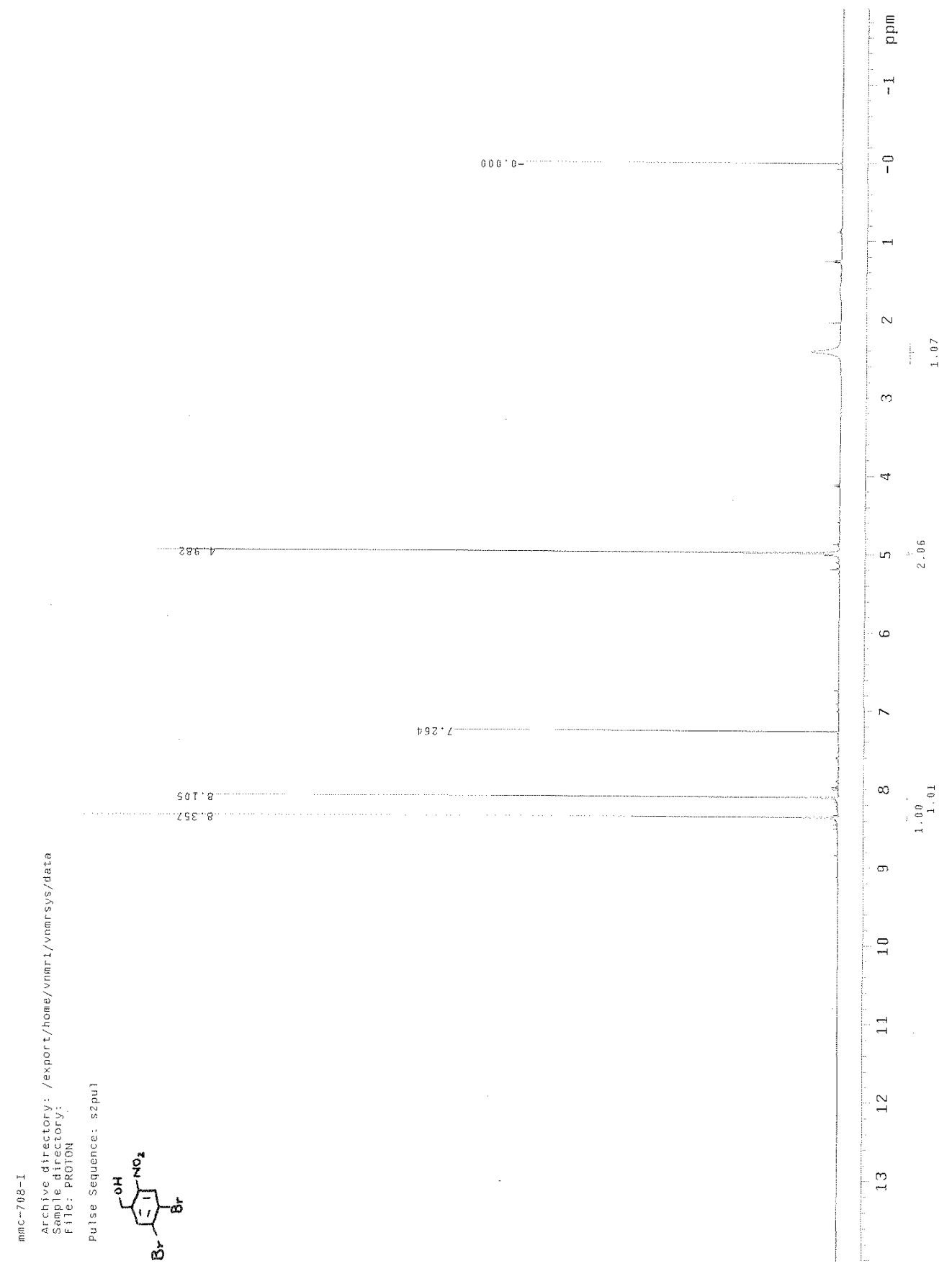

Figure 144: ${ }^{1} \mathrm{H}$ NMR of 4,5-dibromo-2-nitrobenzylalcohol (350) 


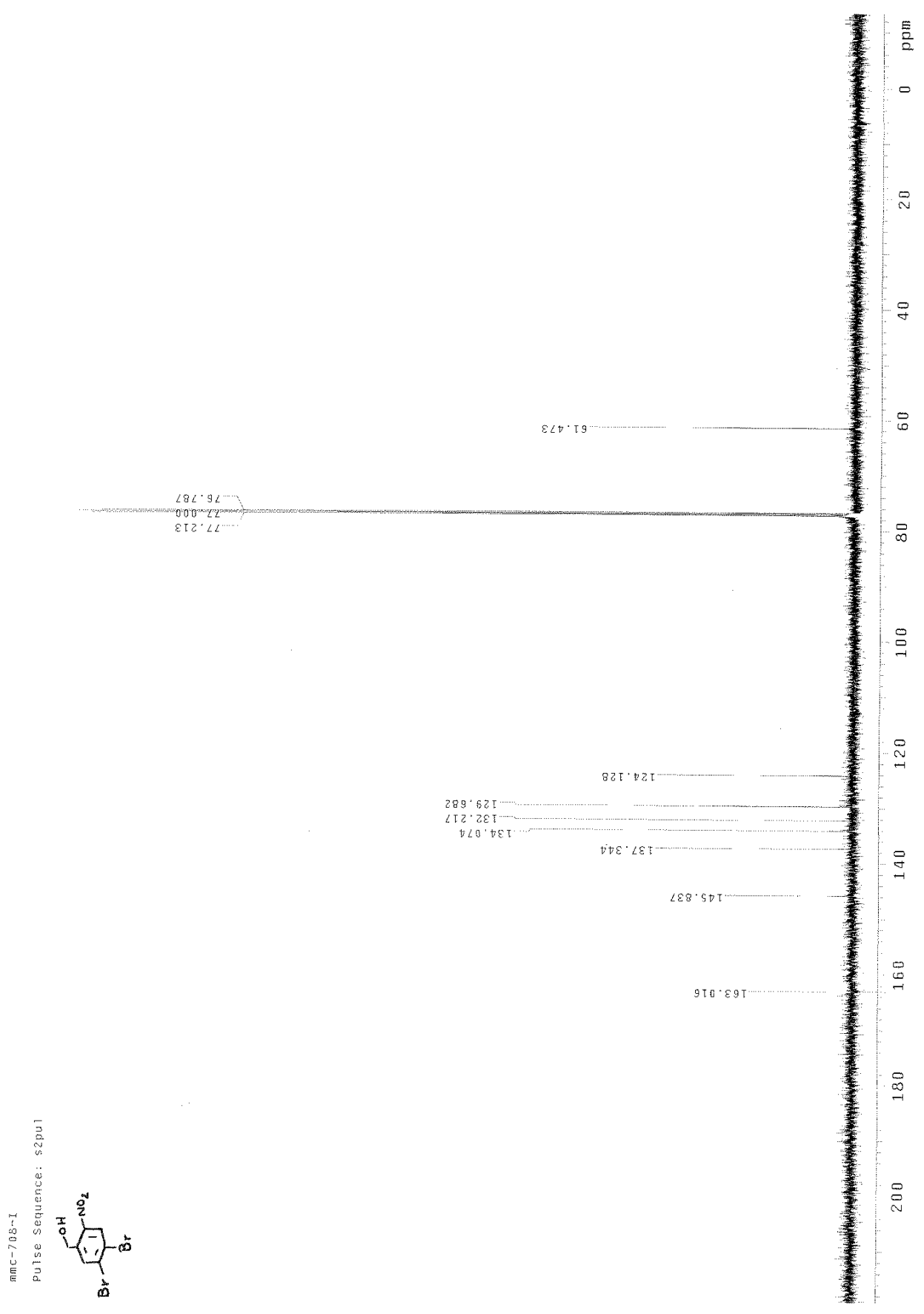

Figure 145: ${ }^{13} \mathrm{C}$ NMR of 4,5-dibromo-2-nitrobenzylalcohol (350) 


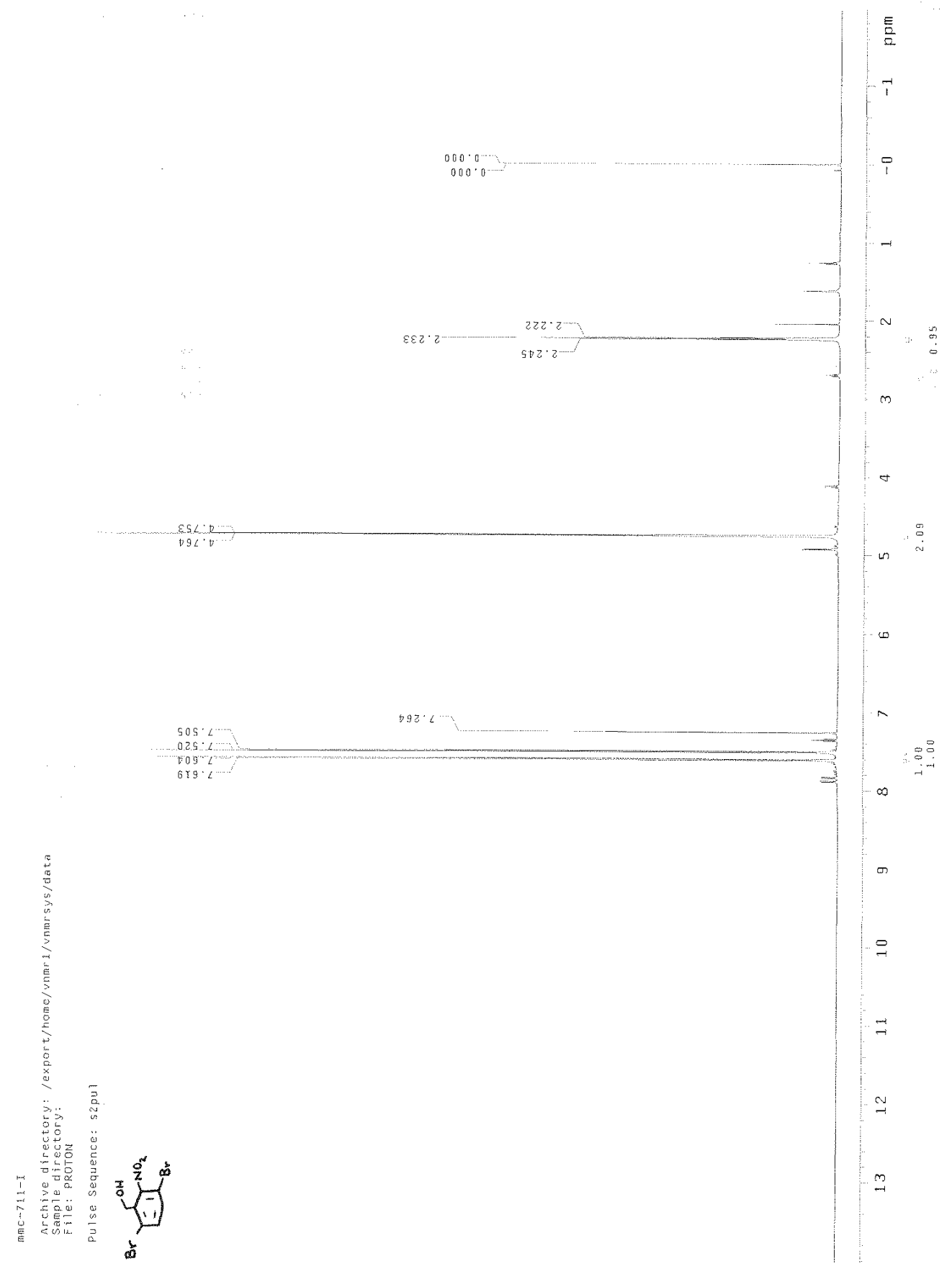

Figure 146: ${ }^{1} \mathrm{H}$ NMR of 3,6-dibromo-2-nitrobenzylalcohol (351) 


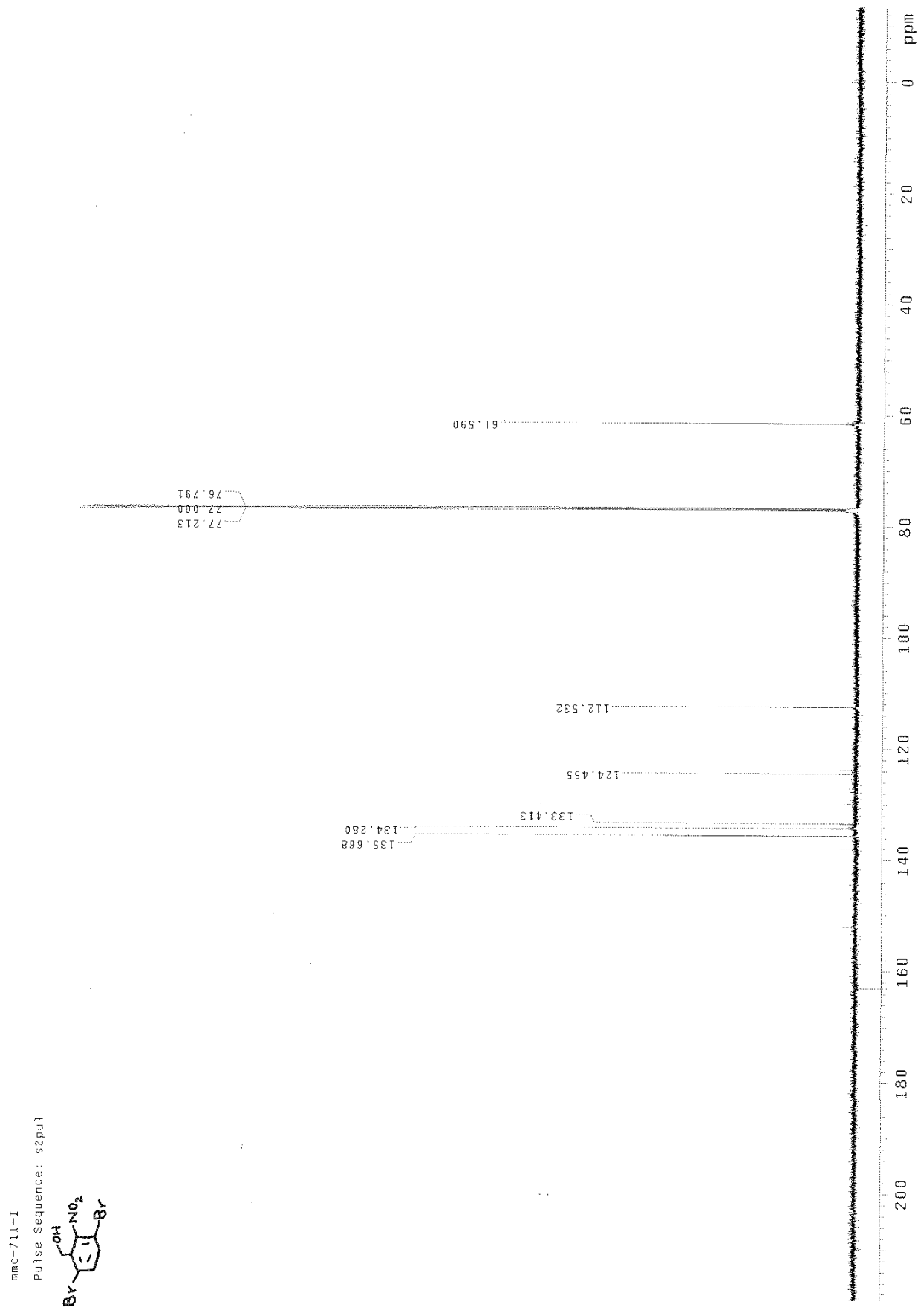

Figure 147: ${ }^{13} \mathrm{C}$ NMR of 3,6-dibromo-2-nitrobenzylalcohol (351) 
${ }^{1} \mathrm{H}$ and ${ }^{13} \mathrm{C}$ NMR for Chapter 6.2: Functionalized Indoles

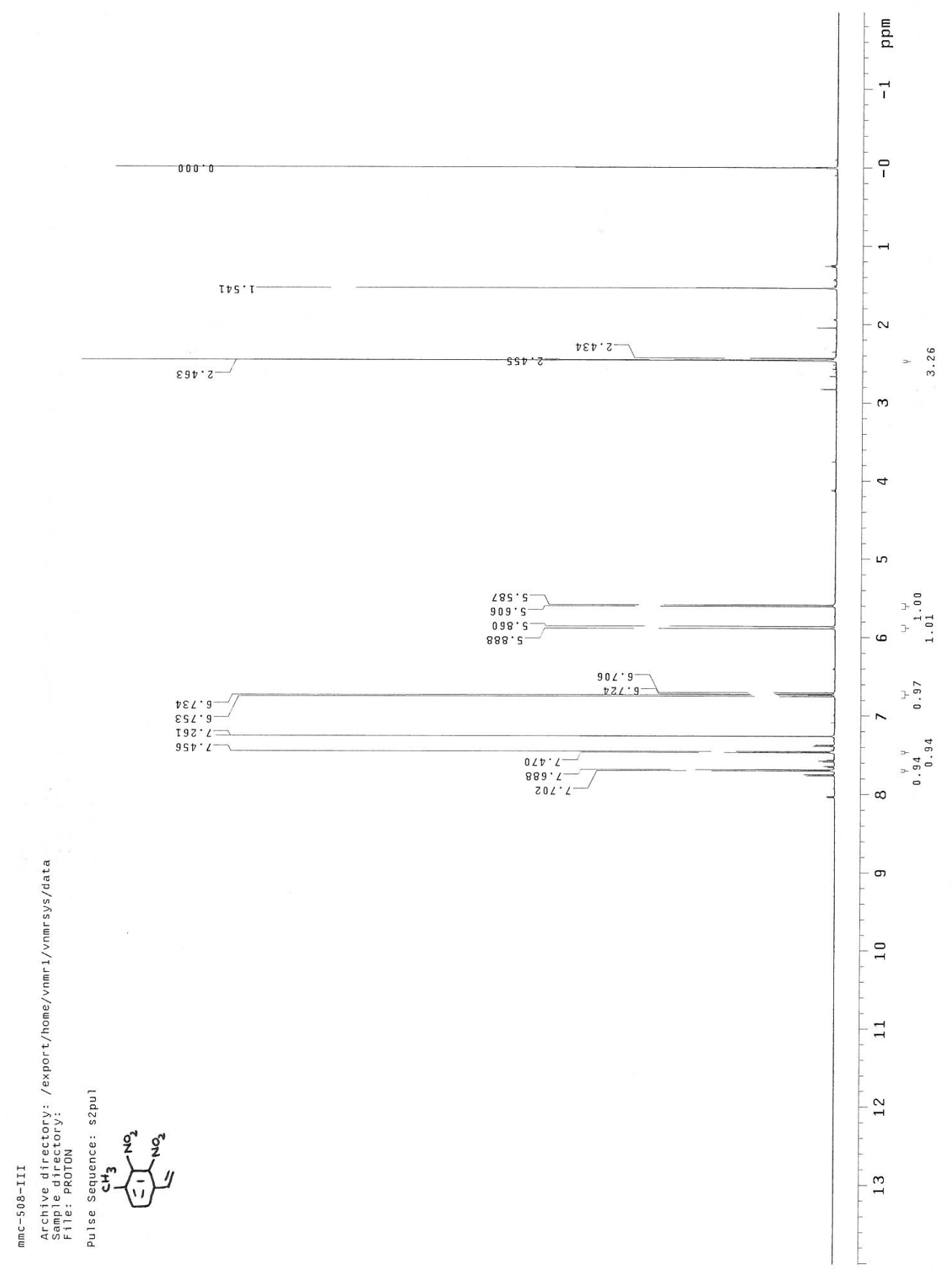

Figure 148: ${ }^{1} \mathrm{H}$ NMR of 2,3-dinitro-4-ethenyltoluene (355) 


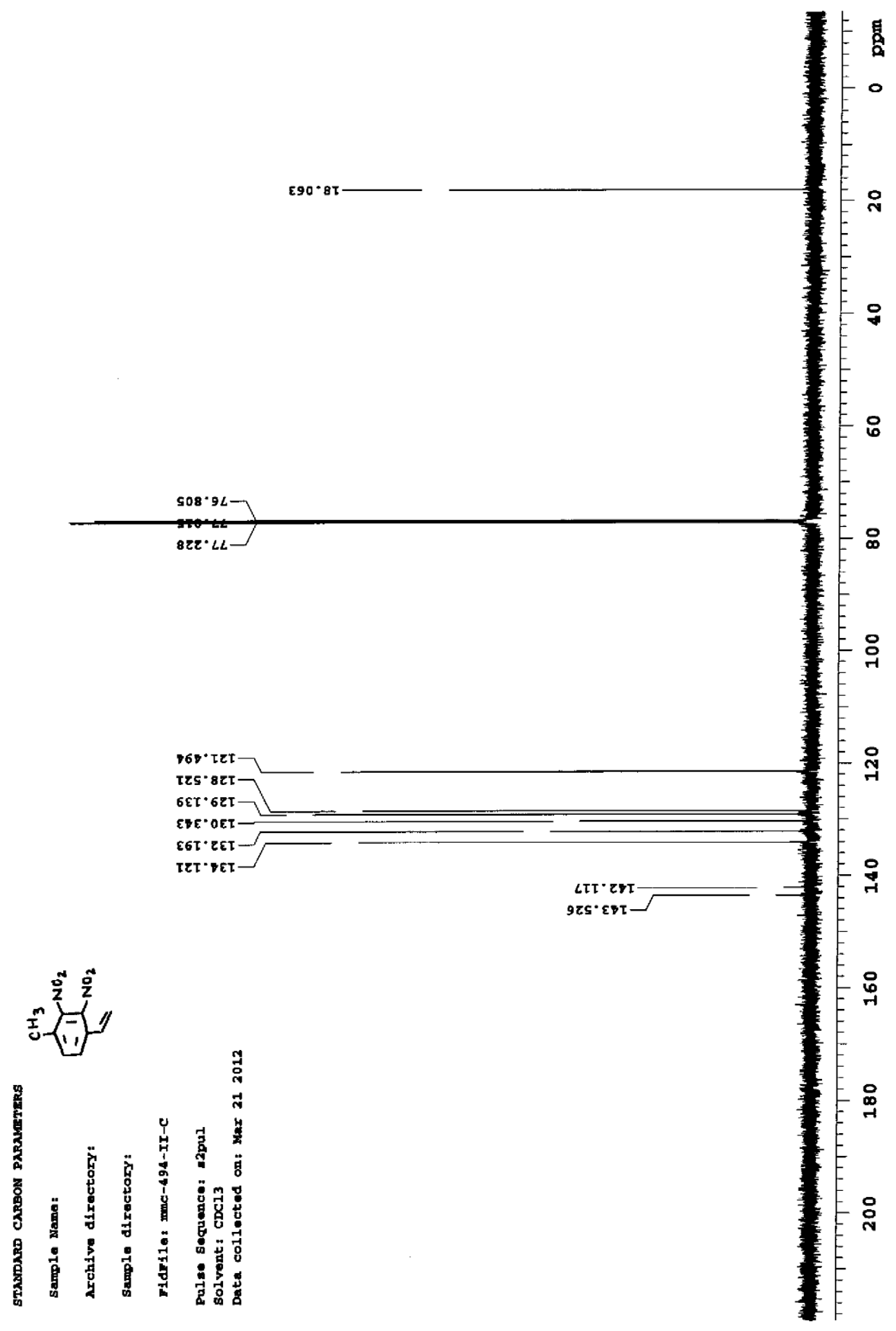

Figure 149: ${ }^{13} \mathrm{C}$ NMR of 2,3-dinitro-4-ethenyltoluene (355) 


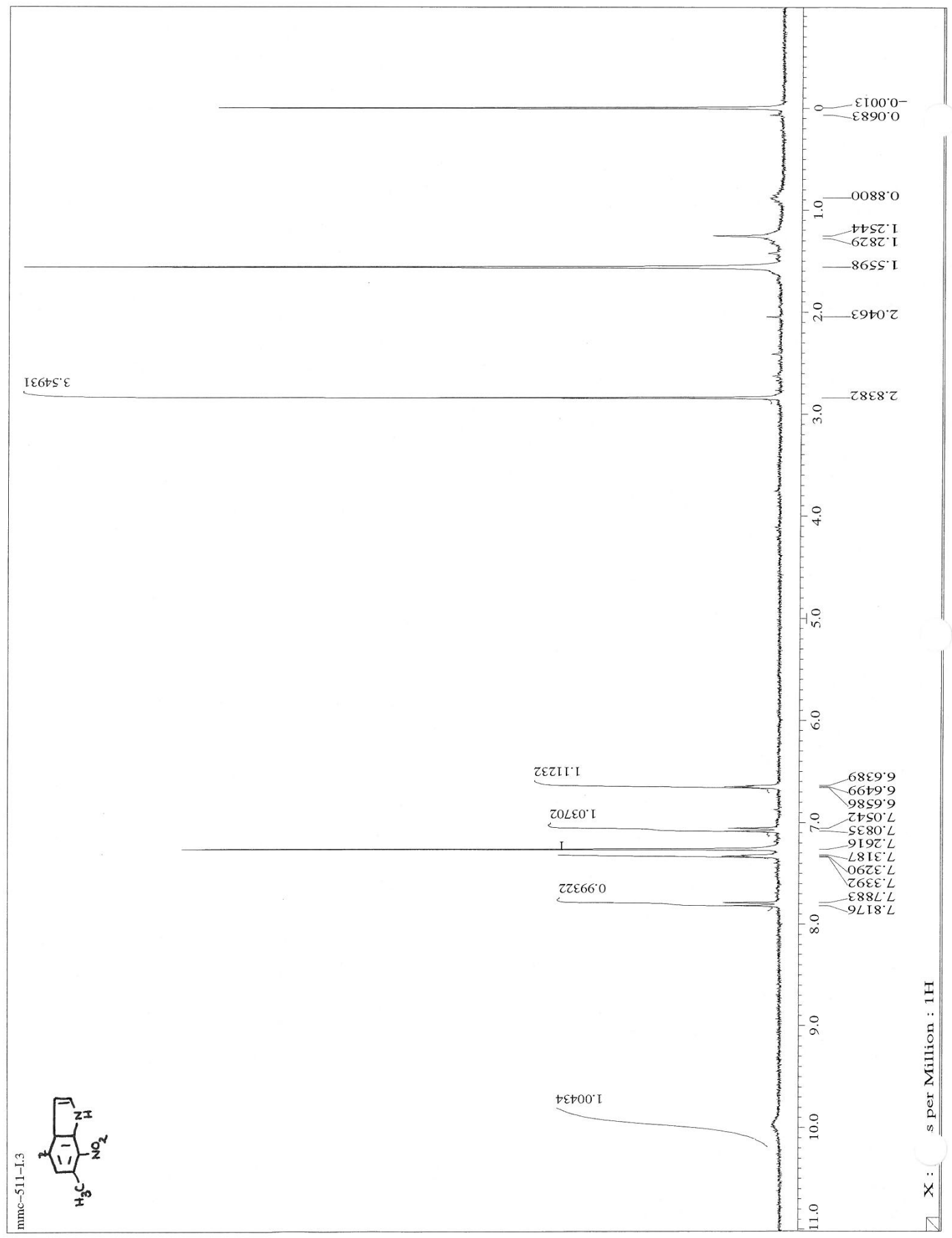

Figure 150: ${ }^{1} \mathrm{H}$ NMR of 6-methyl-7-nitroindole (356) 


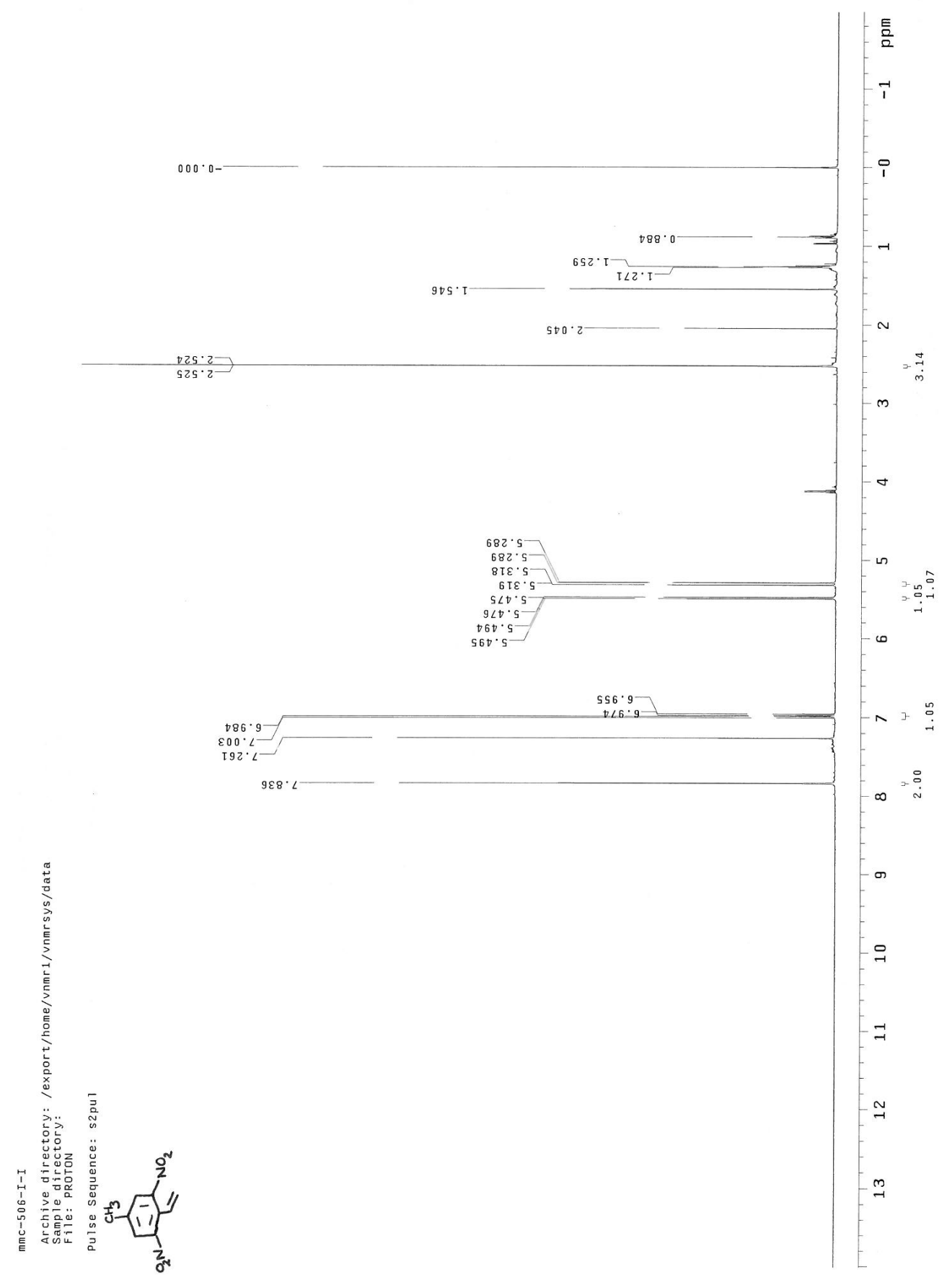

Figure 151: ${ }^{1} \mathrm{H}$ NMR of 3,5-dinitro-4-ethenyltoluene (355) 


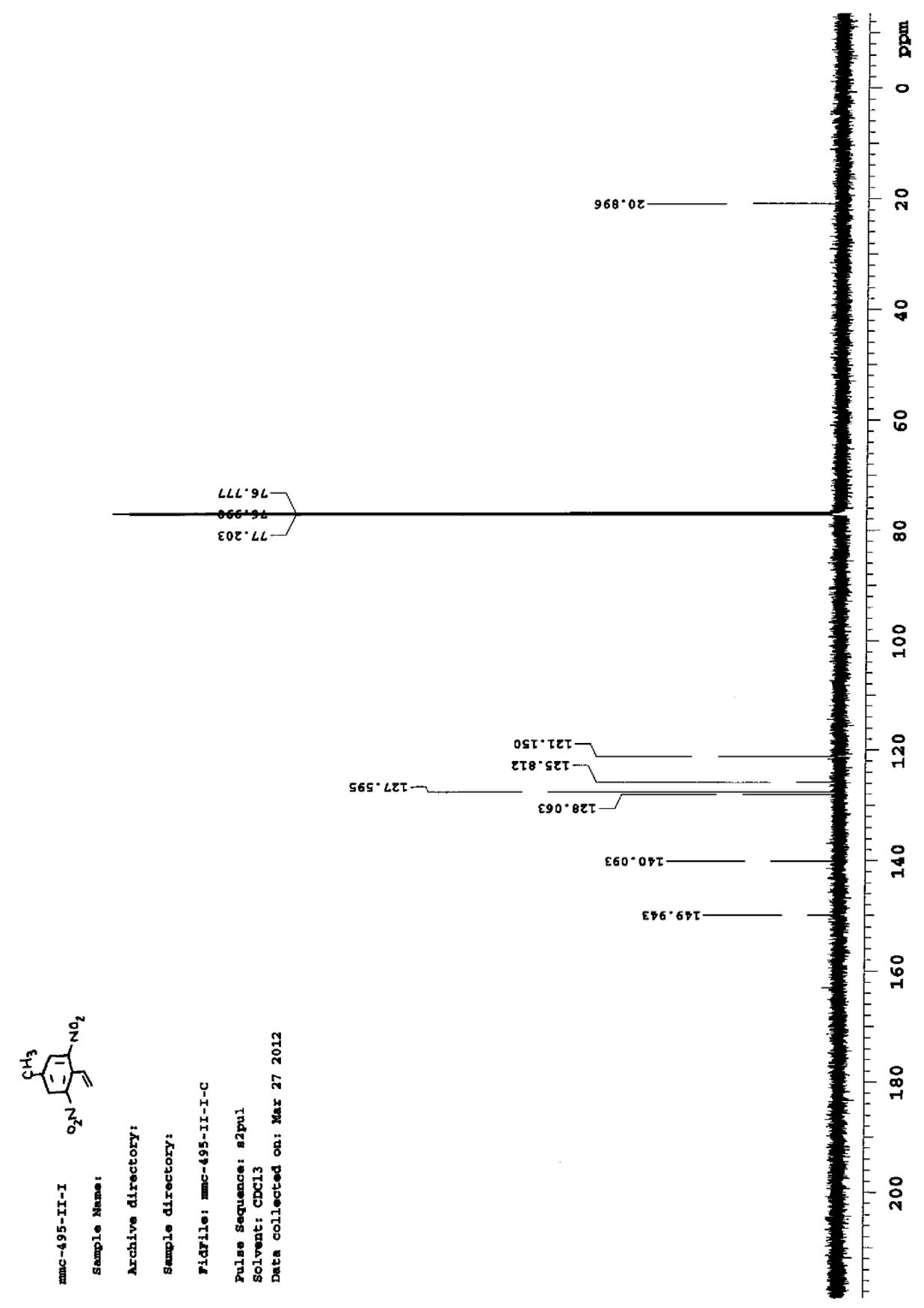

Figure 152: ${ }^{13} \mathrm{C}$ NMR of 3,5-dinitro-4-ethenyltoluene (355) 


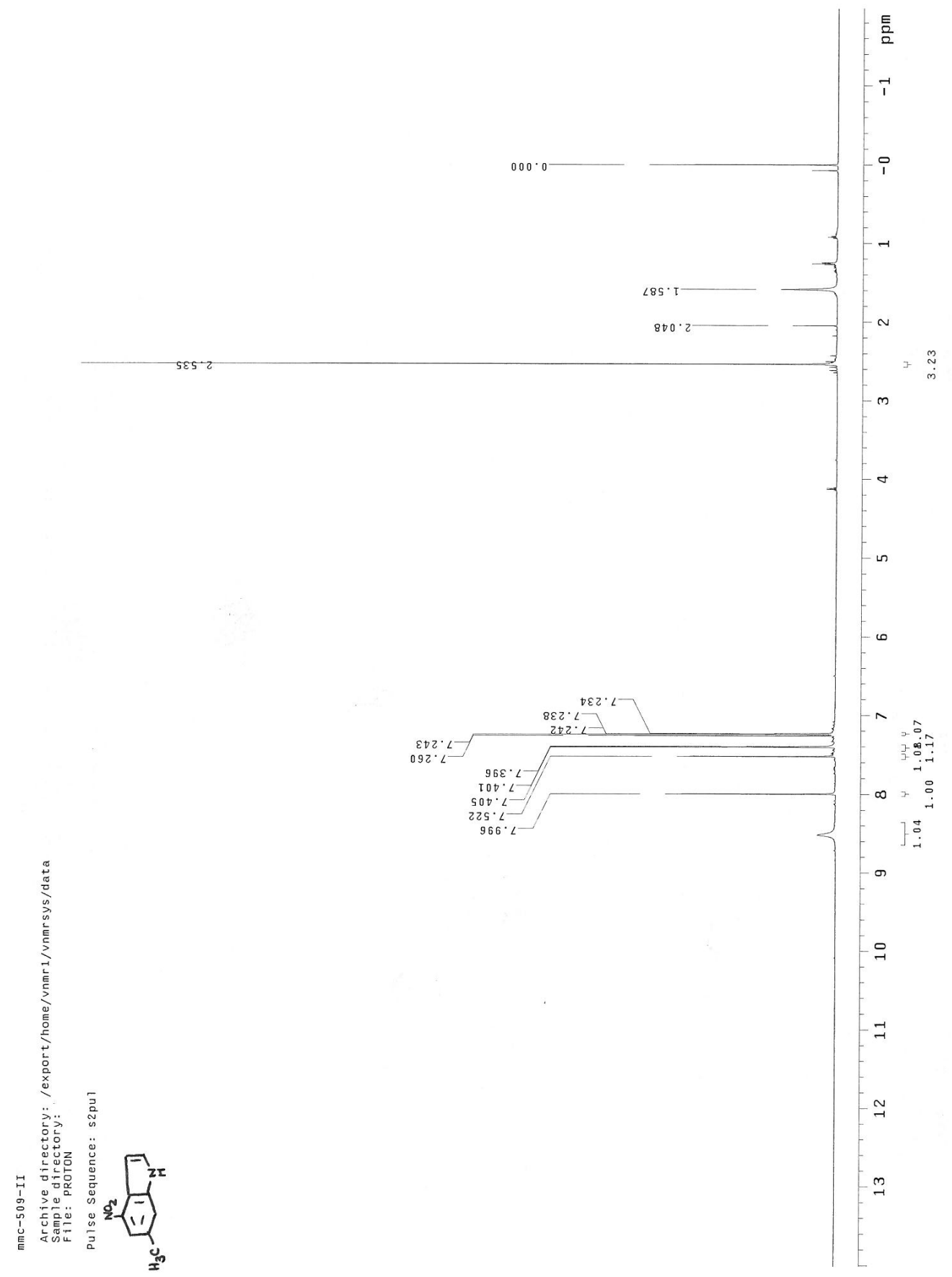

Figure 153: ${ }^{1} \mathrm{H}$ NMR of 6-methyl-4-nitroindole (356) 


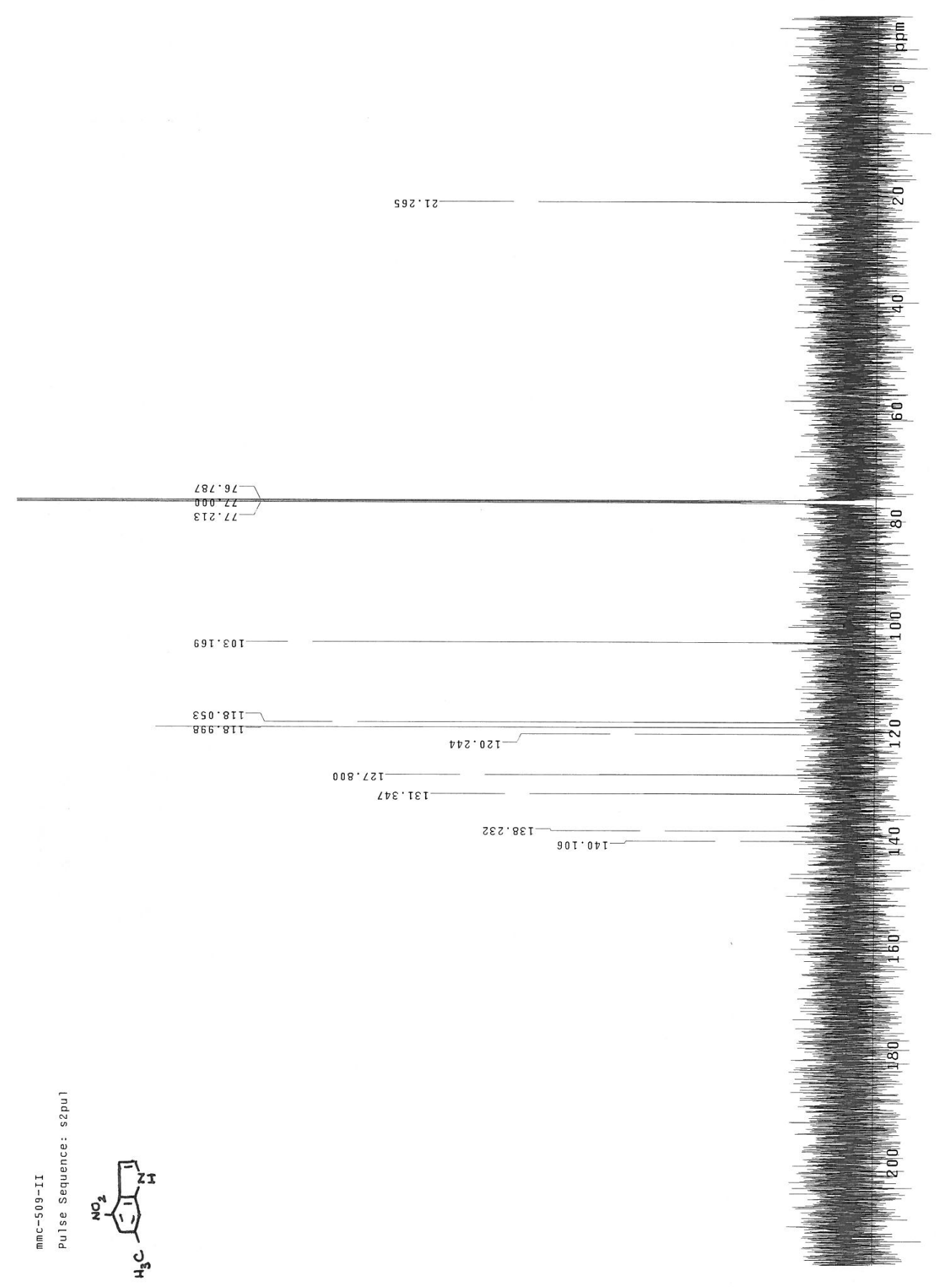

Figure 154: ${ }^{13} \mathrm{C}$ NMR of 6-methyl-4-nitroindole (356) 


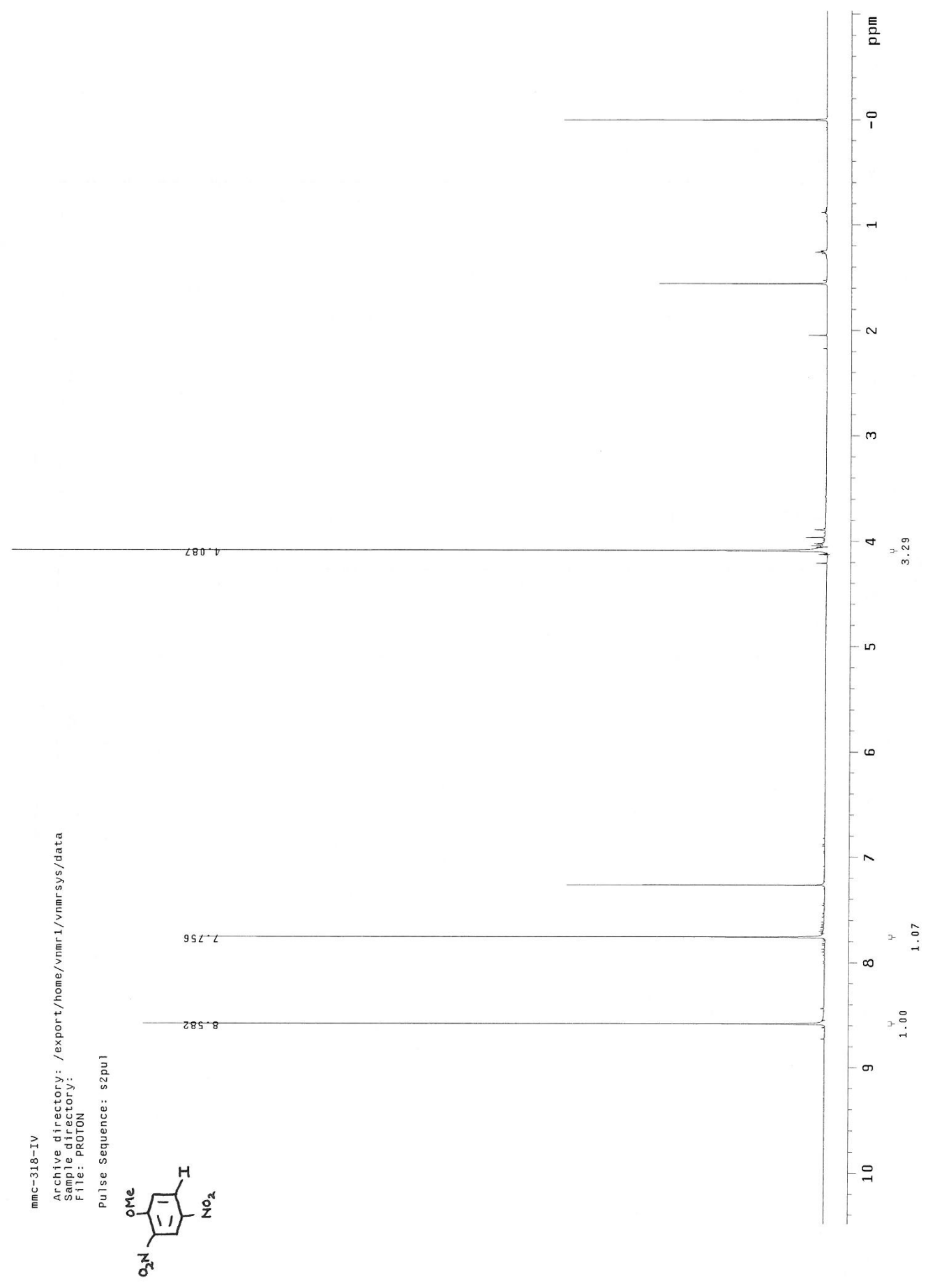

Figure 155: ${ }^{1} \mathrm{H}$ NMR of 5-iodo-2,4-dinitroanisole (363) 


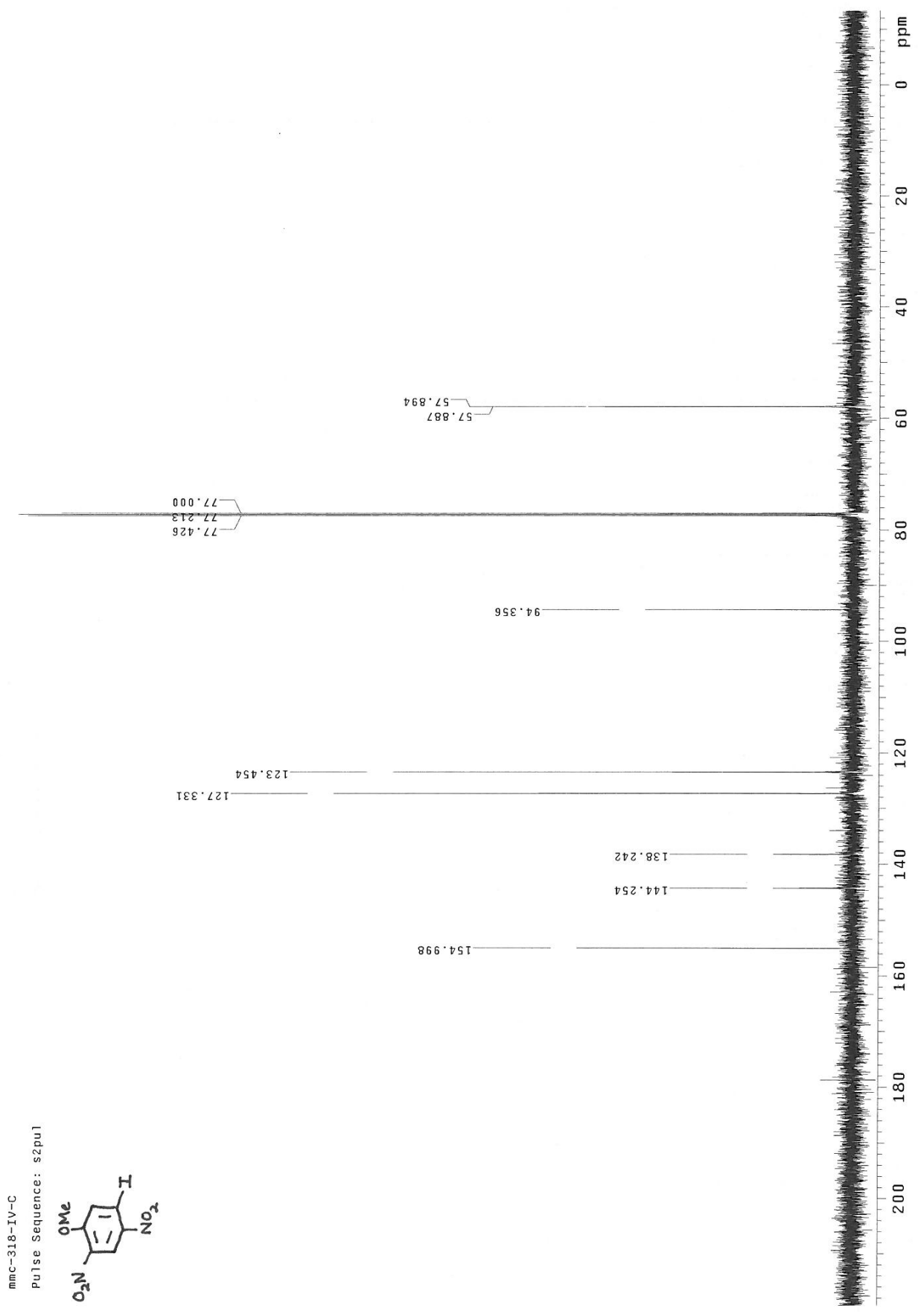

Figure 156: ${ }^{13} \mathrm{C}$ NMR of 5-iodo-2,4-dinitroanisole (363) 


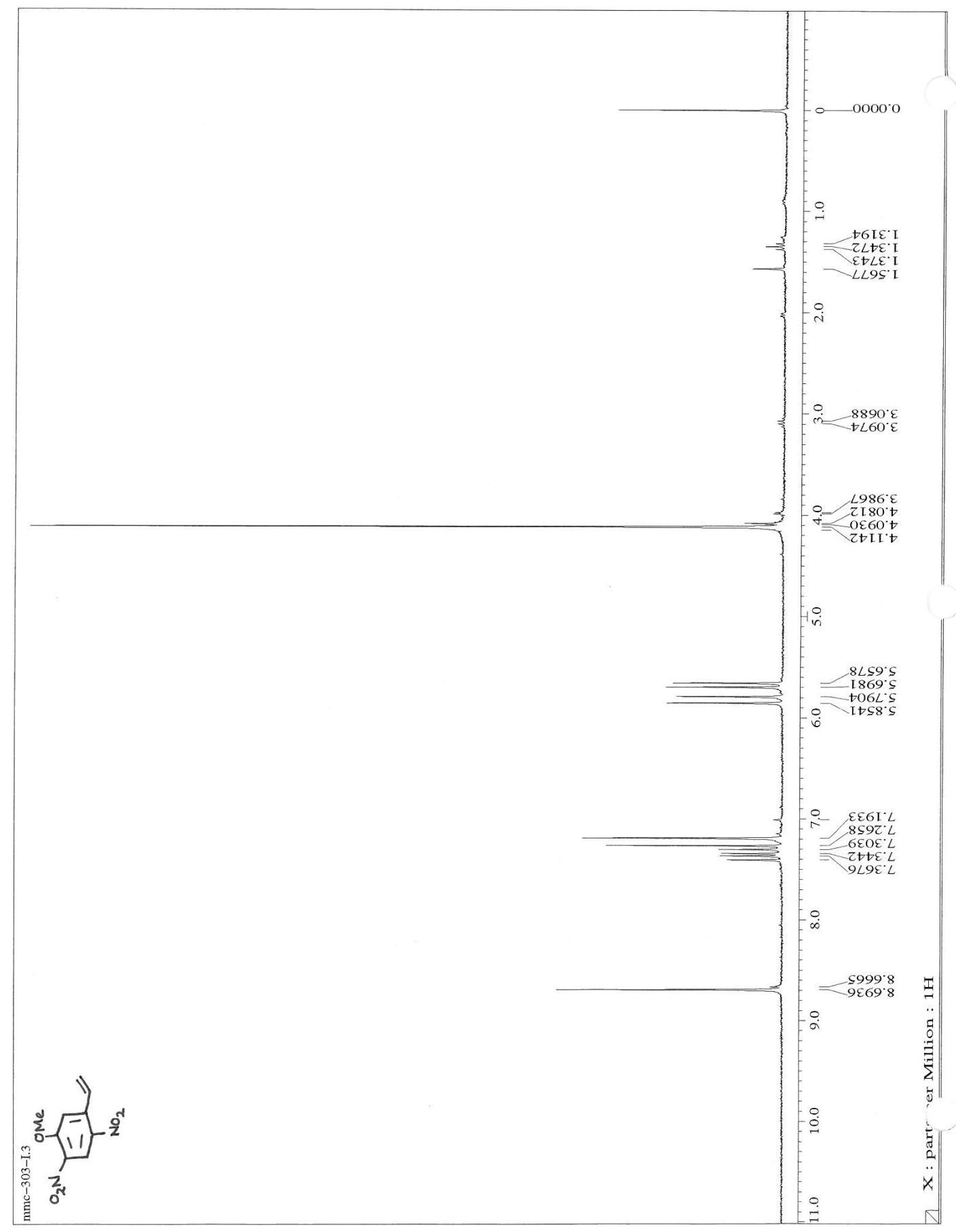

Figure 157: ${ }^{1} \mathrm{H}$ NMR of 5-ethenyl-2,4-dinitroanisole (365) 


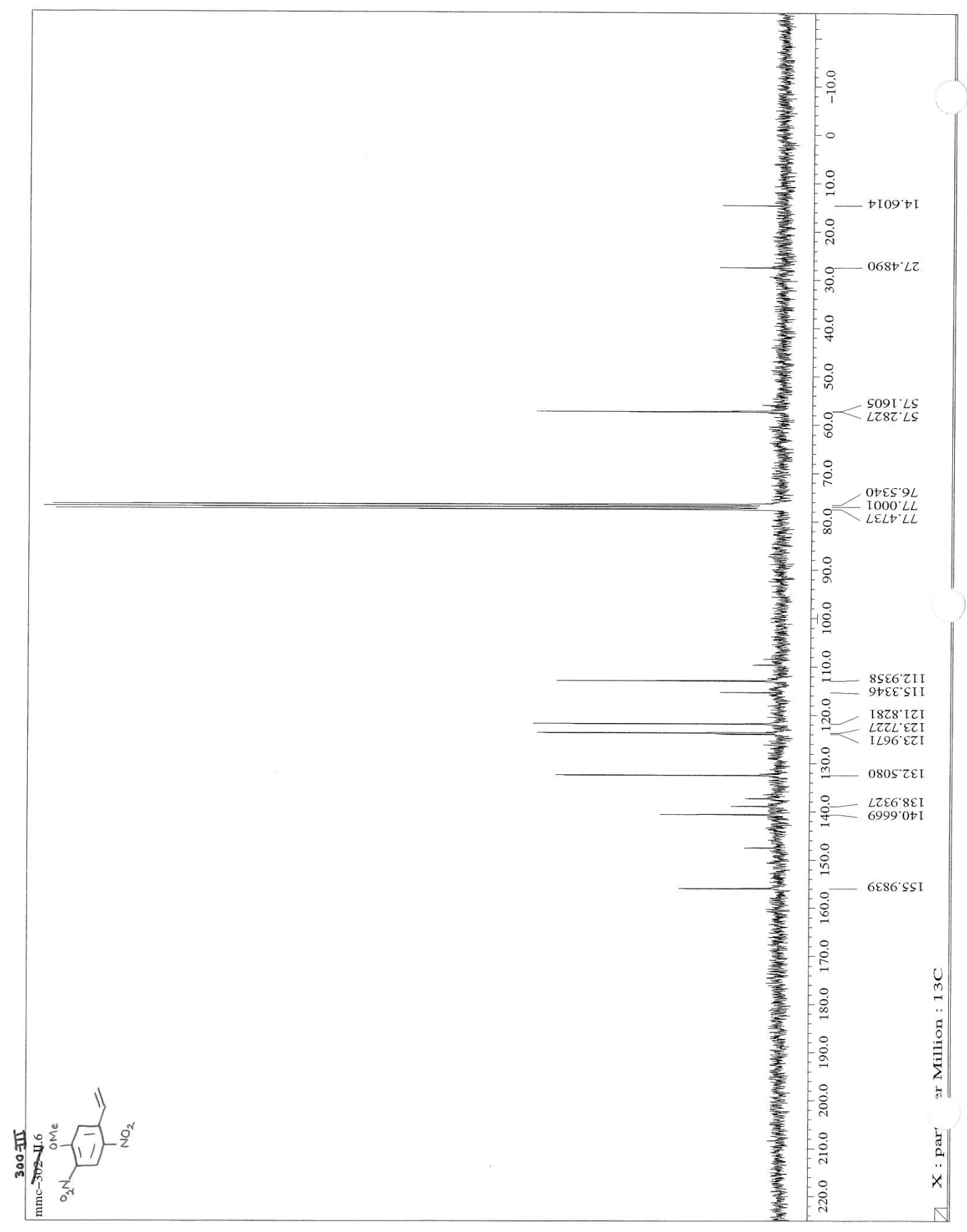

Figure 158: ${ }^{13} \mathrm{C}$ NMR of 5-ethenyl-2,4-dinitroanisole (365) 


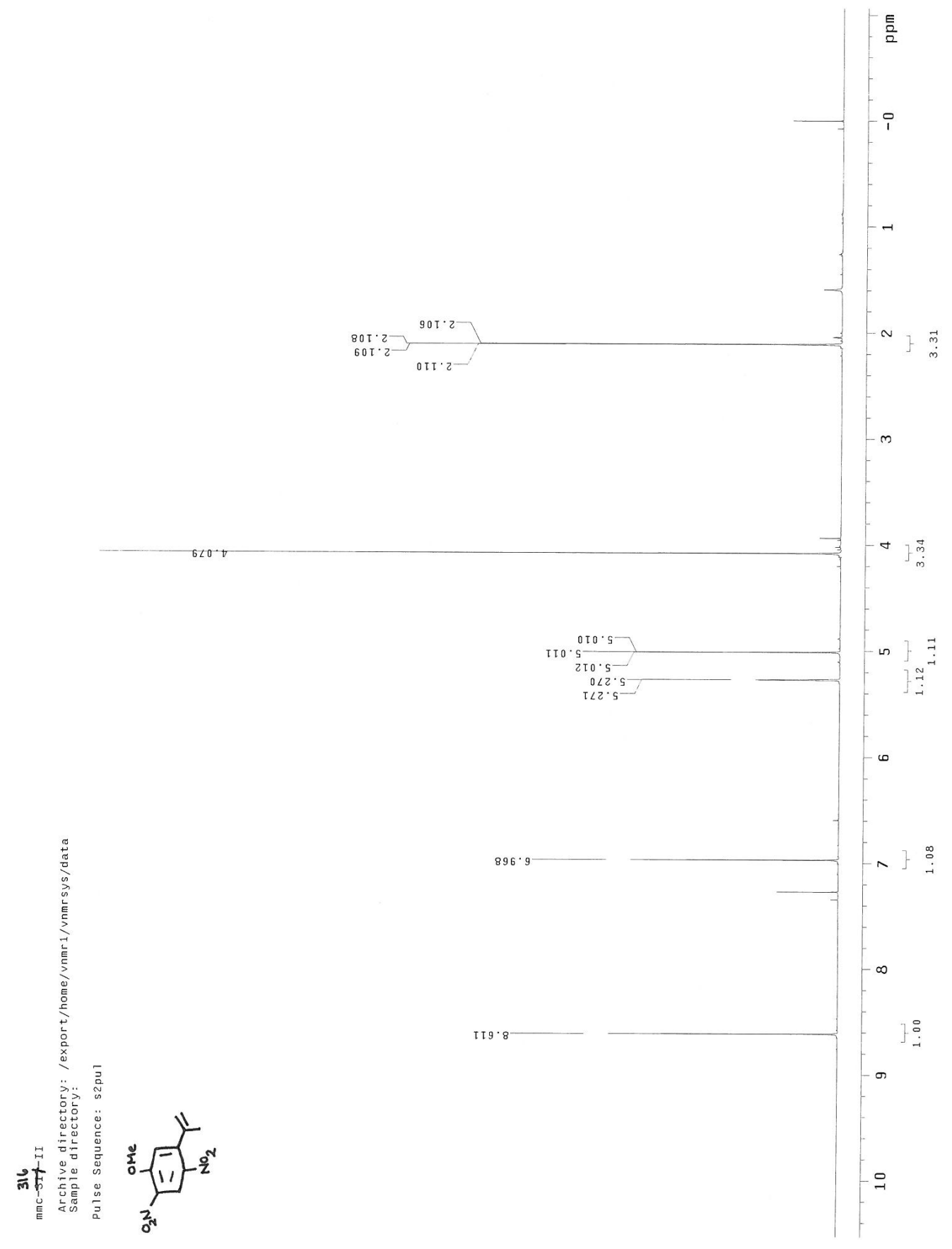

Figure 159: ${ }^{1} \mathrm{H}$ NMR of 2,4-dinitro-5-(prop-1-en-2-yl)anisole (369) 


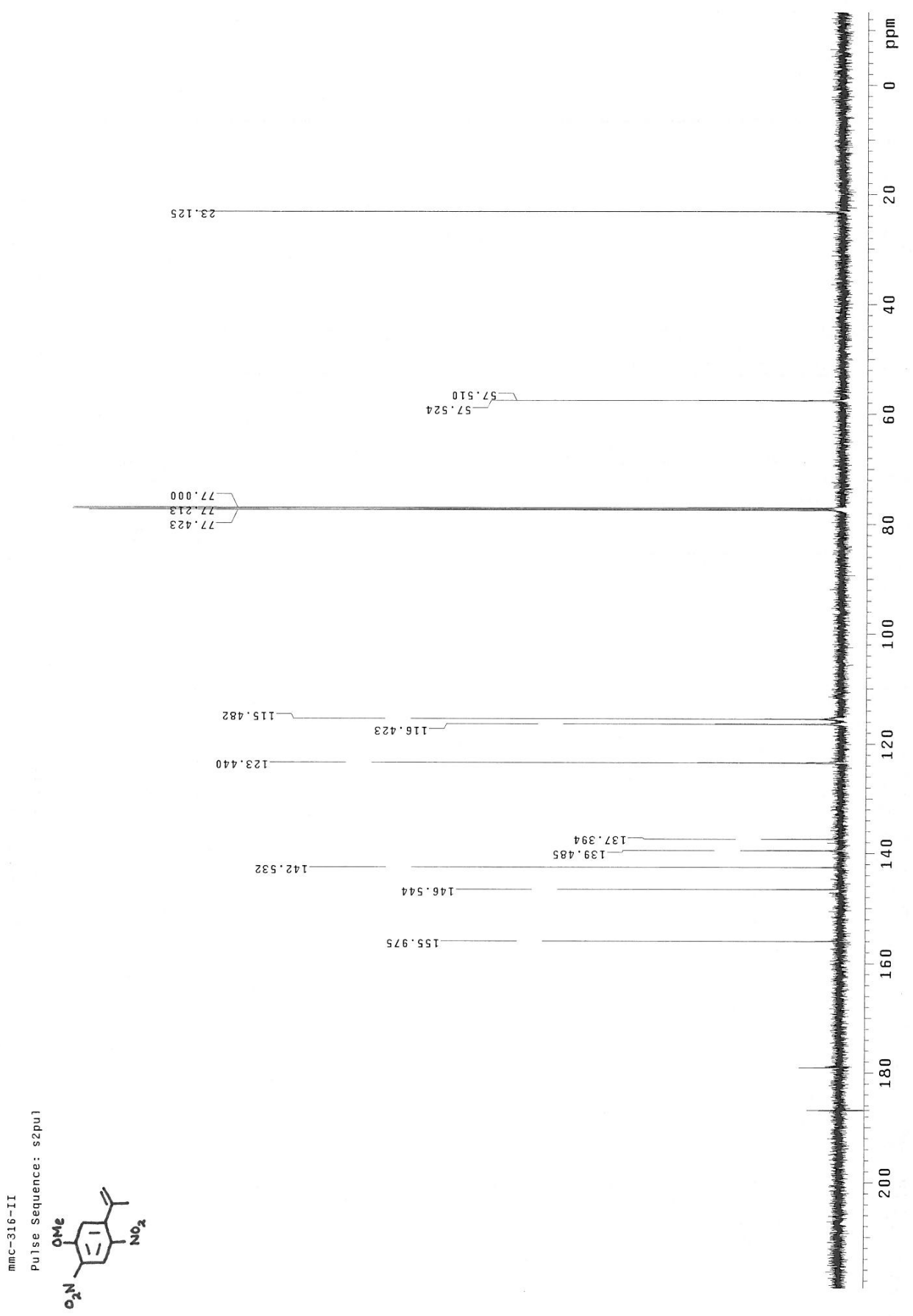

Figure 160: ${ }^{13} \mathrm{C}$ NMR of 2,4-dinitro-5-(prop-1-en-2-yl)anisole (369) 


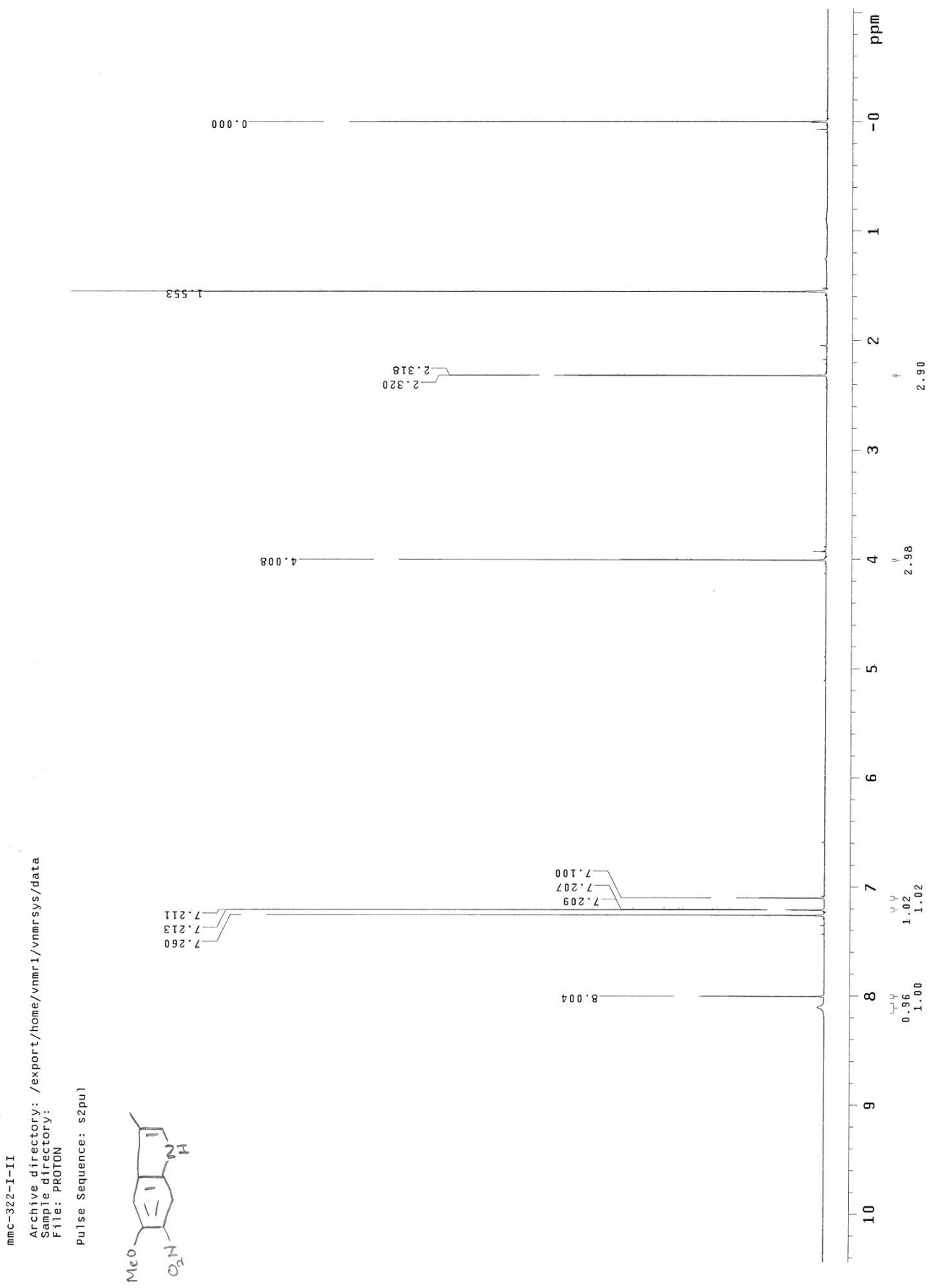

Figure 161: ${ }^{1} \mathrm{H}$ NMR of 3-methyl-5-methoxy-6-nitroindole (370) 


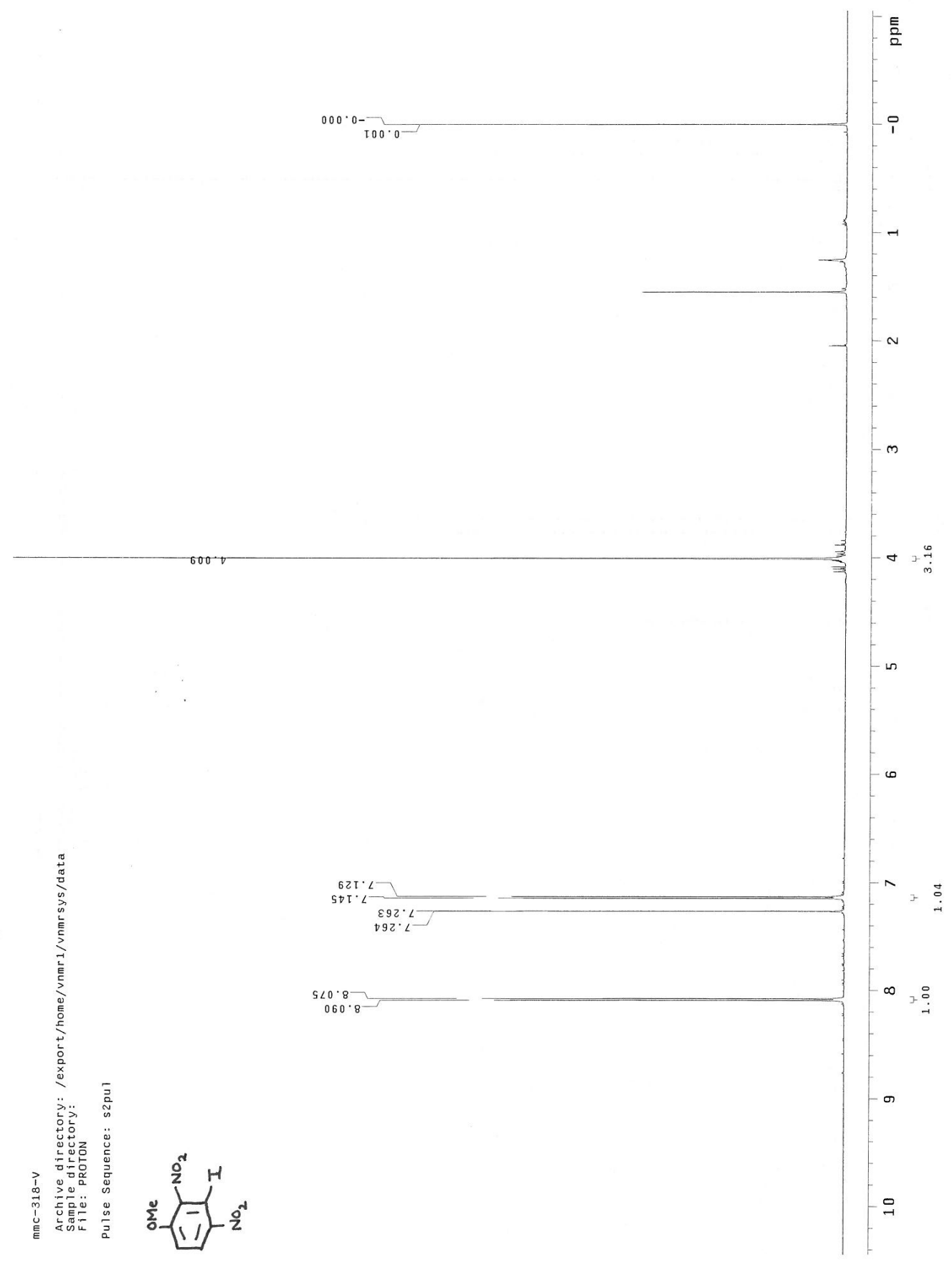

Figure 162: ${ }^{1} \mathrm{H}$ NMR of 3-iodo-2,4-dinitroanisole (364) 


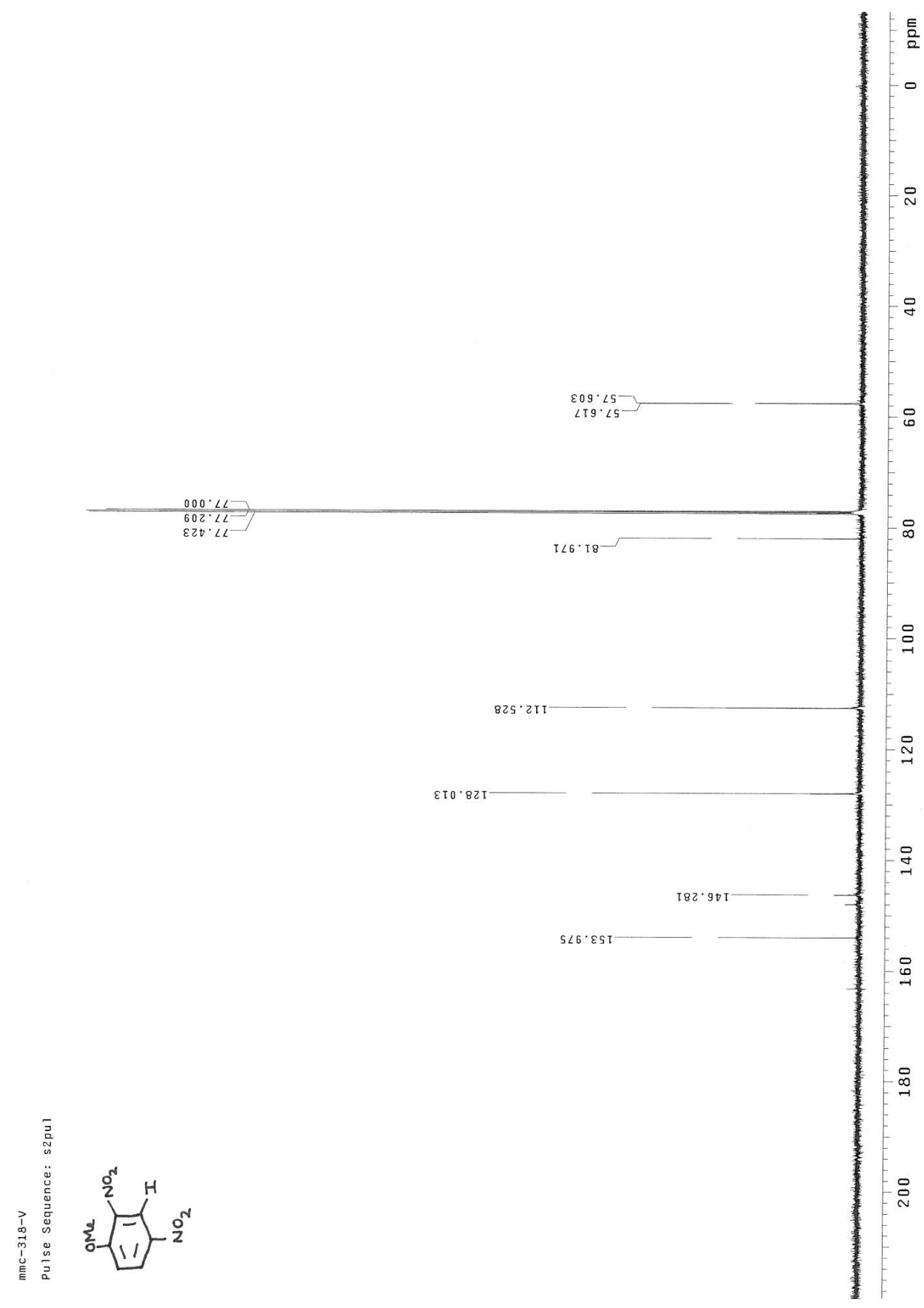

Figure 163: ${ }^{13} \mathrm{C}$ NMR of 3-iodo-2,4-dinitroanisole (364) 


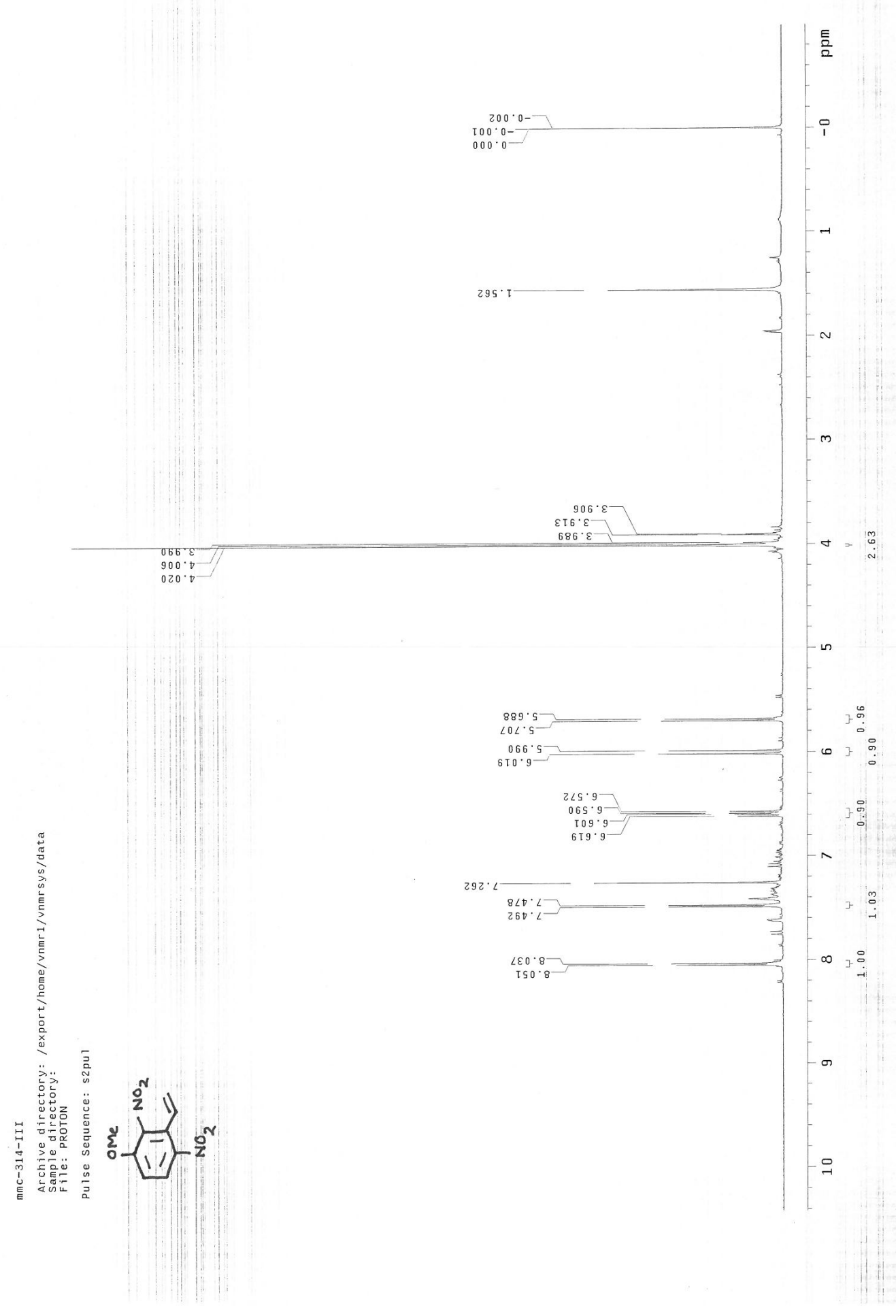

Figure 164: ${ }^{1} \mathrm{H}$ NMR of 3-ethenyl-2,4-dinitroanisole (367) 


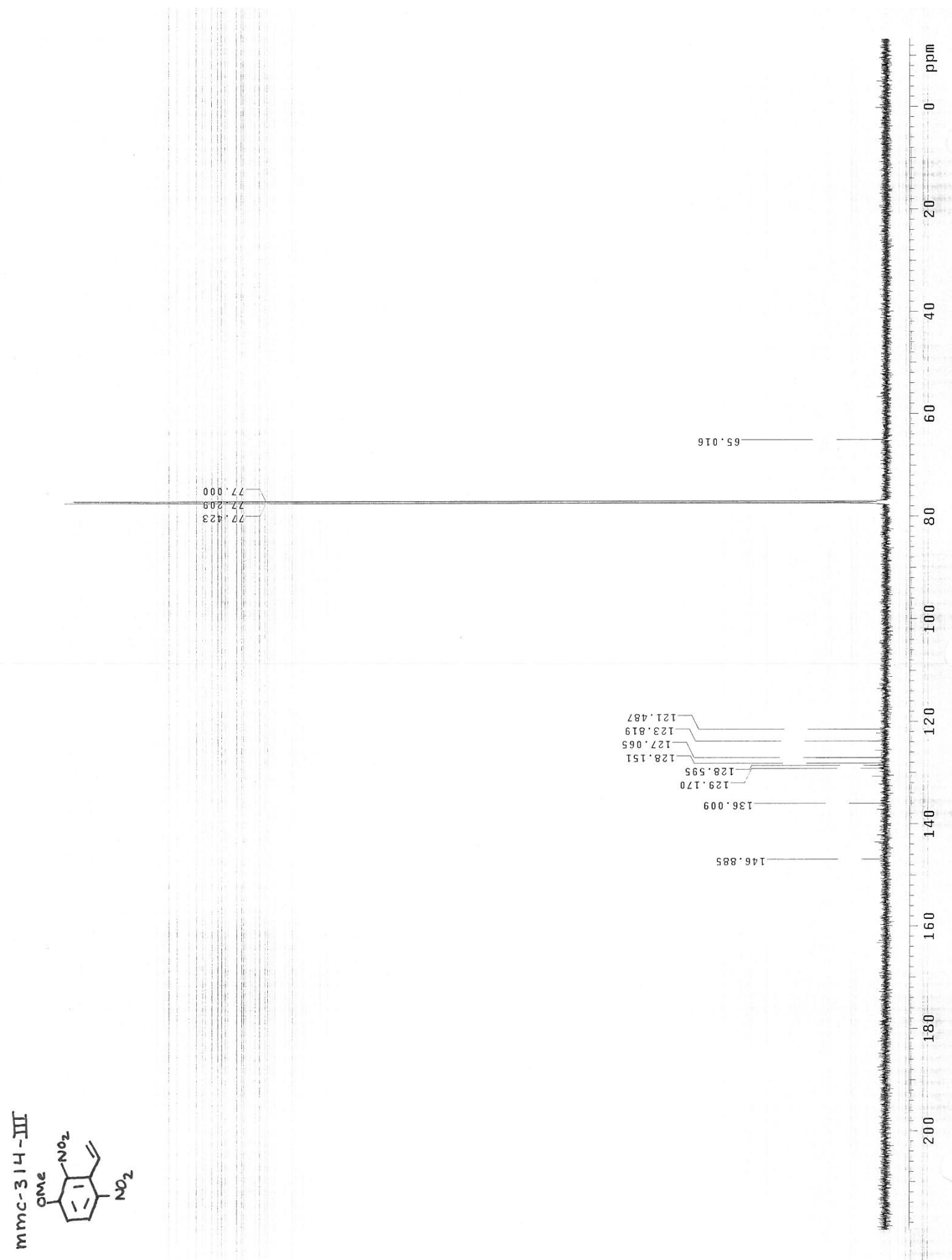

Figure $165:{ }^{13} \mathrm{C}$ NMR of 3-ethenyl-2,4-dinitroanisole (367) 


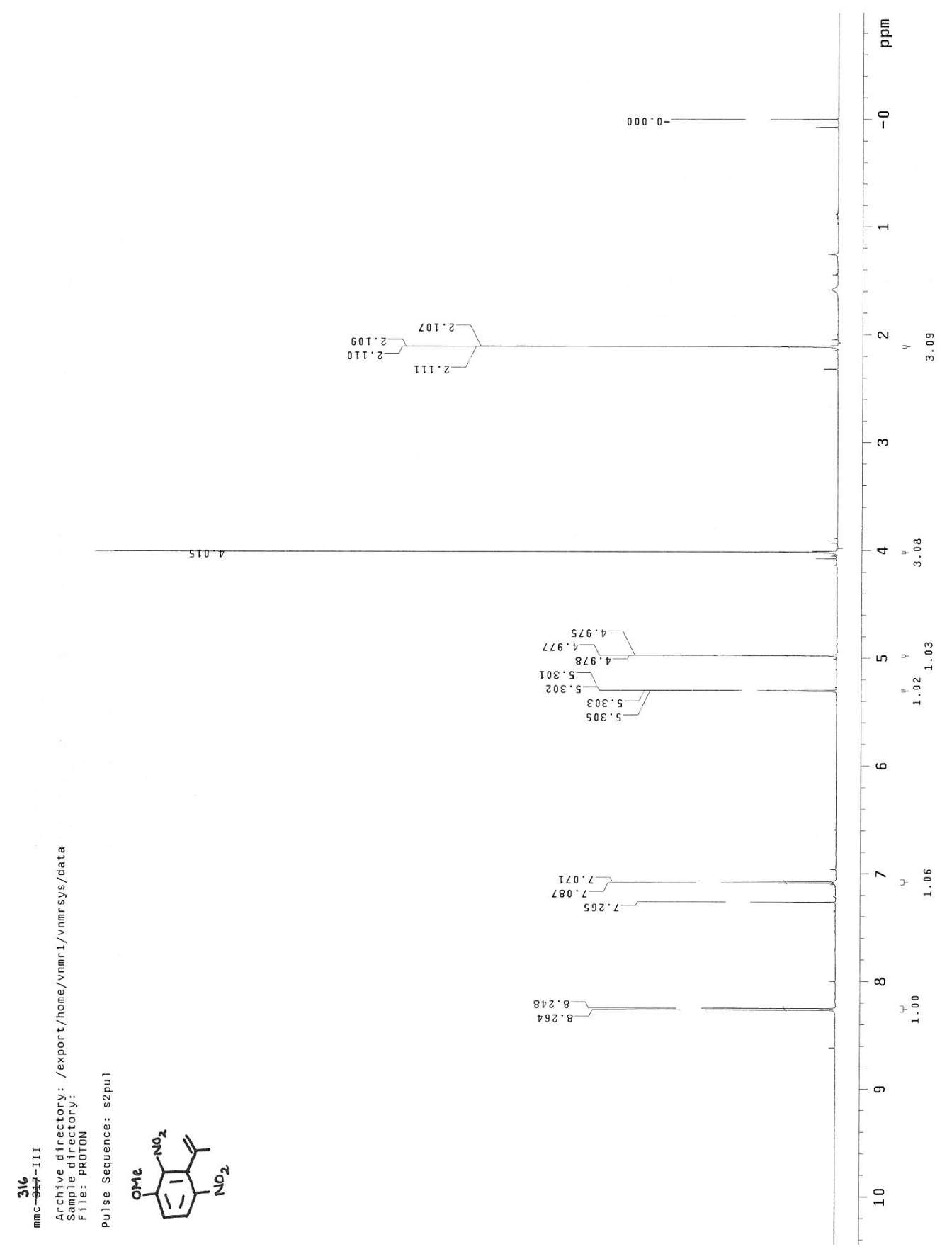

Figure 166: ${ }^{1} \mathrm{H}$ NMR of 3-(prop-1-en-2-yl)-2,4-dinitroanisole (371) 


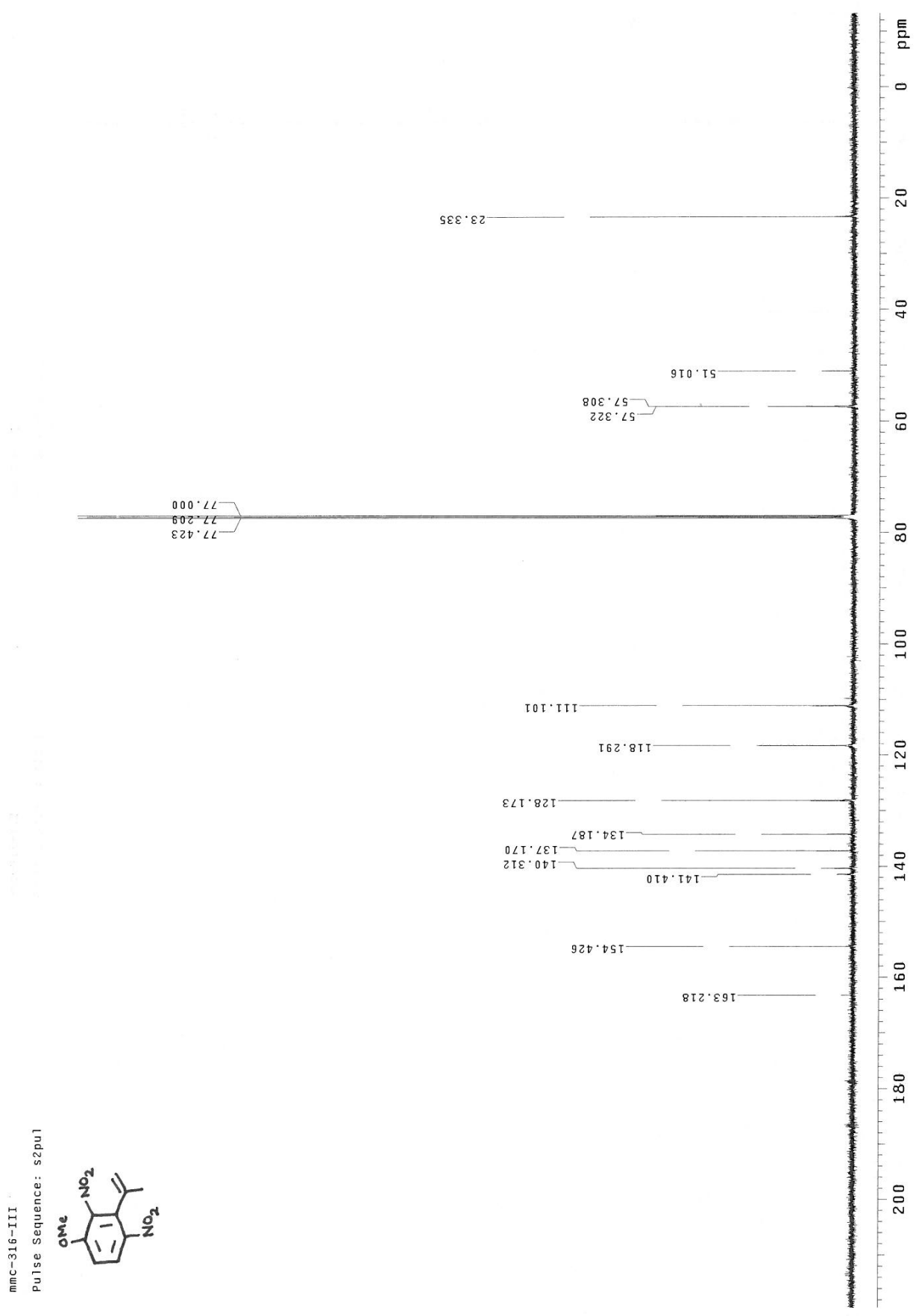

Figure 167: ${ }^{13} \mathrm{C}$ NMR of 3-(prop-1-en-2-yl)-2,4-dinitroanisole (371) 


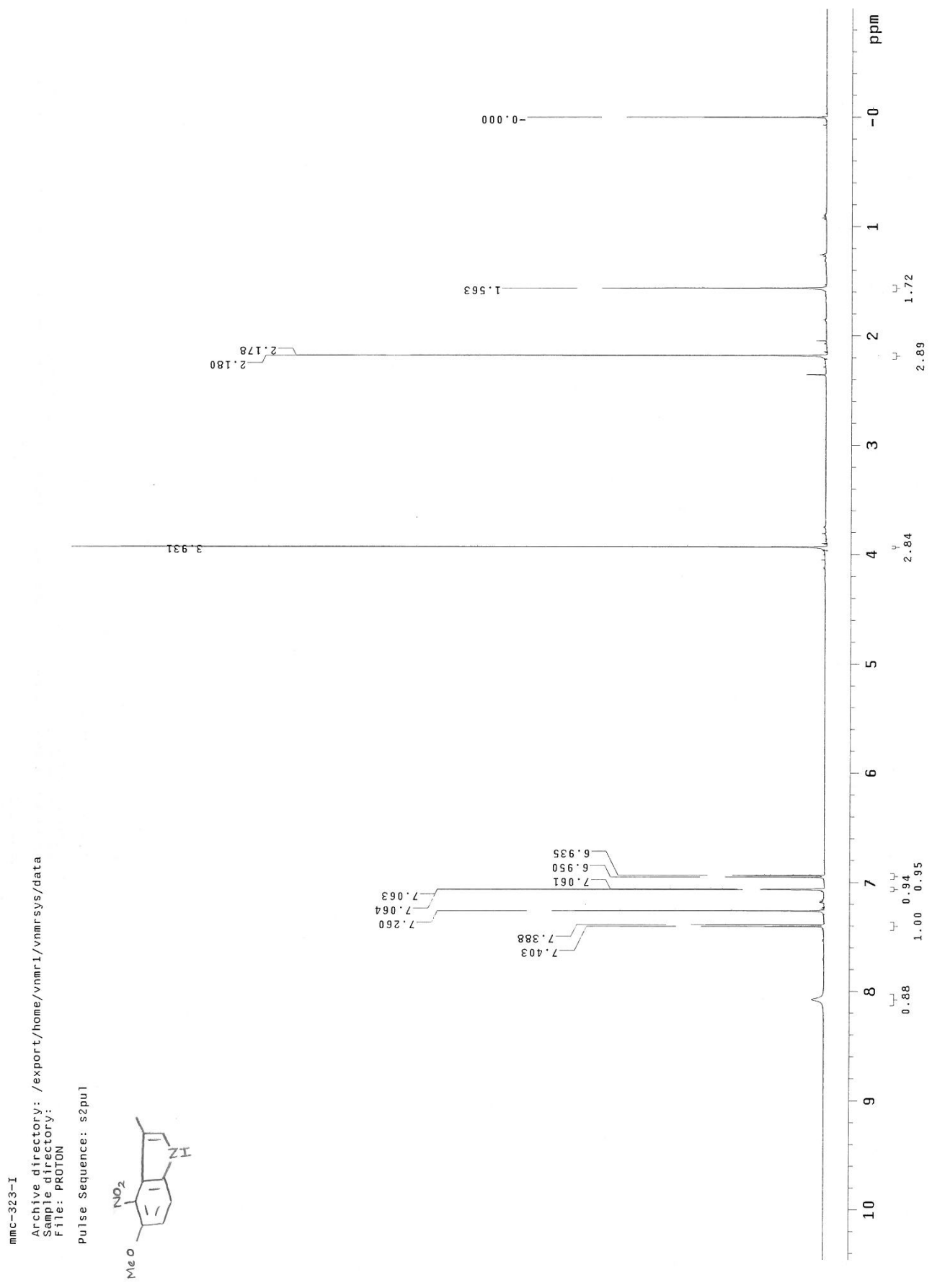

Figure 168: ${ }^{1} \mathrm{H}$ NMR of 3-methyl-5-methoxy-4-nitroindole (372) 


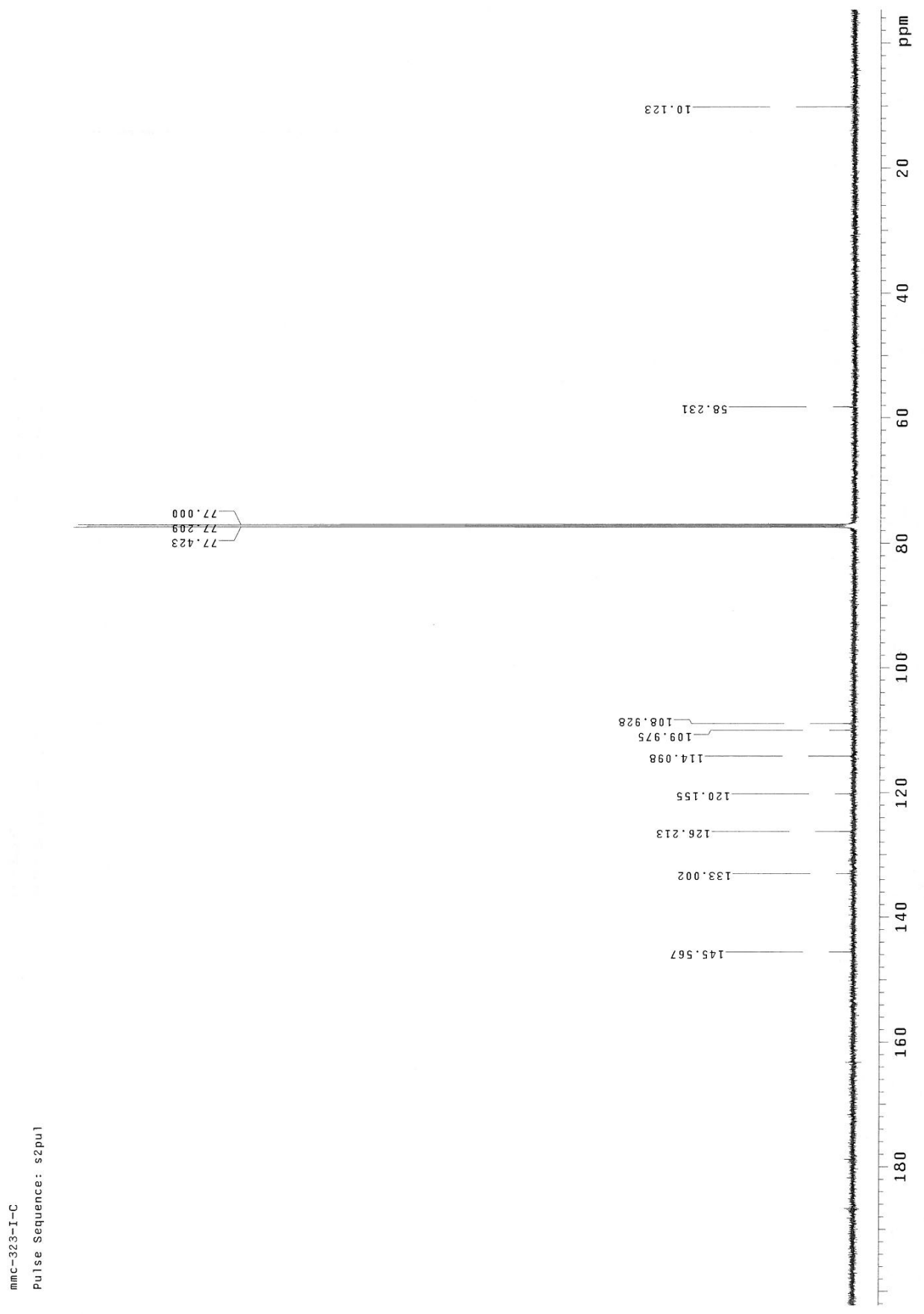

Figure 169: ${ }^{13} \mathrm{C}$ NMR of 3-methyl-5-methoxy-4-nitroindole (372) 
